\title{
DOE/ER/40256--TI PROGRESS IN RESEARCH
}

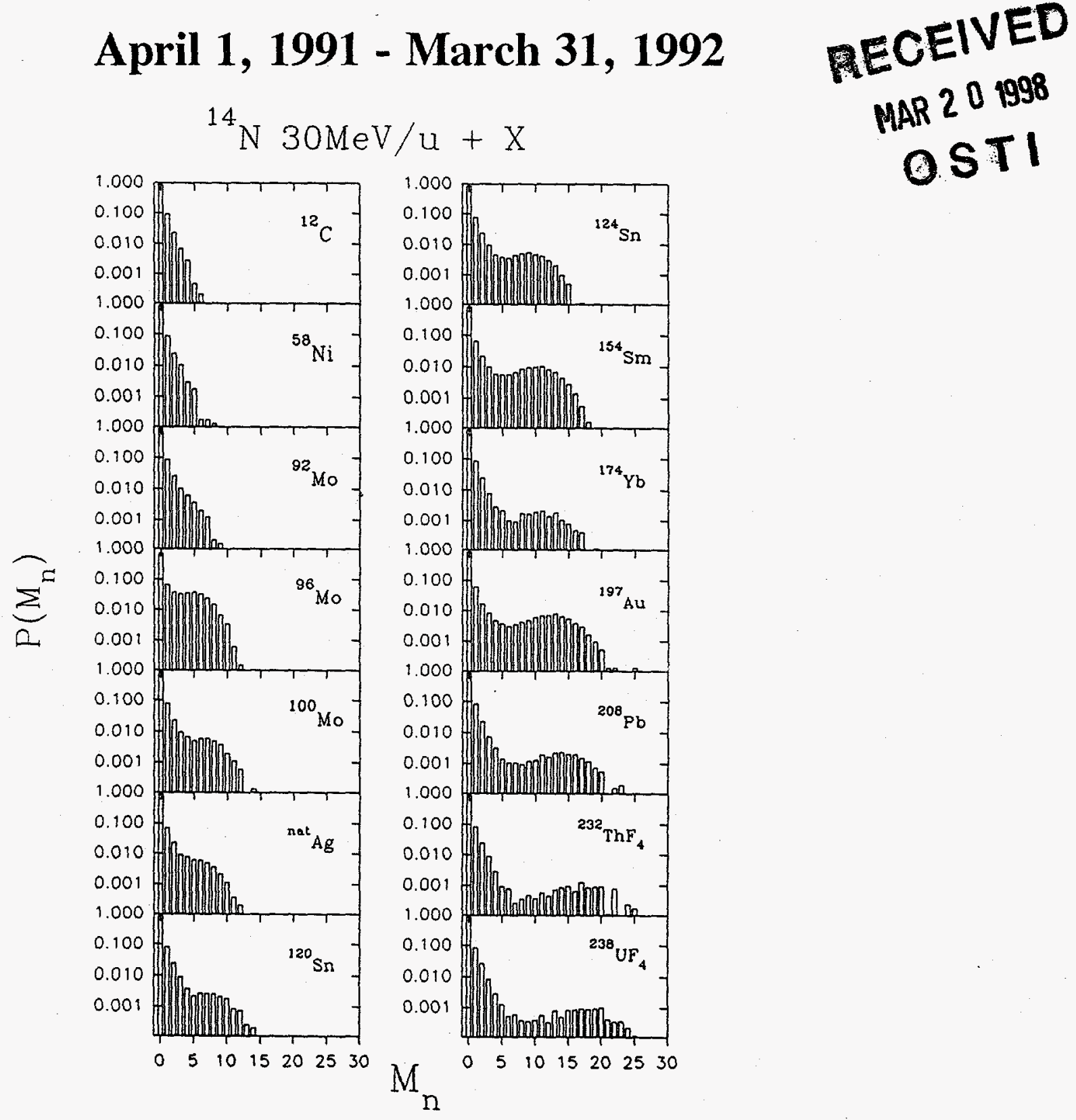

Neutron multiplicity distributions for $30 \mathrm{MeVu}$ ${ }^{14} \mathrm{~N}$ reactions with the indicated targets.

\section{CYCLOTRON INSTITUTE TEXAS A\&M UNIVERSITY COLLEGE STATION, TEXAS}




\section{$D O E / E R / 40256--T /$}

PROGRESS IN RESEARCH

APRIL 1, 1991 - MARCH 31, 1992

Prepared by

The Cyclotron Institute Staff

Texas A\&M University

College Station, TX 77843-3366

OISTRBSUTION OF THIS DOCUMENT IS UNLIMITED Ph

June, 1992 


\section{DISCLAIMER}

This report was prepared as an account of work sponsored by an agency of the United States Government. Neither the United States Government nor any agency thereof, nor any of their employees, makes any warranty, express or implied, or ascumes any legal liability or responsibility for the accuracy, completeness, or usefulness of any information, apparatus, product, or process disclosed, or represents that its use would not infringe privately owned rights. Reference herein to any spe. cific commercial produce, process, or service by trade name, trademarie, manufacturer, or otherwise does not necessarily constitute or imply its endorsemeat, recommendation, or favoring by the United States Government or any ageacy thereof. The views and opinions of authors expressed herein do not necensarily state or reflect those of the United States Government or any agency thereof. 


\section{DISCLAIMER}

Portions of this document may be illegible electronic image products. Images are produced from the best available original document. 


\section{TABLE OF CONTENTS}

\section{HEAVY ION REACTIONS}

THE MULTIFRAGMENTATION OF ${ }^{\circ} \mathrm{Ca}+{ }^{\circ} \mathrm{Ca}$

K. Hagel, M. Gonin, R. Wada, J. B. Natowitz,

B. H. Sa, Y. Lou, M. Gui, D. Utley, G. Nebbia, D. Fabris, G. Prete, J. Ruiz, D. Drain,

B. Chambon, B. Cheynis, D. Guinet, X. C. Hu,

A. Demeyer, C. Pastor, A. Giorni, A. Lleres,

P. Stassi, J. B. Viano, P. Gonthier

NEUTRON MULTIPLICITIES IN THE ${ }^{{ }^{\circ} \mathrm{Ar}}+{ }^{40} \mathrm{Ca}$ SYSTEM.

K. Hagel, R. Wada, J. B. Natowitz, Y. Lou, D.

Utley, $X$. Bin

\section{DYNAMICAL ASPECTS OF INTERMEDIATE MASS FRAGMENT EMISSION IN}

THE REACTION OF ${ }^{32} \mathrm{~S}+\mathrm{Ag}$ AT $30 \mathrm{AMeV}$

R. Wada, M. Gonin, M. Gui, K. Hagel, Y.

Lou, D. Utley, B. Xiao, D. Miller, J. B.

Natowitz, D. Fabris, G. Nebbia, R. Zanon, B.

Chambon, B. Cheynis, A. Demeyer, D. Drain,

D. Guinet, H. C. Hu, C. Pastor, K. Zaid, J.

Alarja, R. Bertholet, A. Giorni, A. Lleres, C.

Morand, P. Stassi, L. Schussler, B. Viano, P.

Gonthier

PRE-SCISSION TIMES AS A FUNCTION OF FRAGMENT MASS ASYMMETRY.

M. Gui, R. Wada, J. B. Natowitz, K. Hagel, Y.

Lou, D. Utley, B. Xiao, G. Enders, W. Kühn,

V. Metag, R. Novotny, O. Schwalb, A. Gobbi,

K. D. Hildenbrand, R. Freifelder, R. Charity, I.

Wessels, W. Henning, R. Mayer, R. S. Simon,

A. Olmi, G. Casini, A. A. Stefanini

FISSION PROCESSES IN THE REACTION ${ }^{63} \mathrm{Cu}+{ }^{100} \mathrm{MO}$ AT 10, 17 AND $25 \mathrm{MeV} / \mathrm{u}$

Y. Lou, L. Cooke, T. Botting, M. Gonin, M.

Gui, K. Hagel, B. Hurst, D. O'Kelly, G.

Mouchaty, J. Natowitz, R. P. Schmitt, W.

Turmel, D. Utley, R. Wada, B. Burch, M. E.

Brandan, D. Fabris, F. Gramegna, A.

Menchaca-Rocha, G. Nebbia, M. Poggi, G.

Prete, J. Ruiz, G. Viesti

HEAVY RESIDUE PRODUCTION IN THE SYSTEM At + Th AT 1600 MEV

D. Utley, X. Bin, M. Gui, K. Hagel, J. Li, Y.

Lou, D. Miller, J. Natowitz, and R. Wada 
B. Xiao, M. Gonin, K. Hagel, R. Wada, J. B. Natowitz, M. Gui, J. Li, Y. Lou, D. Miller, D. Utley, T. Botting, L. Cooke, B. Hurst, D. O'Kelly, G. Mouchaty, W. Turmel, R. P. Schmitt, B. Burch, M. E. Brandan, D. Fabris, F. Gramegna, A. Menchaca-Rocha, G. Nebbia, M. Poggi, G. Prete, J. Ruiz, G. Viesti

ENERGY DEPOSITION SYSTEMATICS IN $30 \mathrm{MeV} / \mathrm{u}^{14} \mathrm{~N}$ AND ${ }^{60} \mathrm{Cu}$ REACTIONS

T. Botting, B. Hurst, L. Cooke, D. O'Kelly, W.

Turmel, R. P. Schmitt

GDR $\gamma$-RAY EMISSION IN $120 \mathrm{MeV}{ }^{16} \mathrm{O}$-INDUCED FISSION OF ${ }^{208} \mathrm{~Pb}$

W. Turmel, T. Botting, L. Cooke, B. Hurst, D.

O'Kelly, R. P. Schmitt, H. Utsunomiya

NEUTRON BALL, AS AN EXIT CHANNEL FILTER

B. Hurst, D. O'Kelly, T. Botting, W. Turmel,

L. Cooke, R. P. Schmitt

INVESTIGATIONS OF ENTRANCE CHANNEL EFFECTS IN THE DECAY OF ${ }^{142} \mathrm{Nd}$ COMPOUND NUCLEUS PRODUCED IN ${ }^{160}+{ }^{120} \mathrm{Te}$ AND ${ }^{22 \mathrm{Ne}}+{ }^{120} \mathrm{Sn}$ REACTIONS

R. K. Choudhury, K. Wolf, R. P. Schmitt, K.

Hagel, R. Wada, R. Erkert, H. Jabs, N. Jevtic,

Y. Lou, D. Miller, J. Shoemaker, W. Turmel,

L. Whitesell, J. B. Natowitz

SEARCH FOR CORRELATED POSITRON-ELECTRON PEAKS IN Xe + AU COLLISIONS

T. E. Cowan, M. W. Clark, J. H. Hartley, J. L. McDonald, J. W. McDonald, R. R. Rohatgi, D.

H. G. Schneider, R. L. Watson, V. Horvat, W. H. Trzaska

\section{NUCLEAR STRUCTURE AND FUNDAMENTAL INTERACTIONS}

DEUTERON ELASTIC SCATTERING

A. C. Betker, C. A. Gagliardi, D. R. Semon,

R. E. Tribble, H. M. Xu, A. F. Zaruba

$M_{n} / M_{p}$ MEASUREMENTS USING $490 \mathrm{MeV}^{14} \mathrm{~N}$

Y.-W. Lui, D. H. Youngblood, W. Liu,

H. M. Xu

RESONANT BREAKUP REACTIONS INDUCED WITH $30 \mathrm{MeV} / \mathrm{u}^{16} \mathrm{O}$ AND ${ }^{20} \mathrm{Ne}$

D. O'Kelly, Y.-W. Lui, H. Utsunomiya, B.

Hurst, T. Botting, L. Cooke, W. Turmel,

R. P. Schmitt 
EXPERIMENTAL PROGRAM IN N-N AND KAON PHYSICS

L. C. Northcliffe, J. C. Hiebert, R. A.

Kenefick, G. Glass, T. Shima, and collaborators

from numerous other institutions

III. NUCLEAR THEORY

TEMPERATURE AND MASS DEPENDENCE OF LEVEL DENSITY PARAMETER

S. Shlomo, J. B. Natowitz

DESCRIPTION OF EVEN-EVEN MO BY PROTON-NEUTRON INTERACTING BOSON MODEL

H. Dejbakhsh, G. Ajupova, D. Latipov,

S. Shlomo

INVESTIGATION OF SHAPE COEXISTENCE OF THE ODD-A Tc ISOTOPES

BY PROTON-NEUTRON INTERACTING BOSON-FERMION MODEL

H. Dejbakhsh, G. Ajupova, D. Latipov,

S. Shlomo

DISAPPEARANCE OF FLOW IN INTERMEDIATE ENERGY NUCLEUS-NUCLEUS COLLISIONS .

Hong Ming Xu

DISAPPEARANCE OF ROTATION IN HEAVY-ION COLLISIONS

Hong Ming Xu

RESIDUE TEMPERATURES AND THE NUCLEAR EQUATION OF STATE

H. M. Xu, P. Danielewicz, W. G. Lynch

VIRTUAL PION CONTRIBUTION IN HEAVY ION REACTIONS

S. V. Akulinichev

PIONS CONTRIBUTION TO $\mathrm{K}^{+}-$NUCLEUS SCATTERING

S. V. Akulinichev

VACUUM ENERGY IN THE NUCLEAR MANY BODY PROBLEM

H. A. Bethe, G. E. Brown, C. M. Ko

PION MULTIPLICITY AS A PROBE OF THE DECONFINEMENT TRANSITION

IN HEAVY-ION COLLISIONS

M. I. Gorenstein, C. M. Ko, S. N. Yang

PHI MESON IN DENSE MATTER

C. M. Ko, P. Lévai, X. J. Qiu, C. T. Li

RHO MESON IN DENSE HADRONIC MATTER

M. Asakawa, C. M. Ko, P. Lévai, S. J. Qiu

QCD SUM RULES OF THE PHI MESON MASS IN HOT AND DENSE MATTER 
DILEPTON PRODUCTION FROM THE DELTA-DELTA INTERACTION IN HEAVY

ION COLLISIONS

X. S. Fang, C. M. Ko, P. J. Siemens,

L. H. Xia

THE $M_{T}$-SCALING IN THE DILEPTON SPECTRUM AS A SIGNATURE FOR THE QUARK-GLUON PLASMA

M. Asakawa, C. M. Ko, P. Lévai

TRANSPORT MODEL WTH KAONS.

X. S. Fang, C. M. Ko, G. E. Brown, V. Koch

COULOMB EFFECTS IN THE ASYMPTOTIC BEHAVIOR OF THE OPTICAL POTENTIAL

I. Scattering Below Breakup Threshold

E. O. Alt, D. M. Latypov, A. M. Mukhamedzhanov

ON THE COULOMB SINGULAR KERNEL OF LIPPMAN-SCHWINGER TYPE EQUATION

D. Latypov, A. M. Mukhamedzhanov

\section{ATOMIC MOLECULAR \& MATERIALS SCIENCE}

KINETIC ENERGY RELEASED IN THE DISSOCIATION OF MULTICHARGED

DIATOMIC MOLECULES PRODUCED IN COLLISIONS WTH $97 \mathrm{MeV} \mathrm{Ar}^{14+}$

G. Sampoll, R. L. Watson, O. Heber,

V. Horvat, K. Wohrer, M. Chabot

DISSOCIATION OF HIGHLY CHARGED CO MOLECULAR IONS PRODUCED IN ELECTRON CAPTURE COLLISIONS OF $97 \mathrm{MeV} \mathrm{Ar}^{14+}$

G. Sampoll, R. L. Watson, V. Horvat,

M. Barrett, B. Griffith

DISSOCIATION FRACTIONS AND BRANCHING RATIOS FOR MULTICHARGED

CO MOLECULAR IONS PRODUCED IN COLLISIONS WTH $97 \mathrm{MeV} \mathrm{Ar}^{14+}$

K. Wohrer, G. Sampoll, R. L. Watson,

M. Chabot, O. Heber, V. Horvat

ORIENTATION DEPENDENCE OF FAST COLLISION INDUCED

MOLECULAR DISSOCIATION

M. Chabot, K. Wohrer, G. Sampoll, V. Horvat,

R. L. Watson 
V. Horvat, G. Sampoll, K. Wohrer, M. Chabot,

R. L. Watson

AUGER ELECTRON SPECTRUM OF $8 \mathrm{MeV} / \mathrm{amu} \mathrm{Ar}^{7+}$

V. Horvat, R. Paramaswaran, G. Sampoll,

R. L. Watson, M. Barrett, B. Griffith,

R. D. DuBois, L. H. Toburen, D. Schneider

LIGHT EMISSION STUDIES WITH SLOW, HIGHLY-CHARGED HEAVY IONS

C. Assad, R. E. Tribble

METASTABLE STATES OF HIGHLY-CHARGED ATOMIC IONS

D. A. Church, LiSheng Yang

\section{SUPERCONDUCTING CYCLOTRON AND INSTRUMENTATION}

OPERATIONS

D. May

K500 BEAM ENERGY RESOLUTION

Y.-W. Lui, D. H. Youngblood, W. Liu,

H. M. Xu, D. P. May

INJECTION LINE BUNCHER

F. P. Abegglen, R. C. Rogers

TEST OF A BIASED PLATE IN THE E.C.R. ION SOURCE.

G. Mouchaty, R. C. Rogers, D. P. May

COMPUTING FACILITIES AT THE CYCLOTRON INSTITUTE

K. Hagel, H. Dejbakhsh

PROTON SPECTROMETER FIELD MAPPING

A. Zaruba, H. Xu, A. Betker, T. Cowden,

G. Derrig, C. Gagliardi

STATUS OF THE PROTON SPECTROMETER

A. C. Betker, C. A. Gagliardi, X. M. Xu,

A. F. Zaruba, P. Smelser, G. Derrig

BARIUM FLUORIDE ARRAY

R. K. Choudhury, J. Shoemaker, H. Jabs, K. L. Wolf

THE MARS PROJECT

R. E. Tribble. J. D. Bronson, W. Liu, X.-G. Zhou

D. H. Youngblood, J. D. Bronson, Y.-W. Lui 
J. L. Blankenship, R. L. Auble, J. R. Beene,

F. E. Bertrand, D. Shapira

PC-BASED ELECTRON SPECTROMETER CONTROL SYSTEM AND PROGRAM ESCAM

V. Horvat

SAMPLING CALORIMETER RESEARCH AND DEVELOPMENT

K. L. Wolf, A. D. Chacon, R. K. Choudhury,

J. E. Simon-Gillo, R. Erkert, M. LaFrance,

B. Sadhwani, S. Johnson, S. Watson, S. Potter,

L. Whitesell, N. Jevtic

MEGA REPORT

C. A. Gagliardi, G. Kim, F. Liu, R. E. Tribble,

X.-L. Tu, L. Van Ausdeln, X.-G. Zhou

A $4 \pi$ CsI DETECTOR OF HEAVY ION REACTION PRODUCTS

B. Xiao, G. Derrig, K. Hagel, R. Wada, J. B.

Natowitz, J. Li, Y. Lou, D. Miller, D. Utley

SIMULATION OF A BEAM PROFILE MONITOR

J. Li, R. Wada, K. Hagel, J. B. Natowitz,

X. Bin, Y. Lou, D. Miller, D. Utley

VI. PUBLICATIONS

ABSTRACTS OF PAPERS PUBLISHED

April, 1991 - March, 1992

ABSTRACTS OF PAPERS SUBMITTED

April, 1991 - March, 1992

VII. APPENDIX

TALKS PRESENTED

CONFERENCE PROCEEDINGS

COLLOQUIA PRESENTED

RESEARCH PERSONNEL AND ENGINEERING STAFF

INSTITUTE COLLOQUIUM SERIES 


\section{INTRODUCTION}

\section{April 1991 - March 1992}

Reports on research activities, facility operation, and facility development of the Texas A\&M Cyclotron Institute for the period 1 April 1991 - 31 March 1992 are presented in this document.

During the report period, the ECR-K500 Cyclotron Combination operated 4377 hours. Of this time, 832 hours was used for beam development, 942 hours was used for tuning and optics, and the beam was available for experiments 2603 hours. This time was used in a variety of studies including elastic and inelastic scattering, projectile break-up, the production and decay of giant resonances, fusion and fission dynamics, intermediate mass fragment emission, $e^{+} e^{-}$production and molecular dissociation. In addition, studies of surfaces and metastable states in highly charged ions were carried out using the ECR source.

Completion of two 19-element $\mathrm{BaF}_{2}$ arrays, of the focal plane detector for the proton spectrometer and installation of the HiLi multidetector have provided significant new experimental capabilities which have been further enhanced by major additions to our computer network. Progress on the Mass Achromat Recoil Spectrometer (MARS) is such that first operation of that device should occur this summer. Funding for installation of the MDM spectrometer was obtained at the beginning of this year. As this report is being completed, the Enge Split Pole Spectrometer is being disassembled and removed to make room for the MDM spectrometer. The split-pole will be shipped to CEBAF for use in experiments there. Installation of the MDM should be completed within the next year. Also expected in the next year is a 92 element plastic-CsI ball. This product of a collaboration between Vanderbilt, ORNL, and TAMU will be used with the HiLi detector and neutron ball for a variety of reaction studies.
The Institute programs are an integral part of the educational program of the College of Science at Texas A\&M University. During the report period, 30 graduate students and 27 undergraduate students have carried out research projects with the Institute research staff and visiting scientists listed in the last section of this Report. There has also been a significant involvement of scientists from ORNL, LLL, Legnaro Laboratory (Italy), and the National University of Mexico in Institute research activities.

In addition to the $\mathrm{K500}$ Cyclotron based research programs, Institute scientists were involved in several major outside collaborations, i.e., MEGA (LAMPF), STAR (RHIC), and Few Nucleon Studies (LAMPF).

The bulk of the research described in this report is funded by the Department of Energy under grants DEFG05-86ER40256 (nuclear) and DE-FG05-84ER13262 (atomic); by the National Science Foundation under grants PHY-8907986 and PHY-9001886 (theory); and by The Rober A. Welch Foundation under grants A-330, A-355, A-558, A-692, A-972, A-1082, A-1110, and A-1159. Operation of the facility is also supported by the University. The Texas A\&M MEGA collaboration is supported under grant DE-FG05-87ER40310. Research in the STAR collaboration is supported under grant DE-AS05-85ER40207. The LAMPF few nucleon studies are funded under grant DE-FG05-88ER40395. MARS, the MDM spectrometer, the $\mathrm{BaF}_{2}$ crystal array, and the computer system are also funded by DOE under grant DE-FG05-86ER40256.

Some of the data and conclusions presented in this report are based upon preliminary analyses of the experiments. Until this research is published, it should not be cited without express consent of the investigators involved. 


\section{HEAVY ION REACTIONS}




\author{
K. Hagel, M. Gonin, ${ }^{\dagger}$ R. Wada, J. B. Natowitz, B. H. Sa, $¥$ Y. Lou, M. Gui, D. Utley, \\ D. Fabris, (1) G. Nebbia, (1) G. Prete, (1) J. Ruiz, (1)
}

B. Chambon, (2) B. Cheynis, (2) A. Demeyer, (2) D. Drain, (2) D. Guinet, (2) X. C. Hu, (2) C. Pastor, (2)

A. Giorni, (3) A. Lleres, (3) P. Stassi, (3) J. B. Viano, (3) P. Gonthier(4)

The analysis of the ${ }^{10} \mathrm{Ca}+{ }^{40} \mathrm{Ca}$ system on which we reported in a previous annual report' has been extended. Comparisons of the various models indicate that the multifragment events which we observe result from the decay of hot nuclei which have undergone significant expansion.

We have used several dynamical models to estimate the starting conditions of of $A$ and $E^{*}$ of the system that we calculated. Landau-Vlasov $\mathrm{v}^{2,3}$ and BUU $\mathrm{BU}^{4,5}$ calculations indicate that at $35 \mathrm{MeV} / \mathrm{u}$ the collision of ${ }^{40} \mathrm{Ca}+{ }^{40} \mathrm{Ca}$ results in an initial compression followed by expansion. This takes about $70 \mathrm{fm} / \mathrm{c}$. During this period significant pre-equilibrium emission occurs. Based on the calculations of reference 2 , on BUU calculations using the codes of references 4 and 5 and on pre-equilibrium emission estimates using the Boltzman Master Equation ${ }^{6}$ we have chosen $A=70, Z=34$, and $E^{*}=420 \mathrm{MeV}$ as the starting point of our calculations.

All model calculations have been filtered through the same detection and analysis criteria as the experimental data. We also corrected for pre-equilibrium emission by adding two protons to each event.

We present in Fig. 1 the experimental charge distribution as solid points and the charge distributions predicted by several statistical models as histograms. The solid line represents the prediction of the simultaneous multifragmentation calculation of $\mathrm{Sa}$ and $\mathrm{Gross}^{7}$ which assumes an expanded starting nucleus with $\rho=\frac{1}{6} \mathrm{q}_{0}$. The dotted line is the prediction of the sequential binary decay model of Richert and Wagner, ${ }^{8}$ and the dashed line is the prediction of the statistical code, GEMINI, ${ }^{9}$ which also treats the fragment emission as sequential. The $J_{c r i}$ for the latter has been chosen to be $60 \mathrm{~h}$ based on the estimate of the BUU calculation.5 For the experimental events, we note a steep falloff in yield between $Z=1$ and 4 , and then a more gradual decrease with increasing $Z$. Of the models considered in Fig. 1, the one which comes closest to describing the trend of this observed Z-distribution is that of Sa and Gross. GEMINI produces the typical Zdistribution expected from a normal statistical model, that is, a relatively large production of $Z=1$ and 2 , a fairly low probability of fragments having $3 \leq Z \leq 15$, and a peak corresponding to heavy residues at higher $Z$. The sequential calculation of Richert and Wagner shows more IMF emission and a significant residue contribution at higher $\mathbf{Z}$.

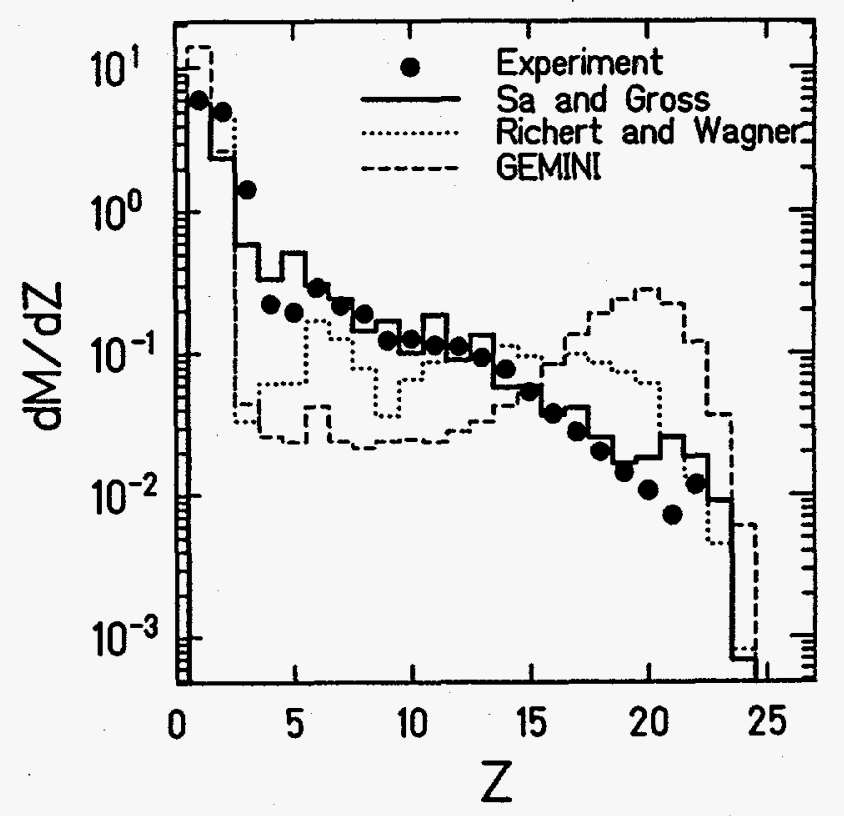

Fig. 1. Elemental charge distributions for the experiment and the model calculations. The points represent the data, the solid line represents the multifragmentation calculation of $\mathrm{Sa}$ and Gross, the dotted line represents the binary decay calculation of Richert and Wagner, and the dashed line represents the GEMINI calculation.

In Fig. 2 we show correlations between the fragment size and multiplicity. The figure shows the probability of detecting at least $n$ fragments which have $Z$ greater than or equal to a specified value which we call $Z_{\text {threshold. }}$ The symbols represent the probabilities for $n=1,2,3,4$ etc. For the data shown in Fig. 2(a), we note that up to 9 fragments having $Z \leq 3$ are observed. Figures 2(b), 2(c) and $2(d)$ show the predictions of the simultaneous multifragmentation model, ${ }^{7}$ the sequential binary code of Richert and Wagner, ${ }^{8}$ and the statistical model, GEMINI, respectively. We note again that of these models, the Sa-Gross model comes closest to reproducing the experimental data. The two standard sequential models show much lower probabilities for multiple fragment emission than the multifragmentation model 
although the model of Richert and Wagner does predict slightly more than GEMINI.

Fig. 2. Probability distributions for emission of at least $\boldsymbol{n}$ fragments, each having $\mathbf{Z} \geq \mathbf{Z}_{\text {bhresh }}$ Symbols are: $n=1,0$; $n=2, \square$; $n=3, \Delta$; $n=4, \diamond$; $n=5, \nabla$ $n=6,+$; $\mathbf{n}=8,4$; $n=9, \triangleright$.

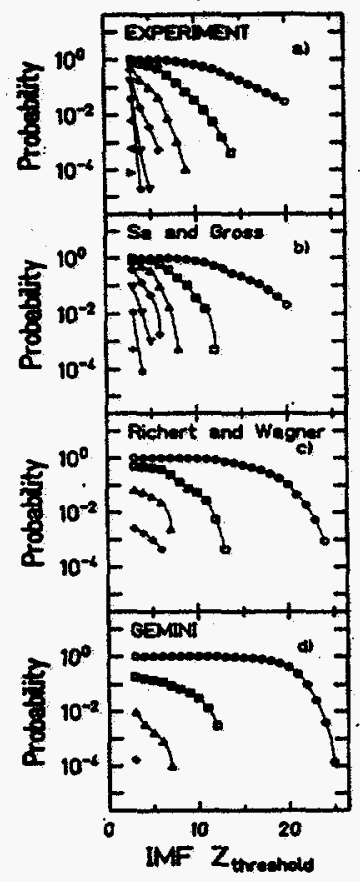

Figure 3 shows a contour plot of the $\ln S_{2}$ vs $\ln Z_{\text {max }}$ distributions as introduced in our previous annual report."

Fig. 3. Log distribution of $Z_{\max }$ vs $S_{2}$ (see text). Each contour represents constant value in units of relative

$$
\frac{d^{2} Y}{d n S_{2}^{\prime \prime} d \ln Z_{\max }}
$$

where $Y$ is the yield.

The outside contour is at a level of 10 , and each inner contour represents a progressive increase in yield of 150 .

(a) Experiment,

(b) Multifragmentation calculation of $\mathrm{Sa}$ and Gross,

(c) Binary decay calculation of Richert and Wagner,

(d) GEMINI calculation.

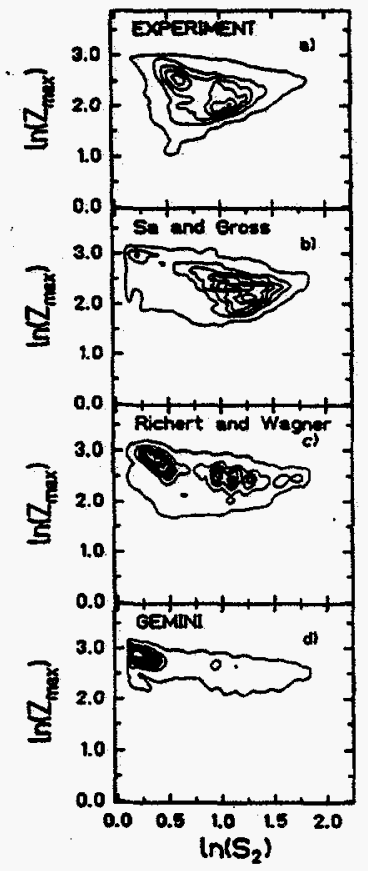

Two peaks occur in the experimental contour plot in Fig.

3(a). One is located at large values of $\ln Z_{\max }$ and small values of $\ln S_{2}^{\prime}$ and the other is located at small values of $\ln Z_{\max }$ and large values of $\ln S_{2}^{\prime}$. Figure 3(b) shows the prediction of the multifragmentation code, ${ }^{7}$ Fig. 3(c) shows the prediction of the Richert and Wagner code, ${ }^{8}$ and Fig. 3(d) shows the prediction of GEMINI.9 The multifragmentation calculation in Fig. 3(b) shows only one peak at large $\ln \mathrm{S}_{2}^{\prime}$ similar to the most prominent peak in Fig. 3(a). The other two calculations, on the other hand, show a large peak only at small values of $\ln S_{2}^{\prime}$. These calculated peaks are at lower values $\ln S_{2}^{\prime}$ than the peak at lower $\ln S_{2}^{\prime}$ in the experimental data, a fact which reflects the dominance of light particle emission in the calculations.

The comparisons of Fig. 1, 2, and 3 suggest that "normal" statistical models are not in agreement with the observations. Since expansion is a key ingredient in the reasonably successful simultaneous multifragmentation model, it is of interest to ask how sequential decay frorn an expanding system compares with the data. Friedman has proposed a sequential decay model in which the deexcitation of an expanding nucleus is treated." In Fig. 4 we present for the schematic model of Friedman, with different assumed values of the expansion energy ( $\mathrm{E}_{\text {expan }}$ ) of the deexciting system, plots like those of Fig. 3. When $E_{\text {expon }}=0$, as shown in Fig. 4(a), the results are qualitatively similar to the GEMINI prediction. However, if $\mathrm{E}_{\text {expan }}=50$ or $100 \mathrm{MeV}$, as shown in Fig. 4(b) and 4(c), respectively, the distributions evolve toward larger values of $\ln S_{2}^{\prime}$ and smaller values of $\ln Z_{\max }$. For $E_{\text {expan }}=100 \mathrm{MeV}$, the results shown in Fig. 4(c) are similar to those of the multifragmentation model presented in Fig. 3(b).

Fig. 4. Same as Fig. 3 for the schematic model of Friedman having $E_{\text {expan }}$ of

(a) $0 \mathrm{MeV}$,

(b) $50 \mathrm{MeV}$, and

(c) $100 \mathrm{MeV}$.

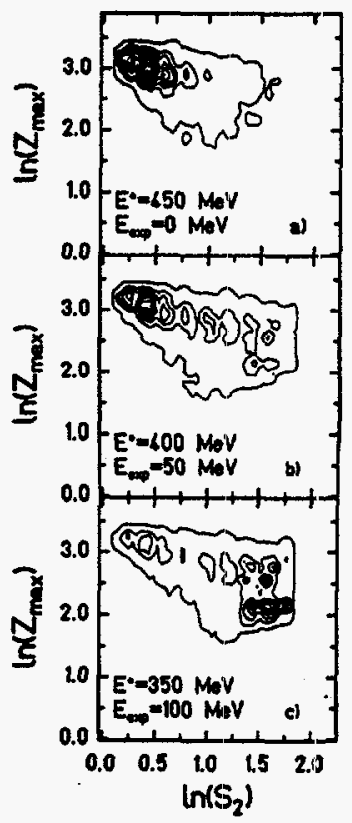

The minimum density reached in the model calculation which corresponds to Fig. $3(\mathrm{c})$ is about $\frac{1}{3} q_{0}$. This may be compared to the value of $\frac{1}{6} q_{0}$ used in the simultaneous multifragmentation calculation. At 35 
$\mathrm{MeV} / \mathrm{u}$ the Landau Vlasov and BUU codes lead to a minimum density near $\frac{1}{2} q_{0}{ }^{2.5}$ It has been pointed out that at such low density the system enters the spinodal region and is subject to mechanical instabilities which could lead to multifragmentation. $2,3,12$

In conclusion, we have shown that model calculations which assume significant expansion of the de-exciting system such as the $\mathrm{Sa}$ and Gross simultaneous multifragmentation model or the expanding sequential decay model of Friedman, are in much better agreement with the observed behavior than are calculations with more standard sequential models. A characteristic of the Friedman model is that the bulk of the IMF emission, though sequential, occurs in a very short time scale at large expansion. We take the success of the two models incorporating expansion as a strong argument that the experimental events reflect the deexcitation from a hot expanded system rather than the equilibrium decay of a hot nucleus of normal density. The reasonable success of the two models does not presently allow a distinction to be made between simultaneous and sequential emission.

† Brookhaven National Laboratory, Upton, New York. \&Institute of Atomic Energy, Beijing, China.

(1)INFN-Legnaro, I-35020, Legnaro, Italy

(2)IPN-Lyon, 69622 Villeurbanne Cedex, France

(3)ISN-Grenoble, 38026 Grenoble-Cedex, France

(4)Hope College, Holland, Michigan 49423
1. K. Hagel et al., Progress in Research 1990-91, Cyclotron Institute, Texas A\&M University, p. 18.

2. K. Sneppen and L. Vinet, Nucl. Phys. A480, 342 (1988).

3. E. Suraud, in Proceedings of the Symposium on Nuclear Dynamics and Nuclear Disassembly, edited by J. B. Natowitz, World Scientific Publishing Company, Singapore, 1989) p. 464.

4. K. Niita et al., Nucl. Phys. A504, 391 (1989).

5. H. M. Xu et al., Phys. Rev. Lett. 65, 843 (1990).

6. M. Blann, Phys. Rev. C31, 1245 (1985).

7. Sa Ban-Hao and D. H. E. Gross, Nucl. Phys.A437, 643 (1985); Xiao-Ze Zhang et al., Nucl. Phys. A461, 641 (1987); Xiao-Ze Zhang et al., Nucl. Phys. A461, 668 (1987).

8. J. Richert and P. Wagner, Nucl. Phys. A517, 399 (1990).

9. R. J. Charity et al., Nucl. Phys. A483, 371 (1988).

10. X. Campi, J. Phys. A19, L917 (1986).

11. W. Friedman, in Proceedings of the International Symposium on Nuclear Dynamics, Niiko, Japan, June 1991 (to be published in Conf. Proc., Amer. Inst. of Physics); W. Friedman, Phys. Rev. Lett. 60, 2125 (1988); W. Friedman, Phys. Rev. C42, 667 (1990).

12. C. J. Pethick and D. G. Ravenhall, Nucl. Phys. A471, 19c (1987).

\title{
NEUTRON MULTIPLICITIES IN THE ${ }^{40} \mathrm{Ar}+{ }^{40} \mathrm{Ca}$ SYSTEM
}

\author{
K. Hagel, R. Wada, J. B. Natowitz, Y. Lou, D. Utley, X. Bin
}

The results of the ${ }^{40} \mathrm{Ca}+{ }^{40} \mathrm{Ca}$ experiment reported elsewhere in this annual report used only data which did not sensitively depend on the calibration of the AMPHORA detector.' In order to push the data further, it would be desirable to have a reliable check on the calibration of the AMPHORA detector. The AMPHORA experiment had two triggers which we might hope to match to the data in this calibration run. One set of data had a multiplicity threshold of 4 , and the other set of data had a multiplicity threshold greater than 14 . The lower multiplicity threshold detected essentially all of the reaction cross section except for the very peripheral collisions, whereas the higher multiplicity threshold which comprises the majority of our data was selected in order to focus on the very violent collisions for the study of multifragmentation.

If neutron multiplicity could be established as an indicator of the degree of violence of the collision in this light system, we could focus on the very violent collisions and compare the energy spectra directly. If the neutron multiplicity cannot, however, be established as an indicator of the violence of the collision, we can still take our data in singles mode and compare it to the AMPHORA data with the lower multiplicity threshold.

In an attempt to check our calibration of the AMPHORA detector, we have bombarded $35 \mathrm{MeV} / \mathrm{u}{ }^{40} \mathrm{Ar}$ ions onto a ${ }^{40} \mathrm{Ca}$ target in the neutron ball. The trigger was the neutron ball. Two 4-element detector telescopes were used to measure the energy spectra of protons and alpha particles at the same angles as detectors in the 
calibrated using a pulser which was normalized to the $5.48 \mathrm{MeV}$ alpha particles from an Am source. Source data were also taken from $3.18 \mathrm{MeV}$ alpha particles from a Gd source and $6.11 \mathrm{MeV}$ alpha particles from a $\mathrm{Cf}$ source. One data run was performed where both detectors were placed at $90^{\circ}$ in order to verify that the calibrations of this run were internally consistent. The CsI detector was calibrated using range energy tables.

Figure 1 shows the proton and alpha particle energy spectra at the various angles. The open circles are the spectra in coincidence with 1 neutron and the closed circles are the spectra in coincidence with 10 neutrons. No background corrections have been made for the neutron ball. We observe in this figure that the spectra for protons and alpha particles have very similar forms regardless of whether one neutron was detected or ten neutrons were detected. If the neutron multiplicity is an indicator of the violence of the collision, we would expect those spectra to change with neutron multiplicity. This insensitivity would indicate that the neutron multiplicity is not a good indicator of the violence of the collision for a light system such as the ${ }^{40} \mathrm{Ar}+{ }^{\circ} \mathrm{Ca}$ system. We do note, however, that the intensity of protons and alpha particles does increase when they are in coincidence with ten neutrons which would indicate more violent collisions.

There is clearly more work to be done in order to understand this possible discrepancy. The analysis of this experiment is in progress.
1. K. Hagel et al., accepted for publication in Phys. Rev. Lett., 1992.

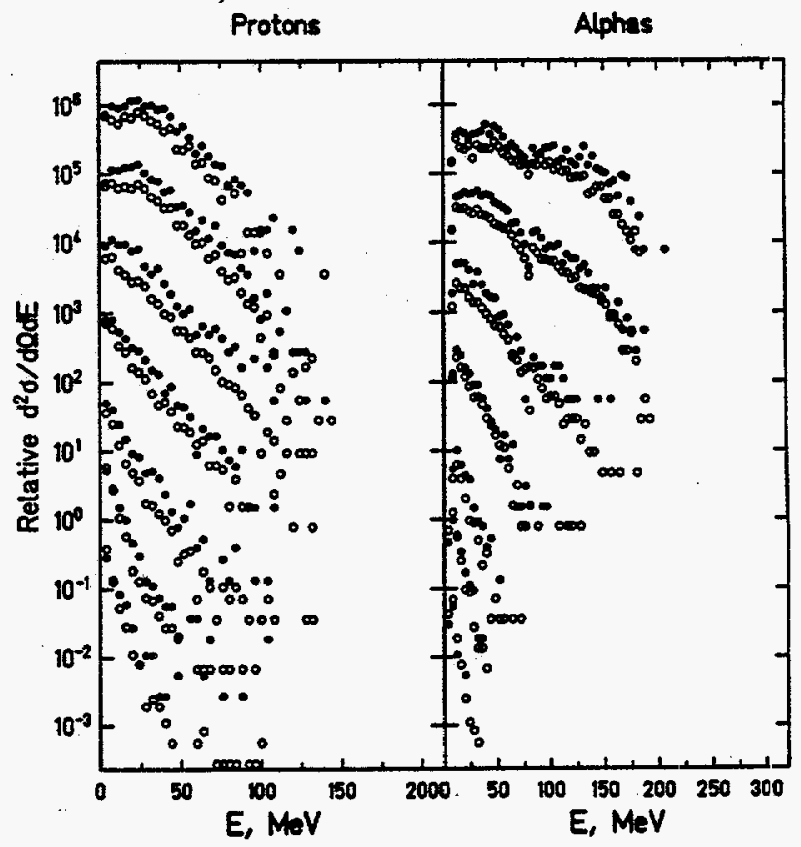

Fig. 1. Energy spectra of protons and alpha particles at the various AMPHORA angles. Open circles represent protons or alpha particles in coincidence with 1 neutron, and solid circles represent protons or alpha particles in coincidence with 10 neutrons. No background correction has been made for the neutroin multiplicity. The top set of spectra are for $\theta=20^{\circ}$, and each one under that is for $\theta=31^{\circ}$, $45^{\circ}, 66^{\circ}, 90^{\circ}, 117^{\circ}, 148^{\circ}$, respectively.

\section{DYNAMICAL ASPECTS OF INTERMEDIATE MASS FRAGMENT EMISSION IN THE REACTION OF ${ }^{32} \mathrm{~S}+\mathrm{Ag}$ AT $30 \mathrm{AMeV}$}
R. Wada, M. Gonin, (1) M. Gui, K. Hagel, Y. Lou, D. Utley, B. Xiao, D. Miller, J. B. Natowitz, D. Fabris, (2) G. Nebbia, (2) R. Zanon, (2) B. Chambon, (3) B. Cheynis, ${ }^{(3)}$ A. Demeyer, (3) D. Drain, ${ }^{(3)}$ D. Guinet, (3) X. C. Hu, (3) C. Pastor, ${ }^{(3)}$ K. Zaid, (3) J. Alarja, ${ }^{(4)}$ R. Bertholet, ${ }^{(4)}$ A. Giorni, ${ }^{(4)}$ A. Lleres, ${ }^{(4)}$ C. Morand, ${ }^{(4)}$ P. Stassi, ${ }^{(4)}$ L. Schussler, ${ }^{(4)}$ B. Viano, ${ }^{(4)}$ P. Gonthier ${ }^{(5)}$

The emission of intermediate mass fragments (IMFs, $3 \leq \mathrm{Z} \leq 14$ ) has been studied in the reaction ${ }^{32} \mathrm{~S}+\mathrm{Ag}$ at $30 \mathrm{AMeV}$. The experiment was performed at the SARA coupled cyclotron facility in Grenoble using a $4 \pi$ array, "AMPHORA." Light charged particles are detected by the AMPHORA with particle identification. Heavier fragments with $Z \geq 3$, including fission fragments, were isolated in the CsI detector without $Z$ identification. In front of four AMPHORA segments at forward angle, $\mathrm{Si}$ detectors $\left(300 \mu \mathrm{m}, 5 \times 5 \mathrm{~cm}^{2}\right)$ were set at a polar angle of $\theta=8^{\circ}$ and azimuthal angles of $\phi=0^{\circ}, 90^{\circ}, 180^{\circ}$ and $270^{\circ}$. Projectile like fragments were observed using these Si $(\Delta \mathrm{E})$ and CsI (E) telescopes. Five of the AMPHORA segments were replaced by telescopes which consisted of a gas ionization chamber IC $(\Delta \mathrm{E})$ and a CsI $(\mathrm{E})$ detector. These telescopes allowed $Z$ identification of the heavier fragments. They were set at $\theta=20^{\circ}, 31^{\circ}, 46^{\circ}, 66^{\circ}, 117^{\circ}$ at an azimuthal angle of $\phi=180^{\circ}$.

The inclusive charge distribution of fragments, which were detected by the IC-CsI telescope at $31^{\circ}$, is plotted in Fig.1 using solid circles. The open symbols show the charge distribution of the fragments at $31^{\circ}$ when an 
additional fragment with $Z \geq 3$ is detected in the angular range $25^{\circ} \leq \theta \leq 75^{\circ}$. For both cases the fragment charge distributions are dominated by light IMFs and show very similar shapes with an exponential fall-off. Calculated IMF yields are also shown for the statistical model code GEMINI ${ }^{1}$ and for a classical dynamical model. ${ }^{2}$ The statistical model underestimates the experimental IMF cross sections by more than a factor of 10 , whereas the dynamical model predicts a factor of $2-3$ smaller than the experiment. For the latter the discrepancy can be explained by the emission from the compound nucleus, which is not taken into account in the dynamical model. This result may indicate a dynamical origin of the observed IMFs.

The azimuthal angular correlation between fragments is an interesting observable which can characterize the emission mechanism. The correlation functions between a PLF observed at $8^{\circ}$ and a fragment detected by the AMPHORA segments at $25^{\circ} \leq \theta \leq 75^{\circ}$ are plotted in Fig. 2, where the azimuthal angle of the PLF detector is taken as $\phi=0^{\circ}$. Two cases are shown, namely that for PLFs with $Z \geq 9$ (closed symbols) and that for PLFs with $Z=5,6$ (open symbols). A strong correlation is observed for the PLFs with $Z \geq 9$. The correlation indicates that the coincident fragment is preferentially emitted in the same plane as the PLF but on the opposite side of the beam. A moving source analysis showed that at angles $\theta \geq 30^{\circ}$ the projectile-like component contributes little to the fragment differential cross sections. For the IMF emission from a strong polarized compound nucleus following an incomplete fusion process, the relative azimuthal correlation between IMF-PLF would show a coplanarity between these two fragments but would also show enhancements at $\Delta \phi_{R z}=0^{\circ}$ and $180^{\circ}$. The latter is not observed experimentally as seen in Fig. 2. The observed azimuthal correlation, therefore, indicates that a dynamical process, such as an incomplete deeply inelastic process, plays an important role for the fragment emission.

The correlation becomes smaller for the PLFs with $Z=5,6$. This may be caused by the sequential particle decay of the excited primary PLF, which smears the primary correlation. Even though the correlation becomes small, one can see that the fragment emission is still enhanced in the reaction plane both on the same side and on the opposite side from the PLF. To shed more light on this correlation, triple coincidence events were examined. The correlation function of the relative azimuthal angle $\left(\Delta \phi_{R E D}\right)$ was examined for two fragments which are observed in the AMPHORA segments at $25^{\circ} \leq \theta \leq 75^{\circ}$ triggered by a PLF having $Z \geq 5$ detected at $8^{\circ}$. The result is shown in Fig. 3. One can clearly see that the two fragments are preferentially emitted around $\Delta \phi_{R E L}=180^{\circ}$. Moreover, in Fig. 4 this azimuthal angle correlation is shown to be a rather general observation for the two fragment emission. In that figure the correlation function of two fragments is plotted by symbols for the events triggered by the IC-CsI telescopes (i.e. no PLF is required). A fragment is detected by one of the IC-CsI telescopes at $\theta=31^{\circ}, 46^{\circ}, 66^{\circ}$ and the other is detected by one of the AMPHORA segments at $25^{\circ} \leq \theta \leq 75^{\circ}$. The azimuthal angle of the IC-CsI telescopes is taken as $\phi=0^{\circ}$ in the figure. Two cases are shown, first one of the fragments detected by the IC-CsI telescope has $Z=5,6$ (open symbols) and second when this fragment has $Z=8-12$ (closed symbols). For both cases a strong azimuthal angular correlation is observed. For the $Z=8-12$ case $40 \approx 50 \%$ of the fragments are emitted in opposite azimuthal directions from each other. As the explanation of this azimuthal angular correlation, several possible mechanisms can be proposed, such as fission of the compound nucleus following incomplete fusion as well

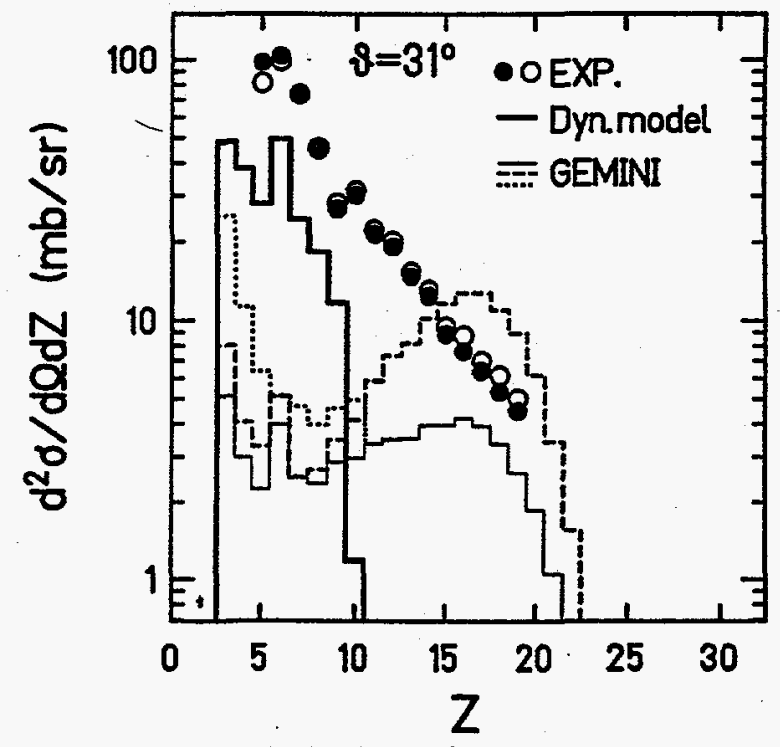

Fig. 1. Charge distributions of the fragments at $31^{\circ}$. Solid symbols show the inclusive distribution. Open symbols show the distribution for fragments in coincidence with a fragment detected at $25^{\circ} \leq \theta \leq 75^{\circ}\left(\mathrm{M}_{\mathrm{F}}=2\right)$. The absolute scale is only for the inclusive data and the coincidence data are arbitrarily normalized to the inclusive data by multiplying by a factor of 4. Histograms are the results of the GEMINI calculation with $J=100 \mathrm{~h}$ for inclusive (thin solid line), $M_{F}=2$ (multiplied by a factor 4 , dashed line) and the primary IMFs (see text, dotted line), respectively. The histogram plotted with a thick solid line shows the result of the extended classical dynamical model. All. calculated results are filtered using the experimental energy thresholds. 
as orbiting or sequential breakup of fragments following deeply inelastic process. The dominance of light MMs in two fragment emission, as seen in Fig. 1, rules out fission as the explanation.

\section{$\Delta \Phi(P L F-F)$}

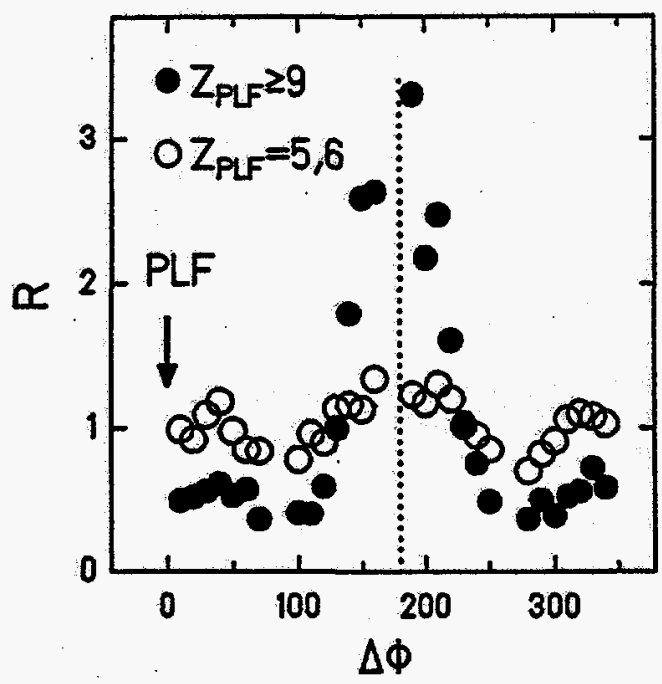

Fig. 2. Azimuthal angular correlation between a PLF observed at $8^{\circ}$ and a fragment with $Z \geq 3$ observed at $25^{\circ} \leq \theta \leq 75^{\circ}$ for different $Z_{P L F}$ triggers. The azimuthal angle of the PLF detector is taken as $\phi=0^{\circ}$, as indicated by the arrow. The dotted line indicates $\phi=180^{\circ}$.

The strong azimuthal angular correlation between PLF-IMF, shown in Fig. 2, and the coplanarity with $\Delta \phi_{R E}=180^{\circ}$ for IMF-IMF emission, shown in Figs. 3 and 4 , are strong constraints for the dominant emission mechanism of IMFs. The classical dynamical model has been applied to nonequilibrium composite light particle emission. ${ }^{2}$

We have applied this model to the IMF emission. Details of the model can be found in Ref. 2. This model assumes a breakup of the projectile into a primary projectile-like fragment $A 1$ and a negatively deflected fragment A2. This fragment A2 may itself fragment after emission as it achieves significant excitation energy during deflection.

For events with IMF emission from (or fission of) fragment $A 2$ one can expect an azimuthal correlation around $\Delta \phi_{R E B}=180^{\circ}$ when fragment $A 2$ is emitted at small angles, which simply results from the momentum conservation. In Fig. 4 the calculated azimuthal correlations for two such IMFs are compared with the data, using the same detection conditions as in the experiment. The calculated results reproduce the observed correlations very well for both fragment cases, although the calculated ratio of double to single emission of fragments $M_{F}=2 / M_{F}=1$ at $25^{\circ} \leq \theta \leq 75^{\circ}$, is 0.030 , whereas the experimental ratio is 0.143 .

- $\Delta \Phi(F-F)$ with PLF

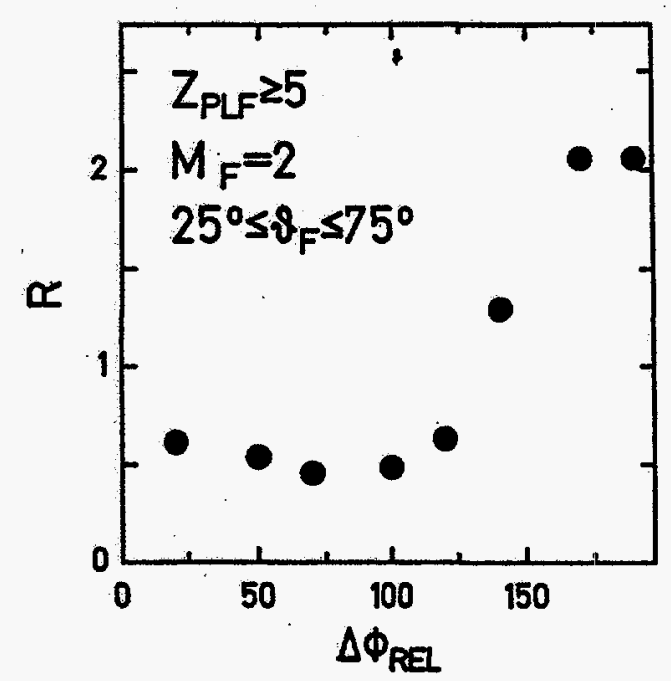

Fig. 3. Relative azimuthal angular correlation between two fragments with $Z \geq 3$ observed at $25^{\circ} \leq \theta$ $\leq 75^{\circ}$ in coincidence with a PLF with $Z \geq 5$ observed at $8^{\circ}$.
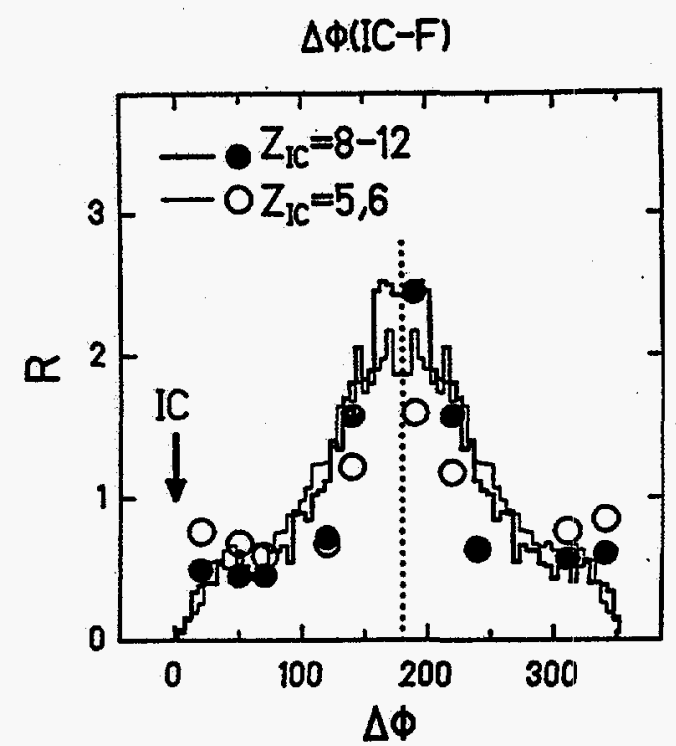

Fig. 4. Azimuthal angular correlation between twro fragments with $Z \geq 3$. One of the fragments is measured by one of the IC-CsI telescopes at $31^{\circ}, 46^{\circ}, 66^{\circ}$. (The azimuthal angle of these telescopes is taken as $\phi=0^{\circ}$.) The other fragment is observed by one of the AMPHOR.A segments at $25^{\circ} \leq \theta \leq 75^{\circ}$. Two different $Z$ groups in the telescopes are selected.

In Fig. 5 we make a comparison between the results of the calculation and the experiment. At the top of Fig. 5 
the calculated initial temperature (assuming an inverse level density parameter $K=8$ ) of the primary fragment $A 2$ is plotted as a function of the charge of PLFs, $Z_{P L F}$. The temperature decreases as $Z_{P L F}$ increases. For lower $\mathrm{Z}$ the calculated temperatures are comparable with those obtained in the measurements of the particle unstable level populations in which a source temperature of 4-5 MeV was determined for intermediate velocity fragments. In the middle of the figure the average differential multiplicity of the fragments with $\mathrm{Z} \geq 3$ observed by the AMPHORA segments is plotted. The multiplicity depends strongly on $Z_{P L F}$, reflecting the increase of the excitation energy with decreasing impact parameter of the collision. The calculated multiplicities at $25^{\circ} \leq \theta \leq 75^{\circ}$ for the primary A2 and the secondary IMFs from A2 are shown by dashed and solid lines, respectively. The calculated multiplicity for the secondary fragments reproduces the general trend of the observed data up to $Z_{P L F} \leq 11$. The discrepancy for $Z_{P L F}=12,13$ may indicate that the thermal excitation energy given to the fragment $A 2$ in proportion to its mass $\left(A=8,6\right.$ for $Z_{P L F}=$ 12,13 , respectively) is too little. In the bottom of the figure the average charge of the fragments with $Z \geq 3$ observed by the IC-CsI telescopes at $31^{\circ}$ and $66^{\circ}$ is plotted as a function of $Z_{P L F}$. The average $Z$ depends slightly on $Z_{P L F}$ and monotonically decreases with increasing $Z_{P L F}$. The calculated results are shown by solid $\left(31^{\circ}\right)$ and dashed $\left(66^{\circ}\right)$ lines. (The calculated results are filtered using the experimental energy thresholds, although this has little effect on the results because of rather high calculated kinetic energy of the IMFs at these angles.) The observed average $Z$ is well reproduced by the calculation. Even though in the comparisons the calculated cross section for two fragment emission is smaller by a factor of 4-5 than for the experiment, reasonable agreement is obtained because the basic characteristics of the fragment emission are dominated by a single fragment emission in this incident energy region.

(i) Present address: BNL, Upton, New York 11973

(2) INFN, 1-35121, Padova, Italy

(3) IPN-Lyon, F-69622 Villeurbanne Cedex, France
ISN-Grenoble, F-38026 Grenoble Cedex, France

Department of Physics, Hope College, Holland, MI 49423

\section{REFERENCES}

1. R. J. Charity et al., Nucl. Phys. A483, 371 (1988).

2. R. Wada et al., Nucl. Phys. A539, 316 (1992).

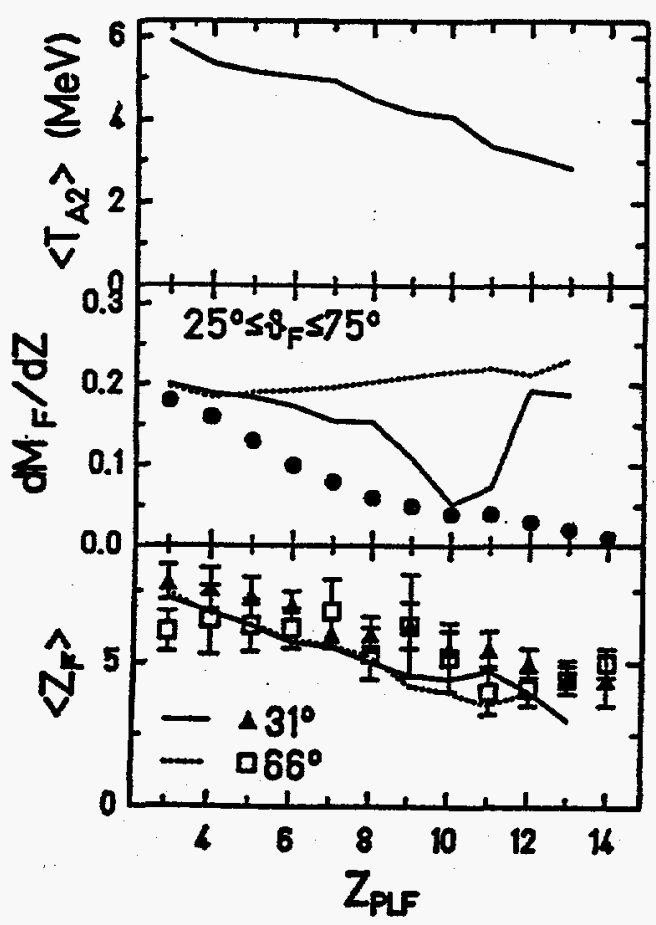

Fig. 5. Results of the extended classical dynamical calculation are compared with the experimental data in coincidence with different PLFs.

Top: Calculated initial temperature distribution of the fragment $\mathrm{A} 2$ when an IMF originating from $\mathrm{A} 2$ is detected at angle $25^{\circ} \leq \theta \leq 75^{\circ}$.

Middle: Differential multiplicity distribution of the fragments detected at $25^{\circ} \leq \theta \leq 75^{\circ}$. Dashed line shows the multiplicity for the $\mathrm{A} 2$ fragment and the solid line is that of the secondary fragments.

Bottom: Average $Z$ distribution of the fragments with $Z \geq 3$ observed at $31^{\circ}$ and $66^{\circ}$. The solid and dashed lines are the calculated results for each angle. 


\author{
M. Gui, R. Wada, J. B. Natowitz, K. Hagel, Y. Lou, D. Utley, B. Xiao, \\ G. Enders, (1) W. Kühn, (1) V. Metag,,(1) R. Novotny,, (1) O. Schwalb, (1) \\ R. Charity, (2) R. Freifelder, (2) A. Gobbi, (2) W.Henning, (2) K. D. Hildenbrand, (2) \\ R.Mayer, (2) R.S.Simon, (2) J. Wessels, (2) G.Casini, (3) A.Olmi, (3) and A.A.Stefanini( ${ }^{(3)}$
}

Systematic experimental data on timescales involved in nuclear fission as a function of excitation energy, temperature, mass-split and total kinetic energy provide new insights into the nature of the fission process. In this work, investigation of the fission timescale as a function of fission fragment mass asymmetry was carried out.

The experiment was performed at the UNILAC accelerator of GSI in Darmstadt, Germany. An 18.5 $\mathrm{MeV} / \mathrm{u}$ (total energy $2516 \mathrm{MeV}$ ) ${ }^{136} \mathrm{Xe}^{21+}$ ion beam bombarded a self-supporting target ${ }^{18} \mathrm{~T}$ with a thickness of $175 \mu \mathrm{g} / \mathrm{cm}^{2}$. Fission fragment velocities were measured. The fragment masses were deduced by means of a kinematic reconstruction. The light charged particles emitted in coincidence with fission fragments were observed. Pre-scission and post-scission $\alpha$ and $p$ multiplicities as a function of fragment mass asymmetry were determined by a three moving source fit method.

An empirical technique was used to obtain the excitation energy at scission of the system from the multiplicities of the emitted light particles.

From our previous observations of charged particle emission, we noted that both the $\alpha$ and the proton multiplicities increase linearly as the excitation energy per nucleon of the system increases,

$$
\langle M\rangle=\kappa\left(E_{x} / A\right)-b
$$

The slope $j$ and intercept $b$ change slightly depending on the mass of the system. In order to determine the excitation energies at scission for different fission fragment mass asymmetries, we have assumed that this property of evaporated $\alpha$ and proton multiplicities increasing linearly with the excitation energy per nucleon of the system is also valid for the present system with $A$ $\approx$ 184. As the symmetric fission is well characterized for our system, we used data for the symmetric fission process to establish the slopes $\kappa_{\alpha}$ and $\kappa_{p}$ for this system and used an intercept formula based on the data from Wada, et a‥ ${ }^{1}$, Gonin, et al. ${ }^{2}$, and Gramegna, et al. ${ }^{3}$ to establish the necessary parameters.

Using this method, we have determined the excitation energy at the scission point for scission with different mass asymmetries from both the post-scission $\alpha$ or proton multiplicities, as well as from the pre-scission $\alpha$ or proton multiplicities. The results are presented in Fig. 1. From these energies, pre-scission times are determined by employing the statistical model computer code EVAP (Nicolis, 1991). In Fig. 2, we show results of calculations $a=A / 10$. The decay by $n, p, d, \alpha, t, 3 H l e$

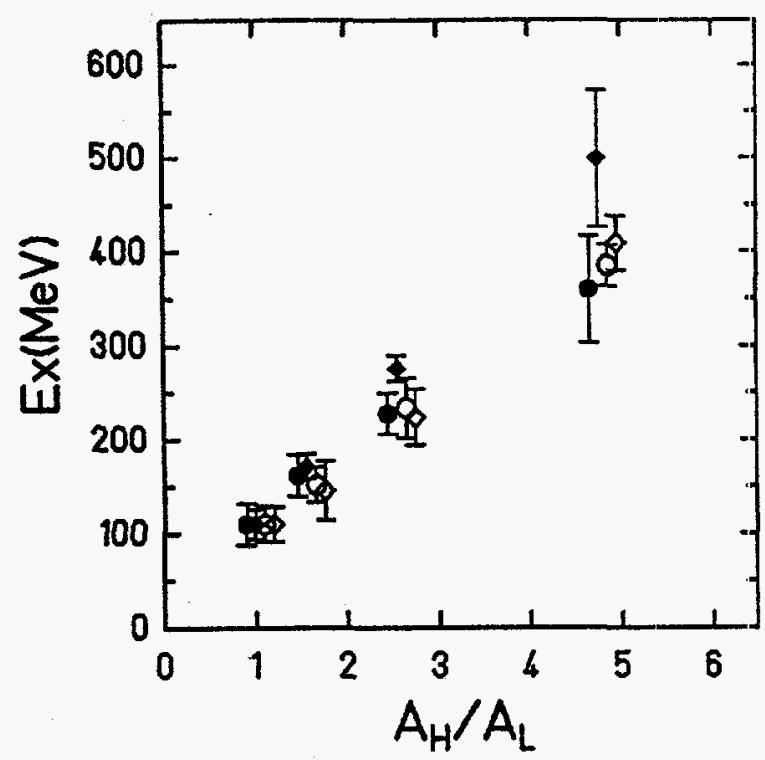

Fig. 1 The excitation energy at scission for different fission fragment mass asymmetries deduced from pre-scission $\alpha$ multiplicity (1), postscission $\alpha$ multiplicity (o), pre-scission protion multiplicity $(\bullet)$ and post-scission protion multiplicity $(\diamond)$ as a function of fission fragment mass asymmetry. For clarity, points are slighily displaced on the mass asymmetry scale.

and ${ }^{6} \mathrm{Li}$ has been considered in the calculation of decay widths. The statistical fission decay was suppressed in the calculation. As it was found previously that the parameler $k=A / a$ increases as excitation energy per nucleon of the system increases, the lifetime and cumulative decay tirne were also calculated as a function of the excitation energy by assuming a temperature dependent level density parameter (Shlomo and Natowitz). These lifetimes are shown in Fig. 2.

Table 1 summarizes our results, where $E_{X}$ represents excitation energy at scission, Time (A/10) represents the pre-scission times obtained by assuming a level density parameter $a=A / 10$, Time $(a(T))$ represents the pre- 
scission times by assuming a temperature dependent level density parameter. These results indicate that for very hot nuclei with $A \propto 180$, having initial excitation energies about $3 \mathrm{MeV} / \mathrm{u}$, symmetric break up still occurs at the very end of the de-excitation chain, at an excitation energy around $100 \mathrm{MeV}$. Symmetrically fissioning nuclei are cold at scission. This is because the time needed for the hot nucleus to change shape into two nearly equal sized nuclei is $1 \times 10^{-20} \mathrm{sec}$. Duing this time, the very rapid evaporation of light particles removes the extra excitation energy, leaving the system relatively cold.

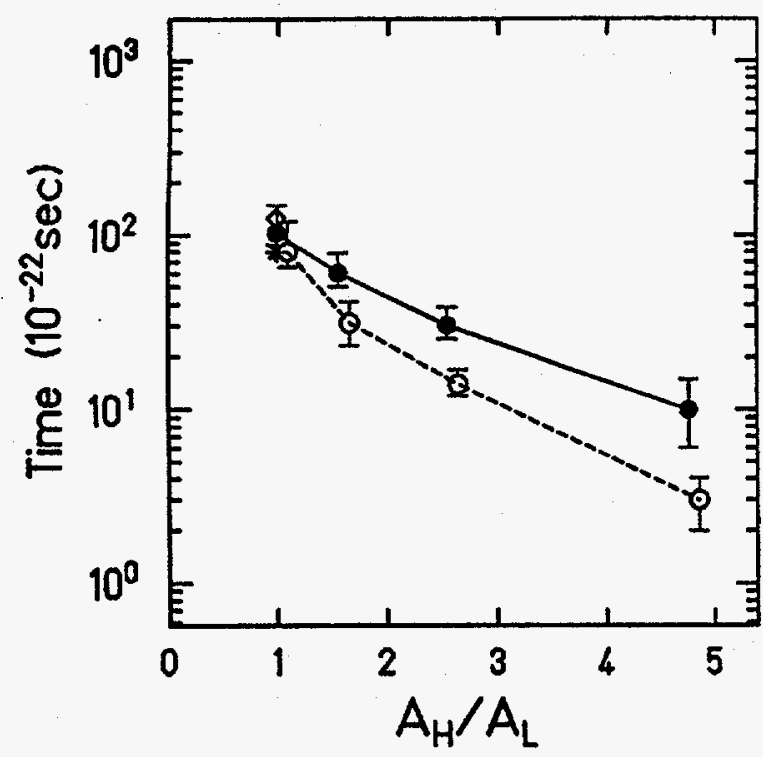

Fig. 2 Pre-scission lifetime as a function of mass asymmetry. ( $\bullet$ represents the pre-scission timescale extracted from our experiment assuming $a=A / 10$. (o) represents the prescission timescale assuming a temperature dependent level density parameter. (For clarity, points are slightly displaced on the mass asymmetry scale.) The lines are to guide the eye. (*) indicates the pre-scission timescale obtained by Hilscher et al. for the system ${ }^{186} \mathrm{Pt}^{*}$, and $(0)$ indicates the pre-scission timescale calculated using the Feldmeier model by assuming $L=120 n$.

Even for completely relaxed processes, asymmetric break-up happens at an earlier stage of the particle deexcitation chain than symmetric fission. The greater the fragment mass asymmetry, the earlier in the de-excitation stage the scission occurs. Some of the asymmetric mass- split processes may be correlated with higher angular momenta, reflecting large fluctuations in energy dissipation or interaction time. For the most asymmetric break up observed here the time to scission is 0.3 to $1.1 \times 10^{-21} \mathrm{sec}$, which is close to the characteristic time of deep-inelastic scattering.

The fission fragment angular distribution observed in this experiment, $(d \sigma / d \theta)$ in the Center of Mass Frame, is flat for symmetric fission, but shows a forwardbackward peaking for the asymmetric fission. In the Center of Mass Frame, the heavier fragment peaks forward while the lighter fragment peaks backward. As our system is a reversed kinematics system, this forward and backward peaking implies that the system at the scission point still has the memory of the entrance channel. This is also true for most detected three body events which were observed to be strongly damped.

Table 1

Excitation Energies and Times

\begin{tabular}{llrl}
\hline$A_{H} / A_{L}$ & $\begin{array}{c}E_{X} \\
(\mathrm{MeV})\end{array}$ & $\begin{array}{c}\text { Time }(A / 10) \\
\left(10^{21} \mathrm{sec}\right)\end{array}$ & $\begin{array}{c}\text { Time }(a(T)) \\
\left(10^{-21} \mathrm{sec}\right)\end{array}$ \\
1.00 & $110 \pm 19$ & $10.5_{-1.5}^{+4.5}$ & $8.0_{-1.5}^{+4}$ \\
1.56 & $158 \pm 22$ & $6.0_{-1.0}^{+1.8}$ & $3.1_{-0.8}^{+1.0}$ \\
2.54 & $240 \pm 25$ & $30.0_{-0.5}^{+0.8}$ & $1.4_{-0.2}^{+0.3}$ \\
4.75 & $413 \pm 46$ & $1.0_{-0.4}^{+0.5}$ & $0.3_{-0.1}^{+0.1}$
\end{tabular}

(1)II. Physikalisches Institüt, Universität Gießen, Germany (2)GSI, Darmstadt, Germany

(3)INFN, Florence, Italy

\section{REFERENCES}

1. Wada, et al., Phys. Rev C31, 1550 (1989).

2. Gonin, et al., Phys. Rev. C42, 2125 (1990).

3. Gramegna, et al., Progress in Research, 1990-1991, Cyclotron Institute, Texas A\&M University, p. 22.

4. S. Shlomo and J. B. Natowitz, Phys. Lett. 252B, 187 (1990). 


\section{FISSION PROCESSES IN THE REACTION \\ ${ }^{63} \mathrm{Cu}+{ }^{100} \mathrm{Mo}$ at 10,17 and $25 \mathrm{Mev} / \mathrm{u}$}

Y.Lou, L.Cooke, T.Botting, M.Gonin,(1) M.Gui, K.Hagel, B.Hurst, D.O'Kelly, G.Mouchaty,

J.Natowitz, R. P. Schmitt, W. Turmel, D.Utley, R.Wada, B.Burch, (2) D.Fabris, (2) G.Nebbia, (2) M.E.Brandan, (3) A.Menchaca-Rocha, (3) F.Gramegna, ${ }^{(4)}$ M.Poggi,(4) G.Prete, (4) J.Ruiz,, (4) G.Viesti( ${ }^{(4)}$

Previous experiments on neutron and light charged particle emission in the ${ }^{60} \mathrm{Ni}+{ }^{100} \mathrm{Mo}$ reaction at 9.2 and $10.9 \mathrm{MeV} / \mathrm{u}$ and on charged light particle emission at 13.3 and $16.7 \mathrm{MeV} / \mathrm{u}$ have shown that the fission decay is a cold process with the fission occurring at an excitation energy near $130 \mathrm{MeV} .^{1}$ To better characterize the deexcitation pathways of the hot compound nucleus deexcitation and to achieve a better understanding of the fission time scale we have looked at the fission decay in coincidence with neutrons and light charged particles for the reaction of ${ }^{63} \mathrm{Cu}+{ }^{100} \mathrm{Mo}$ at bombarding energies of 10, 17 and $25 \mathrm{Mev} / \mathrm{u}$.

For these reactions average linear momentum transfers (LMT) near 100\%, $90 \%$, and $80 \%$ are expected, leading to the excitation energies of 280, 500 and 670 $\mathrm{MeV}$ for the compound nuclei. The hot compound nuclei decay by evaporating light particles (and possibly IMF) both before and after scission.

To detect coincident evaporated species, we position two 7-strip Si detectors on the left (LF) and right (RF) sides of the beam for fission fragment detection and several hodoscopes consisting of an $\mathrm{Si}$ strip detector backed by CsI crystals and several telescopes of $\mathrm{Si}$ detectors for light charge particle detection. The LF strip detector covers from $21.5^{\circ}$ to $42.5^{\circ}$ and the RF strip detector covers from $-21.92^{\circ}$ to $-48.08^{\circ}$. All these detectors are inside the vacuum chamber of the neutron ball, allowing total neutron detection and a neutron angular distribution measurement.

Once the linear momentum transfer is understood, the mass distribution of the fission fragments may be determined from the energy and angles of the detected fragments. Since the evaporation is symmetric in the frame of the evaporating nucleus, we assume that the average velocity of the compound nucleus does not change before scission. Also the post-scission emission is assumed not to affect the average fission fragment velocities. Applying the momentum conservation and assuming the total mass at the scission point is $\mathbf{M}_{\text {tox }}$, we can extract the fission fragment masses.

$$
M 1=\frac{M_{\text {tot }} \times E 2 \times \sin ^{2} \theta_{2}}{E 1 \times \sin ^{2} \theta_{1}+E 2 \times \sin ^{2} \theta_{2}}
$$

$$
M 2=\frac{M_{\text {tot }} \times E 1 \times \sin ^{2} \theta_{1}}{E 1 \times \sin ^{2} \theta_{1}+E 2 \times \sin ^{2} \theta_{2}}
$$

Here $\mathrm{E} 1$ and $\mathrm{E} 2$ are the fission fragment energies and $\theta_{1}$ and $\theta_{2}$ are the angles at which the fission fragnents are detected.

For the $10 \mathrm{MeV} / \mathrm{u}$ experiment Fig. 1 shows the resultant mass spectra for the coincidence matrix of two seven strip Si detectors LF and RF placed on opposite sides of the beam. For this energy the peak of the folding angle for symmetric fission occurs in the upper right portion of the figure. More asymmetric fission events are detected at other angles. The Total Kinetic Energy (TKE) of the two fission fragments in the moving frame is shown in Fig. 2(a) for the $10 \mathrm{MeV} / \mathrm{u}$ experiment. The experimental geometry discriminates against very esymmetric breakups.

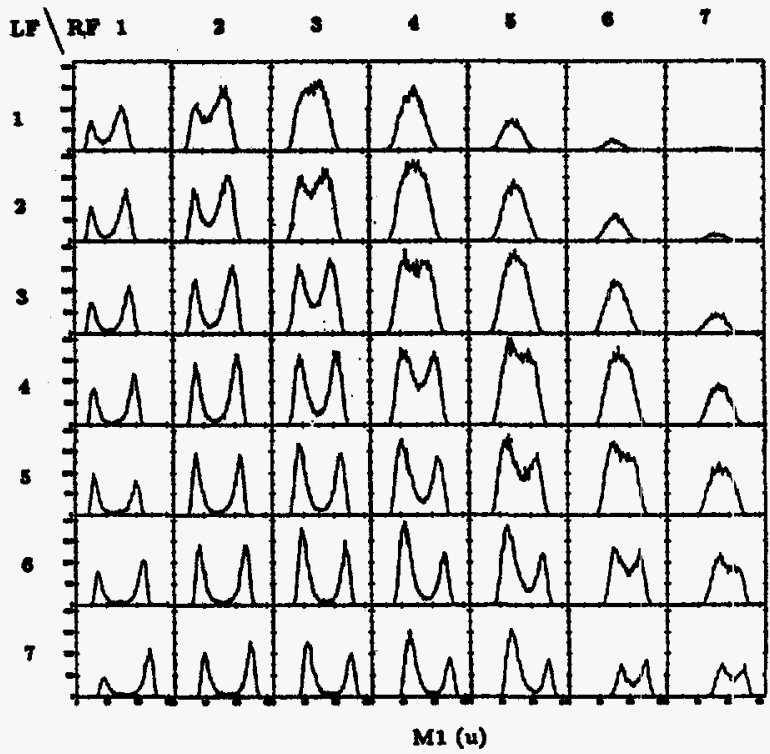

Fig. 1. The mass spectra for coincident fragment detection in two Si strip detectors for the 10 $\mathrm{MeV} / \mathrm{u}$ run.

A simulation was performed to see how the geometry affects the fission fragment detection. In the simulation we let the hot nucleus decay by light particle (protons, alphas and neutrons) evaporation and cool down to 1.30 Mev excitation energy before fissioning. At the scission point we control the width of the fission fragment mass 
distribution. The hot nucleus initially moves at the velocity of the center of mass. We then analyze the simulation results in the same way as the experimental data, reconstructing the masses and the TKE. In Fig. 2(b) we show all the events without geometric restrictions, while Fig. 2(c) shows the result of filtering by the experimental acceptance conditions. The simulation proves to be very useful in understanding the experimental results.

While the simulation starts with the average velocity $V_{c m}$, this may not be the case in the experiment. A range of linear momentum transfers may result from partial fusion at the different impact parameters.

From the experimental folding angle distributions obtained for a symmetric fission fragment window $(\Delta M=20)$ on the $10 \mathrm{MeV} / \mathrm{u}$ data we have extracted the linear momentum transfer (LMT) distribution figures shown in Fig. 3(a). In Fig. 3(b) and Fig. 3(c) we show similar results $(\Delta M=10)$ for 17 and $25 \mathrm{MeV} / \mathrm{u}$ runs. The increasing width of the LMT distribution with increasing energy reflects both a broader range of momentum transfer and evaporation broadening of the primary distribution.

To assist in the interpretation of these results we are employing the simulation. In Fig. 3(a) we show a

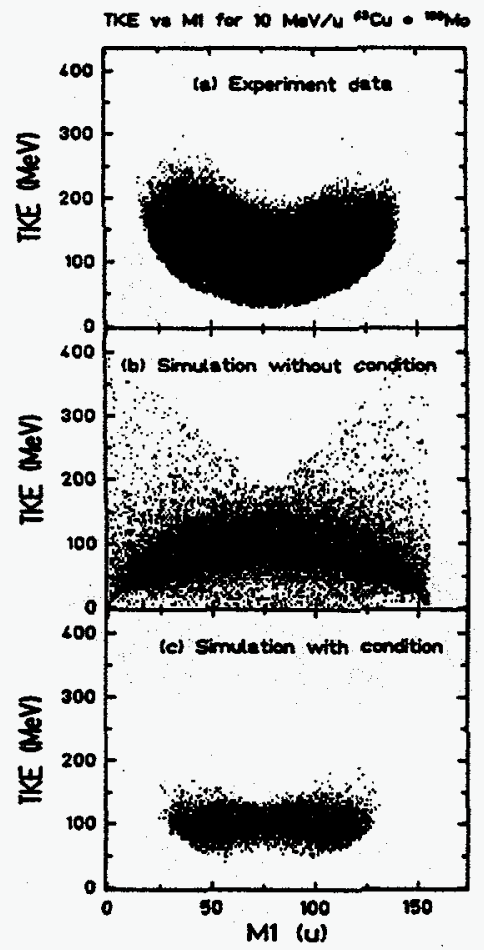

Fig. 2 Total kinetic energy (TKE) of fission fragments vs. fission fragment mass at $10 \mathrm{MeV} / \mathrm{u}$. (a) experimental data. (b) the simulation for $100 \%$ LMT without filtering through the experimental geometry. (c) the simulation with the added condition of the experimental geometry.
${ }^{63} \mathrm{Cu}+{ }^{100} \mathrm{Mo}$ Symmetric fission

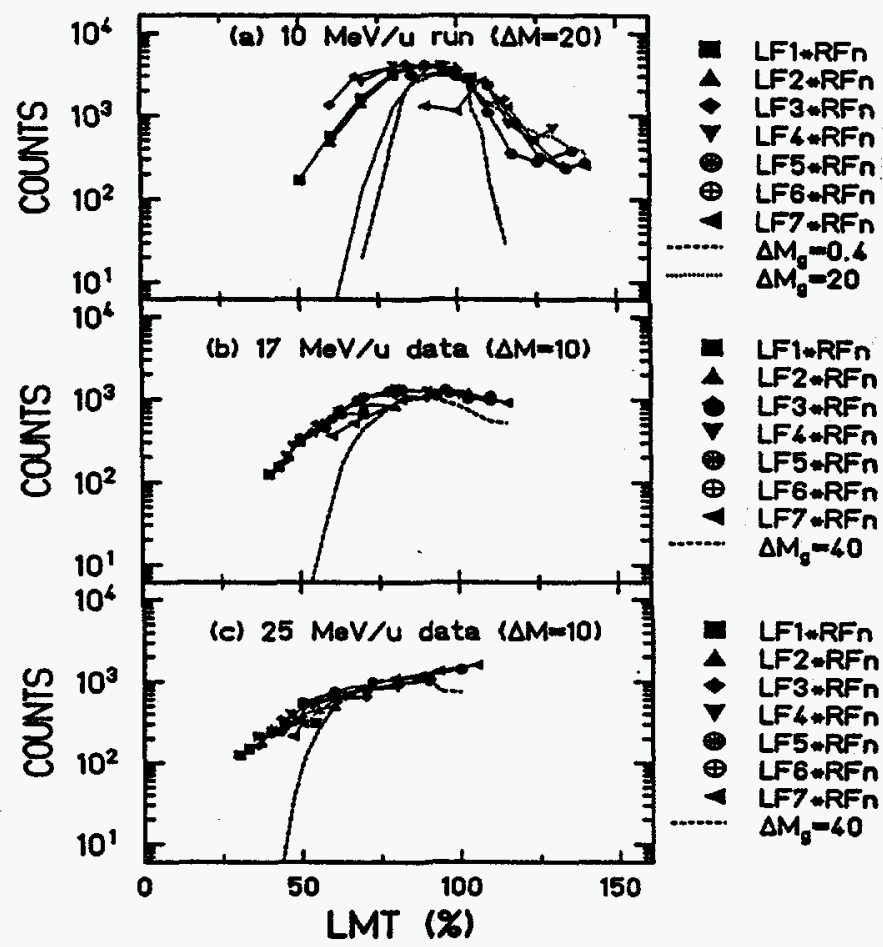

Fig. 3 (a) Linear momentum transfer at $10 \mathrm{MeV} / \mathrm{u}$. The dashed line shows simulation with an initial mass distribution of width $\Delta \mathrm{M}=0.4$ mass units at the scission point. The dotted line shows the simulation with width $\Delta M=20$ mass units at the scission point. As indicated the different symbols represent the results for distributions obtained from observations of fragments in different strips in the LF detector with fragments in the RF detector. (b) Linear momentum transfer at $17 \mathrm{MeV} / \mathrm{u}$. The dashed line shows the simulation with a width $\Delta M^{=}=$ 40 mass unit. (c) Linear momentum transfer at $25 \mathrm{MeV} / \mathrm{u}$. The dashed line shows the simulation with a width $\Delta M_{\mathrm{g}}=\mathbf{4 0}$ mass unit.

simulation of the LMT distribution to be expected at 10 $\mathrm{MeV} / \mathrm{u}$ if the initial LMT is $100 \%$ and the initial width of the fission fragment mass distribution is 0.4 mass units (dashed line) or 20 units (dotted line). Similar calculations for the 17 and $25 \mathrm{MeV} / \mathrm{u}$ simulations are shown in Fig. 3(b) and Fig. 3(c) (dashed line). From comparisons such as this we hope to obtain the primary LMT distributions at 10,17 and $25 \mathrm{MeV} / \mathrm{u}$.

Further analysis of the data is under way.

(I) Brookhaven National Laboratory, Upton, New York

(2) INFN \& Dipartimento di Fisica dell'University di Padova, Padova, Italy

(3) Instituto de Fisica, UNAM, Mexico D.F 01000

(4) INFN, Lab. Naz. Legnaro,I-35020, Legnaro, Italy

(s) INFN \& Dipartimento di Fisica dell'University di Bari, Bari, Italy

\section{REFERENCES}

1. M. Gonin et al., Phys. Rev. C42, 2125 (1990) 


\section{HEAVY RESIDUE PRODUCTION IN THE SYSTEM Ar + Th AT 1600 MEV}

\section{Utley, X.Bin, M. Gui, K. Hagel, J. Li, Y. Lou, D. Miller, J. Natowitz and R. Wada}

Beams from the K-500 superconducting cyclotron have been used in experiments with the neutron ball to explore the limits to excitation energy in compound nuclei formed in the reactions $\mathrm{Ar}+\mathrm{Th}, \mathrm{Au}, \mathrm{Mo}$ and $\mathrm{Ni}$ with projectile energies of $35 \mathrm{MeV} / \mathrm{u}$ and $40 \mathrm{MeV} / \mathrm{u}$.

Experiments with $44 \mathrm{MeV} / \mathrm{u}$ Ar projectiles and Th and Au targets carried out at the Hahn Meitner Institute were interpreted as suggesting an upper limit to the excitation energy of $600-800 \mathrm{MeV}$ for this system. ${ }^{2}$ Calculations completed here show the detector angle of $20^{\circ}$ reported by the Berlin group to be too large to detect the majority of residues which might be produced from collisions central enough to induce excitation energies above $700 \mathrm{MeV}$, should such residues avoid fissioning.

In our experiment at $35 \mathrm{MeV} / \mathrm{u}$ residues with mass greater than $150 \mathrm{u}$ were found to have neutron multiplicities similar to that found in fission. A follow up experiment with the capability of obtaining the light charged particle (LCP) energy spectra, LCP multiplicities and neutron multiplicities in coincidence with heavy residues and projectile like fragments at selected lab angles was performed.

The experiment at $40 \mathrm{MeV} / \mathrm{u}$ was completed in the scattering chamber of the Neutron Ball. Eight LCP telescopes consisting of ionization chambers backed by silicon surface barrier detectors (3) or CsI/PMT detectors (5) were positioned at $20^{\circ}$ intervals in the reaction plane.

Four 300 micron silicon strip detectors $6 \mathrm{~cm} \mathrm{x} 5 \mathrm{~cm}$ each divided into three regions for angular resolution were placed in the forward direction to detect residues: two at $45 \mathrm{~cm}$ and two at $68 \mathrm{~cm}$ from the target. The two detectors at $68 \mathrm{~cm}$ were backed by $0.5 \mathrm{~cm}$ CsI crystals for identification of the projectile like fragments. The residue detectors provided coverage from $3.4^{\circ}$ to $12^{\circ}$ from the beam.

Energy and timing calibrations have been completed for the residue detectors. Preliminary fragment mass distributions based on the lab kinetic energy and time of flight are currently being obtained. Figure 1 illustrates a preliminary mass versus lab kinetic energy distribution for a single detector located at $4^{\circ}$ from the beam axis $68 \mathrm{~cm}$ from the Th target. Corrections for pulse height defect ${ }^{3}$ and timing resolution have not been made in this plot. These corrections are expected to shift the mass distributions significantly. Figure 2 is the background corrected neutron multiplicity for the associated mass distribution of Fig. 1.

Analysis of the spectra of light charged particles ard MFs in coincidence with the massive fragments is in progress. Excitation energies corresponding to the most central collisions will be extracted from these data.

\section{REFERENCES}

1. D. Utley, et al., Progress in Research, 1990-1991, Cyclotron Institute, Texas A\&M University.

2. U. Jahnke, B. Cramer, G. Ingold, E.Schwinn, 19139 Annual Report, Hahn Meitner Institute, Berlin, 1989.

3. S. Kaufman, E. Steinberg, B. Wilkins, J. Unik, and A. Gorski, 1974, Nucl. Inst. and Meth. 115, 47.

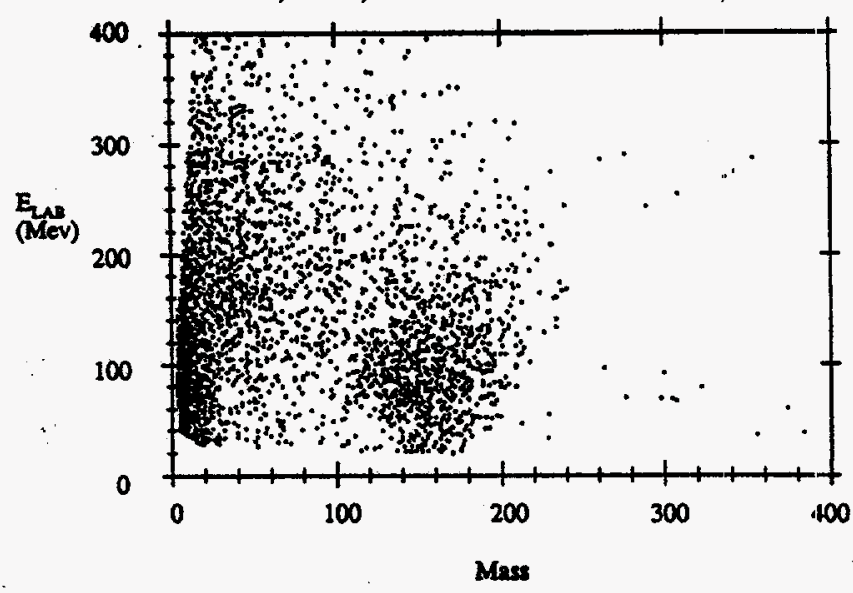

Fig. 1 Mass versus fragment kinetic energy distributions for $\mathrm{Ar}+\mathrm{Th}$ at $1600 \mathrm{MeV}$. Horizontal axis is in AMU. Massive residues and PLF's are clearly soen. Lab angle is $4^{\circ}$.

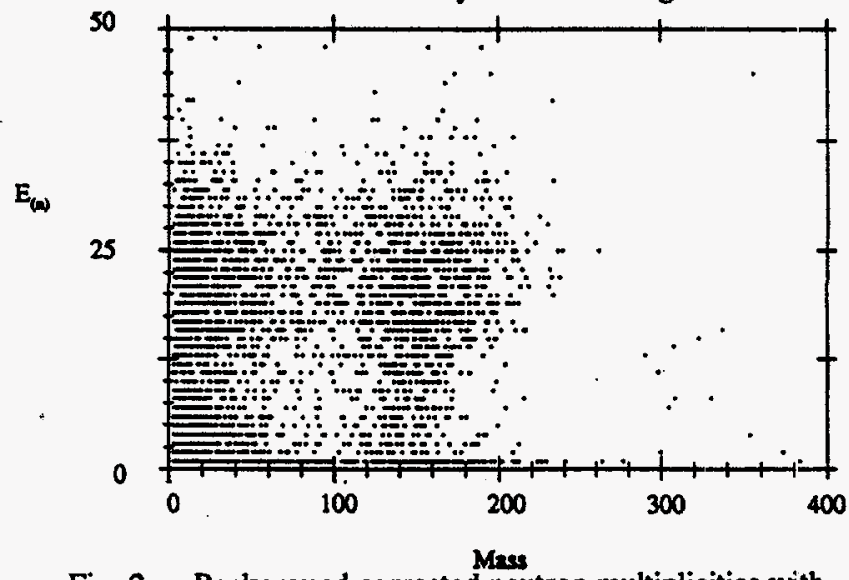

Fig. 2 Background corrected neutron multiplicities with associated mass distributions. The neutron number is not corrected for efficiency. Ar + Th at $1600 \mathrm{MeV}$ with detector at $4^{\circ}$. 


\section{FOLDING ANGLE AND NEUTRON MULTIPLICITY \\ IN THE REACTION ${ }^{20} \mathrm{Ne}+{ }^{144} \mathrm{Sm}$ AT $20 \mathrm{Mev} / \mathrm{u}$}

\section{B.Xiao, M.Gonin,(1) K.Hagel, R.Wada, J.B.Natowitz, M.Gui, J.Li, Y.Lou, D.Miller, D.Utley, \\ T.Botting, L.Cook, B.Hurst, D.O'Kelly, G.Mouchaty, W.Turmel, R.P.Schmitt, \\ B.Burch, (2) D.Fabris, (2) G.Nebbia, (2) M.E.Brandan, ${ }^{(3)}$ A.Menchaca-Rocha, (3) F.Gramegna, (4) M.Poggi, (4) G.Prete, (4) J.Ruiz, (4) G.Viesti(5)}

As a part of our continuing study of the decay properties of hot nuclei with $A \simeq 160$, we have investigated the reaction of $20 \mathrm{MeV}{ }^{20} \mathrm{Ne}+{ }^{144} \mathrm{Sm}$, a much more asymmetric entrance channel than employed in the experiments we performed in Ref. 1, 2.

Fission fragments were detected by $5 \mathrm{Si}$ strip detectors. These detectors are $6.0 \mathrm{~cm}$ long and $4.0 \mathrm{~cm}$ wide. Three of them were placed on the left side of the beam. The LF strip detector was centered at $32^{\circ}$ and covered the angle from $21.5^{\circ}$ to $42.5^{\circ}, \mathrm{LP} 1$ was centered at $61^{\circ}$ and covered from $50.98^{\circ}$ to $71.02^{\circ}$, LP2 was centered at $94^{\circ}$ with angle coverage from $82.48^{\circ}$ to $105.52^{\circ}$. Two strip detectors were placed on the right side of the beam: the RF strip detector centered at $-35^{\circ}$ and covering from $-21.92^{\circ}$ to $-48.08^{\circ}$, and the TOP detector centered at $-75^{\circ}$ with angle coverage from $-62.06^{\circ}$ to $-87.94^{\circ}$. Neutrons, in coincidence with fission fragments, were detected by the $4 \pi$ neutron ball which has top and bottom hemispheres as well as 8 wedges covering $320^{\circ}$ in the reaction plane with $40^{\circ}$ coverage for each wedge. We can measure both the total neutron multiplicity and the angular distribution of the in plane neutrons.

Two measurements of fission fragment folding angle are shown in Fig. 1(a) and Fig. 1(b). In Fig. 1(a), we show the results for fission fragments detected in three different strips of the LF detector LF1 $\left(42.5^{\circ}\right), \operatorname{LF} 4\left(32^{\circ}\right)$, and LF5 $\left(28.5^{\circ}\right)$ in coincidence with fragments detected in the TOP detector. Fig. 1(b) shows the results for fission fragments in strips $\operatorname{TOP} 1\left(-62.06^{\circ}\right)$, TOP2 $\left(-66.34^{\circ}\right)$, and TOP3 $\left(-70.62^{\circ}\right)$ observed in coincidence respectively with fragments in the LP1 detector. These data will be analyzed to get momentum transfer information.

Total neutron multiplicities with different windows on the folding angle are also being studied. The typical neutron multiplicities (uncorrected for efficient) obtained by setting different coincidence trigger requirements are 6-10. The reaction kinematics lead to very forward peaked neutron angular distributions in the lab as shown in Fig. 2. We are exploring the possibility of determining prescission, postcission neutron multiplicities from these angular distributions.
FOLDING ANGLE FOR $20 \mathrm{MeV} / \mathrm{u}^{20} \mathrm{Ne}+{ }^{144} \mathrm{Sm}$

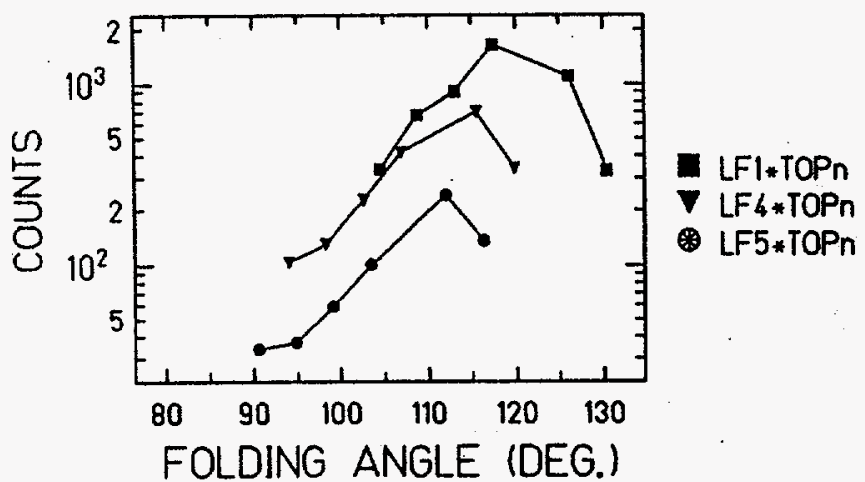

Fig. 1(a). Fission fragment folding angle distribution for LF1*TOPn, LF4*TOPn, and LF5*TOPn

FOLDING ANGLE FOR $20 \mathrm{MeV} / \mathrm{u}^{20 \mathrm{Ne}}+{ }^{\prime \prime} \mathrm{Sm}$

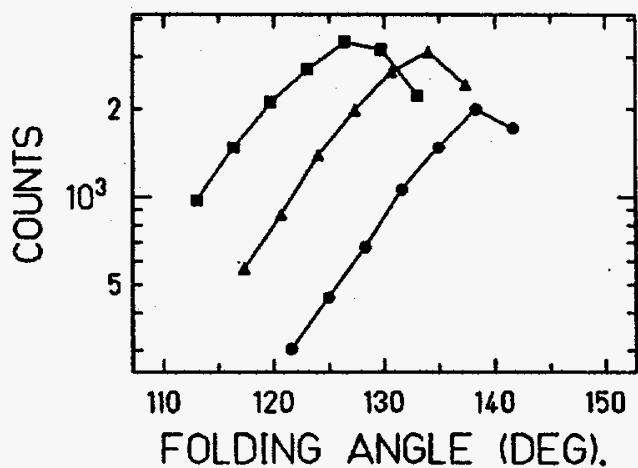

a TOPI*LPIn

A TOP2*LPIn

- TOP3*LPIn

Fig. 1(b). Fission fragment folding angle distribution for TOP1*LP1n, TOP2*LP1n, and TOP3*LP1*

MULIIPLICITY vs WEDGE in $20 \mathrm{MoV} / \mathrm{U}^{20} \mathrm{Ne}+{ }^{\mathrm{w}} \mathrm{S} \mathrm{Sm}$

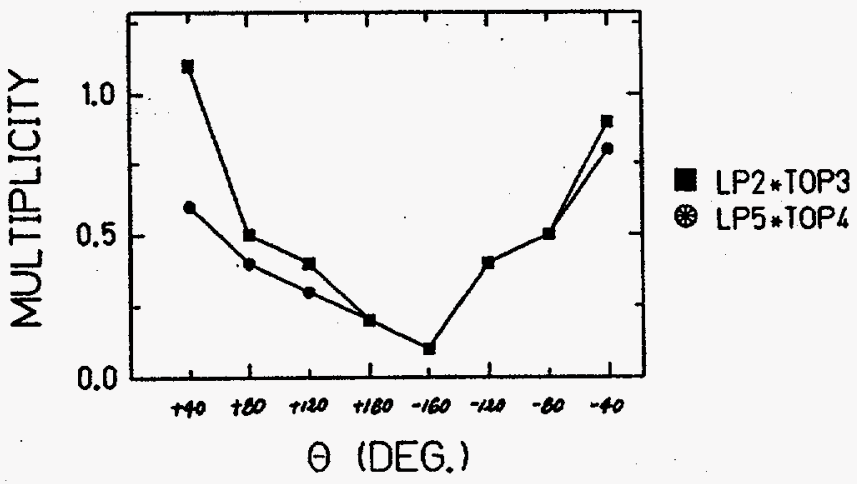

Fig. 2. Comparison of two sets of neutron multiplicities for the wedge detectors. 
(1) Brookhaven National Laboratory, Upton, New York

(2) INFN and Dipartimento di Fisica dell' Universita di Padova, Padova, Italy

(3) Instituto de Fisica, UNAM, Mexico D. F. 01000, Mexico

(9) INFN, Lab. Naz. Legnaro, 1-35020, Legnaro, Italy

(5) INFN and Dipartimento di Fisica dell' Universita di Bari, Bari, Italy

\section{REFERENCES}

1. M. Gonin et al., Phys. Rev. C42, 2125 (1990).

2. Y. Lou et al., Progress In Research, 1990-199!, Cyclotron Institute, Texas A\&M University, p. 6.

\title{
ENERGY DEPOSITION SYSTEMATICS IN $30 \mathrm{MeV} / \mathrm{u}{ }^{14} \mathrm{~N}$ AND ${ }^{63} \mathrm{Cu}$ REACTIONS
}

\author{
T. Botting, B. Hurst, L. Cooke, D. O'Kelly, W. Turmel, and R. P. Schmitt
}

Because of the strong correlations between neutron multiplicity and both momentum transfer and deposited excitation energy, $1 \pi$ neutron detectors are powerful tools for investigating reaction mechanisms and defining the conditions necessary for the production of hot nuclei. ${ }^{2}$ In a recent experiment, we have used our $4 \pi$ neutron ball ${ }^{3}$ to study the neutron multiplicity distributions for a variety of ${ }^{14} \mathrm{~N}$ - and ${ }^{\infty} \mathrm{Cu}$-induced reactions. The objectives of these measurements were two-fold: first, to obtain global systematics on energy deposition, and second, to explore the correlations between the neutron multiplicity and other parameters from the ball, such as the amplitude of the prompt energy signal. Depending on the strength of such correlations, it might be possible to run the detector in a fast mode. This latter mode would be very useful in performing certain classes of measurements which require a simple centrality trigger.

In the experiments $30 \mathrm{MeV} / \mathrm{u}{ }^{14} \mathrm{~N}$ and ${ }^{63} \mathrm{Cu}$ beams from the $K=500$ cyclotron were used to bombard a series of targets ranging from ${ }^{12} \mathrm{C}$ to ${ }^{238} \mathrm{U}$. The neutron ball was operated in a self-triggered mode. When a nuclear reaction occurs inside the ball, a prompt pulse is generally observed in the detector due to the thermalization of neutrons and the interaction of $\gamma$ rays in the detector. This prompt flash is followed by delayed flashes signaling the capture of the thermalized neutrons. When the neutron ball is self-triggered, a significant fraction of the triggers are produced by the detection of background $\gamma$ rays. This is especially a problem when the neutron ball is tuned for high detection efficiency. In the measurements described here, the ball was tuned to $85 \%$ efficiency for ${ }^{252} \mathrm{Cf}$ neutrons. Empirically, it was found that requiring at least four phototubes to fire on the prompt flash reduced the background rate to an acceptable level without significantly biasing the high folds of the neutron multiplicity distribution.

During the measurements a number of signals characterizing the prompt flash were recorded on tapi, including the pulse heights in the hemispheres and the wedges, as well as the multiplicity of phototubes firing in each sector of the detector. The number of delayed capture signals were measured for both hemispheres and all the individual wedges during a $100 \mu \mathrm{sec}$ counting gate (beginning $0.5 \mu \mathrm{sec}$ after the prompt flash) and a $100 \mu \mathrm{sec}$ background gate (beginning 120 $\mu$ sec after the prompt flash). The same information was recorded in ${ }^{252} \mathrm{Cf}$ calibration experiments for three source orientations relative to the median plane. These data will enable us to extract some information on the neutron angular distributions.

The data from the counting gates and the background gates were used to generate background corrected neutron multiplicity distributions for each reaction. Theste distributions were then corrected for losses due to the finite gate widths and pile-up effects. A number of normalized neutron multiplicity distributions, $P\left(M_{n}\right)$, are displayed in Figs. 1 \& 2 . It is interesting to note that each of these distributions was obtained in a relatively short bombardment, typically only about half an hour long.

For the lightest systems, $P\left(M_{n}\right)$ decreasiss monotonically with $M_{n}$. This behavior is partially explained by the relatively low neutron numbers of the systems and the strong competition between neutron and charged particle emission promoted by the low Coulomb barriers. Kinematical effects also contribute to this trend. The excitation energies are expected to be the lowest in the lightest systems. Moreover, the neutrons emitted by these light systems should be forward focused and boosted 


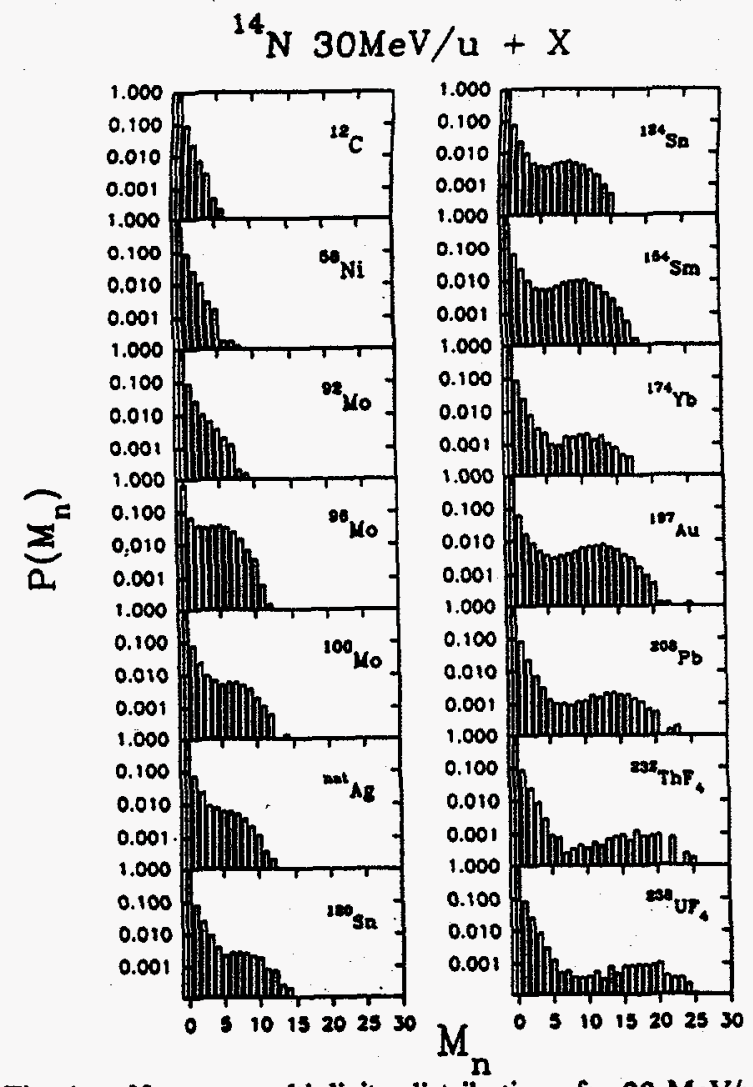

Fig. 1 Neutron multiplicity distributions for $30 \mathrm{MeV} / \mathrm{u}$ $14 \mathrm{~N}$ reactions with the indicated targets.

\section{3}

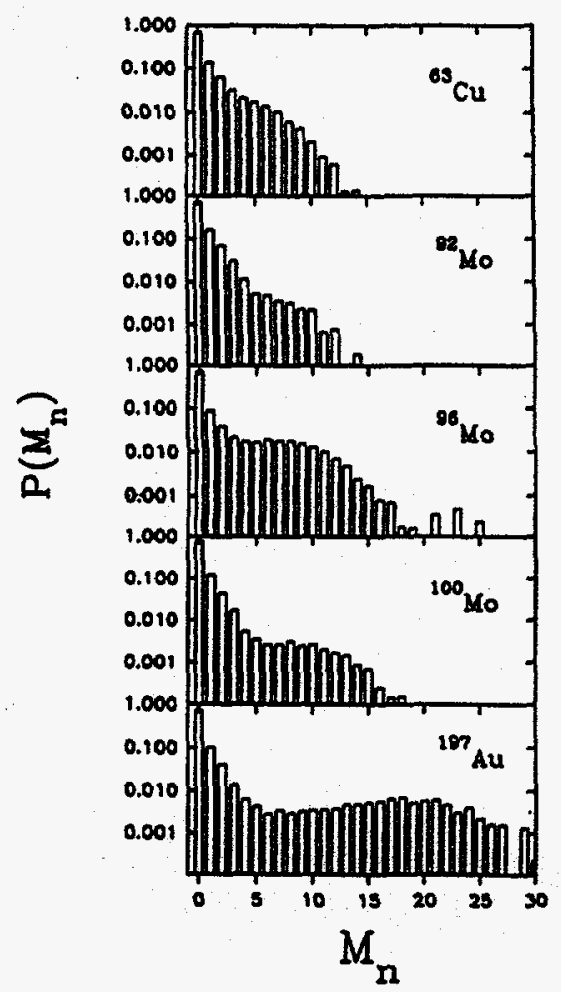

Fig. 2 Neutron multiplicity distributions for a variety of $30 \mathrm{MeV} / \mathrm{u}{ }^{63} \mathrm{Cu}$ reactions with the indicated targets. to high energies due to the relatively high source velocities. A significant fraction of these energetic, forward focussed neutrons can escape from the detector without being captured, depleting the high folds.

For medium mass and heavy targets, the distributions show two distinct components: a low multiplicity tail presumably due to peripheral processes and an approximately Gaussian shaped component which can be attributed to central collisions. One must be cautious in drawing conclusions about the relative probabilities of peripheral and central collisions from these data because self-triggering artificially enhances the yield of 0-fold events. Also, the low multiplicities are certainly enhanced for composite targets, such as $\mathrm{UF}_{4}$, and can also be influenced by possible oxidation of some of the targets. However, these complications should not have much influence on the position of the central collision bump.

In general, the peak of the high multiplicity component shifts towards higher $M_{n}$-values with increasing target mass. This seems easy to understand since an increase in excitation energy, a decrease in charged particle emission probability and, finally, an increase in fission probability are all expected to accompany an increase in target mass. The position of the high multiplicity component has been extracted from all the distributions showing a well-defined bump. These have been approximately corrected for the detector response assuming emission from a source moving with a velocity obtained from momentum transfer systematics. The results for the ${ }^{14} \mathrm{~N}+{ }^{19} \mathrm{Au}$ and ${ }^{63} \mathrm{Cu}+{ }^{197} \mathrm{Au}$ systems are shown in Fig. 3 together with similar data taken at GANIL for $\mathrm{Ar}, \mathrm{Kr}$ and $\mathrm{Pb}$ projectiles with about 30 $\mathrm{MeV} / \mathrm{u}^{2}$ The error bars on our data points mainly reflect

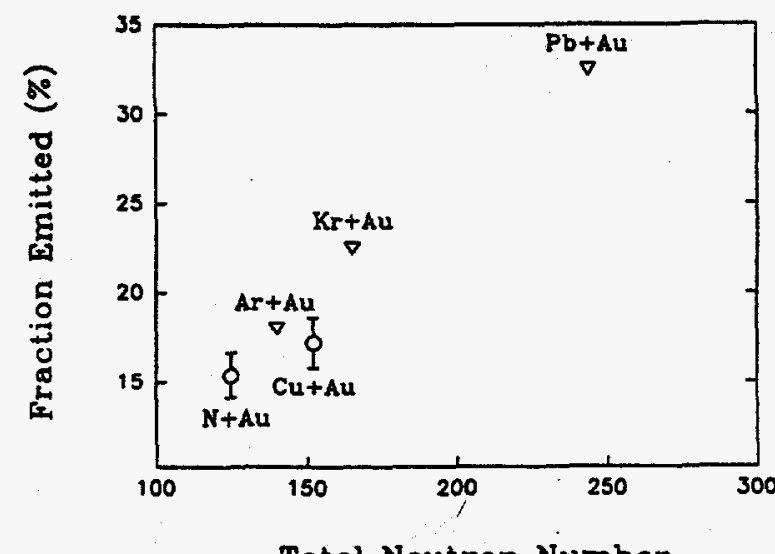

Total Neutron Number

Fig. 3 Mean neutron multiplicities for central collisions for several $30 \mathrm{MeV} / \mathrm{u}$ projectiles incident on a ${ }^{197} \mathrm{Au}$ target. 
the estimated uncertainty in the detection efficiency. The $14 \mathrm{~N}$ point is in good agreement with the results of Piasecki ef al., ${ }^{2}$ but the ${ }^{6} \mathrm{Cu}$ point seems to fall a bit low. Possibly, the detection efficiency has been underestimated for the ${ }^{63} \mathrm{Cu}$-induced reactions.

We are currently in the process of developing a detailed simulation of the neutron ball's response using a highly modified version of the code DENIS. ${ }^{4}$ This version can handle emission from one or more moving sources and portrays the geometry of the neutron ball in detail. The results of this simulation together with the experimental neutron angular distribution data should enable us to obtain a reasonable handle on the energy deposition in the various reactions.

\section{REFERENCES}

1. J. Galin, eㅡ al., Z. Phys. A331, 63 (1988).

2. E. Piasecki, et al., Phys. Rev. Lett. 66, 1291 (1991).

3. Progress in Research, 1990-1991, Cyclotron Institute, Texas A\&M University, p. 104.

4. J. Poitou and C. Signarbieux, Nucl. Inst. Meth. 136, 511 (1976).

\title{
GDR $\gamma$-RAY EMISSION IN $120 \mathrm{MeV}{ }^{16} 0$-INDUCED FISSION OF ${ }^{208} \mathrm{~Pb}$
}

\author{
W. Turmel, T. Botting, L. Cooke, B. Hurst, D. O'Kelly, R. P. Schmitt, H. Utsunomiya
}

Since their discovery, $\gamma$ rays emitted by giant dipole resonances (GDR) built on excited states ${ }^{1}$ have generated much interest because they can provide new information on the shapes and other characteristics of hot nuclei. ${ }^{2}$ Recent studies involving fissioning systems ${ }^{3}$ have shown that the GDR $\gamma$ rays can provide insights into the dynamics of the fission process similar to those obtained in fission-neutron coincidence experiments. ${ }^{4,5}$ Besides yielding the time scale of the fission process, GDR $\gamma$ ray emission can also provide information on the shapes of rapidly rotating nuclei.

As described elsewhere, ${ }^{6}$ we recently performed experiments on GDR $\gamma$ ray emission in $120 \mathrm{MeV}{ }^{16} \mathrm{O}$ induced reactions to explore nuclear shapes and time scales as a function of exit channel mass asymmetry. The results for the $\mathbf{O}+\mathrm{Pb}$ system suggested a mass dependence of the energy of the prefission GDR $\gamma$ ray component. However, because the statistics were somewhat marginal, we repeated the measurement with greatly improved efficiency. To this end, we built an array of four parallel plate avalanche counters (PPAC) each with an active area of $14 \mathrm{~cm} \times 14 \mathrm{~cm}$ and a position resolution of $1 \mathrm{~mm}$. The PPAC's were arranged in a symmetrical manner about the target: two were placed in the horizontal and two in the vertical planes. The PPAC's covered the approximate angular range $50-130^{\circ}$ in the lab system. The gamma rays were detected with 16 of the total energy crystal's $18 \mathrm{Nal}$ segments. These were placed in 4-packs each at a distance of $60 \mathrm{~cm}$ from the target. Two of these clusters were located at $90^{\circ}$ while the other two were placed at $135^{\circ}$. This experimential geometry was chosen to obtain information on both the inplane and out-of-plane $\boldsymbol{\gamma}$-ray angular distributions.

We have made a preliminary analysis of the data. A.s in previous studies, the timing and the position information from the PPAC's were used to kinematically reconstruct the masses of the fission fragments. Because the $\gamma$ rays were timed with respect to the fission events, it was necessary to make corrections for the different flight times of the fragments. This was accomplished by simply adding the time difference for the detection of the two fission fragments to timing signals from the gamma detectors. Time vs. energy contour plots for the uncorrected and the corrected data are shown in Figs. 1 id 2 , respectively. It can be seen that the neutron-gamma separation is quite good in the GDR region. Figure 3 shows the total gamma ray spectrum (i.e., sum of all $\gamma$ ray detectors) gated on fission fragments. One observes a very pronounced bump visible in the spectrum in the region where GDR $\gamma$ rays are expected (about $11 \mathrm{MeV}$ for the compound system and $15 \mathrm{MeV}$ for the fragments).

The initial results indicate differences in $\boldsymbol{\gamma}$-ray spectra gated on different on mass asymmetries. There also appear to be some significant angular distributicin effects. We are presently in the process of refining our kinematical mass calculations to characterize these effects. Given the available statistics, we should be able to extract fission times and shape information for several mass cuts. 
The recent completion of the $\mathrm{BaF}_{2}$ array together with improvements in our fission detector array should greatly facilitate future studies in this area.

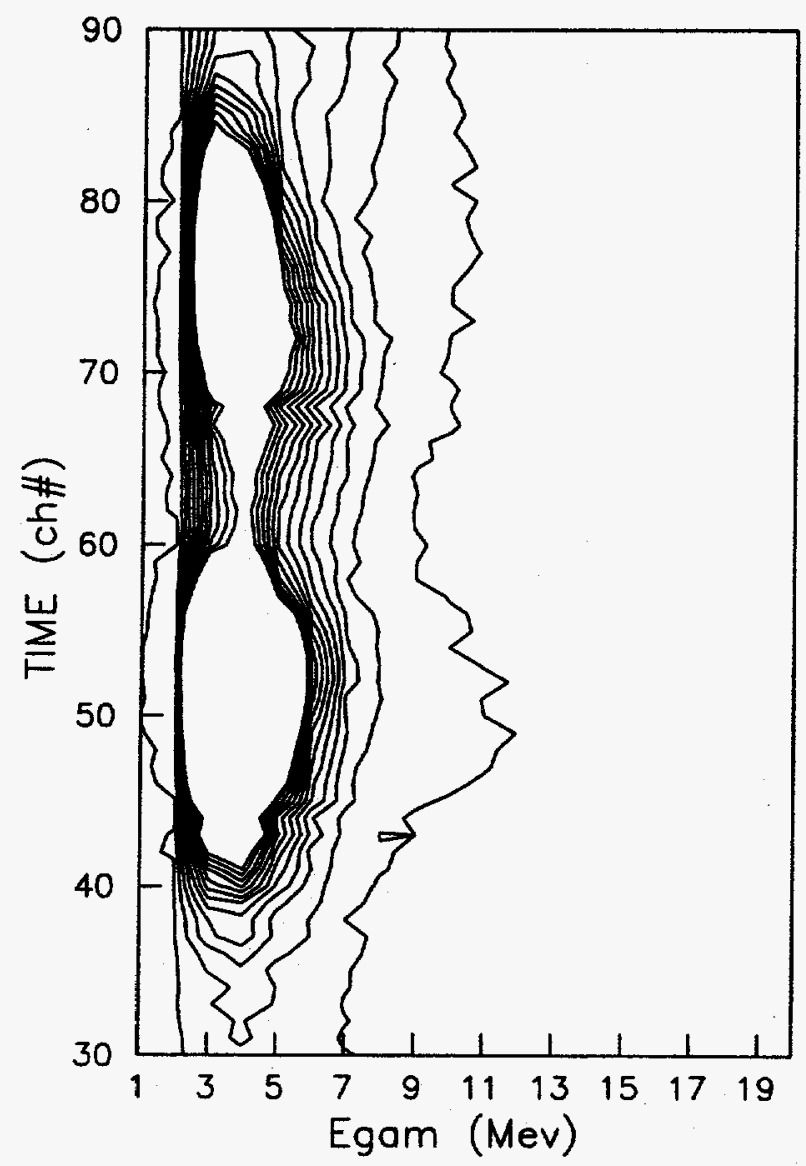

Fig. 1 Contour plot showing the distribution of events in the plane defined by the raw timing and energy.

\section{REFERENCES}

1. J. O. Newton et al., Phys. Rev. Lett. 46, 1383 (1981).

2. K. A. Snover, Ann. Rev. Nucl. Part. Sci., 36, (1986).

3. M. Thoennessen et al., Phys. Rev. Lett. 59, 2860 (1987).

4. A. Gavron et al., Phys. Rev. C 35, 579 (1987), and references therein.

5. D. J. Hinde es al., Phys. Rev. C 45, 1229 (1992).

6. W. Turmel a al., Progress in Research, 1990-1991, Cyclotron Institute, Texas A\&M University, p. 2.

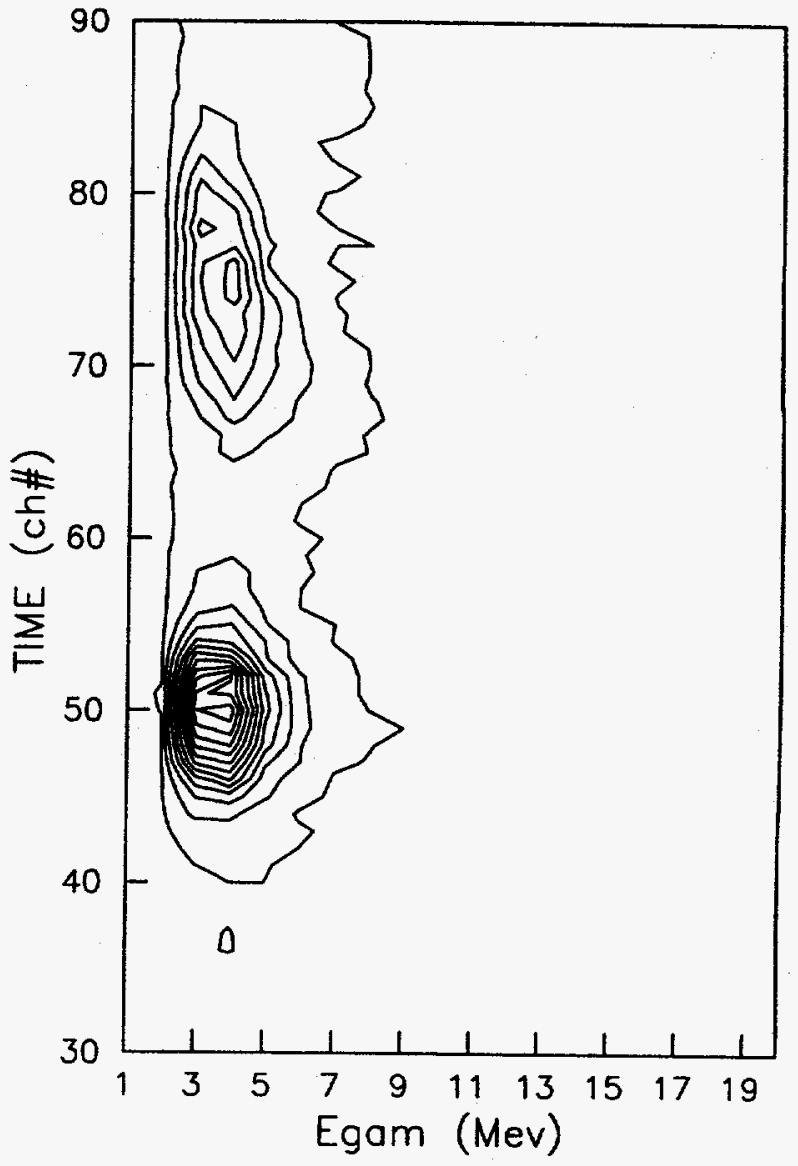

Fig. 2 Same as Fig. 1 after timing corrections.

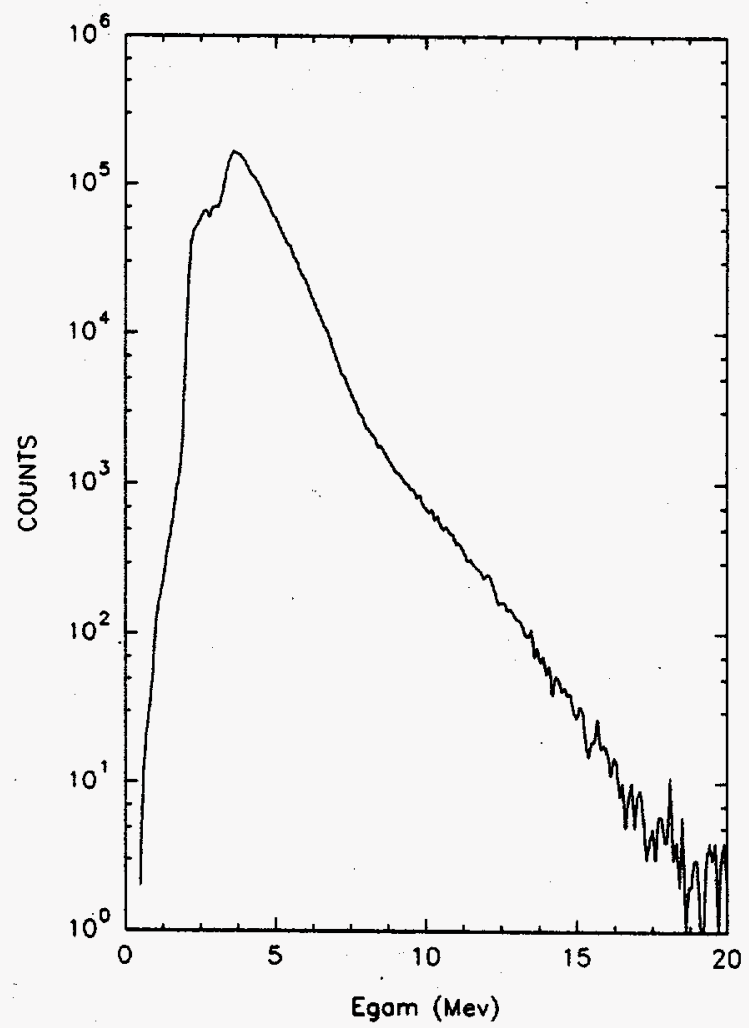

Fig. 3 Sum spectrum of $\boldsymbol{\gamma}$ rays detected in coincidence with fission fragments. 
Because the number of evaporated neutrons is strongly correlated with the excitation energy of medium mass and heavy nuclei, event-by-event neutron multiplicity $\left(\mathbf{M}_{n}\right)$ determinations offer new opportunities in studies of both particle spectroscopy and nuclear reaction mechanisms. As an example, simultaneous measurements of $M_{n}$ and the kinetic energies of projectile-like fragments can help separate transfer reactions from processes involving sequential decay. It should also be possible to study the characteristics of charged particle spectra for specific $M_{n}$ values, providing new, very restrictive tests of the statistical model. Moreover, since $M_{n}$ is expected to be anti-correlated with spin, such studies could also yield insights into charged-particle emission from high-spin nuclei while avoiding troublesome questions about the spin removed by the emitted particles.

We have recently performed a test experiment to investigate some possible applications of our $4 \pi$ neutron ball as an exit channel filter. To this end, we have studied projectile-like fragments produced in the reaction 30 $\mathrm{MeV} / \mathrm{u}{ }^{20} \mathrm{Ne}+{ }^{208} \mathrm{~Pb}$. This particular system was selected to see if we could shed some light on the origin of certain continuum structures reported in a variety of inelastic scattering experiments involving both low and intermediate energy heavy ions. ${ }^{1}$ While these structures have sometimes been cited as evidence for multi-phonon excitations of giant resonances (GR) at high excitation energies, they have also been attributed to various sequential decay processes. ${ }^{2}$

In the experiment, a $30 \mathrm{MeV} / \mathrm{u}^{20} \mathrm{Ne}$ beam was used to bombard a self-supporting $1 \mathrm{mg} / \mathrm{cm}^{2}{ }^{208} \mathrm{~Pb}$ target. The measurements were performed without the most forward wedges of the neutron ball (which subtend $\pm 20^{\circ}$ ) to facilitate the placement of particle detectors at small angles. Projectile-like fragments were detected using two triple-element, charged particle telescopes. Each telescope consisted of a 300 and two $1000 \mu \mathrm{m}$ thick surface barrier transmission detectors collimated to $285 \mathrm{~mm}^{2}$. The telescopes were placed near the grazing angle $\left(\theta_{\text {lab }} \cong\right.$ $\left.10^{\circ}\right)$ about $35 \mathrm{~cm}$ from the target. Energy signals were recorded from each detector of the telescopes. The timing was determined by the middle elements. An event was triggered by the logical OR of the particle telescope timing signals. Energy calibration of the telescopes was accomplished in beam using the elastic peak and graded pulser signals. Detection efficiencies were measured off- line using a calibrated ${ }^{241} \mathrm{Am} \alpha$ source at the target position. The telescopes provided good $\mathbf{Z}$ separation from $\mathrm{Be}$ to $\mathrm{Na}$.

A variety of neutron ball parameters were recordid for each event to test the capabilities of the device. These included the sum energy $\left(E_{\text {som }}\right)$ and the phototube multiplicity $\left(M_{p}\right)$ of the prompt flash, as well as, scalers for the delayed flashes detected during counting and background gates for the top, bottom and median plane sections. Scalers during these two gates were also recorded for the entire ball and each of the eight individual wedge segments. The phototube multiplicity requirement for delayed $\gamma$ rays (capture $\gamma$ 's or background $\gamma$ 's) was made in hardware. We required that two or more tubes fire in the top or bottom hemispheres or one or more tube(s) fire in the median plane. If the logical $C R$ of these conditions was met during an active gate, the appropriate scaler was incremented. The counting and background gates were both $100 \mu s$ wide. They were delayed relative to the prompt flash by .62 and $100 \mu \mathrm{s}$, respectively.

Calibration of the neutron ball was performed in coincidence measurements between fission fragnents and neutrons from a ${ }^{252} \mathrm{Cf}$ source. In order to determine neutron angular distribution data from the median plane detectors, the fragments were measured in different geometries. In one measurement, a collimated $\left( \pm 5^{\circ}\right) \mathrm{Si}$ counter was used to detect fragments emitted normal to the median plane of the ball yielding an isotropic distribution. In the second measurement, the fragments were detected in the median plane at $\theta=90^{\circ}$, which produced an anisotropy of about two in the median plane elements. The overall neutron detection efficiency ranged between $80-85 \%$ for the two source orientations. The results were in good agreements with Monte-Carlo simulations. The neutron ball's response to calibrated ${ }^{60} \mathrm{Co}$ and ${ }^{209} \mathrm{Bi} \gamma$-ray sources was also recorded. These signals were used to aid in matching the gains of the two hemispheres and the median plane so that a total promipt flash sum energy parameter could be made off-line. A total prompt flash phototube multiplicity parameter was also generated in software.

Figure 1 shows the correlation of the centroid of the $E_{\text {aum }}$ of the prompt flash and the $M_{p e}$ threshold. Both of these parameters were gated on $Z=8$ particles with energies between about $300-550 \mathrm{MeV}$. One can see that 
$M_{\mathrm{pk}}$ is approximately linearly correlated with the average sum energy. Initially, we hoped that by requiring both high $M_{p}$-values and high $E_{a m}$, it would be possible to select high $M_{n}$ events, allowing one to run the neutron ball as a fast trigger to pick out central collisions.

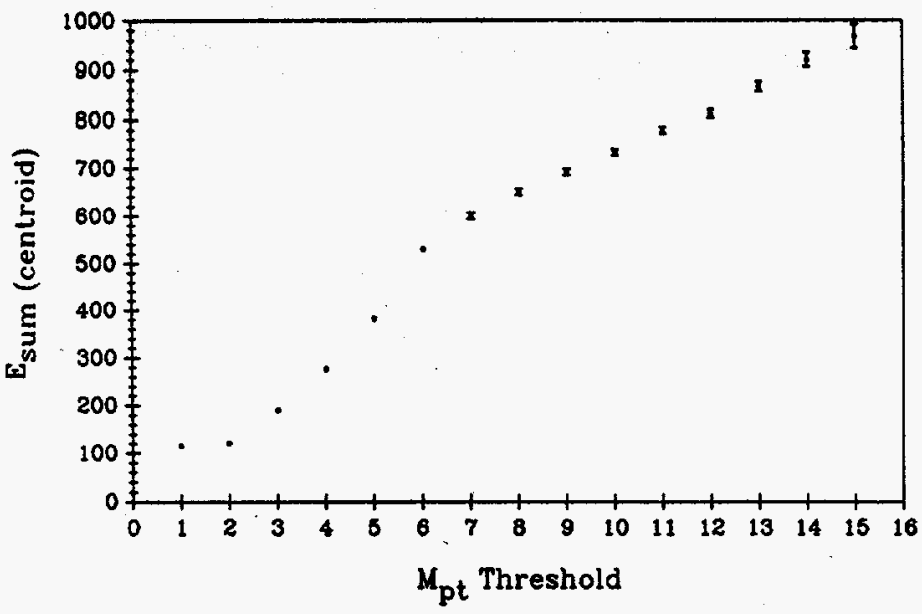

Fig. 1 Centroid of the sum energy signal versus the minimum number of phototubes firing during the prompt flash.

The results of the current test show that the correlation between $M_{z}$ and both $M_{p}$ and $E_{\text {min }}$ are weak for projectile-like fragments, probably due to the relatively low $M_{n}$ values together with the significant background contributions (1-2 per gate) in the measurements. Preliminary results using less biased trigger conditions indicate that $M_{p}$ and $E_{\text {sum }}$ can still be quite useful in using the neutron ball to key on central collisions with other devices, such as the $\mathrm{BaF}_{2}$ array.

The correlation between background corrected $M_{n}$ and the laboratory kinetic energy of projectile-like fragments is illustrated in Fig. 2 for $Z=8$ particles. As seen with other ejectiles, at the highest kinetic energies $M_{n}$ increases rapidly with increasing energy loss, corresponding to about one neutron per 13-15 MeV lost energy. This is compatible with mainly transfers to the target-like fragment. For energy larger than about 50 $\mathrm{MeV}$, this trend gives way to a more gradual increase in $\mathbf{M}_{n}$, presumably because of overlapping contributions from transfer and breakup reactions. Figure 3 shows another correlation of interest, $M_{n}$ verus $Z$ of projectilelike fragments stopped in the second element of the telescopes. The rapid rise in $M_{n}$ with decreasing $Z$ clearly implies increased mass transfers and energy depositions for lighter ejectiles.

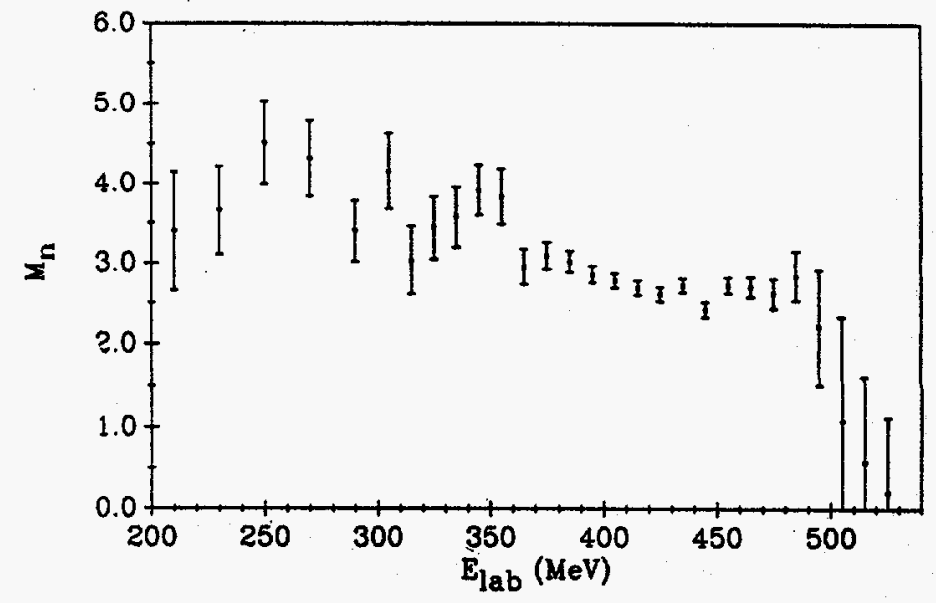

Fig. 2 Neutron multiplicity as a function of kinetic energy for $Z=8$ ejectiles.

We are currently involved in a detailed analysis of the data to better characterize the neutron ball's capabilities as an exit channel filter for both peripheral and central collisions. Somewhat contrary to expectations, it appears that for peripheral reactions the best exit channel selectivity may be obtained when the detector is tuned for relatively low neutron detection efficiencies, in the range of 50-70\%, mainly due to the associated reduction in room and beam related background. We plan to test this further in the near future.

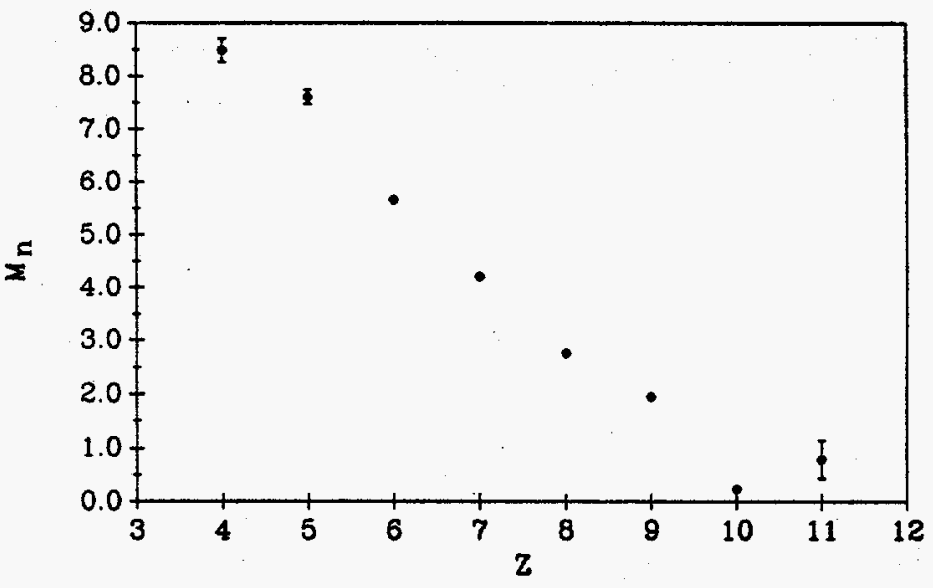

Fig. 3 Neutron multiplicity as a function of $Z$ for projectile-like fragments.

\section{REFERENCES}

1. P. Chomaz, et al., Z. Phys. A318, 41 (1984).

2. D. Hilscher, et al., Phys. Rev. C20, 575 (1979). 


\title{
INVESTIGATIONS OF ENTRANCE CHANNEL EFFECTS IN THE DECAY OF 142Nd COMPOUND NUCLEUS PRODUCED IN ${ }^{16} \mathrm{O}+{ }^{126} \mathrm{Te}$ and ${ }^{22} \mathrm{Ne}+{ }^{120} \mathrm{Sn}$ REACTIONS
}

\author{
R. K. Choudhury, K. Wolf, R. P. Schmitt, K. Hagel, R. Wada, R. Erkert, H. Jabs, N. Jevtic, \\ Y. Lou, D. Miller, J. Shoemaker, W. Turmel, L. Whitesell, J. B. Natowitz
}

In recent years, there has been growing interest in studying the dynamics of the heavy ion reaction processes leading to fusion of two heavy nuclei at intermediate bombarding energies. Experiments are being carried out to study the different aspects of the collision dynamics and to measure the relaxation times in the mass, energy, and shape degrees of freedom of the fusioning system. Entrance channel mass asymmetry may also play some role in governing the reaction dynamics and the fusion time scales in the heavy ion reactions at medium bombarding energies. There have been many attempts to investigate the entrance channel effects in fusion reactions by looking at the differences in the particle and $\gamma$ emission spectra and multiplicities from the same compound system formed by different projectile-target combinations. Recent measurements of Thoennessen, et a‥, ${ }^{1}$ on ${ }^{100} \mathrm{Er}$ and ${ }^{164} \mathrm{Yb}$ compound nuclei produced by collisions of very different entrance channel mass asymmetries indicate significant differences in the $\boldsymbol{\gamma}$ ray energy spectra in the GDR region for both the systems. Since the GDR $\gamma$ rays are predominantly emitted during the initial stages of the compound nucleus decay, they carry direct information on the CN shapes formed in the collision process. In the present work, we have selected the ${ }^{16} \mathrm{O}+{ }^{126} \mathrm{Te}$ and ${ }^{2} \mathrm{Ne}$ $+{ }^{120} \mathrm{Sn}$ reactions producing the ${ }^{142} \mathrm{Nd}$ compound nucleus at $150 \mathrm{MeV}$ excitation energy to look for differences in the GDR and particle spectra with entrance channel mass asymmetry. The above two systems are expected to lie on either side of the Businaro-Gallone critical mass asymmetry and may follow different shape evolutions during the dynamical equilibration leading to the fusion process.

The experiments were performed using the beams from the K-500 cyclotron. The $\gamma$ rays were detected by two 19-pack $\mathrm{BaF}_{2}$ arrays ${ }^{2}$ placed at $90^{\circ}$ and $135^{\circ}$ with respect to the beam direction and at a distance of $65 \mathrm{~cm}$ and $55 \mathrm{~cm}$, respectively, from the target. The compound nuclear residues were detected with two large area parallel plate avalanche counters and one $\mathrm{Si}$ surface barrier detector placed at a distance of about $30 \mathrm{~cm}$ from the target covering the angular range of $6^{\circ}-20^{\circ}$ in the forward direction. Two particle detector telescopes were used at $90^{\circ}$ and $120^{\circ}$ to detect the light charged particles. The $\gamma$ ray energy spectra were recorded in coincidence with the residue and particle detectors and the timing of all the detectors was done with respect to the cyclotron RF. The compound nuclear residues could be identified unambiguously from the TOF spectrum of the residue detectors. Gamma, neutron discrimination in the $\mathrm{BaF}_{2}$ detectors was also done using the TOF spectrum in theste detectors with respect to the cyclotron RF. The $q$ ray energy was obtained by summing the energy output of all the 19 detectors in each pack after suitable gain normalization and the energy threshold for the experiment was set at about $4.0 \mathrm{McV}$ in the sum energy. The charged particle energies were determined from ${ }^{2 / 1} \mathrm{Am} \alpha$ source and pulser calibrations.
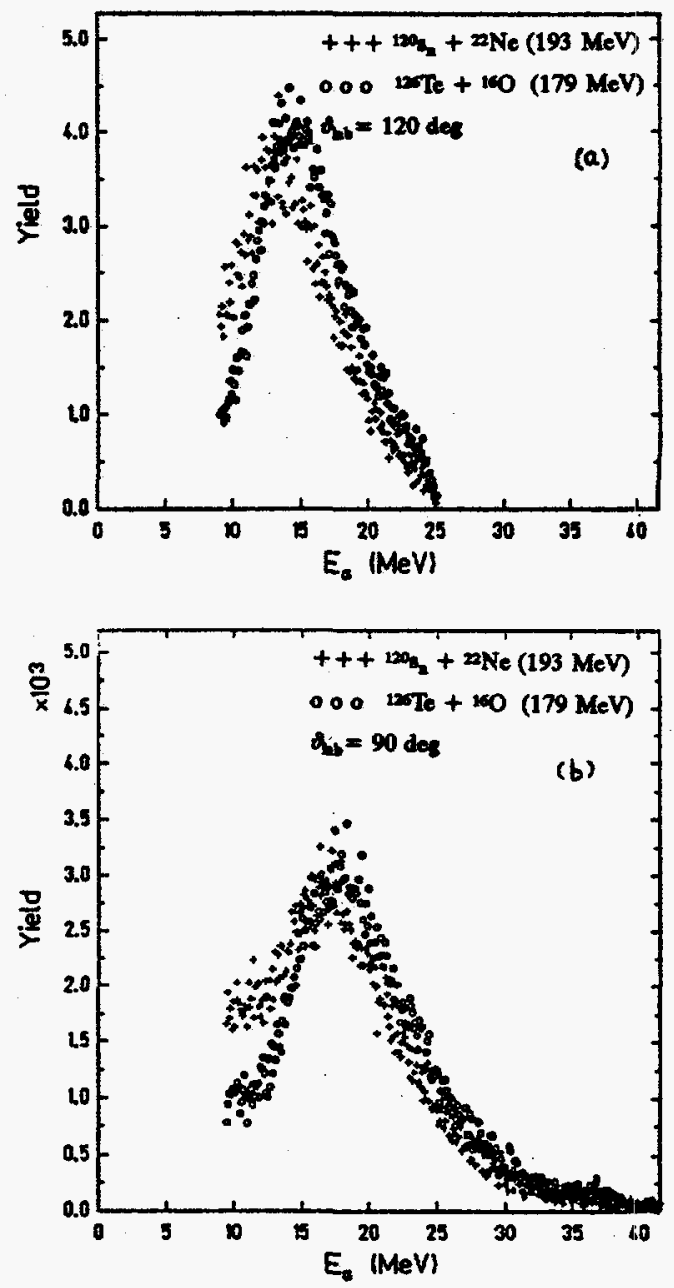

Fig. 1 (a)\&(b) Measured $\alpha$ particle spectra at $120^{\circ}$ and $90^{\circ}$ for the O+Te and $\mathrm{Ne}+\mathrm{Sn}$ reactions. 
Figures 1 (a) and (b) show the energy spectra of the $\alpha$ particles measured at the laboratory angles of $120^{\circ}$ and $90^{\circ}$, respectively, for both systems. The shift in the energy spectra to lower energies for the $\mathrm{Ne}+\mathrm{Sn}$ system as compared to the $\mathbf{O}+$ Te system is consistent with the higher recoil velocity of the compound nucleus in the former case. Figure 2 shows the measured $\gamma$ ray energy aspects for both systems. The statistical $(E<10 \mathrm{MeV})$ and the Bremsatrahlung continuum ( $E>20 \mathrm{MeV}$ ) components were fitted by two exponential distributions as shown by the solid lines in the figure. The GDR yield

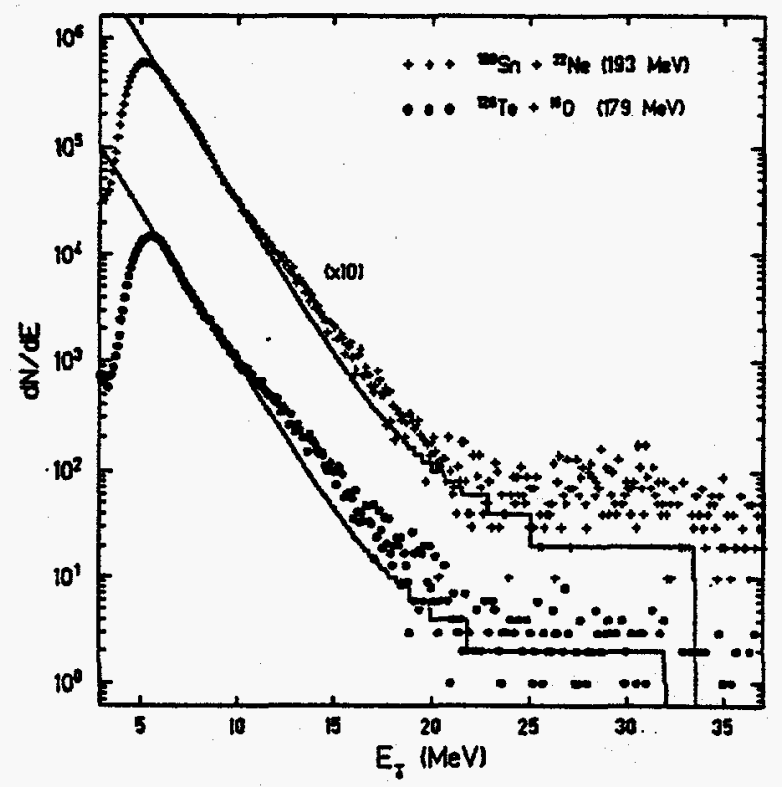

Fig. 2 Gamma ray energy distribution for $\mathrm{O}+\mathrm{Te}$ and $\mathrm{Ne}+\mathrm{Sn}$ reactions in coincidence with compound nuclear residues and light particles.

was obtained by subtracting these components from the measured spectra. It was found that the GDR yield normalized to the total $\boldsymbol{\gamma}$ ray yield is reduced in the case of the $\mathrm{Ne}+\mathrm{Sn}$ system a compared to the $\mathrm{O}+\mathrm{Te}$ system by a factor of about 1.6 to 1.8. The shapes of the GDR spectra were obtained from the ratio of the measured $\gamma$ ray spectra to the smooth fitted lines as shown in Fig. 3. The oxygen data were fitted with a Lorentzian distribution with the resonance energy of $14.88(0.18) \mathrm{MeV}$ and the width parameter of $6.94(0.2) \mathrm{MeV}$, which agree with the available systematics for this mass region. It is seen that within the present statistical errors, there is no discernible difference in the shape of the GDR spectrum for the $\mathrm{Ne}+$ $\mathrm{Sn}$ case. The reduced GDR strength in the case of $\mathrm{Ne}+$ Sn reaction as compared to the $\mathrm{O}+$ Te system needs further investigation. Also, it is seen that for the Ne + Sn system, the $\alpha$ particle spectrum shows somewhat enhanced yield in the low energy region as compared to the $\mathbf{O}+\mathrm{Te}$ system. Further experiments are being carried out to examine these features in more detail.

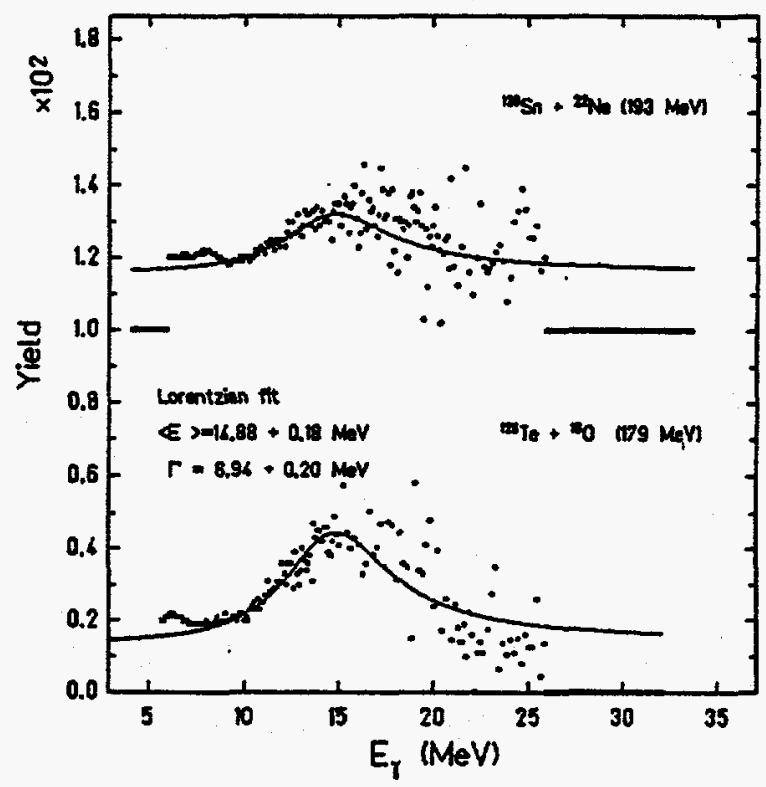

Fig. 3 GDR energy spectra and fits with Lorentzian distributions.

\section{REFERENCES}

1. M. Thoennessen and J. R. Beene, MSUCL Report 799 (1991).

2. J. Shoemaker, K. Wolf, R. Choudhury, Progress in Research, 1990-1991, Cyclotron Institute, Texas A\&M University, p. 108. 
T. E. Cowan,* M. W. Clark, * J. H. Hartley,* J. L. McDonald,* J. W. McDonald,*

R. R. Rohatgi, * D. H. G. Schneider, * R. L. Watson, V. Horvat, W. H. Trzaska

Despite years of study, the origin of the narrow correlated $\mathrm{e}^{+} \mathrm{e}^{-}$peaks discovered in superheavy nuclear collision experiments at GSI Darmstadt, ${ }^{1}$ remains an unsolved mystery in nuclear physics. It has been widely muggested that the $\mathrm{e}^{+} \mathrm{e}^{-}$lines may signal the production and subsequent two-body decays of a new family of particlelike objects having masses of 1-2 $\mathrm{MeV} / \mathrm{c}^{2}$, however no independent support for this hypothesis has yet been found in a variety of particle search experiments. ${ }^{2}$ Additional high-statistics systematic investigations of the $\mathrm{e}^{+} \mathrm{e}^{-}$peaks in heavy-ion collisions are urgently needed to understand this phenomenon. We have developed a new High Efficiency Coincident Lepton Spectrometer (HECLS) which provides almost 25 times greater detection efficiency for the $e^{+} e^{-}$peaks than the GSI instruments, making such investigations possible for the first time. ${ }^{3}$ We installed the HECLS spectrometer in beamline 7 at the K500 in November 1991, and have begun a search for $e^{+} e^{-}$peaks in collision systems much lighter than previously measured at GSI, where the production crosssection is expected to decrease. In this contribution we report the results of a first experiment in ${ }^{120} \mathrm{Xe}+{ }^{19} \mathrm{Au}$ collisions.

\section{Experimental Apparatus}

The HECLS spectrometer, shown in Fig. 1, consists of a 2.7 Tesla superconducting solenoid transport system oriented coaxially to the ion beam. Positrons and electrons emitted from the target site at the center of the high field region, spiral along magnetic field lines to detector arrays both upstream and downstream of the target. Their helical orbits expand and elongate adiabatically as they are transported out of the strong field region. Electrons are separated from positrons as they pass through a transverse magnetic field formed by samarium-cobalt permanent magnets before being collected in separate $\mathrm{Si}(\mathrm{Li})$ detector arrays, as indicated in Fig. 2. Positrons are unambiguously identified by the coincident detection of their $511 \mathrm{keV}$ annihilation quanta in a box-shaped array of large volume NaI crystals (from the Cyclotron Institute's $2 \pi$ sum spectrometer) surrounding the $\mathrm{Si}(\mathrm{Li})$ detectors.

The $30 \times 60 \mathrm{~mm}^{2}$ by $2.5 \mathrm{~mm}$ thick $\mathrm{Si}(\mathrm{Li})$ detectors are segmented in order to accommodate both the high counting rate and large multiplicity of electrons produced

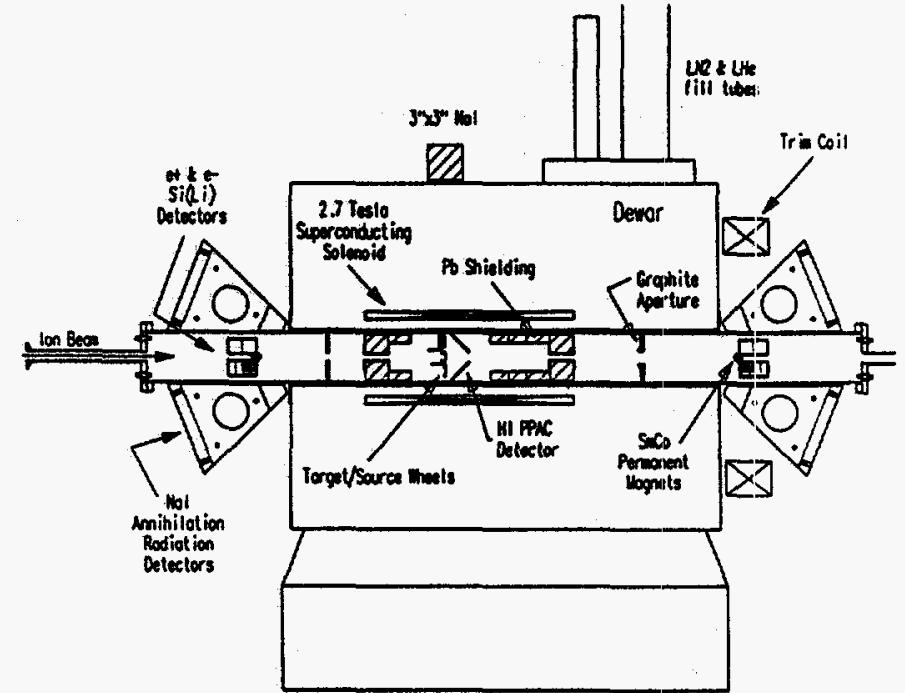

Fig. 1. Diagram of the HECLS spectrometer. Ion bean enters along the axis from left. Positrons and electrons created at target (center) are transported to $S i(L i)$ detector arrays upstream and downstream of target. Scattered heavy-ions are detected in array of gas PPACs.

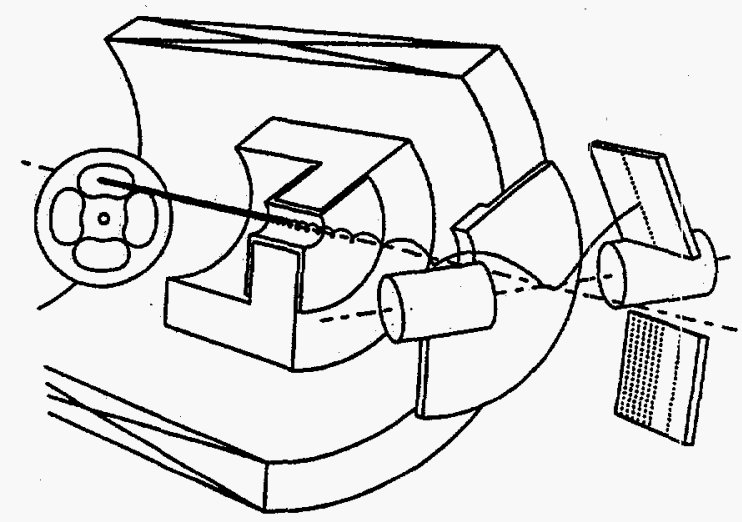

Fig. 2. Ray-tracing calculation of a $400 \mathrm{keV} e+$ in the HECLS spectrometer, showing the adiabatic expansion of its orbit and deflection by SmClo permanent magnets into the $\mathrm{Si}(\mathrm{Li})$ detector. Note the target wheel and graphite and lesid shielding.

in superheavy collisions without pileup limitations. The energy resolution of the alcohol-cooled $\mathrm{Si}(\mathrm{Li})$ 's is $\approx 12$ $\mathrm{keV}$ (FWHM). Lead and graphite shielding inside the vacuum chamber eliminated X-ray, gamma-ray, and external conversion $\mathrm{e}^{+} \mathrm{e}^{-}$backgrounds in the $\mathrm{Si}(\mathrm{Li})$ 's and Nal detectors.

Both the scattered $\mathrm{Xe}$ projectile and $\mathrm{Au}$ recoil ions are detected in kinematic coincidence in a pyramidal array 
of four parallel plate avalanche counters (PPAC's) operated with isobutane at 5 Torr. The $\mathrm{Xe}+\mathrm{Au}$ angular resolution is $=5^{\circ}$, dominated by the beam spot size and multiple scattering in the $0.8 \mathrm{mg} / \mathrm{cm}^{2}$ Au target foils.
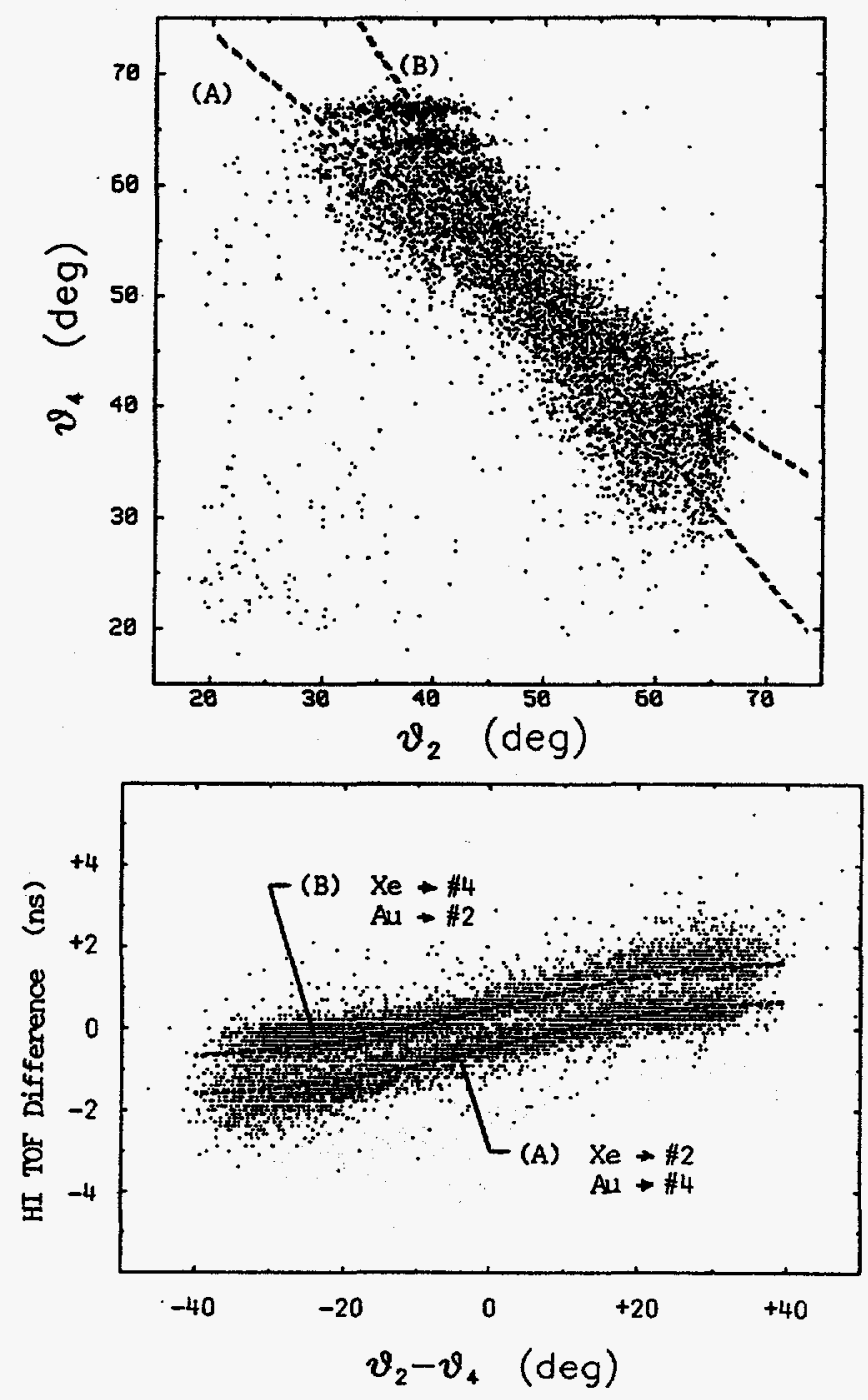

Fig. 3. (A) Angle-angle plot of heavy-ion events, in coincidence with positrons, detected in PPACs "2 \& :4. (B) Time-of-Flight difference for the heavy-ions, plotted versus the difference of their measured scattering angles. Dashed lines denote the kinematic branches. The TOF separation allows determination of the identity of each ion, and hence the CM scattering angle and impact parameter.

(The intrinsic PPAC resolution is $2.4^{\circ}$.) As shown in Fig. $3 \mathrm{a}$, this is not sufficient to resolve the kinematic branches. The PPAC time resolution ( $\approx 600 \mathrm{ps}$ ), however, is sufficient to completely separate the kinematic branches by time-of-flight (Fig. 3b), allowing an unambiguous identification of each ion and determination of the CM scattering angle. The PPAC angular acceptance is $20^{\circ}-67^{\circ}$, corresponding to a $\mathrm{CM}$ scattering angle range of $46^{\circ}-104^{\circ}$ for binary coincidence events.

Nuclear gamma-rays are measured in a $3^{\text {" }} \times 3^{\prime \prime} \mathrm{Nal}$ detector at $90^{\circ}$ w.r.t. the beam. After transit through the spectrometer, the average beam velocity is measured to better than $10^{-3}$ accuracy using an electrostatic convoyelectron spectrometer.

\section{Experimental Results}

The Cyclotron Operations staff developed a high current ${ }^{129} \mathrm{Xe}$ beam for this experiment, which required the early installation of one of three cryopanels which had been planned to improve the median plane vacuum of the $\mathrm{K} 500$. It operated successfully at both $77^{\circ} \mathrm{K}$ and $4.2^{\circ} \mathrm{K}$, and enabled high current extraction of up to 2 particle $n A$ of ${ }^{129} \mathrm{Xe}$ beam. We typically took data with $=0.5-1 \mathrm{pnA}$ of beam on target. Because of the low combined nuclear charge, the delta electron background was much less in $\mathrm{Xe}+\mathrm{Au}$ than in heavier collision systems studied at GSI (e.g., U+Ta \& U+Th). The electron detector count rates ( $\approx 20 \mathrm{kHz}$ in each segment) were much lower than what HECLS can accommodate $(=200 \mathrm{kHz})$. Despite running at only $10 \%$ of the HECLS data-taking capacity, in 4 days of production run we still accumulated a total of $=52500$ $\mathrm{e}^{+} \mathrm{e}^{-}$pairs at 5.9 and $6.0 \mathrm{MeV} / \mathrm{amu}$, more than half of the entire sample from all EPOS Collaboration experiments at GSI.

Figure $4(a, b)$ show the positron and electron energy distributions for the coincident $\mathrm{e}^{+} \mathrm{e}^{-}$pair events. In the heavier collision systems the large delta-electron background obscured individual nuclear transitions, however in these data a strong electron conversion line at $190 \mathrm{keV}$ is apparent. The dependence of its Dopplerbroadened width on ion scattering angle indentifies it as being associated with the $\mathrm{Xe}$ projectile, presumably a highly converted transition at $=220 \mathrm{keV}$. The presence of structure in the electron distribution requires that care be taken so that no sum-energy peaks are artificially produced in the sum-energy and difference-energy projections described below. The total sum-energy distribution of the coincident pairs is rather smooth and structureless, as shown in Fig. 4c.

A "minimum bias" search for two-body decay candidates in this experiment involves examining the subset of $\mathrm{e}^{+} \mathrm{e}^{-}$pairs in which the leptons are emitted with approximately equal energies, into opposite arms of the spectrometer (i.e., a preference for back-to-back emission). Figure 5 shows the $\mathrm{E}_{\mathrm{e}^{+}}$vs. $\mathrm{E}_{\mathrm{e}^{-}}$energy correlation plane for the opposite hemisphere subset of events ( $=40 \%$ of the total sample - same hemisphere pairs constitute the remaining 60\%). The sum-energy 
distribution of events within the wedge-shaped contour " $S$ " is shown in Fig. 6a, where the dashed curve indicates the "background" distribution obtained from the total data sample.
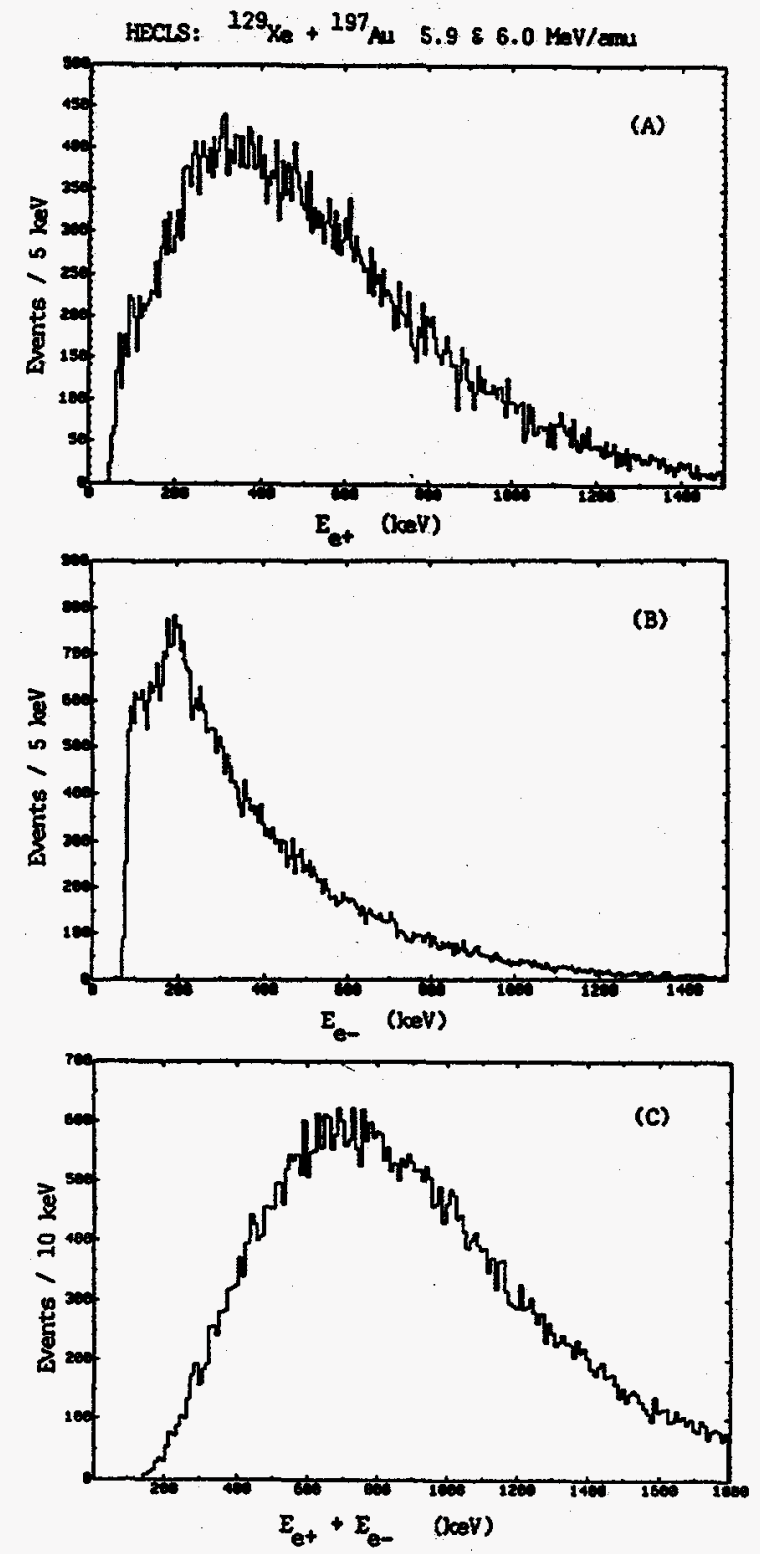

Fig. 4. Positron (A), electron (B), and $\mathrm{e}^{+} \mathrm{e}^{-}$sum-energy (C) spectra of positrons and electrons detected in coincidence in 6.0 and $5.9 \mathrm{MeV} / \mathrm{amu} \mathrm{Xe+Au}$ collisions. A $190 \mathrm{keV}$ electron conversion line is apparent superimposed on smooth exponential electron continuum in (B).

The most prominent feature in this spectrum is a 70 \pm 18 count $(3.8$ o) excess of events in a $20 \mathrm{keV}$ wide peak at a mean sum-energy of $965 \pm 5 \mathrm{keV}$. Figure $6 \mathrm{~b}$ shows the difference-energy spectrum for this line corresponding to events in contour " $D$ " in Fig. 5. This peak exhibits the characteristic narrow sum-energy peak and much wider difference-energy distribution expected for a two-body decay candidate. A peak at this energy has not previously been established in the GSI measurements, and the probability of a statistical fluctuation of this magnitude anywhere within our spectrometer band-pass is approximately 1\%. Ws therefore estimate the statistical significance of this line to be $=99 \%$. The intensity of the $965 \mathrm{keV}$ line $(70$ counts in our $5 \times 10^{4} \mathrm{e}^{+} \mathrm{e}^{-}$pair sample) is comparable to that of previous GSI data (e.g., 40-100 counts in $3 \times 10^{4}$ pair samples), however the relative size of the signal above the continuum background appears to be smaller. Much weaker $(<2 \sigma)$ excesses are present in Fig. $6 \mathrm{a}$ at the energies of previously observed sum-energy lines: $=545,640$, and $800 \mathrm{keV}$.

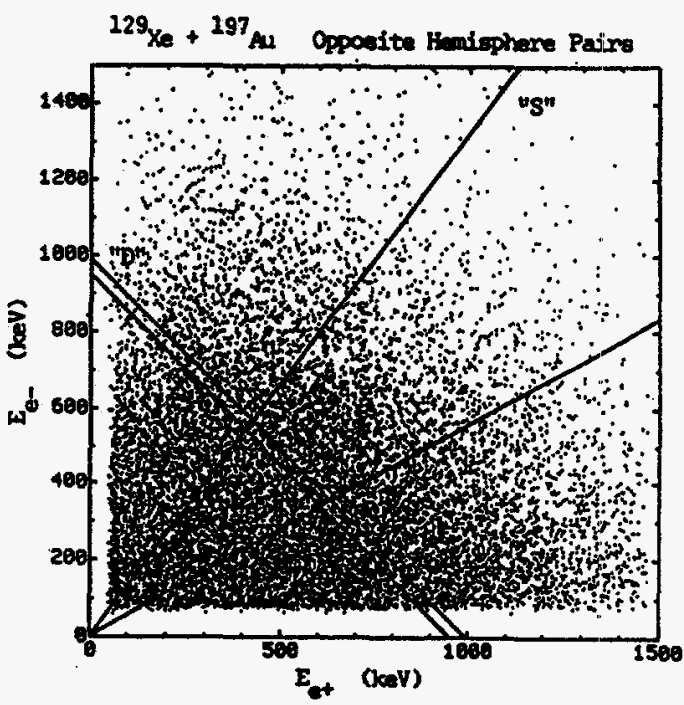

Fig. 5. $\mathrm{Ee}^{+}$vs. $\mathrm{E}_{\mathrm{e}^{-}}$correlation plot of $e^{+} e^{-}$pairs emitted into opposite arms of the spectrometer (i.e., upstream $\mathrm{e}^{+} \&$ downstream $e^{-}$, or downstream $\mathrm{e}^{+} \&$ upstream $\mathrm{e}^{-}$). Contours " $\mathrm{D}^{\text {" }}$ and " $S$ " indicate cuts shown in Fig. 7.

Some of the GSI lines exhibit a correlation with heavy-ion impact parameter. Figure 7a shows data for small impact parameter Xe+Au collisions, corresponding to projectile scattering angles of $60^{\circ}<\theta_{X_{e}}<67^{\circ}$. A 58 \pm 19 count $(3 \sigma)$ sum-energy line at $652 \pm 10 \mathrm{keV}$ is observed within a wedge-cut the same width as shown in Fig. 5 but shifted so as to be symmetric about $\mathrm{E}_{\mathrm{e}^{+}}=\mathrm{E}_{\mathrm{e}^{-}}$.

The corresponding difference-energy spectrum is shown in Fig. $7 \mathrm{~b}$. This line may correspond to the $=620-640 \mathrm{keV}$ line observed at GSI. ${ }^{1}$ Like the GSI data, this peak doess not exhibit solely "back-to-back" lepton emission in the laboratory frame, and both opposite- and same-hemisphere lepton detector combinations are included in these spectra. 
${ }^{129} \mathrm{Xe}+{ }^{197} \mathrm{Au}$ Opposite Henisphere $\mathrm{e}^{+} \mathrm{e}^{-}$Pairs
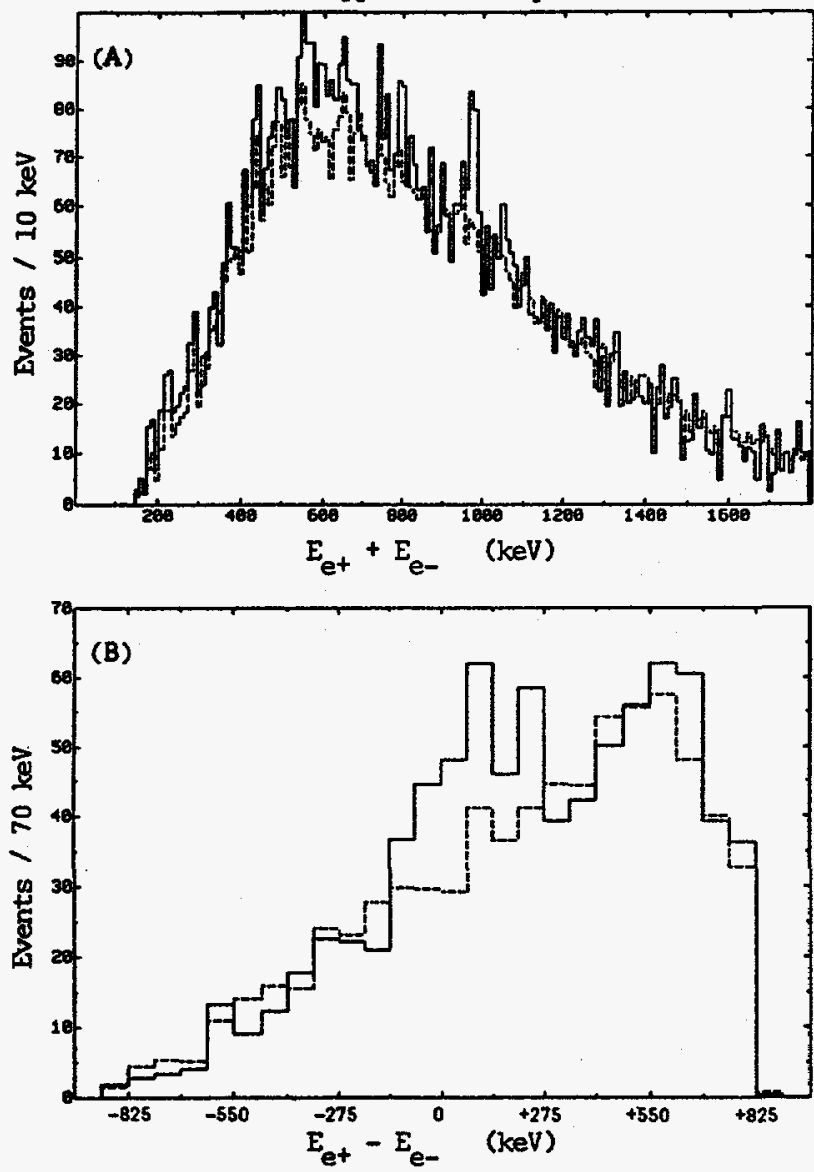

Fig. 6. Coincident $e^{+} e^{-}$pairs emitted into opposite arms of the spectrometer (i.e., upstream $\mathrm{e}^{+} \&$ downstream e; or downstream $\mathrm{e}^{+} \&$ upstream e). (A) Sum-energy distribution for events within wedge-shaped contour " $S$ " in Fig. 5. (B) Difference-energy distribution gated on sumenergies between 945 to $984 \mathrm{keV}$ (contour " $D$ " in Fig. 5). Dashed curves show shape of underlying background events, extracted from neighboring kinematic regions.

\section{Discussion and Summary}

If the existence of these candidate $e^{+} e^{-}$lines at 650 and $965 \mathrm{keV}$ is confirmed in our upcoming run, this discovery of narrow sum-energy peaks in a light collision system could have far reaching consequences for theoretical attempts to explain these lines. The combined nuclear charge of the Xe+Au system $\left(Z_{u=}=133\right)$, is much lower than in the lightest system previously measured $\left(T h+T a, Z_{w}=163\right)$. Many theoretical and phenomenological models (for example, those postulating a new phase of QED) have assumed that the strong Coulomb field of the colliding nuclei is an important or essential ingredient, ${ }^{2}$ and may have to be modified or abandoned. Perhaps coincidentally, these lines lie close to the 1.66 and $2.01 \mathrm{MeV}$ continuum resonances predicted
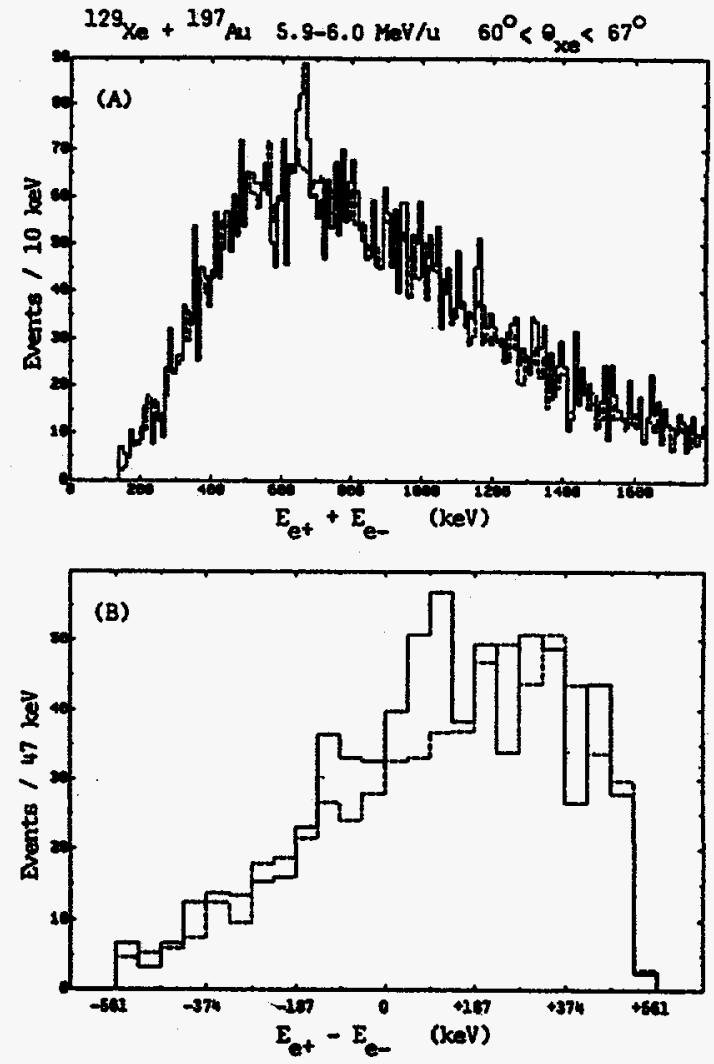

Fig. 7. (A) Sum-energy distribution for $e^{+} e^{-}$pairs detected in coincidence with small impact parameter Xe+Au collisions. (B) Differenceenergy distribution gated on sum-energies between 640 to $669 \mathrm{keV}$. Dashed curves show shape of underlying background events, extracted from neighboring kinematic regions.

by Spence and Vary, ${ }^{4}$ in which the Coulomb field is not required for their existence but does affect the production cross-section via the line width.

In summary, we have completed the construction of the HECLS spectrometer at the K500 and achieved its principal design goal of very high $\mathrm{e}^{+} e^{-}$collection efficiency. In a first search for correlated $\mathrm{e}^{+} e^{-}$emission in low $\mathrm{Z}_{\mathrm{w}}$ collisions, we have observed a candidate twobody decay signal at a $\approx 99 \%$ C.L. at a sum-energy of $965 \mathrm{keV}$, as well as evidence for a $650 \mathrm{keV}$ line which may correspond to lines observed in previous high $\mathrm{Z}_{\mathrm{w}}$ collisions at GSI. In future runs, we plan to expand our base of low $Z_{w e}$ collision data, and begin systematic measurements of the $e^{+} e^{-}$peak production cross-sections versus ion beam energy, scattering angle, and combined nuclear charge.

We are indebted to the operations staff and scientific leadership of the Cyclotron Institute for both their warm 
hospitality and their extensive efforts in developing the ${ }^{129} \mathrm{Xe}$ beam. Development of the HECLS spectrometer has been supported by LLNL and by the DOE Division of Nuclear Physics under contract No. W-7405-ENG-48.

* Department of Physics, Lawrence Livermore National Laboratory, Livermore, California

\section{REFERENCES}

1. T. Cowan et al., (EPOS Collaboration), Phys. Rev. Lett. 56, 444 (1986); W. Koenig et al., (ORANGE Collaboration), Phys. Lett. B 218, 12 (1989);
P. Salabura eㅡ al., (EPOS Collaboration), Phys. Lett. B 245, 153 (1990).

2. Recent reviews of the experimental and theoretical status are given in B. Müller, in Physics of Highly Ionized Atoms, ed. R. Marns (Plenum, New York, 1988); and A. Scherdin at all., Rep. Prog. Phys. 54, 1-52 (1991).

3. T.E. Cowan, "Positron-Electron Spectrosicopy in Heavy-Ion Collisions," Research Proposal to DOIE Office of High Energy and Nuclear Physics, UCRL, PROP-107346 (1991).

4. J.R. Spence, J.P. Vary, Phys. Lett. B 254, 1 (1991). 


\section{NUCLEAR STRUCTURE AND FUNDAMENTAL INTERACTIONS}




\section{DEUTERON ELASTIC SCATTERING}

\section{A. C. Betker, C. A. Gagliardi, D. R. Semon, R. E. Tribble, H. M. Xu, and A. F. Zaruba}

We have measured deuteron elastic scattering crosssections at $110 \mathrm{MeV}$ on $\mathrm{C}$ and ${ }^{208} \mathrm{~Pb}$ with the Texas A\&M K500 cyclotron. The motivation for this experiment is that, except for some limited data above $200 \mathrm{MeV}$, there exists no elastic scattering data above $90 \mathrm{MeV}$. In order to calculate deuteron optical potentials in the energy range accessible by the $\mathrm{K500}, 110-160 \mathrm{MeV}$, these elastic scattering cross-sections must be measured. The immediate interest in this data is to obtain optical potentials with which the $\left(d,{ }^{2} \mathrm{He}\right)$ data taken with the proton spectrometer can be analyzed.

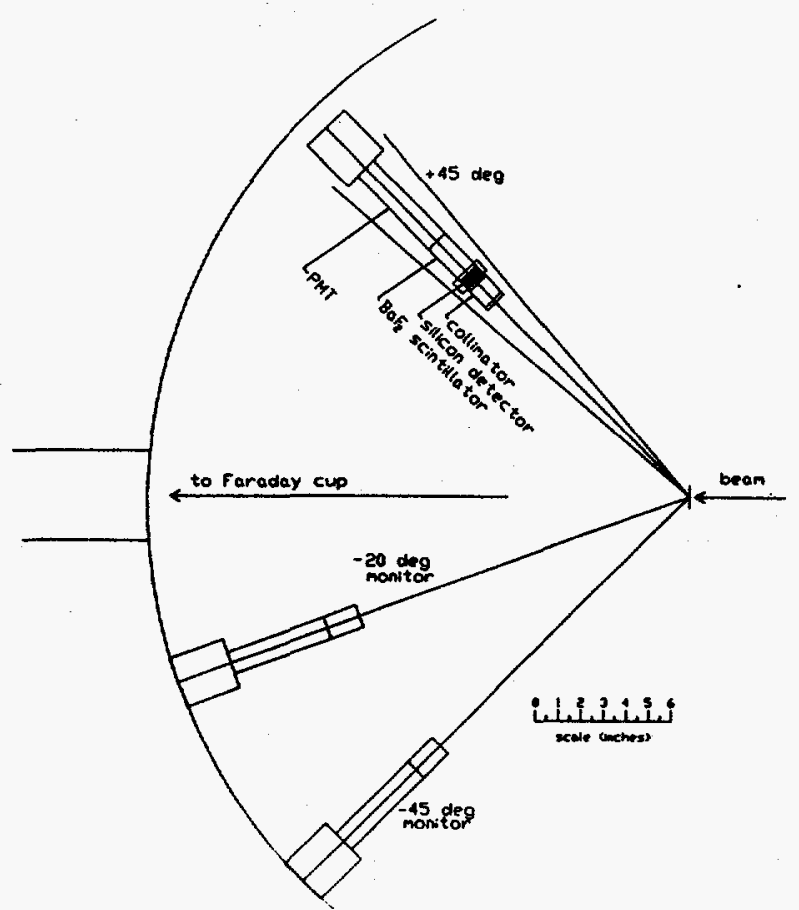

Fig. 1. The experimental apparatus

The experimental setup is shown in Fig. 1. The beam enters from the right. The detector telescope is mounted on a tumtable that was rotated from $-10^{\circ}$ to $+60^{\circ}$. The first element in the telescope is a collimator that, along with the mounting position, defines the solid angle. The solid angles for the two data runs were .159 and $.128 \mathrm{msr}$. After the collimator is a $1 \mathrm{~mm}$ thick transmission mount silicon surface barrier detector for a $\triangle E$ signal, which is followed by a 2 inch thick, $3 / 4$ inch diameter $\mathrm{BaF}_{2}$ scintillator coupled to a Hammamatsu R1397 photomultiplier tube that provides a total energy signal. The energy resolution for this system was about 2
$\mathrm{MeV}$ full width at half maximum. The beam that does not interact with the target goes on to a Faraday cup at $0^{\circ}$ which is connected to an integrator. A monitor detector, another $\mathrm{BaF}_{2}$ scintillator, was mounted at $-20^{\circ}$ for detector angles to $+25^{\circ}$, and at $-45^{\circ}$ for the larger detector angles. The purpose of this detector was to provide a consistency check on the beam current integration and to provide the correct normalization at the smallest angles where the beam intensity was so low that the integrator was unreliable. The overall systematic normalization uncertainty of $5 \%$ arises from target thickness non-uniformity and beam current integration.

There are two deuteron global optical model potentials currently available. The first, from Daehnick, et al., ${ }^{2}$ covers the mass range of $A=27$ to $A=238$. Some ${ }^{12} \mathrm{C}$ and ${ }^{24} \mathrm{Mg}$ data are included at 80 and $90 \mathrm{MeV}$ with reduced weights because not much higher mass data exists at these energies. The full energy range covered is thus 11.8 to $90 \mathrm{MeV}$. Both relativistic and non-relativistic forms of the potential were extracted, but this made little difference when the potentials were extrapolated to our data. The relativistic potential is used in the analysis below.

The other global potential is from Bojowald, et al. ${ }^{3}$ This potential covers the mass range from $A=12$ to $A=208$ and the energy range. from 52 to $85 \mathrm{MeV}$. This group took additional data at 58.7 and $85 \mathrm{MeV}$ on several targets, so their data set includes more higher energy work. Only non-relativistic potentials were extracted.

Both Daehnick and Bojowald optical potentials have the same general form:

$$
\begin{aligned}
V(r)= & V_{R} f\left(r, r_{0}, a_{0}\right)+i W_{s} f\left(r, r_{1}, a_{l}\right)-i a_{l} W_{D} d / d r\left[f\left(r, r_{1}, a_{)}\right)\right] \\
& +V_{L S}(L \cdot S)(1 / r) d / d r\left[f\left(r, r_{L S}, a_{L S}\right)\right]+V_{\text {Coubanb }}
\end{aligned}
$$

where

$$
f\left(r, r_{i}, a_{i}\right)=\left[1+\exp \left(\left(r-r_{i} A^{1 / 3}\right) / a_{j}\right)\right]^{-1}
$$

is the standard Woods-Saxon form.

The higher energy data that is available is from Nguyen Van Sen et al. ${ }^{1}$ Cross-sections along with vector and tensor analyzing powers were measured on ${ }^{58} \mathrm{Ni}$ from 200 to $700 \mathrm{MeV}$, and ${ }^{\circ} \mathrm{Ca}$ at $200 \mathrm{MeV}$. This group found that the Daehnick potential described the shape of their data well, and provided a good starting point for a fit. 
Our carbon data, along with the potentials and fits, are shown in Fig. 2. The error bars are typical statistical errors at the higher angles. Statistical errors at the lower angles are much smaller than the size of the points. The data vary smoothly from the lower energy data and, as expected, are slightly smaller in intensity and slightly more forwand peaked. Note that the Dachnick potential is even more forward peaked than the data, and somewhat larger in intensity. The Bojowald potential has the correct phase, but is also too large in intensity. Both fits, carried out with the optical model search code CUPID 4 and using the two global potentials as starting points, describe the date quite well. The fitting is done by $x^{2}$ minimization.

\section{$12 \mathrm{C}(\mathrm{d}, \mathrm{d}) 12 \mathrm{C}, 110 \mathrm{MeV}$}

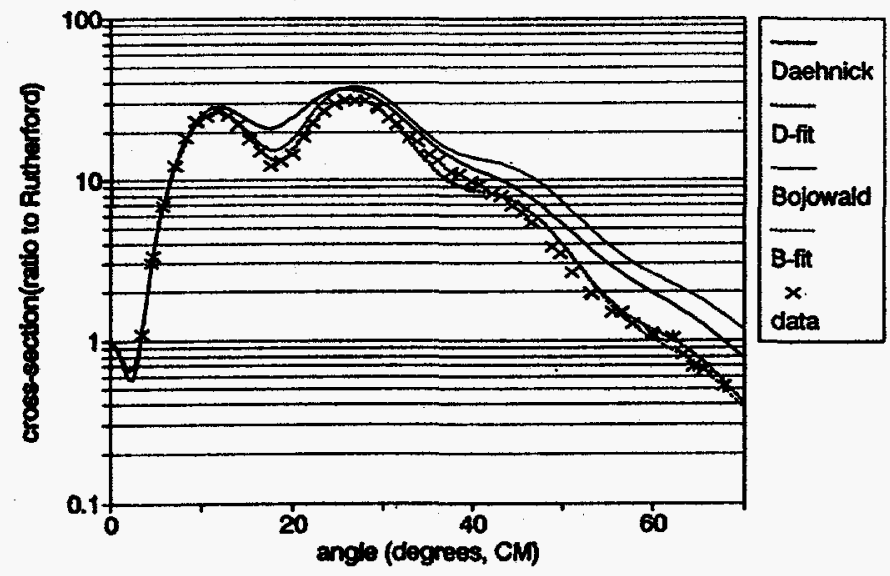

Fig. 2. The Carbon data, along with the predictions of two global optical model potentials, and fits to the data from these potentials. The error bars shown are typical at the larger angles. Small angle error bars are smaller than the points.

Figure 3 shows our lead data. Again, the error bars shown are statistical and typical at those angles. The data are slightly more forward peaked and lower in amplitude than at lower energies, as with carbon. The Daehnick potential is again slightly out of phase with the data, this time with the potential less forward peaked. The intensity of the fit is quite good. The Bojowald potential is too low in amplitude overall, but has the correct phase. Again, both starting points provide fits that agree with the data quite well.

Table 1 shows the optical model parameters from the extrapolated global potentials and the fits on both targets. Both global potentials were qualitatively close. With the limited data that we have, the only significant mass and energy dependent patterns that can be seen are in the imaginary terms. Both carbon fits have the increased absorption in the imaginary volume integral needed to decrease the scattering cross-section. The Bojowald fit on lead has the decreased absorption needed to increase the cross-section. The Daehnick lead fit is very close to its starting point. The mass dependencies in the imaginary terms can be seen by comparing carbon to lead. In Daehnick's potential the difference is all in the diffuseness, $a_{1}$. In Bojowald's potential both $a_{1}$ and the depth of the surface potential, $W_{D}$, change as a function of A. Looking at the fitted parameters, we can see that in the Daehnick case the variation in $a_{1}$ is too large across the mass range at this higher energy. In the Bojowald case, the variation of $a_{1}$ is correct but the overall magnitude is smaller, and the difference in $W_{D}$ is not as large. With the data available, we can fit equally well to either form of the potential, but there is a trend for the mass dependence to be somewhat reduced over what lower energy potentials would imply in both cases.

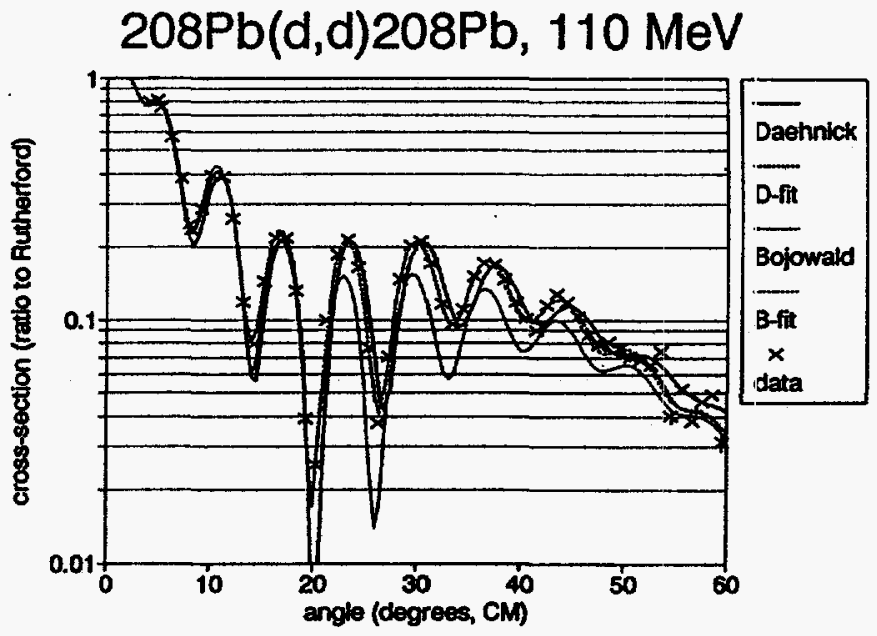

Fig. 3. The Lead data, potentials, and fits, as in Fig. 2.

Since this data were taken we have obtained a new $\mathrm{NaI}$ scintillator to replace the $\mathrm{BaF}_{2}$. We hope that this new detector will give us the increased energy resolution needed to separate the elastic peak from excited states for intermediate mass nuclei such as ${ }^{58} \mathrm{Ni}$ and ${ }^{116} \mathrm{Sn}$. Plans are also underway to repeat these measurements at higher energies when the K500 can provide the higher energy beams.

\section{REFERENCES}

1. Nguyen Van Sen, et al., Phys. Lett. 156B, 185 (1985).

2. W. W. Daehnick, et al., Phys. Rev. C 21, 22:53 (1980).

3. J. Bojowald, et al., Phys. Rev. C 38, 1153 (1988).

4. J. R. Comfort, Computer Phys. Commun. 16, 35 (1978). 
Table 1

Optical Model Parameters

\begin{tabular}{|c|c|c|c|c|c|c|c|c|}
\hline & \multicolumn{4}{|c|}{${ }^{12} \mathrm{c}$} & \multicolumn{4}{|c|}{$208_{\mathrm{Pb}}$} \\
\hline & Daehnick & Daehnick-fit & Bojowald & Bojowald-fit & Daehnick & Daehnick-fit & Bojowald & Bojowald-fit \\
\hline$v_{R}$ & -59.18 & -60.00 & -58.67 & -60.17 & -69.05 & -68.37 & -74.71 & -68.17 \\
\hline$r_{0}$ & 1.17 & 1.16 & 1.18 & 1.14 & 1.17 & 1.18 & 1.18 & 1.19 \\
\hline$a_{0}$ & 0.849 & 0.765 & 0.716 & 0.748 & 0.849 & 0.811 & 0.843 & 0.810 \\
\hline$w_{s}$ & -10.82 & -11.34 & -8.58 & -9.12 & -10.82 & -10.94 & -8.58 & -8.14 \\
\hline$w_{0}$ & -18.38 & -19.01 & -16.29 & -17.36 & -18.38 & -18.61 & -31.41 & -29.31 \\
\hline$r_{I}$ & 1.271 & 1.319 & 1.270 & 1.356 & 1.271 & 1.295 & 1.270 & 1.273 \\
\hline $\mathbf{a}_{1}$ & 0.666 & 0.697 & 0.816 & 0.741 & 0.895 & 0.873 & 0.892 & 0.845 \\
\hline$v_{\text {LS }}$ & -7.36 & -7.39 & -12.00 & -10.88 & -7.36 & -10.47 & -12.00 & -13.01 \\
\hline$r_{\text {LS }}$ & 1.07 & 1.12 & 0.867 & 0.921 & 1.07 & 1.11 & 1.01 & 1.07 \\
\hline$a_{L S}$ & 0.66 & 0.7 & 0.867 & 0.902 & 0.66 & 1.30 & 1.01 & 1.14 \\
\hline \multicolumn{9}{|c|}{ Volume Integrals (J/A) } \\
\hline Real & -793.8 & -721.3 & -684.8 & -682.4 & -531.8 & -538.3 & -588.0 & -545.1 \\
\hline Imaginary & -268.3 & -315.1 & -278.8 & -302.8 & -164.9 & -172.3 & -184.1 & -167.8 \\
\hline spin-orbit & -19.0 & -19.9 & -26.0 & -25.0 & -2.8 & -4.15 & -4.3 & -5.0 \\
\hline
\end{tabular}

\title{
$\mathrm{M}_{\mathrm{n}} / \mathrm{M}_{\mathrm{p}}$ MEASUREMENTS USING $490 \mathrm{MeV}{ }^{14} \mathrm{~N}$ COULOMB
}

\author{
Y. -W. Lui, D. H. Youngblood, W. Liu and H. M. Xu
}

It is known that the deformation parameters $B$ are widely different from experiments using different projectiles and this systematic difference is explained by Madsen aㅡ a‥' for single-closed-shell nuclei and later extended to the open-shell nuclei. ${ }^{2}$. These variations are due to different contributions of the isoscalar and isovector parts of the collective deformation parameters $\left(B_{p}\right.$ and $\left.B_{q}\right)$ from different transition mechanisms. It is commonly assumed that the deformation parameter is an intrinsic property of the target nucleus, therefore, independent of its excitation by different projectiles. However, Bernstein et al. $^{3}$ show that $B$ is in fact dependent on the type of projectiles as well as the target neutron $\left(M_{v}\right)$ and proton $\left(M_{p}\right)$ matrix elements. Results from the inelastic scattering of $\boldsymbol{x}^{+}$and $\boldsymbol{\pi}$ indicated that there is a large isovector component in the region where the giant quadrupole resonance (GQR) is located. ${ }^{48}$ This contradicts both theoretical prediction" and experimental results from other inelastic hadron scattering ${ }^{10,11}$ that the GQR is a pure isoscalar resonance. Recently, Beene et al. ${ }^{12}$ and Horen et al..$^{13}$ used beams of $84 \mathrm{MeV} / \mathrm{u}^{17} \mathrm{O}$ ions to investigate the isospin character of the GQR on several nuclei. Their technique uses the interference between nuclear and Coulomb interaction to determine the ratio of the neutron to proton matrix elements $\left(M_{a} / M_{p}\right)$. Horen et al. ${ }^{14}$ later used the same technique to further investigate the isospin character of transitions to low-lying $2^{+}$and $3^{-}$ states of ${ }^{204,206,200} \mathrm{~Pb}$ by inelastically scattered $375 \mathrm{MeV}$ ${ }^{17} \mathrm{O}$ ions. Since heavy ions can excite both nuclear and Coulomb strongly, it is a good probe to study the isospin mixtures of the low lying states as well as the giant resonances. As high energy heavy ion beams become more generally available, it is interesting to use other heavy ion projectiles to further test the validity of projectile dependence on deformation parameter, especially on those nuclei with large discrepancies.

A $490 \mathrm{MeV}{ }^{14} \mathrm{~N}$ beam from the $\mathrm{K} 500$ cyclotron was used to bombard ${ }^{90} \mathrm{Zr}$, ${ }^{2} \mathrm{Mo}$ and ${ }^{144} \mathrm{Sm}$ targets. Elastic and inelastic scattering of the ${ }^{14} \mathrm{~N}$ ions were measured using the Enge split-pole magnetic spectrograph and a 
focal plane detector system. This detector system includes a vertical drift chamber (VDC), an ionization chamber and $\mathrm{BC} 400$ plastic scintillator and provides information on position, angle, timing as well as particle identification for light heavy ions. The typical energy resolution during these measurements was maintained less than $400 \mathrm{KeV}$ in order to separate the first excited state from the ground state. A large solid angle opening with angular acceptance $\pm 1.6^{\circ}$ was used.

Analyses were done by dividing each angle setting to nine angle bins. Between each angle setting two or three overlapping angles were obtained which provide relative normalization of cross-section between each angle setting. Figure 1 shows the angular distribuions of the differential cross-section of elastic scattering.

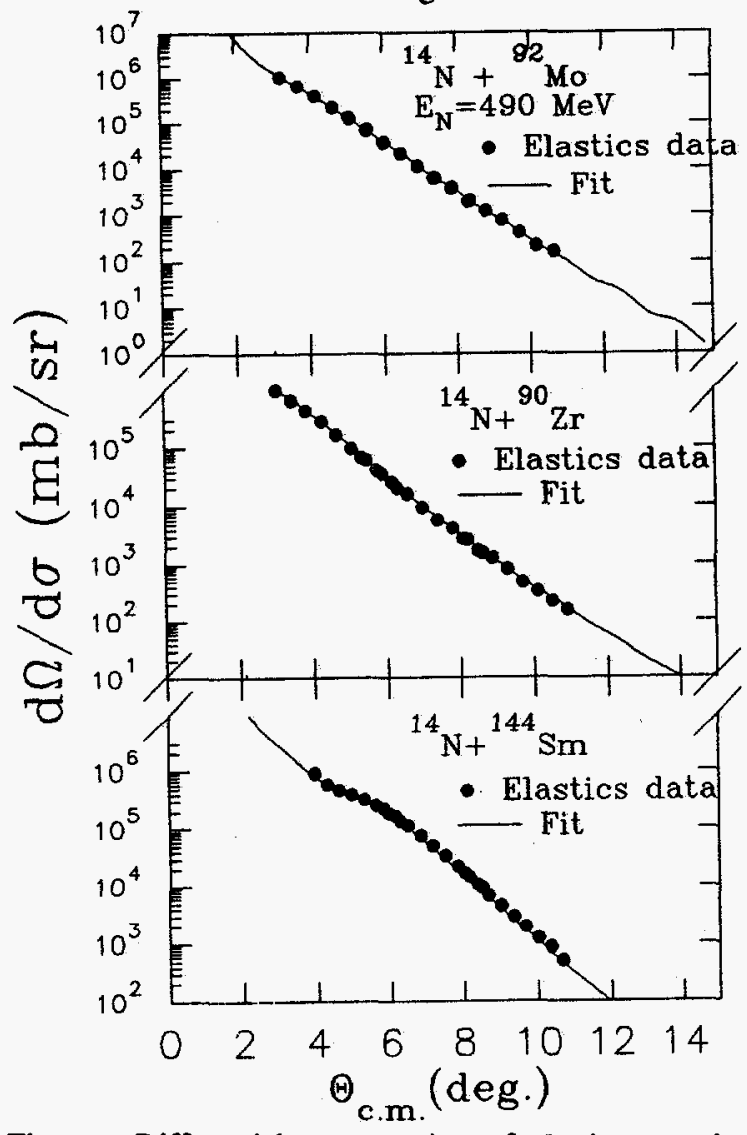

Fig. 1 Differential cross-section of elastic scattering. Solid lines are the optical model fits.

The solid lines are the optical model fits. The data were normalized to the calculations. The normalization factor was included as one of the fitting parameters and the same normalization factor was applied to the inelastic scattering cross-section. The angular distributions of differential cross-section of inelastically scattered ${ }^{14} \mathrm{~N}$ from ${ }^{0} \mathrm{Z}$, ${ }^{22} \mathrm{Mo}$ and ${ }^{144} \mathrm{Sm}$ are shown in Fig. 2 and the solid lines were the corresponding cross-section calculations. Both the optical model analyses and the inelastic scattering calculations were performed with the prograin PTOLEMY. ${ }^{15}$ Optical model potentials obtained from the fitting process were used in calculating the inelastic crosssection.

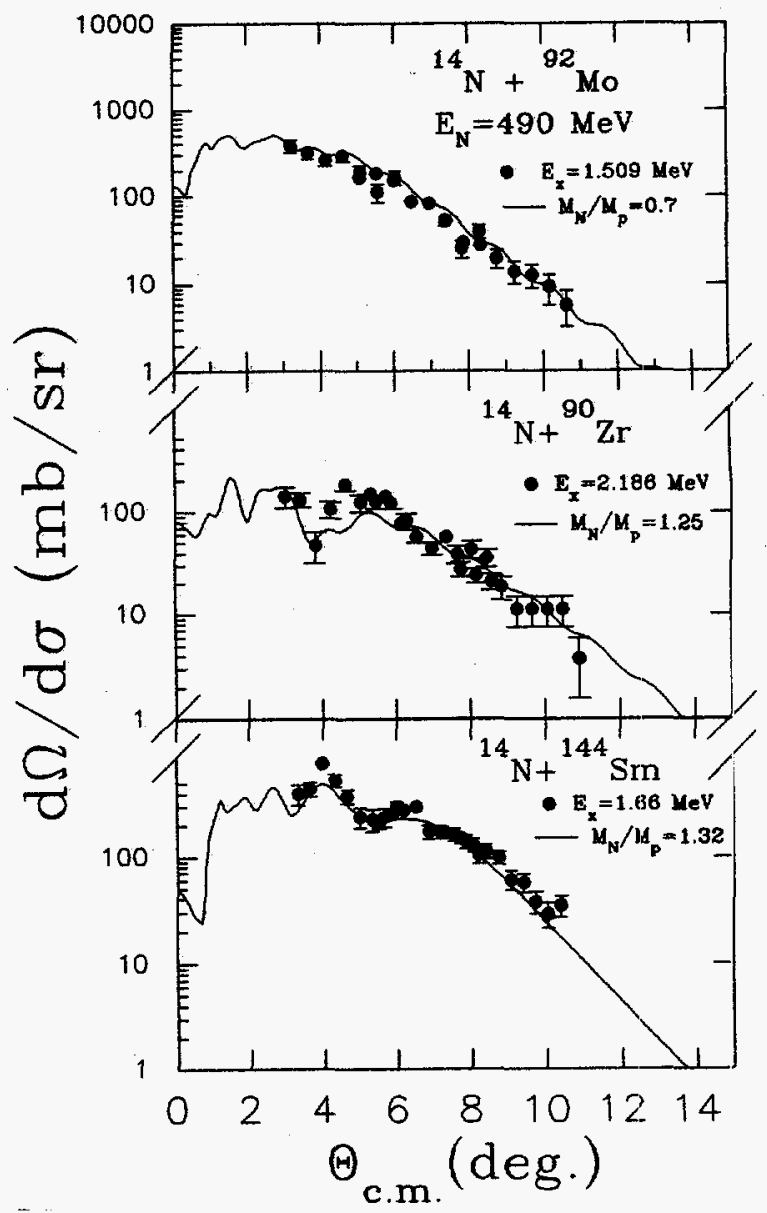

Fig. 2 Differential cross-section of inelastic seattering. Solid lines are the corresponding coupled channel calculations.

Calculations clearly show that the absolute crosssections which relate to the deformation length (BR), cin be changed by a factor of three from different optical potentials. However, the shape of the angular distributions are quite similar to each other as long as the same ratio of $M_{n}$ to $M_{p}$ was used. The ratio of $M_{n}$ to $M_{p}$ is determined by varying $M_{n}$ and $M_{p}$ to fit the experimental data, especially in the region just inside the grazing angle where it is dominated by the interference between the nuclear and Coulomb interactions. Preliminary results indicate that the ratio of $M_{n}$ to $M_{p}$ for the first excited states in both ${ }^{90} \mathrm{Zr}$ and ${ }^{144} \mathrm{Sm}$ is nearly $M_{n} M_{p}=N / Z$, suggesting a collective excitation, however the result for the first excited state in ${ }^{92} \mathrm{Mo}$ can be reproduced by $M_{n} / M_{p}=0.7$, which indicates the state is less collective. In ${ }^{22} \mathrm{Mo}$, the result is in good agreement with schematic model prediction by Madsen and Brow/n, ${ }^{2}$ 


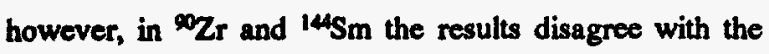
prediction. In order to determine an accurate ratio of $\mathbf{M}_{\mathbf{w}}$ and $M_{p}$, more detailed analyses are required, especially in ${ }^{90} \mathrm{Zr}$, where the first excited states was not clearly separated from the second excited state.

\section{REFERENCES}

1. V.A. Madsen, V.R. Brown and J.D. Anderson, Phys. Rev. C12, 1205 (1975).

2. V.A. Madsen and V.R. Brown, Phys. Rev. Lett. 52, 176 (1984).

3. A.M. Bernstein, V.R. Brown and V.A. Madsen, Phys. Lett 106B, 259 (1981).

4. J.L. Ullmann, J.K. Kraushaar, T.G. Masterson, R.J. Peterson, R.S. Raymond, R.A. Ristinen, N.S. P. King, R.L. Boudrie, C.L. Morris, W.W. True, R.E. Anderson, and E.R. Siciliano, Phys. Rev. Lett. 51, 1038 (1983).

5. C.L. Morris, S.J. Seestrom-Morris and L.C. Bland, in Proc. of the International Symposium on Highly Excited States and Nuclear Structure, Orsay, France, edited by N. Marty and N. Van Gai [j. Phys.(Paris) Colloq. 45, C4-327 (1984)].

6. J.L. Ullmann, J.K. Kraushaar, T.G. Masterson, R.J. Peterson, R.S. Raymond, R.A.Ristinen, N.S.P. King, R.L. Boudrie, C.L. Morris, R.E. Anderson, and E.R. Siciliano, Phys. Rev. C31, 177 (1985).

7. S.J. Seestrom-Morris, C.L. Morris, J.M. Moss, T.A. Carey, D. Drake, J.C. Dousse, L.C. Bland, and G.S. Adams, Phys. Rev. C33, 1847 (1986).
8. J.L. Ullmann, P.W.F. Alons, B.L. Clausen, J.J. Kraushaar, J.H. Mitchell, R.J. Peterson, R.A. Ristinen, R.L. Boudrie, N.S.P. King, C.L. Morris, J.N. Knudson, and E.F. Gibson, Phys. Rev. C35, 1099 (1987).

9. A. Bohr and B.R. Mottleson, Nuclear Structure (Benjamin, NY, 1975), Vol. 2.

10. D.H. Youngblood, J.M. Moss, C.M. Rozsa, J.D. Bronson, A.D. Bacher, and D.R. Brown, Phys. Rev. C13, 994 (1976).

11. C.M. Rozsa, D.H. Youngblood, J.D. Bronson, Y.W. Lui, and U. Garg, Phys. Rev. C21, 1252 (1980).

12. J.R.Beene, F.E. Betrand, D.J. Horen, R.L. Auble, B.L. Burks, J. Gomez del Campo, M.L. Halbert, R.O. Sayer, W. Mittig, Y. Schutz, J. Barrette, N. Alamanos, F. Auger, B. Fernandez, A. Gillibert, B. Haas, and J.P. Vivien, Phys. Rev. CA1, 920 (1990).

13. D.J. Horen, F.E. Bertrand, J.R. Beene, G.R. Satchler, W. Mittig, A.C.C. Villari, Y. Schutz, Zhen Wenlong, E. Plagnol, and A. Gillibert, Phys. Rev. C42, 2412 (1990).

14. D.J. Horen, R.L. Auble, J.R. Beene, F. E. Bertrand, M. L. Halbert, G. R. Satchler, M. Thoennessen, R. L. Varner, V. R. Brown, P. L. Anthony and V. A. Madsen, Phys. Rev. C44, 128 (1991).

15. M.H.Macfarlane and S.C. Pieper, Argonne National Lab. Rept. \#ANL-76-11 (Rev.1) 1978 (unpub.); M. Rhoades-Brown, M.H.Macfarlane, and S.C.Pieper, Phys. Rev. C21, 2417 (1980); C21, 2436 (1980).

\title{
RESONANT BREAKUP REACTIONS INDUCED WITH $30 \mathrm{MeV} / \mathrm{u}{ }^{16} \mathrm{O}$ and ${ }^{20} \mathrm{Ne}$
}

\author{
D. O'Kelly, Y.-W. Lui, H. Utsunomiya, B. Hurst,
} T. Botting, L. Cooke, W. Turmel, and R. P. Schmitt

The study of projectile dissociation reactions continues to be an active area of heavy ion research. Sequential breakup reactions, which populate discrete, resonant states in the emitting system, are rather well documented. However, so-called direct breakup reactions are not well characterized. ${ }^{1,2,3}$ A direct breakup mechanism has been attributed to a continuous distribution of relative energies between the two breakup fragments. Such continus have been observed near the particle thresholds in the dissociation of ${ }^{6} \mathrm{Li}$ and ${ }^{\mathrm{Zi}}$, ${ }^{4,5,6}$ but have yet to be seen with heavier projectiles. The excitation of continuum states in the projectile-like system are of considerable interest beyond their possible mechanistic implications. It has been suggested ${ }^{7}$ that the Coulomb contribution to the cross section for such continuum excitations can, through the detailed balance approximation, provide information on radiative capture reactions down to extremely low relative momenta, which are of considerable astrophysical interest. As described elsewhere, we have previously studied the projectilebreakup reaction of $\mathrm{Li}_{\mathrm{i}}$ into $\alpha+\mathrm{t}$ at two incident energies. 5 To further our understanding of projectile- 
breakup reactions and better define its astrophysical implications, we have conducted additional experimental investigations of heavy ion breakup reactions at intermediate energies.

Using an Enge split pole magnetic spectrograph, we have performed separate experiments on breakup reactions of $30 \mathrm{MeV} / \mathrm{u}{ }^{10} \mathrm{O}$ and ${ }^{20} \mathrm{Ne}$ projectiles with a series of target nuclei. Both studies employed a co-linear breakup geometry, which selects breakup partners traveling either parallel or anti-parallel with respect to the initial ejectile direction. This configuration is very sensitive because the detection threshold in relative kinetic energy can be reduced to low values. Also, in this geometry the energy resolution benefits from the fact that small changes in the relative kinetic energy results are amplified into large changes in the laboratory kinetic energies of the fragments. An additional feature of the spectrograph method is that it allows easy access to forward angles without the interference of the elastic scattering, which is of obvious importance in coincidence measurements.

In the first experiment, an ${ }^{16} \mathrm{O}$ beam from the Texas A\&M superconducting cyclotron was used to irradiate self-supporting metallic foils of ${ }^{58} \mathrm{Ni},{ }^{120 \mathrm{Sn}}$ and ${ }^{208} \mathrm{~Pb}$. The ${ }^{20} \mathrm{Ne}$ experiment employed targets of ${ }^{12} \mathrm{C},{ }^{58} \mathrm{Ni},{ }^{120} \mathrm{Sn}$, and ${ }^{200} \mathrm{~Pb}$. As described previously, ${ }^{8}$ two focal plane detectors were mounted in the Enge split pole to measure the two particle correlations. Both these detectors, which consisted of $40 \mathrm{~cm}$ long resistive wire proportional counters backed by plastic phoswiches, were mounted on the high B-o side of the focal plane. An $11 \mathrm{~cm}$ dead space was left between the counters to accommodate elastically scattered projectiles. This gap resulted in a detection threshold of $200 \mathrm{keV}$ (relative kinetic energies) for the $\alpha$ $+{ }^{12} \mathrm{C}$ and the $\alpha+{ }^{16} 0$ channels in the two measurements. The phoswich detectors were comprised of a thin layer of BC400 fast plastic and a thick layer of BC444 slow plastic. Together, the signals from these two scintillators provided complete isotope separation for "light" heavy ions when combined with the $p / q$ selection of the spectrograph. The focal plane detectors were position and energy calibrated using a $30 \mathrm{MeV} / \mathrm{u} \mathrm{HD} \mathrm{HD}^{+}$beam. The beam energy was accurately determined by applying the cross-over method to ${ }^{12} \mathrm{C}\left(\mathrm{d}, \mathrm{d}^{\prime}\right)^{12} \mathrm{C}$ and ${ }^{12} \mathrm{C}(\mathrm{d}, \mathrm{t})^{11} \mathrm{C}$ reactions.

As described previously, the initial analysis of the ${ }^{16} \mathrm{O}$ data concentrated on the $\alpha+{ }^{12} \mathrm{C}$ channel in view of its astrophysical significance. However, the data from the 100 run also showed strong correlations for a variety of $\alpha+$ H.I. pairs: $\alpha$ particles were seen in coincidence with isotopes of $B, C, N$, and $O$, indicating a variety of different processes preceding breakup. As an illustration, Fig. 1 shows a sum energy spectrum for ot - ${ }^{12} \mathbf{E}$ coincidences obtained with a ${ }^{208} \mathrm{~Pb}$ target summed over all angles $\left(3,5,8\right.$, and $\left.11^{\circ}\right)$. The mean energy of this peal: corresponds to about $130 \mathrm{MeV}$ total kinotic energy loss.

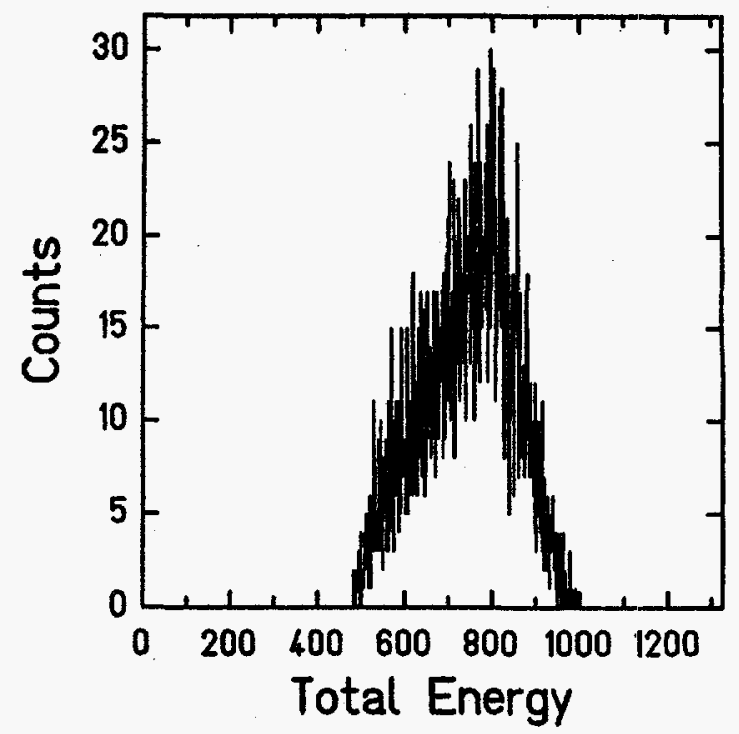

Fig. 1. Sum energy spectrum for $\alpha-{ }^{12} B$ coincidences obtained with a ${ }^{20} \mathrm{Pt}$ target summed over all angles $\left(3,5,8\right.$, and $\left.11^{\circ}\right)$.

The broad structure seen in Fig. 1 corresponds to many different inelastic breakup channels. A gate was set on this structure in order to generate a relative kinetic energy spectrum for the different breakup processes. The relative kinetic energy spectrum can be seen in Fig. 2. Three distinct peaks can be discerned in this spectrum, corresponding to excitation energies of 14.502, 16.044, 17.727 MeV, respectively. The spin and parity of these

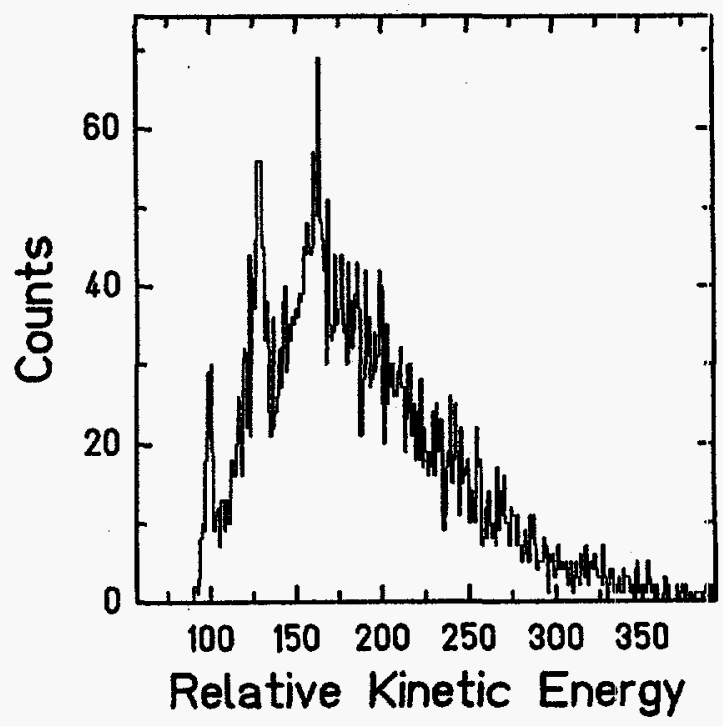

Fig. 2. Relative kinetic energy spectrum displaying resonant states in ${ }^{16} \mathrm{~N}^{*}$. 
states have not been determined. It is interesting to note that apparently none of these peaks have been identified in previous work on the ${ }^{16} \mathrm{~N}^{*}$ system.

We have recently begun the analysis of the ${ }^{20 \mathrm{Ne}}$ data. Fig. 3 shows a $\Delta-E$ versus position scatter plot for the high-p detector (Counter 1). As can be seen from this figure, $Z$ resolution for $Z=2$ through 10 was obtained. The low $\rho$ detector gave similar $Z$ resolution. Gates were set around $Z=2$ in the high- $\rho$ detector and various other $Z$ 's in the low $p$ counter to investigate the various particle correlations, particularly the $\alpha+{ }^{16} 0$ channel. Figure 4 shows a scatter plot of the $\alpha-{ }^{16} \mathrm{O}$ coincidence events for the ${ }^{208} \mathrm{~Pb}$ target in the plane defined by the positions of the $\alpha$ and the ${ }^{10}$. A number of structures are apparent in this plot. The band with a negative slope seen in the upper right hand corner arises from breakup reactions in which the target nucleus remains in its ground state (i.e., elastic breakup). There are several heavy concentrations of

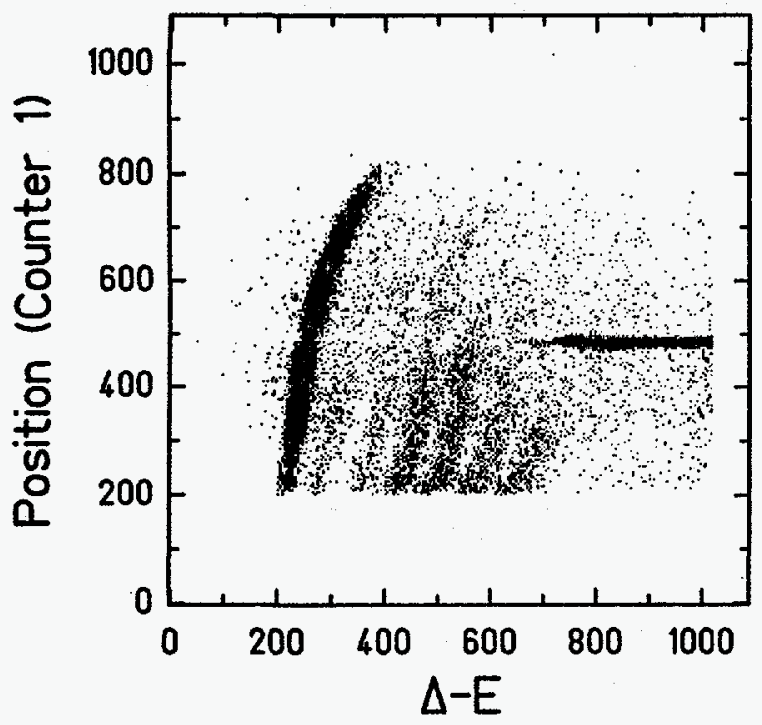

Fig. 3. Scatter plot of $\Delta-E$ versus position for the high-p detector showing $Z$ resolution from $Z-2$ through 10.

events along this line of constant energy. These are associated with various particle unbound resonances in the ${ }^{20} \mathrm{Ne}^{*}$ system. A number of bands with positive slopes are also seen in Fig. 4. The bands arise from the same states in ${ }^{20} \mathrm{Ne}^{*}$, but correspond to varying degrees of target excitation.

The distribution of events in Fig. 4 suggests that elastic breakup is more important than inelastic breakup. This is clearly evident in the sum energy spectrum shown in Fig. 5, which is dominated by a narrow peak corresponding to $\alpha+{ }^{16} 0(Q=-4.73 \mathrm{MeV})$. The broad structure at lower total energies is due to the various inelastic breakup channels.

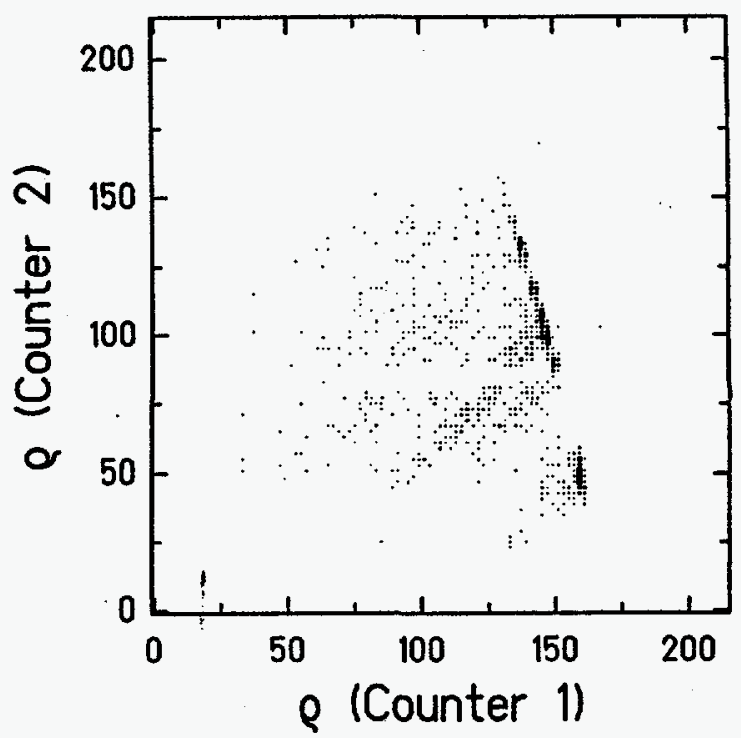

Fig. 4. Scatter plot of $\rho 1$ versus $\rho 2$ for $\alpha-16$ coincidence events for a ${ }^{20} \mathrm{~Pb}$ target at $5^{\circ}$.

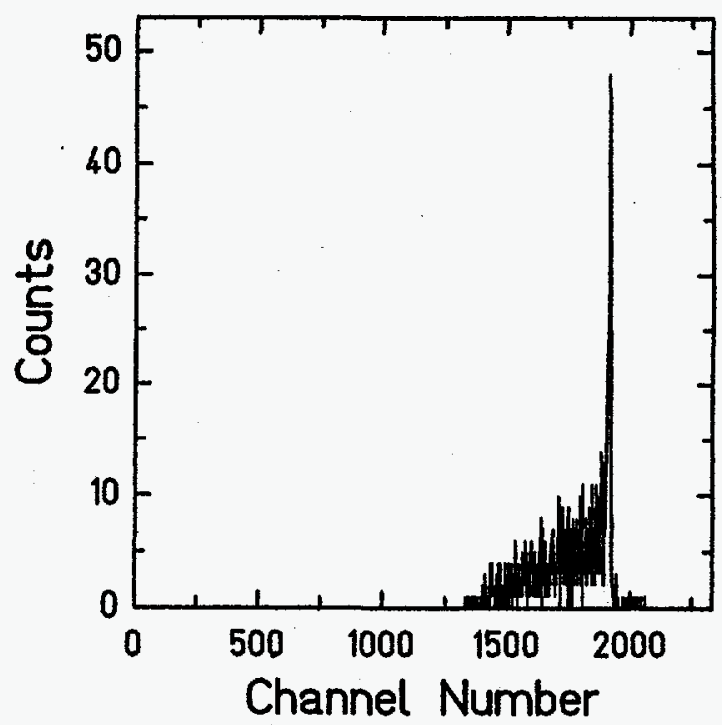

Fig. 5. Sum energy spectrum for $\alpha-160$ coincidences. The narrow peak corresponds to the breakup of ${ }^{20} \mathrm{Ne}$.

After placing a gate on the sum energy, a relative kinetic energy spectrum was generated (Fig. 6). This spectrum shows distinct peaks corresponding to resonant states in ${ }^{20} \mathrm{Ne}$. The first discrete peak corresponds to an excitation energy of $5.52 \mathrm{MeV}$. The second, third, and fourth peaks correspond to known states at $6.73\left(0^{+}\right)$, $7.16\left(3^{\circ}\right)$, and $7.42\left(2^{+}\right) \mathrm{MeV}$, respectively. The fifth broad peak is possibly a triplet corresponding to the $8.453\left(5^{\circ}\right), 8.708\left(1^{\circ}\right)$, and $9.03\left(4^{+}\right)$resonant states in ${ }^{20} \mathrm{Ne}$.

Although the analysis of the ${ }^{20} \mathrm{Ne}$ data is still underway, preliminary indications are that the breakup 
spectra are dominated by discrete resonances, as is the case in the ${ }^{16} \mathrm{O}$ data. In contrast to the ${ }^{7} \mathrm{Li}$ experiment, no significant continua were observed in the elastic breakup channels with either of these projectiles. Hopefully, a more thorough analysis of the ${ }^{16} \mathrm{O}$ and ${ }^{20} \mathrm{Ne}$ data will cast further light on the differences in the underlying reaction mechanisms.

\section{REFERENCES}

1. W. D. Rac e a‥, Phys. Rev. Lett. 45, 884 (1980).

2. R.K. Bhowmik et al., Nucl. Phys. A390, 117 (1982).

3. W. D. Rac et gl., Phys. Rev. C30, 158 (1984); Phys. Lett. 105B, 417 (1981).

4. A. C. Shotter et al., Phys. Rev. Lett. 46, 12 (1981); ibid. 53, 1539 (1984).

5. H. Utsunomiya et al., Phys. Lett. 211B, 24 (1988); Nucl. Phys. A511, 379 (1990).

6. S. B. Gaze, J. E. Mason, R. B. Roberts and S. G. Teichmann, Phys. Rev. Lett. 68, 150 (1992).

7. G. Baur, C. A. Bertulani, and H. Rebel, Nucl. Phys. A458, 188 (1986).
8. H. Utsunomiya, Y.-W. Lui, and R. P. Schmit, Nucl. Instr. Meth. A278, 744 (1989).

9. Progress in Research, 1990-1991, Cyclotron Institute, Texas A\&M University, p. 31.

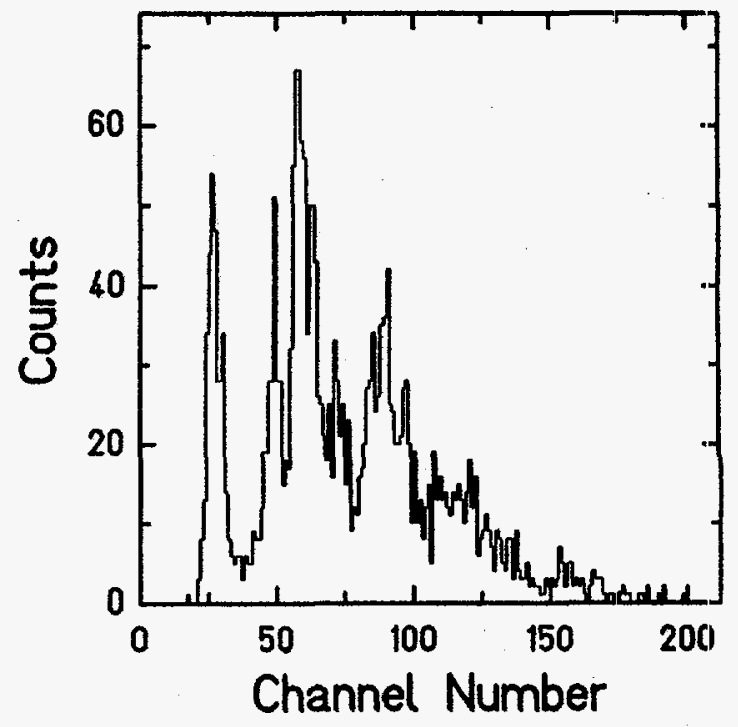

Fig. 6. Total relative kinetic energy apectrum summed over all Q-values. Peaks correspond to excited states in ${ }^{20} \mathrm{Ne}$.

\title{
EXPERIMENTAL PROGRAM IN N-N AND KAON PHYSICS
}

\author{
L. C. Northcliffe, J. C. Hiebert, R. A. Kenefick, G. Glass, T. Shima and \\ collaborators from numerous other institutions
}

\section{A. Experiments at LAMPF (Clinton P. Anderson Meson Physics Facility)}

\section{Neutron-proton elastic spin transfer} measurements from 485 to $788 \mathrm{MeV}$

This program (LAMPF experiment E876) was initiated in the summer of 1990 with the measurement of $K_{L L}, K_{L S}, K_{s L}$ and $K_{s s}$ at $788 \mathrm{MeV}$. These initial data have been published.' E876 was continued during 1991 and measurements were made of these same spin transfer coefficients at 485 and $635 \mathrm{MeV}$. These data have a significant impact on the phase shift analyses and there are now sufficient data near these energies to over-determine the elastic nucleon-nucleon amplitudes. A manuscript has been submitted ${ }^{2}$ on the most recent data set. E876 is continuing in the summer of 1992 with the measurement of $\mathrm{K}_{\mathrm{NN}}$ at 635 and $788 \mathrm{MeV}$. The entire program has been in collaboration with persons from Argonne National Laboratory (ANL), Los Alamos National Lab (LANL), Rice University (RiU), Rutgers University (RuU), the
Univ. of Central Arkansas (UCA), the University of Montana (UM), the University of Texas at Austin (U1.) and Washington State University (WSU). Dr H. L. Woolverton, who received her $\mathrm{PhD}$ in 1981 based on an n-p measurement at the Cyclotron Institute, has recently joined the faculty at UCA and received financial suppoit from our research grant in order to join this collaboration.

\section{Spin transfer parameter $\mathrm{K}_{\mathrm{LL}}$ at $0^{\circ}$ for the $\mathrm{D}(\mathrm{p}, \mathrm{n}) 2 \mathrm{p}$ reaction from 305 to $788 \mathrm{MeV}$}

The E876 spin transfer measurements revealed a 10 $16 \%$ normalization discrepancy with previous measurtments of the polarization of the LAMPF neutron beam. Consequently, experiment E1234 was proposed to remeasure the $D(p, n) 2 p$ spin transfer parameter $K_{L, ~}$ at zero degrees at beam energies of $305,485,635,722$ and $788 \mathrm{MeV}$. These measurements were completed in the summer of 1991 by the collaboration of A.1 with an additional collaborator from the University of Colorado. A manuscript has been submitted to Phys. Rev. C. $^{3}$ 
3. Single pion production in np scattering: LAMPF E1097

The cylindrical drift chamber detector for E1097 was installed downstream of the E876 setup for testing during the summer of 1991. E1097 will measure the differential cross section and the spin observables $A_{\text {NO }}, A_{L O}, A_{\text {so }}$, $A_{\mathrm{OL}}, A_{\mathrm{SL}}, A_{\mathrm{NL}}$, and $A_{\mathrm{LL}}$ for the single pion production reaction np-ppx at neutron beam energies in the range $500-800 \mathrm{MeV}$. The four independent channels available in the $\mathrm{NN} \rightarrow \mathrm{NN} \pi$ reaction can be identified in terms of the total isospin of the initial (I) and final (I') state nucleons (I,I') with $I$ or $I^{\prime}=0,1$. The dominant channel for pion production is through the $(1,0)$ resonance, since the $\Delta^{++}$ $(I=3 / 2, S=3 / 2)$ can be excited through this channel. The role of the non-resonant amplitudes, particularly the $(0,1)$ channel is not well-determined. The np $\rightarrow p p \pi$ reaction proceeds through the $(1,1)$ and $(0,1)$ channels which cannot excite the $\Delta^{++}$, thereby making passible the study of the non-resonant contributions to pion production. The data acquired in E1097 should permit a first order partial wave analysis of the $I=0$ inelastic amplitudes.

The first phase of E1097, scheduled for the summer of 1992, will use the polarized neutron beam and a liquid hydrogen target. Upon completion of phase I a polarized hydrogen (frozen spin) target is required for the measurement of the spin correlation parameters.

Participating institutions in this LAMPF experiment include ANL, California State University, the University of Houston, LANL, the University of Manitoba, the University of Alberta, RiU, UTA and TAMU.

\section{Absolute pp-elastic cross sections} from 492 to $793 \mathrm{MeV}$

Absolute pp-elastic differential cross sections have been measured at incident energies $492,576,642,728$, and $793 \mathrm{MeV}$ from about $30^{\circ}$ to $90^{\circ} \mathrm{c.m}$. The total uncertainty was determined to be less than $1 \%$, made possible by particle counting for beam normalization and extensive cross-checks of systematic effects. The new data are consistent with previous data above $600 \mathrm{MeV}$ but have uncertainties about a factor of ten smaller. Near 500 MeV these data are consistent with $90^{\circ}$ data from TRIUMF, but differ significantly from similar data from PSI. A manuscript reporting these results will be submitted in the near future. This work was done in collaboration with persons from LANL, UCLA, RiU, $\mathrm{RuU}$, and UT and forms the basis for the PhD dissertation of Anthony J. Simon, which should be completed in 1992.
5. Neutron-proton elastic scattering spin correlation parameter measurements between $500-800 \mathrm{MeV}$

A manuscript based on this experiment has been submitted for publication.4 A second manuscript has been prepared and is about to be submitted. The work was done in collaboration with persons from ANL, NMSU, LANL, UM, and WSU.

\section{Differential cross section for $n-p$ elastic scattering at $459 \mathrm{MeV}$}

A manuscript on this experiment has been submitted

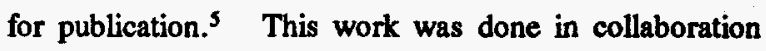
with persons from LANL and UTA.

\section{B. Experiments at Brookhaven National Laboratory (BNL)}

\section{1. $\mathrm{K}^{+}$total cross sections as a test for nucleon "swelling"}

The final manuscript reporting results of E835 at the Zero Gradient Synchrotron (ZGS) has been submitted. ${ }^{6}$ This experiment was a collaboration with persons from Tel Aviv University, BNL, Vassar College, Osaka University, and TRIUMF. A PhD. degree was awarded to R. A. Krauss in May, 1991, for a dissertation based on this experiment.

\section{Experiments at KEK (National Laboratory for High Energy Physics, Tsukuba, Japan)}

\section{Energy dependence of the analyzing power for the $p p \rightarrow p p$ and $p p \rightarrow d \pi^{+}$reactions in the energy region $500-800 \mathrm{MeV}$}

Two final manuscripts have been submitted for publication based on the experiments conducted at KEK. ${ }^{7,8}$ Participating institutions included KEK, Kyoto University, Tohoku University, and the Tokyo Institute of Technology.

\section{REFERENCES}

1. M. W. McNaughton, et al., np Elastic Spin Transfer Measurements at $788 \mathrm{MeV}$, Phys. Rev. C44, 2267 (1991).

2. M. W. McNaughton, et al., $n p$ Elastic Spin Transfer Measurements at 485 and $635 \mathrm{MeV}$, Submitted to Phys. Rev. (see: Abstracts of Papers Submitted, this report). 
3. M. W. McNaughton, et al., ${ }^{2} \mathrm{H}(p, n)$ Spin Transfer from 485 and $635 \mathrm{MeV}$, Submitted to Phys. Rev. C (see: Abstracts of Papers Submitted, this report).

4. W. R. Ditzler, et al., Neutron Proton Elastic Scattering Spin-Spin Correlation Parameter Measurements Between 500 and $800 \mathrm{MeV}$, Submitted to Phys. Rev. D (1992) (See: Abstracts of Papers Submitted, this report).

5. L. C. Northeliffe, et al., Differential Cross Section for $n-p$ elastic scattering in the angular region $50^{\circ}<$ $\theta<180^{\circ}$ at $459 \mathrm{MeV}$, Submitted to Phys. Rev. C (1991) (See: Abstracts of Papers Submitted, this report).
6. R. A. Krauss, et al., $K^{+}$Total Cross Sections and Medium Effects in Nuclei, Submitted to Phys.Rev. C (1992) (See: Abstracts of Papers Submitted, this report).

7. Y. Kobayashi, et al., Energy Dependent Measurements of the $p-p$ Elastic Analyzing Power and Narrow Dibaryon Resonances, Submitted to Nucl. Phys. A (1991) (See: Abstracts of Papers Submitted, this report).

8. H. Y. Yoshida, et al., Energy Dependence of the Analyzing Power for the $p p \rightarrow \pi^{+} d$ Reaction in the Energy Region 500-800 MeV, Submitted to Nucl. Phys. A. (1991) (See: Abstracts of Papers Submitted, this report). 
NUCLEAR THEORY 


\section{S. Shlomo and J. B. Natowitz}

Very recently, there has been an increasing interest in the nuclear level density parameter, $a$, stimulated by experimental data ${ }^{1}$ on the temperature dependence of $a$ for nuclei with $A \approx 160$. The temperature dependence of the level density parameter, $a$, was deduced from coincidence measurements between heavy residues and evaporated light particles. It was found that $a$ decreases from $A / 8$ at low temperature to $A / 13$ for $T \neq 5 \mathrm{MeV}$. This observation stimulated a significant amount of theoretical and experimental work. Temperature dependent HartreeFock $^{2}$ or semiclassical ${ }^{3}$ calculations with various types of effective interaction fail to reproduce the trend of the data. It has been recognized ${ }^{46}$ that the observed $T$ dependence of $a$ can be accounted for by including the effects of correlations (collectivity), i.e., the $T$-dependence of the frequency dependent effective mass, $m_{\omega}$. In Ref. [4] we have presented a simple and realistic model for calculating $a$, as a function of $T$, taking into account the finite size of the nucleus, the continuum states, shell effects, the momentum and frequency dependence of the effective mass and the variation of these effects with $T$. Using this model, a reasonable agreement with the experimental data in the $A \simeq 160$ mass region was obtained. More recently, several experimental investigations have been carried out $^{7-10}$ to determine the temperature dependence of $a$ for various mass regions. The purpose of this work is to present results of a calculation of $a$ for a wide range of temperature and nuclear mass, using the model described in Ref. [4].

We first give a short review of the model we use to calculate the level density parameter $a$. We adopt the definition,

$$
a(T)=E_{X} / T^{2}
$$

where

$$
E_{X}(T)=E(T)-E(0) \text {. }
$$

In our calculation the internal energy $E(T)$ is obtained from,

$$
E(T)=\int \operatorname{cg}(\varepsilon) f(\varepsilon, \mu, T) d \varepsilon,
$$

where $g(\varepsilon)$ is the single particle level density and

$$
f(\varepsilon, \mu, T)=\frac{1}{1+\exp [(\varepsilon-\mu) / T]}
$$

is the occupation probability. The chemical potential $\mu$ is determined by the conservation condition

$$
A=\int g(\varepsilon) f(\varepsilon, \mu, T) d \varepsilon .
$$

To calculate the single particle level density, we adopt the Thomas-Fermi approximation corrected for the continuum effect. As demonstrated recently, ${ }^{11}$ the $\hbar^{2}$ corrections for a smooth potential, such as the WoodSaxon potential, are relatively small and can be accounted for by slightly reducing the potential depth (by 3 to 4 $\mathrm{MeV}$ ). Moreover, a good approximation for the total level density parameter, $a=a_{n}+a_{p}$, can be obtained by using the mean field associated with a neutron (which includes the symmetry potential) for the $A$ nucleon system (without Coulomb interaction). Thus, for a local mean field, $V$, modified by an effective mass, $m^{*}$, we use the expression

$$
\begin{aligned}
& g(c)=\frac{1}{\pi^{2}} \int d^{3} r\left[\frac{2 m^{*}}{\hbar^{2}}\right]^{3 / 2} \\
& {\left[\left[c-\frac{m}{m^{*}} v(r)\right]^{1 / 2}-\theta(\varepsilon) \sqrt{\varepsilon}\right],}
\end{aligned}
$$

where $\theta(\varepsilon)=0$ or 1 for $\varepsilon<0$ or $\varepsilon>0$, respectively.

For the local potential we adopt the Woods-Saxon form,

$V(r)=\left[\begin{array}{cl}\frac{V_{0}}{[1+\exp [(r-R) / d]} & r<R_{\max } . \\ \infty, & r>R_{\max } .\end{array}\right.$

and for the effective mass $m^{*}=m\left(m_{k} / m\right)\left(m_{\omega} / m\right)$ we use the forms ${ }^{12}$

$$
\begin{aligned}
& m_{k} / m=1-\alpha n(r) \\
& m_{\omega} / m=1-\beta \frac{d n}{d r} .
\end{aligned}
$$

where

$$
n(r)=\frac{q(r)}{\rho_{0}}=\frac{1}{1+\exp [(r-c) / 2]} .
$$

To determine the $A$ dependence of the mean field parameters, we follow the prescription of Ref. [13]. Considering the matter density $\rho(r)$, we first determine the equivalent sharp radius $R_{\rho}$ which is defined as the radius of a uniform distribution having the same bulk density $\rho_{0}$ and volume integral as $\rho(r)$. Assuming $\rho_{0}=0.17 \mathrm{fm}^{-3}$, we find using $\frac{4}{3} \pi R_{\rho}^{3} \rho_{0}=A$, that 
$R_{p}=r_{0} \wedge^{1 / 3}, \quad r_{0}=\left(\frac{3}{4 \pi p_{0}}\right)^{1 / 3}=1.12 \mathrm{fm}$.

Therefore, the parameters of the Fermi density distribution, Eq. (9), are taken to be

$P_{0}=0.17 \mathrm{fm}^{-3}, \quad z=0.54 \mathrm{fm}$,

$c=\frac{1.12 A^{1 / 3}}{\left[1+(\pi 2 / C)^{2}\right]^{1 / 3}} \mathrm{fm}$.

The value of $C$ is determined by iteration. For $\pi z<C$. we have $C=1.12 A^{1 / 3}-0.857 A^{-1 / 3}$ fm. The root mean square radii $\left\langle r^{2}\right\rangle^{1 / 2}$ of (9) using (11) agree nicely with experimental values. ${ }^{14}$ The parameters of the single particle potential well are determined by taking the equivalent sharp radius of the potential well, $R_{V}$, to be $e^{13}$

$$
R_{V}=R_{\rho}+b
$$

where $b$ is a constant. We therefore use the following parameters for the mean field, for $T=0$,

$$
\begin{aligned}
& V_{0}(\text { Mev })=-47+33 \frac{N-2}{A} \\
& d=0.70 \mathrm{fm} \\
& R=\frac{R_{V}}{\left[1+(\pi d / R)^{2}\right]^{1 / 3}}, \\
& R_{V}=1.12 A^{1 / 3}+0.8 \mathrm{fm},
\end{aligned}
$$

and 12

$$
\begin{aligned}
& \alpha(T=0)=0.3, \\
& \beta(T=0)=0.4 \mathrm{~A}^{1 / 3} .
\end{aligned}
$$

For the temperature dependence of the mean field parameters we adopt the expressions used in Ref. [4] (see also Refs. 2,5,6,12,15-17).

$$
\begin{aligned}
& d(T)=d(T=0)\left(1+0.01 T^{2}\right) \\
& R(T)=R(T=0)\left(1+0.0005 T^{2}\right) \\
& \alpha(T)=\alpha(T=0)\left(1-0.005 T^{2}\right) \\
& \beta(T)=\beta(T=0) \exp \left[-(T / \gamma)^{2}\right] .
\end{aligned}
$$

We use the value of $\gamma=21 / A^{1 / 3}(\mathrm{MeV})$. For $A \approx 200$ we have $\gamma \simeq 3.5(\mathrm{MeV})$ in agreement with the results of Refs. [6] and [16]. The $A$ dependence of $\gamma$ is introduced to take into account the well-known variation with $A$ of the excitation energies of the low lying collective states. ${ }^{18}$
To calculate $a(T)$, we follow the procedur: introduced in Ref. [4], which takes into account the variation of the mean field and the effective mass with $T$. For each fixed value of $\mathrm{T}$ we determine $g(\varepsilon)$ from (6) using $V$ and $m^{*}$ given by Eqs. (7) - (15). The resulting $g(\varepsilon)$ is then used in (3) to determine both $E(T)$ and $E(0)$. The value of $a(T)$ is then obtained from (1). We have carried out calculations for various nuclei along the stability line with mass number $A=40,60,110,160$ and 210. In Table 1 we present the values of the parameters $V$ and $m^{*}$ for these nuclei at $T=0$. We also include in this table the density average of the effective mass defined by,

$$
\left\langle m^{*}\right\rangle=\int m^{*}(\bar{r}) n(\bar{r}) d \bar{r} / \int n(\bar{r}) d \bar{r} .
$$

Note that $\left\langle m_{k} / m\right\rangle=0.8$ and $\left\langle m_{\omega} / m\right\rangle=1.4$ in agreement with Refs. [6] and [16]. In Fig. 1 we present the results for the parameter

$$
K(T)=A / a(T) \text {, }
$$

for the nuclei with $A=40,60,110,160$ and 210 .

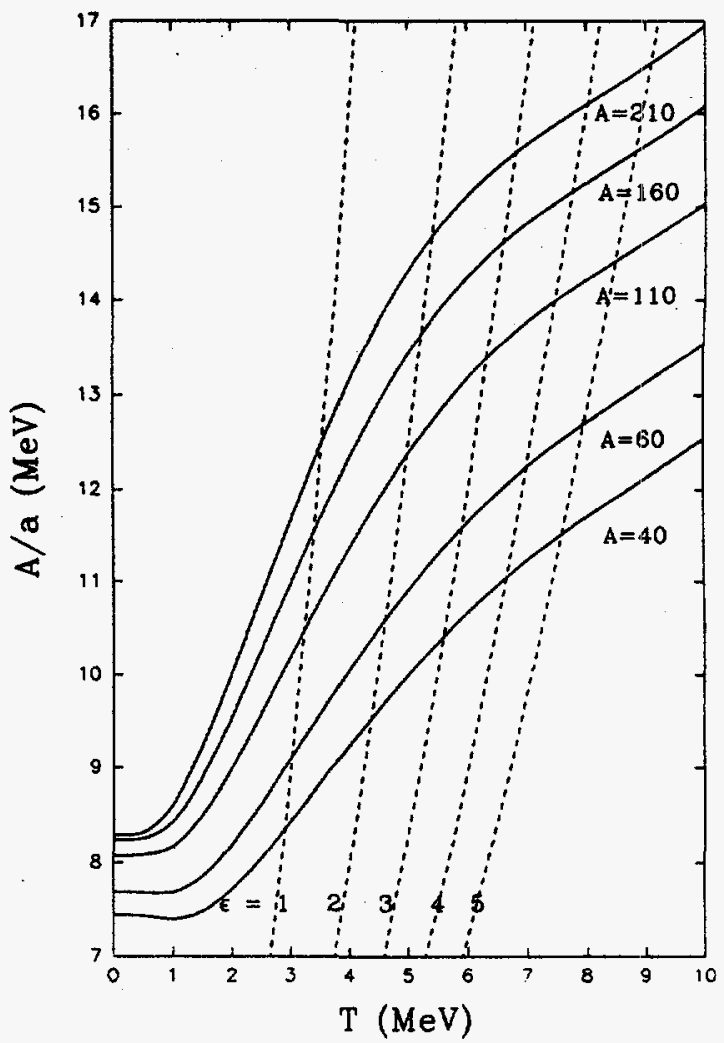

Fig. 1. Calculated inverse level density parameter $\boldsymbol{K}=$ $A / a$ as a function of Temperature for various nuclei with mass $A$ along the line of stability. See text for the values of the parameters used. Also indicated by the dashed lines are the losi corresponding to constant excitation energies per nucleon of 1-5 MeV. 
Values of $K(T)$ are now available for several mass regions. ${ }^{1,7-10}$ These values are measured over a range of excitation energies. Any comparison of these results with the present calculation should take cognizance of the excitation energy range involved. To facilitate this comparison we also indicate in Fig. 1 the loci of lines of constant excitation energy per nucleon, $\varepsilon$, of 1-5 MeV where $e=1 / K T^{2}$.

Table I

Potential and effective mass parameters at $T=0$

\begin{tabular}{|c|c|c|c|c|c|}
\hline$A$ & 40 & 60 & 110 & 160 & 210 \\
\hline $2(\mathrm{fm})$ & 0.54 & 0.54 & 0.54 & 0.54 & 0.54 \\
\hline$C(\mathrm{fm})$ & 3.58 & 4.17 & 5.19 & 5.92 & 6.51 \\
\hline$d(f m)$ & 0.70 & 0.70 & 0.70 & 0.70 & 0.70 \\
\hline$R(\mathrm{fm})$ & 4.28 & 4.87 & 5.91 & 6.65 & 7.24 \\
\hline$v_{0}($ MeV $)$ & -45.0 & -44.0 & -43.0 & -42.0 & -41.0 \\
\hline$\alpha$ & 0.3 & 0.3 & 0.3 & 0.3 & 0.3 \\
\hline$\beta\left(\mathrm{fm}^{-1}\right)$ & 1.37 & 1.57 & 1.92 & 2.17 & 2.38 \\
\hline$\gamma(\mathrm{MeV})$ & 5.84 & 5.36 & 4.38 & 3.87 & 3.53 \\
\hline$\varepsilon_{F}(\operatorname{MeV})$ & -5.85 & -5.28 & -4.65 & -4.01 & -3.37 \\
\hline$<m_{x} / m>$ & 0.82 & 0.81 & 0.79 & 0.78 & 0.77 \\
\hline$\left\langle m_{w} / m\right\rangle$ & 1.37 & 1.39 & 1.43 & 1.45 & 1.47 \\
\hline $\mathrm{cm} * / \mathrm{m}>$ & 1.12 & 1.12 & 1.13 & 1.13 & 1.13 \\
\hline
\end{tabular}

For $A \propto 160$, measurements up to $\varepsilon=2 \mathrm{MeV}$ show a strong decrease in a consistent with our calculations.' For nuclei with $A \approx 110$ to 120 at similar excitation energies, a higher value of $a$ is predicted. The values near $A / 11$ which are reported 7,8 are in reasonable agreement with the calculations. This value does not appear to decrease with increasing temperature. Note, however, that for higher excitation energies per nucleon the onset of intermediate mass fragment emission becomes increasingly important and, as a result, establishing the required temperature - excitation energy correlations is more difficult. ${ }^{19}$

For $A=40$ at excitation energies up to $1.7 \mathrm{MeV} / \mathrm{u}$, values of $a=A / 8$ are derived from particle spectra.9 Again this result is consistent with the trends presented in Fig. 1. For similar light mass systems at even higher excitation energy, little change in a is found. ${ }^{10}$ But here again the onset of intermediate mass fragment emission may affect the results.

\section{ACKNOWLEDGMENTS}

We would like to thank F. Bortignon for fruitful discussions. This work was supported in part by the National Science Foundation Grant No. PHY-9001886, the Department of Energy under Contract \#DE-FG0585ER40256 and The Robert A. Welch Foundation.

\section{REFERENCES}

1. G. Nebbia et al., Phys. Lett. 176B, 20 (1986); K. Hagel et al., Nucl. Phys. A486, 429 (1988); M. Gonin et al., Phys. Lett. 217B, 406 (1989).

2. P. Bonche, S. Levit, D. Vautherin, Nucl. Phys. A436, 265 (1985).

3. E. Suraud, Nucl. Phys. A462, 109 (1987).

4. S. Shlomo, J. B. Natowitz, Phys. Lett. 252B, 1987 (1990).

5. R. Hasse, P. Schuck, Phys. Lett. 179B, 313 (1986).

6. P. F. Bortignon and C. H. Dasso, Phys. Lett. 189B, 381 (1987).

7. R. Wada et al., Phys. Rev. C 39, 497 (1989).

8. A. Chbihi, L. G. Sobotka, N. G. Nicolis, D. G. Sarantites, D. W. Stracener, Z. Majka, D. C. Hensley, J. R. Beene and M. L. Halbert, Phys. Rev. C 43, 666 (1991).

9. B. Fornal et al., Contribution A-28, International Conference on Nucleus - Nucleus Collisions, Kanazawa, Japan (June, 1991).

10. J. Peter et al., Contribution A-44, International Conference on Nucleus - Nucleus Collisions, Kanazawa, Japan (June, 1991).

11. S. Shlomo, Submitted to Nucl. Phys. A.

12. M. Prakash, J. Wambach and Z. Y. Ma, Phys. Lett. 128B, 141 (1983)

13. W. A. Myers, Nucl. Phys. A204, 465 (1973).

14. A. Bohr and B. R. Mottelson; Nuclear Structure, Vol. I and II (Benjamin, Reading, MA, 1969 and 1975).

15. G. Sauer, H. Chandra and U. Mosel, Nucl. Phys. A269, 221 (1976).

16. D. Vautherin and N. Vin Mau, Orsay Report/PNO/TH 87-03 (1987).

17. A. Lejeune, P. Grangé, M. Martzoloff and J. Cugnon, Nucl. Phys. A453, 189 (1986).

18. F. Bortignon, Private communication.

19. J. B. Natowitz et al., To be published in Proceedings of the International Conference on Nucleus - Nucleus Collisions, Kanazawa, Japan (June, 1991). 


\section{H. Dejbakhsh, G. Ajupova, D. Latipov, and S. Shlomo}

The neutron-rich even-even Mo isotopes in mass 100 region have been investigated using the proton-neutron interacting boson model (IBM-2). Two different approaches were used in these calculations. The first investigation is based on the validity of the $Z=38$ subshell closure and the second considers only $Z$ and $N=50$ as valid shells. The results from both calculations are in good agreement with the experimental data.

The $\mathrm{O}_{2}^{+}+$levels in Mo isotopes are low in energy and have a minimum in ${ }^{9} \mathrm{Mo}$ and are near or below the $2_{1}^{+}+$ energies. This feature has been associated with shape coexistence in these nuclei. The origin of the strong deformation and coexistence of two shapes in this region can be attributed to the gaps in the single-particle spectrum at $Z=40, B=0$ and $Z=38, B=0.28$, which stabilizes the nuclear shape. However, the deformed state can coexist with a nearly spherical configuration in a delicate balance.

The Sambataro et al. ${ }^{\prime}$ investigation is the first attempt to explore the nature of the shape transitions in even-even Mo isotopes in mass 100 region. In their investigation the authors have argued that no reasonable set of parameters is able to predict the $\mathrm{O}_{2}^{+}+$levels in these nuclei, considering the $Z=40$ subshell closure. Therefore, the authors considered the mixing of two boson configurations. The first configuration consists of one proton boson and two to five neutron bosons ( $A=96$ to 104) in the single-particle orbits near the closed shells of $Z=40$ and $N=50$. The second configuration corresponds to the excited pair of proton bosons out of the $Z=\mathbf{4 0}$ shell resulting in 3 proton bosons, two proton bosons and one proton hole boson. The mixing of two boson configurations by Sambataro et al. ${ }^{1}$ has provided a good agreement with the known properties of these nuclei.

The Mo isotopes have been investigated in the framework of IBM-1 assuming an effective boson number derived from configuration mixing or $N_{n}{ }^{*} N_{p}$ scheme. ${ }^{2}$ The parameter $\chi$ which is related to quadrupole strength has a large negative value, -1.12 , in contrast with the mixing calculation result $\left(x=\left(x_{\pi}+x_{\nu}\right) / 2=-0.3\right)$. The overall agreement with data is reasonable, but a large discrepancy exists between the theoretical and some of the experimental $B(E 2)$ values in the case of an effective boson calculation. This is an indication of the limitation of the effective boson calculation.
The microscopic investigation of the $\mathrm{Zr}$ and $\mathrm{Mo}$ isotopes carried out by Federman and Pittel $^{3}$ in the shell model and Hartree-Fock-Bogoliubov framework considered $\mathrm{Z}=\mathbf{3 8}$ and $\mathrm{N}=\mathbf{5 0}$ as closed shells for protons and neutrons respectively, in their calculation. Based on this investigation we considered $Z=38$ as a proton subshell closure instead of $Z=40$, which had been chosen in the Sambataro et al. ${ }^{1}$ study, as a core. The calculation was carried out for the Mo isotopes based on the $Z=318$ proton and $\mathrm{N}=50$ shell, the preliminary results support the microscopic investigation of the Federman and Pittel that the $n-p$ interaction of valance particles outside the core ${ }^{8 r}(Z=38$ and $N=50)$ is sufficient to describe these nuclei. The number of proton bosons for Mo isotopes, outside the ${ }^{88} \mathrm{Sr}$ core, is 2 .

The IBA-2 Hamiltonian used in this calculation is written as follow:

$$
H=H_{\pi}+H_{\nu}+H_{\nu, \pi}
$$

where

$$
\begin{aligned}
& H_{\rho}=\epsilon_{\rho} n_{d_{\rho}}+k\left(a_{\nu} \cdot a_{\pi}\right)+\kappa_{\rho}\left(a_{\rho} \cdot a_{\rho}\right)+1 / 2 \xi_{2} \\
& \left(d_{\nu}^{\dagger} s_{\pi}^{\dagger}-d_{\pi}^{\dagger} s_{\nu}^{\dagger}\right) \cdot\left(\tilde{d}_{\nu} s_{\pi}-\tilde{d}_{\pi} s_{\nu}\right)+\Sigma_{K=1,3} \xi_{K}\left(\left[d_{\nu}^{t} d_{\pi}^{\dagger}\right](K)\right. \\
& \left.\cdot\left[\tilde{d}_{\pi} \tilde{d}_{\nu}\right](K)\right)+1 / 2 \Sigma_{L=0,2,4} c_{\rho}^{(L)}\left(\left[d_{\rho}^{\dagger} d_{\rho}^{\dagger}\right](L) .\right. \\
& {\left[\tilde{d}_{\rho} \tilde{d}_{\rho}\right](L),}
\end{aligned}
$$

and

$$
\begin{aligned}
& Q_{\rho}=d_{\rho}^{\dagger} s_{\rho}+s_{\rho}^{t} \tilde{d}_{\rho}+x_{\rho}\left[d \rho \tilde{d}_{\rho}\right](2) \\
& \rho=\nu, \pi
\end{aligned}
$$

The parameters obtained in the first calculation using $N_{\pi}=2$ and $N_{\nu}=2$ to 6 are presented in Table 1 . 
Table 1.

The neutron-proton interacting boson model parameters for the even-even Mo isotopes.

The $N_{\pi}=2$ and $C_{0 \pi}, C_{2 \pi}$, and $C_{4 \pi}$ are equal to zero.

\begin{tabular}{cccccccccccc}
\hline$N$ & $W_{\nu}$ & $\varepsilon_{\pi}$ & $\varepsilon_{\nu}$ & $\kappa$ & $x_{\pi}$ & $x_{\nu}$ & $c_{0 \nu}$ & $c_{2 \nu}$ & $c_{4 \nu}$ & $\xi_{1}-\xi_{3}$ & $\xi_{2}$ \\
\hline 54 & 2 & 1.22 & 0.74 & -0.10 & -1.2 & -1.2 & -0.4 & 0.1 & 0.0 & 0.035 & 0.08 \\
56 & 3 & 1.25 & 0.75 & -0.08 & -1.2 & -1.2 & -1.0 & 0.2 & 0.06 & & \\
58 & 4 & 0.81 & 0.62 & -0.059 & -1.2 & -1.2 & -0.52 & 0.18 & 0.12 & & \\
60 & 5 & 0.60 & 0.52 & -0.08 & -1.05 & -1.05 & -0.18 & 0.07 & 0.12 & & \\
62 & 6 & 0.39 & 0.55 & -0.115 & -0.68 & -0.68 & 0.07 & 0.0 & -0.01 & & \\
\hline
\end{tabular}

The interaction between the protons in a $1 \mathrm{~g}_{9 / 2}$ orbit and the neutrons in a $1 \mathrm{~g}_{7 / 2}$ is an important deformation driving force in the Mo isotopes and will increase as the number of neutrons increases. This has been reflected through the values of $\varepsilon_{\nu}$ and $\varepsilon_{\pi}$ which decreases as the number of neutrons increases (see Table 1). In Ref. [1] the energy of the proton and neutron $d$ bosons $\left(\varepsilon_{n}=\varepsilon_{\nu}\right)$ were chosen equal, the authors also have chosen proton quadrupole, $x_{\pi}$ and neutron quadrupole strength, $x_{\nu}$ for Mo isotopes to be the same as those in the $\mathrm{Ru}$ isotopes. Since these parameters $x_{\pi}$ reflect the direction of the shape transition, we expect these values to be different from those in the $R u$ isotopes. In our calculations we used different values for $\varepsilon_{\pi}$ and $\varepsilon_{\nu}$ following the shell model structure of these nuclei. The values of $x_{\pi}$ and $x_{\nu}$ have the same sign and amplitude which is an indication of a different symmetry for Mo isotopes from those in Sambataro's $\left(x-\left(x_{\pi}+x_{y} / 2=-0.3\right)\right.$ calculation.

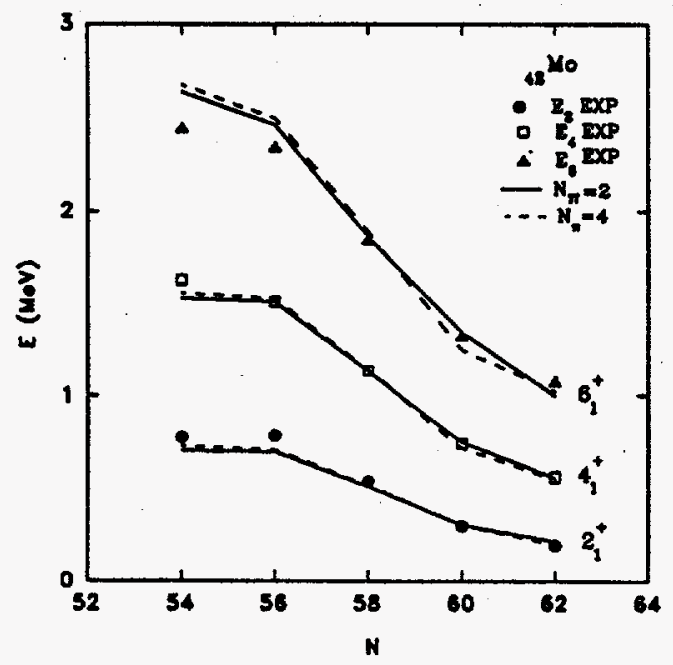

Fig. 1) Systematics of experimental and IBM-2 calculation with $N_{\pi}=2$ (solid line) and $N_{\pi}=4$ (dashed line) of the yrast band in the Mo isotopes.
Agreement of the result from IBM-2 with $\mathrm{N}_{\pi}=2$ with the experimental result is good. Figure 1 shows the results of the calculation for the yrast band in ${ }^{6-104} \mathrm{Mo}$ isotopes. In Fig. 2, the calculations are compared with the experimental results for other states in these nuclei. This shows that by considering $\mathrm{Z}=38$ as a proton closed shell, the IBM-2 can properly predict the property of the Mo isotopes. This result will also strengthen the IBM-2 model and its microscopic foundations, which we are planning to investigate further.

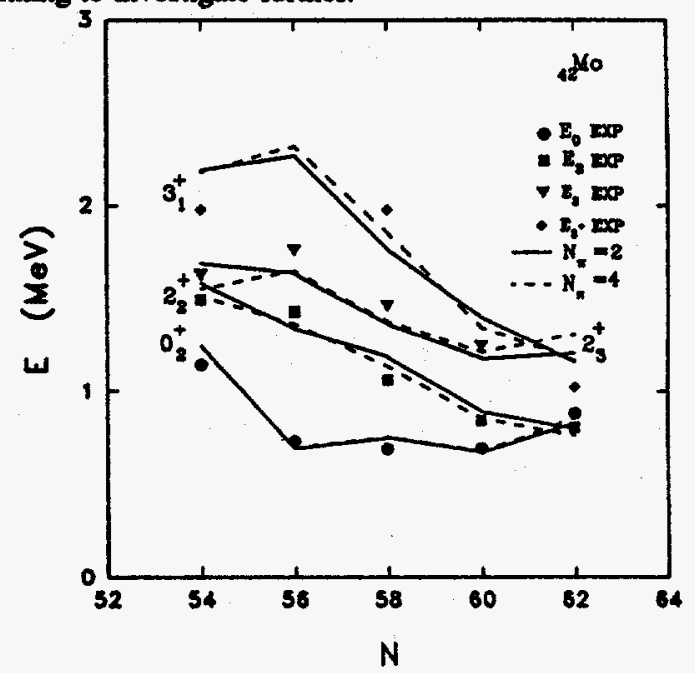

Fig. 2) The same as Fig. 1 but for a different set of states in the Mo isotopes.

In order to investigate further the validity of the $Z=38$ subshell closure in comparison with the $Z=50$ closed shell, we investigated the neutron-proton interacting boson model for Mo isotopes considering the $\mathrm{Z}=\mathbf{5 0}$ and $\mathrm{N}=50$ closed shell. In this case, the number of proton-hole bosons is 4 for the Mo isotopes. The parameter for the IBM-2 calculation with $N_{\pi}=4$ is given in Table 2. The results from this investigation for the yrast band are also shown in Fig. 1 and for the other states, in Fig. 2. 
Table 2.

The neutron-proton interacting boson model parameters for the even-even Mo isotopes.

The $K_{\pi}=4$ and $C_{0 \pi}, C_{2 \pi}$, and $C_{4 \pi}$ are equal to zero.

\begin{tabular}{rccccccccccc}
\hline$N$ & $N_{\nu}$ & $\varepsilon_{\pi}$ & $\varepsilon_{\nu}$ & $\kappa$ & $x_{\pi}$ & $x_{\nu}$ & $c_{0 \nu}$ & $c_{2 \nu}$ & $c_{\zeta_{\nu}}$ & $\xi_{1}=\xi_{3}$ & $\xi_{2}$ \\
\hline 54 & 2 & 1.28 & 0.68 & -0.06 & -1.2 & -1.2 & -0.4 & -0.1 & 0.0 & 0.035 & 0.05 \\
56 & 3 & 1.36 & 0.70 & -0.06 & -1.1 & -1.1 & -1.0 & 0.2 & 0.06 & & \\
58 & 4 & 1.02 & 0.65 & -0.06 & -1.1 & -1.1 & -0.6 & -0.1 & 0.10 & & \\
60 & 5 & 0.70 & 0.55 & -0.06 & -1.0 & -1.0 & -0.1 & -0.05 & 0.10 & & \\
62 & 6 & 0.58 & 0.50 & -0.088 & -0.55 & -0.55 & 0.08 & 0.05 & 0.10 & &
\end{tabular}

In conclusion the systematic investigation of eveneven Mo isotopes in the framework of the IBM-2 has been carried out for two proton bosons and four proton-hole boson configurations. The calculated results are in good agreement with the experimental data. This investigation shows that the low-lying structure of these nuclei can be well reproduced by the IBM-2 without introducing mixing of two configurations. The $B(E 2)$ values from both calculations for Mo isotopes are in reasonable agreement with the experimental data.

\section{REFERENCES}

1. M. Sambataro, and G. Molnar, Nucl. Phys. A376, 201 (1982).

2. J.M. Arias, C.E. Alonso, and M. Lozano, Nucl. Phys. A466, 295 (1987).

3. P. Federman, S. Pittel, Phys. Rev. C 20, 820 (1979).

\title{
INVESTIGATION OF SHAPE COEXISTENCE OF THE ODD-A TC ISOTOPES BY PROTON-NEUTRON INTERACTING BOSON-FERMION MODEL
}

\author{
H. Dejbakhsh, G. Ajupova, D. Latipov, S. Shlomo
}

The odd-A neutron-rich Tc isotopes with $Z=43$ lie in a region with an active $Z=38,40$ and $N=56$ subshell closure. The presence of these subshell closures has a strong effect on the shapes and shape transition of these nuclei.

The Tc isotopes have been investigated based on Interacting Boson Fermion model-1 (IBFM-1) and Interacting Boson Fermion model-2 (IBFM-2). In the IBFM-1 calculation the even-even core nuclei were investigated based on interacting boson model (IBM-1) which does not distinguish between proton and neutron bosons. In the IBFM-1 calculation the odd particle is coupled to the IBM-1 core. A more realistic interacting boson model (IBM-2) is the case when proton and neutron degrees of freedom are taken into account along with the interaction between them. In the IBFM-2 model the odd particle is coupled to an IBM-2 core. In all of the previous investigations of the Tc isotopes, the authors considered the even-even $\mathrm{Ru}$ isotopes as the core, assuming that the only valid shell is $Z=50$ and investigating the $\mathrm{Tc}$ isotopes as hole states. Our investigation reported here is based on the validity of the $\mathrm{Z}=38$ subshell closure with the states being considered a.s particle states. New experimental results at the TAMUJ Cyclotron Institute enable us to investigate Tc isotopes systematically.

We have investigated the low lying structure of the Tc isotopes within the framework of the IBFM-2 model. In our investigation we considered $\mathrm{Z}=38$ and $N=50$ घ|s proton and neutron closed shells respectively. The result for the even-even Mo isotopes, obtained within the IBM-2 model, was used as a core to investigate the odd-A Tic isotopes. The results of the calculation for Mo isotopes are reported in another section.

The IBFM-2 Hamiltonian can be written as follows:

$$
H=H_{B}+H_{F}+H_{B F}
$$


where $H_{B}$ is the IBM-2 Hamiltonian which has been discussed in another section of the progress report; for details see "The Interacting Boson Model" by lachello and Arima. The fermion Hamiltonian, $\mathrm{H}_{F}$, is written as:

$$
H_{F}=\left\{\Sigma_{j, m} E_{j} a_{j, m}^{\dagger} a_{j, m}\right\}_{\rho}, \rho=\pi, \nu
$$

where $E_{j} s$ are quasi-particle energies. The BosonFermion interaction for the odd-proton is written as:

$$
n_{B F}=A_{\pi} n_{d} n_{\pi}+r_{\pi}\left(0_{B}, \nu(2) \cdot q_{\pi}^{(2)}\right)+A_{\pi} F_{\pi \nu}
$$

where $Q_{m}$ is the neutron-boson quadrupole operator,

$$
a_{B \nu}(2)=(s t x d+d t x s)_{\nu}(2)+x_{\nu}(d t x d)_{\nu}(2)
$$

The $\mathrm{H}_{\mathrm{BF}}$ (boson fermion interaction) consists of a monopole-monopole, a quadrupole-quadrupole interaction between bosons and fermions and an exchange term with corresponding interaction strength, $A_{\pi}, r_{\pi}, \Lambda_{\pi}$, respectively. $A$ large number of parameters are involved in many $j$ orbitals. In order to reduced the number of free parameters, the matrix elements $\Gamma_{j j}^{\pi}$ and $\Delta^{\pi} j_{j} j^{\prime}$ ' have been expressed in term of physical quantities, such as occupation probabilities and quasi-particle energies, as follows:

$$
\begin{aligned}
& F_{\pi \nu}=a_{B \nu}(2)\left\{\Sigma_{j j ! j}, a_{j j},\left(u_{j} v_{j}+v_{j} u_{j}\right) \beta_{j}+j\right. \\
& \left.(1 /(2 j+1)) \cdot\left[\left(d t \times a_{j},\right)^{(j)}\left(a_{j}, \times s\right)\left(j^{\prime}\right)\right]^{(2)}\right\}_{\pi}
\end{aligned}
$$

$$
\begin{aligned}
& r_{j j}{ }_{j \prime}=r_{\pi}\left(u_{j} v_{j},-v_{j} u_{j}\right) Q_{j j}
\end{aligned}
$$

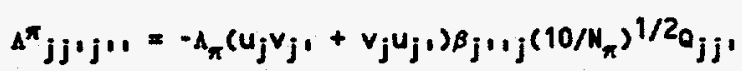

where:

$$
\begin{aligned}
& \beta_{j j^{\prime}}=\left\langle u_{j} v_{j}+v_{j} u_{j}\right\rangle Q_{j j} \\
& Q_{j j^{\prime}}=\left\langle 11 / 2 j\left|\gamma^{(2)}\right| 1{ }^{\prime} 1 / 2 j \cdot\right\rangle
\end{aligned}
$$

The quasi-particle energies, $E_{j}$, and occupation probabilities, $v_{j}$, were obtained by solving the BCS equations:

$$
\begin{gathered}
E_{j}=\left[\left(\epsilon_{j}-\lambda\right)^{2}+\Delta\right]^{1 / 2} \\
v_{j}=\left\{1 / 2\left[1-(e-\lambda) / E_{j}\right]\right\}^{1 / 2}, u_{j}=\left(1-v^{2}\right)^{1 / 2}
\end{gathered}
$$

where $\epsilon_{j}$ are single-particle energies, $\lambda$ is the Fermi energy and we have used $\Delta=12 \mathrm{~A}^{-12} \mathrm{MeV}$ for the pairing gap. In our work the single-particle energies, $\epsilon$, were taken from Ref. 2.

Both configurations $\left(\pi g_{9 n}\right.$ and $\left.\pi p_{1 / 2}\right)$ have been investigated using the same boson fermion interaction parameters, $A_{\pi}, \Gamma_{\pi}$, and $A_{\pi}$. The single particle orbitals used for the $T c$ isotopes are $2 p_{1 / 2}, 2 p_{3 / 2}, 1 f_{5 / 2}, 1 f_{12}$ for negative parity and $1 g_{9 / 2}, 2 d_{5 / 2}$ for the positive parity band. We only report here the result from the positive parity configuration. The neutron-proton interacting boson-fermion model parameters used in this calculation are as follows:

$$
A=-0.1 \mathrm{MeV}, \quad \Gamma=0.87 \mathrm{MeV}, \text { and } \Lambda=-4.0 \mathrm{MeV}
$$

The result for the BCS calculation and the parameters used to investigate the Tc isotopes in the present work are presented in Table 1. We have obtained very good agreement with the experimental energy levels especially for the low lying levels, $5 / 2^{+}$and $7 / 2^{+}$, which most of the previous calculations failed to predict. The experimental and calculated result from the IBFM-2 for positive parity are shown in Fig. 1.

\section{REFERENCES}

1. F. Iachello and A. Arima, The Interacting Boson Model (Cambridge University Press, 1987).

2. J.M. Arias, C.E. Alonso, and M. Lozano, Nucl. Phys. A466, 2955 (1987).

\section{Table 1.}

Result from the BCS calculation for positive parity orbitals along with the single particle energies used in the present calculation.

\begin{tabular}{cccc}
\hline nlj & $\epsilon_{j}$ & $E_{j}$ & $v_{j}^{2}$ \\
\hline $1 g_{9 / 2}$ & 3.20 & 1.24 & 0.40 \\
$2 d_{S / 2}$ & 7.20 & 4.42 & 0.02 \\
\hline
\end{tabular}


Fig. 1. Experimental (circle, square, triangle symbols) and calculated (lines) results of the positive parity states in the $T c$ isotopes. The $j^{\pi}$ for each state is shown on the left.

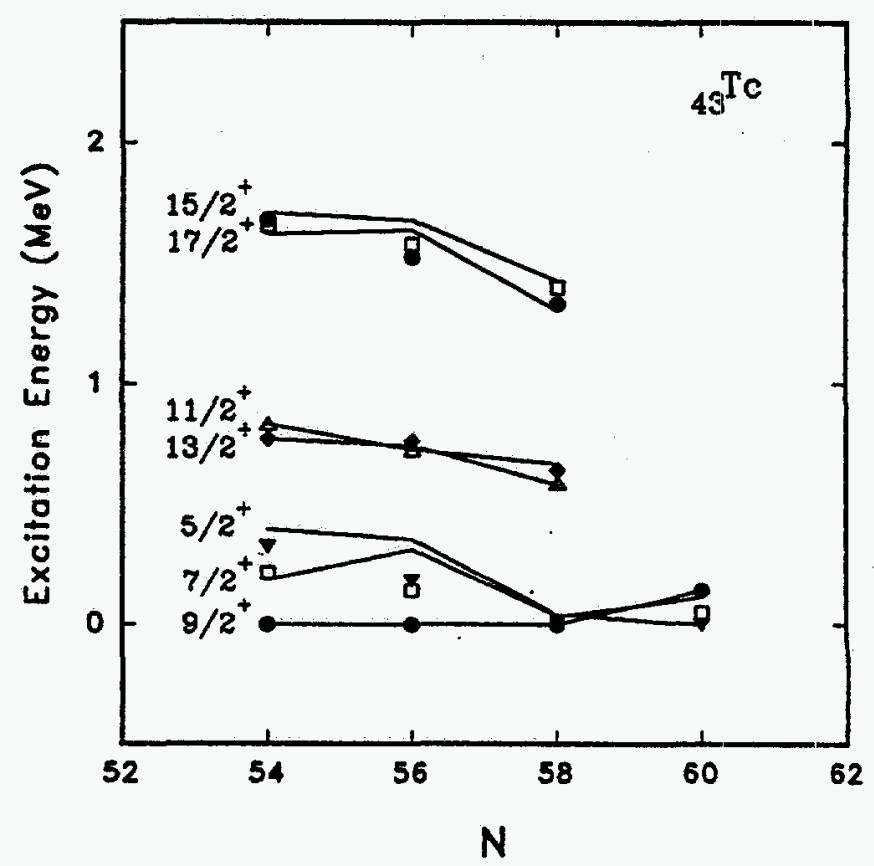

\section{DISAPPEARANCE OF FLOW IN INTERMEDIATE ENERGY NUCLEUS-NUCLEUS COLLISIONS}

\section{Hong Ming Xu}

Flow, or, the in-plane transverse momentum distribution of emitted particles, can carry important information about reaction dynamics of nucleus-nucleus collisions and the nuclear equation of state. At incident energies of a few tens of $\mathrm{MeV}$ per nucleon, the interaction between nucleons is dominated by the attractive part of the nuclear mean field and the particles are deflected to negative angles. ${ }^{1}$ At energies of a few hundred $\mathrm{MeV}$ to a $\mathrm{GeV}$ per nucleon, the individual nucleon-nucleon scattering and the repulsive part of the nuclear mean field become important and the particles are emitted to positive angles. ${ }^{2-4}$ At a certain intermediate incident energy, $E_{b a l}$, referred to as the energy of balance,,$^{5-8}$ the attractive part and the repulsive part of interactions are expected to balance each other and the flow crosses zero, changing from a negative sign at low energies to a positive sign at high energies. 912 Measurements of flow at different energies could, in principle, provide quantitative information concerning the nuclear equation of state at both low (attractive) and high (repulsive) densities. Significant efforts were made recently to study the equations of state at high densities. Whether there is any noticeable sensitivity of flow to equations of state at low densities remains unclear.
To investigate the sensitivities of $E_{b a l}$ to the equation of state and to the in-medium nucleon-nucleon cross section and to determine whether one can separate these dual dependencies, we have performed improved BUU calculations ${ }^{12.13}$ for ${ }^{{ }^{0}} \mathrm{Ar}+{ }^{27} \mathrm{Al}$ collisions. The upper-left panel of Fig. 1 shows the in-plane transverse momentum distributions of free nucleons for an incident energy $E / A=$ $65 \mathrm{MeV}$ with an in-medium nucleon-nucleon cross section of $\sigma_{\mathrm{NN}}=25 \mathrm{mb}$ (triangles), $35 \mathrm{mb}$ (circles), and $45 \mathrm{mb}$ (diamonds), respectively. For all values of $q_{\mathrm{NN}}$, the momentum distributions are characterized by negative slopes in the mid-rapidity region, indicating the importance of the attractive mean field at this energy. Calculations with larger $\alpha_{\mathrm{NN}}$ yield less steep slopes since nucleon-nucleon scattering tends to make the emission more isotropic. The evolution of flow with incident energy can be clearly seen in the calculations with $\sigma_{\mathrm{NN}}=$ $35 \mathrm{mb}$ (solid circles). In the mid-rapidity region, the slope becomes less negative at $E / A=85 \mathrm{MeV}$ (upper-right panel), flat at $\mathrm{E} / \mathrm{A}=100 \mathrm{MeV}$ (lower-left panel), and changes to positive sign at $\mathrm{E} / \mathrm{A}=125 \mathrm{MeV}$ (lower-right panel). This gradual change reflects the increasing importance of the repulsive part of the nuclear equation of state. The higher the incident energy, the larger the 


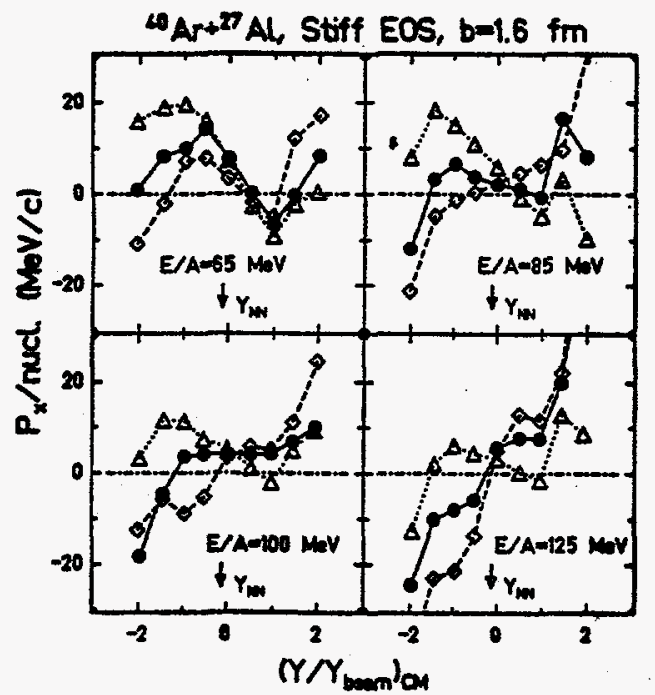

Fig. 1: The transverse momentum distribution as a function of longitudinal rapidity for ${ }^{10} \mathrm{Ar}+{ }^{27} \mathrm{Al}$ collisions calculated with a stiff equation of state at impact parameter $b=1.6 \mathrm{fm}$ and incident energies $E / A=65$ (upper left panel), 85 (upper right), 100 (lower left), and 125 (lower right) $\mathrm{MeV}$, respectively. The triangles, circles, and diamonds indicate calculations with $\sigma_{\mathrm{NN}}=25$, $35,45 \mathrm{mb}$, respectively. The lines are used to guide eyes. The arrows indicate the nucleonnucleon center of mass rapidity, $\mathbf{Y}_{\mathrm{NN}}$.

nuclear compression, and therefore, the stronger the repulsive mean field. The role of nucleon-nucleon scattering is clearly evident in all incident energies: more positive emission is observed with a larger value of $q_{w N}$. It is interesting to note here that for this asymmetric system, the momentum distribution becomes flat at a nonzero value (in the projectile side), in contrast to collisions between symmetric nuclei. ${ }^{5}$

To better characterize flow for this asymmetric system and to allow for a comparison with data, we follow Ref. [7] and define the flow parameter as the slope in the mid-rapidity region multiplied by $\left(\mathrm{Y}_{\text {beam }}-\mathrm{Y}_{\mathrm{NN}}\right) / \mathrm{Y}_{\text {beam }}$ Here $Y_{\mathrm{NN}}$ indicates the nucleon-nucleon center of mass rapidity and the slope is extracted by a linear fit of the momentum distribution within $\left|\mathrm{Y} / \mathrm{Y}_{\text {beam }}\right| \mathrm{CM} \leq 1$ (CM denotes the nucleus-nucleus center of mass). In Fig. 2, we display the flow parameters as a function of incident energy for ${ }^{40} \mathrm{Ar}+{ }^{27} \mathrm{Al}$ collisions at impact parameters $b=1.6,3$, and $5 \mathrm{fm}$, respectively. The solid- diamonds, circles, and triangles correspond to calculations with $\sigma_{\mathrm{NN}}$ $=25,35$, and $45 \mathrm{mb}$, respectively, and with a stiff equation of state. The open circles are the calculated results with $q_{\mathrm{NN}}=35 \mathrm{mb}$ and a soft equation of state. Clearly, at all impact parameters, the calculated flow parameter is very sensitive to the in-medium nucleon-

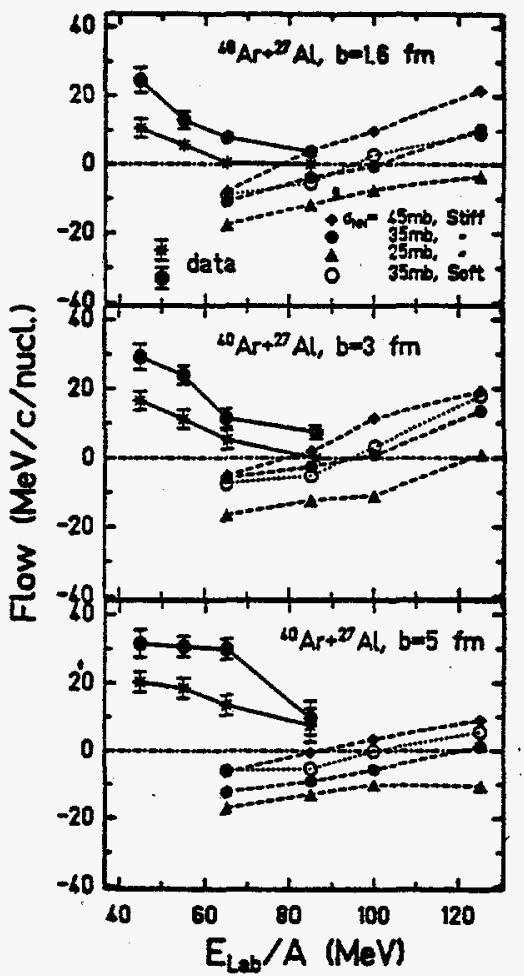

Fig. 2: The flow parameter as a function of energy for ${ }^{40} \mathrm{Ar}+{ }^{27} \mathrm{Al}$ collisions calculated at $\mathrm{b}=1.6$ (top window), 3 (center) and $5 \mathrm{fm}$ (bottom), respectively. The solid- triangles, eircles, and diamonds display the BUU calculations with a stiff equation of state and $\sigma_{\mathrm{NN}}=25,35,45 \mathrm{mb}$, respectively. The open circles are calculations with a soft equation of state and $a_{\mathrm{NN}}=35 \mathrm{mb}$. The open and closed stars indicate experimental data for particles with charge $Z=1$, and $Z=2$, respectively, taken from Ref. [7]. The lines are used to guide eyes.

nucleon cross section. For the calculations at $b=1.6 \mathrm{fm}$ and $3 \mathrm{fm}$, the flow parameter appears to be in-sensitive to the equation of state, allowing $q_{\mathrm{NN}}$ to be determined from data. The sensitivities to the equation of state and to $\sigma_{\mathrm{NN}}$ become comparable at impact parameters $b \geq 5 \mathrm{fm}$. This sensitivity to the EOS at large impact parameters could arise from the difference of EOS at low densities. ${ }^{13} \mathrm{~A}$ stiffer EOS has higher surface tensile strength ${ }^{14}$ and the emitted nucleons are therefore deflected to more negative angles (the corresponding $\mathrm{E}_{\mathrm{bal}}$ is higher). At smaller impact parameters, the sensitivity to the EOS at subnuclear density is washed out mainly because: 1) collisions among nucleons are more frequent, and 2) surface effects due to the different EOS are reduced (the average density in the participant region is closer to $\rho_{0}$, where the gradients of mean field for both EOS are about zero. If $q_{\mathrm{vN}}$ could be determined from the flow in small impact parameter collisions, the equation of state could, in turn, be extracted from the flow in large impact parameter collisions. 
The open and closed stars in Fig. 2 depict the experimental data for particles with charge $Z=1$ and $Z=2$, respectively, taken from Ref. [7]. Due to the experimental limitations, ${ }^{2}$ only the absolute values were extracted. The average flow per nucleon should be intermediate between the flow extracted for $Z=1$ and $Z=2$ since these two charges constitute the dominant part of the observed multiplicities. ${ }^{8}$ Based on coalescence models, one also expects that particles with charge $Z=1$ and $Z=2$ have the same value of $E_{\text {bal }}$, at which the flow vanishes. The calculations with $\mathrm{q}_{\mathrm{NN}}=35-45 \mathrm{mb}$ yield a value of $E_{\text {bor }} / A=80-100 \mathrm{MeV}$ at $b=1.6$ and $3 \mathrm{fm}$, consistent with the experimental data. The calculations seem to exclude values of $\alpha_{\mathrm{NN}}$ below $25 \mathrm{mb}$. Clearly, more data at higher energies are needed to determine the upper limit of $E_{b a l}$ (lower limit of $\sigma_{\mathrm{NN}}$ ). Experimental tests of whether particles with different charges and masses produce different values of $E_{b a l}$ would also provide a stringent test for coalescence models.

In summary, we have studied the disappearance of flow for ${ }^{0 \mathrm{Ar}}+{ }^{27} \mathrm{Al}$ collisions ${ }^{12}$ with improved BUU calculations. The influence of the attractive- and the repulsive- part of the nuclear mean field, and of the inmedium nucleon-nucleon scattering to the flow is investigated. Within the present parameterization for the EOS, the calculated energy of balance, $\mathrm{E}_{\text {bal }}$, is insensitive to the equation of state but quite sensitive to the inmedium nucleon-nucleon cross section, at impact parameters of less than $3 \mathrm{fm}$. This lack of sensitivity to the nuclear equation of state could provide a direct observable to extract the in-medium nucleon-nucleon cross section from experimental data. The sensitivity to both the equation of state and $\alpha_{\mathrm{vN}}$ becomes comparable at larger impact parameters. Although the change of flow from a negative sign at low energies to a positive sign at high energies reflects the increasing importance of the repulsive part of the $E O S$, the sensitivity of $E_{b a l}$ to the EOS at large impact parameters turns out to be determined by the EOS at low densities. Compared with the available data, the calculations indicate an in-mediun nucleon-nucleon cross section in the range of $q_{\mathrm{NN}} \approx$ 25-45 mb. Clearly, more experimental data in the high energy region are called for to determine the lower limit of $\sigma_{\mathrm{NN}}$. On the theoretical side, further calculations are needed to investigate the influence of the detailed algorithm of Pauli Blocking and of the surface energy to the predicted flow.

\section{REFERENCES}

1. M. B. Tsang, et al., Phys. Rev. Lett. 57, 559 (1986).

2. P. Danielewicz, 르 al., Phys. Lett. 157B, 146 (1985); Phys. Rev. C 38, 120 (1988).

3. K. G. Doss, et al., Phys. Rev. Lett. 59, 2720 (1987).

4. J. W. Harris, et al., Nucl. Phys. A 471, 241c (1987).

5. D. Krofcheck, et al., Phys. Rev. Lett. 63, 2028 (1989).

6. C. A. Ogilvie, et al., Phys. Rev. C 40, 2592 (1989); Phys. Rev. C 42, R10 (1990); Phys. Rev. C 40, 654 (1990); D. Krofcheck, e 씨., Phys. Rev. C 43, 350 (1990).

7. J. P. Sullivan, et al., Phys. Lett. 249B, 8 (1990).

8. J. Péter, et al., Nucl. Phys. A 519, 611 (1990); Phys. Lett. 237B, 187 (1990).

9. J. J. Molitoris and H. Stöker, Phys. Lett. 162B, 47 (1985).

10. A. Bonasera and L. P. Csernai, Phys. Rev. Lett. 59, 630 (1987).

11. G. F. Bertsch, et al., Phys. Lett. 189B, 384 (1987).

12. H. M. Xu, Phys. Rev. Lett. 67, 2769 (1991).

13. H. M. Xu, et al., Phys. Rev. Lett. 65, 843 (1990); Phys. Lett. 261B, 240 (1991).

14. G. Bertsch and D. Mundinger, Phys. Rev. C 17, 1646 (1978). 


\section{DISAPPEARANCE OF ROTATION IN HEAVY-ION COLLISIONS}

\section{Hong Ming Xu}

The azimuthal distributions of emitted particles in nucleus-nucleus collisions can carry important information concerning the reaction dynamics and the nuclear equation of state (EOS). ${ }^{1}$ At incident energies below E/A $=100$ $\mathrm{MeV}$, the azimuthal distributions have been investigated by using large angle particle-particle correlations and the observed in-plane enhancements could be well explained by models incorporating the decay of a hot rotating source. ${ }^{2}$ On the other hand, $\gamma$-ray circular polarization measurements ${ }^{3}$ indicated that particles were preferably emitted to negative angles because of the attracting mean field. Moreover, the in-plane transverse momentum distributions of emitted particles were predicted, 47 and later observed $d^{8-10}$ to change sign at a certain intermediate energy, referred to as the energy of balance. ${ }^{8-10}$ This transverse motion due to attractive nuclear mean field at low energies or repulsive mean field at high energies was referred to as the directed transverse motion.

Recently, in an effort to investigate relative contributions from the collective rotation and the directed transverse motion, azimuthal distributions of emitted particles were measured by Wilson et al. ${ }^{11}$ for ${ }^{40} \mathrm{Ar}+$ ${ }^{51} \mathrm{~V}$ collisions at energies from $\mathrm{E} / \mathrm{A}=35$ to $85 \mathrm{MeV}$ using the Michigan State University $4 \pi$ array. It was demonstrated that the observed in-plane enhancement gradually decreased with energy and the spectra became nearly isotropic at energies around $\mathrm{E} / \mathrm{A}=80 \mathrm{MeV}$. However, due to incomplete coverage of angles and large detector thresholds, the measured spectra exhibited large distortions and it was rather difficult to examine effects of rotational motion and directed transverse motion. Even for an ideal detecting system with no loss of efficiency, it is still difficult to get a detailed insight into the reaction dynamics which governs the final observables, mainly because experiments only provide data in the final exit channels. This task could, in principle, be assessed from dynamical model calculations.

To investigate effects of the collective rotation and the directed transverse motion and whether azimuthal distributions are sensitive to the equation of state and the in-medium nucleon-nucleon cross section, we have performed improved $\mathrm{BUU}^{7}$ calculations for ${ }^{40} \mathrm{Ar}+{ }^{51} \mathrm{~V}$ collisions. In Fig. 1, we show both $F_{\text {ip }}$ (solid symbols) and $F_{p s}$ (open symbols) as a function of incident energy. The first parameter is the fraction of particles emitted "in plane" defined as those within $45^{\circ}$ of the reaction

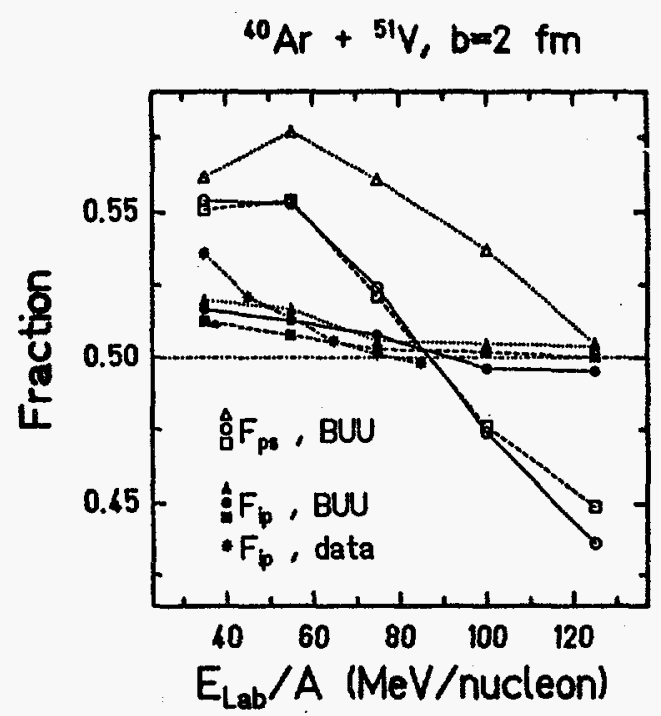

Fig. 1. The azimuthal fractions of $F_{\text {ip }}$ (solid symbols) and $F_{p}$ (open symbols) are shown as a function of incident energy calculated at $b=2 \mathrm{fm}$. The asterisks indicate experimental values of $F_{i p}$ taken from Ref. 11. The circles and the squares depict calculations with the stiff EOS and the soft EOS, respectively, and with $\sigma_{\mathrm{NN}}=41 \mathrm{mb}$. The triangles depict calculations with the stiff EOS and $\sigma_{\mathrm{NN}}=25 \mathrm{mb}$. The lines are used to guide eyes. Details concerning $F_{i p}$ and $F_{p w}$ are discussed in the text.

plane (satisfying $|\phi| \leq 45^{\circ}$ or $\left|\phi-180^{\circ}\right| \leq 45^{\circ}$; with $\phi$ $=0$ defined by the momentum vector pointing from the center of the projectile towards that of the target, when the impact parameter $b \neq 0$ ), and the second is the fraction of particles satisfying $|\phi| \leq 90^{\circ}$ or those on the "projectile side" as referred to in Ref. 11. To accumulate sufficient statistics, we have included all emitted nucleons satisfying $\mathbf{Y} \geq \mathbf{Y}_{\mathrm{c.M}}$; here $\mathbf{C . M}$. denotes the nucleusnucleus center of mass. The asterisks indicate $F_{i p}$ extracted experimentally for charged particles with $Z=2$, taken from Ref. 11.

For both equations of state, the predicted $F_{i p}$ decreases with incident energy, and becomes nearly isotropic at high energies, $\mathrm{E} / \mathrm{A} \geq 75 \mathrm{MeV}$, similar to the trends established by the experimental data. The noticeable differences between the calculated and measured values of $F_{i p}$ at low energies are as expected because (1) clusters, not incorporated by BUU calculations, tend to show larger in-plane enhancements than nucleons, and (2) emission due to a rotating source in the final stages could provide additional enhancements, 
particularly at low incident energies. We will come back to this point. The predicted values of $F_{i p}$ show little sensitivity to both the equation of state and the in-medium nucleon-nucleon cross section. In contrast, the predicted values of $F_{p}$ show little sensitivities to the EOS, but significant sensitivities to $\sigma_{\mathrm{NN}}$, with $\sigma_{\mathrm{NN}}=25 \mathrm{mb}$ producing significant larger values of $F_{p s}$ Thus measurements of energy dependent $F_{p}$ could in principle provide a powerful tool to pin down $\sigma_{\mathrm{NN}}$ accurately by comparing model calculations with experimental data.

Because of negative deflections at low energies and positive deflections at higher energies, the predicted $F_{p s}$ would only have a value of $F_{p}=0.5$, consistent with azimuthally isotropic emission, at a certain fixed energy (for $q_{\mathrm{NN}}=41 \mathrm{mb}$ here, this energy is at $\mathrm{E} / \mathrm{A}=87 \mathrm{MeV}$; for $g_{\mathrm{NN}}=25 \mathrm{mb}$, it is higher than $125 \mathrm{MeV}$ ). For later reference, we denote this energy as $E_{\text {ino. }}$. It is interesting to note here that $E_{\text {ino }}$ is higher than the energy of balance (for $q_{\mathrm{NN}}=41 \mathrm{mb}, \mathrm{E}_{\mathrm{bal}} / \mathrm{A} \approx 80 \mathrm{MeV}$ ) determined by the slope of transverse momentum distributions.? This difference arises because of the mass asymmetry between the projectile and the target. As shown in Ref. 7, the transverse momentum distribution becomes flat at a value away from zero (in normal kinematics, a negative value) and thus at $\mathrm{E}_{\mathrm{bol}}$ ( $\approx 80 \mathrm{MeV}$ per nucleon), $\mathrm{F}_{\mathrm{p} \text { at rapidity }}$ $Y \geq Y_{\text {C.M. }}$ is still consistent with negative deflections. As the mass asymmetry between projectile and target increases, the difference between $E_{i \text { iso }}$ and $E_{b e l}$ is expected to increase, and could therefore provide an additional observable for the extraction of $a_{\mathrm{NN}}$ from collisions between asymmetric systems.

To examine how effects of residue rotations depend on the incident energy, we display in Fig. 2 the angular momenta of bound residues $\left(\rho(r) \geq 10 \% \rho_{0}\right)$ as a function of time for ${ }^{40} \mathrm{Ar}+{ }^{5 i} \mathrm{~V}$ collisions at $\mathrm{E} / \mathrm{A}=35 \mathrm{MeV}$ (circles), $75 \mathrm{MeV}$ (squares), and $125 \mathrm{MeV}$ (triangles), respectively. The numerical accuracies for angular momentum conservation are demonstrated by the open symbols which depict the total entrance channel angular momenta in the center of mass. For calculations at all three energies, the angular momenta left in residues exhibit two distinct stages, a rather steep decrease at a early stage ( $\leq 120 \mathrm{fm} / \mathrm{c}$ ) and a slow decrease afterwards. This result is not a surprise since one would expect the earlier non-equilibrium stage could have a higher emission rate than that in the later evaporation stage. The interesting point here is that the angular momentum drops at the early non-equilibrium stage are so large at higher energies, $\mathrm{E} / \mathrm{A}=75$ and $125 \mathrm{MeV}$, that the angular momenta left in residues are even smaller than
${ }^{60} \mathrm{Ar}+{ }^{51} \mathrm{~V}$, Stiff EOS, $b=2 \mathrm{fm}$

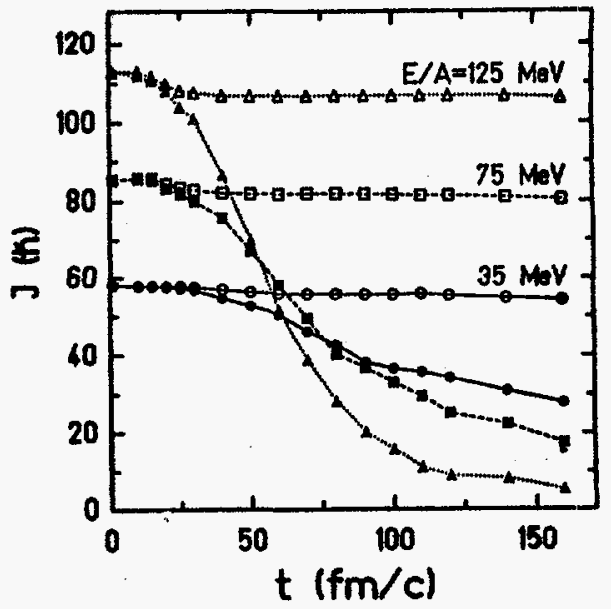

Fig. 2. The angular momenta of residues (solid symbols) as a function of time calculated at $E / A$ $=35 \mathrm{MeV}$ (circles), $75 \mathrm{MeV}$ (squares), and 125 $\mathrm{MeV}$ (triangles). The open symbols show the corresponding total angular momenta in the nucleus-nucleus center of mass. The lines are used to guide eyes.

that at $\mathrm{E} / \mathrm{A}=35 \mathrm{MeV}$ after a certain elapse of time ( $t=$ $75 \mathrm{fm} / \mathrm{c}$ for $E / A=75 \mathrm{MeV}$ and $t=60 \mathrm{fm} / \mathrm{c}$ for: $E / A=$ $125 \mathrm{MeV}$ ). Several points are immediately clear. First, because residues have not made a full cycle of rolation on the time scale which we follow, the contributions to azimuthal asymmetry from later residue rotations would be smaller at higher energies, simply because they have smaller angular momenta. At low energy, $E / A=35$ $\mathrm{MeV}$, because the residue still has a non-negligible angular momentum, $\mathrm{J} \approx 35 \hbar$, emission from residue rotation could provide additional in-plane enhancement to $F_{\text {ip }}$ (see Fig. 1). Second, the residue at energies above $\mathrm{E} / \mathrm{A}=75 \mathrm{MeV}$, if any, would have a much shorter life time than that at lower energies. Even for the earlier norequilibrium stage, the calculations appear to indicate that a hot equilibrated rotating source may not exist at high energies, E/A $\geq 75 \mathrm{MeV}$, due perhaps to multifragmentation which is not incorporated in the present calculations. This result is further supported by the nearly isotropic emission (although the instant angular momentum in the non-equilibrium stage is very larg()) indicated by parameter $F_{\text {ip }}$ as shown in Fig. 1.

In conclusion, with improved BUU calculations, we have investigated the dynamical origins of the azimuthal asymmetry in central ${ }^{40} \mathrm{Ar}+{ }^{51} \mathrm{~V}$ collisions. W/e demonstrated that dramatically different aspects of 
reaction dynamics could be revealed by constructing two simplified azimuthal parameters, $F_{\mathbf{p}}$ and $F_{p p^{*}}$. The calculations indicated that the contributions to azimuthal asymmetry from collective rotating residues decrease with incident energy and could eventually vanish at energies $\mathrm{E} / \mathrm{A} \geq 75 \mathrm{MeV}$. In contrast, the directed transverse motion due to the nuclear mean field remains important at all energies. The predicted azimuthal distributions appear not sensitive to the equation of state, but can be very sensitive to the in-medium nucleon-nucleon cross section, if they are properly constructed. We therefore propose that measurements of $E_{\text {wo }}$ at which the azimuthal distributions are consistent with isotropic emission as revealed by $F_{p e}$, could provide an important tool to pin down accurately the in-medium nucleon-nucleon cross section.

\section{REFERENCES}

1. G. M. Welke, M. Prakash, T. T. S. Kuo, S. Das Gupta and C. Gale, Phys. Rev, C 38, 2101 (1988).
2. M. B. Tsang, eㅡ a․, Phys. Rev. Lett. 52, 1967 (1984); Phys. Lett. 148B, 265 (1984); Phys. Rev. C 42, R15 (1990); Phys. Rev. C 44, 2065 (1991).

3. M. B. Tsang, 로 aㅛ., Phys. Rev. Lett. 60, 1479 (1988); Phys. Rev. Lett. 57, 559 (1986).

4. J. J. Molitoris and H. Stöcker, Phys. Lett. 162B, 47 (1985).

5. G. F. Bertsch, W. G. Lynch and M. B. Tsang, Phys. Lett. 189B, 384 (1987).

6. A. Bonasera and L. P. Csernai, Phys. Rev. Lett. 59, 630 (1987).

7. H. M. Xu, Phys. Rev. Lett. 67, 2769 (1991); and to be published.

8. D. Krofcheck, et al., Phys. Rev. Lett. 63, 2028 (1989).

9. C. A. Ogilvie, 뜨 al., Phys. Rev. C 42, R10 (1990);

D. Krofcheck, et al., Phys. Rev. C 43, 350 (1991).

10. J. P. Sullivan, et al., Phys. Lett. 249B, 8 (1990).

11. W. K. Wilson, eㅡ al., Phys. Rev. C 41, R1881 (1990).

\title{
RESIDUE TEMPERATURES AND THE NUCLEAR EQUATION OF STATE
}

\author{
H. M. Xu, P. Danielewicz* and W. G. Lynch*
}

Intermediate energy nucleus-nucleus collisions provide excellent opportunities for producing hot nuclear systems at excitation energies and temperatures near the values beyond which metastable nuclei cannot exist. Many experimental investigationa suggest a limiting temperature in the range of $T=4-6 \mathrm{MeV} .^{1 \cdot 12}$ Such observations could reflect either the thermal instabilities of metastable hot nuclei: or some dynamical limits to the energy which can be deposited into these residues via the reactions being studied.

To investigate the possible dynamical limits to the excitation energies of hot residual nuclei, and the sensitivity of residue excitation energies to the nuclear equation of state and the in-medium nucleon-nucleon cross section, calculations were performed with an improved Boltzmann-Uehling-Uhlenbeck (BUU) model for ${ }^{\circ} \mathrm{Ar}+$ ${ }^{124} \mathrm{Sn}$ reactions at a variety of incident energies. ${ }^{13}$ Figure 1 shows the predicted thermal excitation energy/nucleon as a function of the impact parameter for ${ }^{0} \mathrm{Ar}+{ }^{12} \mathrm{Sn}$ collisions at $E / A=35 \mathrm{MeV}$ (top window) and $\mathrm{E} / \mathrm{A}=65$ $\mathrm{MeV}$ (bottom window), respectively. A comparison of the thermal excitation energies calculated for the two equations of state and

$$
q_{N N}=\int \frac{d \sigma_{n n}}{d n} d n=41 m b
$$

are shown in the left hand panels. The thermal excitation energies calculated for the stiff EOS (open circles) are significantly larger than those calculated for the soft EOS (solid circles). This dependence can be attributed to the later freezeout time for calculations with the soft EOS, since it allows a longer time during which energy can be carried away by preequilibrium nucleon emission. The thermal excitation energies also depend more strongly upon impact parameter at the higher incident energy, where the target nucleus is less effective in capturing nucleons from the projectile, than at $E / A=35 \mathrm{MeV}$. The right hand side of the figure shows the sensitivity of the residue thermal excitation energies to the in-medium nucleon-nucleon cross section for calculations assuming a soft equation of state. Calculations with $g_{\mathrm{NN}}=41 \mathrm{mb}$ (solid circles) are slightly larger than the corresponding 
calculations with $\mathrm{q}_{\mathrm{NN}}=20 \mathrm{mb}$ (open circles). This difference is comparable, however, to the uncertainty in the calculated thermal excitation energy $\delta \mathrm{E} / \mathrm{A}=0.3$ $\mathrm{MeV}$ due to uncertrintien in defining the freezeout time.

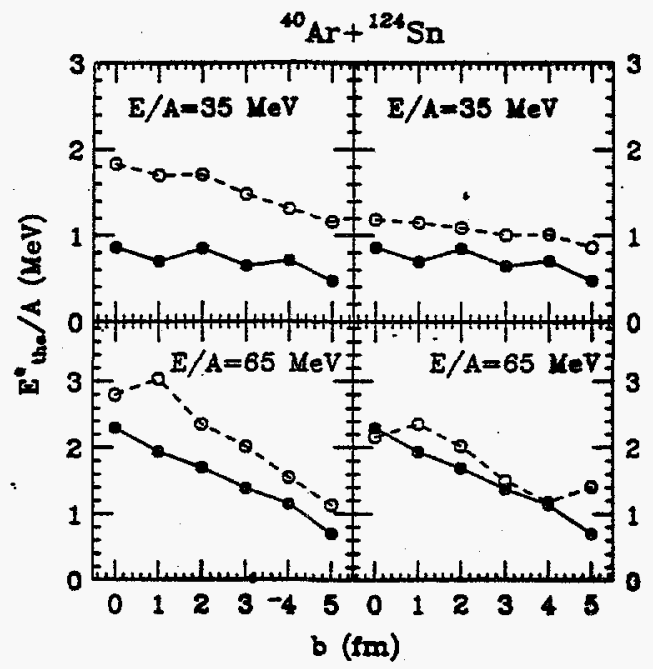

Fig. 1: Senuitivities of the temperature to the EOS (left panels) and $\alpha_{\mathrm{NN}}$ (right panela) for ${ }^{0} \mathrm{Ar}+{ }^{12} \mathrm{sn}$ collisions at $\mathrm{E} / \mathrm{A}=35 \mathrm{MeV}$ (top panels) and 65 $\mathrm{MeV}$ (bottom panels). Details are discussed in the text.

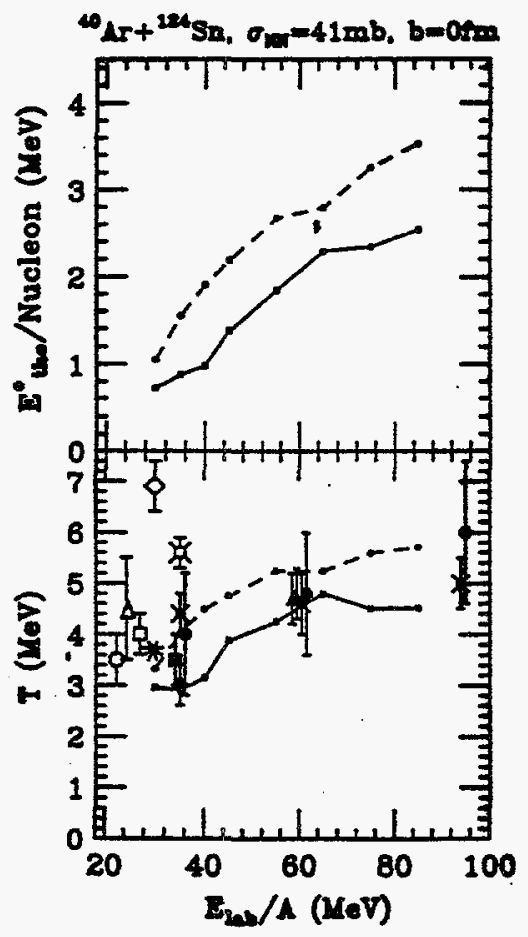

Fig. 2: Dependence of thermal excitation energies (top panel) and temperatures (bottom) on the incident energy for ${ }^{10} \mathrm{Ar}+{ }^{124} \mathrm{Sn}$ collisions at $\mathrm{b}=0$. The dashed lines are results for the stiff EOS and the solid lines are results for the soft EOS. The other symbols in the bottom panel are experimental data discussed in the text.
The top panel of Fig. 2 shows the energy dependence of the thermal excitation energy/nucleon at $b=0$ fm for the two equations of state and $\alpha_{\mathrm{NN}}=41 \mathrm{mb}$. The bottom panel shows corresponding estimates of the residte temperatures, calculated by integrating the Fermi-gts expression $\epsilon^{*}\left(T, \epsilon_{F}(\rho)\right)$ over the density distributions and equating the integrated value to the thermal energy from the numerical simulations. $\left(\epsilon_{F}(0)\right.$ is the Fermi energy for a nuclear system at density $\rho$.) Assuming equal Ferrai energies for protons and neutrons and the low temperaturc limit, this procedure yields:

$E_{\text {the }}^{*}=a r^{2}=\left(\frac{m}{3 \pi^{2}}\left(\frac{3 \pi^{2}}{2}\right)^{1 / 3} \int \alpha^{3} r \rho^{1 / 3}(\vec{r})\right) r^{2}$

where the integration is performed over the volume of the reaction residue, defined by the requirement $\rho(\vec{r}) \geq 0.1 \mu_{0}$. (Except at the highest incident energies, $E / A \geq 65 \mathrm{MeV}$, Eq. (1) provides a level density parameter close to $A / 10$.) In both panels of the figure, the dashed lines represent calculations using the stiff $E O S$ and the solid lines represent calculations using the soft EOS. Both equations of state predict a gradual increase in the residue thermal excitation energies and temperatures as the incident energy is raised from $\mathrm{E} / \mathrm{A}=30 \mathrm{MeV}$ to $85 \mathrm{MeV}$. The rate of increase, however, becomes very small at the highest incident energies and the calculated temperatures are nearly constant at $E / A \geq 65 \mathrm{MeV}$.

A saturation has been reported in the excitation energies deduced from measurements of the multiplicities of neutrons and $\alpha$ particles. ${ }^{1,2}$ Since pre-equilibrium emission carries away more nucleons in our calculations than assumed in the analysis of neutron and $\alpha$ particle multiplicities, we do not know whether our calculations are truly comparable to the data, and therefore, we refrain from making this comparison. Limiting temperatures have also been deduced from the energy spectra of light particles ${ }^{3-8}$ and from the relative populations of excited states of emitted complex fragments. ${ }^{9 / 2}$ In the bottom panel, we include the temperatures extracted from energy spectra of light charged particles by Refs. 6 (open diamond), 7 (open cross), 3 (solid triangles), 5 (open square), 8 (open triangle); neutron energy spectra by $R$ ef. 4 (star), and the emission temperatures extracted from excited states by Refs. 9 (solid- circles and crosses), 10 (open circle), 11 (solid diamond), and Ref. 12 (solid square), because such temperatures have proven relatively insensitive to the total mass of the colliding systen. Except for the few data points represented by an open diamond and an open cross, the calculated temperatures 
are similar to the measured values. This comparison must, nevertheless, be regarded as speculative, because measured temperatures are expected to be strongly impact parameter dependent and the influence of impact parameter averaging on these measurements is poorly understood.

In summary, thermal excitation energies of residues have been calculated as functions of the impact parameter for central ${ }^{40} \mathrm{Ar}+{ }^{124} \mathrm{Sn}$ collisions over a range of incident energies. These thermal excitation energies are evaluated at freezeout times determined from the time dependencies of the thermal excitation energy and the emission rate of nucleons. Both these thermal excitation energies and temperatures, obtained assuming Fermi gas level densities, are sensitive to the nuclear equation of state and the impact parameter. Surprisingly little sensitivity is observed to the in-medium nucleon-nucleon cross section.

\section{REFERENCES}

1. J. Galin, Nucl. Phys. A488, 297C (1988).
2. D. X. Jiang, et al., Nucl. Phys. A503, 560 (1989).

3. S. Song, et al., 130B, 14 (1983).

4. D. Hilscher, et al., Phys. Rev. C 36, 208 (1987).

5. D. Jacquet, et al., Phys. Rev. C 32, 1594 (1985); D. Jacquet, Thesis, Orsay, 1987.

6. R. Wada, et al., Phys. Rev, C 39, 497 (1988).

7. K. Hagel, et al., Nucl. Phys. A486, 429 (1988).

8. A. Chbihi, et al., Phys. Rev. C 43, 666 (1991).

9. Z. Chen, et al., Phys. Rev. C 36, 2297 (1987); Nucl. Phys. A473, 564 (1987).

10. H. M. Xu, et al., Phys. Rev. C 40, 186 (1989); Phys. Lett. 182B, 155 (1986)

11. C. Bloch, et al., Phys. Rev. C 36, 203 (1987).

12. T. K. Nayak, et al., Phys. Rev. C 45, 132 (1992).

13. H. M. Xu, P. Danielewicz and W. G. Lynch, Phys. Lett. B (submitted).

*National Superconducting Cyclotron Laboratory and Department of Physics, Michigan State University, East Lansing, MI 48824

\section{VIRTUAL PION CONTRIBUTION IN HEAVY ION REACTIONS}

\section{S. V. Akulinichev*}

In a system of nucleons interacting via effective meson exchange currents, there is a certain excess of virtual mesons. ${ }^{1,2}$ The direct contribution of such virtual pions, for example, to the deuteron electrodisintegration has been observed. ${ }^{1}$ This example shows that virtual nuclear pions can absorb and generate quanta of external fields. We demonstrated in an earlier paper ${ }^{3}$ that the contribution of virtual nuclear pions can explain the $\mathrm{K}^{+} \mathrm{A}$ total cross section enhancement at intermediate energies. Here we will use the same conjecture to explain some puzzles of heavy ion reactions at high energies. The kaons produced in the collisions of virtual pions with nucleons from a colliding nucleus are different from kaons produced in standard channels in their rapidity distribution and isospin content. The contribution of virtual pions can be very important at AGS energy, but also can be seen at CERN energies. If we further assume that virtual pions can evaporate in high energy collisions, then it can lead to a low $P_{1}$ enhancement of produced pions and photons, seen in many experiments.
The amount of virtual pions per nucleon, $n_{\pi}$, can be estimated in many different ways, with slightly different results. In a model ${ }^{2}$ with a realistic nucleon-nucleon interaction, $n_{n}=0.12$ was obtained for intermediate nuclei. In other papers, a smaller excess of pions was claimed (see Ref. 3 for further references). For the qualitative estimates of this paper, we will use the above value for definitiveness. Our main assumption is that at high energies, when the projectile energy is much larger than any energy of intranuclear interactions, the virtual nuclear pions interact with a projectile like free particles. From this point of view, there is no difference between nuclear nucleons and pions. In fact, nuclear nucleons and pions are both described by interacting fields and therefore are both virtual. Note that each excess pion is produced coherently by the interaction of several nucleons. ${ }^{2}$ For definitiveness we will assume an equal number of $\pi^{\circ}, \pi^{+}$, and $\pi^{-}$mesons in nuclei. This assumption is not very important here in contrast to the case of $\mathrm{K}^{+}$A scattering. ${ }^{3}$ According to our conjecture, in 
heavy ion collisions at high energies, the kaons are produced not only in the initial collisions of baryons and in the fireball, but also in $\mathbf{N}+\boldsymbol{\pi}$ and $\pi+\mathbf{N}$ collisions (here the first particle belongs to the projectile nucleus and the second one to the target nucleus). Note that the $\mathrm{K}^{-}$-production in $\mathbf{N} \mathbf{T}$ interactions is restricted by the isospin. This is the possible explanation of the $\mathrm{K}^{+} / \mathrm{K}^{-}$ relative yield enhancement at AGS energies. ${ }^{4}$ The total cross section for the $\mathrm{K}^{+}, \mathrm{K}^{\circ}$-production in the $\pi+N$ channel now can be written

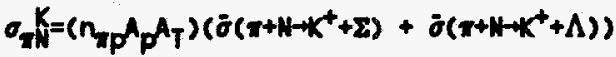

where the subseript $p$ or $T$ means projectile or target. $\bar{\sigma}\left(\pi+N+k^{+}+\Sigma\right)$ and $\bar{\sigma}\left(\pi+N+k^{+}+\Lambda\right)$ are isospin-averaged ${ }^{5}$ elementary cross sections. The total cross section for the $N+\pi$-channel is given by $\sigma_{\pi}^{K}=\sigma_{\pi N}^{K}\left(n_{\pi T} / n_{\pi p}\right) \approx \sigma_{T N}^{K}$ for two similar nuclei. The parametrization of elementary cross sections for Brookhaven energies can be found in Ref. 5. As it follows from the data, the AGS energy is very close to the resonance for $\mathrm{K}^{+}$and $\mathrm{K}^{\circ}$-production in $\pi \mathrm{N}$ collisions. Therefore the discussed contribution can be very important. The elementary $\pi+N$ cross section $\delta\left(\pi+N+K^{+}+X\right)$ for the beam energy $14.6 \mathrm{GeV} / \mathrm{A}$ is equal to $0.16 \mathrm{mb}$. For a large energy, we may neglect the intranuclear motion of constituents. From Eq. (1) we obtain $\sigma_{x N}^{K}(S i+A U) \approx 0.1 b$. We don't know experimental cross section for this reaction, but a comparison with the available data ${ }^{6}$ for other nuclei and lower energies shows that contribution of virtual pions is very important at AGS energy. The production of pions in the $\pi+N$ and $N+\pi$ channels must be peaked at the corresponding center of mass rapidities

$$
Y_{\pi N}=0.9, Y_{N \pi}=2.6 \text {. }
$$

The maximum at $Y=Y_{x N}$ was clearly observed ${ }^{7}$ and no other model has yet explained it. This peak is below the fireball rapidity $Y_{\text {part }}=1.25$ and below the $N N$ c.m. rapidity $Y_{N N}=1.7$. The peak at $Y=Y_{\pi N}$ observed by the same group for the $p+A u$ reaction may be connected to the fireball rapidity in this reaction because in the last case the projectile nucleon hits several target nucleons. There is no data for $\mathrm{K}^{+}$-production at $\mathrm{Y}=\mathrm{Y}_{\mathrm{Nx}}$. The observation of a peak in this region could be a strong support of our conjecture. Since negative kaons are not produced in $\pi \mathrm{N}$ interactions, their rapidity distribution should be different from that for other kaons: $\mathrm{K}^{-}$-mesons are only produced in baryon-baryon collisions and must be concentrated at midrapidity. This was observed in the data. 7,8 Note that for all kaons produced in the fireball, the rapidity distribution should be almost the sane. We can conclude that at Brookhaven energy the nuclear pion contribution to the strangeness production is very important and leads to some observed but previously unexplained effects.

At the CERN energy the elementary cross sectionis $\sigma(\pi+N-K+X)$ become relatively small, as does the role of this channel. The main contribution to the strangeneas production comes from the fireball and is strongly peaked at the central rapidity. We are not aware of reliable daia for elementary cross sections in this energy region. The simple continuation of the parametrization from Ref. 5 to the energy $200 \mathrm{GeV} / \mathrm{A}$ gives $\bar{\sigma}_{\pi N}(\pi+1-k+x)=0.01 \mathrm{mb}$, In reality, we may expect a larger value since all the hadronic cross sections become almont constant at smaller energies. We will use the above cross section only to estimate the minimal contribution of pions. For the $S+S$ reaction at $200 \mathrm{GeV} / \mathrm{A}$ we obtain $\alpha_{\pi}^{K}(s+s)=\alpha_{\pi}^{K}(s+s)$ $\geq 1.2 \mathrm{mb}$. This is a negligible contribution to the total yield of kaons. The dominant part of the kaons in the central collisions of these nuclei is produced at: $\mathbf{Y}_{\text {part }}=$ $\mathbf{Y}_{\mathrm{NN}}=3$. Therefore we may expect the observation of the pionic contribution, if any, in the rapidity dependence of the ratio $R(Y)=[\sigma(S+S) / \sigma(P+P)]$. This ratio strongly enhances all the non-central contributions. The c.rn. rapidities for two pionic channels are given by

$$
r_{\pi N} \simeq 2, Y_{N \pi} \simeq 4(3)
$$

As it follows from Ref. 9, the enhancement of $R(y)$ was observed at $Y \propto Y_{x N}$ ! We don't know any other possible source for this peculiarity and believe that here we have an indication of the virtual pion contribution, if the experimental results are confirmed. We also may expect that since the pionic channel is less important at this energy, the relative $K^{+} / K^{*}$ yield will be smaller than at AGS energy. The comparison of the data ${ }^{7,10}$ shows this trend. To make a firm conclusion, we need elementary cross sections and a more detailed rapidity dependence of the production cross section. The direct measurement of the strangeness of production at $Y \simeq Y_{N R}$ is very important:

It is interesting to note that the region of the low $p_{t}$ enhancement of pions," produced in heavy ion reactions, coincides with the momentum distribution of the excess pions in nuclei. ${ }^{2}$ It is a simple exereise to show that the transverse momentum dependence of the excess pions ${ }^{2}$ and of the low $p_{t}$-enhancement ${ }^{11}$ is quite similar. This suggests that we may assume some mechanism of the pions release (evaporation) in heavy ion collisions at high 
energies. In that case, pions will keep their initial transverse momentum distribution, but their rapidity distribution will be somehow modified. We find, however, that the amount of virtual pions in nuclei is at least 5 times smaller than needed to reproduce the absolute value of the low $p_{t}$ enhancement, ${ }^{12}$ provided we describe the whole spectrum with only one temperature. Thus the pion evaporation can produce a low $P_{t}$ enhancement, but not of the magnitude reported.

We demonstrated that virtual nuclear pions have a remarkable effect for the strangeness production in heavy ion collisions at high energies. This effect can be hardly reproduced by other models. These results are in contradiction with the papers ${ }^{13}$ claiming that there is no excess of pions in nuclei. It is important to clear up this problem to better understand nuclear properties.

I am grateful to the Cyclotron Institute at Texas A\&M University for the kind hospitality.

* Permanent address: Institute for Nuclear Research, 60-th October Anniversary Prospect 7a, Moscow 117312, Russia

\section{REFERENCES}

1. J. F. Mathiot, Nucl. Phys. A446, 123c (1985).

2. B. L. Friman eㅡ al., Phys. Rev. Lett. 51, 763 (1983).

3. S. V. Akulinichev, Cyclotron Institute Preprint N9114, Texas A\&M University (1991).

4. T. Abbott et aㅣ. (E802 Collaboration), Nucl. Phys. A498, 67c (1989).

5. T. Cugnon et al., Nucl. Phys. A422, 635 (1984).

6. S. Schnetzer et al., LBL-preprint 11118 (1981).

7. T. Miake (E802 Collaboration), Nucl. Phys A525, 231c (1991).

8. R. T. Ledoux, Nucl. Phys. A498, 205c (1989).

9. H. Ströbele (NA35 Collaboration), Nucl. Phys. A525, 59c (1991).

10. P. Sonderegger (NA38 Collaboration), Z. Phys. C38, 129 (1988).

11. E. V. Shuryak, Phys Rev. D42, 1764 (1990).

12. H. Ströbele (NA35 Collaboration), Z. Phys. 38, 89 (1988).

13. D. M. Alde et al., Phys. Rev. Lett. 64, 2479 (1990).

\title{
PIONS CONTRIBUTION TO K+-NUCLEUS SCATTERING
}

\author{
S. V. Akulinichev*
}

As a result of its long mean free path, the $\mathrm{K}^{+}$meson is a promising probe of the nuclear interior. Contrary to the expectation, there is a persistent discrepancy of about $10 \%$ between "conventional" optical model calculations of the $\mathrm{K}^{+}$-nucleus cross section and the available experimental data. Namely, the experimental cross sections in the $P_{L}=450-1000 \mathrm{MeV} / \mathrm{c}$ region are systematically larger than the optical model results. ${ }^{1-3}$ The widely accepted explanation of this discrepancy was based on the mechanism of "swelling" or partial deconfinement of nucleons in nuclei.3,4 A similar mechanism can be used to explain the European Muon Collaboration (EMC) effect in the deep inelastic lepton scattering from nuclei.5 This led many authors to the conclusion that the $\mathrm{K}^{+}$-nucleus scattering and the EMC effect reflect the same properties of nucleons in the nucleus.

Here we show that the $\mathrm{K}^{+}$-nucleus data can be explained without assuming the "swelling" of nucleons, provided the contribution of virtual nuclear pions is taken into account. As is known, ${ }^{6}$ some amount of virtual pions in nuclei is necessary to fulfill the energy-momentum sum rule for the deep inelastic scattering from nuclei composed of bound nucleons. We show here that the $\mathrm{K}^{+} \pi$ cross section can be larger than the $K^{+} \mathbf{N}$ cross section in the considered energy region. This makes important the pionic contribution to the $\mathrm{K}^{+}$-nucleus cross section.

At low (resonance) energies the $\mathrm{K}^{+} \mathbf{N}$ interaction is relatively weak because of its quark content. The quark structure of the $\mathrm{K}^{+}$meson is $|\bar{u}\rangle$ and it does not permit the s-channel resonances with the nucleon. But such resonances contribute to the scattering of $\mathrm{K}^{+}$meson from $\pi^{-}$and $\pi^{0}$, since their structure is represented by $\mid \mathrm{d} i \boldsymbol{\nu}$ and $(1 / \sqrt{2} \mid d a-u \bar{u}>$, respectively. Therefore one might expect a large $\mathrm{K}^{+} \pi$ cross section.

Direct experimental results for the $\mathrm{K}^{+} \pi$ cross sections are not available, but these cross sections can be reconstructed from the partial-wave analysis of the $K^{+} p$ 
scattering. This analysis gives phenomenological $K_{\pi}$ phase shifts $\delta_{L}^{2 I}$, where $I$ and $L$ are the total isospin and angular momentum of the $K_{\pi}$ system. The experimental results can be fitted to an effective range form for the phase shifts

$$
q^{2 L+1} \cot \delta_{L}^{2 I}=\frac{1}{L_{L}^{2 I}}+\frac{1}{2} r^{2 I} q^{2}
$$

where $q$ is the c.m. momentum and the phenomenological parameters $a_{L}^{21}$ and $r_{L}^{21}$ are given by

$$
\begin{aligned}
& a_{S}^{1}=2.39 \mathrm{GeV}^{-1}, \quad r_{S}^{1}=-1.76 \mathrm{GeV}^{-1} \\
& a_{S}^{3}=-1.00 \mathrm{GeV}^{-1}, \quad r_{S}^{3}=-1.76 \mathrm{GeV}^{-1} .
\end{aligned}
$$

The contribution of partial waves with $L \neq 0$ is very small and can be neglected in the energy region under consideration. From (1) and (2) we obtain the results for the $I=\frac{1}{2}$ and $I=\frac{3}{2}$ cross sections presented in Fig. 1, where the data for the $K^{+} \mathrm{p}$ cross section are also shown. As we can see from this figure, the $I=\frac{1}{2}$ experimental cross section for $\mathrm{K} \pi$ scattering is almost three times larger than the $K^{+} \mathbf{p}$ cross section.

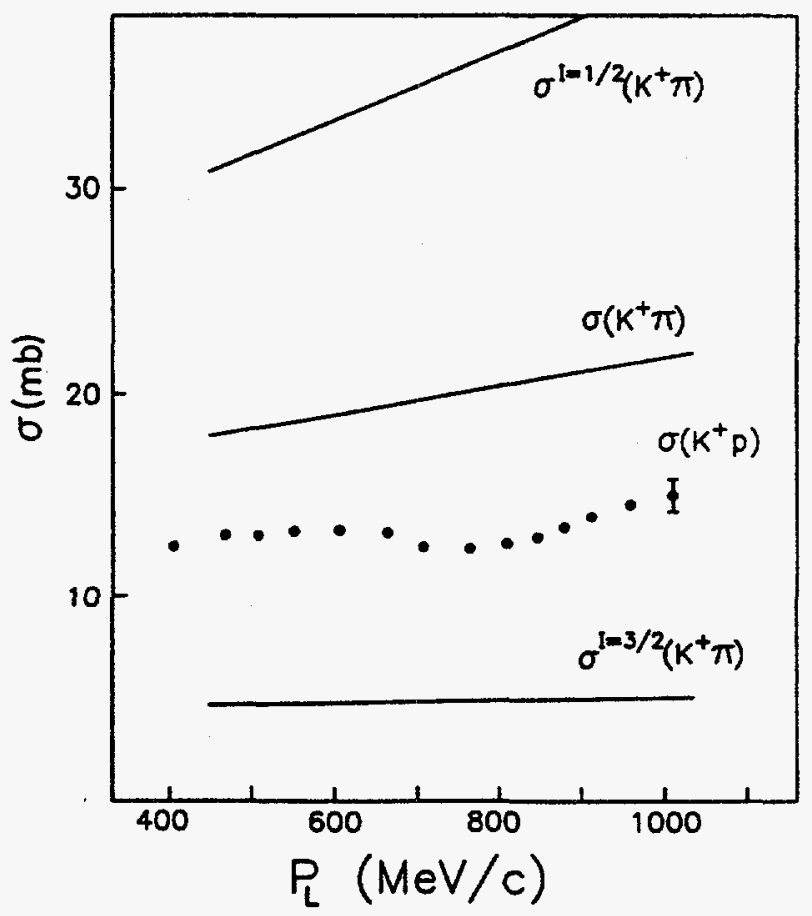

Fig. 1. Phenomenological fit for the $\mathrm{K}^{+} \pi$ cross section. The intermediate line is the average of the two possible isospins, $I=\frac{1}{2}$ and $I=\frac{3}{2}$, of the $K^{+} \pi$ system. $P_{L}$ is the lab momentum of $K^{+}$.

The optical model results ${ }^{2,3}$ for the ratio

$$
R=\frac{\sigma\left(K^{+12} c\right)}{\sigma \sigma\left(K^{+} d\right)}
$$

are shown in Fig. 2 by the lower band. This band represents the uncertainty of the "conventional" calculations. Even with many possible sources of uncertainty of the conventional mechanism taken into account, there is still a discrepancy of about $10 \%$ between the calculated and experimental results.

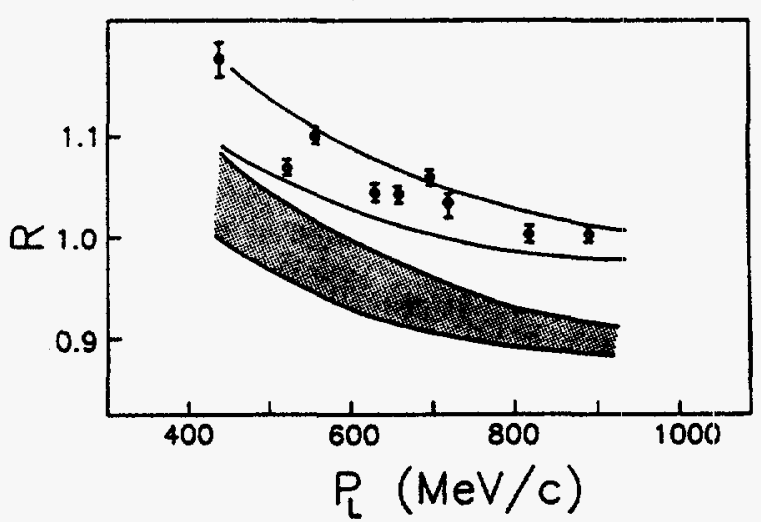

Fig. 2. The total cross section ratio for the $\mathbf{K}^{+}$-nucleus scattering. The data for ${ }^{12} \mathrm{C}$ are from Ref. 11 . The lower band shows the optical model results. ${ }^{3}$ The upper band is the result of this work.

We now show that this discrepancy can be eliminated by the contribution of virtual pions. Some amount of virtual mesons is present in a system of bound nucleons interacting by the effective mesonic field. The contribution of these mesons is not included in the elementary projectile - nucleon amplitudes used in the multiple scattering formalism. The number of pions por one nucleon can be related to the enhancement factor $K$ of the photonuclear sum rule ${ }^{8}$

$$
n_{\pi}=\frac{3}{8} k /<\omega_{\pi}^{-1}>m_{\pi} .
$$

where $\left\langle\omega_{\pi}^{-1}\right\rangle$ is an average pion inverse energy. If we take $^{9}\left\langle\omega_{\pi}\right\rangle=400 \mathrm{MeV}$ then from (4) we obtain ${ }^{8} n_{\pi}$ * 0.07 for nuclear matter. Using energy-momenturn conservation it was found in Ref. 6 that

$$
n_{\pi}=(\langle\epsilon\rangle-\mu) /\left\langle\omega_{\pi}\right\rangle,
$$

where $\langle\epsilon\rangle$ and $\mu$ are the average separation energy and the chemical potential. If we use for nuclear matter $\langle\mathrm{E}\rangle=40 \mathrm{MeV}, \mu=8 \mathrm{MeV}$ and $\left\langle\omega_{\pi}\right\rangle=400 \mathrm{MeV}$, we obtain the estimate $n_{\pi} \approx 0.08$, which is very close to the result of Eq. (4). In a recent work, Nakano anid Wong ${ }^{10}$ obtained the value of $n_{\pi}=0.057$. Therefore, for 
the average number of pions per nucleon, we use the intermediate value of

$$
n_{\pi}=0.07 .
$$

The energy momentum distribution of pions and the off-mass-shell continuation of the $\mathrm{K}^{+} \pi$ amplitude are unknown. But as a first approximation, we can neglect them because of the smooth behavior of the $\mathrm{K}^{+} \pi$ cross sections. Instead of (3) we now can write,

$$
R=R_{\text {opt }}+n_{\pi} \sigma\left(K^{+} \pi\right) / \sigma\left(K^{+} N\right) \text {, }
$$

where $\mathbf{R}_{\text {opt }}$ is the optical model result of Ref. 3 and $\sigma\left(K^{+} \pi\right)$ is the average $K^{+} \pi$ cross section. For the latter we use the expression,

$$
\sigma\left(K^{+} \pi\right)=\frac{\sigma^{I=1 / 2}\left(K^{+} \pi\right)+\sigma^{I=3 / 2}\left(K^{+} \pi\right)}{2}
$$

since the isospin content of virtual pions is unknown. The last equation is, of course, an approximation that may be improved in later publications.

Numerical results are presented in Fig. 2 by the upper band. As we can see from this figure, the experimental data are now inside the theoretical band.

Let us now briefly discuss the uncertainties and the limitations of this model. First, we do not try to describe the differential cross sections. As pointed out in Ref. 3, there are large additional uncertainties in the experimental and theoretical (optical model) differential cross sections. The clean observable is the ratio of total cross sections. The average separation energy $\langle\varepsilon\rangle$ of ${ }^{12} \mathrm{C}$ is smaller than that of nuclear matter. Therefore $n_{\pi}$ for carbon should be correspondingly smaller than the nuclear matter value. On the other hand, some authors claim larger values of $n_{\pi}{ }^{12}$ and $\left.\langle\varepsilon\rangle\right\rangle^{13,14}$ Therefore we expect that (6) does not significantly overestimate the effect. Equation (7) is certainly an approximate result. Besides usual limitations of the optical model, it is assumed that the multiple scattering from nucleons and the projectilepion interactions develop independently. It is difficult to assess here the validity of this approximation, but it is likely that the error is smaller than the bare pionic contribution. The large uncertainty comes from Equation (8), as it follows from Fig. 1. For $\sigma\left(K^{+} \pi\right)$ we simply used a value at the center of the corresponding uncertainty band. This value significantly overestimates the cross section for $\pi^{0}$ mesons but it is close to the average cross section for the mixture of $\pi^{-}$and $\pi^{+}$mesons. More detailed analysis of the isospin content of virtual pions as well as of the $K \pi$ phase shifts are needed. Note that results (1) and (2) are based on the elastic unitarity assumption ${ }^{7}$ and may underestimate the $K \pi$ cross sections.

We conclude that the discrepancy between the "conventional" calculations and the data for the $\mathrm{K}^{+}$. nucleus scattering can be explained by the contribution of virtual pions. These pions are the result of the binding nuclear forces. It was shown ${ }^{6}$ that these forces can significantly change the nuclear structure function due to the binding correction. Thus the nuclear effects in the deep inelastic scattering and in the $\mathrm{K}^{+}-\mathrm{A}$ scattering indeed may have the same physical origin.

I would like to thank S. Shlomo for useful discussions.

* Supported in part by the National Science Foundation Grant N PHY-9001886.

** On leave from the Institute for Nuclear Research of the Academy of Science, Moscow - 177312, 60th October Anniversary Prospect $T^{*}$, Russia

\section{REFERENCES}

1. M. J. Paez, R. H. Landau, Phys. Rev. C 24, 1120 (1981).

2. P. B. Siegel, W. B. Kaufmann, W. R. Gibbs, Phys. Rev. C 30, 1256 (1984).

3. P. B. Siegel, W. B. Kaufmann, W. R. Gibbs, Phys. Rev. C 31, 2184 (1985).

4. G. E. Brown, C. B. Dover, P. B. Siegel, W. Weise, Phys. Rev. Lett. 60, 2723 (1988); W. Weise, Nuovo Cim. 102A, 265 (1989).

5. R. Jaffe, F. Close, R. Roberts and G. Ross, Phys. Lett. 134B, 449 (1984).

6. S. V. Akulinichev, S. A. Kulagin, G. M. Vagradov, NBI-preprint, 8520 (1985); Phys. Lett. 158B, 485 (1985).

7. P. Estabrooks et al., Nucl. Phys. B133, 490 (1978).

8. M. Ericson and M. Rosa-Clot, Phys. Lett. 188B, 11 (1987).

9. V.R. Pandharipande, Nucl. Phys. A446, 189 (1985).

10. K. Nakano, S. S. M. Wong, Phys. Lett. 257B, 10 (1991).

11. E. Piasetzky, Nuovo Cim. 102A, 281 (1989).

12. S. Glazek, M. Schaden, Z. Phys. A 323, 451 (1986).

13. S. Shlomo, G. M. Vagradov, Phys. Lett. 232B, 19 (1989).

14. M. Baldo et al., Preprint ULG-PNT-91-5-P, University of Liege, Belgium (1991). 


\section{H. A. Bethe, (1) G. E. Brown, (2) and C. M. Ko}

Chiral symmetry is broken through the development of a quark condensate in the physical vacuum. The zero temperature quark condensate is determined by the cut off $\Delta$ in momentum and the mass $m_{Q}$ of the constituent quark. At finite temperature, the condensate is reduced by the excitation of quarks from the negative to the positive energy states. If $\Lambda>>m_{Q}$, as in consideration to date, then the condensate depends on the temperature quadratically. This quadratic temperature dependence is also given uniquely by chiral invariance. ${ }^{1}$ By comparing the two results, one obtains $A \approx 607 \mathrm{MeV}$. When the covariant momentum cut off is used, we expect it to have a larger value and thus be comparable to the scale for chiral symmetry breaking $\approx 1 \mathrm{GeV}$.

The magnitude of the zero temperature quark condensate is known from the Gell-Mann, Oakes, Renner relation ${ }^{2}$ to be $\langle 0|\bar{u} u| 0\rangle=-(240 \pm 20 \mathrm{MeV}) .{ }^{3}$ Using the above momentum cutoff, one can then determine the constituent quark mass to be $m_{Q} \approx 329 \mathrm{MeV}$, which is about $1 / 3$ of the nucleon mass.

In the broken symmetry mode of chiral symmetry where quarks are condensed in negative energy states, mesons, not quarks and gluons, are the correct variables to use. We consider the constitutent quarks to be held together in the condensate by interactions involving scalar meson exchange. This gives us the expression for the vacuum energy obtained in the Nambu-Jona-Lasinio theory, ${ }^{3,4}$

$$
\begin{aligned}
& { }_{x S R}^{-8}=E_{v a c}=-12\left[\int_{0}^{A} \frac{d^{3} k}{(2 \pi)^{3}} \sqrt{k^{2}+m_{Q}^{2}}-\frac{1}{2} \int_{0}^{\Lambda}\right. \\
& \left.\frac{d^{3} k}{(2 x)^{3}} \frac{m_{Q}^{2}}{\sqrt{k^{2}+m_{Q}^{2}}}\right]+\frac{3}{2} \frac{\Lambda^{4}}{x^{2}}=-\frac{3}{x^{2}}\left\{\frac{1}{2} \Lambda\left(\Lambda^{2}+m_{Q}^{2}\right)^{3 / 2}\right. \\
& \left.-\frac{3}{4} m_{Q}^{2} \Lambda \sqrt{\Lambda^{2}+m_{Q}^{2}}+\frac{1}{4} m_{Q}^{4} \ln \left[\frac{\Delta+\sqrt{\Lambda^{2}+m_{Q}^{2}}}{m_{Q}}\right]-\frac{\Lambda^{4}}{2}\right\} .
\end{aligned}
$$

The last term on the right hand side subtracts off the energy of the perturbative vacuum. Vector mesons couple to baryon number and their effect will cancel out in the subtraction of the energy of the perturbative vacuum. With the values of $\Lambda$ and $m_{Q}$ determined above, we find $-E_{\text {vac }}=B_{\chi S R} \approx(161 \mathrm{MeV})^{4} \approx 88 \mathrm{MeV} / \mathrm{fm}^{3}$, just in the range of values usually taken from the MIT bag model. As $\Lambda \rightarrow \infty$, the vacuum energy diverges logarithmically with $\Lambda$. If both $\Lambda$ and $m_{Q}$ scale the same way with density, ${ }^{6} m_{Q}^{*} / m_{Q}=\Lambda^{*} / \Lambda=\Phi^{*}$, then $B_{\chi S R}^{*}=\left(\Phi^{*}\right)^{4} B_{\chi S P P}$ Choosing the energy of our physical vacuum to be zero, we find that the vacuum energy at finite density, is

$E_{\text {vac }}=B_{X S R}\left[1-\left(\Phi^{*}\right)^{4}\right]=B_{\chi S R}\left[1-\left(\frac{m^{*}}{m}\right)^{4}\right]$.

where $m *$ represents any of the hadron masses, other than that of the pion, which have common scaling. The vacuum energy is presently being incorporated inio calculations of the nuclear equation of state.

(1) Laboratory of Nuclear Studies, Cornell University, Ithaca, NY 14853

(2) Department of Physics, State University of New York, Stony Brook, NY 11794

\section{REFERENCES}

1. J. Gasser and H. Leutwyler, Phys. Lett. B184, \&3 (1989).

2. M. Gell-Mann, R. J. Oakes, and B. Renner, Phys. Rev. 175, 2195 (1968).

3. M. Asakawa and K. Yazaki, Nucl. Phys. A504, 668 (1989).

4. S. Li, R. S. Bhaleroa, and R. K. Bhaduri, Int. J. of Mod. Phys. A6, 501(1991).

5. For nucleons as variables, this was first done by $\mathbf{G}$. E. Brown, Phys. Rept. 163, 167 (1988).

6. G. E. Brown and M. Rho, Phys. Rev. Lett. 66, 27200 (1991). 


\title{
PION MULTIPLICITY AS A PROBE OF THE DECONFINEMENT TRANSITION IN HEAVY-ION COLLISIONS
}

\author{
Mark I. Gorenstein, (1) C. M. Ko, and S. N. Yang(2)
}

Pion production from relativistic heavy ion collisions is studied in the hydrochemical model with self-similar expansion.' For hadronic matter we use the ideal gas equation of state for a gas of pions, nucleons and deltas modified by the excluded volume corrections. ${ }^{2}$ To describe the quark-gluon plasma phase we use the bag model equation of state with massless $u, d$-quarks and the bag constant $B=400 \mathrm{MeV} / \mathrm{fm}^{3}$. The phase diagram for our two-phase system is constructed according to the Gibb's procedure for systems with a first-order phase transition. We find reasonable values for the phase transition parameters between the hadronic $(\mathrm{H})$ and quarkgluon $(Q)$ phases for baryon hard-core radii $r=$ $(0.5-0.7) \mathrm{fm}$ in the hadronic phase. The $T-n$ phase

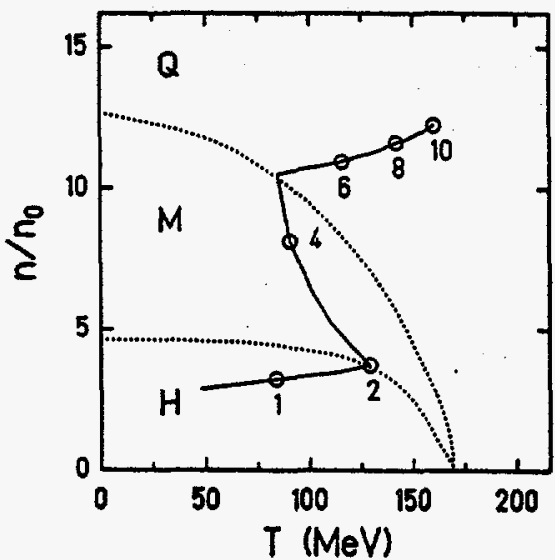

Figure 1. Phase diagram (dotted curves) in the $T-n$ plane. The solid eurve is the shock adiabat with the numbers near the open circles being the incident energies in $\mathrm{GeV} /$ nucleon.

diagram, where $\mathrm{n}$ is the baryonic number density, for $\mathrm{r}=$ $0.65 \mathrm{fm}$ is shown in Fig. 1 by the dotted curves.

We assume that via the compression shock model $^{3}$ a baryon-rich fireball is formed in the central collision of two heavy ions. The compression shock adiabat in the $T$ - $n$ plane is shown in Fig. 1 by the solid curve. The numbers near the open circles, which correspond to the entry points in the phase diagram, stand for the values of the incident energy per nucleon in units of GeV/nucleon.

From a given initial state the fireball expands up to the freeze out at some critical baryonic density which we choose as $n_{f}^{\text {th }}=0.5 n_{0}$. In the quark-gluon and mixed phases we assume both thermal and chemical equilibriums. For the expansion in the hadronic phase, we include the reactions $\mathbf{N}+\pi * \Delta$ and $N+N-N+$
$\Delta$. So the chemical equilibrium among hadrons can be violated and the total entropy of the fireball becomes a nonconserved quantity.

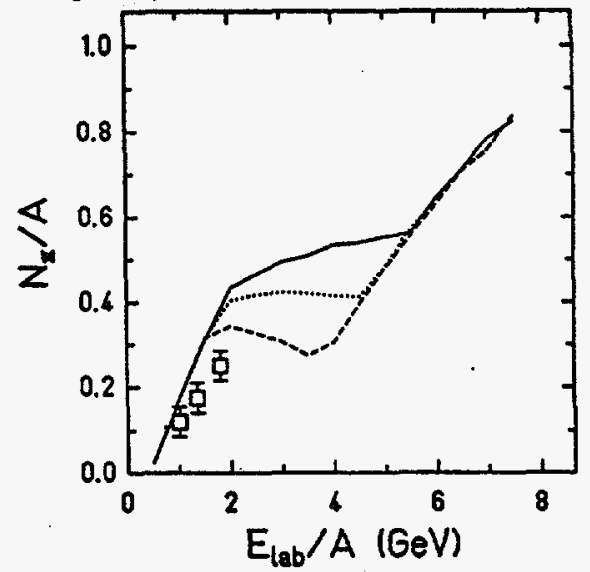

Figure 2. The incident energy dependence of the pion multiplicity per nucleon. The solid, dotted, and dashed curves denote results obtained with the baryon radius parameter $r=0.60,0.65$ and $0.70 \mathrm{fm}$, respectively. The data points given by the open squares are from Ref. 4.

In Fig. 2, we show the results of our calculation for the pion multiplicity per nucleon, $N_{\pi} \quad / A$, as a function of the incident energy per nucleon, $E_{\text {lab }} / A$, for three different values of baryon radius. The solid, dotted, and dashed curves correspond to $r=0.60,0.65$ and $0.70 \mathrm{fm}$, respectively. The pion multiplicity is calculated as a sum of pion and delta numbers at the thermal freeze-out when the baryonic number density is $\mathrm{n}=\mathrm{n}_{\mathrm{r}}^{\text {th }}$ We see that when the initial state of the fireball is in the mixed phase we find a plateau-like structure in the dependence of pion multiplicity on the incident energy. The physical origin of this phenomenon is the anomalous thermodynamical properties of the mixed phase. With increasing initial collision energy both baryonic density and energy density on the compression shock adiabat inside the mixed phase increase but the fireball temperature decreases. Besides the structure in pion multiplicity function this temperature decrease also leads to the suppression of dilepton production in the corresponding range of the initial energies. ${ }^{1}$ It is thus of interest to study experimentally these signals for the mixed phase.

(1) Institute for Theoretical Physics, Kiev-130, USSR

(2) Department of Physics, National Taiwan University, Taipei, Taiwan 10764, ROC 


\section{REFERENCES}

1. L. H. Xia, C. M. Ko, and C. T. Li, Phys. Rev. C 41, 572 (1990); C. M. Ko and L. H. Xia, Phys. Rev. C 37, 179 (1988); Phys. Rev. Lett. 62, 1695 (1989).
2. D. H. Rischke aㅡ al., Preprint UFTP 252/1990, Frankfurt, 1990.

3. K. A. Bugaev, M. I. Gorenstein, B. Kämpfer and V. I. Zhdanov, Phys. Rev. D 40, 2903 (1989).

4. J. W. Harris et al., Phys. Lett. 153B, 377 (1985).

\title{
PHI MESON IN DENSE MATTER
}

\author{
C. M. Ko, P. Lévai(1), X. J. Qiu(2) and C. T. Li(3)
}

In relativistic heavy-ion collisions, the nuclear matter can be compressed to densities which are many times the normal nuclear density. This has recently generated great interests in theoretical studies of hadron properties under such extreme conditions. We have studied the property of the phi meson in dense matter using the vector dominance model. ${ }^{1}$
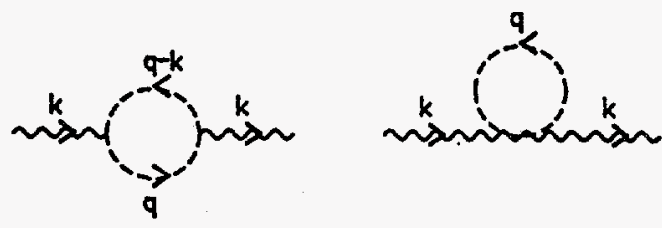

Figure 1. The phi meson self-energy diagrams. The wavy and dashed lines denote, respectively, the phi meson and the kaon.

In this model, the one-loop self-energy of the phi meson is shown by the two diagrams in Fig. 1, in which the dashed line denotes the kaon. According to Refs. $[2,3]$, the kaon effective mass in the medium decreases and vanishes at the critical density $2.5 \rho_{0}<\rho_{\mathrm{c}}<5 \rho_{0}$, depending on the strangeness content of the nucleon.

Choosing $\rho_{c} \approx 5 \rho_{0}$, corresponding to zero strangeness content for the nucleon, we have calculated the phi meson mass and width in the matter. It is seen in Fig. 2 that the phi meson mass decreases slightly with increasing density except near the critical density $\rho_{c}$ where the phi meson mass increases again with the density but remains below the free mass. The width shown in Fig. 3 increases with the density and becomes an order of magnitude larger than its value in free space. We note that the phi meson mass and width remain unchanged beyond the critical density. The change in the phi meson property in dense matter can be investigated experimentally via the dilepton invariant mass spectrum in high energy heavy-ion collisions in which a dense matter is formed in the initial stage.

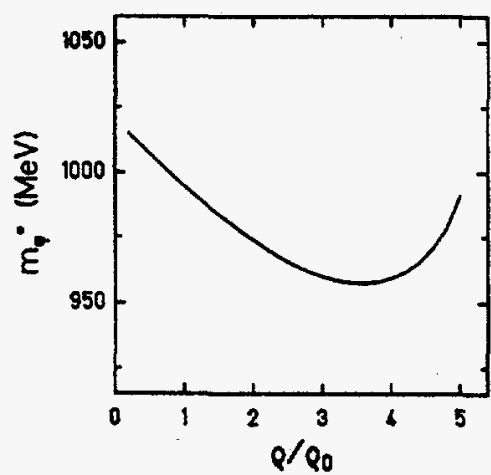

Fig. 2. The mass of phi meson as a function of the matter density.

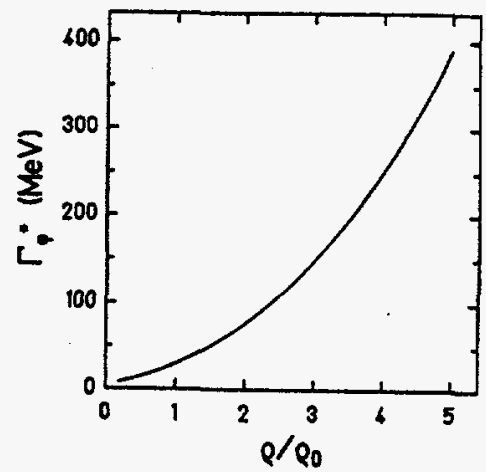

Fig. 3. The width of phi meson as a function of the matter density.

(1) On leave from KFKI Budapest, Hungary

(2) Institute of Nuclear Research, Academia Sinica, Shanghai 201800, China

(3) Physics Department, National Taiwan University, Taipei, Taiwan 10764, China

\section{REFERENCES}

1. J. J. Sakurai, Currents and Mesons, (University of Chicago Press, 1969).

2. D. B. Kaplan and A. E. Nelson, Phys. Lett. 175B, 57 (1986).

3. G. E. Brown, K. Kubodera and M. Rho, Phys. Lett. 192B, 273 (1987). 


\section{RHO MESON IN DENSE HADRONIC MATTER}

\section{Asakawa, C. M. Ko, P. Lévai(1) and X. J. Qiu(2)}

We have studied the property of a tho meson at rest in nuclear matter using the Vector Dominance Model (VDM). ' The self-energy of a rho meson is then determined by its coupling to the pions, which are modified by the delta-hole polarization of nuclear matter. ${ }^{2}$

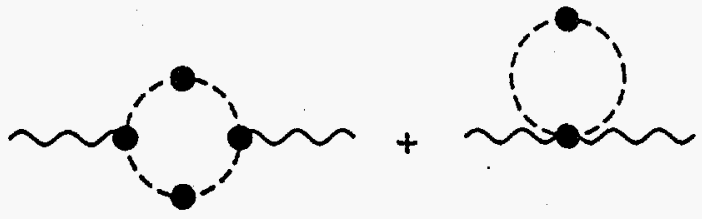

Figure 1. The rho meson self-energy diagrams. The wavy and dashed lines with a solid circle denote the rho meson and the pion, respectively.

This is shown in Fig. 1, where the second diagram in the figure results from treating the tho meson as a gauge boson in the VDM. Both the $\rho \pi \pi$ and $\rho \rho \pi \pi$ vertices also include the vertex corrections from the delta-hole polarization in order to preserve the gauge invariance.

The imaginary part of the rho meson self-energy is finite, but the real part is divergent and needs to be renormalized. This is done by writing the self-energy $\Sigma^{\mu \nu}$ as $\operatorname{Re} \Sigma^{\mu \nu}=\left(\operatorname{Re} \Sigma^{\mu \nu}-\operatorname{Re} \Sigma_{0}^{\mu \nu}\right)+\operatorname{Re} \Sigma_{0}^{\mu \nu}$. The difference shown in the bracket between the real part of the rho meson self-energies in the medium and in free space is finite as the pion self-energy in nuclear matter vanishes at large momenta. The divergent rho meson self-energy in free space $\operatorname{Re}_{0}^{\mu \nu}$ given by the last term is then replaced by the square of the measured rho meson mass $m_{\rho}=770$ MeV.

The property of a rho meson in the medium can be expressed by its spectral function which is given by $2 \pi$ times the imaginary part of its propagator. We have calculated the rho meson spectral function at different nuclear densities. They are shown in Fig. 2. We find that as the nuclear density increases the rho peak shifts to larger invariant masses and its width also becomes larger. At high nuclear densities we have also found a peak at masses around three times the pion mass, corresponding approximately to the sum of the pion mass and the deltahole energy.

But hadron masses decrease in the medium due to the scaling property of $\mathrm{QCD}^{3}$ This effect can be included by using the density-dependent hadron masses in the effective Lagrangians. Assuming that all hadron masses in the medium decrease as the empirical density-dependent nucleon effective mass, we have repeated the above

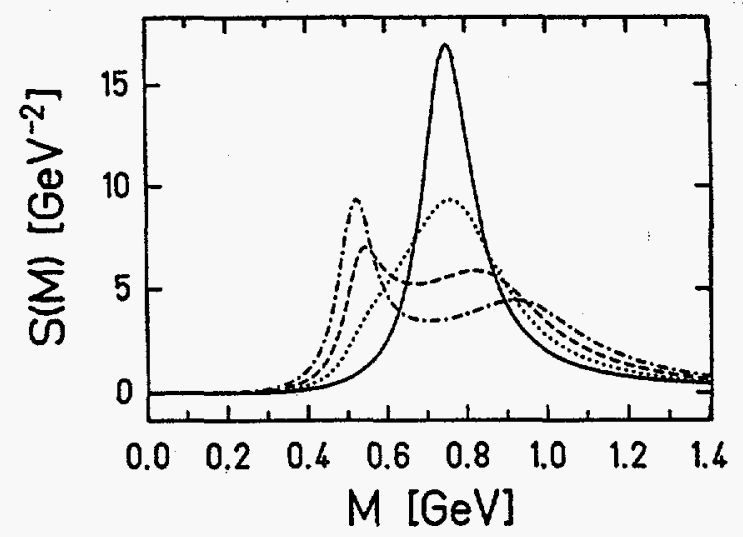

Fig. 2. The spectral function of a rho meson. The solid curve is for a tho meson in free space. For a rho meson in the medium, the dotted, dashed, and dash-dotted curves correspond, respectively, to nuclear densities of $\rho_{0}, 2 \rho_{0}$ and $3 \rho_{0}$, where $\rho_{0}$ is the normal nuclear matter density.

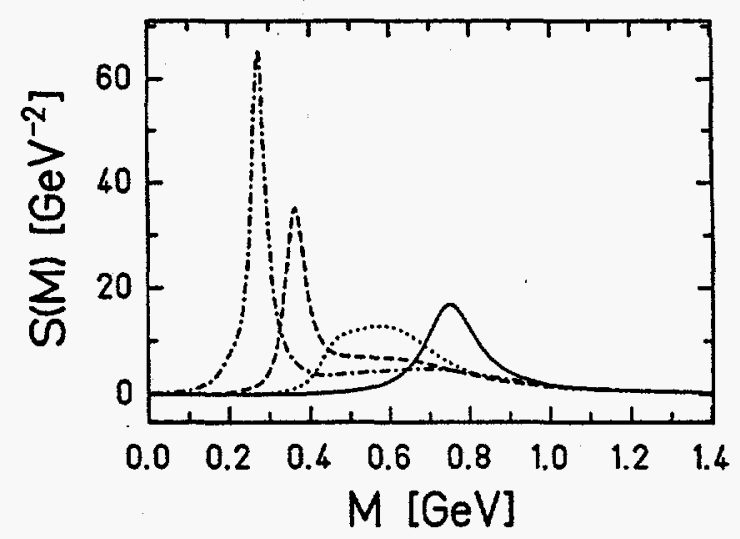

Fig. 3. Same as Fig. 2 with the density-dependent hadron masses in the effective Lagrangians.

calculations and the results are shown in Fig. 3 . It is seen that the rho peak in the spectral function moves to a smaller invariant mass with diminishing strength when the density becomes higher. The low mass peak also shifts down with increasing density but becomes more pronounced than in the case with bare hadron masses. Future experiments to measure the dilepton invariant mass spectrum from heavy-ion collisions should provide excellent opportunities to find out experimentally how the property of the rho meson is modified in dense nuclear matter. 
1. J. J. Sakurai, "Currents and Mesons", (University of Chicago Press, 1969).

2. B. Friedmann, V. R. Pandharipande and Q. N. Usmani, Nucl. Phys. A372, 483 (1981).
3. G. E. Brown and M. Rho, Phys. Rev. Lett. 66, 2720 (1991).

(1) KFKI, Budapest, Hungary

(2) Institute of Nuclear Research, Academia Sinica, Shanghai 201800, People's Republic of China

\title{
QCD SUM RULES OF THE PHI MESON MASS IN HOT AND DENSE MATTER
}

\author{
M. Asakawa, C. M. Ko and T. Hatsuda*
}

According to QCD sum rules, the mass of the phi meson $^{1}$ is related to the strangeness condensate in the $Q C D$ vacuum $<\dot{s} s>_{0}$, which has a value of about $-(265$ $\mathrm{MeV}^{3}$. In dense nuclear matter, the strangeness condensate $\langle\bar{s} s\rangle_{\rho}$ is reduced by the strangeness content of the nucleon $\langle\dot{s s}\rangle_{N}$; i.e. $\langle\dot{s} s\rangle_{\rho} \approx\langle\dot{s}\rangle_{0}+\langle\dot{s} s\rangle_{N} \rho$, where $\rho$ is the density of the matter. Since the empirical strangeness content of the nucleon $\langle\dot{s}\rangle_{N}=0.5-0.9$ is small, the phi meson mass in dense matter is thus not much affected. The reduction of the phi meson mass at three times the normal nuclear matter density is at most $5 \%$.

However, the high density matter formed in high energy heavy ion collisions consists of many strange particles as observed in both AGS and CERN SPS experiments. These strange particles, mostly kaons and lambdas, have appreciable strangeness content.

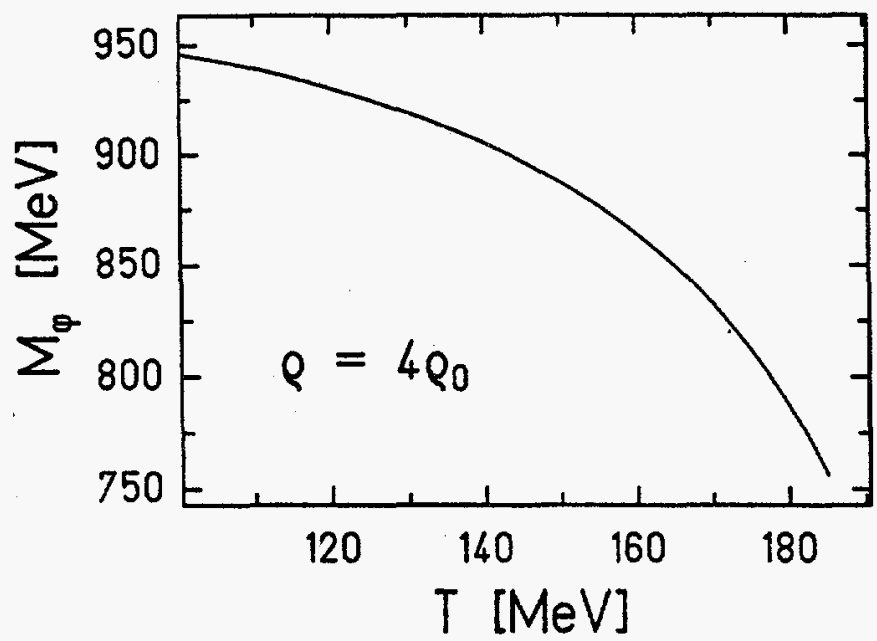

Figure 1. The phi meson mass as a function of the temperature of the hadronic matter at four times normal nuclear density.
According to [2], the strangeness content is about 1.83 and 1.73 for kaons and lambdas, respectively. Their effect on the phi meson mass is thus expected to be significant. This effect can be included by considering the dense matter at finite temperature and assuming that all hadrons are in equilibrium. The results from the detailed QCD sum rules calculation is given in Fig. 1 where the phi meson mass is shown as a function of the temperature of the hadronic matter at four times normal nuclear density. The strangeness content is taken to be 0.74 , $1.73,1.83$ and 0.0 for nucleon, lambda, kaon, and pion, respectively. We see that the phi meson mass is reduced substantially in high density matter when the temperature is high. In the effective Lagrangian approach, ${ }^{3}$ the phi meson mass also decreases in dense matter. The relation between the two approaches needs to be studied. It has been shown ${ }^{4}$ that the decreasing phi meson mass in dense matter enhances its production in heavy-ion collisions and may account for the large $\phi / \omega$ ratio measured in the CERN experiments. 5

"Institute for Nuclear Theory, University of Washington, Seattle, Washington 98195

\section{REFERENCES}

1. L. J. Reinders and H. R. Rubinstein, Phys. Leth. 145B, 108 (1984).

2. T. Kunihiro and T. Hatsuda, Phys. Lett. 240B, 20:7 (1990); Nucl. Phys. B, in press.

3. C. M. Ko, P. Levai, X. J. Qiu and C. T. Li, Phys. Rev. C 45, 1400 (1992).

4. C. M. Ko and B. H. Sa, Phys. Lett. 258B, 6 (1991).

5. NA38 Collaborations, J. P. Guilland 으 a‥ in Quark Matter '90, Nucl. Phys. A525, 449c (1991). 


\section{ANTILAMBDA ENHANCEMENT IN ULTRARELATIVISTIC HEAVY ION COLLISIONS}

\section{M. Ko, M. Asakawa and P. Lévai*}

In the NA35 experiment at CERN SPS for the collision between sulfer nuclei at $200 \mathrm{GeV} /$ nucleon, ${ }^{1}$ the antilambda yield is 1.5 per event and is 115 times greater than that in p-p collisions at the same energy. Compared with the 36-fold enhancement of the negatively charged particles, most of them being negative pions, there is a factor three enhancement of antilambda yield in heavy ion collisions.

Since there are many mesons produced in the collision, the reaction $\mathrm{KM} \rightarrow \bar{N} \mathrm{~N}$, where $\mathrm{M}$ denotes either a pion or a tho meson, is favored as it is not suppressed by the OZI rule. This cross section in free space is, however, small due to the large threshold energy. But the antilambda mass in the medium is reduced according to the Walecka model, ${ }^{2}$ and the cross section is thus expected to increase with the density.

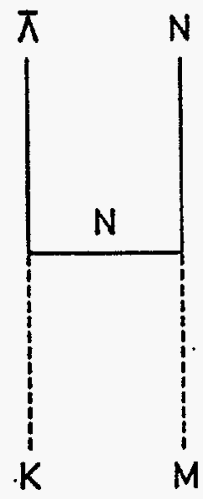

Fig. 1. The Feynman diagram for $\mathrm{KM} \rightarrow T N$, where $M$ denotes either a pion or a rho meson.

The Feynman diagram for $\mathrm{KM} \rightarrow \bar{\Lambda} \mathrm{N}$ is shown in Fig. 1. Using the in-medium antilambda mass, the energy dependence of the cross section is shown in Fig. 2 for different densities. It is seen that in dense matter not only the threshold of the reaction is reduced but also the magnitude of the cross sections is indeed increased. We note that $\sigma_{\mathrm{K} \rho \rightarrow \bar{\Lambda} N}$ is larger than $\sigma_{\mathrm{K} \pi \rightarrow \bar{\Lambda} N}$.

For the collision between two sulfur nuclei at 200 $\mathrm{GeV} /$ nucleon, we assume that a fire-cylinder is formed in the expansion stage. Its cross section is given approximately by $\pi R^{2}=40 \mathrm{fm}^{2}$, where $R=3.5 \mathrm{fm}$ is the radius of the sulfur nucleus. The number of baryons in the fire-cylinder is inferred from the measured nucleon distribution in the central rapidity, ${ }^{1} \mathrm{dN} / \mathrm{dy}=6$, to be about 36. The initial density and temperature are taken to be $2 \rho_{0}$ and $190 \mathrm{MeV}$, respectively. To describe the longitudinal expansion of the system, we use the simplified hydrochemical model ${ }^{3}$ with linear scaling ansatz for the velocity profile. The hadronic matter is assumed to be in thermal equilibrium and the thermal energy is converted to flow energy as the system expands.
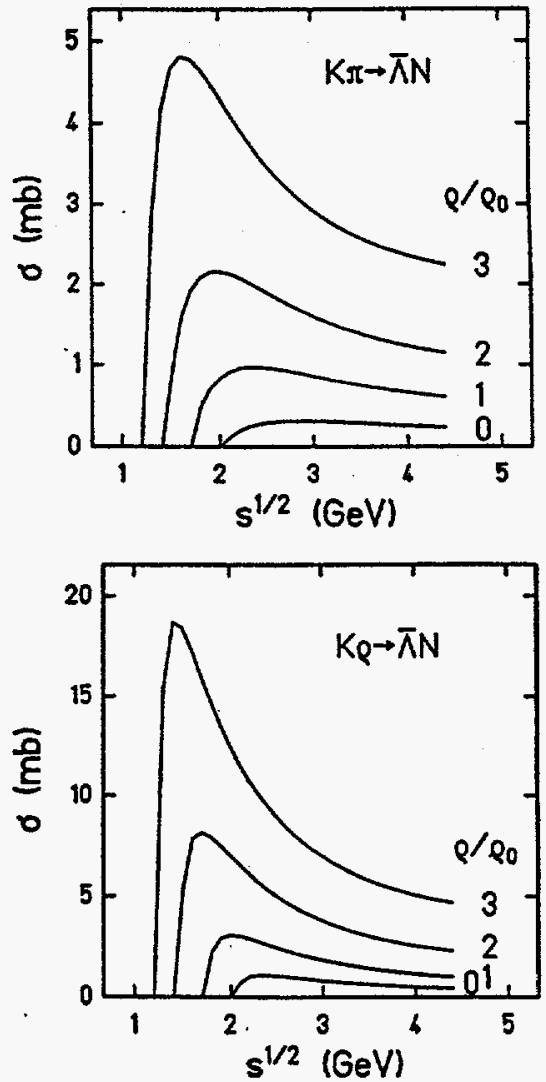

Fig. 2. The energy dependence of (a) $\sigma_{\mathrm{K} \pi \rightarrow \bar{\Lambda} N}$ and (b) $S_{K \rho \rightarrow \bar{h} N}$ at different densities.

The masses of non-strange hadrons are assumed to decrease in hot dense matter, ${ }^{4}$ and they are further assumed to be in chemical equilibrium. But the strange hadrons, including both kaons and lambdas as well as their antiparticles, are not in chemical equilibrium and their abundance is determined by rate equations. ${ }^{3}$

The results are shown in Fig. 3. We see that at freeze out the number of thermal pions is about 85 while the number of pions from the decays of both rho mesons and baryon resonances is about 165 . Altogether we have about $\mathbf{2 5 0}$ pions, and this number is slightly smaller than the $=300$ pions measured in the CERN experiment. The final kaon number is about 14 and again is smaller than the measured number of $\approx 20$ kaons. The lambda number is about 4 and is less than the measured number of 
- 8. The smaller numbers of pions, kaons, and lambdas from our model than the measured ones may be attributed to particle production from the initial nonequilibrium stage and from the spectator matter which have been ignored in the study. But the antilambda number is about two and is comparable to the measured value of $1.5 \pm 0.4$. The enhanced antilambda production measured in ultrarelativistic heavy ion collisions at the CERN energy thus offers the possibility to study the property of hadrons in dense matter formed in the compression stage of the collision.

KFKI, Budapest, Hungary

\section{REFERENCES}

1. J. Bartke et al. (NA35 Collab.), Z. Phys. C 48, 191 (1990); R. Stock et al. (NA35 Collab.), Nucl. Phys. A525, 221c (1990).

2. N. K. Glendenning and S. A. Moszkowski, Phys. Rev. Lett. 67, 2414 (1991).

3. C. M. Ko and B. H. Sa, Phys. Lett. 258B, 6 (1991).

4. G. E. Brown and M. Rho, Phys. Rev. Lett. 66, 2720 (1991).
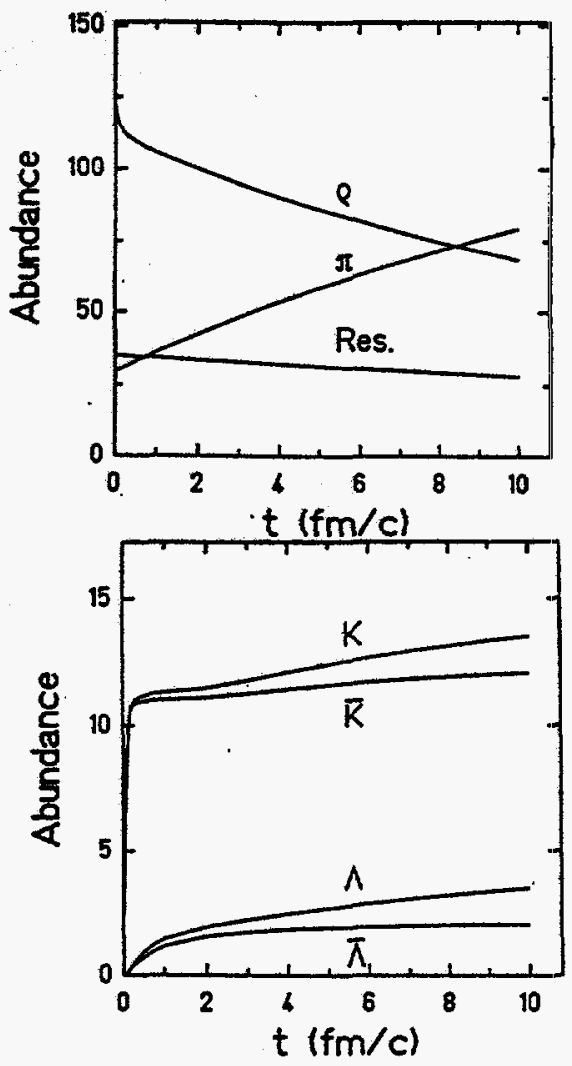

Fig. 3. The time dependence of the abundance of (a) nonstrange and (b) strange hadrons.

\section{MOMENTUM DEPENDENCE IN PAIR PRODUCTION BY AN EXTERNAL FIELD}

\section{Asakawa}

The problem of fermion pair production is very old. Its origin can be traced back to the foundation of the Dirac theory and the discovery of the positron. In QED, fermion pair production by an external field has been formulated in many ways since the 1930 's.'

Particle creation by an external field has also been used in understanding multi-particle production in nuclear reactions. ${ }^{2}$ In particular, it is an important ingredient in models for the production of quark-gluon plasma in ultrarelativistic nuclear collisions. ${ }^{3}$ To incorporate pair production into such models, it is necessary to introduce the transverse and longitudinal momentum distributions of the created particles. The transverse momentum distribution has been studied using various methods ${ }^{4}$ such as the WKB method and the mode expansion. But the longitudinal momentum dependence has not been included.

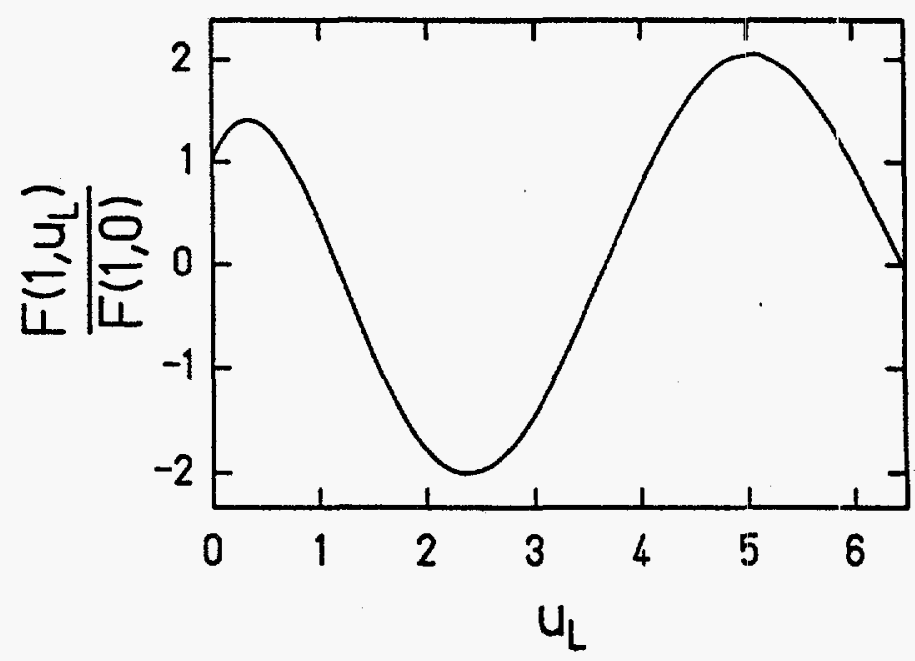

Fig. 1. The behavior of $F\left(u_{T}, u_{L}\right)$ at $u_{T}=1$. 
Including both the transverse and longitudinal momentum dependence, we have calculated the differential fermion pair production rate under a uniform static electric field, $d w / d p_{T}^{2} d p_{L}$, by the Green's function method. The behavior of $d w / d p_{T}^{2} d p_{L}=F\left(u_{T}, u_{L}\right)$, where $u_{T}=p_{T}^{2} / e \varepsilon_{0}$ and $u_{L}=\left(p_{L}+e \varepsilon_{0} t\right)^{2} / e \varepsilon_{0}$ with $\varepsilon_{0}$ the field atrength, is shown in Fig. 1 for a typical transverse momentum $u_{T}=1$. It is seen that $F\left(u_{T}, u_{L}\right)$ is oscillatory and not positive definite. This oscillatory behavior is a result of the interference between the transmitted and the reflected waves from the external field in which particles are created.

\section{REFERENCES}

1. W. Heisenberg and H. Euler, Z. Phys. 98, 714 (1936); J. Schwinger, Phys. Rev. 82, 664 (1951).

2. B. Andersson, G. Gustafson and T. Sjöstrand, Z. Phys. C6, 235 (1980).

3. K. Kajantic and T. Matsui, Phys. Lett. 164B, 373 (1985); A. Bia et al., Nucl. Phys. B296, 611 (1988); M. Asakawa and T. Matsui, Phys. Rev. D 43, 2871 (1991).

4. A. Casher, H. Neuberger and S. Nussinov, Phys. Rev. D 20, 179 (1979); R. C. Wang and C. Y. Wong, Phys. Rev. D 38, 348 (1988); Th. Schönfeld et al., Phys. Lett. 247B, 5 (1990).

\title{
DILEPTON PRODUCTION FROM THE DELTA-DELTA INTERACTION IN HEAVY ION COLLISIONS
}

\author{
X. S. Fang, C. M. Ko, P. J. Siemens ${ }^{*}$ and L. H. Xia*
}

The experimental data ${ }^{1,2}$ from the Bevalac have shown that dileptons of invariant mass greater than twice the pion mass are mainly produced from the pion-pion annihilation. ${ }^{3}$ Since the pion electromagnetic form factor is dominated by the tho meson, which is modified in dense matter, ${ }^{4}$ dilepton production from the pion-pion annihilation offers the passibility of studying the property of the rho meson in dense matter. But in the transport model description of high energy heavy ion collisions, most pions appear after the initial compression stage when the density is not very high. In the high density region, deltas are more likely to be created and they eventually decay into pions when the density becomes low. The collision between deltas can also lead to the production of dileptons but in studies to date only the bremstrahlung contribution has been taken into account and it is appreciable at relatively low invariant masses.

We have studied the exchange current contribution to dilepton production from the delta-delta interaction as shown in Fig. 1. To remove the singularity when the exchanged pion becomes on shell, we include in the pion propagator an imaginary part to take into account its finite life time due to the absorption by nucleons. From [5] the mean free path of a pion being absorbed in nuclear matter at twice the normal density $2 \rho_{0}$ is about $1 \mathrm{fm}$, so the pion mass acquires an imaginary part of about $200 \mathrm{MeV}$.

We have calculated dilepton production in the fireball model in which nucleons, deltas, and pions are assumed to be in equilibrium. Using a temperature of $100 \mathrm{MeV}$ and a

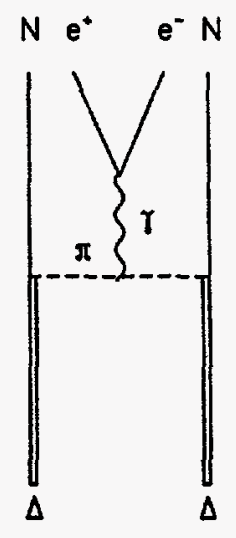

Fig. 1. The Feynman diagram for dilepton production from the delta-delta interaction.

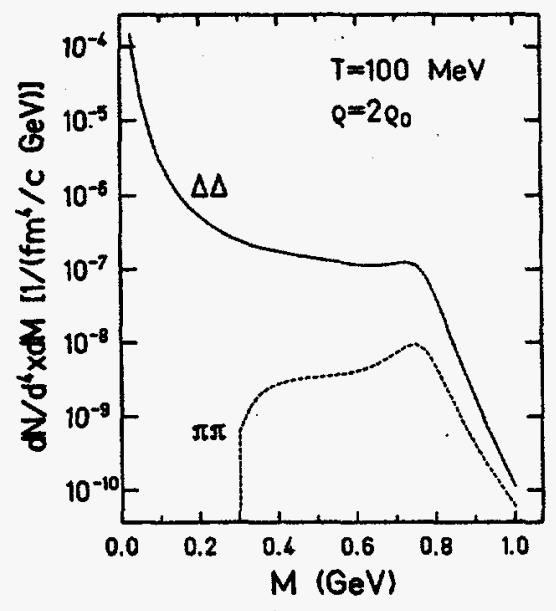

Figure 2. Dilepton production rate in the fireball from the pion-pion and the delta-delta interactions. 
density of $2 \rho_{0}$, the results are shown in Fig. 2 . We find that the contribution from the delta-delta interaction to dileptons with invariant mass around the rho meson mass is important. It is thus possible to study the rho meson property in dense matter from the dilepton spectrum in heavy ion collisions. Work is in progress to include dilepton production from the delta-delta interaction using the relativistic transport $\operatorname{model}^{6}$ so more realistic predictions can be made.

"Physics Department, Oregon State University, Corvallis, OR 97331

\section{REFERENCES}

1. G. Roche et al., Phys. Lett. 226B, 228; Phys. Riv. Lett. 61, 1069 (1989).

2. C. Naudet et al., Phys. Rev. Lett. 62, 2652 (1989).

3. L. Xiong, Z. G. Wu, C. M. Ko and J. Q. VVu, Nusl. Phys. A512, 772 (1990).

4. M. Asakawa, C. M. Ko, P. Lévai and X. J. Qiu, submitted to Phys. Rev. C.

5. C. M. Ko, Phys. Rev. C 20, 757 (1979).

6. C. M. Ko, Q. Li and R. Wang, Phys. Rev. Lett. 59, 1084 (1987); Q. Li and C. M. Ko, Mod. Phys. Lett. A3, 465 (1988); C. M. Ko and Q. Li, Phys. Rev. C 37, 2270 (1988); Q. Li, J. Q. Wu and C. M. Ko, Phys. Rev. C 39, 84 (1989); C. M. Ko, Nucl. Phy's. A495, 321c (1989).

\title{
THE $M_{\mathrm{r}}$-SCALING IN THE DILEPTON SPECTRUM AS A SIGNATURE FOR THE QUARK-GLUON PLASMA
}

\author{
M. Asakawa, C. M. Ko and P. Lévai*
}

In general, the dilepton spectrum from nuclear reactions depends on both the transverse momentum $q_{T}$ and the transverse mass $M_{T}=\sqrt{M^{2}+q_{T}^{2}}$ where $M$ is the invariant mass of the pair. But for a quark-gluon plasma under the boost-invariant one-dimensional flow ${ }^{1}$ and with the Boltzmann thermal distribution, the dilepton spectrum has been shown to depend only on the transverse mass. ${ }^{2,3}$ But this $M_{T}$-scaling is broken at the later stage of the time evolution when the transverse expansion becomes appreciable and extra mass scales appear in dilepton production from the hadronic matter because of the vector meson dominance.

In order to determine whether the $M_{T}$-scaling in the dilepton spectrum from the quark-gluon plasma can be observed in ultrarelativistic heavy-ion collisions, we have carried out a study to include not only the effect of dilepton production from the hadronic matter but also the transverse flow of the system. This is done using the hydrodynamical code of [4]. We take the initial proper time to be $1 \mathrm{fm}$, the critical temperature to be $180 \mathrm{MeV}$, and the freeze out temperature $120 \mathrm{MeV}$. The initial temperature is varied to obtain different charge particle rapidity density. The initial radial velocity at the surface of the cylinder is chosen to be $v_{0}=0$. In Fig. 1, we show by the solid curve the ratio $R$ of $d N / d M_{T}^{2} d y d q_{T}^{2}$ for fixed $M_{T}=2.6 \mathrm{GeV}$ at $\mathrm{q}_{\mathrm{T}}=2 \mathrm{GeV}$ to that at $\mathrm{q}_{\mathrm{T}}=0$ as a

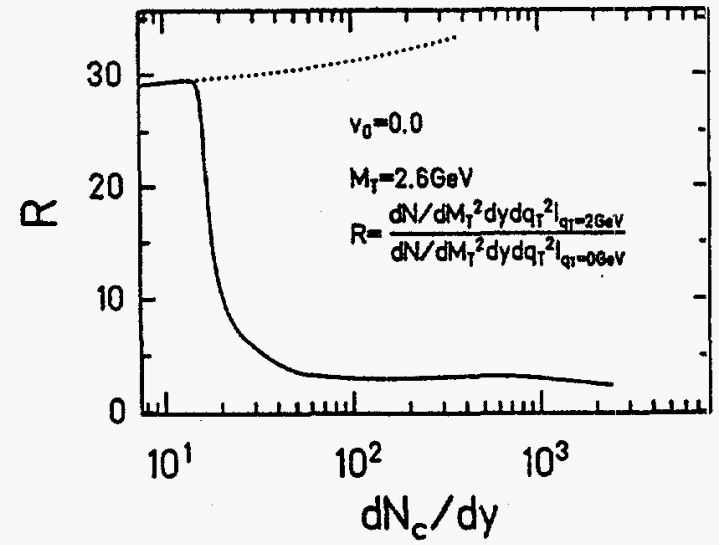

Figure 1. The ratio of the differential dilepton yield $\mathrm{dN} / \mathrm{dM}_{\mathrm{T}}^{2} \mathrm{dydq} \mathrm{q}_{\mathrm{T}}^{2}$ at $\mathrm{q}_{\mathrm{T}}=2 \mathrm{GeV}$ to that at $\mathrm{q}_{\mathrm{T}}=0$. The solid curve is the results with the initial state in the quark-gluon phase if the temperature is above the critical temperature while the dotted curve is obtained by assuming that the initial state is always in the hadronic phase.

function of the charge particle multiplicity per unit rapidity $d N_{c} / d y$. We note that for $q_{T}=2 \mathrm{GeV}$ the invariant mass approximately corresponds to the $\rho(1700)$ peak. For low rapidity densities corresponding to initial temperatures below the critical temperature, the system starts in the hadronic phase and remains so during the expansion. The ratio $R$ is about 30 and there is thus no $\mathbf{M}_{\mathbf{r}}$-scaling. For high rapidity densities, the initial 
temperature is above the critical temperature and the system is initially in the quark-gluon phase. In this case, the ratio $R$ is about 3 and the $M_{T}$ scaling is almost realized. If we assume that the initial state is in the hadronic phase even if the temperature is above the critical temperature, the ratio $R$ is large as shown by the dotted curve and the $M_{T}$-scaling is badly violated. The $M_{T}$-8caling is thus a plausible signature for the formation of the quark-gluon plasma in ultrarelativistic heavy-ion collisions. It would be useful to design detectors at RHIC with the capability of detecting dileptons with different transverse momenta for transverse masses between $\phi$ and $\mathbf{J} / \boldsymbol{\psi}$.
* KFKI, Budapest, Hungary

\section{REFERENCES}

1. J. D. Bjorken, Phys. Rev. D 27, 140 (1983).

2. L. D. MeLerran and T. Toimela, Phys. Rev. D 31, 545 (1985).

3. M. Asakawa, Ph.D. thesis, University of Tokyo, 1990.

4. P. Lévai and B. Müller, Phys. Rev. Lett. 67, 1519 (1991).

\title{
TRANSPORT MODEL WITH KAONS
}

\author{
X. S. Fang, C. M. Ko, G. E. Brown ${ }^{*}$ and V. Koch ${ }^{*}$
}

In all transport models to date, kaons are treated as free particles. But in the linear chiral perturbation theory, both kaon and antikaon masses decrease in dense matter ${ }^{1,2}$ because nucleons act on the kaon as an effective scalar field. There is also a repulsive vector potential for the kaon and an attractive one for the antikaon. ${ }^{3}$ These effects can be included in the relativistic transport model ${ }^{4}$ in which nucleons propagate in a self-consistent meanfield potential generated by the scalar and vector mesons. The scalar and vector mesons couple both to the kaon but with different strengths. The effective coupling constant of the kaon to the scalar field is equal to half the $\mathrm{KN}$ sigma term ${ }^{1,2}$ while its coupling to the vector meson is $1 / 3$ of that to the nucleon. We have already shown that the opposite signs of the vector potential for kaons and antikaons can account for the difference in the measured slope parameters of $\mathrm{K}^{+}$and $\mathrm{K}^{-5}$

The generalized transport model is used to study the expansion stage of relativistic heavy ion collisions. We assume that initially a highly excited and compressed fireball is formed in the collisions. The fireball is assumed to have a temperature of $180 \mathrm{MeV}$ and a density of $4 \rho_{0}$, where $\rho_{0}$ is the normal nuclear matter density. This initial condition corresponds approximately to that one expects for heavy-ion collisions at the AGS energies. In the initial fireball, we include only nucleons, deltas, pions and assume that they are in thermal and chemical equilibrium. Furthermore, all particles are assumed to be uniformly distributed inside the fireball of radius -3.5 fm. Then the fireball expands and both kaons and antikaons are produced from $M M \rightarrow K \bar{K},{ }^{6}$ where $M$ denotes either a pion or a rho meson. The cross section for this reaction is about $3 \mathrm{mb}$ in the free space but is enhanced in the medium. The average in-medium cross section has a value of about $40 \mathrm{mb}$.

Both kaons and antikaons undergo collisions with other particles. The interaction of the kaon with the nucleon is relatively weak and the cross $\operatorname{section}^{7}$ is $\sigma_{\mathrm{KN}}$ $10 \mathrm{mb}$. Due to the resonance nature of the interaction, the antikaon-nucleon cross section is much larger and can be approximately taken to be ${ }^{7} \sigma_{\overline{\mathrm{K} N}}-40 \mathrm{mb}$. Both the kaon and antikaon can interact with the pion via the $K^{*}$ resonances. The isospin averaged cross section has a value $60 \mathrm{mb}^{8}$ Antikaons can also be converted to lambdas via the reaction $\bar{K} N \rightarrow \Lambda \pi$ with an average inmedium cross section of about $40 \mathrm{mb} .^{6}$

Unlike the hydrodynamical model, ${ }^{6}$ there is no need to introduce a freeze out density in the transport model. We simply let the system expand to a very low density, $\sim 0.3 \rho_{0}$ in the present calculation, when it is practically free of both mean-field potential and particle collisions. The fireball reaches such a low density after about $10 \mathrm{fm} / \mathrm{c}$. The final particle rapidity and transverse kinetic energy distributions are shown in Fig. 1. We see that our results are comparable to the measured data. Work is in progress to extend the calculation to include in the initial stage two approaching nuclei in order to take into account the nonequilibrium stage of the collision.

* Department of Physics, State University of New York, Stony Brook, New York 11974 
(a)

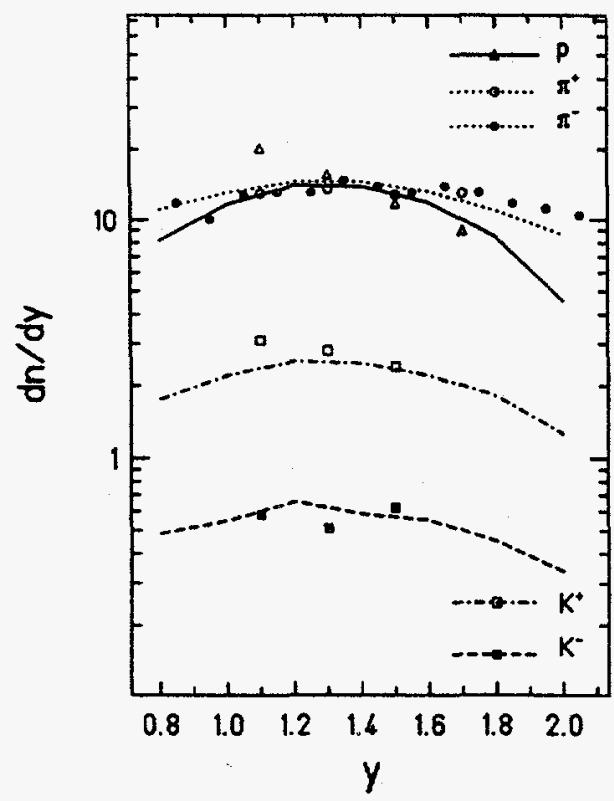

(b)

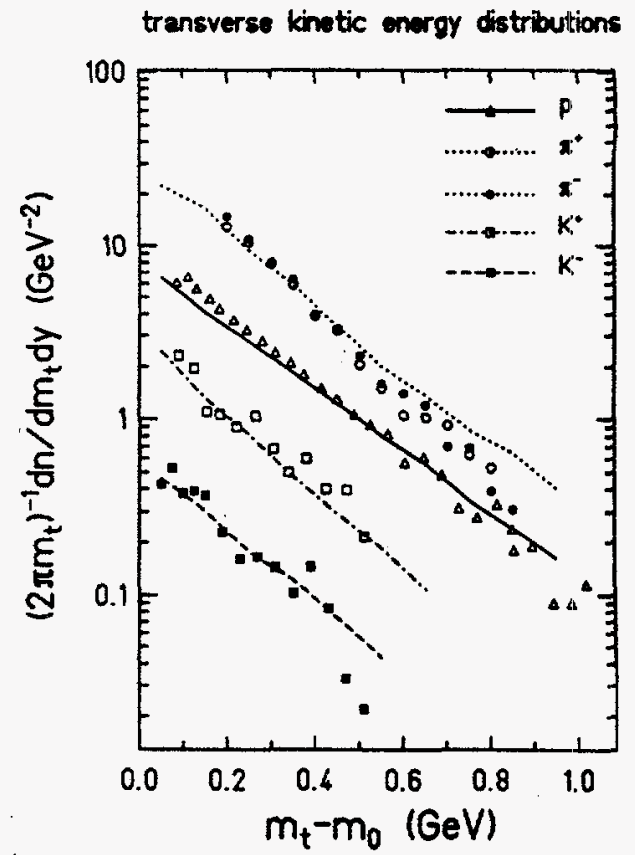

Fig. 1. (a) Rapidity and (b) Kinetic Energy spectra

\section{REFERENCES}

1. D. B. Kaplan and A. E. Nelson, Phys. Lett. 175B, 57 (1986); A. E. Nelson and D. B. Kaplan, Phys. Lett. 192B, 193 (1987).

2. G. E. Brown, K. Kubodera and M. Rho, Phys. Lett. 193B, 273 (1987).

3. G. E. Brown, C. M. Ko and K. Kubodera, Z. Phys. A341, 301 (1992).

4. C. M. Ko, Q. Li and R. Wang, Phys. Rev. Lett. 59, 1084 (1987); Q. Li and C. M. Ko, Mod. Phys. Lett. A3, 465 (1988); C. M. Ko and Q. Li, Phys. Rev. C
37, 2270 (1988); Q. Li, J. Q. Wu and C. M. K.o, Phys. Rev. C 39, 84 (1989); C. M. Ko, Nucl. Phys. A495, 321c (1989).

5. Progress in Research, 1990-1991, Cyclotron Institute, Texas A\&M University, p. 87.

6. C. M. Ko, G. E. Brown, Z. G. Wu and L. H. Xia, Phys. Rev. Lett. 66, 2577 (1991); G. E. Brown, C. M. Ko, Z. G. Wu and L. H. Xia, Phys. Rev. C 43, 1881 (1991).

7. C. B. Dover and G. E. Walker, Phys. Rep. 89, 1 (1982).

8. C. M. Ko, Phys. Rev. C 23, 2760 (1981).

\title{
COULOMB EFFECTS IN THE ASYMPTOTIC BEHAVIOR OF THE OPTICAL POTENTIAL I. SCATTERING BELOW BREAKUP THRESHOLD
}

\author{
E. O. Alt*, D. M. Latypov, and A. M. Mukhamedzhanov
}

The optical potentials (OP) are of wide use in nuclear physics. These potentials are introduced to describe the interaction of compound clusters regarded as structureless objects. The formal expression for an $O P$ may be obtained, for instance, using the Feshbach projection method. ${ }^{1}$ This expression shows that the short-range part of OP is very complicated, it is non-local, complex, and energy-dependent. Other possible definitions of an $\mathrm{OP}^{2-7}$ despite giving the same asymptotics of $O P$, lead to the different representations at finite distances.

For many problems, however, the structure of the OP at finite distances is not essential and only its asymptotic behavior is important. For instance, longrange terms in $O P$ may generate angular and threshold singularities of scattering amplitudes, ${ }^{8}$ renormalization of the low-energy parameters (scattering lengths, effective ranges), ${ }^{9}$ and result in a slow convergence of partial expansions of wave functions.

The simplest case of such a problem is the scattering of a charged particle by the ground state of two bounded charged particles at energies below breakup threshold. This problem has been considered for a long time both experimentally and theoretically. The long-ranige behavior of the OP for the energies below the first 
excitation threshold was found by means of perturbation theory 4 and the method of strong channel coupling. ${ }^{3}$

The rigorous approach to the problem is based on dynamic equations of a three-body scattering theory. The integral Faddeev equations for this problem have been used in Ref. [5-7]. In these works, several simplified restrictions were made. First, it was assumed that the target, formed by one charged and one neutral particle, has no excited states. Second, it was assumed that the projectile particle and a neutral particle of the target do not interact.

The purpose of the present work is to consider the problem on the basis of the AGS quasiparticle method. ${ }^{10}$ In this method, the original three-body problem is replaced by fully equivalent Lippmann-Schwinger-type matrix equations for effective two-body operators. This makes the AGS-method particularly suited for the following investigations. In the next paper," we will show that the AGS-method allows the straight-forward generalization of the present results for energies above the breakup threshold.

We consider a scattering of a charged particle 1 on a bound pair (23) formed by a neutral particle 3 and a charged particle 2 . For simplicity of notation, we assume that the short-range forces acting between the particles are described by separable potentials of rank one and in each channel $\alpha$ precisely one stable bound s-state exists with binding energy $\dot{E}_{\alpha}$. This requires the particles 1 and 2 to be equally charged. Later we will show how to get rid of these assumptions.

To describe the system, we make use of the AGSequation ${ }^{10}$

$$
\begin{aligned}
& s_{\beta \alpha}\left(\vec{q}_{\beta}, \vec{q}_{\alpha} ; z\right)=\nu_{\beta \alpha}\left(\vec{a}_{\beta}, \vec{a}_{\alpha} ; z\right)+\sum_{\gamma} \int \\
& \nu_{\beta \gamma}\left(\vec{q}_{\beta}, \vec{q}_{\gamma}^{\prime \prime} z\right) \\
& \times \frac{\dot{s}_{\gamma}\left(z-q_{\gamma}^{n 2}\right)}{z-q_{\gamma}^{\prime 2}-\dot{E}_{\gamma}} J_{\gamma \alpha}\left(\vec{q}_{\gamma}^{\prime \prime}, \vec{q}_{\alpha} ; z\right) d \vec{q}_{\gamma}^{\prime \prime}
\end{aligned}
$$

with effective potential

$$
\begin{aligned}
& \nu_{\beta \alpha}\left(\vec{q}_{\beta}^{\prime}, \vec{q}_{\alpha} ; z\right)=<\vec{q}_{\beta}\left|<x_{\beta}\right|\left\{\overline { \delta } _ { \beta \alpha } \left[G_{0}(z)+\right.\right. \\
& \left.\left(\delta_{\beta 3}+\delta_{\alpha 3}+\bar{\delta}_{\beta 3} \bar{\delta}_{\alpha 3}\right) \times G_{o}(Z) T_{c}(Z) G_{o}(Z)\right]+ \\
& \left.\delta_{\beta \alpha} \bar{\delta}_{\alpha 3} G_{0}(Z) T_{c}(Z) G_{0}(z)\right\}\left|x_{\alpha}>\right| q_{\alpha}>\text {. }
\end{aligned}
$$

The structure of the effective potential (2) has been investigated to some extent in Ref. [12]. There it was shown that it is singular at small transfer momenta $\vec{p}$ $=\mathrm{q}_{\alpha}-\mathrm{q}_{\alpha}$ and that its singular part, $\nu_{\alpha \alpha}^{\mathrm{s}}$ originates only from the last term in parenthesis (2). Eq. (1), except for the terms which are not singular at small transfer momenta $\vec{p}$, has a form of Lippmann-Schwinger equation with effective potential $\dot{\mathrm{s}}_{\alpha}\left(\mathrm{z}-q_{\alpha}^{i}\right) \nu_{\alpha \alpha}^{\mathrm{s}}\left(\vec{q}_{\alpha}, \vec{q}_{\alpha i} z\right)$. Consequently, the singular part of the optical potential coincides with that of the effective potential $\dot{\mathrm{s}}_{\alpha} \nu_{\alpha \alpha}^{\mathbf{s}}$.

At the negative energies $Z=E, E=q_{0}^{2}+\vec{E}_{\alpha}<0$, the effective potential $\hat{s}_{\alpha}\left(z-q_{\alpha}^{\prime 2}\right) \nu_{\alpha \alpha}^{s}\left(\vec{q}_{\alpha}^{\prime}, \vec{q}_{\alpha} ; z\right)$ can be shown to be a function of $q_{0}^{2}-q_{\alpha}^{2}, q_{0}^{2}-q_{\alpha}^{2}$ and $|\vec{p}|$. This function can be expanded in powers of its variables. The corresponding expansion is the kernel of some integral operator. This operator has the same singular structure as $V_{\text {opt }}$ when considered on the solution $f_{q o}\left(\vec{q}_{\alpha}\right)$ of the two-body Schrödinger equation

$$
\begin{aligned}
& \left(q_{0}^{2}-q_{\alpha}^{2}\right) f_{\vec{q}_{0}}\left(\vec{q}_{\alpha}\right)=\int v_{\text {opt }} \\
& \left.\left(\vec{q}_{\alpha}, \vec{q}_{\alpha}^{\prime}\right) f_{\vec{q}_{0}}\left(\vec{q}_{\alpha}^{\prime}\right)\right) \vec{q}_{\alpha}^{\prime}
\end{aligned}
$$

has the same singular structure as $V_{\text {opt }}$. This fact allows us to find the next asymptotic formula for $V_{\text {opt }}\left(\vec{q}_{\alpha}^{\prime}, \vec{q}_{\alpha}\right)$

$$
v_{\text {opt }}\left(\vec{a}_{\alpha}^{\prime}, \vec{a}_{\alpha}\right)=\frac{z_{1} z_{2} e^{2}}{\left|\vec{q}_{\alpha}^{\prime}-\vec{q}_{\alpha}\right|^{2}}+o\left(\left|\vec{a}_{\alpha}^{\prime}-\vec{a}_{\alpha}\right|\right) \text {. }
$$

Taking the Fourier transform of both sides of (4), one can find asymptotic behavior of the OP in the coordinate space

$$
v_{\text {opt }}(r)=\nu_{c}(r)+o\left(r^{-4}\right)
$$

In numerical calculations, the $T_{c^{-m a t r i x}}$ in Eq. (1) (2) is replaced by its first Born term $\mathbf{v}_{\mathbf{c}}$. It is therefore of interest to examine the asymptotic behavior of the OP for such a model. One can repeat all our calculations and find that in this model both dipole and quadropole terms are present in the asymptotics of OP.

Let us now briefly describe how to get rid of some of the assumptions we made in our derivation.

We consider a system of three particles interacting via local potentials which give rise to $N_{\gamma}$ bound states in two-body $r$-subsystems. The AGS-equations for the elastic and rearrangement processes then are as follows ${ }^{12}$

$$
\begin{aligned}
& J_{\beta n, \alpha \in m}\left(\vec{q}_{\beta n}, \vec{q}_{\alpha \times m} ; z\right)=\nu_{\beta n, \alpha M n}\left(\vec{a}_{\beta n}, \vec{q}_{o \alpha m} ; 2\right) \\
& +\sum_{\gamma} \sum_{\kappa, \ell=1}^{N \gamma} \nu_{\beta n, \gamma \kappa}\left(\vec{a}_{\beta n}, \vec{q}_{\gamma \kappa}^{\prime \prime} ; z\right) \Delta_{\gamma, \kappa \ell}\left(Z-q_{\gamma}^{n 2}\right)
\end{aligned}
$$

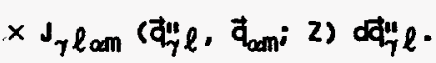


To examine the singular structure of these equations, we recall two results of Ref. [12]. The first is that the matrix elements $\nu_{\beta n, \text { an }}$ of the effective potential with $\alpha \neq \beta$ are nonsingular and that the strongest singularity of those with $\alpha=\beta, m \neq n$ is proportional to $\left|\bar{q}_{\alpha n}-\bar{c}_{\alpha m}\right|^{-1}$. The second one is that the matrix elements $\dot{\Delta}_{\alpha}$, rs with $r \neq s$ are nonpolar. Using these results it is easy to find that the singular part of the kernel of (6) is due to only its diagonal part $(\alpha=\beta, m=n)$. Thus, this problem is reduced to the present one. The case of attractive Coulomb force was also shown (below breakup threshold) to be reduced to the present problem.

\section{CONCLUSION}

In the present work, we considered the long-range behavior of the optical potential in the problem of the lowenergy scattering of a charged particle on a bound state formed by one neutral and one charged particle. To treat this three-body problem, we used the AGS quasiparticle method. The singular part of the effective potential in these equations below breakup threshold allows the expansion in powers of transfer momenta, which is uniform with respect to the energy. The singular part of this expansion, considered in the two-body scattering state with the same scattering amplitude as that of the initial three-body problem, coincide with the singular part of OP.

The long-range behavior of the OP can then be found by means of a Fourier transform. For the case of a spherically symmetric bound state, we arrive at the wellknown asymptotics value, $v_{o p t}(r)=v_{c}(r)+O\left(r^{-4}\right)$.

The advantage of the AGS-method for this problem is that instead of using a complicated $O P$, one can use an effective potential. The effective potential of the AGS:equations is a two-particle quantity and its singular structure can be analyzed in detail. In the next paper, ${ }^{11}$ we will use this fact to generalize present analysis for the energies above breakup threshold.

*Institut für Physik, Universität Mainz, Mainz, Germany Institute for Nuclear Physics, Tashkent 702132, Uzbekistan

\section{REFERENCES}

1. H. Feshbach, Ann. Phys. (N.Y.)5, 357 (1958); 19, 287 (1962).

2. C. J. Kleiman, Y. Kahn, and L. Spruch, Phys. Rev. 165, 53 (1968).

3. M. J. Seaton, L. Steenman-Claric, J. Phys. B11, 293 (1978).

4. G. F. Drukarev, Collisions of Electrons with Atoms and Molecules. NY, Plenum Press, 1987.

5. V. F. Kharchenko, S. A. Shadchin, Yad. Fiz. 45, 333 (1987) [Sov. J. Nucl. Phys. 45, 210 (1987)].

6. V. F. Kharchenko, S. A. Shadchin, Few-Body Systems 6, 45 (1989).

7. M. L. Zepalova, Preprint ITP-87-21R, Kiev, 1987.

8. A. A. Kvitsinsky, I. V. Komarov, S. P. Merkuriev, Yad. Fiz 38, 101 (1983) [Sov. J. Nucl. Phys. 38, 58 (1983)].

9. R. O. Berger, L. Spruch, Phys. Rev. B138, 1106 (1965).

10. E. O. Alt, P. Grassberger, W. Sandhas, Nucll. Phys. B2, 167 (1967).

11. E. O. Alt, D. M. Latypov, A. M. Mukhamedzhanov. In preparation.

12. E. O. Alt, W. Sandhas, H. Ziegelmann, Phys. Rev. C17, 1981 (1978).

\section{ON THE COULOMB SINGULAR KERNEL OF THE LIPPMANN-SCHWINGER TYPE EQUATION}

\section{M. Latypov and A. M. Mukhamedzhanov*}

Lippmann-Schwinger type equation with Coulomb singular kernel is considered. It is shown that all the solutions are singular on the energy shell, $\mathrm{k}^{2} / 2 \mu=\mathrm{E}$. In order for this equation to have a solution with a singularity of the type $\left(E-k^{2} / 2 \mu+i c\right)^{i \alpha}, \alpha$ is shown to be equal to the Coulomb parameter $\eta$. The Coulomb singular kernel in the given class of functions is found to split into a $\delta$ function and a kernel which smoothes the singularity.

It is well known that the conventional scattering theory' should be modified if the particles interact via a long-range Coulomb force. In a time-dependent theory, for instance, the usual wave operator ${ }^{1}$ has to be replaced by one introduced by Dollard. ${ }^{2}$ In coordinate representation of the stationary theory, the Coulomb interaction changes the asymptotic behavior of the wave function.' In the stationary theory based on integral equations (Lippmann-Schwinger ${ }^{1}$, Faddeev, FaddeevYakubovsky $^{3}$ and Alt-Grassberger-Sandhas') the Coulomb interaction results in strong singularities in the kernels of these equations. In the following we shall refer to such kernels as Coulomb singular kernels.

The treating of equations with Coulomb singular kemels is one of the most serious problems of the few body scattering theory. For the two cluster collision at 
energies below the break-up threshold into three or more particles, this problem has been solved. ${ }^{5,6}$ In this case, the above mentioned singularities have a two-body origin and their inversion results in a system of Fredholm type equations. The inversion can be done by means of solving a number of effective two-body Coulomb problems. Since they have an explicit analytic solution, the kernels of the modified equations can also be found analytically.

At energies above the break-up threshold, new singularities appear in the kernels of the equations. These singularities have truly few body origin and cannot be inverted explicitly. It might therefore be worthwhile to develop a method of treating such equations without depending on inversion procedure.

The aim of this paper is to suggest such a method and examine it for a Lippmann-Schwinger type equation. We consider some general properties of the corresponding Coulomb singular kernel and find a functional class in which one can look for solutions of the equation.

Our method may also be applied to the Coulomb singular kernels which appear in Faddeev or AltGrassberger-Sandhas equations. In this way one can find, for example, the structure of singularities of the resolvent for the three-body energy operator. ${ }^{7}$

\section{Lippmann-Schwinger-type Equation with Coulomb Singular Kernel}

The Lippmann-Schwinger equation

$$
\begin{aligned}
& t\left(k, k^{\prime}, E+i_{\varepsilon}\right)=v\left(k-k^{\prime}\right)+\frac{1}{(2 \pi)^{3}} \int \frac{v(k-q)}{E-q^{2} / 2 \mu+i \varepsilon} \\
& t\left(q, k^{\prime}, E+i_{\varepsilon}\right) d q
\end{aligned}
$$

with potential $v(\mathbf{k})$ satisfying the Hölder condition

$$
\begin{aligned}
& |v(q+h)-v(q)| \leq A(1+|q|)^{-1-\theta}|h|^{\alpha}, \\
& \theta>0, \alpha>0, \quad A \in R_{f}
\end{aligned}
$$

has been well investigated. ${ }^{8}$ In this case, Eq. (1) is of the Fredholm type and the properties of its solutions can be described in detail. However, for the Coulomb potential

$$
v(q)=\frac{4 \pi e_{1} e_{2}}{q^{2}}
$$

with $e_{1}$ and $e_{2}$ being particle charges, condition (2) is not fulfilled because of the singularity of (3) in the origin. In this case, the kernel of Eq. (1)
$K(k, q, E+i \varepsilon)=\frac{4 \pi e_{1} e_{2}}{|k-q|^{2}} \frac{1}{E-q^{2} / 2 \psi+i \varepsilon}$

has a singularity which coincides on the energy shell $\mathrm{k}^{2} / 2 \mu$ $=\mathrm{E}$ with that of the resolvent $\left(E-q^{2} / 2 \mu+i \varepsilon\right)^{-1}$. The resulting singularity is of the type $(k-q)^{-3}$ and therefore non-integrable. For this reason all the solutions of the Lippmann-Schwinger type equation

$f\left(k, E+i_{\varepsilon}\right)=f_{0}(k)+\frac{1}{(2 \pi)^{3}} \int K\left(k, q, E+i_{\varepsilon}\right) f\left(q, E, i_{\varepsilon}\right) d q$

are singular in the neighborhood of the energy shell. Besides this singularity, they may have a singularity related to that of $f_{0}(\mathbf{k})$. In the following we assume that these singularities do not coincide. For Eq. (1) with potential (3), this implies that $E \neq 0$.

Let us now define the class $\Xi_{\alpha}$ of functions in which we shall look for a solution of Eq. (5). We also find the action of the operator with the kernel (4) on a member of the class.

A function $h(k, E+i c)$ is said to be of class $C$ if it is continuous in the vicinity of the energy shell $\mathrm{k}^{2} / 2 \mu=\mathrm{E}$. A function $f(k, E+i c)$ is said to be of class $\Xi_{\alpha}$ if it may be represented as

$f(k, E+i c)=\left(E-k^{2} / 2 \mu+i \varepsilon\right)^{i \alpha} \tilde{f}(k, E+i c)$,

where $\tilde{f}(k, E+i c) \in C$ and $\alpha$ is an arbitrary real number.

Lemma. The integral operator $\hat{k}$ with the kernel (4) acts on $f \in \Xi_{\alpha}$ as follows

$(\hat{k} f)\left(k, E+i_{\varepsilon}\right)=\frac{e_{1} e_{2} \mu}{k \alpha} f\left(k, E+i_{\varepsilon}\right)+h\left(k, E+i_{\varepsilon}\right)$,

where $h(k, E+i \varepsilon) \in C$.

Proof. Let us split the integral

$(\hat{K} f)(k, E+i c)=\frac{4 \pi e_{1} e_{2}}{(2 \pi)^{3}} \int \frac{d q}{|k-q|^{2}} \frac{\tilde{f}\left(q, E+q_{c}\right)}{\left(E-q^{2} / 2 \mu+i \varepsilon\right)^{1-\alpha}}$

in two parts:

$(\hat{k} f)(k, E+i c)=\frac{4 \pi e_{1} e_{2}}{(2 \pi)^{3}}\left\{\int \frac{d q}{|k-q|^{2}}\right.$

$\left.\frac{\tilde{f}\left(q, E+i_{\varepsilon}\right)-\tilde{f}\left(k, E+i_{\varepsilon}\right)}{\left(E-q^{2} / 2 \mu+i_{\varepsilon}\right)^{1-i \alpha}}+\tilde{f}\left(k, E+i_{\varepsilon}\right) I\right\}$ 
$I=\int \frac{d q}{|k-q|^{2}}\left(E-q^{2} / 2 \mu+i \varepsilon\right)^{i \alpha-1}$.

The integrand in the first integral in (9) is smoothed by subtracting the value of $\tilde{f}$ in the singularity point and the whole integral may be shown to belong to $C$. The integral $I$, by introducing a new variable $p=q-k$ and performing the integration over the angular variables, can be reduced to the radial one

$I=\frac{2 \pi \mu}{i k \alpha} \lim _{\delta \rightarrow+0} \int_{-\infty}^{\infty} \frac{d}{p}\left[E-(p-k)^{2} / 2 \mu+i \varepsilon\right]^{i \alpha-\delta}$.

This integral can be evaluated by choosing the contour shown in Fig. 1. One finds that

$I=\frac{2 \pi^{2} \mu}{k \alpha}\left(E-k^{2} / 2 \mu+i \epsilon\right)^{i \alpha}+g(k, E)$,

where $g(k, E) \in C$. For details of the calculation and the explicit expression of $\mathbf{g}(\mathbf{k}, \mathrm{E})$ see the Appendix. Inserting (12) into (9) we arrive at the Eq. (7).

Q.E.D. We are now in the position to prove the next

Lemma. For the integral equation (5) with the kernel (4) to have a solution in $\Xi_{\alpha}$, it is necessary that $\alpha$ be equal to the Coulomb parameter $\eta=e_{1} e_{2} \sqrt{\mu / 2 E}$

$\alpha=\eta$

Proof. Let us compare the terms in both sides of Eq. (5) which are singular on the energy shell. Taking into account (7), we immediately get (13).

Q.E.D.

One can see now from (7) and (13) that the kernel $\mathbf{K}(\mathbf{k}, \mathbf{q}, \mathbf{E}+\mathbf{i \varepsilon})$ in $\Xi_{\eta}$ may be written as follows:

$\left.k(k, q, E+i \varepsilon)\right|_{\Xi_{\eta}}=\frac{k}{k} \delta(k-q)+\tilde{k}\left(k, q, E+i_{\varepsilon}\right)$

where $k_{0}=\sqrt{2 \mu E}$ and $\tilde{k}(k, q, E+i e)$ is a kernel which transforms a function from $\Sigma_{\eta}$ to a function from $C$.

In conclusion, we have shown that all solutions of the Lippmann-Schwinger type equation with the Coulomb singular kernel are singular on the energy shell. We have derived the necessary condition for this equation to have a solution in a class of functions with a singularity of the type $\left(\mathrm{E}-\mathrm{k}^{2} / 2 \mu+\mathrm{i \varepsilon}\right)^{\mathrm{i \alpha}}$. The Coulomb singular kernel in this class is shown to split into a delta function and a smooth kernel.
The integrand in (11) has a pole at the point $p=0$ and branching points at $p=p_{1}, p_{2}$

$p_{1}=k+\sqrt{2 \mu E}+i \sqrt{\mu / 2 E} \varepsilon$

$P_{2}=k-\sqrt{2 \mu E}-i \sqrt{\mu / 2 E}$ c

Applying the residue theorem we have

$I=\frac{2 \pi^{2} \mu}{k \alpha}\left(E-k^{2} / 2 \mu+i \varepsilon\right)^{i \alpha}+i \frac{2 \pi \mu}{k \alpha}\left(1-e^{-2 \pi \alpha}\right)(-2 \mu)^{-i \alpha} J_{1}$

where $\mathrm{J}$ is the integral over the upper rim of the cut from $p_{1}$ to $\infty+i \sqrt{\mu / 2 E} \varepsilon$ :

$$
J=\lim _{\delta \rightarrow 0} \int_{p_{1}}^{\infty+i \sqrt{\mu / 2 E} \varepsilon} \frac{d p}{p}=\left[\left(p-p_{1}\right)\left(p-p_{2}\right)\right]^{i \alpha-\delta}
$$

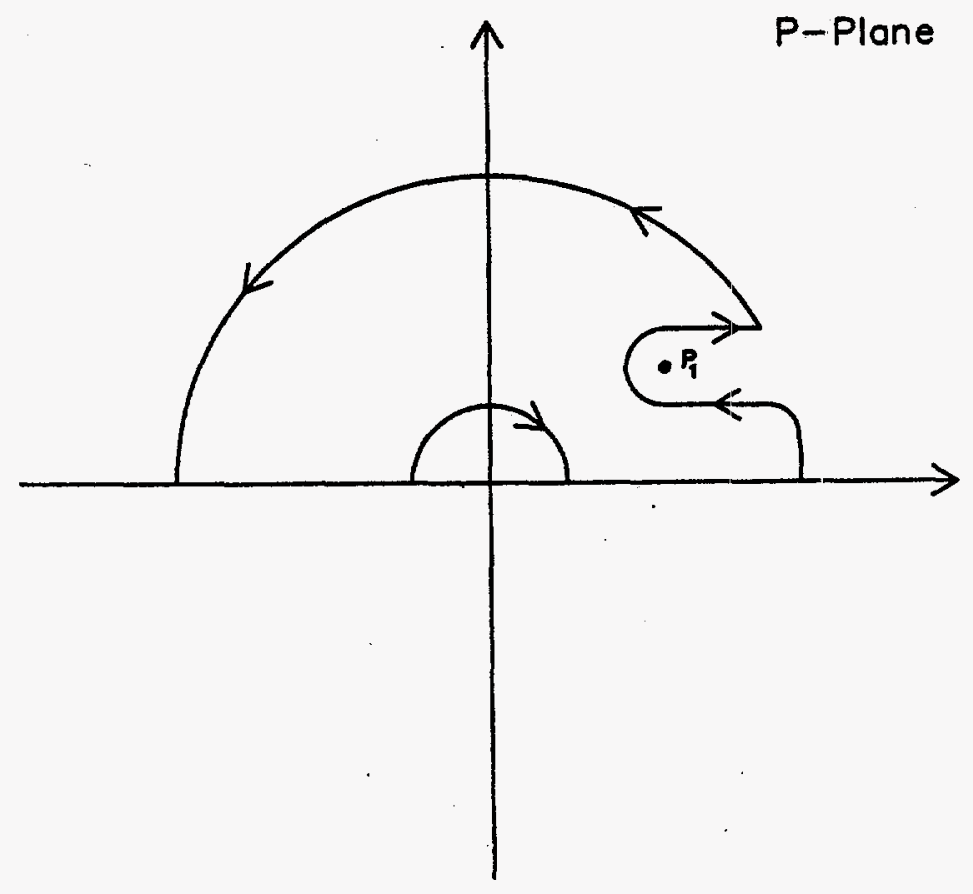

Fig. 1. Contour for evaluating integral (11).

Making use of the substitution

$p=p_{1}+\sqrt{8 \mu E} \frac{y}{1-y}$

the integral J may be transformed to

$J=(8 \mu E)^{i \alpha} \gamma^{-1} \lim _{\delta \rightarrow+0} \int_{0}^{1} d y(1-y)^{-1-2 i \alpha+\delta} y^{i \alpha}$ 


$$
\left[1-\frac{x-1}{1} y\right]^{-1}
$$

where

(A.7)

The integral in (A.6) may be expressed in terms of hypergeometrical $[F(a, b, c, z)]$ and $\operatorname{Beta}\left[B\left(z_{1}, z_{2}\right)\right]$ functions':

$J=(8 \mu E)^{i \alpha} \gamma^{-1} B(-2 i \alpha, 1+i \alpha) F\left(1,1+i \alpha, 1-i \alpha, \frac{y-1}{y}\right)$

$$
\text { (A.8) }
$$

Inserting (A.8) into (A.3) we get finally:

$$
\begin{aligned}
& I=\frac{2 \pi^{2} \mu}{k \alpha}\left(E-k^{2} / 2 \mu+i \varepsilon\right)^{i \alpha}+i \frac{2 \pi \mu}{k \alpha}\left(1-e^{-2 \pi \alpha}\right) \gamma^{-1} \\
& x(-4 E)^{i \alpha} B(-2 i \alpha, 1+i \alpha) F\left(1,1+i \alpha, 1-i \alpha, \frac{y-1}{\gamma}\right) .
\end{aligned}
$$

We note that the second term is continuous in the neighborhood of the energy shell and equal to

$$
\text { i } \frac{2 \pi \mu}{k \alpha}\left(1-e^{-2 \pi \alpha}\right)(-4 E)^{i \alpha} B(-2 i \alpha, 1+i \alpha)
$$

when $k^{2} / 2 \mu=E$.
* Institute for Nuclear Physics, Tashkent 702132 Uzbekistan

\section{REFERENCES}

1. R. G. Newton. Scattering Theory of Waves and Particles, McGraw-Hill, NY (1966).

2. J. D. Dollard, J. Math. Phys. 5, 729 (1964).

3. S. P. Merkuriev, L. D. Faddeev. Quantum Scattering Theory for Systems of Few Particles (in Russian), Nauka, Moscow (1985).

4. E. O. Alt, P. Grassberger, W. Sandhas, Nucl. Phys. B2, 167 (1967).

5. A. M. Veselova, Teor. Mat. Fiz 3, 326 (1970).

6. E. O. Alt, W. Sandhas, H. Ziegelman, Phys. Rev. C17, 1981 (1978).

7. D. M. Latypov, A. M. Mukhamedzhanov, Sov. J. Nucl. Phys. 55, 174 (1992).

8. L. D. Faddeev. Mathematical Aspects of the ThreeBody Problem in the Quantum Scattering Theory, Jerusalem, Israel Program for Scientific Translations (1965).

9. I. S. Gradshteyn, I. M. Ryzhik. Table of Integrals, Series, and Products, Academic Press (1980). 


\section{ATOMIC MOLECULAR AND MATERIALS SCIENCE}




\title{
KINETIC ENERGY RELEASED IN THE DISSOCIATION OF MULTICHARGED DIATOMIC MOLECULES PRODUCED IN COLLISIONS WITH $97 \mathrm{MeV} \mathrm{Ar}^{14+}$
}

\author{
G. Sampoll, R. L. Watson, O. Heber, V. Horvat, K. Wohrer, and M. Chabot
}

A study of the dissociation of transient $\mathrm{CO}^{\mathrm{Q}}, \mathrm{N}_{2}^{\mathrm{Q}+}$, and $O_{2}^{Q+}$ (where $Q$ ranged from 2 to 7 ) molecular ions produced in collisions with $97 \mathrm{MeV} \mathrm{Ar}^{14+}$ ions was completed recently. The resulting dissociation products were identified by coincidence time-of-flight (TOF) spectroscopy and the differences in the times of flight of the two ions formed in these binary dissociation events were used to deduce the total kinetic energy distributions for various charge division pathways. The data obtained for $\mathrm{CO}$ are shown in the form of a two-dimensional plot of ion TOF versus ion-pair time difference in Fig. 1(a). Projection of this data onto the TOF axis [Fig. 1(b)] yields the total TOF spectrum, while projection onto the timedifference axis [Fig. 1(c)] yields the total time-difference spectrum.

By placing windows around the various TOF peaks in the total TOF spectrum and projecting only events within these windows onto the time-difference axis, separated time-difference spectra were obtained for each of the observed dissociation-production charge states.

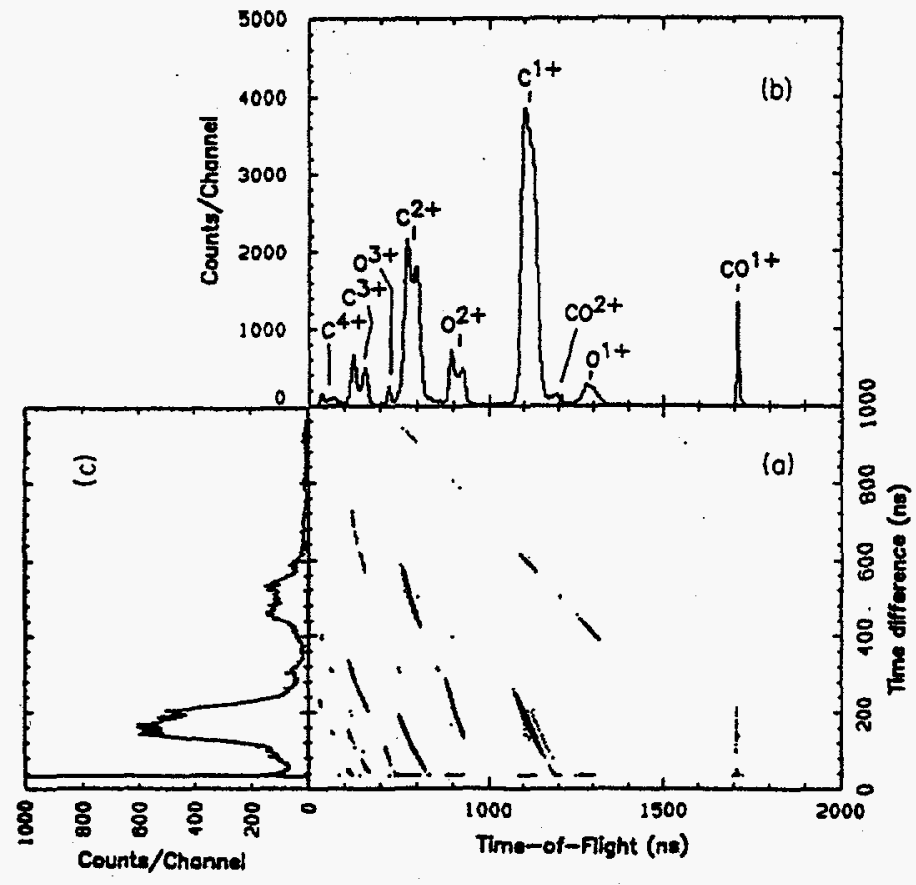

Fig. 1. A two-dimensional display of the data for the dissociation of multicharged $\mathrm{CO}$ molecular ions.

Separated time-difference spectra for $\mathrm{C}^{3+}$ and $\mathrm{C}^{4+}$ ions are shown in Fig. 2. In these spectra, well defined timedifference distributions are visible for the dissociation products produced in the dissociation reactions

$$
\mathrm{CO}^{\mathrm{Q}} \longrightarrow \mathrm{Cl}_{1}^{\mathrm{+}}+\mathrm{O}_{2}^{+} \text {, }
$$

where $Q=4$ to 7 .

Methods were devised to transform the ion-pair timedifference distributions into total kinetic energy distributions. The first step of this process involved calculating the ion-pair time-difference distributions expected for specific values of the total kinetic energy release. A computer program was developed for this purpose and it incorporated a Monte Carlo procedure to simulate statistical distributions of the starting coordinates and velocity components of the dissociation product ions.
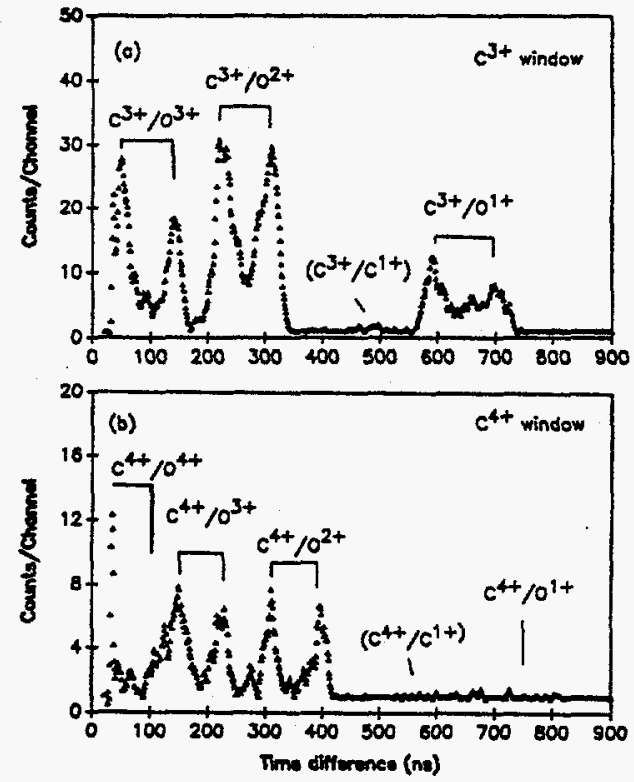

Fig. 2. Sorted ion-pair time difference spectra obtained by projecting only those events within the TOF peaks for the (a) $\mathrm{C}^{3+}$ ions and the (b) $\mathrm{C}^{4+}$ ions.

Response matrices were constructed for all the dissociation reactions having sufficient statistics for a meaningful analysis. Each response matrix consisted of an array of time-difference distributions in energy intervals which varied from $0.45 \mathrm{eV}$ to $4.39 \mathrm{eV}$, depending on the range of kinetic energies required. A schematic representation of the response matrix for the case of

$$
\mathrm{CO}^{2+} \longrightarrow \mathrm{C}^{1+}+\mathrm{O}^{1+}
$$


is shown in Fig. 3. The relative yield of each timedifference distribution in the response matrix was proportional to the average transmission efficiency for its total kinetic energy.

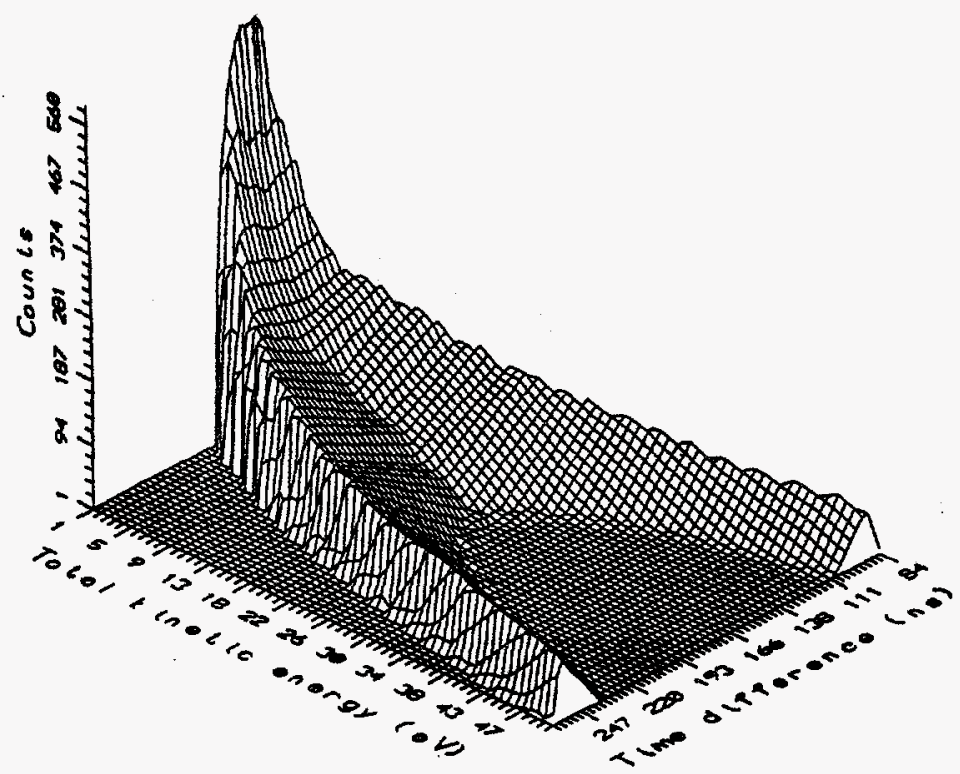

Fig. 3. Response matrix for $\mathrm{CO}^{2+} \longrightarrow \mathrm{C}^{1+}+\mathrm{O}^{1+}$ showing the dependence of the time difference distribution on total kinetic energy.

The resulting kinetic energy distributions may be characterized by their widths and averages, both of which are listed in Table 1. Potential energy curves for $C O$ molecular ions having charges greater than 2 have not yet been calculated. Even so, it is clear from the curves calculated for $\mathrm{CO}^{2+}$ by Lablanquie, et al. ${ }^{\prime}$ that the density of states increases rapidly with charge state. Hence, it is not surprising that the total kinetic energy distributions obtained in the present work for the dissociation of $\mathrm{CO}$ molecular ions having charges of $3+$ or greater are rather featureless. The rapid increase in the widths of the kinetic energy distributions indicates that the potential energy curves become more repulsive in the region of the FranckCondon zone (i.e. they rise more steeply) as electrons are removed from the $\mathrm{CO}$ molecule.

A "theoretical" estimate of the amount of kinetic energy released in a dissociation event may be obtained from the point charge Coulomb potential between the two product ions at the average internuclear separation for the neutral (ground state) molecule. This estimation procedure assumes that the dissociative state is formed in a vertical transition during the ionizing collision and it neglects effects associated with the overlap of the electron clouds of the two ions. The collision time is fast $\left(\approx 10^{-17} \mathrm{~s}\right)$ relative to the dissociation time (it takes at least
$=10^{-14} \mathrm{~s}$ for the distance between the $\mathrm{C}$ and $\mathrm{O}$ ions to expand to twice the equilibrium bond length). Therefore, the assumption of a vertical transition is valid. It should be noted, of course, that electron-electron interaction and exchange can cause the real potential to deviats: considerably from a point charge Coulomb potential, especially for low charge states.

Table 1.

Average values and widths of the total kinetic energy distributions.

\begin{tabular}{|c|c|c|c|}
\hline $\begin{array}{l}\text { Parent Molecular } \\
\text { Ion }\end{array}$ & $\begin{array}{l}\text { Product Ion } \\
\text { Pair }\end{array}$ & $\mathrm{E}_{\text {ave }}(\mathrm{eV})$ & FWHM(eV \\
\hline $\mathrm{CO}^{2+}$ & $\mathrm{C}^{1+10^{1+}}$ & $20 \pm 1$ & 20 \\
\hline \multirow[t]{2}{*}{$\mathrm{CO}^{3+}$} & $\mathrm{C}^{2+} / \mathrm{O}^{1+}$ & $37 \pm 3$ & 43 \\
\hline & $\mathrm{C}^{1+} / \mathrm{O}^{2+}$ & $43 \pm 5$ & 50 \\
\hline \multirow[t]{2}{*}{$\mathrm{CO}^{4+}$} & $\mathrm{C}^{3+} / \mathrm{O}^{1+}$ & $65 \pm 5$ & 70 \\
\hline & $\mathrm{C}^{2+} / \mathrm{O}^{2+}$ & $68 \pm 6$ & 57 \\
\hline \multirow[t]{2}{*}{$\mathrm{Co}^{5+}$} & $\mathrm{C}^{3+} / \mathrm{O}^{2+}$ & $105 \pm 10$ & 79 \\
\hline & $\mathrm{C}^{2+} / \mathrm{O}^{3+}$ & $132 \pm 12$ & 97 \\
\hline \multirow[t]{2}{*}{$\mathrm{CO}^{6+}$} & $\mathrm{C}^{4+} / \mathrm{O}^{2+}$ & $127 \pm 12$ & 128 \\
\hline & $\mathrm{C}^{3+} / \mathrm{O}^{3+}$ & $162 \pm 17$ & 80 \\
\hline $\mathrm{CO}^{7+}$ & $\mathrm{C}^{4+} / \mathrm{O}^{3+}$ & $162 \pm 21$ & 161 \\
\hline $\mathrm{O}_{2}^{3+}$ & $\mathrm{O}^{2+} / \mathrm{O}^{1+}$ & $36 \pm 2$ & 38 \\
\hline $\mathrm{O}_{2}^{4+}$ & $0^{3+} / 0^{1+}$ & $63 \pm 3$ & 41 \\
\hline $\mathrm{O}_{2}^{5+}$ & $0^{3+} / 0^{2+}$ & $100 \pm 3$ & 76 \\
\hline $\mathrm{O}_{2}^{6+}$ & $\mathrm{O}^{4+} / \mathrm{O}^{2+}$ & $141 \pm 7$ & 54 \\
\hline $\mathrm{O}_{2}{ }^{7+}$ & $0^{4+} / 0^{3+}$ & $199 \pm 10$ & 141 \\
\hline $\mathbf{N}_{2}{ }^{3+}$ & $\mathbf{N}^{2+} / \mathbf{N}^{1+}$ & $41 \pm 4$ & 41 \\
\hline $\mathrm{N}_{2}^{4+}$ & $\mathrm{N}^{3+} / \mathrm{N}^{1+}$ & $76 \pm 7$ & 44 \\
\hline $\mathbf{N}_{2}{ }^{5+}$ & $\mathrm{N}^{3+} / \mathrm{N}^{2+}$ & $112 \pm 7$ & 80 \\
\hline $\mathrm{N}_{2}^{6+}$ & $\mathrm{N}^{4+} / \mathrm{N}^{2+}$ & $151 \pm 10$ & 102 \\
\hline $\mathbf{N}_{2}{ }^{7+}$ & $\mathbf{N}^{4+} / \mathbf{N}^{3+}$ & $197 \pm 11$ & 115 \\
\hline
\end{tabular}

A comparison of the average total kinetic energies obtained in this work for various dissociation reactions of $\mathrm{CO}, \mathrm{O}_{2}$, and $\mathrm{N}_{2}$ with those predicted by the above mentioned point-charge model is shown in Fig. 4. It is evident from this figure that all of the measured average total kinetic energies exceed the point-charge energies. The few theoretical results that are currently available concerning potential energy curves for multicharged molecular ions tend to indicate that, in the Franck-Conclon zone, the point-charge Coulomb potential lies above most of the states which connect to low energy asymptustic configurations. Therefore, this observation may indicate 
that most of the dissociation channels populated in heavyion collisions lead to highly excited product ions.

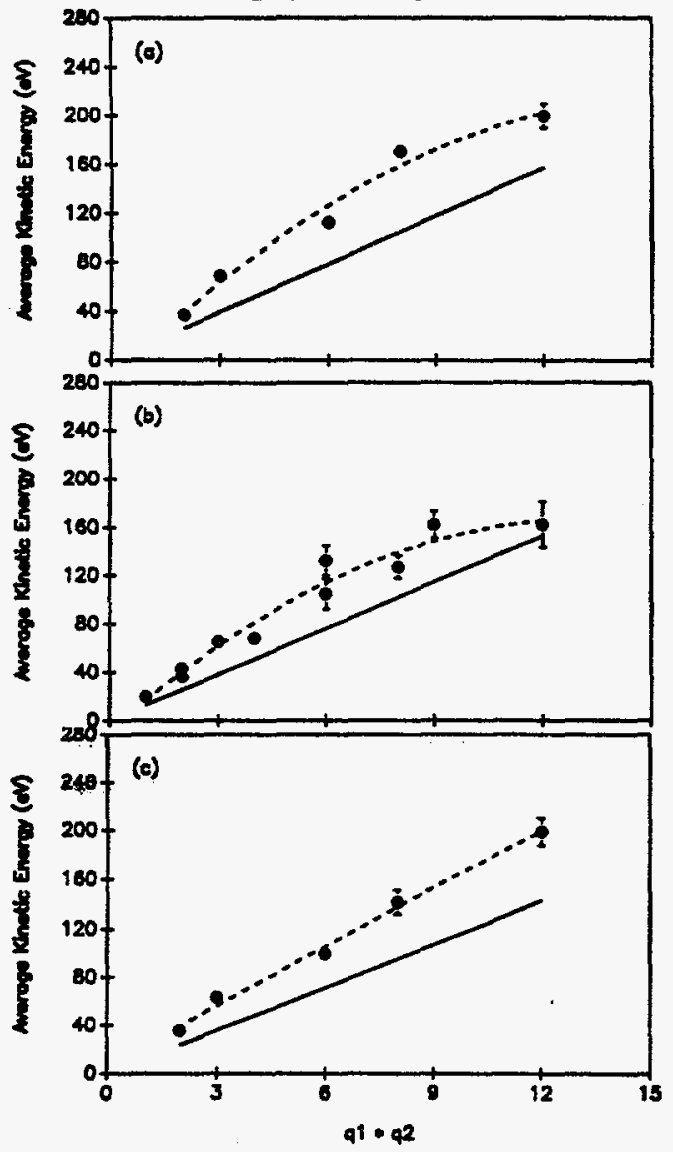

Fig. 4. Comparison of the experimental average total kinetic energies (data points) with the pointcharge Coulomb energies (solid lines) for (a) $\mathrm{N}_{2}$, (b) $\mathrm{CO}$, and (c) $\mathrm{O}_{2}$.

It is noteworthy that the experimental average energy for $\mathrm{CO}$ appears to be converging with the point-charge Coulomb energy at the highest values of $q_{1} q_{2}$. This behavior is reasonable because in the limit where all of the electrons are removed from the molecule, the total kinetic energy must correspond to the Coulomb potential between the two bare nuclei. The data for $\mathrm{N}_{2}$ also suggest the beginning of a "turn-over" at $q_{1} q_{2}=12$. Apparently in the case of $\mathrm{O}_{2}$, the molecule with the most electrons, somewhat higher values of $q_{1} q_{2}$ are required for the onset of convergence with the point-charge Coulomb energy.

The average excitation energy of the parent molecular ion can be estimated using the average total kinetic energies (Table 1) and the separated atom ionization energies. This procedure, of course, assumes the dissociation product ions are left in their ground states. Estimates of the average excitation energies, along with the energies of the corresponding (ground state) asymptotic limits, are presented graphically in Fig. 5. A noteworthy feature of the average excitation energies is that they increase approximately linearly with the number of electrons removed for $Q \geq 3+$. The slopes of the linear relationships for $\mathrm{CO}, \mathrm{N}_{2}$, and $\mathrm{O}_{2}$ are 78,90 , and 96 eV/electron, respectively.

\section{REFERENCES}

1. A. P. Hitchcock, P. Lablanquie, P. Morin, E. Lizon, A. Lugrin, M. Simon, P. Thirty, and I. Nenner, Phys. Rev. A37, 2448 (1988).
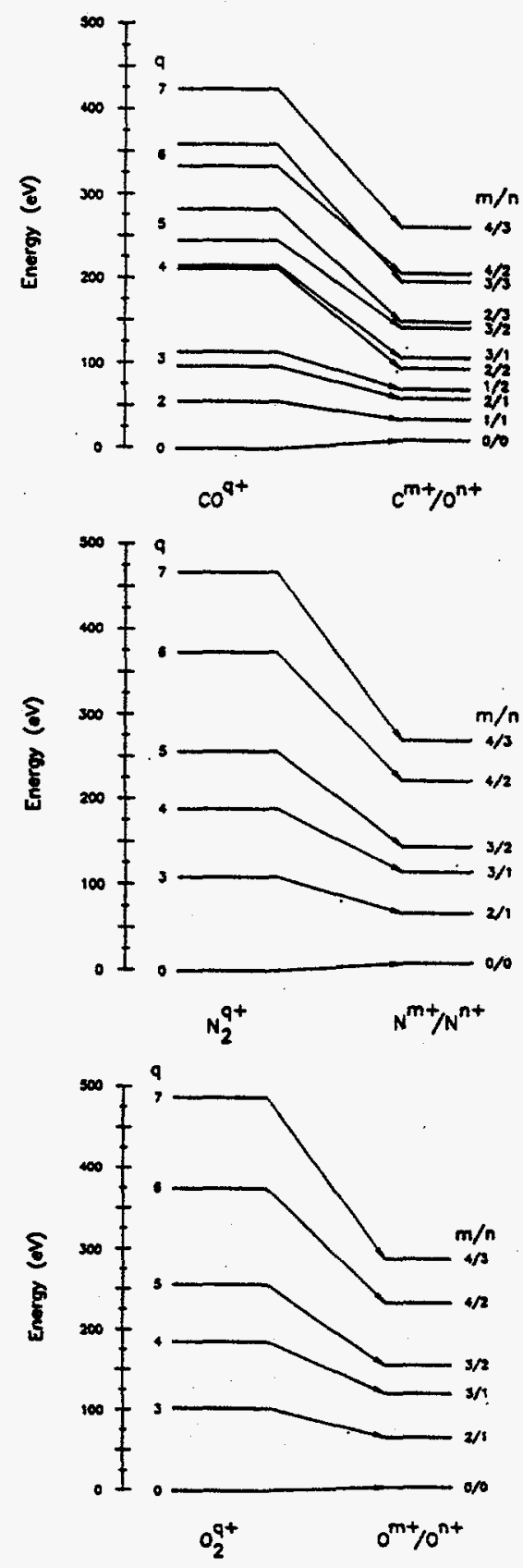

(b)

Fig. 5. Estimated average excitation energies for (a) $\mathrm{CO}$, (b) $\mathrm{N}_{2}$, and (d) $\mathrm{O}_{2}$ molecular ions. 


\section{DISSOCIATION OF HIGHLY CHARGED CO MOLECULAR IONS PRODUCED IN ELECTRON CAPTURE COLLISIONS OF $97 \mathrm{MeV}^{\mathrm{Ar}}{ }^{14+}$}

\section{G. Sampoll, R. L. Watson, V. Horvat, M. Barrett, and B. Griffith}

Electron capture collisions occur at much smaller impact parameters, on average, than collisions in which the charge of the projectile remains unchanged. The socalled transfer ionization (T) process, in which electron capture to the projectile is accompanied by additional Coulomb ionization of the target, leads to an enhancement of the higher charge state of the target relative to the charge state distribution typically produced by pure ionization (P) collisions. This property has been utilized recently in an experiment designed to investigate the dissociation of highly charged $\mathrm{CO}$ molecular ions.

The experiment was performed using the same differentially pumped gas cell system described in Ref. [1]. A time-of-flight (TOF) spectrometer was employed to measure the TOF of the first dissociation product ion to reach the microchannel plate (MCP) detector and the flight time difference between the first ion and its dissociation partner in coincidence with $97 \mathrm{MeV} \mathrm{Ar}{ }^{14+}$ projectiles. The TOF spectrometer consisted of two acceleration stages identical to those described in [1], and a modified $2.5-\mathrm{cm}$ diameter flight tube $15-\mathrm{cm}$ in length attached to a $2-\mathrm{cm}$ diameter chevron MCP detector. Behind the gas cell was placed a projectile charge state

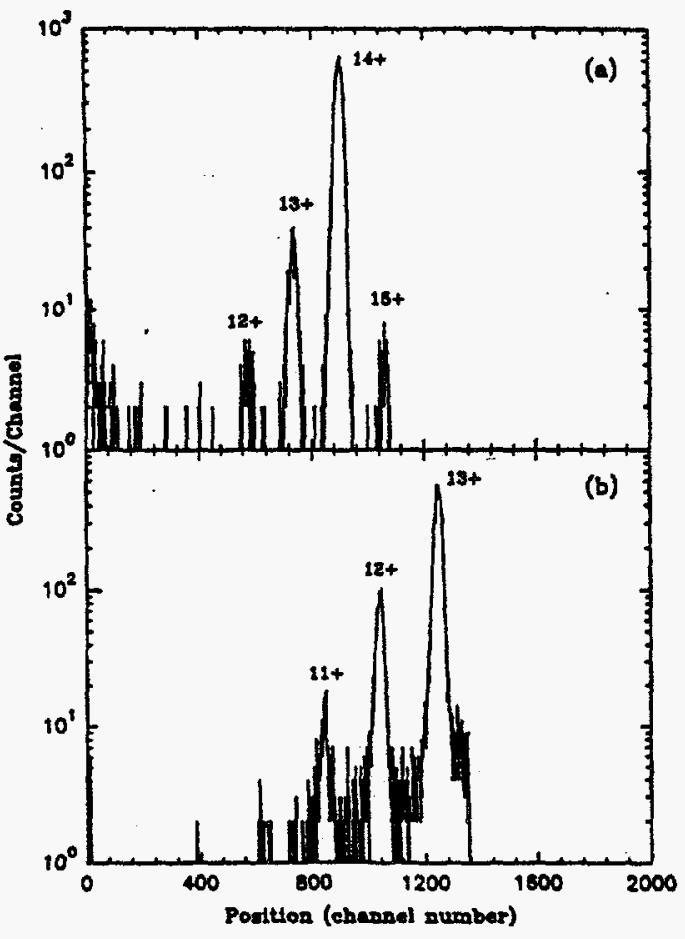

Fig. 1. Position pectra howing the charge state diatribution of the $\mathrm{Ar}^{14+}$ projectiles after passing through the gas cell taken (a) without and (b) with the detector mask. analyzer which consisted of a bending magnet and a onedimensional position sensitive MCP $(1 \times 4-\mathrm{cm})$. The three parameter data were acquired on a MICROVAX II computer using the Los Alamos Q-system software and recorded on magnetic tape event-by-event. The position spectrum showing the charge state distribution of the $\mathrm{Ar}^{\mathrm{l4}+}$ projectiles after passing through the gas cell is presented in Fig. 1 (a). In order to increase the counting rate for electron capture collisions, projectiles with exit charge states $\geq 14+$ were prevented from hitting the position sensitive MCP by a mask during part of the run. The resulting position spectrum is shown in Fig. 1 (b).

Calibration of the TOF spectrometer $\left(\mathrm{M} / q^{1 / 2}\right.$ sanle (where $M$ and $q$ are respectively the ion's mass and charge) was accomplished by measuring TOF spectra for an Ar target gas. The spectra obtained for charge unchanged (PI) collisions and electron capture collisions (TI) are shown in Fig. 2 (a) and (b). From these spectra, it is apparent that charge states up to $13+$ are produced for this collision system. In the TI spectrum [Fig. 2(b)], the charge states in the vicinity of $8+$ are greatly enhanced relative to the low charge atates. In fact, the yields of $\mathrm{Ar}^{1+}, \mathrm{Ar}^{2+}$, and $\mathrm{Ar}^{3+}$ in this spectrum are almost entirely attributable to PI collisions of projectiles

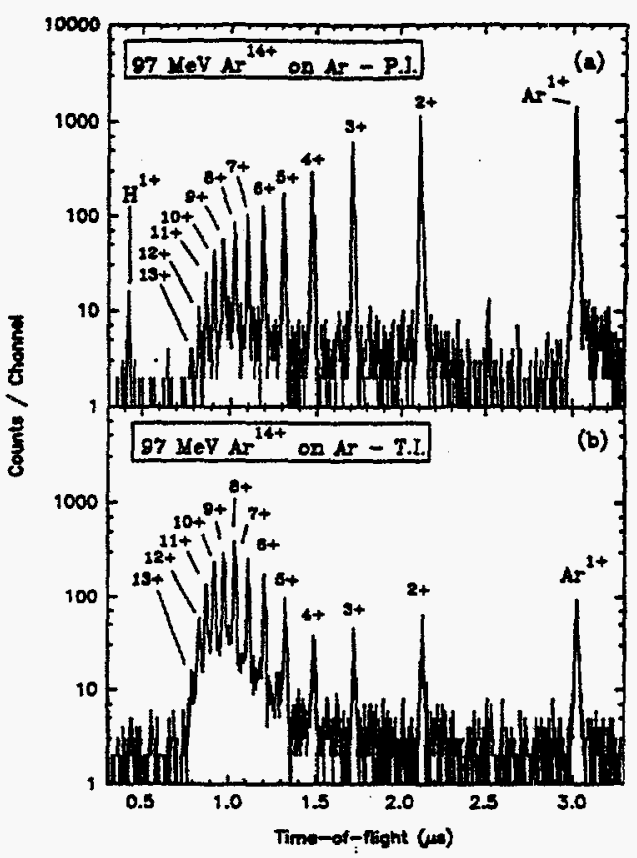

Fig. 2. Time-of-tight spectrum of Ar ions produced by (a) pure ionization (PI) and (b) transfer ionization (TI) collisions of $97 \mathrm{MeV} \mathrm{Ar}{ }^{24+}$ with Ar. 
that had captured an electron prior to entering the gas cell. The ratio of $\mathrm{Ar}^{13+}$ to $\mathrm{Ar}^{14+}$ in the incident beam was found to be $2.0 \times 10^{-2}$.

A two-dimensional display of the TOF versus timedifference data obtained for ions produced in electron capture collisions of $97 \mathrm{MeV} \mathrm{Ar}^{14+}$ with $\mathrm{CO}$ molecules is shown in Fig. 3(a). Projection of this data onto the TOF axis gives the first-ion TOF spectrum shown in Fig. 3(b), while projection onto the time-difference axis gives the total ion-pair time-difference spectrum shown in Fig. 3(c). CO (Capture data)

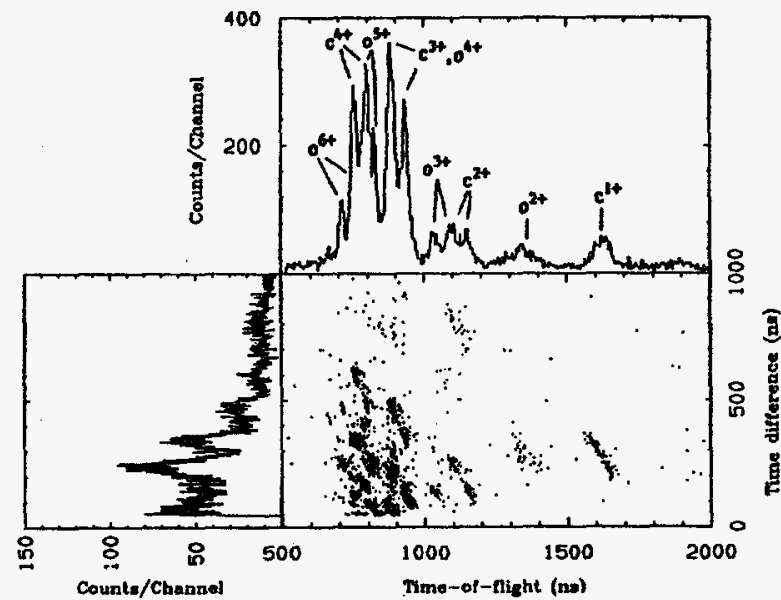

Fig. 3. Two-dimensional diplay of the data for CO diesociation product ions produced in electron capture collisions.

Comparison of Fig. 3 with Fig. 1 in report IV-A confirms the prediction that in $T$ collisions with molecules the higher charge states are greatly enhanced relative to the distribution of ions resulting from PI collisions. Nevertheless, it is apparent that the highest charge observed for $\mathrm{C}$-ions is $4+$ and the highest charge observed for $\mathrm{O}$-ions is $6+$, implying that $\mathrm{K}$-vacancy production is very improbable.

By placing windows around each of the twodimensional data "islands" appearing in Fig. 3(a) and projecting only events within these islands onto the timedifference axis, separate ion-pair time-difference distributions were obtained for each of the statistically significant dissociation channels within the observation range of the experiment. The various ion-pair timedifference distributions associated with electron capture collisions are shown in Figs. 4 and 5 . The time consuming process of transforming these time-difference distribution into total kinetic energy distributions is currently in progress. The goal of this effort is to compare the average total kinetic energies for specific dissociation channels excited in PI, one-electron $\mathrm{TI}$, and two-electron $\mathrm{TI}$ to see if the excitation energies of the parent molecular ions are significantly different.

\section{REFERENCES}

1. G. Sampoll, R. L. Watson, O. Heber, H. Horvat, K. Wohrer, and M. Chabot, Phys. Rev. A 45, 2903 (1992).

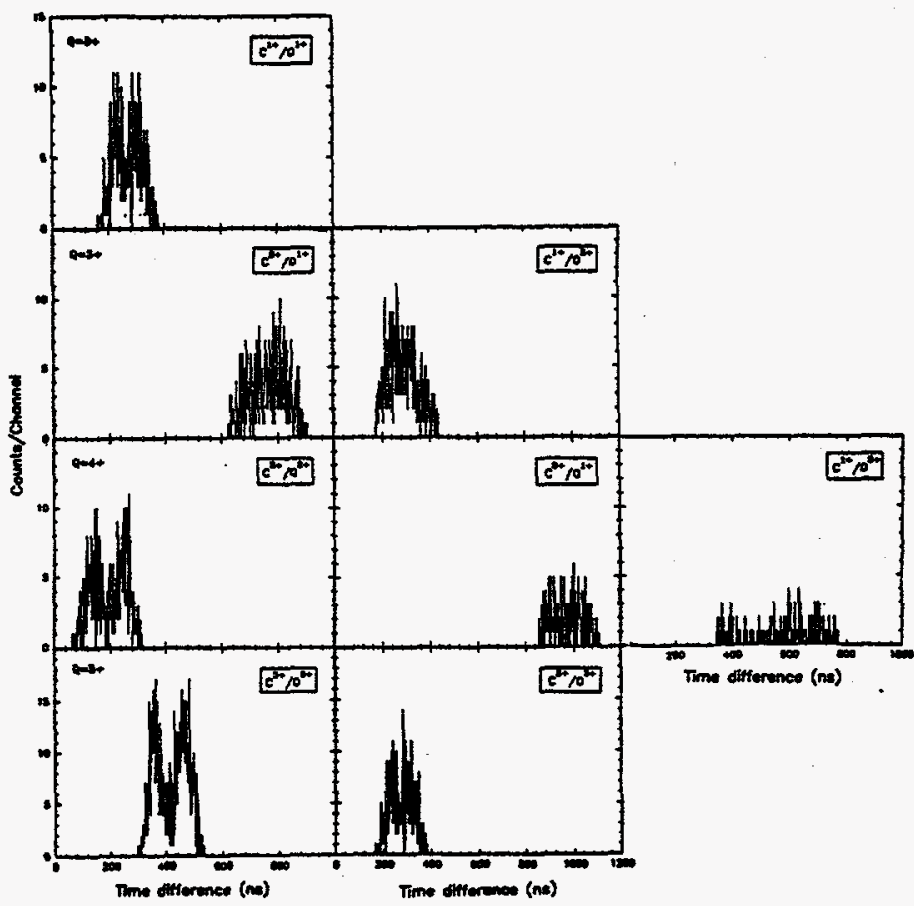

Fig. 4. Sorted ion-pair time-difference distributions for the statistically significant disuociation channels of multicharged $\mathrm{CO}^{\mathrm{Q}+}$ molecular ions producod in electron capture collisions by $97 \mathrm{MeV} \mathrm{Ar}^{14+}$, where $Q$ $=2$ to 5 .

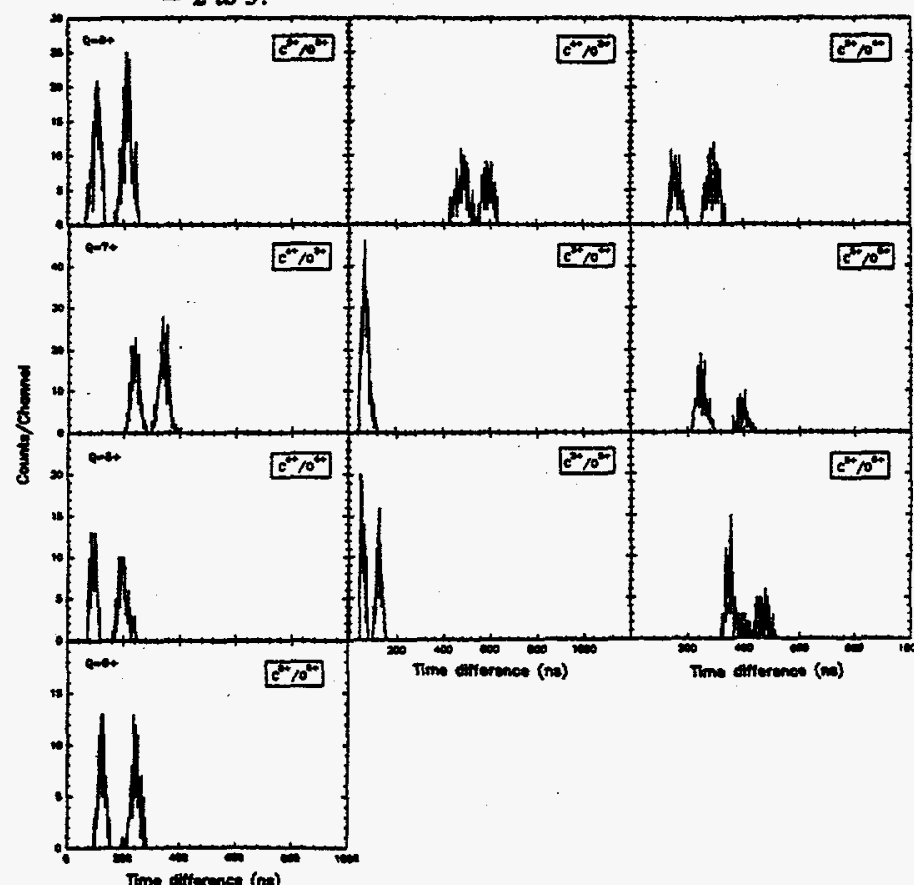

Fig. 5. Sorted ion-pair time-difference distributions for the statistically significant dissociation channels of multicharged $\mathrm{COQ}^{+}$molecular ions produced in electron capture collisions by $97 \mathrm{MeV} \mathrm{Ar}{ }^{14 t}$, where $Q$ $=6$ to 9 . 


\title{
DISSOCIATION FRACTIONS AND BRANCHING RATIOS FOR MULTICHARGED CO MOLECULAR IONS PRODUCED IN COLLISIONS WITH $97 \mathrm{MeV} \mathrm{Ar}^{14+}$
}

\author{
K. Wohrer, G. Sampoll, R. L. Watson, M. Chabot, O. Heber, and V. Horvat
}

The dissociation product yields obtained for $\mathrm{CO}$ in the experiments described in this section, p. 73 were used to determine dissociation fractions for $\mathrm{CO}^{1+}$ and $\mathrm{CO}^{2+}$, and branching ratios for the different charge division pathways observed in the dissociation of $\mathrm{CO}^{3+}$ to $\mathrm{CO}^{7+}$. Total detection yields [N(I)] of single ions were determined by integrating the corresponding peaks in the singles TOF spectrum after subtraction of a linear background. In cases where two or more peaks overlapped, a Gaussian peak fitting program was used to calculate the individual yields. The single-ion production yields [Y(I)] were then obtained using the relationship

$$
Y(I)=N(I)\left[T_{18} T_{g} \epsilon_{d}\right]^{-1},
$$

where $T_{1}$ is the single-ion transmission probability through the TOF spectrometer, $T_{3}$ is the product of the transmission probabilities through the set of grids at the entrance and at the exit of the flight tube $(0.77)$, and $\epsilon_{d}$ is the detection efficiency of the MCP ion detector at the end of the flight tube.

Total detection yields of ion-pairs $\left[N\left(I_{1}+I_{2}\right)\right]$ were determined by integrating the corresponding ion-pair time-difference distributions. These yields were corrected for background and for contributions due to double collisions by the projectile while passing through the gas cell. The ion-pair production yields were then obtained using the relationship

$$
Y\left(I_{1}+I_{2}\right)=N\left(I_{1}+I_{2}\right)\left[T_{12} T_{2}^{2} \epsilon_{1} \epsilon_{2}\right]^{-1},
$$

where $T_{12}$ is the ion-pair transmission probability through the spectrometer and the other quantities are as defined above.

In the following analysis, the total dissociation yield for a specific parent molecular ion $Y_{D}(M)$ is defined as the sum of the production yields of all possible dissociation product pairs formed within a time interval determined by the flight time of the molecular ion to the entrance of the flight tube. The time restriction is imposed by the fact that molecular ions which dissociated after entering the flight tube were still detected as molecular ions since, at that point, they had been fully accelerated. In particular, the dissociation yields for $\mathrm{CO}^{1+}$ and $\mathrm{CO}^{2+}$ are
$Y_{D}\left(\mathrm{CO}^{1+}\right)=\mathrm{Y}\left(\mathrm{C}^{1+}+\mathrm{O}\right)+\mathrm{Y}\left(\mathrm{C}+\mathrm{O}^{1+}\right)$

$\mathrm{Y}_{\mathrm{D}}\left(\mathrm{CO}^{2+}\right)=\mathrm{Y}\left(\mathrm{C}^{2+}+\mathrm{O}\right)+\mathrm{Y}\left(\mathrm{C}^{1+}+\mathrm{O}^{1+}\right)+\mathrm{Y}\left(\mathrm{C}+\mathrm{O}^{2+}\right)$

and the pertinent time intervals are 0.30 and $0.21 \mu \mathrm{s}$, respectively. Then the fractions $\alpha$ of molecular ions that dissociate within these time intervals are given by

$$
\begin{aligned}
& \alpha\left(C O^{1+}\right)=\frac{Y_{D}\left(\mathrm{CO}^{1+}\right)}{Y_{D}\left(\mathrm{CO}^{1+}\right)+Y\left(C O^{1+}\right)} \\
& \alpha\left(\mathrm{CO}^{2+}\right)=\frac{Y_{D}\left(\mathrm{CO}^{2+}\right)}{Y_{D}\left(\mathrm{CO}^{2+}\right)+Y\left(\mathrm{CO}^{2+}\right)}
\end{aligned}
$$

Since $\mathrm{CO}^{1+}$ has a stable ground state and the ground state of $\mathrm{CO}^{2+}$ is known to have a lifetime greater than 15- $\mu \mathrm{s}, 1$ the dissociation fractions determined by the short acceleration time scale of this experiment provide measures of the total excitation probabilities to short-lived autodissociative states.

Since only ions were detected in the present experiments, the production yields of dissociation product pairs in cases where one of the products was neutral had to be determined using the single-ion production yield of the charged partner and the ion-pair production yields for all ion-pairs involving that partner. For example, the production yields of $\left(\mathrm{C}^{1+}+\mathrm{O}\right)$ and $\left(\mathrm{C}+\mathrm{O}^{1+}\right)$ pairs from the dissociation of $\mathrm{CO}^{1+}$ were obtained using the following expressions:

$Y\left(\mathrm{C}^{1+}+\mathrm{O}\right)=\mathrm{Y}\left(\mathrm{C}^{1+}\right)-\sum_{\mathrm{n} \neq 0} \mathrm{Y}\left(\mathrm{C}^{1+}+\mathrm{O}^{\mathrm{n}}\right)$

$Y\left(C+\mathrm{O}^{1+}\right)=Y\left(\mathrm{O}^{1+}\right)-\sum_{\mathrm{n} \neq 0} \mathrm{Y}\left(\mathrm{C}^{\mathrm{n}+}+\mathrm{O}^{1+}\right)$.

The dissociation fractions and ion-pair production yields for $\mathrm{CO}^{1+}$ and $\mathrm{CO}^{2+}$ are listed in Table 1. Since direct Coulomb ionization of valence electrons is expected to be the dominant ion-production mechanism for collisions of $2.4 \mathrm{MeV} / a m u-\mathrm{Ar}^{14+}$ ions, ${ }^{2}$ these results ure compared with those obtained by Hitchcock et a‥ ${ }^{3}$ for valence-electron photoionization by $280 \mathrm{eV}$ synchrotron radiation, and by Shah and Gilbody for direct valence- 
Table 1.

Dissociation fractions and production yield ratios for $\mathrm{CO}^{1+}$ and $\mathrm{CO}^{2+}$.

\begin{tabular}{|c|c|c|c|c|}
\hline \multirow[t]{2}{*}{ Ratio } & \multirow{2}{*}{$\begin{array}{l}\text { Present work } \\
\text { 96-MeV Arr }\end{array}$} & \multicolumn{2}{|c|}{ Shah and Gilbody ${ }^{4}$} & \multirow{2}{*}{$\begin{array}{l}\text { Hitchcock et al. } \\
280-\mathrm{eV} \text { photons }\end{array}$} \\
\hline & & $16-\mathrm{keV} \mathrm{H} \mathrm{H}^{1+}$ & 261-keV He${ }^{2+}$ & \\
\hline$\alpha\left(\mathrm{CO}^{1+}\right)$ & $0.33 \pm 0.08$ & $0.44 \pm 0.04$ & $0.22 \pm 0.04$ & 0.8 \\
\hline $\mathrm{Y}\left(\mathrm{C}^{1+}+\mathrm{O}\right) / \mathrm{Y}\left(\mathrm{CO}^{1+}\right)$ & $0.31 \pm 0.08$ & $0.58 \pm 0.08$ & $0.23 \pm 0.03$ & 1.6 \\
\hline $\mathbf{Y}\left(\mathrm{C}+\mathrm{O}^{1+}\right) / \mathrm{Y}\left(\mathrm{CO}^{1+}\right)$ & $0.21 \pm 0.11$ & $0.19 \pm 0.03$ & $0.06 \pm 0.01$ & 1.6 \\
\hline $\mathrm{Y}\left(\mathrm{C}^{1+}+\mathrm{O}\right) / \mathrm{Y}\left(\mathrm{C}+\mathrm{O}^{1+}\right)$ & $1.8 \pm 0.7$ & $3.1 \pm 0.8$ & $3.8 \pm 1.0$ & 1.0 \\
\hline$\alpha\left(\mathrm{CO}^{2+}\right)$ & $0.95 \pm 0.02$ & & & 0.9 \\
\hline $\mathrm{Y}\left(\mathrm{C}^{1+}+\mathrm{O}^{1+}\right) / \mathrm{Y}\left(\mathrm{CO}^{2+}\right)$ & $14.5 \pm 4.3$ & \pm 1.8 & \pm 2.9 & 6.5 \\
\hline $\mathrm{Y}\left(\mathrm{C}^{2+}+\mathrm{O}\right) / \mathrm{Y}\left(\mathrm{CO}^{2+}\right)$ & $3.2 \pm 2.2$ & & & 3.0 \\
\hline $\mathrm{Y}\left(\mathrm{C}+\mathrm{O}^{2+}\right) / \mathrm{Y}\left(\mathrm{CO}^{2+}\right)$ & $0.7 \pm 0.7$ & & & 1.0 \\
\hline
\end{tabular}

* These values are for transfer ionization.

electron ionization by $16 \mathrm{keV} / \mathrm{amu}-\mathrm{H}^{1+}$ and 65 $\mathrm{keV} / \mathrm{amu}-\mathrm{He}^{2+}$ ions. The ion-molecule systems all have nearly the same values of the parameter

$$
K=\left(Z_{p} / Z_{t}\right)\left(v_{e} / v_{p}\right)
$$

where $Z_{p}, Z_{t}, v_{e}$, and $v_{p}$ are respectively the projectile and target atomic numbers, and the valence electron and projectile velocities. The quantity $K$ is a measure of the strength of the perturbation presented to the valence electrons during a collision. ${ }^{3}$ It is apparent that all three ion-molecule systems display fairly similar characteristics with the $\left(\mathrm{C}^{1+}+\mathrm{O}\right)$ dissociation channel being considerably preferred over the $\left(\mathrm{C}+\mathrm{O}^{1+}\right)$ dissociation channel. In the case of $\mathrm{CO}^{2+}$, the symmetric charge division pathway leading to $\left(\mathrm{C}^{1+}+\mathrm{O}^{1+}\right)$ is greatly preferred over the asymmetric charge division channels and it is evident also that the branching ratio for producing doubly ionized $\mathrm{C}$ ions is considerably enhanced relative to the branching ratio for producing doubly ionized $O$ ions. This is understandable on the basis of the atomic ionization energies since $48.8 \mathrm{eV}$ is required to remove 2-electrons from oxygen while only $35.6 \mathrm{eV}$ is required to remove 2electrons from carbon. The minimum excitation energy required by the $\left(\mathrm{C}^{1+}+\mathrm{O}^{1+}\right)$ dissociation branch is nearly the same as that for the $\left(\mathrm{C}^{2+}+\mathrm{O}\right)$ branch because in addition to the ionization energies of $\mathrm{C}$ and $\mathrm{O}(24.9 \mathrm{eV})$, the Coulomb repulsion energy $(\approx 12.8 \mathrm{eV})$ must also be supplied.

The photoionization results of Hitchcock, et $\underline{\text { al }} .{ }^{3}$ for single ionization of $\mathrm{CO}$ are quite different than those obtained by ion-impact ionization. In particular, the dissociation fraction for $\mathrm{CO}^{1+}$ is much larger, implying that valence-electron photoionization at $280 \mathrm{eV}$ is considerably more effective in populating short-lived autodissociative states than ion-impact ionization. On the other hand, the dissociation fractions for $\mathrm{CO}^{2+}$ are essentially the same for both ionization mechanisms.

The relative production yields of ion-pairs produced in the dissociation of $\mathrm{CO}^{Q+}$ with $Q=2$ to 7 are listed in Table 2. In general, the same preference for charge symmetric or nearly charge symmetric dissociation channels as was observed for $\mathrm{CO}^{2+}$ is evident in this data. It is to be expected that, in most collisions, the ionization will be localized along the projectile trajectory. This means that most of the electron vacancies produced in a collision initially will be localized about either the $C$

Table 2.

Relative production yields of ion-pairs from the dissociation of $\mathrm{CO}^{\mathrm{Q}+}$.

\begin{tabular}{|c|c|c|c|c|}
\hline $\mathbf{Q}$ & $\left(I_{1}+I_{2}\right)$ & \multicolumn{3}{|c|}{$Y\left(I_{1}+I_{2}\right) / Y\left(C^{1+}+O^{1+}\right)$} \\
\hline 2 & $\left(\mathrm{C}^{1+}+\mathrm{O}^{1+}\right) 1$ & & & \\
\hline \multirow[t]{2}{*}{3} & $\left(\mathrm{C}^{2+}+\mathrm{O}^{1+}\right)$ & 0.45 & \pm & 0.04 \\
\hline & $\left(\mathrm{C}^{1+}+\mathrm{O}^{2+}\right)$ & 0.20 & \pm & 0.02 \\
\hline \multirow[t]{2}{*}{4} & $\left(\mathrm{C}^{3+}+\mathrm{O}^{1+}\right)$ & 0.059 & \pm & 0.006 \\
\hline & $\left(\mathrm{C}^{2+}+\mathrm{O}^{2+}\right)$ & 0.26 & \pm & 0.03 \\
\hline \multirow[t]{2}{*}{5} & $\left(\mathrm{C}^{3+}+\mathrm{O}^{2+}\right)$ & 0.13 & \pm & 0.01 \\
\hline & $\left(\mathrm{C}^{2+}+\mathrm{O}^{3+}\right)$ & 0.13 & \pm & 0.03 \\
\hline \multirow[t]{2}{*}{6} & $\left(\mathrm{C}^{3+}+\mathrm{O}^{3+}\right)$ & 0.29 & \pm & 0.04 \\
\hline & $\left(\mathrm{C}^{4+}+\mathrm{O}^{2+}\right)$ & 0.024 & \pm & 0.006 \\
\hline 7 & $\left(\mathrm{C}^{4+}+\mathrm{O}^{3+}\right)$ & 0.04 & \pm & 0.04 \\
\hline
\end{tabular}


or the $\mathrm{O}$ atom and hence considerable rearrangement must occur during the initial stages of the dissociation process.

To aid in the following discussion, the estimated minimum excitation energies required for all of the possible charge division channels are displayed in Fig. 1. These excitation energies were calculated with the formula

$$
E_{\min }\left(q_{1}, q_{2}\right)=D_{0}+\sum_{i=1}^{q_{1}} B_{1}(C)+\sum_{j=1}^{q_{2}} B_{j}(O)+C E,
$$

where $q_{1}$ and $q_{2}$ are respectively the charges of the carbon and oxygen ion dissociation products, $D_{0}$ is the dissociation energy of the neutral molecule (11.1 eV), $B_{i}(C)$ is the ith ionization energy of the carbon atom, $B_{j}(O)$ is the jth ionization energy of the oxygen atom, and CE is the point-charge Coulomb potential energy, $q_{1} q_{2} / R$,

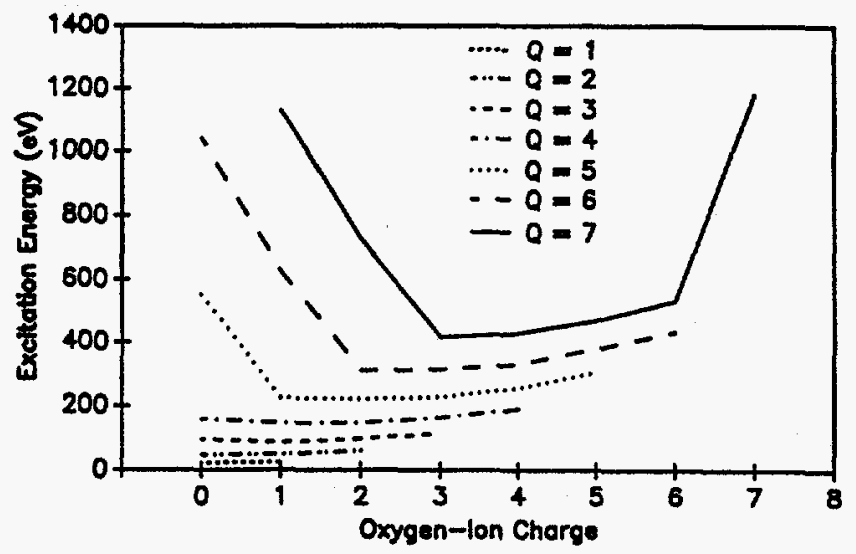

Fig. 1. The estimated minimum excitation energy required to produce ion-pairs $\left(\mathrm{C}_{11}+\mathrm{O}_{2}^{9}\right)$ versus the charge $\left(q_{2}\right)$ of the oxygen ion, where $Q=q_{1}$ $+q_{2}$.

where $R$ is the neutral molecule bond length $(1.128 A)$. Figure 1 shows that, for a given total charge $Q$, the lowering of the total ionization energy that results from equalizing the carbon and oxygen ion charges is, for the most part, canceled by the attendant increase in the Coulomb energy. The net result is that there is not much of a change in the minimum excitation energy required for any charge division pair (having the same $Q$ ) unless formation of the pair involves the removal of a $\mathrm{K}$-shell electron. Nevertheless, for $Q=3$ the minimum excitation energy required for the $\left(\mathrm{C}^{2+}+\mathrm{O}^{1+}\right)$ channel is approximately $11 \mathrm{eV}$ lower than that for the $\left(\mathrm{C}^{1+}+\mathrm{O}^{2+}\right)$ channel and the data in Table 2 clearly indicate that the former channel is favored. On the other hand, for $Q=4$, the data in Table 2 indicate there is a very strong preference for the symmetric $\left(\mathrm{C}^{2+}+\mathrm{O}^{2+}\right)$ channel over the asymmetric $\left(\mathrm{C}^{3+}+\mathrm{O}^{1+}\right)$ channel while the estimated minimum excitation energies for these two dissociation channels are both the same. Moreover, for $Q=5$, the: estimated minimum excitation energies required for the three channels $\left(\mathrm{C}^{4+}+\mathrm{O}^{1+}\right),\left(\mathrm{C}^{3+}+\mathrm{O}^{2+}\right)$, and $\left(\mathrm{C}^{2+}+\mathrm{O}^{3+}\right)$ are all within $7 \mathrm{eV}$ of each other. Experimentally, the: $\left(\mathrm{C}^{3+}+\mathrm{O}^{2+}\right)$ and $\left(\mathrm{C}^{2+}+\mathrm{O}^{3+}\right)$ channels are equally probable while the $\left(\mathrm{C}^{4+}+\mathrm{O}^{1+}\right)$ channel is barely observable.

Using the data given in Tables 1 and 2, along with the ratio of the $\mathrm{CO}^{2+}$ and $\mathrm{CO}^{1+}$ production yields, which was $0.019 \pm 0.002$, the relative probability for removing $\mathrm{Q}$ electrons in collisions of $97 \mathrm{MeV} \mathrm{Ar}^{14+}$ ions with $\mathrm{CO}$ molecules may be obtained. These probabilities, expressed as the ratios $Y(Q) / Y(1)$ where $Y(Q)$ is the total yield of all products resulting from the removal of $Q$ electrons from $\mathrm{CO}$, are compared with the yield ration obtained for the ionization of $\mathrm{Ne}$ atoms by $97 \mathrm{MeV} \mathrm{Ar}{ }^{14--}$ collisions in Fig. 2. It is apparent from this comparison that the jonization probabilities for $\mathrm{CO}$ molecules agree quite well up to $Q=4$, but become increasingly larger than those for Ne atoms beyond this point.

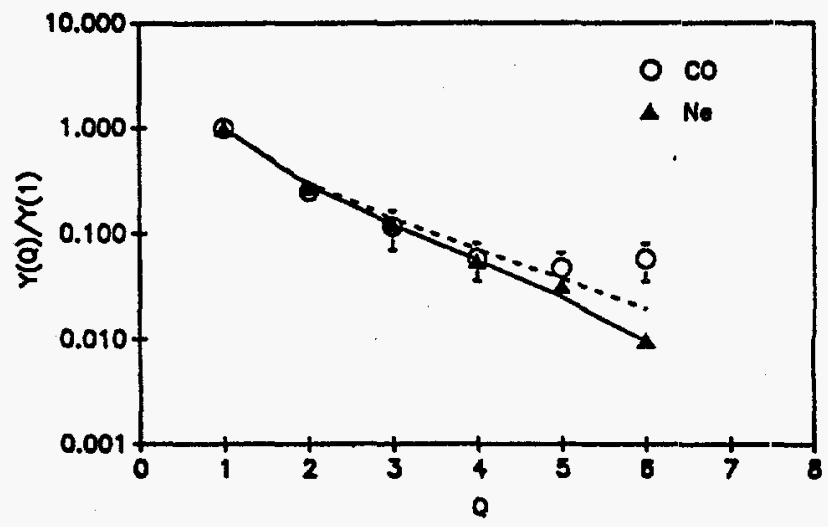

Fig. 2. The ratio of the total ionization yield for charge $Q, Y(Q)$, to the total ionization yield for $Q=1$. The solid and dashed lines show the results of independent electron approximation calculations for $\mathrm{Ne}$ and $\mathrm{CO}$, respectively.

The solid line in Fig. 2 shows the relative ionization cross sections for $\mathrm{Ne}$ given by an independent electron approximation calculation, similar to that described in Ref. 6, in which the impact-parameter-dependent singleelectron ionization probability was represented by the simple exponential function $p_{0} \exp \left(-b / r_{L}\right)$. The value of the parameter $p_{0}$ that gave the best fit to the data was 0.7 . The relative ionization cross sections were found to be quite insensitive to the value used for the radius parameter 
$r_{L}$. The present calculations for Ne were performed using the most probable $L$-electron radius, $0.335 \AA$, for this parameter. Corresponding cross sections for $\mathrm{CO}$ were calculated using the same $p_{0}$ value as for $\mathrm{Ne}$, a radius parameter value of $1.13 \AA$, and 10 as the number of valence electrons (instead of 8 as for $\mathrm{Ne}$ ). These results are shown by the dashed line in Fig. 2 and they indicate that the cross section enhancements cannot be entirely attributed to the fact that $\mathrm{CO}$ has more valence electrons than $\mathrm{Ne}$ and is a somewhat larger target.

Part of the enhancement of the ionization probability for $\mathrm{CO}$ might be due to $\mathrm{K}$-shell vacancy production followed by Auger decay. Similar charge multiplication effects caused by Auger decay of $\mathrm{L}$ vacancies have been observed in collisions of 1-MeV/amu $O$ and $F$ ions with Ar. ${ }^{6}$ The dominant mechanism for $\mathrm{K}$ vacancy production in the $\mathrm{Ar}^{\mathrm{I4}+}+\mathrm{CO}$ collision system is electron capture to the Ar L-shell. ${ }^{7}$ However, this same mechanism must also contribute to the Ne results. In fact, the electron capture cross sections are expected to be larger for Ne than for $\mathrm{CO}$ since the $\mathrm{K}$-binding energy of Ne more closely matches the L-binding energy of Ar.

From the above discussion, it must be concluded that much of the ionization probability enhancement is caused by a mechanism that is unique to $\mathrm{CO}$. One such possibility is linked to the electron exchange that must occur between the ion with the lowest charge and its more highly charged partner during the early stages of the dissociation process in order to symmetrize the charge division. According to the classical overbarrier model of charge exchange, ${ }^{8}$ the exchange of electrons is expected to proceed from the L-shell of the lower charged ion to the M-shell of the higher charged ion.

Thus, one of the dissociation products might be left in an excited state that would subsequently decay by an LMM Auger transition, thereby increasing its charge. One problem with this hypothesis is that the excited state would have to contain at least two $M$-shell electrons in order to undergo LMM Auger decay. However, such configurations could arise as a consequence of the transfer of two or more electrons in the rearrangement process, or as a result of electron excitation during the collision followed by one-electron transfer. The yield data for $Q=$ 4 and $Q=5$ in Table 2 provide some support for this scenario. The arguments based on minimum excitation energies presented above lead one to expect equal yields of $\left(\mathrm{C}^{2+}+\mathrm{O}^{2+}\right)$ and $\left(\mathrm{C}^{3+}+\mathrm{O}^{1+}\right)$ ion pairs while experimentally the yield of the former is a factor of 3.4 larger than the yield of the latter. However, only the asymmetric charge division pairs will have a tendency to undergo charge exchange, which means that most of the $\left(\mathrm{C}^{3+}+\mathrm{O}^{1+}\right)$ and $\left(\mathrm{C}^{1+}+\mathrm{O}^{3+}\right)$ pairs produced in the collision will end up as $\left(\mathrm{C}^{3+}+\mathrm{O}^{2+}\right)$ and $\left(\mathrm{C}^{2+}+\mathrm{O}^{3+}\right)$ pairs as a result of electron transfer followed by $L M M$ Auger decay. The net effect of this will be to reduce the yields of the asymmetric $Q=4$ ion pairs and increase the yields of the $Q=5$ ion pairs.

\section{REFERENCES}

1. A. S. Newton and A. F. Sciamanna, J. Chem. Phys. 53, 132 (1970).

2. R. Olsen, J. Phys. B12, 1843 (1979).

3. A. P. Hitchcock, P. Lablanquie, P. Morin, E. Lizon, A. Lugrin, M. Simon, P. Thiry, and I. Nenner, Phys. Rev. A37, 2448 (1988).

4. M. B. Shah and H. B. Gilbody, J. Phys. B: At. Mol. Opt. Phys. 23, 1491 (1990).

5. E. Merzbacher, Fundamental Processes in Energetic Atomic Collisions (NATO ASI, 1983) Vol. 103, p. 319.

6. O. Heber, G. Sampoll, B. B. Bandong, R. J. Maurer, R. L. Watson, I. Ben-Itzhak, J. L. Shinpaugh, J. M. Sanders, L. Hefner, and P. Richard, Phys. Rev. A39, 4898 (1989).

7. S. Andriamonje, J. F. Chemin, J. Roturier, B. Saboya, J. N. Scheurer, R. Gayet, A. Salin, H. Laurent, P. Aguer, and J. P. Thibaud, Z. Phys. A317, 251 (1984).

8. H. Ryufuku, K. Sasaki, and T. Watanabe, Phys. Rev. A21, 745 (1980). 


\title{
ORIENTATION DEPENDENCE OF FAST-COLLISION-INDUCED MOLECULAR DISSOCIATION
}

\author{
M. Chabot, K. Wohrer, G. Sampoll, V. Horvat, and R. L. Watson
}

An experiment designed to investigate the orientation dependence of the cross section for ionization of $\mathrm{N}_{2}$ by 97 $\mathrm{MeV} \mathrm{Ar}^{14+}$ has been performed. Orientation dependent cross sections can occur as a result of interference between the scattering amplitudes associated with the different atomic centers, ${ }^{\prime}$ and as a result of geometrical effects. ${ }^{2}$

A well collimated beam of $\mathrm{Ar}^{14+}$ ions was directed through a gas jet which was enclosed within a uniform electric field. The jons produced in single collisions of the beam particles with molecules in the gas jet were accelerated by the electric field into the second stage of the ion-collection/time-of-flight (TOF) spectrometer system, where they were further accelerated by a second uniform electric field. After entering the third stage, the ions drifted in a field free region into a $3-\mathrm{cm}$ diameter chevron microchannel plate detector (MCP) system equipped with a resistive anode. This detector system provided a start signal for the TOF measurement and four amplitude signals (one from each comer of the resistive anode) for the position determination of each detected ion. Several low transmission grids were mounted in front of the MCP to reduce the probability of detecting both ions from the same binary dissociation event. The stop signal for the TOF measurement was provided by another MCP used to directly detect the beam particle after it passed through the gas jet.

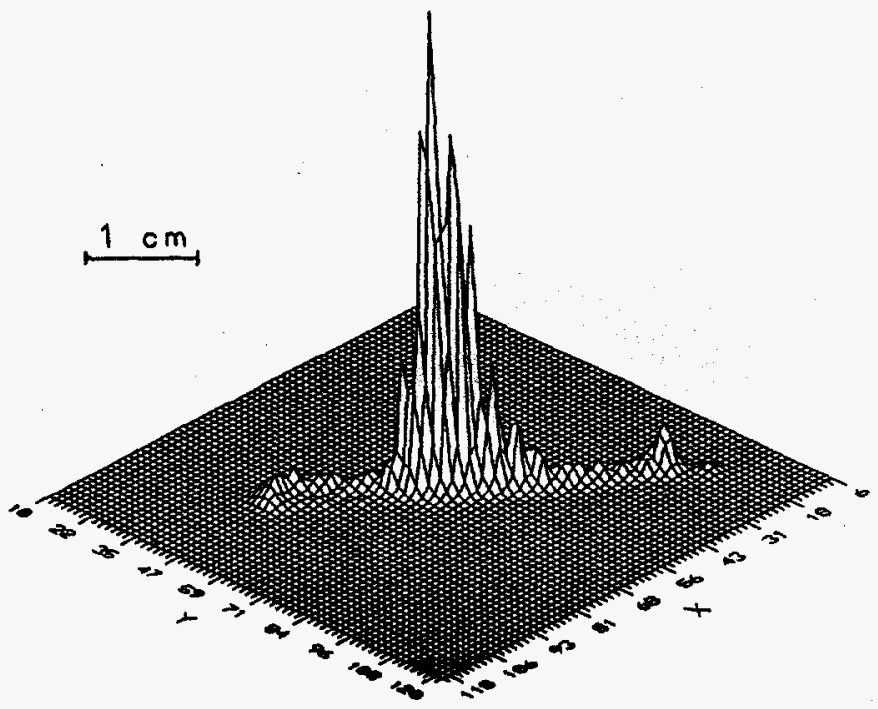

Fig. 1. Position distribution of $\mathrm{N}_{2}^{1+}$ showing the profile of the gas jet where it overlapped the Ar beam.
A profile of the gas jet in the region where it: overlapped with the ion beam is shown by the position distribution obtained for $\mathrm{N}_{2}^{1+}$ molecular ions in Fig. 1 . These ions are produced with near-thermal energivs since the recoil energy associated with single ionization is negligible. ${ }^{3}$ The sharp structure in the main peak was caused by the grids in front of the MCP. This figure also shows the contribution from residual gas background along the beam path. The small peaks at both ends of the beam track are caused by focusing effects associated with nonuniformities in the electric fields near the periphery of the collection region.

The grid structure is shown more clearly in Fig. 2(8i) where the $\mathrm{N}_{2}^{1+}$ position distribution has been projected onto the $\mathrm{X}$ axis. The character of the background due to the residual gas is shown by the $X$ projection of the position distribution of $\mathrm{H}_{2} \mathrm{O}^{1+}$ in Fig. 2(b).
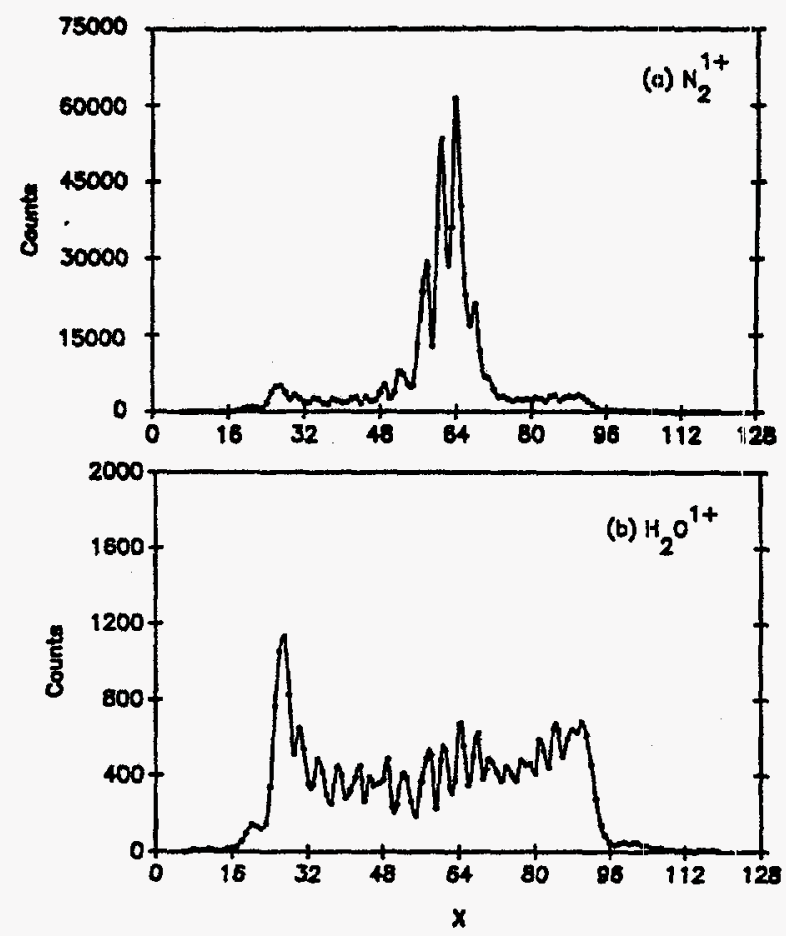

Fig. 2. Projections onto the $\mathrm{X}$ axis of (a) the $\mathrm{N}_{2}^{1+}$ position distribution and (b) the $\mathrm{H}_{2} \mathrm{O}^{1+}$ position distribution. 
The focusing effect encountered in the present experiments limited the analysis to $\mathrm{N}^{1+}$ and $\mathrm{N}^{2+}$ ions, for which the kinetic energy release was low enough that the peripheral region did not play a significant role. In order to assess the magnitude of any orientation effects, the position distributions were analyzed using a polar coordinate $(R, \theta)$ representation. The angles $\theta=45$ and 135 degrees were defined by the beam axis and $R=0$ was defined by the intersections of the beam and gas jet axes. The method of analysis consisted of comparing the $\mathbf{R}$ and $\theta$ projections of the data with detailed simulations computed with different values of the asymmetry parameter $\alpha$ for an assumed angular dependence $\mathrm{do} / \mathrm{d} \Omega=$ $k(1+\alpha \sin \theta)$, where $\theta$ is the angle between the molecular axis and the ion beam axis. These simulations required the ability to calculate accurate trajectories of ions through the ion-collection/TOF spectrometer system, which in turn required a detailed knowledge of the electrostatic field profile, the jet density distribution over the interaction region, the kinetic energy of the ions of interest, and the background. The electrostatic field profile was generated using the electrostatic lens program SIMION.4 The density distribution of the gas jet and the background were obtained from information provided by the position distributions for $\mathrm{N}_{2}^{1+}, \mathrm{H}_{2} \mathrm{O}^{1+}$, and $\mathrm{H}^{1+}$. The kinetic energy distributions were estimated from the results of previous

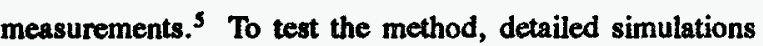
of the position distribution for isotropically emitted $\mathrm{N}_{2}^{1+}$ were performed. The experimental and simulated position distributions in $\mathbf{R}$ and $\boldsymbol{\theta}$ coordinates are shown for $\mathbf{N}_{2}^{1+}$ in Pig. 3.

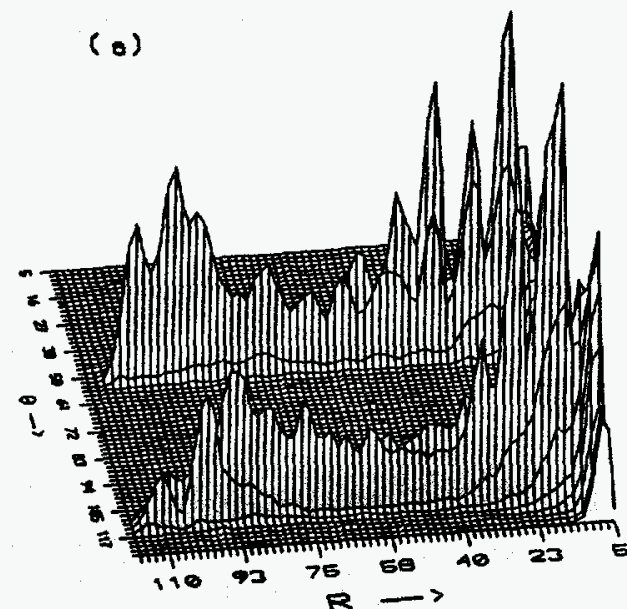

Although the $R$ and $\theta$ units shown in this figure are arbitrary, the two angular positions at which the $\mathbf{R}$ distributions are the broadest correspond to the beam axis (i.e. $\theta=45$ and 135 degrees).

It was finally concluded that the sensitivity of the method is insufficient to detect asymmetries of $\alpha<0.5$. In the present case of $\mathrm{N}^{2+}$, coming primarily from the dissociation of $\mathrm{N}_{2}^{3+}$ molecular ions produced in direct ionization collisions, it was determined that $\alpha<0.5$, and hence no evidence was found for an orientation dependence of the ionization cross section. This result is evidently at variance with the results obtained by Varghese, et al. ${ }^{6}$ via one-electron capture collisions with $19 \mathrm{MeV} \mathrm{F}^{9+}$ projectiles.

\section{References}

1. S. Cheng, C. L. Cocke, V. Frohne, E. Y. Kamber, and S. L. Varghese, Nucl. Instrum. Meth. B56, 78 (1991).

2. K. Wohrer and R. L. Watson, Progress in Research, 1990-1991, Cyclotron Institute, Texas A\&M University, p. 54.

3. O. Heber, R. L. Watson, and G. Sampoll, Nucl. Instrum. Meth. B56, 232 (1991).

4. D. A. Dahl and J. E. Delmore, Idaho National Engineering Laboratory report EGG-CD-7233 Rev. 1 (1987).

5. G. Sampoll, R. L. Watson, O. Heber, V. Horvat, K. Wohrer, and M. Chabot, Phys. Rev. A45, 2903 (1992).

6, S. L. Vargese, C. L. Cocke, S. Cheng, E. Y. Kamber, and V. Frohne, Nucl. Instrum. Meth. BAO, 266 (1989).

(b)

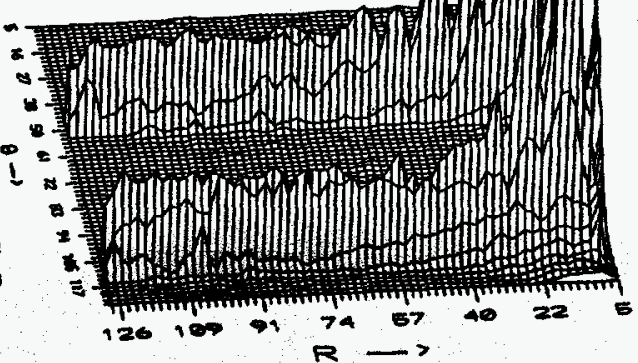




\title{
K-SHELL IONIZATION OF INTERMEDIATE Z ELEMENTS BY $30 \mathrm{MeV} / \mathrm{amu}$ $\mathrm{H}, \mathrm{N}, \mathrm{He}, \mathrm{AND}$ Ar IONS
}

\author{
V. Horvat, G. Sampoll, K. Wohrer, M. Chabot, and R. L. Watson
}

The mechanisms that contribute to the production of $\mathbf{K}$-shell vacancies in heavy ion-atom collisions are numerous, and their relative importance depends on the values of the many parameters that characterize the process. A universal theory covering all the possible parameter regimes still does not exist, but most of the available experimental data for low $Z$ projectiles, spanning a large range of relative velocities and target atomic numbers, are successfully described in a consistent way by the ECPSSR theory. This formulation utilizes the plane-wave Born approximation in treating direct $\mathbf{K}$-shell ionization ${ }^{1}$ and the OBK approach in calculating the contribution of $\mathrm{K}$-vacancies produced by non-radiative $\mathrm{K}$ electron capture to the projectile. ${ }^{2}$ In addition, it takes into account the Coulomb deflection of the projectile, the recoil of the target atom, the increased binding of the target K-shell electrons due to the projectile nuclear charge, and the polarization of the electrons in the target atom. The ECPSSR formulation is non-relativistic, but it incorporates corrections for relativistic effects.

While the ECPSSR theory has been rather thoroughly tested with low $Z$ projectiles below 10-MeV/amu, it is not known how well it works for intermediate and high energy heavy ions. One important benchmark has been established by Liatard, et $a{ }^{1} .{ }^{3}$ who recently performed measurements on solid targets ranging in $Z$ from 27 to 90 using 30-MeV/amu Ne and Ar projectiles. Their results, while yielding good overall agreement with the predictions of the CPSSR theory (an earlier version of the ECPSSR theory that did not take into account the recoil of the target atom), displayed small, but systematic deviations from the theoretical projectile and target $\mathbf{Z}$ dependences. They also revealed that $\mathbf{K}$-electron capture to the projectile contributed significantly to the total K-shell ionization cross sections for Ar.

The measurements described in this report were undertaken to further examine the projectile and target $Z$ dependences of $\mathrm{K}$-shell ionization at $30-\mathrm{MeV} / \mathrm{amu}$. Beams of $30-\mathrm{MeV} / \mathrm{amu} \mathrm{HD}{ }^{+}, \mathrm{N}^{5+}, \mathrm{Ne}^{7+}$, and $\mathrm{Ar}^{14+}$ were extracted from the Texas A\&M K500 superconducting cyclotron and focused to a spot size less than 1-mm-diam by viewing a $\mathrm{ZnS}$ phosphor mounted in the target holder with a television camera. A $2.5-\mathrm{cm}$-long carbon collimator with a 3-mm-diam opening was positioned 4.5$\mathrm{cm}$ in front of the target to prevent scattered beam particles from hitting the target and to limit changles in the detection solid angle due to movement of the beam. The beam intensity was regulated using three pairs of remotely adjustable slits, all located upstream from a bending magnet. The vacuum in the target chamber was maintained below $5 \times 10^{-5}$ - Torr.

The targets were mounted on a target wheel that was coupled to a remotely controlled stepping motor. All of the targets were self supporting, commercially available, $25-\mu$ m-thick metal foils except the Ge target, which was prepared by vacuum evaporation onto a $6-\mu \mathrm{rn}$ (1.6?$\mathrm{mg} / \mathrm{cm}^{2}$ ) thick Al foil. The nominal error in target thickness was $10 \%$ for the metal targets and $20 \%$ for the germanium target.

A Si(Li) detector was positioned at $90^{\circ}$ with respect to the beam axis and viewed the front surface of the target at an angle of $45^{\circ}$ relative to the surface normal through a $1.3 \times 10^{-3} \mathrm{-cm}$ thick beryllium window. The detectior cryostat was coupled directly to the target vacuum chamber with a distance of $2.5-\mathrm{cm}$ between the detector (Si) crystal and the target. The $x$-ray detector system gave an energy resolution of $260-\mathrm{eV}$ full width at half maximum (FWHM) at 6.4-keV.

A microchannel plate detector (MCPD) in Chevron configuration was placed $5-\mathrm{cm}$ behind the target directly in the beam path to count the beam particles in coincidence with the $K x$-rays. The MCPD signals were fed directly into a fast amplifier, without going through: a preamplifier. The main limitation on the beam intensity was imposed by the desire to maintain the counting rate in the $x$-ray spectrometer at or less than 1000-counts-pirsecond. As a consequence of the coincidence requirement, the background was reduced and the need to measure the efficiency of the MCPD was eliminated.

A FORTRAN program was written to calculate $K$ shell ionization cross sections using the ECPSSR theoretical formulation. The program runs on \& $P C(3 \& 16)$ and it gives the values of all the defined variables and allows for the exclusion of any number of effects from the calculations, so that it is possible to compare the results with all previously published calculations, including those based on the predecessors of the ECPSSR formulation, which took into account some, but not all of the effects included in the ECPSSR theory. The results of the program were checked by comparing them with the 
ECPSSR calculations of Lapicki in his compilation of measured and calculated values of the $\mathrm{K}$-shell ionization cross sections for systems involving hydrogen and helium ion projectiles.

The scaled experimental ionization cross sections $o_{K} / Z_{1}^{2}$ for $N$, Ne, and $A r$ projectiles divided by the cross sections for $H$ projectiles are shown in Fig. 1 . It is evident that the data display significant deviations from the simple Born approximation $Z_{1}^{2}$ scaling law for all the projectiles. The experimental data obtained in the present work are all larger than unity except those for $\mathrm{Al}$, indicating that a crossover to values less than unity occurs in the vicinity of $Z_{2}=13$. The cross sections of Liatard, et al. ${ }^{3}$ for $A r$ and Ne projectiles have been divided by the ECPSSR cross sections for protons and these ratios (included in Fig. 1) indicate that another crossover occurs in the vicinity of $Z_{2}=60$. The cross section ratios predicted by the ECPSSR calculations are shown by the dashed curves in Fig. 1. The agreement with the experimental ratios is within experimental error for all projectile/target combinations except $\mathrm{Ar}$ on $\mathrm{Al}$ and $\mathrm{Ar}$ on $Z_{2}$ from 26 to 40 .

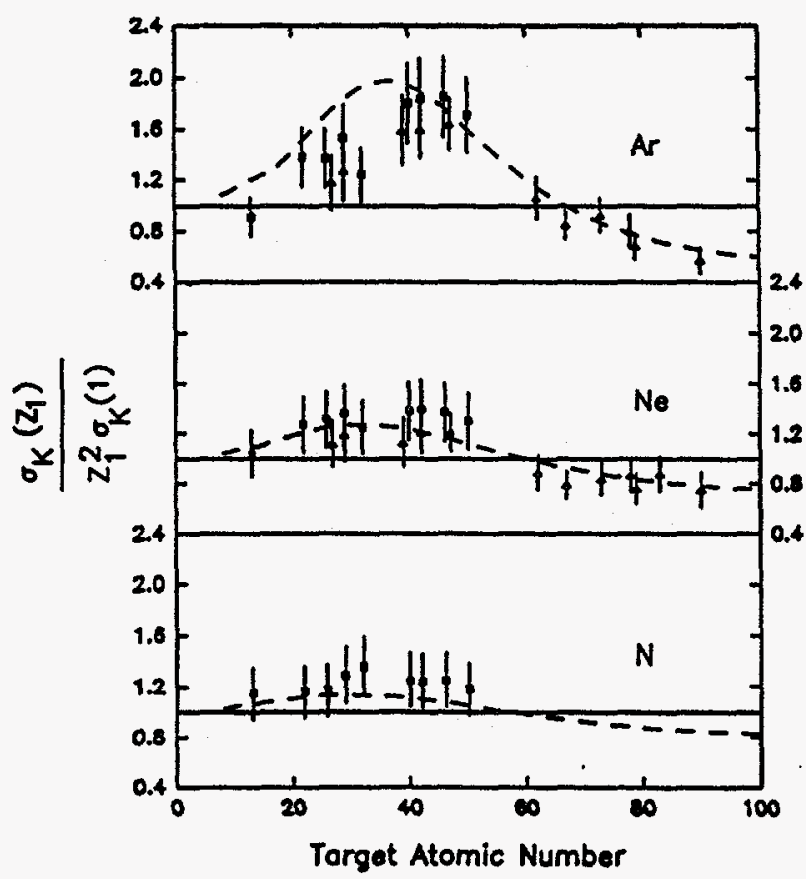

Fig. 1. Scaled experimental $\mathrm{K}$-shell ionization cross section $\sigma_{K} / Z_{1}^{2}$ divided by the cross section for protons as a function of the target atomic number for $\mathrm{Ar}, \mathrm{Ne}$, and $\mathrm{N}$ projectiles. The present results are shown by the squares. The cross sections of Liatard, et al. ${ }^{3}$ have been divided by proton cross sections calculated using the ECPSSR formulation and are shown by the triangles. The calculated (ECPSSR) ratios are shown by the dashed curves.
The reason for the Al deviation is not known at this time. It should be noted that increasing the fluorescence yield to correct for multiple $\mathbf{L}$-shell ionization causes the deduced cross sections to drop even further below the ECPSSR curve. The deviations around $Z_{2}=30$, however, can be explained by considering the relative contribution to the total target $\mathbf{K}$-shell ionization cross section of electron capture from the target $K$-shell to the projectile $\mathrm{K}$-shell [EC(K)]. As shown in Fig. 2, the (theoretical) relative $\mathrm{EC}(\mathrm{K})$ contribution is quite large in the vicinity of $Z=30$ and reaches its maximum value around $Z=40$. In the ECPSSR calculations, it was

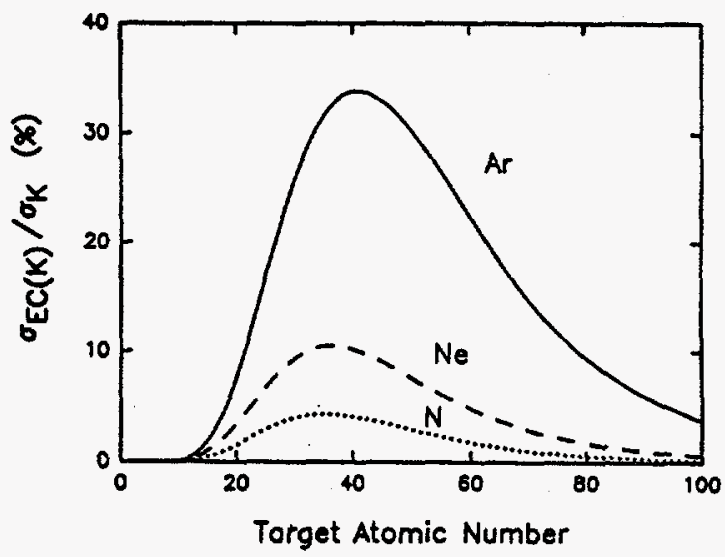

Fig. 2. Calculated relative contribution to the total Kshell ionization cross section of electron transfer from the target $\mathrm{K}$-shell to the projectile K-shell.

assumed that the projectile was fully stripped and, therefore, that all of the projectile $K$-shell states were available to capture K-electrons from the target. In reality, the average projectile charge changes from its initial value (e.g. 14+ in the case of $A r$ ) to its equilibrium value at some depth within the target. The charge distributions for $26 \mathrm{MeV} / \mathrm{amu} \mathrm{Ar}^{13+}$ and $\mathrm{Ar}^{18+}$ recently have been measured as a function of carbon target thickness by Awaya, et al . 5 These measurements show that although the average charge of the equilibrated beam is very close to $18+$, the carbon thickness required to reach equilibrium is $\approx 1$ to $5 \mathrm{mg} / \mathrm{cm}^{2}$. Moreover, in the case of $\mathrm{Ar}^{13+}$, the average charge changes from approximately 16.9 at $0.1 \mathrm{mg} / \mathrm{cm}^{2}$ to 17.9 at $0.5 \mathrm{mg} / \mathrm{cm}^{2}$. Therefore, unless the charge distributions are quite different for targets with $Z>6$, it must be concluded that the average charge of the Ar beam used in the present measurements was less than 18 over a significant fraction of the $x$-ray production path length. Under these conditions, the resulting $\mathrm{K}$-shell ionization cross sections would reflect the reduced contribution from $E C(K)$ due to the partial occupancy of the projectile K-levels, and hence 
the cross sections deduced from these measurements would be lower than those predicted by the ECPSSR calculations. The effect should be especially prominent for the Ge target, which was much thinner than the equilibration thickness. The cross section obtained for $\mathrm{Ge}$ does indeed display the largest deviation in Fig. 1, thereby providing strong support for the above hypothesis.

Reliable energy analysis of the hypersatellite peaks was limited by the $x$-ray detector resolution to the four highest-Z targets investigated. The energy difference between the peak containing the $\mathrm{K} \alpha$ hypersatellite lines and the peak containing the $\mathbf{K} \boldsymbol{\alpha}$ diagram plus satellite lines is shown for each of these four targets in Fig. 3. Under the assumptions that (a) the L-vacancy distribution is independent of the number of K-vacancies and (b) the shift per L-vacancy is approximately the same for $\mathbf{K} \alpha \mathbf{x}$-rays from the decay of single $K$-vacancy states as from the decay of double K-vacancy states, these energy differences may be compared with the hypersatellite shifts calculated by Chen, et al. ${ }^{6}$ which are shown by the solid line. The fact that the measured energy differences were independent of projectile $Z$ supports the validity of the above assumptions. Hypersatellite shifts determined in

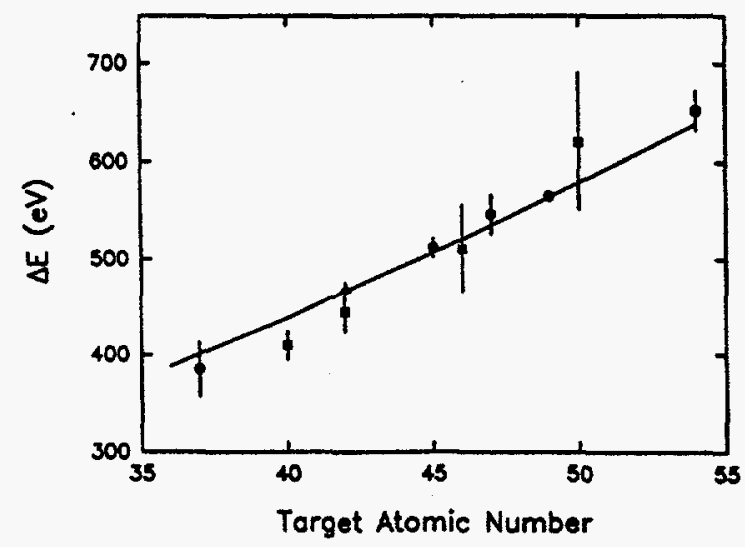

Fig. 3. Measured energy differences $\Delta \mathrm{E}$ between the peaks containing the $\mathrm{K} \alpha$ hypersatellite lines and the peaks containing the $K \alpha$ diagram plus satellite lines for the four highest $Z$ targets examined in the present work (squares). $\mathrm{K} \alpha$ hypersatellite energy shifts determined in measurements of $\mathrm{K}$ X-ray emission induced by electron bombardment ${ }^{7}$ and in radioactive decay ${ }^{8}$ are shown by the triangles and circles, respectively. Theoretical $\mathbf{K} \alpha$ hypersatellite shifts ${ }^{6}$ are shown by the solid line.

measurements of $\mathbf{K}$ x-ray emission induced by electron bombardment ${ }^{7}$ and by radioactive decay ${ }^{8}$ also are shown in Fig. 3. Within experimental error, the present hypersatellite shifts, which are the only ones in this region of $\mathbf{Z}$ that have been measured for $\mathrm{X}$-ray emission induced by heavy ion collisions, are in reasonably good agreement with the other experimental hypersatellite shifts and the theoretical predictions.

The ratios of the hypersatellite-to-satellite $x$-ray yields obtained for the $\mathrm{Zr}, \mathrm{Mo}, \mathrm{Pd}$, and Sn targets using $\mathrm{Ar}, \mathrm{Ne}$, and $\mathrm{N}$ projectiles are shown in Fig. 4. The ratios for a particular target approximately increase quadratically with projectile $Z$. This behavior is consistent with a twostep mechanism for double K-vacancy production, rather than a one-step (shakeoff) mechanism, for which the yield ratios would be expected to increase linearly with $Z_{1}$.

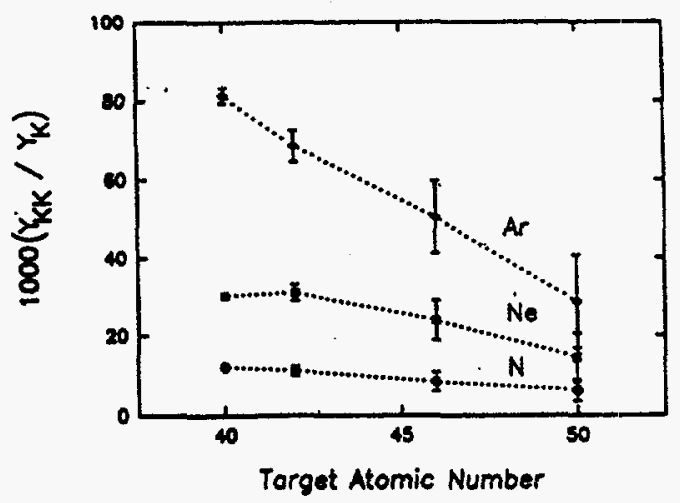

Fig. 4. Measured ratio of the $\mathrm{K} \alpha$ hypersatellite yield to $K \alpha$ diagram plus satellite yield as a function of atomic number for $\mathrm{Ar}, \mathrm{Ne}$, and $\mathrm{N}$ projectiles.

\section{REFERENCES}

1. W. Brandt and G. Lapicki, Phys.Rev. A23, 17:17 (1981).

2. G. Lapicki and F. D. McDaniel, Phys. Rev. A23, 975 (1981).

3. E. Liatard, J. F. Bruandet, F. Glasser, T. U. Chan, G. J. Costa, C. Gerardin, C. Heitz, M. Samri, and R. Selz, Z. Phys. D2, 223 (1986).

4. G. Lapicki, J. Phys. Chem. Ref. 18, 111 (1989).

5. Y. Awaya, T. Kambara, Y. Kanai, A. Hitachi, T. Mizogawa, and K. Kuroki, Nucl. Instrum. Methods B53, 375 (1991).

6. M. H. Chen, B. Crasemann, and H. Mark, Phys. Rev. A25, 391 (1982).

7. S. I. Salem, Phys. Rev. A21, 858 (1980).

8. G. Schupp and H. J. Nagy, Phys. Rev. C29, 1414 (1984) for $Z=37$; C. W. E. van Eijk, J. Wijnhorst, and M. A. Popelier, Phys. Rev. A20, 1749 (1979) for $Z=45$; V. Horvat and $K$. llakovac, Phys. Rev. A31, 1543 (1985) for $Z=47$; C. W. E. van Eijk, J. Wijnhorst, and M. A. Popelier, Phys. Rev. A24, 854 (1981) for $Z=49 ; K$. Ilakovac, $M$. Veskovic, V. Horvat, and S. Kaucic, Phys. Rev. A42, 3984 (1950) for $Z=54$. 


\section{Horvat, R. Paramaswaran, G. Sampoll, R. L. Watson, M. Barrett, B. Griffith, R. D. DuBois, (1) L. H. Toburen, (1) and D. Schneider(2)}

A parallel-plate, tandem-type, zero-degree electron spectrometer, together with a collision chamber (including two sets of four-jaw adjustable slits and a $1500 \mathrm{~L} / \mathrm{s}$ turbomolecular pumping system), on loan from Lawrence Livermore National Laboratory, is being used to study the spectra of electrons resulting from the interaction of highenergy multiply-charged ion beams from the K-500 cyclotron with gas and solid targets. A schematic diagram of the system is shown in Fig. 1. A channeltron has been adapted to the spectrometer to serve as the electron detector. A Faraday cup with a biased electron-suppresser ring provides normalization of the counting intervals during the spectrometer voltage scans. The gas cell is attached to a gas supply and control system that includes an automatic valve and a capacitance manometer. Precise alignment of the spectrometer, the gas cell, and the slits is achieved visually by means of a transit.

Operation of the spectrometer is controlled by a PCbased control and data acquisition system, described in Section V, p. 113 of this report. A simple electron gun was constructed to test the electron spectrometer system and to accurately calibrate the energy scale. In the calibration procedure, the voltages on the spectrometer and the electron gun were measured using a four-digit precision digital voltmeter. The spectrometer was

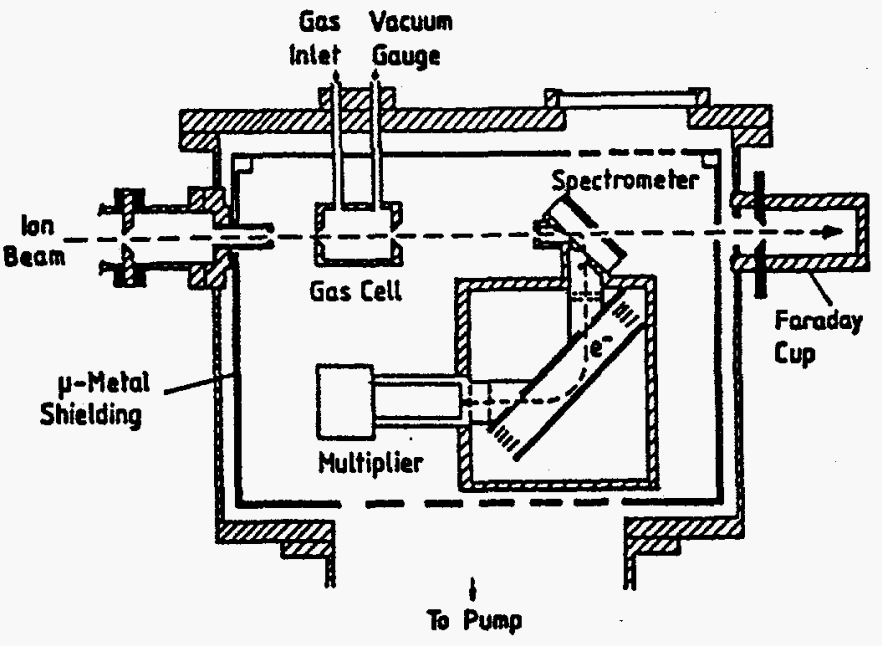

Fig. 1. Schematic diagram of the electron spectrometer system (taken from Ref. 1).

tested both in the low resolution (high transmission) mode and in the high resolution (low transmission) mode.
A typical spectrum of electrons from the electron gun measured in the high resolution mode is shown in Fig. 2. With $2 \mathrm{~mm}$ slits at the entrance and exit of the second stage of the electron spectrometer, the energy resolution was found to be $2.4 \%$ in the low resolution mode, and $2.4 \%$ of the pass energy in the high resolution mode.

The performance of the electron spectrometer system was tested in preliminary measurements of electrons from 8.05 MeV/amu $\mathrm{Ar}^{7+}$ projectiles interacting with an Ar gas target at a pressure of $\mathbf{4 0} \mathrm{mTorr}$. The spectrum of Auger electrons emitted from projectiles in the backward $\left(180^{\circ}\right)$ direction was recorded. The beam energy was accurately determined from the cusp electron spectrum and used to transform the Auger spectrum to the projectile rest frame.

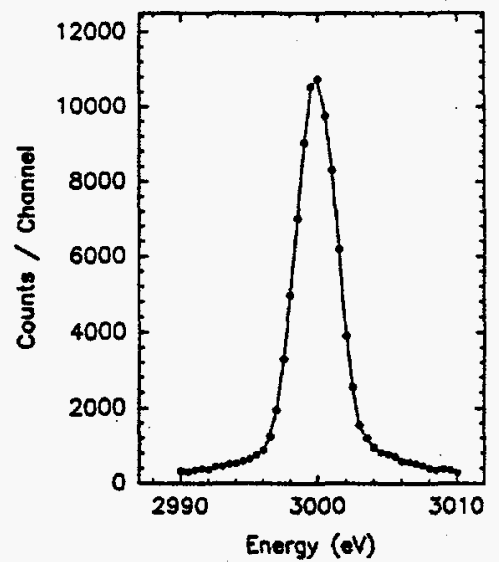

Fig. 2. Spectrum of electrons from the electron gun measured in high resolution mode with a pass energy of $100 \mathrm{eV}$.

The resulting spectrum is shown in Fig. 3. Four groups of peaks, labeled A, B, C, and D, are observable in this spectrum. To identify the transitions responsible for these peaks, the multiconfiguration Dirac-Fock program of Desclaux ${ }^{2}$ was employed to calculate the transition energies between the possible initial and final state configurations. Since the projectile electron configuration prior to the interaction with the target is predominantly $1 s^{2} 2 s^{2} 2 p^{6} 3 s$, L-Auger electrons from the projectile can be emitted only via the excitation of one (or more) of the Lshell electrons into one of the higher shells. On the basis of a comparison of the calculated transition energies with the measured peak energies, it was concluded that all of the observed groups correspond to transitions from initial 
states formed by $2 p \rightarrow 3 s$ (group $A$ ), $2 p \rightarrow 3 p$ (group B), $2 p \rightarrow 3 d$ (group C), and $2 p \rightarrow 4 d$ (group D) excitation, to the final state $\left(1 s^{2} 2 s^{2} 2 p^{6}\right)^{1} S_{0}$.

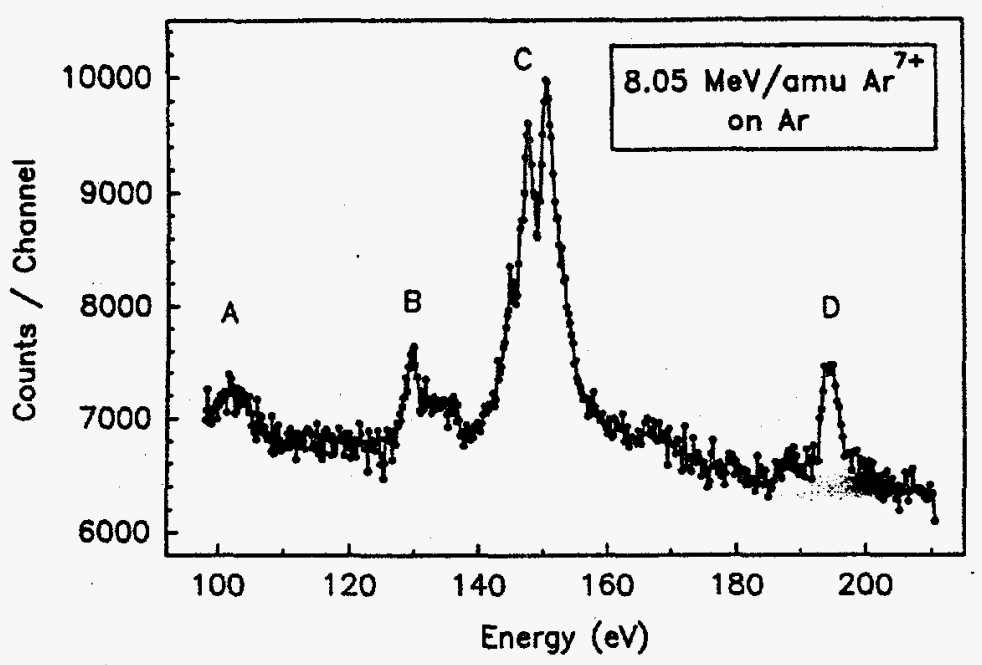

Fig. 3. Spectrum of L-Auger electrons from 8 $\mathrm{MeV} / \mathrm{amu} \mathrm{Ar}^{7+}$ measured at $180^{\circ}$.

The peak widths in the laboratory frame are larger than those in the projectile rest frame due to the kinematic stretching effect. ${ }^{3}$ This effectively enhances the resolution of the spectrometer. On the other hand, the lines are broadened due to the finite acceptance solid angle of the spectrometer and the variation of the laboratory energy with observation angle. The latter effoct dominated in the present case and hence transitions between individual multiplet states could not be fully resolved. The production cross section for the most prominent peak in group $\mathrm{C}$ was estimated to be of the order of $1 \mathrm{~kb}$.

(1) Collaborators from Battelle Pacific Northwest Laboratories, Richland, WA

(2) Collaborator from Lawrence Livermore Laboratory, Livermore, CA

\section{REFERENCES}

1. D. Bruch, N. Stolterfoht, S. Datz, P. D. Miller, P. L. Pepmiller, Y. Yamazaki, H. F. Krause, and J. K. Swenson, Phys. Rev. A35, 4114 (1987).

2. J. P. Descalux, Comp. Phys. Comm. 9, 31 (1975).

3. N. Stolterfoht, Phys. Rep. 146, 317 (1987).

\section{LIGHT EMISSION STUDIES WITH SLOW, HIGHLY-CHARGED HEAVY IONS}

\section{Assad and R. E. Tribble}

During this past year, we have continued our work on light emission from the interaction between multiplycharged heavy ions and surfaces with a study of the surface emission from $\mathrm{NaCl}$ targets. The groundwork and results of our previous work on a silver target are discussed in Ref. [1]. Most of the experimental setup and concepts are similar to that reported in Ref. [1]. However, there are some subtle differences, which makes the alkali-halide target a more interesting and difficult one to work with.

The main focus of these investigations has been to look for possible electronic contributions to the sputtering process and further to determine the mechanism by which ions neutralize as they approach and interact with the surface. Electron emission has been used to study the charge neutralization process on metallic targets as a function of ion charge for kinetic energies ranging from a few hundred eV to several keV.2.3 The yield of emitted electrons is observed to depend on both the kinetic energy and the potential energy of the incoming ion. The neutralization of the incoming ion as suggested from these experiments occurs through a transfer of charge from the surface atoms to the incoming ion via a series of resonsunt transfer or Auger transfers of electrons accompanied by Auger deexcitation in the surface and incident atoms.

Bombardment light emission, the process where light is emitted by surfaces bombarded with charged particles, was actively studied in the 1970 's but problems in interpreting the data frustrated attempts to fully understand the process. ${ }^{4}$ Within the past few years, Postawa, 스 $\mathbf{g i l .} .^{5}$ studied light emission by atoms sputtered from alkali halide targets for both electron and (singly charged) ion bombardment. They concluded that light emission resulting from heavy ion bombardment is die to the excitation of a sputtered atom as it leaves the surface. Tribble, Prior and Stokstad ${ }^{6}$ looked at light emission from $\mathrm{Na}$ atoms sputtered from a $\mathrm{NaCl}$ target with highly charged Ar ions. They observed no dependence of the light yield with the charge state of the incoming heavy ion from the sputtered atoms that decayed at least $0.5 \mathrm{~mm}$ 
from the target. They did observe an increase in the light yield with increasing charge state for atoms that decayed very near the surface of the target. However, the geometry used to view the light was optimized for studying light emission from sputtered atoms that drifted at least $0.5 \mathrm{~mm}$ from the surface before they decayed and not the light emitted by atoms that decayed closer than this to the surface.

The work reported in Refs. [5] and [6] focused on the light produced by sputtered atoms which depends on the sputtering yield and on the probability that a sputtered atom will be excited as it leaves the surface. For light emission from atoms sputtered off of an insulating surface, both the sputtering rate and the electronic excitation of the sputtered atom could depend, in principle, on the charge state of the incident ion. The sputtering yield could be increased by a direct electronic ejection of a surface atom - the postulated Coulomb explosion accompanying the passage and neutralization of an incident ion.? The probability that a sputtered ion will undergo an electronic excitation as it leaves the target could be affected by the status of the surface and in particular it could depend on whether the local charge depletion due to the neutralization of the incoming ion has itself been neutralized. Sputtering yields for metallic, ${ }^{8}$ semi-conducting 9 and insulating ${ }^{8}$ target materials have been measured with Ar beams of fixed energy but varying incident charge state within the past few years. The results for both metals and semi-conductors show no enhancement in the total sputtering yield with increasing ion charge. The results for insulators do indicate a slight enhancement in the total sputtering yield as the charge state of the incident ion is increased. However, the effect is small and it provides no direct evidence to support the Coulomb explosion mechanism as being important in the sputtering rate of neutral atoms. As we noted above, the results from Ref. [6] do not show any indication of an enhanced light yield with ion beam charge state from the alkali halide target. Thus it appears that neither of the two mechanisms that could enhance this yield are important.

Bombardment light emission from surface atoms that are displaced from the condensed phase lattice could be sensitive to the way that the potential energy of the highly charged incoming ion is dissipated by the target. In fact, the results from Ref. [6] suggest that there may be other mechanisms that could lead to enhanced light emission at or near the surface of the $\mathrm{NaCl}$ target. The nature of the light emitted from displaced surface atoms is likely to be correlated to the condition of the surface. We report below our results for the potential and kinetic energy dependence of the light yield to a discrete transition in neutral $\mathrm{Na}$ atoms induced by $\mathrm{Ar}$ ions on a $\mathrm{NaCl}$ target.

One of the advantages of the silver was its high electric conductivity. As the Ar ions from our Electron Cyclotron Resonance (ECR) ion source would approach and then embed themselves in the Ag target, their positive charge would very quickly dissipate to ground. ${ }^{10}$ This is of course not possible with an insulating target such as $\mathrm{NaCl}$. Ion impact on poorly conducting materials will generally result in charge buildup on the bombarded sample. The magnitude of the effect depends on such parameters as the resistivity of the sample, and the charge state, energy, and impact angle of the incident particle. Hence, the surface of the crystal charged up, which was destructive in two ways. First, the charging of the surface makes controlled bombardment of the sample no longer possible. Second, once the voltage reached high enough levels, sparks were produced between the surface and the grounded target holder. The latter flooded our spectrometer with a high photon flux over most of the visible electromagnetic spectrum, which in turn increased our background noise to an unacceptable level. We had to make several changes to our setup to counteract this problem.

In the developmental stages of the experiment, an electron flood gun aimed at the surface was initially chosen as the means by which the target would be neutralized." Low energy electrons ( $1 \mathrm{eV}-10 \mathrm{eV}$ ) would flood the target surface, canceling the positive charges. The gun's current intensity was to be controlled by a feedback system which would monitor the incoming Ar beam (via a grid system upstream, which is discussed later) and adjust the electron flux accordingly.

For this purpose, a model FRA-2X1-2 electron gun was purchased from Kimbal Physics. We built a power supply which provided several options for controlling the gun current thus allowing for the most flexible use of the gun. The low energy electron gun is a triode design. An acceleration grid enhances the beam current at low energies. The FRA-2X1-2 can produce modest beam currents at energies as low as $5 \mathrm{eV}$, and currents as high as $220 \times 10^{-6} \mathrm{~A}$ was tested in our vacuum system. Some of the data from the tests are presented in Fig. 1. We carried out a preliminary experiment with the $\mathrm{NaCl}$ target and the electron gun in place. Unfortunately, for the gun to be effective we needed a high gun filament current, which in turn produced enough light on the target so that the target assembly could easily be seen with the naked eye. We are primarily interested in the Sodium D lines, $3 \mathrm{p}\left({ }^{2} \mathrm{P}_{1 / 2}\right) \rightarrow 3 \mathrm{~s}\left(\mathrm{~S}_{1 / 2}\right)$ and $3 \mathrm{p}\left({ }^{2} \mathrm{P}_{3 / 2}\right) \rightarrow 3 \mathrm{~s}\left({ }^{2} \mathrm{~S}_{1 / 2}\right)(589.6 \mathrm{~nm}$ 
and $589.0 \mathrm{~nm}),{ }^{12}$ which matched closely the yellowish light emanating from the electron gun. Hence, no data
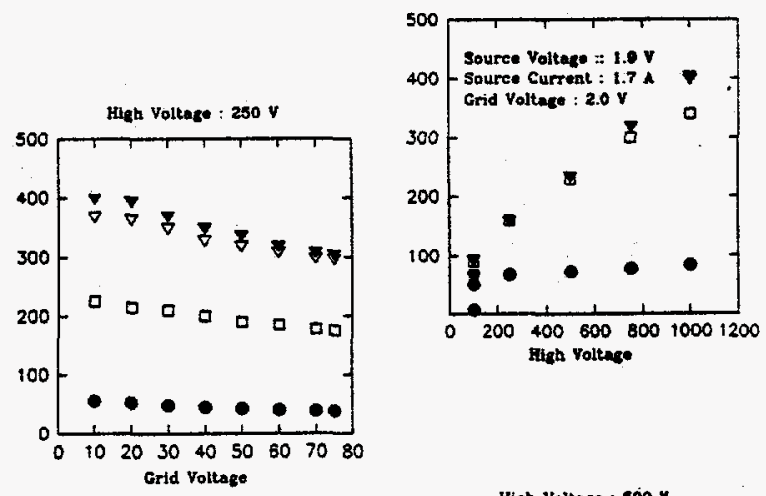

Souren Voltage $=1.0 \mathrm{~V}$ Source Current : 1.7 A
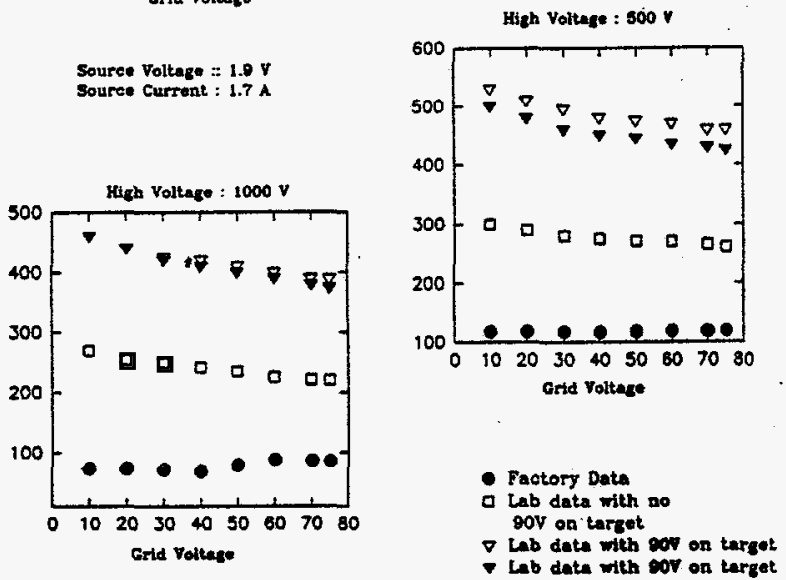

Fig. 1: Electron Gun yield as a function of various gun settings. The ordinates are expressed in $\mu \mathrm{A}$. The E-Gun uses triode design principles.

could have been taken under such conditions. However, an important observation emerged from that set of experiments. We noticed that no sparking was taking place even when the electron gun was turned off. Since the target assembly was heated to $350^{\circ} \mathrm{C}$, we found that heating the crystal not only stabilized the sputtering rate (the original reason why the target was heated ${ }^{13}$ ) but also increased the electric conductance ${ }^{14}$ as is shown in Fig. 2. Hence, we did not use the electron gun for the set of measurements discussed below. We do have some future uses for it, however, which we discuss below.

Our apparatus was setup in a general purpose UHV scattering chamber that is located on the beam line from ECR source for atomic and surface science experiments. A description of the beam line can be found in Ref. [15]. The light emission from the surface of the target was observed with a JARELL-ASH (Model 82-000) series Ebert Scanning Spectrometer which was coupled with a C31034 GaAs photomultiplier tube by Burle. The
C31034 is a low noise tube especially suited for single photon counting with a quantum efficiency that is optimized for wavelengths around $600 \mathrm{~nm}$. For even lower background noise, the tube was cooled to $-30^{\circ} \mathrm{C}$ with a solid state cooling device, which also needed external water cooling for better heat exchange. We used a $-5^{\circ} \mathrm{C}$ alcohol $+\mathrm{H}_{2} \mathrm{O}$ based solution circulating in the hest exchanger to provide the additional cooling. The phototube was operated with negative high voltage (-1750 VDC). Output signals from the anode were amplified by an Ortec Model 579 Fast Filter Amplifier and then sent to a PS Model 715 Timing Discriminator. The discriminator level was adjusted to be above the noise of the amplifier but below the voltage output level from the amplifier corresponding to a single photo-electron striking the photo-cathode. The discriminator output signals were sent through a logic converter and then counted by a Tennelec Model TC-532 Counter. The spectrometer was used in a mode where a single transition was observed at a fixed grating angle. The grating angle was adjusted manually 10 look at $589.3 \mathrm{~nm}$ wavelength (the middle of $\mathrm{Na} \mathrm{D}$ lines). The calibration on the JARREL-ASH spectrometer coupled with the phototube was done with a sodium vapor lamp. During the experiments the light sensitivity of the system was checked against a $100 \mathrm{~W}$ semi-opaque light bulb kept at $22.00 \mathrm{VDC}$. The light output of the bulb wals constant but very sensitive to changes as small as \pm 0.01 VDC.

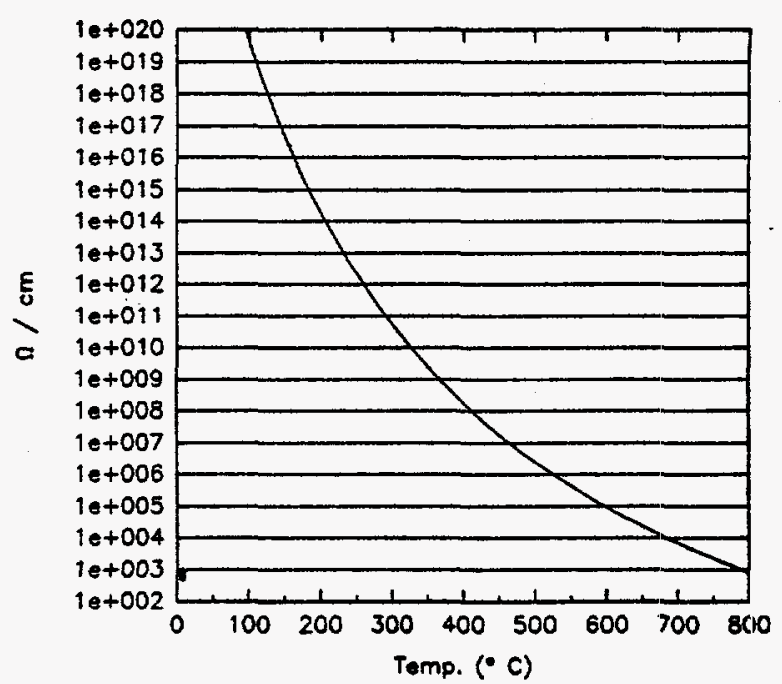

Fig. 2: The resistivity of $\mathrm{NaCl}$ erystal as a function of temperature. The ordinate is expressed in ohn per $\mathrm{cm}$, the abscissa in degrees $\mathrm{C}$.

A schematic view of the apparatus is shown in Fig. 3. The beam spot on the target was determined by a series of collimators. A $10 \mathrm{~mm}$ wide by $25 \mathrm{~mm}$ high aperture in the scattering chamber limited the acceptance 
into the photon spectrometer from the target location. The actual slit defining the entrance to the photon spectrometer was $1 \mathrm{~mm}$ wide by $14.5 \mathrm{~mm}$ high. The spectrometer was coupled to the vacuum chamber through a UHV flange with a quartz window. The photon spectrometer was aligned with the target by using a Neon laser in place of

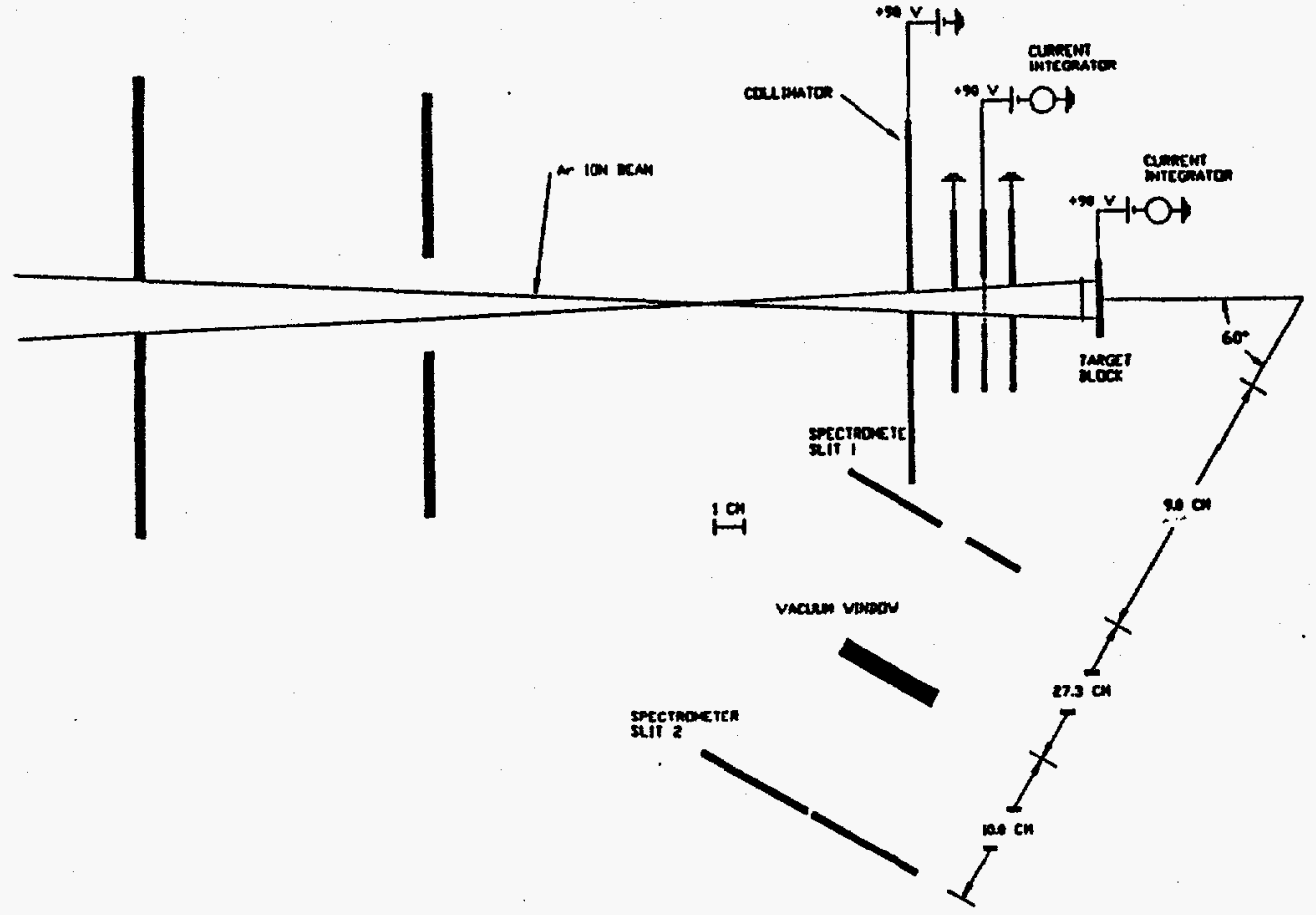

Fig. 3: Schematic of the experimental setup inside of the UHV scattering chamber.

the photomultiplier tube to illuminate the target. A small aperture was used at the quartz window to center the spectrometer optical axis on the vacuum flange. During data acquisition, the entrance and exit slits in the spectrometer were set to give a resolution of approximately $0.4 \mathrm{~nm}$.

$A 1 \mathrm{~cm} \times 1 \mathrm{~cm} \times 0.2 \mathrm{~cm} \mathrm{NaCl}$ target was mounted on a oxygen free high conductivity (OFHC) $\mathrm{Cu}$ target ladder. The target ladder was attached with a ceramic insulator to a linear motion feedthrough whose movement was perpendicular to the beam direction. A Faraday cup, made from a $1.5 \mathrm{~cm}$ diameter OFHC $\mathrm{Cu}$ nipple, was also attached to the target ladder to allow us to check the beam current integration. The entrance collimator and the target ladder were biased with +90 volts to keep secondary electrons produced by the beam from escaping. A ground plate with a $9.5 \mathrm{~mm}$ diameter hole electrically isolated a grid plate at +90 volts and the target ladder. An additional ground plate with a $9.5 \mathrm{~mm}$ diameter hole electrically separated the grid plate and the entrance collimator.

The beam current was monitored on the grid plate, both ground plates and the target ladder during data acquisition. The target, grid and ground plates currents were respectively digitized by an Ortec Model 439, a BIC Model $1000 \mathrm{C}$ and a BNI Current Digitizers. The output of the digitizers were counted by Tennelec Scalers. By comparing the beam current on the grid and the Faraday

$$
\text { (1) }
$$


study the potential energy dependence. In Fig. 4, we show the light yield as a function of charge state for incident beams of charges $4^{+}$to $11^{+}$. The relative yield for each data point has been normalized to the number of incident Ar atoms on the target. The overall scale used in the figure is arbitrary. The uncertainties for the data points were estimated to be at $8 \%$. This estimate includes effects of background, statistics, beam integration and geometry as well as other (surface) effects which we have found give rise to additional fluctuations in the light yield of around 5\%. It is clear from Fig. 4 that, contrary to the silver case, ${ }^{1}$ the light yield does change with increasing ionic charge state of the beam. We observe an increase in light yield of about 1.45 from $\mathrm{Ar}^{4+}$ to $\mathrm{Ar}^{11+}$. These results show reasonably good agreement with the results of Tribble, ex al. ${ }^{6}$ where they found an increase of a factor 1.30 from $\mathrm{Ar}^{4+}$ to $\mathrm{Ar}^{12+}$ with a geometry that was not optimized to view the surface of the crystal.

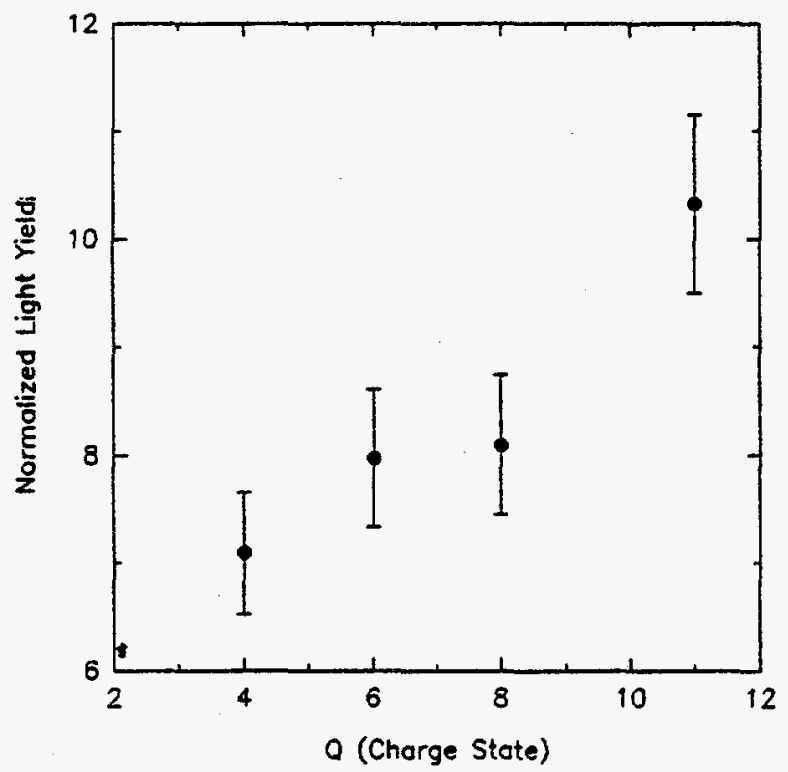

Fig. 4: $\mathrm{NaCl}$ light yield as a function of the incident ion potential energy. The normalization and uncertainties contributing to the error bars are described in the text. All of the incident beams had a fixed kinetic energy of $48 \mathrm{keV}$.

The kinetic energy dependence of the light yield was studied over a range of incident energies from $8 \mathrm{keV}$ to 99 keV. The results of the measurements as a function of incident energy are shown in Fig. 5. The data were taken with several different charge states as noted in the figure. We normalized the data such that only the kinetic energy dependence remained; to remove the potential energy dependence, all points were normalized with respect to the $\mathrm{Ar}^{8+}$ at $48 \mathrm{keV}$. The datum point for $\mathrm{Ar}^{8+}$ at $88 \mathrm{keV}$ had an anomalous set of readings on the current integrators and hence we do not believe that it is correct. Again contrary to the results for the Ag target where we observed nearly a factor of 10 increase in light yield over the same kinetic energy range, ${ }^{1}$ the light yield from the $\mathrm{NaCl}$ target is relatively flat.

Given this set of results, we would like to repeat the Alkali-halide experiment with a new approach. Tests are underway to make a $\mathrm{NaCl}$ target which would heat up

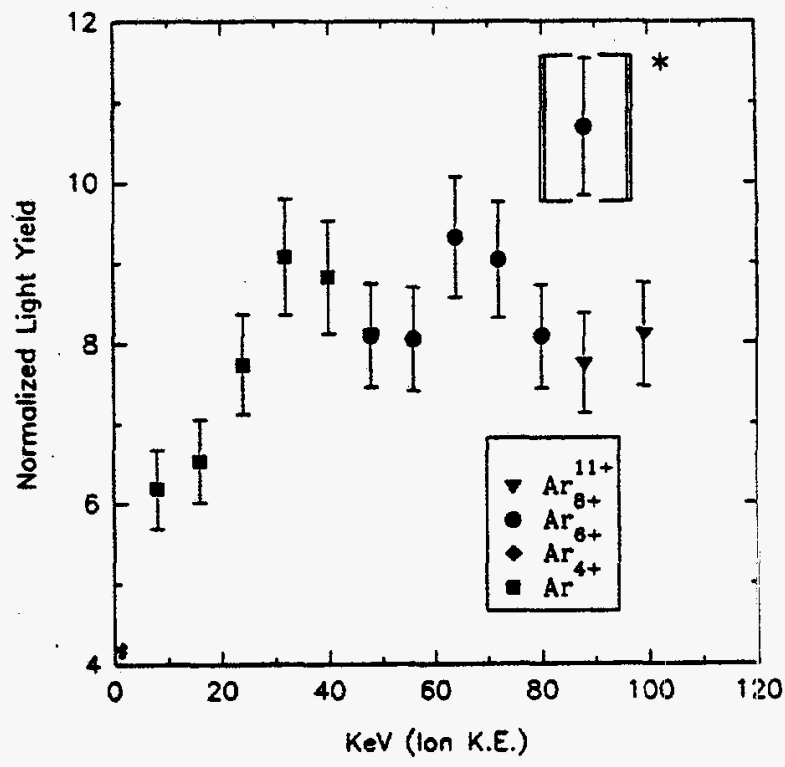

Fig. 5: $\mathrm{NaCl}$ light yield as a function of the incident isn kinetic energy. The normalization and uncertainties contributing to the error bars are described in the text. [ $]^{*}$ The $\mathrm{Ar}^{\beta+}$ datum point at $88 \mathrm{keV}$ had an anomalous set of readings as was noted in the text.

evenly and have a higher electrical conductivity. With these characteristics we hope to stabilize the target and minimize the unknown surface effects mentioned earlier. Initial attempts to grow a crystal around a copper grid have not been successful; the salt has a tendency to precipitate out of solution rather than forming a crystal around a seed. Recently we have melted $\mathrm{NaCl}$ and successfully solidified it around the copper grid. This technique needs some refinements, however the preliminary results look promising.

Next, we hope to repeat the silver and $\mathrm{NaCl}$ experiments, this time measuring the electron emission yield and energy spectrum. Ion-surface interaction studies by means of electron emission measurements have been of interest for many years. ${ }^{16}$ We already have an electron spectrometer, Model AC-902 from Comstock Inc. The double focusing electrostatic analyzer has an acceptance angle of 0.002 steradians, a resolution of $0.2 \%$ and a 
kinetic energy scan over a minimum range of 0 to $5 \mathrm{eV}$ to a maximum range of 0 to $140 \mathrm{eV}$.

\section{REFERENCES}

1. C. Assad, W. Liu and R. E. Tribble, Nucl. Instrum. Methods B62/2, 201 (1991).

2. F. W. Meyer, C. C. Havener, S. H. Overbury, K. J. Snowdon, D. M. Zehner, W. Heiland and $H$. Hemme, Nucl. Instrum. Methods B23, 234 (1987).

3. S. T. de Zwart, Nucl. Instrum. Methods B23, 239 (1987).

4. For a review of the subject, see G. E. Thomas, Surf. Sci. 90, 381 (1979).

5. Z. Postawa, J. Rutkowski, A. Poradzisz, P. Czuba and M. Szymonski, Nucl. Instrum. Methods B18, 574 (1987).

6. R. E. Tribble, M. H. Prior, and R. G. Stokstad, Nucl. Instrum. Methods B44, 412 (1990).

7. I. S. Bitenskii, M. N. Murakhmetov, and E. S. Parilis, Sov. Phys. Tech. Phys. 24, 618 (1979).
8. D. L. Weathers, T. A. Tombrello, M. H. Prior, R. G. Stokstad and R. E. Tribble, Nucl. Instrum. Methods B42, 307 (1989).

9. S. T. de Zwart, T. Fried, D. O. Boerma, R. Hoekstra, A. G. Drentje and A. L. Boers, Surf. Sci. 177, L939 (1986).

10. P. Varga, Applied Physics A44, 31 (1987).

11. H. W. Werner and A. E. Morgan, Jou. of App. Physics Vol. 47, No. 4, 1232 (1976).

12. P. A. Tipler, Modern Physics, Worth Publishers Inc., New York (1978), p. 270.

13. M. Szymonski, J. Rutkowski, A. Poradzisz, Z. Postawa and B. Jorgensen, Springer Series in Surface Science, Vol. 4, Springer-Verlag, Berlin, Heidelberg (1984) p. 160.

14. W. Lehfeldt, Z. Physik, 85, 717 (1933),

15. R. L. Watson, D. A. Church, R. E. Tribble, L. Yang, B. B. Bandong and T. Lotze, Nucl. Instrum. Methods B56/57, 223 (1991).

16. H. D. Hagstrum, Phys. Rev. 96, 325 \& 336 (1954); B. Baragiola, Radiat. Eff. 61, 47 (1982).

\title{
METASTABLE STATES OF HIGHLY-CHARGED ATOMIC IONS
}

\author{
D. A. Church and LiSheng Yang \\ Physics Department, Texas A\&M University
}

The capabilities of the Electron Cyclotron Resonance Ion Source at the TAMU Cyclotron Institute for production of intense beams of highly-charged ions are being used to study the mean lives of highly metastable electronic states of these ions. The ground states of many ions are usually composed of several terms with the same parity; electric dipole transitions between these terms are forbidden, although the term separations correspond to photon emission in or near the visible spectral region. These terms are generally populated in the ion source. Magnetic dipole (M1) or electric quadrupole (E2) transitions can occur, but with mean lives ranging from milliseconds to seconds. These transitions are observed in astrophysical and magnetic fusion plasmas; they can play a role in estimates of plasma densities and temperatures, as well as in identification of plasma components. The E2 transitions also remain a challenge to the best manyelectron theories.

To study these long-lived decays, beam ions are captured in an electrostatic (Kingdon) ion trap, in which they orbit stably for times exceeding the mean lives of the

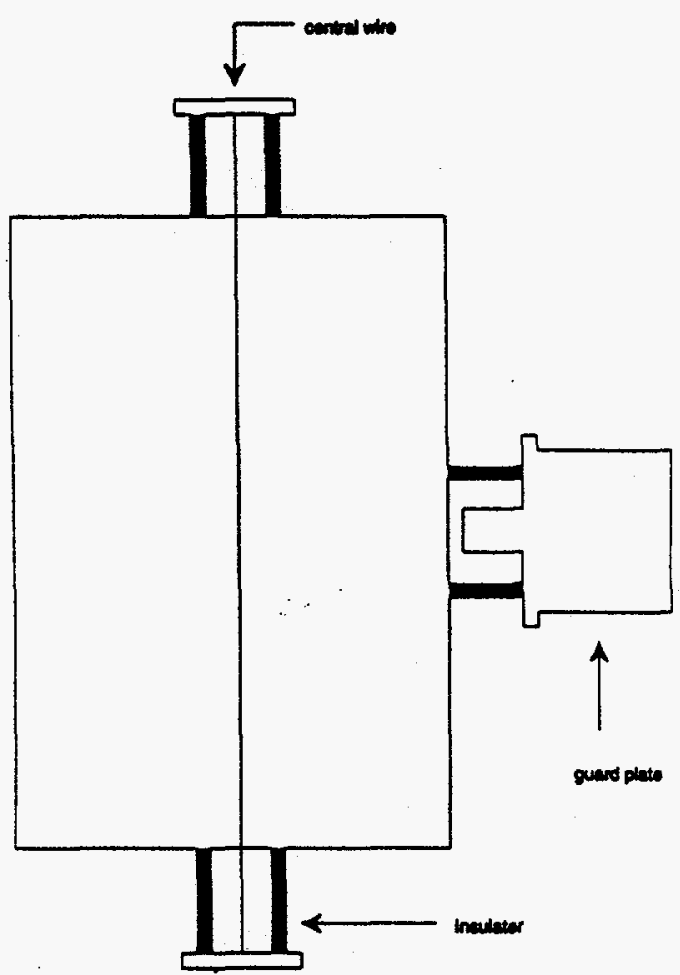

Fig. 1 
metastable states. Figure 1 shows a diagram of a trap, composed of a cylinder with a coaxial central wire at different potentials. Electrically biased end electrodes prevent axial escape by the ions. Beam ions are decelerated by the guard plate and focused through an aperture in the cylinder. Ions are captured when the potential difference between wire and cylinder is rapidly changed. Photons emitted by stored metastable ions are collected through another aperture in the cylinder (not shown) using a lens, wavelength analyzed with an interference filter, and counted by a photomultiplier. After several time constants the ions are dumped and the cycle repeated to acquire good statistics. An example of data for the $\mathrm{Ar}^{9+}$ ground state transition near $553 \mathrm{~nm}$ is shown in Fig. 2; the lifetime is near $10 \mathrm{~ms}$. Collisional quenching of the metastable state during ion confinement is studied by measuring the mean life vs. residual gas pressure. A plot of the decay rate vs. pressure extrapolated to zero pressure yields the natural decay rate.

Initial measurements have been focused on several argon ion charge states. Measurements on $\mathrm{Ar}^{2+}$ are in excellent agreement with results by other techniques. Our technique permits measurements on charge states which cannot be reached by other methods.

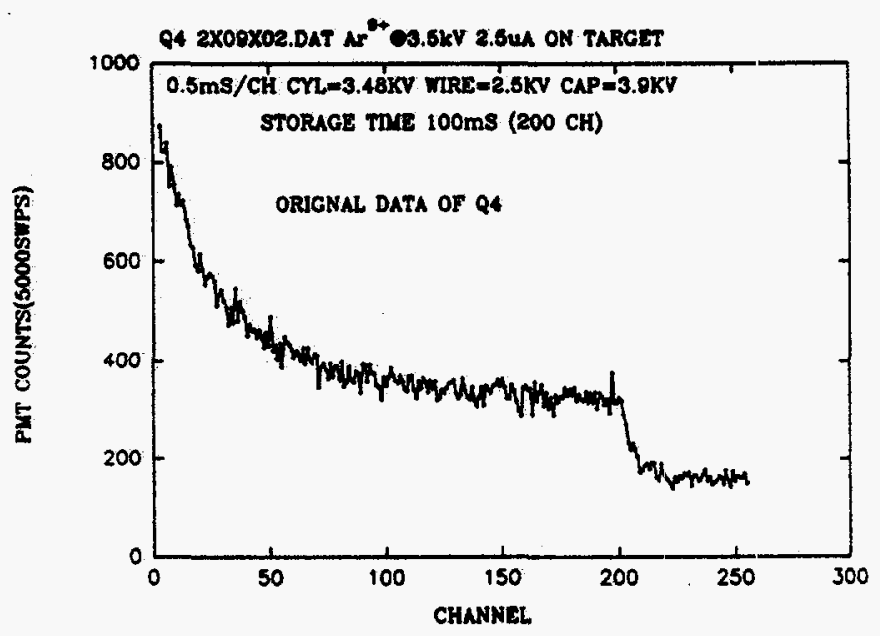

Fig. 2 


\section{SUPERCONDUCTING CYLOTRON AND INSTRUMENTATION}




\section{K500 OPERATIONS AND DEVELOPMENT}

D. P. May

There have been several significant developments in K500 operations in the last year. Two of these, the installation of a single cryopanel inside the " $\mathrm{C}$ " dee shell and the installation of a low-energy beam buncher in the axial injection line, have increased the intensity of beams dramatically. There has also been further K500 main-coil lowering, beam development in the lowest range of the $\mathrm{rf}$ frequency and ECR ion source development especially with solid beams.

\section{Buncher and Cryopanel}

The installation of these two devices was driven by the need to supply higher intensity $6 \mathrm{MeV} / \mathrm{amu}$ xenon beams. The buncher, described elsewhere in this report, was installed in August 1991. The cryopanel was installed in October 1991. The buncher multiplies the extracted beam by as much as factor of 5 with a factor of between 3 and 4 being typical. For the $6 \mathrm{MeV} / \mathrm{amu}$ xenon beam and other low energy beams, the factor is only about 2 . With the xenon beam, it is possible to see a significant rise in the $\mathrm{K500}$ vacuum pressure with more intense accelerated beam, but space-charge effects on the low-velocity beam coming from the ECR may be the main reason for the degradation. With a high intensity, high voltage ${ }^{16} \mathrm{O}^{5+}$ beam, the factor becomes around 2, and as the intensity is reduced, the factor increases with no discernible affect on the $\mathrm{K500}$ vacuum.

The cryopanel is one of an array of three slated to be installed in the three dee shells. It is designed with two circuits, one for liquid nitrogen and one for liquid helium, but initially it was tested by placing the two in series and running $\mathbf{L N}_{2}$ through both. As measured by an ion gauge near the \#1 turbomolecular pump, the pressure decreased from approximately $2 \times 10^{-7}$ to $4 \times 10^{-8}$ torr. Dewars for the $\mathbf{L N}_{2}$ and LHe must be lowered into the K500 pit and attached to the lines which run up the " $C$ " dee stem. Running with $\mathrm{LN}_{2}$ exclusively has become a common mode and a 160 liter $\mathrm{LN}_{2}$ dewar is generally sufficient for about 24 hours. Intensity for the $6 \mathrm{MeV} / \mathrm{n}^{129} \mathrm{Xe}$ beam has increased by a factor of 5 due to the better vacuum with $\mathbf{L N}_{2}$; this despite the fact that the pressure increases from $4 \times 10^{-8}$ to $8 \times 10^{-8}$ torr when approximately 200 na of the beam is accelerated.

\section{Main Coil Lowering}

As described in the last progress report, a small lowering of the superconducting main coil of the K500 has enabled the development of all the higher energy and higher field beams. This lowering, which is most probably a centering of the coil, was last tried in the successful development of a $65 \mathrm{MeV} / \mathrm{amu}$ deuteron beam in July of 1991 . To date, this beam would be the most sensitive to the centering of the main coil. It has now been lowered by a total of 0.135 inch since October of 1990. Further centering, if necessary, will occur with development of higher $\mathrm{MeV} / \mathrm{amu}$ beams.

\section{Low Frequency Beam Development}

The $65 \mathrm{MeV} / \mathrm{amu}$ deuteron beam is the highest $\mathrm{MeV} / \mathrm{amu}$ beam and the highest $\mathrm{rf}$ frequency (25.3 MHz) beam developed to date. In an effort to run the lowest energies possible on the second harmonic of the rf, the rf was tuned to the lowest frequency allowed by the positioning of the dee-stem sliding shorts. This proved to be $9.37 \mathrm{MHz}$ with which a $2.00 \mathrm{MeV} / \mathrm{amu}{ }^{40} \mathrm{Ar}^{3+}$ beam was developed.

\section{Solid Beams from the ECR Ion Source}

The first solid beam from the ECR was developed as a result of a request for a beam for atomic physics. A thin cylindrical rod of nickel supported on a stainless steel rod was introduced to the plasma through a port on the side. The support rod was fed through an o-ring, which did not cause a vacuum problem. Running with a low-pressure, oxygen-supported plasma, the rod needed repositioning after approximately 8 hours. When the source was run with a high pressure, helium-supported plasma in order to produce the lower charge-states, the rod needed to be repositioned every $1 / 2$ hour. When withdrawn, the rod proved to be melted into a rounded blob, with thin flakes of nickel deposited on the vacuum chamber wall near the rod feed.

The next solid beam developed used a thin rod of tantalum with an oxygen plasma. A stainless steel support proved inadequate in that it cooled the tantalum by evaporating into the source. With a tantalum support, however, a good charge-state-distribution of tantalum was 
produced. Three of the charge states were injected into the cyclotron and one was extracted at $6 \mathrm{MeV} / \mathrm{amu}$.

Finally, lead beams were developed in the ECR using the same rod feeds. This time a lead wire was mounted in a tantalum crucible. The lead evaporated into the source at a position which was much more withdrawn than the position with which tantalum was produced. Table I lists the ECR beams available at a source voltage of $10 \mathrm{kV}$.

Table 1.

ECR Beams at $10 \mathrm{kV}$

\begin{tabular}{|c|c|c|c|c|}
\hline Ion & Q & \multicolumn{2}{|c|}{ Intensity $(c \mu a)$} & Feeds \\
\hline \multirow[t]{2}{*}{${ }^{14} \mathrm{~N}$} & 5 & \multicolumn{2}{|c|}{46} & \multirow[t]{2}{*}{$\mathrm{N}_{2}+\mathrm{He}$} \\
\hline & 6 & 19 & & \\
\hline \multirow[t]{2}{*}{160} & 6 & \multicolumn{2}{|l|}{84} & \multirow{2}{*}{$\mathrm{O}_{2}+\mathrm{He}$} \\
\hline & 7 & \multicolumn{2}{|l|}{11} & \\
\hline \multirow[t]{7}{*}{${ }^{\infty} \mathrm{Ar}$} & 8 & \multicolumn{2}{|l|}{31} & \multirow[t]{7}{*}{$\mathrm{Ar}+\mathrm{O}_{2}$} \\
\hline & 9 & 25 & & \\
\hline & 11 & \multicolumn{2}{|l|}{12} & \\
\hline & 12 & \multicolumn{2}{|l|}{6.5} & \\
\hline & 13 & \multicolumn{2}{|l|}{2.0} & \\
\hline & 14 & \multicolumn{2}{|l|}{0.5} & \\
\hline & 16 & \multicolumn{2}{|c|}{0.006} & \\
\hline \multirow[t]{5}{*}{${ }^{58} \mathrm{Ni}$} & 13 & \multicolumn{2}{|l|}{7.5} & \multirow[t]{5}{*}{$\mathrm{Ni}+\mathrm{O}_{2}$} \\
\hline & 14 & \multicolumn{2}{|l|}{6.0} & \\
\hline & 15 & \multicolumn{2}{|l|}{4.0} & \\
\hline & 16 & \multicolumn{2}{|l|}{2.5} & \\
\hline & 17 & \multicolumn{2}{|l|}{1.5} & \\
\hline \multirow[t]{5}{*}{${ }^{181} \mathrm{Ta}$} & 24 & \multicolumn{2}{|l|}{5.7} & \multirow[t]{5}{*}{$\mathrm{Ta}+\mathrm{O}_{2}$} \\
\hline & 25 & \multicolumn{2}{|l|}{3.9} & \\
\hline & 26 & \multicolumn{2}{|l|}{2.6} & \\
\hline & 27 & \multicolumn{2}{|l|}{1.1} & \\
\hline & 28 & \multicolumn{2}{|l|}{0.5} & \\
\hline \multirow[t]{5}{*}{$\begin{array}{l}{ }^{200} \\
{ }^{200} \mathrm{~Pb}\end{array}$} & 27 & \multicolumn{2}{|c|}{3.0 (all isotopes) } & $\mathrm{Pb}+\mathrm{O}_{2}$ \\
\hline & 28 & 2.2 & $n$ & \\
\hline & 31 & 0.7 & $"$ & \\
\hline & 32 & 0.4 & $"$ & \\
\hline & 33 & 0.26 & $"$ & \\
\hline
\end{tabular}

Table 2 is a representative list of beams as of March 31, 1992. Those that are post-buncher are indicated.

Table 2.

Representative Beams Developed as of March 31, 1992

\begin{tabular}{|c|c|c|c|c|c|}
\hline Ion & $\begin{array}{c}\mathrm{E} / \mathrm{A} \\
(\mathrm{MeV} / \mathrm{n})\end{array}$ & $\begin{array}{c}\text { I } \\
\text { extracted } \\
\text { (ena) }\end{array}$ & Ion & $\underset{(\mathrm{MeV} / \mathrm{n})}{\mathrm{E} / \mathrm{A}}$ & $\begin{array}{c}\text { I } \\
\text { extracted } \\
\quad \text { (ena) }\end{array}$ \\
\hline $\mathbf{H}-\mathrm{H}^{+}$ & 53 & 5.5 & $\begin{array}{l}{ }^{20} \mathrm{Ne}^{4+} \\
{ }^{20} \mathrm{Ne}^{4+} \\
20 \mathrm{Ne}^{6+}\end{array}$ & $\begin{array}{l}14.5 \\
15 \\
20\end{array}$ & $\begin{array}{r}40 \\
20 \\
3\end{array}$ \\
\hline $\begin{array}{l}\mathrm{D}^{+} \\
\mathrm{D}^{+}\end{array}$ & $\begin{array}{l}52 \\
55\end{array}$ & $\begin{array}{r}5 \\
11\end{array}$ & ${ }^{20} \mathrm{Ne}^{7+}$ & 30 & 10 \\
\hline $\begin{array}{l}D^{+} \\
D^{+}\end{array}$ & $\begin{array}{l}60 \\
65\end{array}$ & $\begin{array}{l}25 \\
15\end{array}$ & ${ }^{27} \mathrm{Al}^{9+}$ & 30 & 140 \\
\hline $\mathrm{HD}^{+}$ & 30.5 & 72 & $32 S^{11+}$ & 30 & 6 \\
\hline $\mathrm{HD}^{+}$ & 35 & 15 & $\begin{array}{l}{ }^{\infty} \mathrm{Ar}^{3+} \\
{ }^{\infty} \mathrm{Ar}{ }^{4+}\end{array}$ & $\begin{array}{l}2.0 \\
2.4\end{array}$ & $\begin{array}{r}14 \mathrm{~B} \\
1.2\end{array}$ \\
\hline $\begin{array}{l}{ }^{4} \mathrm{He}^{+} \\
{ }^{4} \mathrm{He}^{+}\end{array}$ & $\begin{array}{l}15 \\
30\end{array}$ & $\begin{array}{r}600 \\
3\end{array}$ & $\begin{array}{l}{ }^{0} \mathrm{Ar}^{12+} \\
{ }^{0} \mathrm{Ar}^{12+}\end{array}$ & $\begin{array}{l}20 \\
30\end{array}$ & $\begin{array}{l}20 \\
65\end{array}$ \\
\hline${ }^{4} \mathrm{He}^{2+}$ & 53 & 28 & ${ }^{0} \mathrm{Ar}^{12+}$ & 35 & 25 \\
\hline $\begin{array}{l}{ }^{4} \mathrm{He}^{2+} \\
{ }^{4} \mathrm{He}^{2+}\end{array}$ & $\begin{array}{l}55 \\
56\end{array}$ & $\begin{array}{r}40 \\
2\end{array}$ & ${ }^{4} \mathrm{Ar}^{14+}$ & 40 & 3.5 \\
\hline${ }^{4} \mathrm{He}^{2+}$ & 65 & 15 & $\begin{array}{l}{ }^{63} \mathrm{Cu}^{13+} \\
{ }^{63} \mathrm{Cu}^{15+}\end{array}$ & $\begin{array}{l}10 \\
16.7\end{array}$ & $\begin{array}{l}4.5 \\
3.5\end{array}$ \\
\hline $\mathrm{H}-{ }^{4} \mathrm{He}^{+}$ & 9 & $3 \mathrm{~B}$ & $\begin{array}{l}{ }^{63} \mathrm{Cu}^{17+} \\
{ }^{63} \mathrm{Cu}^{18+}\end{array}$ & $\begin{array}{l}25 \\
30\end{array}$ & $\begin{array}{l}5 \\
1.5\end{array}$ \\
\hline $\begin{array}{l}{ }^{11} \mathrm{~B}^{+} \\
{ }^{10} \mathrm{~B}^{3+}\end{array}$ & $\begin{array}{l}2.5 \\
30\end{array}$ & $\begin{array}{l}6 \\
0.4\end{array}$ & ${ }^{63} \mathrm{Cu}^{19+}$ & 35 & 4 \\
\hline${ }^{12} C^{2+}$ & 5.4 & 1.0 & $\begin{array}{l}{ }^{84} \mathbf{K} \mathbf{r}^{11+} \\
{ }^{84} \mathbf{K} \mathbf{r}^{11+}\end{array}$ & $\begin{array}{l}5 \\
6\end{array}$ & $\begin{array}{r}4.5 \\
83 \mathrm{~B}\end{array}$ \\
\hline${ }^{12} C^{3+}$ & 15 & 2.5 & ${ }^{84} \mathrm{Kr}^{19+}$ & 15 & 0.5 \\
\hline${ }^{12} C^{3+}$ & 20 & 4 & & & \\
\hline${ }^{12} \mathrm{C}^{4+}$ & 30.6 & 2 & ${ }^{129} \mathrm{Xe}^{17+}$ & 6 & $59 \mathrm{~B}$ \\
\hline${ }^{12} C^{5+}$ & 50 & 3 & $\begin{array}{l}{ }^{129} \mathrm{Xe}^{18+} \\
{ }^{129} \mathrm{Xe}^{18+}\end{array}$ & $\begin{array}{l}5 \\
6\end{array}$ & $\begin{array}{l}2 \\
6\end{array}$ \\
\hline${ }^{14} \mathrm{~N}^{2+}$ & 5.4 & 14 & & & \\
\hline${ }^{14} N^{3+}$ & 10 & 1.9 & ${ }^{181} \mathrm{Ta}^{24+}$ & 6 & $21 \mathrm{~B}$ \\
\hline $\begin{array}{l}{ }^{14} N^{4+} \\
{ }_{14} N^{5+}\end{array}$ & 20 & 30 & & & \\
\hline $\begin{array}{l}{ }^{14} \mathrm{~N}^{5+} \\
{ }^{14} \mathrm{~N}^{5+}\end{array}$ & $\begin{array}{l}30 \\
35\end{array}$ & $\begin{array}{r}200 \\
90\end{array}$ & & & \\
\hline${ }^{14} \mathrm{~N}^{6+}$ & 40 & 7 & & & \\
\hline${ }^{14} \mathrm{~N}^{6+}$ & 43.4 & 5.7 & & \multirow{7}{*}{\multicolumn{2}{|c|}{$\mathrm{B}=$ buncher }} \\
\hline${ }^{16} \mathrm{O}^{3+}$ & 7.5 & 105 & & & \\
\hline${ }^{16} \mathrm{O}^{4+}$ & 15 & 2.5 & & & \\
\hline${ }^{16} \mathrm{O}^{4+}$ & 20 & 50 & & & \\
\hline${ }^{16} \mathrm{O}^{6+}$ & 30 & 40 & & & \\
\hline${ }^{16} \mathrm{O}^{6+}$ & 35 & 180 & & & \\
\hline${ }^{16} \mathrm{O}^{7+}$ & 45 & 5 & & & \\
\hline
\end{tabular}

\section{Operations}

For the period of April 1, 1991 to March 31, 199:2, the operational time is summarized in Table 3, while schedule time is listed in Table 4. 
Table 3

Operational Time

\begin{tabular}{|c|c|c|}
\hline & Hours & X Time \\
\hline Bean on Target & 2309 & 39.4 \\
\hline Tuning, Optics & 942 & 16.1 \\
\hline Beem Development & 832 & 14.2 \\
\hline Scheculed Maintenance & 736 & 12.6 \\
\hline Unscheduled Maintenance & 726 & 12.4 \\
\hline Idle Tine & 294 & 5.0 \\
\hline Cool Down, Transfer & 17 & 0.3 \\
\hline
\end{tabular}

Table 4.

Scheduled Beam Time

\begin{tabular}{lrr}
\hline & Hours & $\underline{\underline{X} \text { Time }}$ \\
Nuclear Physics & 1879 & 36.8 \\
Nuclear Chemistry & 1136 & 22.3 \\
Atomic Physics & 622 & 12.2 \\
Outside Collaborations & 456 & 8.9 \\
Outside Users & 176 & 3.5 \\
Beam Development & 832 & 16.3 \\
\hline
\end{tabular}

\title{
K500 Beam Energy Resolution
}

\author{
Y.-W. Lui, D. H. Youngblood, W. Liu, H. M. Xu, and D.P. May
}

The energy resolution of the beam from the $\mathrm{K500}$ cyclotron has been measured using the Enge split-pole spectrograph. A $490 \mathrm{MeV}^{14} \mathrm{~N}$ beam was delivered to the split pole spectrograph for studies of elastic and inelastic scattering on several targets. For these experiments the beam was focused to an approximately $1.5 \mathrm{~mm}$ wide by 3 mm high spot in the target chamber, and slits were used before and after a 30 degree bend to restrict the beam phase space and improve energy resolution. The solid angle defining slits were set at $1 / 2^{\circ}$ horizontally and the scattered ${ }^{14} \mathrm{~N}$ ions were detected in a hybrid detector consisting of a vertical drift chamber for position and angle measurement, an ionization chamber for energy loss and a scintillator for total energy and timing information. The detector position resolution was $.41 \mathrm{~mm}$ (150 keV) and anglular resolution was $0.22^{\circ}$. Excellent rejection for all ions other than ${ }^{14} \mathrm{~N}$ was obtained.

A spectrum taken under these conditions is shown in

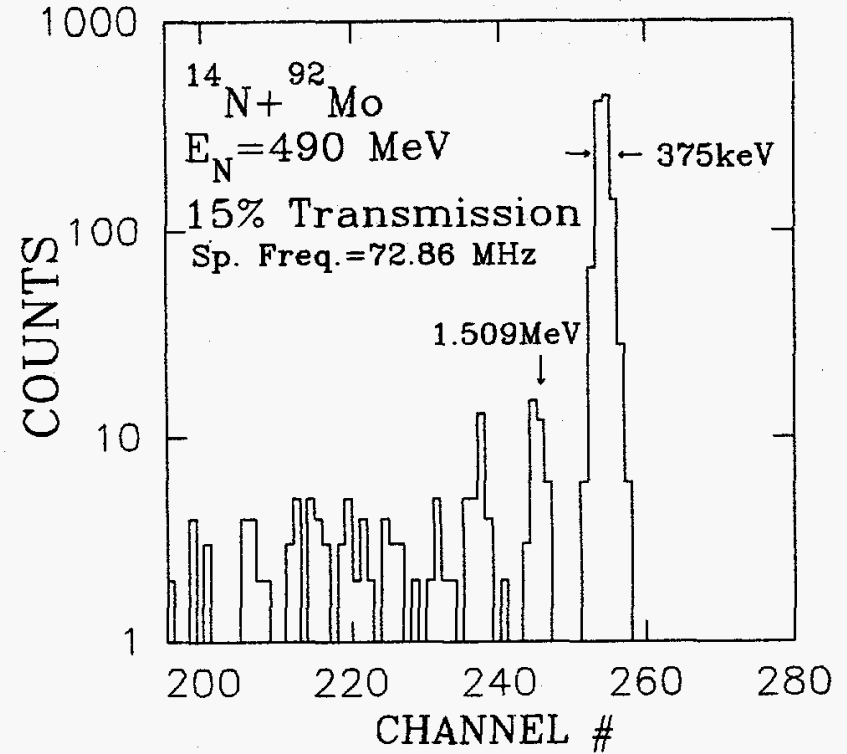

Fig. 1. Position spectrum of run 691026
Fig. 1 and the width (FWHM) of the elastic peak is 376 $\pm 20 \mathrm{keV}$. In addition to beam energy resolution, other contributions are detector (150 keV), spot width (180 $\mathrm{keV}$ ) and target thickness (50 KeV). This results in a beam energy spread contribution of $290 \mathrm{keV}$ (.06\%). Approximately $15 \%$ of the beam was transported from the cyclotron to the target.

The slits were then opened and the beam refocused to provide approximately $95 \%$ transmission of beam from the K500 cyclotron to the target. The beam spot increased horizontally to approximately $3 \mathrm{~mm}$ across. A spectrum taken under these conditions is shown in Fig. 2 and shows an overall energy resolution of $785 \pm 36 \mathrm{keV}$. After corrections for detector resolution, beam spot size, and straggling in the target, the beam energy spread contribution was $675 \mathrm{keV}$. This corresponds to $0.14 \%$ energy resolution for the $490 \mathrm{MeV}^{14} \mathrm{~N}$ ions from the cyclotron.

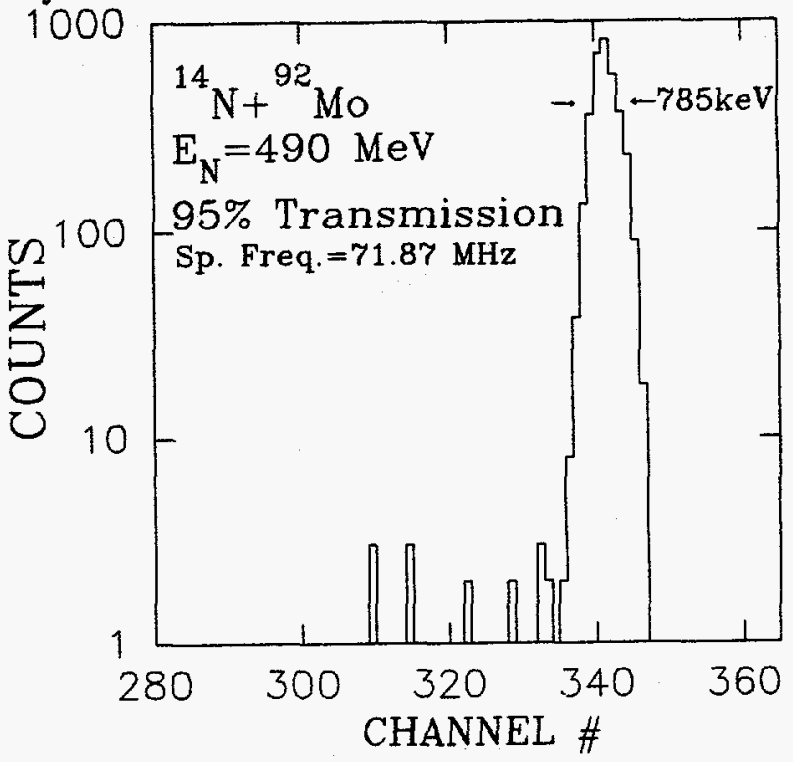

Fig. 2. Position spectrum of run 691065 


\section{INJECTION LINE BUNCHER}

\section{F. P. Abegglen and R. C. Rogers}

A buncher structure was added to the low energy injection line that couples the ECRIS to the K500 cyclotron (Fig. 1). The basic mechanical design of the buncher, shown in Fig. 2, follows that of the buncher at the National Superconducting Cyclotron Laboratory. The most notable change is the use of equal size fieldcorrecting cones at the entrance and exit to the buncher grids. This reflects the intent to use only a single driving waveform rather than first and second harmonic sinusoidal waveforms.

Figure 3 is a block diagram of the buncher electronic circuitry. At the present time, a sinusoidal waveform is being used to drive both grids of the buncher in a biphase

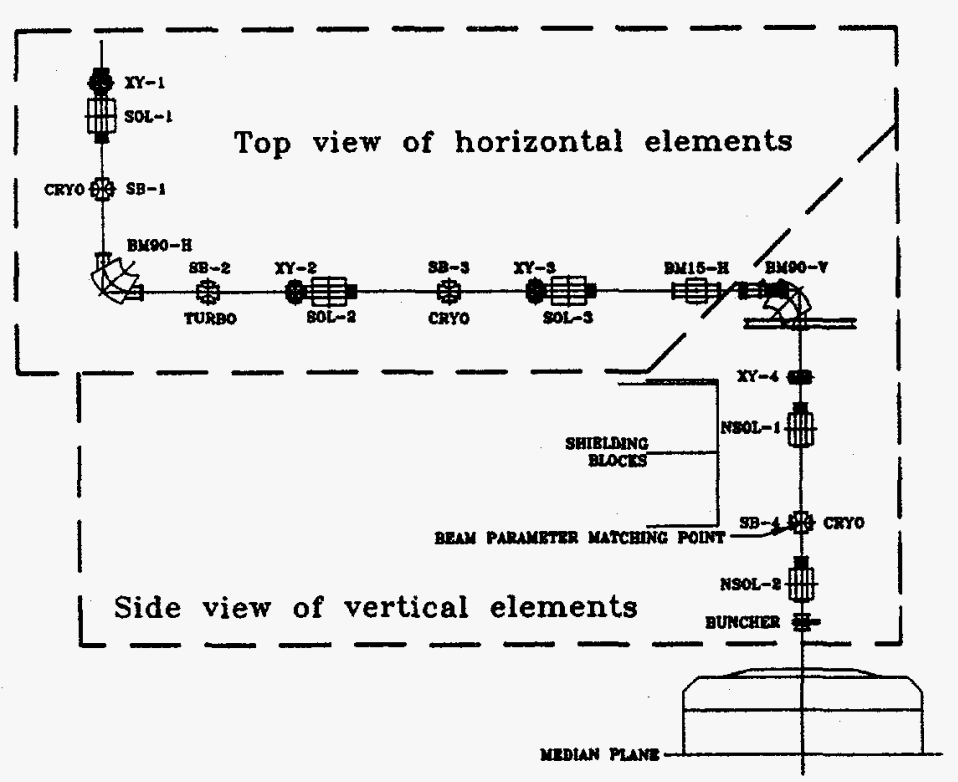

Fig. 1. Schematic view of injection line showing major elements and location of buncher. mode. This driving technique reduces the required drive power by a factor of two as well as reducing the effects of the input and output fields. The two $50 \mathrm{ohm}$ water cooled loads, shown in Figs. 2 and 3, provide a suitable load at all frequencies so that no tuning of the buncher grids is required.

The driving amplifier is a modified ENI model A-300 which provides the necessary drive power to each grid. The maximum power required is approximately 400 walts per grid. This power demand is determined by the distance of the buncher from the median plane of the cyclotron and the required injection velocity. The buncher give the usual gain of 3-5 in beam intensity.

Fig. 2. Cut-away drawing of buncher structure.
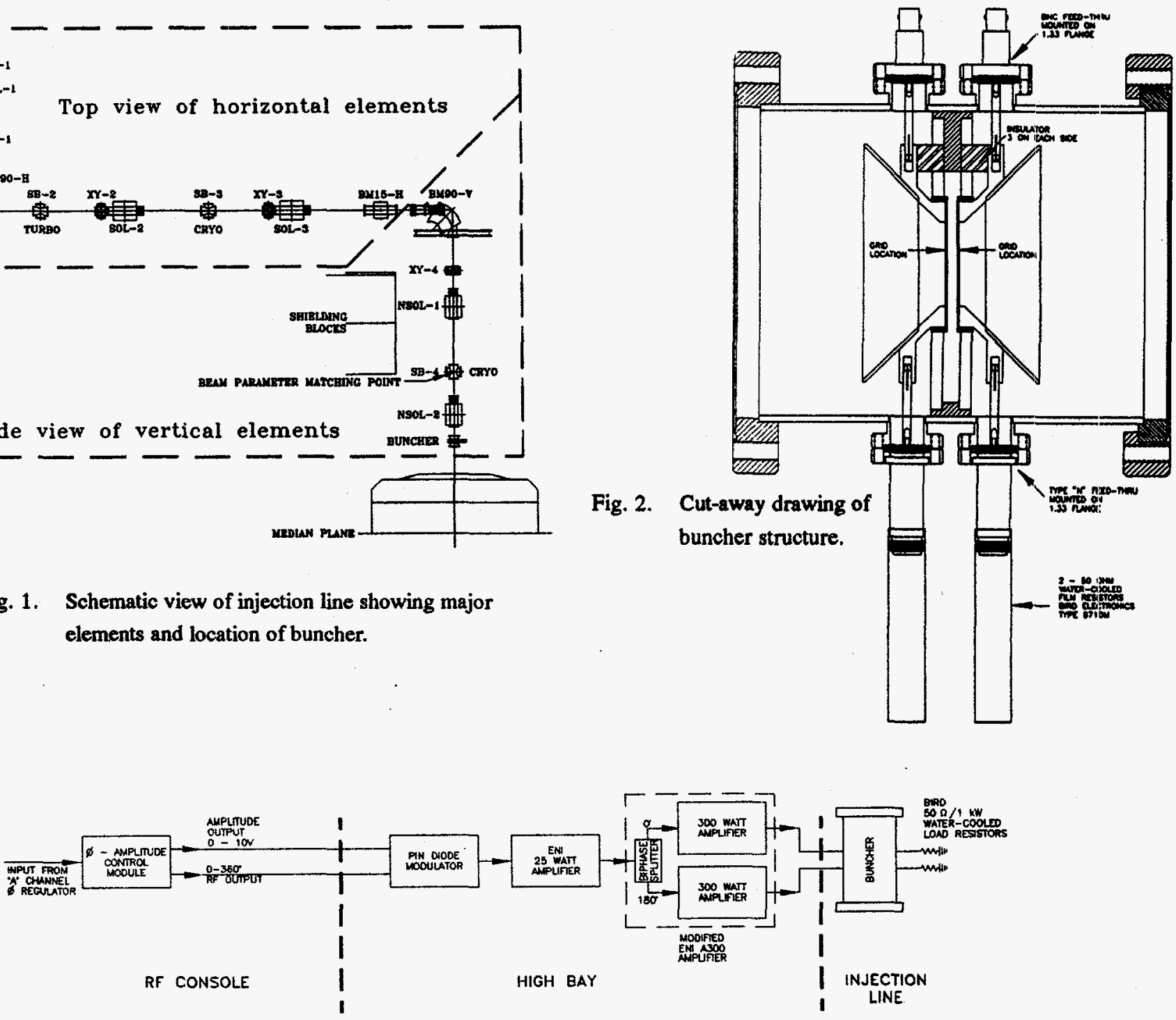

Fig. 3. Block diagram of buncher electronics. 


\section{G. Mouchaty, R. C. Rogers, and D. P. May}

As discussed earlier, ${ }^{1}$ the Texas A\&M E.C.R.I.S. will be upgraded in the near future. Our laboratory is a production one and minimal time is available for system research and development. Therefore only well established ideas have been incorporated in the actual source. ${ }^{2}$ This orthodoxy resulted in a stable source with an excellent production and reliability record. ${ }^{3}$ However, keeping cost effectiveness in mind and following the rapidly evolving body of E.C.R.I.S. science, makes minimal experimentation unavoidable.

Among the multitude of questions that have to be addressed when designing the upgrade of the source is the necessity of a first stage. And, if at all, what should replace it. Experiments with biased plates ${ }^{4,5}$ and with an electron gun ${ }^{6}$ have shown substantial increase of the output of the source when these devices were used with a main stage only. We have tested a $3.5 \mathrm{~cm}$ diameter copper plate in our source and found a substantial shift towards higher charge states and higher currents. Figure 1 shows the currents of the different charge states for an oxygen-helium plasma as a function of the plate bias. The currents were measured on a plate intercepting the beam after a $90^{\circ}$ analysis magnet. Due to budget and time limitations, these results were obtained using a battery to provide the bias. The battery degraded with time and allowed measurements at increasingly lower voltages. The data dictates further experimentation with a voltage regulated power supply. An effort is underway to understand these data and infer the appropriate conclusions for the source upgrade. ${ }^{6}$

\section{REFERENCES}

1. G. Mouchaty, The Texas A\&M E.C.R.I.S. Design and Justification Unpublished Report (1986). See also G. Mouchaty, The Texas A\&M E.C.R.I.S., An Alternative First Stage Unpublished report (1986).

2. D. P. May, et al., International conference on ECR Ion Sources and Their Applications, East Lansing Michigan (1987), p. 364.

3. G. Mouchaty, et al., To be published (Vancouver meeting 1992).

4. S. Gammino, et al., 4th International Conference on Ion Sources ICIS 91, Bensheim, Germany (1991).

5. G. Melin, et al., 10th International Workshop on ECR Ion Sources (1990), p. 17.

6. C. Lyneis, et al., 10th International Workshop on ECR Ion Sources (190), p. 47.

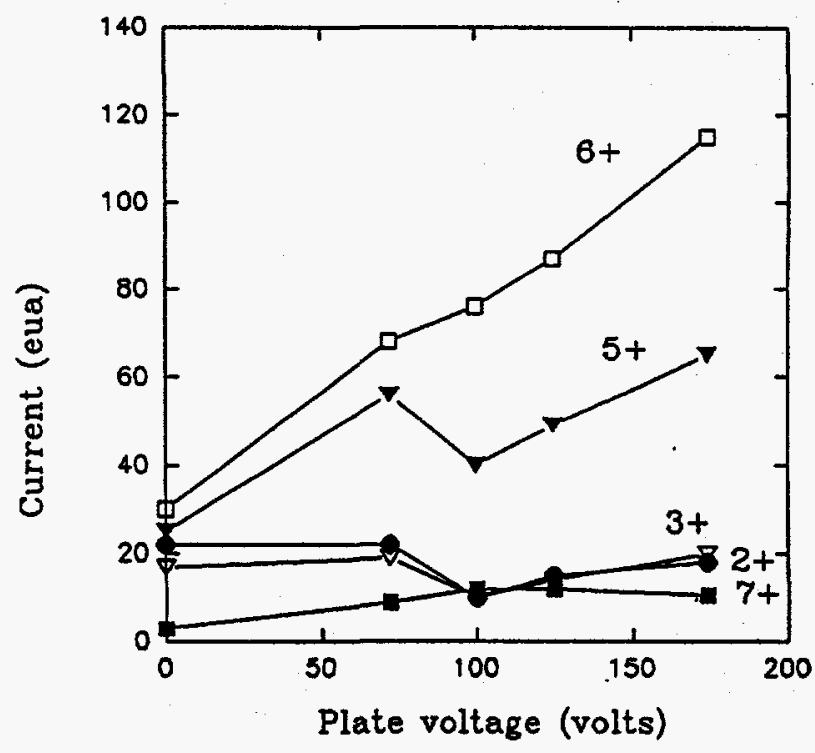

Fig. 1 Different charge state currents measured on a plate intercepting the beam after a $90^{\circ}$ analysis magnet as a function of the plate voltage. 


\section{COMPUTING FACILITIES AT THE CYCLOTRON INSTITUTE}

\section{K. Hagel and H. Dejbakhsh}

The first installment of a three year grant to upgrade the computer system in the Cyclotron Institute coupled with the failure of the VAX 11/780 which had been the backbone of our computing system enabled (forced) us to change our computer environment from essentially a single central computer to a VAXCluster environment. The increases in technology since the debut of the VAX $11 / 780$ in late 1979 mean that the slowest computer in our 4 computer cluster is a mere 3 times faster than the 780 . We have also in the past year continued our journey into the unix world with the purchase of two DECstation $5000 / 200$ 's.

Figure 1 depicts the computers system currently in the Cyclotron Institute. Systems not discussed in this report have been purchased in previous years.

Our VAXCluster now contains one VAXstation 3100 model 76, one VAXstation 3100 model 38 , one VAXstation 3100 model 30 and one VAXstation 3520. Three of these machines were basically inherited by the cyclotron from a group which has departed, and the VAXstation 3100/76 was purchased with the computer upgrade fund. The VAXstation 3520 is the boot node for the cluster and acts as the file server for the system. Two Kennedy 9400 nine track tape drives in addition to the system and user disks are connected to the bootnode. The other VAXstation 3100's which had already existed were each upgraded to have $1 \mathrm{~GB}$ of disk scratch space connected locally and one $8 \mathrm{~mm}$ tape drive. The VAXstation $3100 / 76$ contains $32 \mathrm{MB}$ of main memory, and the other two VAXstation 3100's contain $16 \mathrm{MB}$ of main memory. The VAXstation 3520 has only $8 \mathrm{MB}$ of memory which limits its performance severely but due to the price structure of DEC memory, a memory upgrade is not cost effective. The "COMP" cluster, which is now the main backbone of our computer system, is equivalent to $18 \mathrm{VAX} 11 / 780$ 's.

One other VAXstation 3100/76 was purchased with the computer upgrade fund. This system contains $16 \mathrm{MB}$ of main memory, $1 \mathrm{~GB}$ of disk space and an $8 \mathrm{~mm}$ tape drive and was added to another cluster which had been removed from the main computer system in order to isolate the intensive work of that cluster from the rest of the computer system.

The VAXCluster environment enables all computers to be easily managed from a single node. In addition, almost all peripherals except tape drives are shared across the cluster. This means that users can $\log$ onto arly machine in the cluster and everything except the speed of the machine will be transparent to this user. In order to avoid a large amount of network activity, it is, in fact, necessary to educate the general user to use disics connected to the computer which he is logged onto if a large amount of data is to be transferred from that disk. This has proven to date not to be a problem.

All of the computers in the building are connected via Ethernet. Two terminal servers which are connected to the Ethernet have also been purchased to add to the one that we already had. All terminals lines in the building which had previously been connected directly into the VAX 11/780 are now connected to the terminal server. This enables the efficient connection of a given terminal to any computer at the Cyclotron or on the Texas A\&M campus.

In addition to terminals connected to the terminial server, we also added two Hewlett Packard Laser printers (HP LaserJet IIISi's) connected to the termingl server. Although the cluster environment allows for the printers to be accessed across the cluster even though they are connected directly to one of the computers, being connected to the terminal server allows these printers to be accessed by any computer on the network. Each of the HP LaserJet IIISi's have the PostScript option which can be turned on in software mode.

The unix systems we have purchased consist of two DECstation 5000/200's, one configured with $32 \mathrm{MB}$ of main memory, and the other configured with $16 \mathrm{MB}$ of main memory. The one with $32 \mathrm{MB}$ of memory acts as a server for the other and will be the main server for any other unix machines that we may purchase in the future. Each of these systems has an $8 \mathrm{~mm}$ tape drive connectiod locally. One GB of disk space is currently available on these systems.

At present we are in the second year of our three year computer upgrade. This year we will purchase several more workstations as well as a color postscript printer. Network capabilities will also be upgraded.

Besides the major upgrade of the computing system at the Cyclotron, we have implemented the first phase of the data acquisition and network upgrades. The MicroVAX CPU, one of the two main data acquisitions systems at the Cyclotron, has been upgraded to a VAXStation 3200 CPU. The memory also has been 
replaced and increased to $16 \mathrm{Mbytes}$ for this system. The Network adapter for this computer was one of the early Ethernet adapters and must be replaced to allow the system to function with future DECnet versions. The disk space on this system has been increased from 300 $\mathrm{MB}$ to over $2 \mathrm{~GB}$.

To utilize the MicroVAX CPU with its associated memory, and to increase the capability of the off line detector testing and experimental set up at the Cyclotron, we set up a third data acquisition system. We purchased a used Q-bus chassis along with a used network adapter and a terminal controller which constitute a complete $Q$-bus system along with a Q-bus to SCSI bus DISK/TAPE controller. At present a $1 \mathrm{~GB}$ disk has been logically divided to a $332 \mathrm{MB}$ system/user disk and $776 \mathrm{MB}$ scratch disk. One Exabyte $8 \mathrm{~mm}$ tape drive is attached to the system. The following data acquisition hardware has been purchased to complete a third data acquisition set: $Q-U$ converter adapter, CAMAC crate, crate controller, branch highway, Micro Branch Driver (MBD) and event trigger module. All the above equipment has been purchased from the first year upgrade funds. A Desta unit, which converts the thickwire to thinwire Ethernet, has been purchased to allow this system to maintain its connectivity regardless of its location. The third data acquisition will be portable to different experimental locations. The disk for the other VAXStation $\mathbf{3 2 0 0}$ data acquisition system has been upgraded from a $760 \mathrm{MB}$ system and user disk to a total of 2 GB. All three of the data acquisition systems run as stand alone systems and use the same peripherals, such as the printer and terminals, as the "COMP" cluster.

Early last year the Cyclotron became part of a subnet which includes a few large departments: Physics, Mechanical Engineering, and Geosciences. This posed some problems, especially for devices such as the terminal server where performance is sensitive to network traffic. To isolate the network traffic at the Cyclotron and in accord with our computer upgrade plan we purchased a CISCO bridge/router (BR). This BR placed the Cyclotron in a subnet by itself. The bridge router is the best solution for the Cyclotron because it allows us to communicate using our terminal server and its associated LAT software. At the present time the CISCO unit only implements the Ethernet media, but it can be upgraded to allow a Fiber Optic (FO) connection to the campus FO backbone for a higher speed communication link. At present we are routing only a couple of the protocols and rely on the bridge for the other types of communication. The system is capable of a greater number of protocol routings if the need arises in the future. At present only one member of the cluster is running INET software for BITnet connectivity, but all the computers, including the data acquisition systems, are running TCP/IP software which provides connectivity to all educational sites as well as national laboratories in the state and other parts of the world. This allows both fast file transfer and remote login to be independent of the operating system. This specific software allows personal computer (PC) systems which are being used by some of the Cyclotron faculty and staff to communicate with the "COMP" cluster and data acquisition systems. During the last year the University has replaced the campus HEPnet link using a leased 9600 baud line with a $T 1$ connection. This allows the Cyclotron experimental groups to communicate more efficiently with their collaborators at national labs, such as Fermi Lab and Los Alamos.

The Cyclotron PC system also was upgraded last year. One experimental group purchased two $50 \mathrm{MHZ}$ 486 PC systems for data analysis - each with $8 \mathrm{MB}$ of memory, 760 MB SCSI disk drives, and Exabyte 8200 tape drive with controllers and associated software.

In conclusion, this past year has seen a tremendous improvement in the computing capabilities of the Cyclotron Institute. It is anticipated that conditions will improve dramatically in the coming year with the purchases planned for this second year of upgrade.

Fig. 1. Block Diagram of the computer systems currently in the Cyclotron Institute. (Page 102.) 

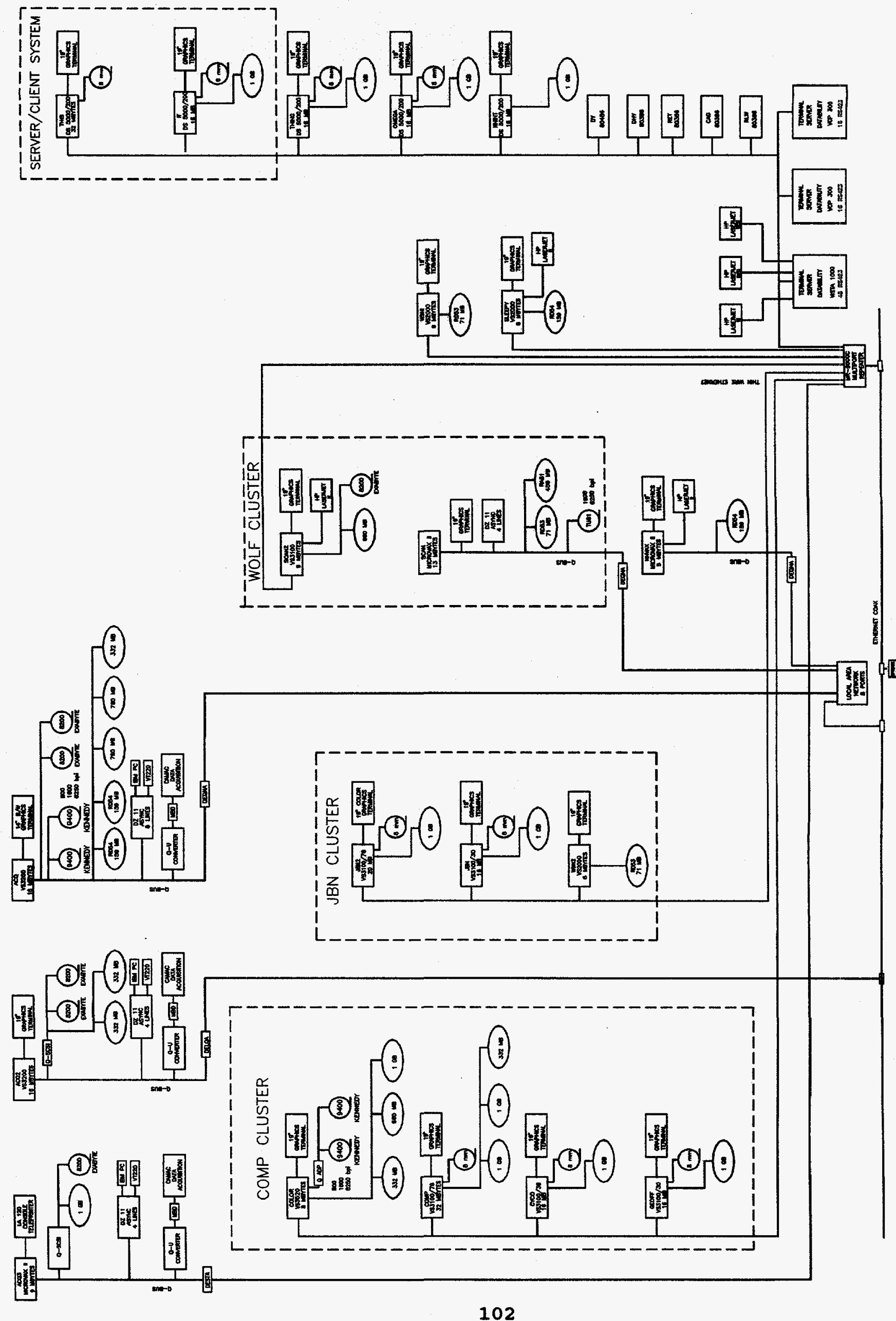


\section{PROTON SPECTROMETER FIELD MAPPING}

\section{A. Zaruba, H. Xu, A. Betker, T. Cowden, G. Derrig, and C. Gagliardi}

The detailed field mapping of the Proton Spectrometer is complete. The system originally described' was designed using results from POISSON ${ }^{2}$ and RAYTRACE. ${ }^{3}$ The two-dimensional nature of POISSON required simplification of the three-dimensional problem of determining the true fringing fields present. Preliminary results from our data show good agreement with our calculated expectations, both in field magnitudes and shapes. Detailed analysis is currently underway to determine the higher-order multipole components of the central field for varying field levels, the effective field boundaries necessary for ray-tracing calculations, and the magnitude and shape of fringe fields at both the entrance and exit of the magnet. When this analysis is complete, we will know the extent to which the Proton Spectrometer matches the optical properties which were assumed when it was designed.

The Proton Spectrometer was designed to run at magnetic fields of up to $17 \mathrm{kG}$. Four field levels $-9,11$, 14.5, and $16.5 \mathrm{kG}$ - were chosen for mapping. These covered the anticipated range of experimental use and provided good points for interpolation on the magnet's saturation curve, which is shown in Fig. 1. These field levels were also chosen to correspond with those originally used for theoretical calculations, 11 and $17 \mathrm{kG}$. Two NMR probes have been permanently installed inside the magnet to provide baseline fixed-point field measurements. Tests of the magnet's standard highcycling showed a B vs. I curve for the NMR probes that was reproducible to \pm 2 gauss.

\section{Magnetic Field For PSP}

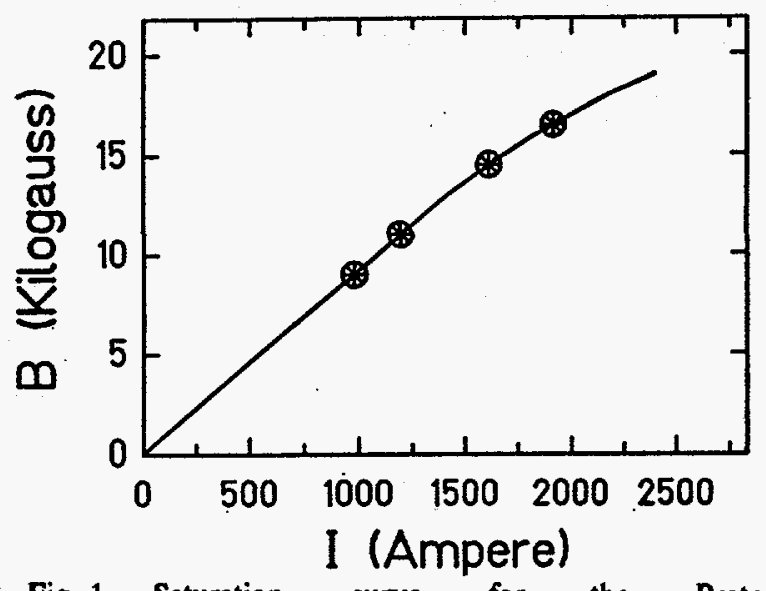

Fig. 1. Saturation curve for the Proton Spectrometer. The four points marked show the four mapped field levels.
The procedure for field mapping was controlled to a large extent by the inherent physical boundaries of the magnet itself. After a $17.8 \mathrm{~cm}$ (horizontal) entrance flange, the central ray traverses a $112.0 \mathrm{~cm}$ radius over $70.7^{\circ}$, to a $66 \mathrm{~cm}$ exit flange. The angular acceptance $\left( \pm 7^{\circ}\right)$ and the momentum acceptance $\left( \pm 14 \% \Delta p / p_{\alpha}\right)$ of the Proton Spectrometer about this central ray determine the critical area to be mapped inside the magnet, plus the exterior areas to the target and detector chambers. This irregular are was split into the 7 Cartesian mapping regions indicated in Fig. 2. These were designed to cover the critical area as efficiently as possible given the limits imposed by the vacuum chamber and return yoke on access to the interior of the magnet. Each map region sufficiently overlapped adjacent regions so that all the data may be combined into a global magnet map for each field level. All of the data for a given nominal field level was, in fact, taken at a constant energizing current. The data from the various mapping regions are normalized to the desired magnetic field with the simultaneous NMR readings.

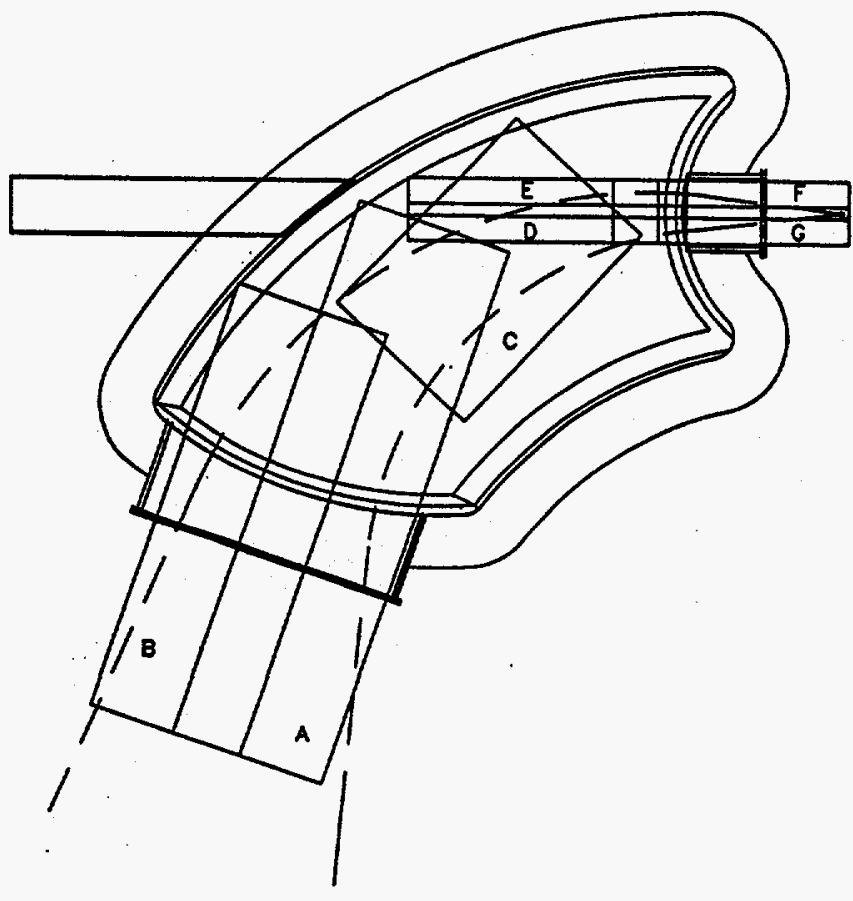

Fig. 2. The regions covered by the field mapping. The dashed lines bound the critical area of the beam. Position of the target is marked by the small cross. 
The mapping apparatus itself consisted of a $0.01 \%$ accurate temperature-stabilized Hall probe mounted in the magnet's median plane on a transverse and longitudinal brass feed-screw arrangement. The feed screws assured positional accuracy and reproducibility of $<0.01$ " on a $0.1^{\prime \prime} \times 0.1^{\prime \prime}$ grid. The transverse screw, which allowed a maximum travel of $16^{\prime \prime}$, was mounted on the $60^{\text {" long }}$ longitudinal screw. A position wire fixed to the longitudinal screw base and free to move in length with the transverse screw provided a digital readout for marking the longitudinal coordinate. The Hall probe was affixed to one of several carriages which traveled along the transverse screw. The transverse motion was motordriven under computer control. The computer controlled the transverse travel via an optical reader (attached also to the probe carriage) which scanned a fixed ruled glass rod. At each increment indicated by the computer's programming - in this case, every 0.4 inches - the mapper paused for probe stabilization and input a field strength directly from the Hall probe. The mapper carriage continued across, taking the designated number of readings, and saved the complete transverse scan at that longitudinal position only if the entire scan was clean. At the end of one scan across, the computer returned the probe to the start position. The mapper carriage system was moved longitudinally to the next position by means of manual rotations of the feed screw. The longitudinal coordinate of the mapper was manually entered into the data set, then the computer was again allowed to move the probe across. In the interior regions, the longitudinal increment was $1^{n}$. Of special interest while assembling the field map grid for each field level was the behavior of the field at the entrance and exit boundaries of the magnet. This determines the true shape of the Proton Spectrometer. For each map that traversed the apparent boundary, a finer-grid of half-inch longitudinal increments was used. The true effective field boundary for each field level will be part of the calculations.

Access to the interior of the magnet was limited.

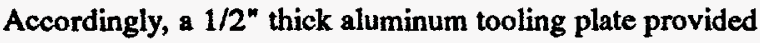
a frame upon which to mount the mapping apparatus. The mapper track was pinned to the plate to ensure precise relocation whenever the apparatus was removed for system reconfiguration or repair. The aluminum plate provided a fixed frame of reference for the apparatus within the magnet, and also a way for translating the mapping apparatus frame to the actual magnet frame of reference.
Initially, the plate was to have been uniquely located on the pole tip by means of three pins resting on the bevel of the pole tip. However, the plate was finally pinned to a support bar outside the magnet because the interior access problems made unique location impossible to ascertain otherwise.

The system was transformed in several ways to fit each of the 7 different mapping regions. Access to the largest regions was available through the larger exit flange. For regions $A$ and $B$, the assembly was fixed on the plate, and the mapping of the different regions was accomplished with a slight change to the probe carriagr. For region $C$, the mapper plate was removed, the entire assembly was transferred and pinned in a new location on the plate, and a different probe carriage was attached. For the remaining four regions, access to the mapping regions was through the six inch $0^{\circ}$ hole in the return yoke. The mapper plate remained in place while the majority of the assembly was fixed separately outside the hole. The transverse feed screw remained outside the magnet where it controlled a long extension arm into the magnet with the probe carrier attached to the end insids. This extended framework was guided in part by a groove in the fixed plate meant to control any sideways travel of the arm.

The frame of reference of the probe was controlled by the mapping apparatus. The precision of the location of the probe was determined both by the pinning of the mapper assembly to the aluminum plate, which was in turn pinned to an exterior support of the magnet, and by transiting the probe and mapper assembly with respect to fiducial marks on the magnet. The translation of each of the 7 different coordinate systems into one greater frame of reference is a primary step in analysis of the complete data for the four mapped field levels.

Preliminary analysis, as said, shows promising results, sufficient to clear the way for permanent emplacement of the interior entrance slits, Faraday cup, and the rest of the vacuum chamber. More detailed analysis of the complete mapping data is currently underway.

\section{REFERENCES}

1. A. C. Betker and C. A. Gagliardi, Nuc. Instrun. Methods A283, 67 (1989).

2. R. F. Holsinger, unpublished.

3. S. Kowalski and H. A. Enge, unpublished. 


\section{A. C. Betker, C. A. Gagliardi, H. Xu, A. F. Zaruba, P. Smelser and G. Derrig}

During the past year we have made significant progress on the Proton Spectrometer project, culminating in a test run which examined the properties of the back drift chamber. A major accomplishment was the completion of the magnetic field mapping, which is described in detail elsewhere in this progress report. The vacuum chamber of the spectrometer magnet was leak tested when it was first assembled. But due to space constraints, the "focal plane" vacuum chamber, which houses the front drift chamber and extends the vacuum system to a final window located approximately $2 \mathrm{~cm}$ in front of the back drift chamber, could not be installed until the mapping was complete. Furthermore, the vacuum pump for the Proton Spectrometer, which is mounted on the $0^{\circ}$ exit port, had to be removed to facilitate access for the mapper. The entire vacuum system has now been assembled, leak checked, and exercised thoroughly.

During the past year, we have also checked out the beam line optics, completed construction of the drift chambers and much of their electronics, and assembled most of the trigger scintillator hodoscope. The data room that the Proton Spectrometer shares with HILI, and ultimately with additional experiments performed in the new K500 experimental area, is now complete, including installation of clean electrical power and cabling into the Proton Spectrometer cave. Although it was not there during the test run, as of this writing we have an Ethernet connection to the new data room to allow the data acquisition computer to communicate with the rest of the Cyclotron Institute computer network. It is not yet clear whether one of our data acquisition computers will be kept in the new data room or only be moved there on an "as needed" basis. The rest of this report describes our progress during the past year in greater detail.

Last August we had a short run with a $110 \mathrm{MeV}$ deuteron beam to check our beam line optics. The actual emittance of high velocity beams from our K500 had not been investigated previously. Rather, the beam line had been installed based on calculations that utilized K500 emittance predictions from $\mathrm{MSU}^{1}$ as the starting point. During deuteron elastic scattering measurements which are described elsewhere in this progress report, we had found that the deuteron beam emittance at the White Chamber is $<8 \mathrm{~mm}-\mathrm{mr}$ in both the $\mathrm{x}$ and $\mathrm{y}$ directions. This is consistent with the MSU predictions. The beam transmission from the $\mathrm{K500}$ to the White Chamber was approximately $35 \%$. Essentially the entire transmission loss occurred at the C-01 slits (near the exit of the K500 cyclotron), which needed to be closed down to eliminate "wings" on the beam spot.

During our test run, we found that the deuteron beam at the C-01 slits consisted of two distinct components of nearly equal intensity, together with a small amount of additional beam providing a continuum between them. It appears that the elastic scattering measurements used the C-01 slits to select one of the major components for transport. The beam line from the cyclotron to the Proton Spectrometer was intentionally made as short as reasonably practical in order to allow for the future installation of a neutron beam facility. As such, we only have a limited ability to remove slit scattered particles in the beam before they reach the target. $35 \%$ transmission will not be acceptable for $0^{\circ}\left(\mathrm{d},{ }^{2} \mathrm{He}\right)$ scattering experiments. We found that, by retuning the magnetic channel elements of the $\mathrm{K} 500$ beam extraction system, we could restrict the extracted beam to only one of the two intense components at the expense of reduced extraction efficiency. We had over $10 \mathrm{nA}$ of beam available, nonetheless, without making use of the injection line beam buncher. This is far more beam than required for the Proton Spectrometer. We were then able to transport the remaining beam to the Proton Spectrometer target with minimal losses, while simultaneously achieving the desired beam spot. Although the total beam emittance is consistent with the MSU predictions, the $x-\theta$ and $x-\Delta p$ correlations are quite different. Fortunately, we had built enough flexibility into the beam line configuration to compensate for this.

During the past year, we completed the construction of both drift chambers for the Proton Spectrometer. Figure 1 shows the front drift chamber after all of the wires and internal electronics had been mounted, but before the front vacuum window had been installed. We have also tested both of the chambers with sources and further tested the back detector with protons produced in an $(\alpha, p)$ reaction. Analysis of the proton tracking data is currently underway. Preliminary analysis indicates that its performance is similar to that of our prototype detector. $^{2}$ The front detector, which sits within the vacuum system and is designed to operate at $1 / 2 \mathrm{~atm}$, was not installed during this initial test run even though it was 


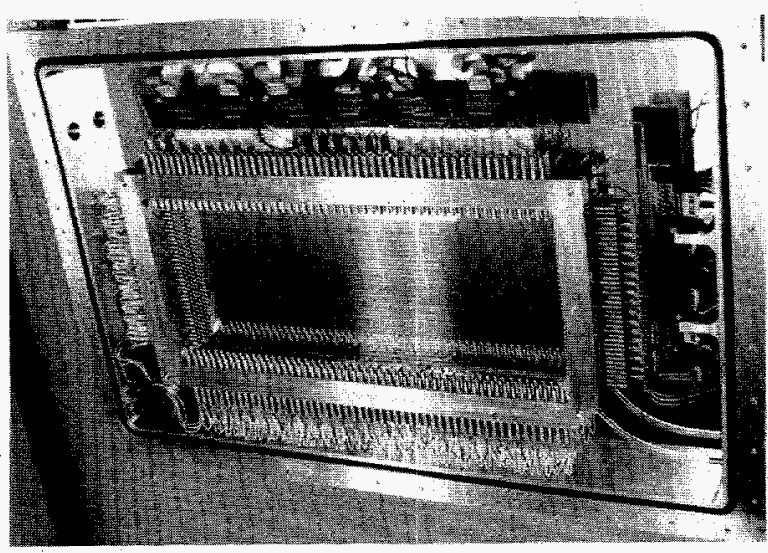

Fig. 1. A photograph of the front drift chamber, without its front window, after it was assembled.

ready, because we were not yet fully confident of the integrity of the exit window of the "focal plane" vacuum chamber. Originally, this window was to be made of 0.25 $\mathrm{mm}$ thick lexan. Long-term durability tests of the lexan window via repeated cycling of the vacuum system showed that it would craze after a number of cycles, and eventually fail. Prior to the test run, the window support frame was modified slightly, and the lexan was replaced by a $0.25 \mathrm{~mm}$ thick mylar window. This configuration is now working very well, but it had not been tested sufficiently prior to the test run to risk placing the front drift chamber inside the vacuum. The front drift chamber was installed after the first run, and the entire vacuum and chamber gas system has now been exercised fully. After it has stabilized, the gas system maintains the front drift chamber at its operating pressure of 370 torr to within 0.1 torr. A run to test the entire drift chamber system is scheduled for May.

We completed the design and construction of the trigger scintillator hodoscope during the past year. There are $16 \mathrm{x}$ scintillators and $8 \mathrm{y}$ scintillators. The scintillators are $3.2 \mathrm{~mm}$ thick and made of Bicron BC 400 . Each $x$ scintillator, which is $26 \mathrm{~cm}$ long, is read out at one end by a Hamamatsu R1355 photomultiplier tube. Each y scintillator, which is $67 \mathrm{~cm}$ long, is read out by a pair of phototubes, one at each end. The $x$ scintillator array has been assembled and tested. When the $y$ scintillators arrived from Bicron, we found that they were missing one light guide each. They were returned to Bicron to be completed, and are now expected to arrive in mid May. The y scintillator array will be assembled and tested at that time.

At present, the Proton Spectrometer system is essentially complete. Unfortunately, due to RF and deflector problems with the K500 cyclotron, we have not had deuteron beams available for more than a few hours at a time since last fall. These problems now seem to be resolved. If so, we will take the first physics data with the system shortly.

\section{REFERENCES}

1. E. Fabrici, D. Johnson and F. G. Resmini, Michigan State University Report MSUCP-33 (1980).

2. A. C. Betker, R. H. Burch, and C. A. Gagliardi, Nucl. Instrum. Methods A294, 549 (1990).

\section{BARIUM FLUORIDE ARRAY}

\section{R. K. Choudhury, J. Shoemaker, H. Jabs, and K. L. Wolf}

The $\mathrm{BaF}_{2}$ crystals were procured and tested for their energy resolution and timing characteristics. The crystals were coupled to R2059 photomultiplier tubes purchased from Hamamatsu Corporation. Three support structures were fabricated to hold the 19-pack assemblies. With the available detectors, only two of these assemblies could be installed inside the support structures. Figure 1 shows a photograph of the integrated $\mathrm{BaF}_{2}$ assemblies ready for use in an experiment. The electronics hardware for pulse processing and data recording from the $\mathrm{BaF}_{2}$ detectors have also been procured. The energy signal from each detector is recorded with fast $(100 \mathrm{nsec})$ and slow $(2 \mu \mathrm{sec})$ gating requirements for $\gamma, \mathrm{n}$ and charged particle discrimination. Figure 2 shows a typical 2-D plot of the

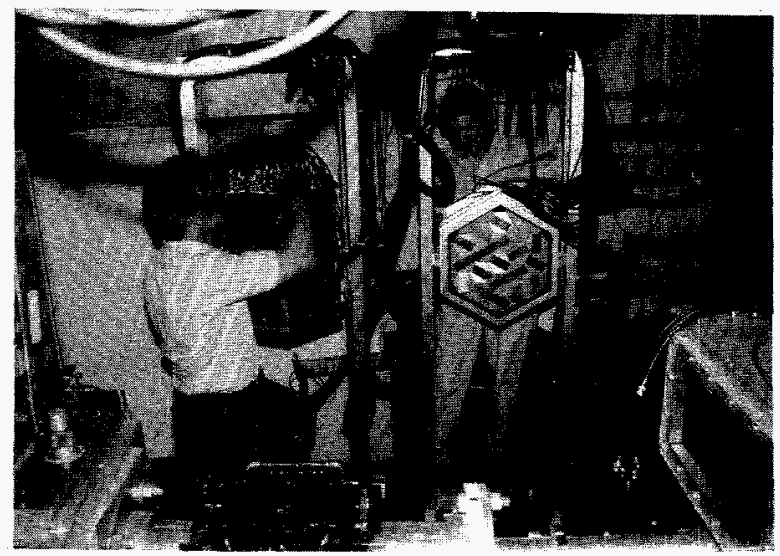

Fig. 1. Integrated $\mathrm{BaF}_{2}$ assemblies. 
fast vs. slow ADC outputs, where the separation between the $\gamma$ rays and charged particles can be clearly seen. The slow gate energy signal is also recorded in two gain ranges to cover different regions of interest in $\gamma$ ray energies. The total energy deposited in the 19 detectors is obtained by either hardware linear sum of all signals or by software analysis using the calibration of each detector.

Figure 3 shows the sum energy spectrum obtained with a Pu-Be source which gives $4.413 \mathrm{MeV} \gamma$ rays from the ${ }^{9} \mathrm{Be}\left({ }^{4} \mathrm{He}, \mathrm{n}\right)^{12} \mathrm{C} *$ reaction. The full energy peak is prominently seen with very little of the escape component. The lower energy bumps are due to crystal radioactivity and room background.

The two $\mathrm{BaF}_{2}$ packs were used in an experiment to study the GDR $\gamma$ emission from compound nuclei produced by ${ }^{16} \mathrm{O}+{ }^{126} \mathrm{Te}$ and ${ }^{22} \mathrm{Ne}+{ }^{120} \mathrm{Sn}$ reactions at 179 and $193 \mathrm{MeV}$ bombarding energies, respectively. The detectors were placed at a distance of $50 \mathrm{~cm}$ from the target. The compound nuclear residues were detected

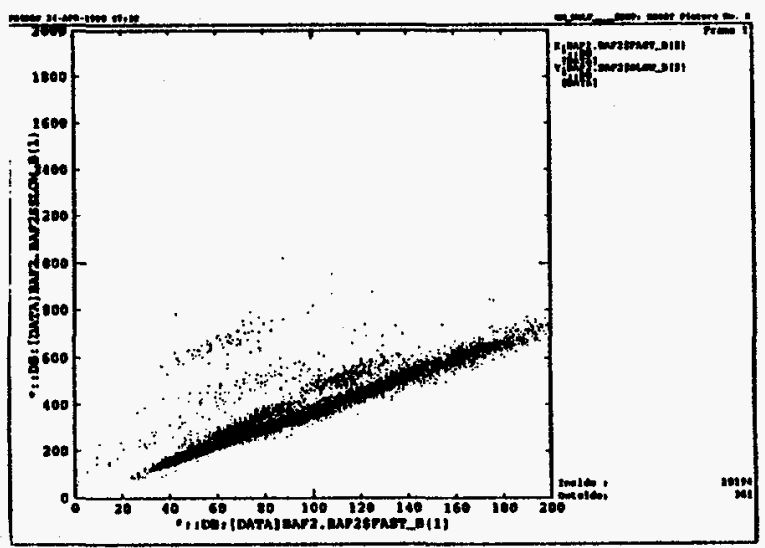

Fig. 2. 2-D plot of the fast vs. slow ADC outputs.

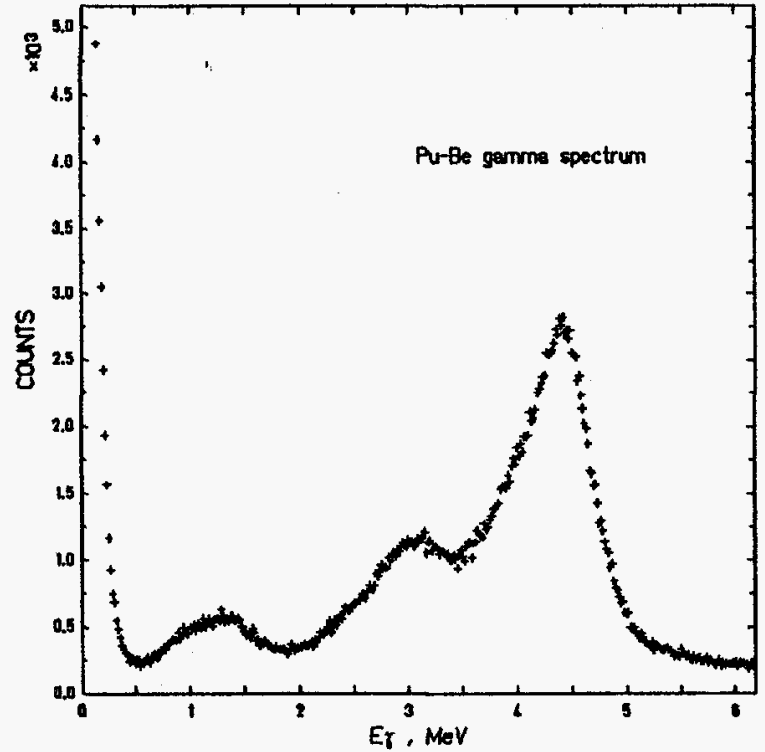

Fig. 3. Sum energy spectrum obtained with a $\mathrm{Pu}-\mathrm{Be}$ source. with two PPAC and one Si detector mounted in the forward $\left(6^{\circ}\right.$ to $\left.20^{\circ}\right)$ direction at a distance of $30 \mathrm{~cm}$ from the target. The RF signal of the cyclotron was used to derive the start time for all the detectors. Typical TOF spectra of the Si detector and one of the $\mathrm{BaF}_{2}$ detectors are shown in Figs. 4 and 5, respectively.

The timing energy resolution is mainly decided by the spread in the RF time from the cyclotron. Preliminary results of the GDR experiment using the $\mathrm{BaF}_{2}$ arrays have been reported elsewhere in the report. $A$ number of experiments to study the gamma ray emission in the GDR $\left(\mathrm{E}_{\gamma}<20 \mathrm{MeV}\right.$ ) and the continuum bremsstrahlung region $\left(20 \mathrm{MeV}<\mathrm{E}_{\gamma}<150 \mathrm{MeV}\right.$ ) are being planned using the $\mathrm{BaF}_{2}$ arrays.

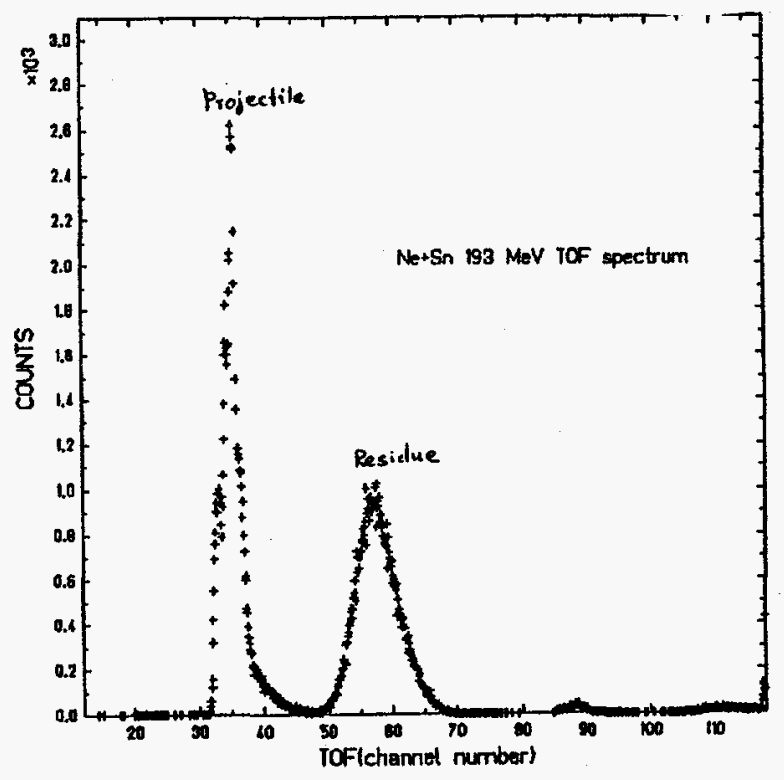

Fig. 4. Typical TOF spectra of the Si detector.

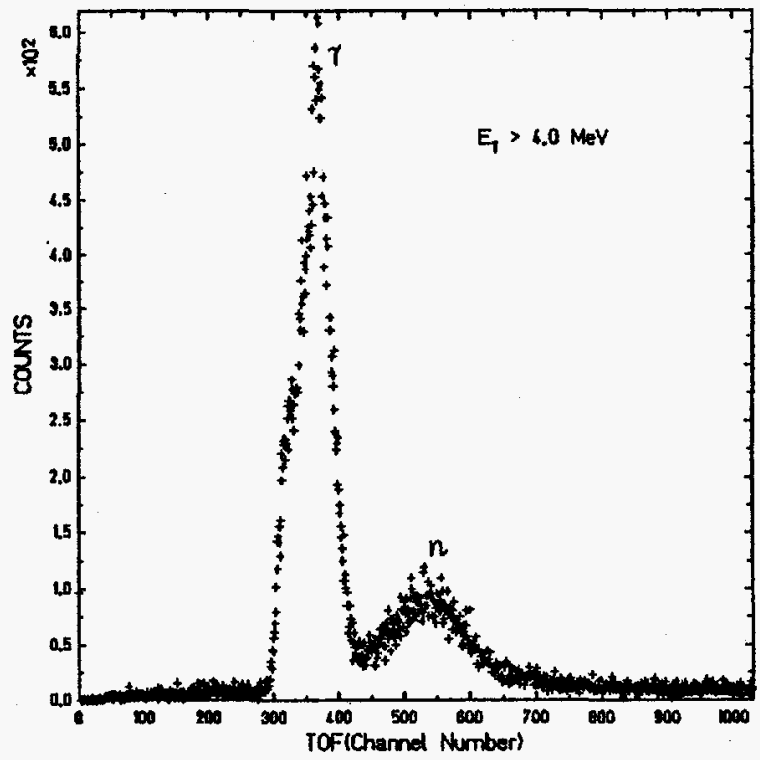

Fig. 5. Typical TOF spectra of one of the $\mathrm{BaF}_{2}$ detectors. 


\section{R. Tribble, J. D. Bronson, W. Liu, and X.-G. Zhou}

In the 1989 Annual Report, a detailed description was provided of the ion optics for the recoil spectrometer MARS. As we noted in last years Annual Report, two papers have since been published outlining the optics and the status of the device. 1,2 Some minor modifications were made to the original design and these are discussed in Ref. [2]. Below, we update the progress on the construction of MARS since last year's Annual Report. For convenience, we have once again divided the discussion into three sections to highlight the various major components.

\section{(1) Magnetic Systems}

MARS uses three dipole magnets. Two of them form the momentum achromat and the third provides a vertical bend after the velocity filter to cancel the momentum dispersion of the recoil ions, thus producing a mass focus. The contract for the construction of the three dipoles was awarded to Inland Steel early in '91. At that time, the schedule called for an August ' 91 delivery of the three dipoles. The dipoles actually arrived just after the beginning of ' 92 , about four months behind the original schedule. The coils for all three dipoles had already been wound by our technical staff. Once the dipoles arrived, the outside surfaces were painted and preparations began to put on the coils. During the first two months of '92, cables were run from the magnet power supplies, which are located in the basement of the building which housed the $224 \mathrm{~cm}$ cyclotron, to Cave 5 where MARS is located. Following this, the technical staff began putting the coils onto the dipoles. The first assembly that has been completed is D2 - the second of the two magnets that form the momentum achromat. Work is now proceeding to complete the assembly of D1 - the first magnet in the achromat section. Magnetic field maps of both D1 and D2 will be underway before the end of April and completed early in May. The field mapping will focus on the fringe field region at the entrance and exit of the two dipoles. As was noted in last year's Annual Report, the field shape and effective field boundary at the entrance and exit of these two magnets is important in determining the ion optics of the system. A field clamp was added to the two magnets to stabilize the effective field boundary and to produce a fringe field region that looks like the "short tail" field in RAYTRACE. It is important to determine how closely the actual field profile follows the prediction that was made with the computer cocle POISSON. By mapping the field along the beam direction for the entrance and exits, we will be able to determine the exact location of the effective field boundary and the fringe field shape parameters that are used in RAYTRACE. The contract to construct the vacuum chambers for the three dipoles was awarded in Decembir '91. At that time, the expected date for delivery of the chambers was the end of February '92. The vendor encountered some problems during the construction of the chambers and has revised the delivery date to the beginning of May ' 92 . The field mapping should be completed by the time the vacuum chambers arrive so that we can immediately begin assembling the full systems. The magnet stands for the three dipoles were completed during this past year and both D1 and D2 are being assembled on their stands.

A fourth dipole magnet is needed as part of a beam swinger for MARS. Initially this dipole was configured ats a large aperture magnet that would accept beams over the full angular range provided by an upstream bending magnet. Two beam line quadrupole magnets were to be mounted on a movable stand so that the beams from the upstream bending magnet would always pass through the quadrupoles on their optical axis. This system was redesigned so that now a small bending magnet, which moves with the two beam line quadrupoles, replaces the large bore stationary magnet. All of the particle bearis enter the small magnet at the same location with this configuration. By adjusting the magnetic field from -15 $\mathrm{kG}$ to $+15 \mathrm{kG}$, we are able to provide the full range of scattering angles $\left(0^{\circ}\right.$ to $\left.30^{\circ}\right)$ for which the previous system was configured. POISSON calculations for the small magnet have been completed and the magnet geometry has been fixed. The final mechanical drawings for this magnet were completed in the fall of ' 91 and the construction of the magnet began early in the ' 92 after the iron arrived from Inland Steel. The new design is sufficiently simple and compact that it is being built in one of the local TAMU shops.

The five large quadrupoles that are part of MARS were shipped to us from Brookhaven National Laboratory in the spring of ' 89 (we actually received seven of these quadrupoles but two were returned). As we noted in last year's Report, the quadrupoles were refurbished by replacing water hoses, cleaning out the coils and adding 
new temperature sensors and flow meters. Also the water lines and fittings were pressure checked. The first doublet and the singlet are now in place in Cave 5 on their stands and aligned. They have utilities connected (power and water) and field mapping of one of the quadrupoles is presently underway. As for the dipoles, we are carrying out a field map in the fringe field region of the quadrupole to determine the effective field boundary and the coefficients that describe the fringe field fall off for use in RAYTRACE. The remaining two quadrupoles are to be mounted on the movable detector arm that follows the third dipole. The construction of this movable arm has been completed and the drive mechanism to move it has been delivered. The mount pivots about the center of dipole D3 and it will be put in place once this dipole is installed.

Machining of the pole pieces and return yokes for the two correction sextupole magnets was completed by March '91. The coils were wound on the pole pieces after the last of the dipole coils was completed and they were assembled during this past year. The stand for the first of the two sextupoles has been constructed and put in its proper location along the beam line. The second sextupole sits on the movable detector assembly with the quadrupole doublet.

The power supplies for the dipoles and the first two large quadrupoles arrived on schedule this past fall. All of the supplies have been checked out and the power cables to connect them to the magnet elements have been run. In addition, the cables that are needed to operate the other magnets from existing power supplies are in place.

\section{(2) Velocity Filter}

During the fall, we completed the modifications to the velocity filter that were required for making the transition from the conducting glass plates to anodized $\mathrm{Al}$. The motivation for this change was discussed in last year's Annual Report. The anode and cathode plates were mechanically aligned to a tolerance of approximately

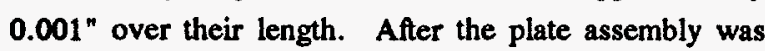
inserted into the housing, the system was put in place in Cave 5 and aligned. A small vacuum leak was found in one of the spool pieces that connects the high voltage decks to the vacuum system. After the leak was repaired, we have achieved a steady-state vacuum of about $5 \times 10^{-7}$ Torr in the device using a single cryopump. We discovered this past summer that the high voltage decks had both suffered some slight damage from internal sparking. Several modifications were made to the negative high voltage deck to eliminate possible internal spark points and both decks were refurbished. The decks were mounted on the velocity filter after the plate assembly was inserted this past fall. We have conditioned the system, at high vacuum, up to a voltage of $300 \mathrm{kV}$. A problem arose with the negative supply while the system was running at this voltage and it is now being repaired. From all of the indications that we have, the plates appear to be conditioning just as we would expect. Thus operation up to design goal of $500 \mathrm{kV}$ appears to be achievable.

As we noted last year, the magnetic field for the velocity filter is provided by energizing a main coil and four smaller dipole coils, two at each end. All of the coils were designed to run in series so that a single power supply could be used. The main magnet power supply from the $224 \mathrm{~cm}$ cyclotron will be used to produce the magnetic field in the velocity filter. In checking the current and voltage requirements, we found that we would be voltage limited to magnetic fields of about $700 \mathrm{G}$ with the present coils. Since we plan to run the fields up to a $1000 \mathrm{G}$, we replaced two of the small dipole coils with larger conductor. The number of ampere-turns remained constant but the resistance of the coils was substantially reduced. This modification makes operation up to $1000 \mathrm{G}$ feasible with our existing power supply. The cable run to connect up the velocity filter to the old cyclotron power supply was completed this past fall.

\section{(3) Vacuum and Support Systems}

The final engineering design for all of the components in the vacuum system has now been completed. This includes the beam swinger and target chamber system, the detector chamber movable arm and a modular detector chamber and the beam line section in the momentum achromat where most of the beams will be stopped for $0^{\circ}$ operation of MARS. We have decided to begin studies with MARS using a simple $0^{\circ}$ scattering chamber instead of the more complex general purpose chamber. The $0^{\circ}$ chamber has been built and is now in place on the scattering chamber post. The system was specifically designed to make it modular so that we can easily remove one chamber and replace it with another. The design for the beam line section where we will stop beams in $0^{\circ}$ operation has evolved during the engineering design phase to consist of a $24^{\prime \prime}$ diameter stainless steel tube that will be fitted with movable Faraday cups along each side. A pair of fixed cups will intercept beams that hit at the back of the tube and a system of slits and a movable Faraday "finger" will be inserted on a flange from the top of the tube. The tube and connecting flanges 
have already been built. Several penetration flanges need to be welded onto the tube and then it will be ready for installation. Construction of the remaining parts for the vacuum system is being carried out by our mechanical shop and other local TAMU shops. Pumps, valves and other hardware for the vacuum system arrived during the summer and fall of ' 91 .

\section{REFERENCES}

1. R. E. Tribble, R. H. Burch and C. A. Gagliardi, Nucl. Instr. and Methods A285, 441 (1989).

2. R. E. Tribble, C. A. Gagliardi and W. Liu, Nucl. Instr. and Methods (In Press).

\section{MDM Magnetic Spectrometer}

\section{H. Youngblood, J. D. Bronson, and Y.-W. Lui}

Funding was approved in January 1992 for installation of a $\mathrm{K}=315$ large solid angle magnetic spectrometer ${ }^{1,2}$ in place of the $K=150$ Enge split pole spectrometer in Cave 3. The MDM spectrometer including the scattering chamber and heavy ion detector system ${ }^{3}$ was obtained from Oxford University in 1991. Vacuum pumps and power supplies were not obtained. The detector, with addition of a scintillator to stop ions up to $80 \mathrm{MeV} /$ nucleon, will be appropriate for many planned experiments and has been refurbished and tested at Oak Ridge. ${ }^{4}$

The project is in two stages. The first, funded for calender 1992 and to be completed in November, 1992 will have the spectrometer and beam line in place and operational for experiments, with several limitations. The second stage, to be funded for calender 1993 will provide new pumps to replace the 23 year old pumps in the beam line, a large vacuum chamber to house a variety of detectors, connection of the power supplies to the K500 cyclotron computer control system, and dedicated electronics for the detector system.

The beam line layout for the spectrometer is shown in Fig. 1. Figure 2 shows TRANSPORT calculations of the beam profiles for this line. A system to provide high resolution analyzed beams to the spectrometer is planned for construction in 1993 and 1994 and the spectrometer beam line has been configured to provide dispersion matched beams from this analysis system to the MDM spectrometer. The optics for the spectrometer line were calculated assuming a focus just before the wall where the beam enters the spectrometer cave. For the two year period from end of 1992 until the analysis system is completed, beams will be transported to this focus through an existing line. $A$ beam resolution of $.06 \%$ has been measured through this line into the Enge spectrometer with $490 \mathrm{MeV}{ }^{14} \mathrm{~N}$ beam from the $\mathrm{K} 500$ cyclotron, though with considerably reduced intensity. Several minor improvements in this line are planned to enhance the transmission.

The median plane of the MDM is $50 \mathrm{~cm}$ above the median plane of the K500 cyclotron and our present beam lines. As is apparent in Fig. 1, two bending magnets are configured with each bending 10 degrees to raise the beam $50 \mathrm{~cm}$. These magnets are presently under construction in house. All components for the magnets are in house: the iron is finished, and the coil winding forms are nearly complete. These magnets will be completed by mid Junt.

On June 8, removal of the Enge spectrometer will begin and it will be loaded directly on trucks that week to go to CEBAF where it will be used as a beam tag magnet.

The MDM dipole will be powered by the main magnet power supply from the 88 inch cyclotron and the quadrupole will be powered by the Enge spectrometier power supply. New power supplies have been purchased for the exit multipole and are in house. Turbo pumps for the spectrometer and target vacuum chambers have been purchased and are in house. An NMR system to monitor the dipole field is on order.

\section{REFERENCES}

1. D. M. Pringle et al., Nucl. Inst. and Meth. A245, 230 (1986)

2. J. D. Bronson and D. H. Youngblood, Progress in Research, 1990-1991, Cyclotron Institute, Texsis A\&M University, p. 119.

3. J. S. Winfield et al., Nucl. Inst. and Meth. A251, 297 (1986)

4. J. D. Blankenship, et al., Progress in Research, 1991-1992, Cyclotron Institute, Texas A\&M University, p. 112.

5. Y.-W. Lui, et al., Progress in Research, 1991-1992, Cyclotron Institute, Texas A\&M University, p. 30. 
$M D M-2$

TARGET CHAMBER
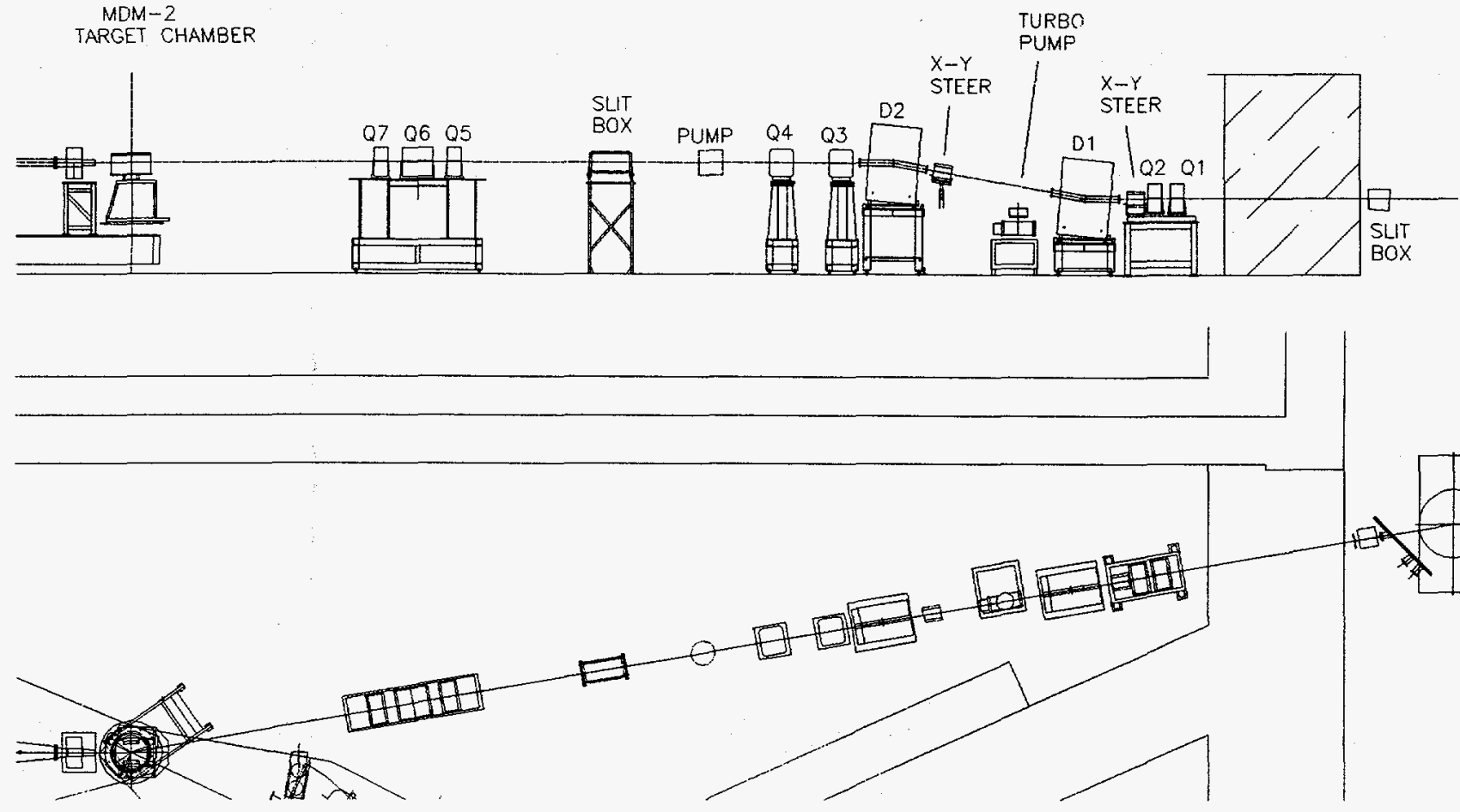

Fig. 1. Beam line layout for the spectrometer.

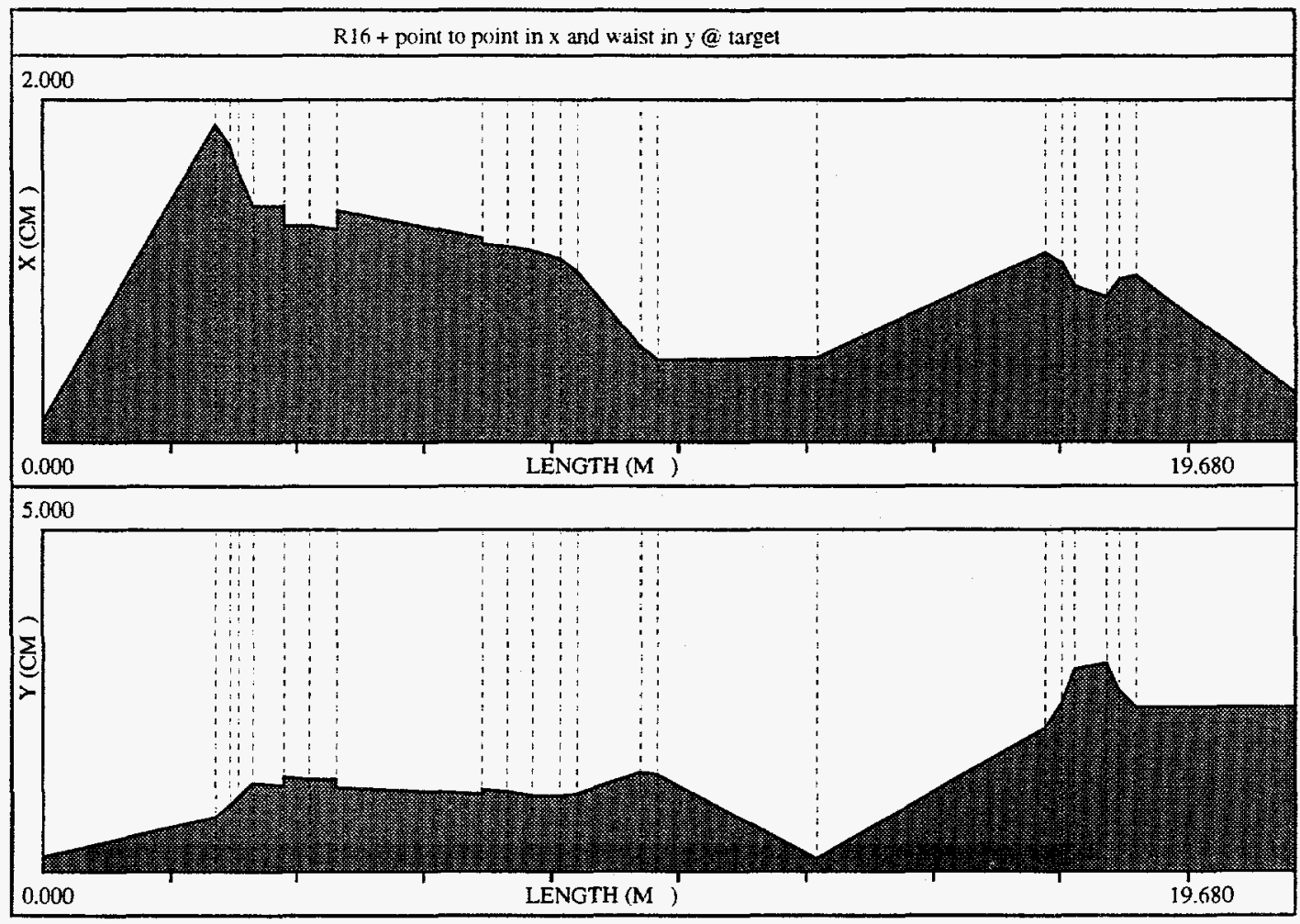

Fig. 2. One example of TRANSPORT calculations of the beam profiles for spectrometer line. 


\title{
HYBRID ION CHAMBER FOR THE MDM SPECTROMETER
}

\author{
J. L. Blankenship, ${ }^{1}$ R. L. Auble, J. R. Beene, F. E. Bertrand, and D. Shapira \\ Physics Division, Oak Ridge National Laboratory, ${ }^{2}$ Oak Ridge, Tennessee 37831
}

Texas A\&M University (TAMU) is replacing its Enge Spectrograph with an MDM magnetic spectrometer originally designed and built for use at Oxford University. The focal plane detector for the MDM spectrometer is a hybrid ion chamber with a sensitive volume of $10.5-\mathrm{cm}$ high, 49.5-cm deep, and $37-\mathrm{cm}$ wide. The existing entrance window has a clear opening of $6 \mathrm{~cm} \times 30 \mathrm{~cm}$. The total energy signal is obtained from the charge induced on the cathode, frisch grid, and field shaping electrodes by the remaining positive ions after the electrons have drifted to the anode. Measurements of particle energy loss ( $\Delta E$ ) are made by two anode segments, each of which are $6.7-\mathrm{cm}$ deep. The electrons collected from the remaining anode depth are not sensed electronically. The horizontal location of the track is measured at two depths by position-sensitive proportional counters (PSPC's). The two PSPC's are spaced $15 \mathrm{~cm}$ apart, with the two $\Delta \mathrm{E}$ anodes between them.

At ORNL, the hybrid ion chamber has been tested, and changes in design have been made to improve detector performance and adapt the chamber to the energy range of the TAMU Superconducting Cyclotron. New front and back cover plates have been designed. A plastic scintillator will be located at the rear window to stop particles which pass through the ion chamber, and thus will provide part of the total energy information. The new front cover plate will provide coupling to the transition chamber which will continue the vacuum flight path from the last spectrometer element to the entrance window of the ion chamber. Drawings of these parts are complete, and construction is underway at TAMU.

The design of the field shaping electrode structure has been changed to provide a more robust construction and to allow quick replacement of field shaping wires at the front and rear windows. Stainless steel rods of 1.6$\mathrm{mm}$ diameter are used as field shaping electrodes on each side of the drift region. The voltage divider resistors which fix the potential of the field shaping electrodes are now located on a terminal strip, along with bypass capacitors, and the charge signal from the field shaping electrodes is now available for addition to the charge signals from the cathodes and the frisch grid. Using a movable, collimated alpha source, we found that the charge signal induced by the positive ions was strongly dependent on horizontal position if the charge signal w'as obtained only from the anode and frisch grid. At $15 \mathrm{~cm}$ from the chamber center, measured horizontally, the charge signal was found to drop to 70 percent of that signal measured at the chamber center. This distance corresponds to the edges of the entrance window. This measurement demonstrates the importance of including the charge signal induced on the field shaping electrodes.

The large quantity of Delrin plastic used in the original construction of the ion chamber presents a challenge to keeping the gas free of impurities which trap electrons and reduce electron mobility. After a month of conditioning, the reduced electric field at which nearcomplete electron collection could be obtained in isobutane was found to be $2.5 \mathrm{~V} /$ Torr-cm. This value u/as found to hold between $\mathbf{5 0}$ and 150 Torr of isobutane. This condition is achieved at 100 Torr for $3500 \mathrm{~V}$ on the cathode, $700 \mathrm{~V}$ on the frisch grid, and anode at ground potential.

An improved system of grounding was installed in the ion chamber to reduce crosstalk between the proportional counters, the anodes, and the frisch grid. A 2.5-cm-wide strip of printed circuit board, clad on both sides with copper, extends from the front plate along both outer sides of the Delrin support structure to the rear, 3 $\mathrm{mm}$ below the anode plane, and is electrically bonded to the front cover. Filter capacitors are bonded to this ground bus with minimum lead length, thus minimizing lead inductance.

Modification of the ion chamber at ORNL has been completed, and the chamber has been returned to TAMU. Following completion of the new front and rear chamber windows/covers, beam tests will be made.

1 Partial support from the Joint Institute for Heavy Ion Research, ORNL.

2 Managed by Martin Marietta Systems, Inc. under contract DE-AC05-840R214100 with the U.S. Department of Energy. 


\section{PC-BASED ELECTRON SPECTROMETER CONTROL SYSTEM AND PROGRAM ESCAM}

\section{Horvat}

An electron spectrometer control and data acquisition system was designed to automate the operation of the tandem-type, parallel-plate, zero-degree electron spectrometer, to process the signals from detectors, and to perform rudimentary data analysis operations. The system utilizes a CAMAC interface and a personal computer. The basic hardware consists of a Kinetic System Corporation (KSC) CAMAC crate, a KSC 3933 Parallel Bus Camac Crate Controller that controls the operation of CAMAC-type electronic modules, and a KSC 2926-21A IBM PC Interface W/DMA card that enables communication between the personal computer and the crate controller.

As shown in the schematic diagram in Fig. 1, the electron spectrometer consists of two parallel-plate capacitors. The role of the first set of plates is to deflect the electrons from the beam path without affecting the beam. Due to its large apertures, the energy range of the deflected electrons is quite broad and the transmission is very high. The second set of parallel plates analyzes the energy of the incoming electrons in high resolution at the cost of significantly reduced transmission. The design and operation of the electron spectrometer has been described in detail in Refs. [1] and [2].

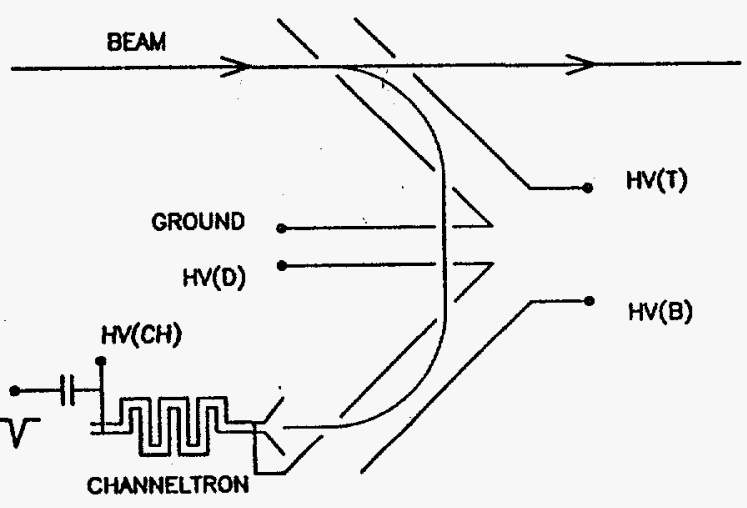

Fig. 1. Schematic diagram of the tandem-type, parallel plate, zero-degree electron spectrometer.

The entrance plate of the first stage of the spectrometer is normally operated at ground potential and voltages are applied to the other three plates of the system. These voltages are denoted $\mathrm{HV}(\mathrm{T}), \mathrm{HV}(\mathrm{B})$, and $H V(D)$ in Fig. 1, where T, B, and D stand for the top, bottom, and decelerating plate, respectively. If a channeltron electron multiplier is used to detect the electrons emerging from the spectrometer (as in the present application), it is advantageous from the standpoint of background reduction to maintain the front at voltage $H V(D)$ and supply an additional voltage $\mathrm{HV}(\mathrm{CH})$ to the back. All the voltages mentioned above depend on the energy of the electrons being transmitted through the spectrometer and the desired resolution. When the spectrometer is scanned to accumulate an electron spectrum, these voltages must be stepped up and down over the appropriate voltage intervals.

One of the functions of the spectrometer control system is to automate the voltage stepping cycles. This is accomplished by using a KSC 3195 6-channel, 16-bit, CAMAC digital-to-analog converter (DAC) to provide control voltages to a set of programmable high voltage power supplies (TENNELEC TC 952), as shown schematically in Fig. 2. The voltage provided by each power supply is the sum of the manual dial setting (offset) and the input (DAC) control voltage multiplied by a preset gain factor of 100 or 300 . Also, the voltage may be removed remotely by grounding the disable input.

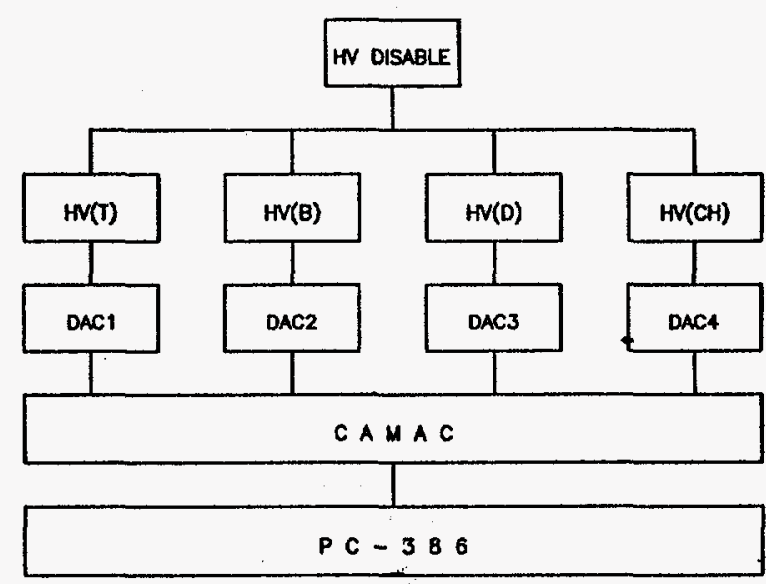

Fig. 2. Schematic diagram of the spectrometer voltage control system.

A schematic diagram of the signal-processing part of the system is shown in Fig. 3. Signals from a $60 \mathrm{~Hz}$ pulser are sent to channel 1 of a LeCroy Research Systems (LRS) 2551 12-channel scaler and used to measure the live time of the system. The signals from the channeltron electron multiplier (CHAN) are sent through a Canberra 2003B preamplifier (PA) to an Ortec 571 Spectroscopy Amplifier (A), converted into logic signals using a timing single-channel analyzer (TSA) and counted by channel 2 of the scaler. 
A Faraday cup (FC) is normally placed behind the detector in measurements with particle beams to provide normalization for the voltage scan counting intervals. The FC current is integrated using a Brookhaven Instruments Corporation (BIC) Current Integrator (Cl). The signals from the $\mathrm{CI}$ are sent through a logic signal shape converter (LSSC) to channel 3 of the scaler.

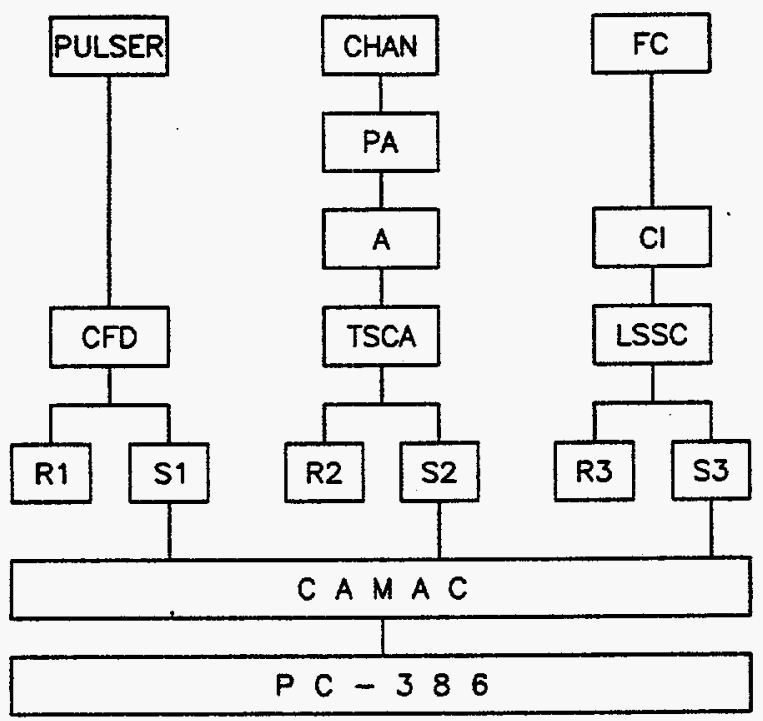

Fig. 3. Schematic diagram of the signal processing system.

The software for the acquisition and control system consists of the program ESCAM and a package of programs for displaying, plotting, and analyzing the acquired data, and for file conversions. ESCAM was written in Microsoft FORTRAN (Ver. 3.3) using also the KSC Camac Driver model 6110-1BAP, the KSC library of driver routines, and a special utility routine library. The positions of CAMAC modules in the CAMAC crate, and their characteristics are defined in a configuration file so that these can be changed without having to change the program itself. The program provides for the automated fine adjustment of the voltages that are applied to the spectrometer during the scan cycles after coarse adjustment of the starting voltages has been performed manually. The required voltages on the spectrometer are calculated automatically, depending on the desired resolution (high or low), the spectrometer constant, the electron energy range, the pass energy, and the selected power supply gain factor. A warning sound is made if the gain factor needs to be changed.

The program ESCAM is designed to minimize the amount of input required from the operator, to be simple to use, and to document all the operator initiated actions. After the first session, in which pre-defined default values of the parameters are assumed, the default values are automatically updated to the ones used in the preceding session. Selection of each menu item is logged and date/time stamped. The current sweep, current live time, and real time (in seconds clocked from the beginning of the session) are logged as well. The same logging procedure is enacted when preset count or preset time conditions are met, or when the acquisition is terminated by the operator. The values of all relevant parameters (including the accumulated spectra) are kept on the hard disk to reduce the risk of losing data due to power failure, program bugs, or inappropriate usage. In most of the cases of abnormal termination of the program (such as those resulting from power failure, calculational or input/output error, or invoking ${ }^{~} \mathrm{C}$ ), it is possible to restart ESCAM with a minimal loss of data and/or other information.

The program ESCAM traps most of the input errors and handles them without abnormal termination. Usually, re-entering the input or typing ${ }^{\wedge} Z$ will correct the problem. The message DISK WRITE ERROR will be reported and program execution stopped if the disk on which a write was attempted did not have enough space available. However, no data will be lost, so that after some cleaning of the disk space it will be possible to resume program execution.

Except when the input parameters need to be entered, the up-to-date spectrum graph, and the values of all the control parameters that define it are shown on the screen with a resolution of $320 \times 200$ pixels. The flow of the program is menu-driven and the selection is made by pressing the appropriate (pre-defined) easy-to-remember "active" keys on the alphanumeric keyboard. Low graphics resolution and keyboard input were chosen to avoid unnecessary hardware dependability of the program and to increase the speed of operation and execution.

The dats acquisition can be either stopped immediately or at the end of the current sweep. It will stop automatically if the preset count or preset time condition is met. In that case, the preset values will be automatically incremented by the initial values to enable continuation of the data acquisition. During dala acquisition, one of the program options enables the time period between successive events in the FC scaler to be continuously monitored and a warning to the operator generated whenever the period exceeds the selected time duration. Exit from the program is possible at any time without any loss of data or information, since before exiting all the data are automatically stored onto the disk and read later when the program is restarted. 
REFERENCES

1. G. A. Harrower, Rev. Sci. Instr. 26,850 (1955).
2. A. Itoh, T. Schneider, G. Schiwietz, Z. Roller, H. Platten, G. Nolte, D. Schneider, and N. Stolterfoht, J. Phys. B16, 3965 (1983).

\title{
SAMPLING CALORIMETER RESEARCH AND DEVELOPMENT
}

\author{
K. L. Wolf, A. D. Chacon, R. K. Choudhury, J. E. Simon-Gillo, R. Erkert, M. LaFrance, \\ B. Sadhwani, S. Johnson, S. Watson, S. Potter, L. Whitesell, and N. Jevtic
}

Among the various methods used for calorimeter readout, the scheme of wavelength shifting optical fiber coupled to plastic scintillator plates is one of the most efficient of the known techniques in terms of hermeticity and response. An optical fiber readout scheme for sampling calorimetry has been developed in an extension of earlier work performed at Texas A\&M for the Participant Calorimeter project. ${ }^{1}$ The original scheme outlined by Albrow, et al. ${ }^{2}$ was extended to provide much better, more reliable response from plastic scintillator plates, into each of which a single $1.5 \mathrm{~mm}$ diameter plastic optical fiber was bonded along the plane of the plate. The green fiber absorbs UV and blue light, shifts the light and transmits through the length of the clad fiber. With the proper scintillator and bonding methods, rather high response levels were obtained, up to 10-15 photoelectrons for a minimum ionizing electron through a $3 \mathrm{~mm}$ thick scintillator plate, read out with a $7 \%$ efficient phototube (in the green), or over 200 photons collected. This type of light collection in terms of photons rivals conventional lightguides, but has the advantage of a high concentration of light into a small fiber. Many plates can be read out with a single phototube, and dead regions in the calorimeter can be minimized.

For conventional hadronic calorimetry, the superior response obtained allows the use of much thinner scintillator plates and thus can allow an increase in the energy resolution through the parallel use of thinner lead plates. High resolution electromagnetic calorimeters can be more easily constructed in a compact manner and still maintain good photoelectron statistics. In the present project, instead of pursuing the scheme of one bonded fiber per plate, which is quite labor intensive and relatively expensive in requirements for the active readout device, a solution was developed whereby the light from an entire calorimeter tower is concentrated into one optical fiber. Wavelength shifting fibers run perpendicular to the scintillator plane in a tile-type structure and a single fiber can read out many plates, e.g. up to 75 here as shown in Fig. 1 for the test section under construction. (This is called a "shish kebab" design in later high energy physics developments.) In terms of an integrated solution, the current scheme essentially eliminates dead regions and allows the use of a small-area type readout devices, such as photodiodes, solid state phototubes, or as chosen here, segmented anode phototubes. The cost of the active readout device is reduced approximately one order of magnitude compared to conventional phototubes, which is most significant in a finely segmented device. In the development project it was found that sufficient photoelectron statistics are obtained despite limited fiber contact area, to allow a relatively high resolution device to be made, i.e., somewhat better than lead-glass. Problems with uniformity of response with a similar scheme ${ }^{3}$ have been solved with appropriate scintillator, reflector, and optical fiber combinations, and reduced to the few percent level. In the test section a response of 5 pe/mip is obtained from a $3 \mathrm{~mm}$ thick scintillator. Collection is made with a single $1.5 \mathrm{~mm}$ diameter unbonded fiber in perpendicular geometry.

The original goals in the construction of the test section included the proof of the technology in a cost effective device which is hermetic (for EM energy here). The use of inexpensive construction methods was emphasized with the future application for a large $10^{5}$ tower device for RHIC. ${ }^{4}$ A goal of the original proposal for high energy resolution from a sampling calorimeter, at $4.5 \% \sqrt{E}$ was later modified when simulations for RHIC were started, with particle identification (EM energy separation) being considered more important in the RHIC environment. Thus the energy resolution target was relaxed to $7.5-8 \% / \sqrt{E}$ with provision for $3-4$ fold variable longitudinal segmentation in a highly compact hermetic device. This reduced energy resolution was considered more practical for energy calibration/stabilization reasons and for compactness, since $2 \mathrm{~mm}$ thick lead could be used instead of twice the number of planes of $1 \mathrm{~mm}$ lead and 3 
mm thick scintillator. There is no reason found, however, that a $4-5 \%$ resolution device cannot be built with the present technology. It would require the use of bonded optical fibers for enhanced light collection. Bonding of the optical fiber results in an increased light collection amounting to a factor of 2-3 in all cases studied.

The layout of the test section shown in Fig. 1 features a tower area of $2.5 \mathrm{~cm} \times 2.5 \mathrm{~cm}$ and is well matched to the problem of EM shower reconstruction and neutral pion measurements. Monitoring is performed by feeding blue light into a clear fiber that runs parallel to the WLS fibers. Table I gives some measurements of the response of the coupling scheme. More details of the development of the technology for the WLS fiber optic light collection are given elsewhere. ${ }^{5}$

\section{REFERENCES}

1. J. E. Simon-Gillo, A. Farooq, M. Rawool-Sullivan, A. Ray, J. Shoemaker, J. P. Sullivan, K. L. Wolf, E. F. Barasch, J. G. Bossevain, A. Gavron, K. Holzcheiter, B. V. Jacak, T. Lopez, J. Kapustinsky, W. Sondheim, J. W. Sunier, H. Van Hecke, and B. Wolf, NIM A309, 427 (1991).

2. M. Albrow, Nucl. Phys. A461, 417e (1987).
3. H. Fessler, P. Freund, J. Gebauer, K. M. Glas, K. P. Pretzl, P. Seyboth, and J. Seyerlein, NIM A254, 26 (1987).

4. S. Aronson, M. Fatyga, X. Felgeyrolles, A. Franz, H. Gordon, W. Guryn, S. Kahn, D. Lissauer, W. Lope, S. Margetis, S. Persson, F. Plasil, P. Thomson, L. Waters, and K. L. Wolf, Proceedings of the Third Workshop on Experiments and Dletectors for RHIC, BNL 52185, 31 (1988).

5. K. L. Wolf, Progress Report for Grant DE-ASOS85ER40207, Calorimeter and TPC Measurements in Relativistic Heavy Ion Collisions (1990-91).

Table 1.

Response (in photoelectrons) of single plates of $\mathrm{BC}-408$ plastic scintillator to minimum ionizing electrons for light collection with a single $1.5 \mathrm{~mm}$ diameter WLS optical fiber running through and perpendicular to the plate. From 5 to 200 samples have been tested for each entry.

\begin{tabular}{|c|c|c|c|}
\hline $\begin{array}{l}\text { Plate } \\
\text { Area } \\
\left(\mathrm{cm}^{2}\right) \\
\end{array}$ & $\begin{array}{l}\text { Thickness } \\
\text { (mm) }\end{array}$ & Coupling & $\begin{array}{l}\text { Response } \\
\text { (pe/mip) }\end{array}$ \\
\hline 6.2 & 3 & unbonded & $5.1 \pm 0.1$ \\
\hline 25.0 & 3 & unbonded & 1.9 \\
\hline 6.2 & 6 & unbonded & 11.5 \\
\hline 25.0 & 3 & bonded & 3.9 \\
\hline 40.0 & 3 & bonded & 2.9 \\
\hline
\end{tabular}

\section{RIP CALDRIMETER}

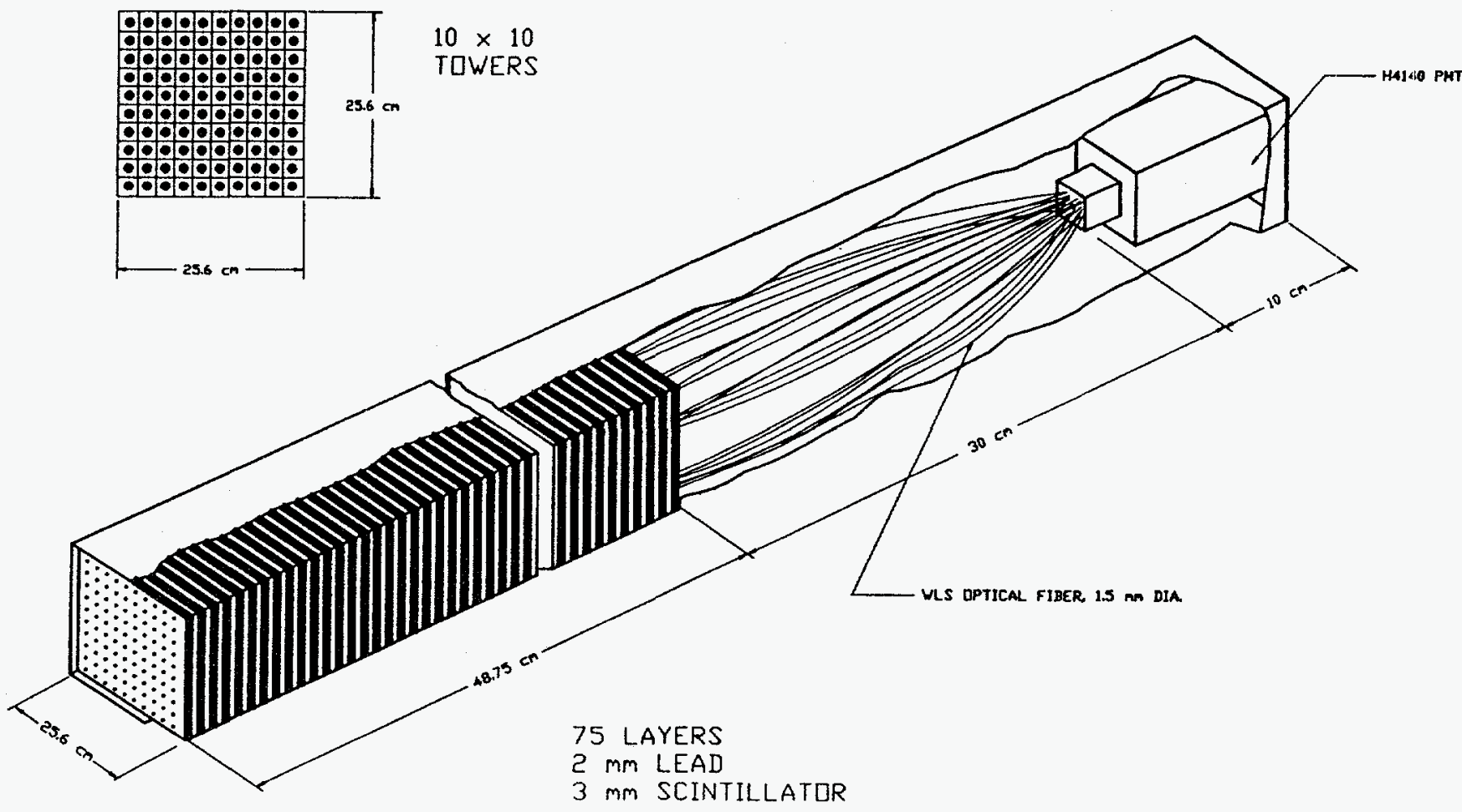




\section{A. Gagliardi, G. Kim, F. Liu, R. E. Tribble, X-L. Tu, L. Van Ausdeln, and X-G. Zhou}

During the past year, we have continued our work on the photon detector for the MEGA experiment at Los Alamos. The MEGA photon detector is designed to observe the $52.83 \mathrm{MeV}$ photon produced in a $\mu \rightarrow$ e $\gamma$ decay with an energy resolution of $1.25 \mathrm{MeV}$, a position resolution of $2 \times 5 \mathrm{~mm}^{2}$, a directional resolution of $10^{\circ}$, a time resolution of $500 \mathrm{ps}$, and an efficiency of about 5.4\%. (All quoted resolutions are FWHM.) It will consist of three independent concentric cylindrical pair spectrometers mounted within a $1.5 \mathrm{~T}$ magnetic field produced by a superconducting solenoid magnet. Each pair spectrometer includes two thin $(0.045$ radiation lengths each) $\mathrm{Pb}$ foils to convert photons into $\mathrm{e}^{+} \mathrm{e}^{-}$pairs. The two smaller pair spectrometers will have three drift chamber layers to track the $\mathrm{e}^{+} \mathrm{e}^{-}$pairs and thereby determine both their locations and their vector momenta. The third pair spectrometer will have four layers of drift chamber, together with a larger turning region, to provide better tracking information for high energy photons such as those from the $x^{0} \rightarrow 2 \gamma$ decay. The inner dritt chamber layer in each of the spectrometers includes a delay line cathode to determine the $\mathrm{z}$ coordinates needed for track reconstruction. An MWPC located between the two $\mathrm{Pb}$ layers identifies the conversion layer so that energy loss corrections may be applied, while plastic scintillators provide timing information. The scintillators and MWPC's also participate in the first-stage trigger, which requires a hit pattern that is characteristic of showers produced by photons with transverse momenta of $>35$ $\mathrm{MeV} / \mathrm{c}$. The two outer drift chamber layers also provide the information necessary for the second-stage trigger, which rejects a large fraction of the first-stage triggers that are due to accidental coincidences while passing nearly all of the true $e^{+} e^{-}$pairs associated with high energy photons.

Our group, together with a group from the University of Houston, is responsible for the design and construction of the photon detector, as well as developing the computer codes necessary for Monte Carlo simulations and data analysis. The division of labor between the two groups has the Houston group responsible for the construction of the wire chambers and their associated electronics, while our group is responsible for software development and construction of the scintillators. We also were given the task of developing the delay line cathode read-out system for the drift chambers.
During the past year, our efforts have focused primarily on software development, completion of the delay line tests, and development of electronics for the scintillators and delay line read-out. A run at LAMPF during the summer of 1990 contributed important data for these tasks, although delay line bench tests have also proven to be invaluable. Optical windows were glued onto the light guides required for the third pair spectrometer during the fall of ' 91 , thus completing the construction of all of the light guides that are needed for the experiment. In addition, we have made major contributions to the development of a proposal to measure the Michel parameter, $\rho$, using the MEGA positron detectors. The rest of this report describes our recent work on the tasks outlined above.

\section{Drift Chamber Delay Line Cathode Z Read-out}

In order to achieve our target photon resolution goals as described above, we must be able to determine the positions of the hits along the anode wires (the $\mathrm{z}$ direction) of the innermost drift chamber layers to within $\approx 5 \mathrm{~mm}$ FWHM. In last year's progress report, we described the tests which had been carried out to determine the properties of 174-cm long delay lines. Two different types of delay lines were used: (1) full length lines produced by Buckbee-Mears (BM), and (2) half length lines provided by Tech-Etch (TE) which were spliced together to form full length lines. The beam studies of the delay line test chamber in the MEGA magnet provided our first realistic look at the performance of full-length lines. However, because of the tracking ambiguities through the chambers and the subsequent uncertainty in the $z$ assignment, only a small region, the middle $20 \mathrm{~cm}$ of the delay lines, was tested in detail. After the beam studies, the delay line testing was extended to the full length of the delay lines using a collimated ${ }^{90} \mathrm{Sr}$ source. From the source tests, we found that there existed substantial signal attenuation and rise-time degradation through the long delay lines. This resulted in rapid deterioration of the $z$ resolution away from the middle of the delay line. (In the middle, both the BM and TE delay lines achieved $z$ resolution of $5 \mathrm{~mm}$ (FWHM) or better in the source tests). The deterioration was much worse for the BM delay lines than for the TE lines. The resolution (quoted as FWHM deviation from a fixed $z$ ) of the TE lines varied from less than $5 \mathrm{~mm}$ in the middle of the delay lines to about $8 \mathrm{~mm}$ 
toward the ends while the BM line also achieved $5 \mathrm{~mm}$ in the middle but was about $13 \mathrm{~mm}$ near the end. It was decided in the June ' 91 MEGA collaboration meeting that we would use the Tech-Etch delay lines on the layer two pair spectrometer and would continue investigating other options involving full length lines for the other pair spectrometers.

As we noted in last year's report, two important parameters of the delay lines are the line impedance $(Z)$ and the propagation velocity (v). $Z$ and $v$ are related to the distributed inductance $(L)$ and capacitance $(C)$ of the delay line by: $Z=\sqrt{L / C}$, and $v=1 \sqrt{\mathrm{LC}}$. In the BNL internal report by $R$. Bosshard et al., ${ }^{1}$ the delay line inductance is shown to be proportional to $a(3+5 \sqrt{s / w})$, while the capacitance is $\epsilon_{\mathrm{r}} a(1+w / e)$, where $a$ is the delay line width, $s$ is the trace to trace spacing, $w$ is the width of copper trace, $e$ is the thickness of the dielectric material, and $\epsilon_{\mathrm{r}}$ is the relative dielectric constant of the substrate. For the BM delay lines, $a=10.05 \mathrm{~mm}, s=w=250 \mu \mathrm{m}$, $e=100 \mu \mathrm{m}$, and $\epsilon_{\mathrm{r}}=3.6$, and for the $T E$ delay lines, $a=9.5 \mathrm{~mm}, \mathrm{~s}=w=250 \mu \mathrm{m}, \mathrm{e}=125 \mu \mathrm{m}$, and $\epsilon_{\mathrm{r}} \approx 3.6-4.0$. In order to increase the impedance of the line which improves both its rise-time and signal to noise ratio, it would be advantageous to increase the inductance because this will boost $Z$ and lower the transmission velocity at the same time. The inductance has already been increased several times due to the half-period shift of the top and bottom traces, ${ }^{1,2}$ and as given by the equation above, is fixed by the delay line geometry. The next possibility is to lower the capacitance, which may be accomplished by either using a substrate with a smaller dielectric constant or by increasing the thickness of the substrate. The dielectric material used in the BM delay lines is polyimide, a KAPTON-like film, and for the TE delay lines it is KAPTON film from du Pont. The dielectric constant for the two films is given as 3.6 to 4.0 at $1 \mathrm{MHz}$. Teflon, polyethylene, and rexolite (polystyrene) are possibilities for the substrate, with dielectric constants ranging from 2.1 to 2.5. They also exhibit a factor of 10 better dielectric loss than KAPTON, which would help in reducing signal loss. Unfortunately we were not able to find a vendor who could laminate a sheet of copper over these materials. The other difference between the BM and the TE lines which we found to be important was the adhesive that was used to bond the copper onto the dielectric film. The BM lines used an adhesive which had a substantially higher dielectric constant than that used by du Pont for the TE lines. Since the adhesive is $>30 \%$ of the substrate, this difference could account for the larger degradation observed in the BM lines. We were able to locate a supplier (Sheldahl) of copper laminated KAPTON that used an adhesive with similar properties to that of the TE lines. Buckbee-Mears made ten long delay lines for us with this material for testing. We found that the new lines had nearly identical performance to the spliced TE lines. However, the materials and manufacturing costs would be nearly four times that of the spliced TE lines. The labor involved in making the connections would not make up the production cost differential. Consequently, we have decided to use spliced Tech-Etch delay lines in all of the photon pair spectrometers.

The Tech-Etch delay lines for the layer two pair spectrometer were delivered to Texas A\&M in August and September '91. They required substantial preparatory work before they were ready to be mounted on the drift chamber cylinder fabricated by our collaborators from the University of Houston. The major steps in delay line preparation were:

(1) Make electrical connections from the bottom of the delay line to the top. Because the bottom side of the delay line would be inaccessible to electrical connection after the delay lines were epoxied on the chamber cylinder, we used two side by side copper pads with top-to-bottom electrical connections provided using small, $25 \mu \mathrm{m}$ thick, copper pieces as shown in Fig. 1.

(2) Splice two $87 \mathrm{~cm}$ long delay lines to form one $1 \% 4$ $\mathrm{cm}$ length. Because the TE delay lines were produced in $87 \mathrm{~cm}$ lengths, two sections neeted to tie spliced together. Figure 1 illustrates the steps involved in the splicing process. Two important cautionary points are (a) to keep the spliced delay lines straight (we had a special table with long straight parallel lines for this task), and (b) to make the solder joints facing the anode wires as smooth $8: \mathrm{s}$ possible in order to minimize any potential sparking problems.

(3) Repair small breaks in the delay line traces. Generally the quality of the TE delay lines was very good. The second photon spectrometer has 320 anode wires, which means preparing $640 \mathrm{TE}$ delay lines. Out of 640 lines only about 40 had at most one small break in a trace. These were repaired sis illustrated in Fig. 2.

After the delay lines were prepared, the actual installation on the chamber cylinder was done by our collaborators at University of Houston. The delay lines for the innermost pair spectrometer were put together this spring following the procedures outlined above. Again the quality of the lines that we received from Tech-Etch was found to be quite good. 
(a)

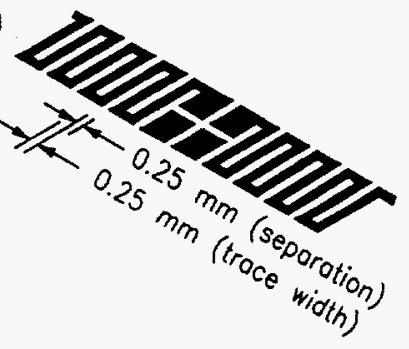

(b)

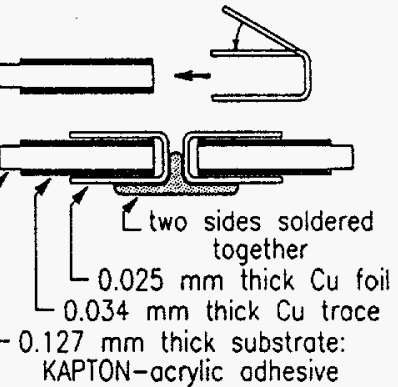

(c)

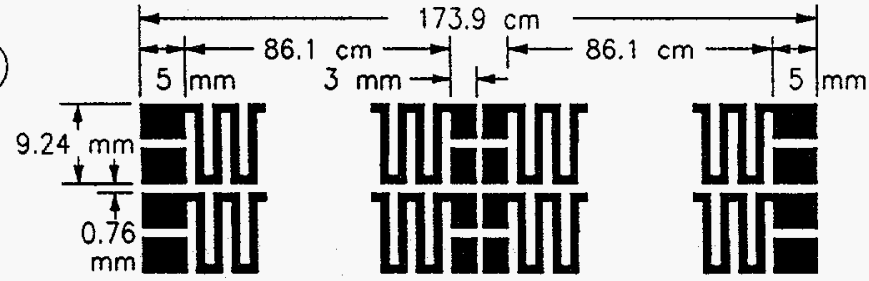

Fig. 1. A schematic view of the delay lines. Inserts (a) and (c) show the geometry of the line while insert (b) shows the steps involved in splicing two lines together.

(a)

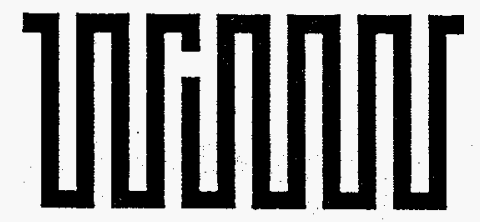

(b)

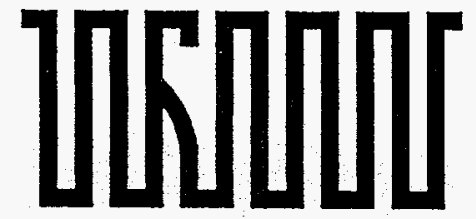

Fig. 2. Insert (a) shows the appearance of a typical broken delay line and (b) demonstrates how it would be repaired.

\section{Data Analysis}

As was noted in last year's report, a new computer workstation, a DECstation 5000/200, was delivered to the Cyclotron Institute for MEGA data analysis in December '90. This system was chosen to provide compatibility with the four-node DECstation 5000/200 microprocessor farm that was recently purchased at LAMPF both to serve as the first installment of the MEGA on-line software trigger and to provide off-line analysis capabilities. Following the installation of the Unix operating system, which was completed by early February, we began porting the MEGA REPLAY code to the DECstation. Not including the Single Event Display (SED), this code consists of well over 20,000 lines of FORTRAN, which originally was intended to run under the VAX VMS Version 5.0 operating system (and consequently included many VMS system calls), together with extensive use of CERNLIB and $Q$ routines and the Template deviceindependent graphics library. Fortunately, CERNLIB is available for the DECstation. Also the Template graphics library is available for the DECstation, and during this past year the MEGA collaboration decided to purchase the DECstation license in order to continue using the software developed assuming support from Template-based graphics. The $Q$ routines which primarily supported tape operations were rewritten.

The REPLAY code, without SED, was ported to the DECstation after about 2 months of work. With REPLAY operational for off-line use, analysis of the photon data obtained from inner Bremsstrahlung muon decay during the summer ' 90 run was able to get underway. The photon chamber that was used in the ' 90 run had several deficiencies which impacted the track finding algorithms. Three major problems were: (1) the MWPC part of the chamber was not operational; (2) the drift chamber efficiency was typically $95 \%$ or less; (3) we had no $z$-read-out. The lack of a working MWPC removed a part of the tracking routines that Monte Carlo (MC) studies indicate will be particularly useful to locate the vertex of an event for the full chamber. The rather poor efficiency resulted in many more missing cells than we had planned on in the MC studies. Also missing (broken) wires presented problems when they occurred in an important part of the event, such as near the vertex or edge. The lack of z-read-out meant that only the perpendicular momentum could be found. This deficiency made the attempt to look at photon resolution with $\pi^{\circ} \rightarrow \gamma$ $+\gamma$ useless. We began the analysis with installation and debugging of the pattern recognition code (PRC) which we had developed over the past few years based on MC simulations. Using a GKS version of the SED routine (see last year's report for details), we were able to do low statistics checks of the PRC to determine what types of problems were not being handled properly. From this visual examination, we found that nearly all of the events which were being mis-identified either had noise hits or multiple hits due to cross talk in both the chamber and the electronics. The MC based algorithms required only very minor modifications to eliminate these problems.

The next level of problem occurred when we began to do circle fitting. Determining the drift chamber cells that are to be fit for the first pass of the electron and positron pair is also a part of the PRC. Again, a few 
minor modifications were needed in the tagging routines to eliminate noise hits and to limit the acceptable width of an event where only one member of the pair made it into the third drift chamber layer. A least squares circle fitting routine, similar to the one used in the original MC analysis code, was used to determine the circle radii. In order to extract a perpendicular momentum, it was necessary to calibrate the drift chamber response. This was done using cosmic ray data that had been taken with the magnetic field on, thus including the $\vec{E} \times \vec{B}$ effect in the time to distance profiles. (We plan to use this calibration data as a "starting point" for the drift chamber calibration in the summer of '92.) While looking at the calibration data in detail, it became apparent that we could significantly reduce cross talk in the chamber (charge leaking from one drift chamber cell to a neighboring cell) by imposing a cut on the drift time. The effect of such a cut was to "clean up" events by removing extra hits. Of course, such a cut also modified the efficiency of the chamber. Thus a systematic study was carried out to determine the best cutoff, maximizing the chamber efficiency and the fits done by the track finding algorithms. In order to compare the muon data to MC simulations, the MC generator was modified to include all of the defects, except the $\vec{E} \times \vec{B}$ drift response, that we had in the test chamber. Also, positron annihilation in flight was included in the event generator and a set of events were produced. The MC data were analyzed with exactly the same routines that were used for the muon data analysis. Figure 3 shows a comparison of the inner Bremsstrahlung data from muon decay to the $M C$ prediction. A $\chi^{2}$ cut was imposed on both the $M C$ and muon data to remove the poor fits from the data set. The results are quite encouraging; we find a good fit to the data over the entire energy range. There is a high energy tail present at the few percent level in both the real and the $M C$ data sets. The $M C$ reproduces the intensity of this tail to within about $10 \%$. The high energy tail that is seen in both data sets is due in part to circle fits that tend to make a series of hits tangent to a large circle. This effect is an artifact of the circle fitting algorithm and is not something indicative of a problem in the detector system. The defects that we noted above reduced the efficiency of the system below what we have quoted. To see how this translates to a "final detector," we have run off $\mathbf{5 2 . 8 3}$ MeV MC events without the detector defects and processed them with the modified algorithms. We find an expected efficiency of about $1.7 \%$ for the innermost pair spectrometer, which is close to the value that we were expecting based on previous MC data.
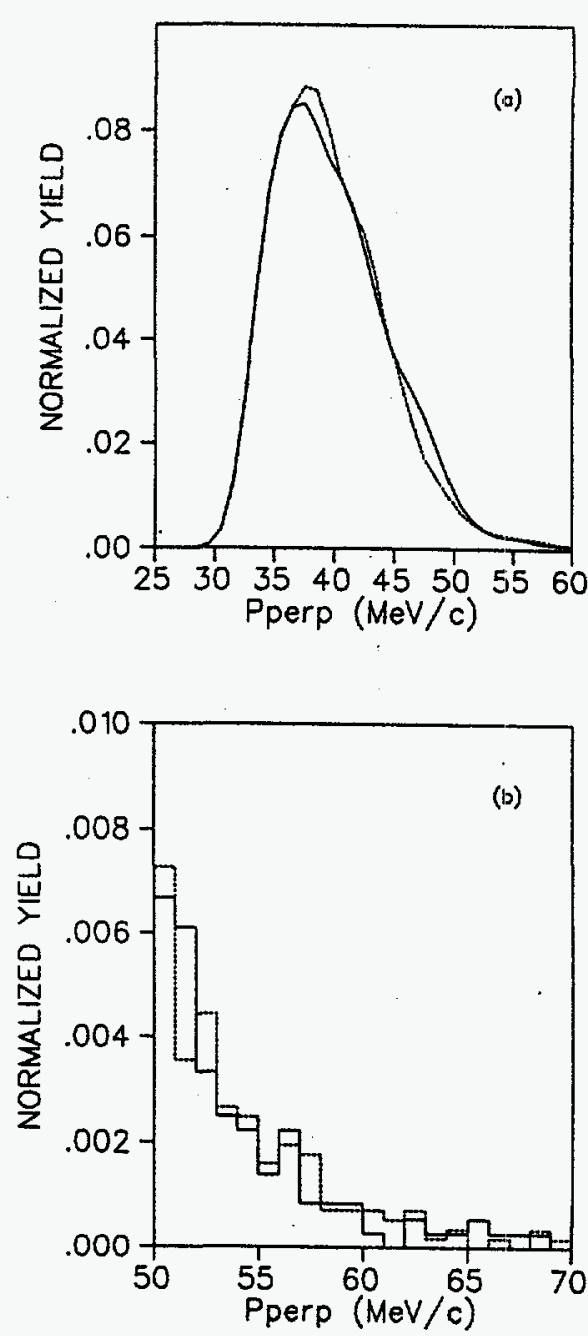

Fig. 3. Results from the analysis of the inner Bremsstrahlung data (solid line) taken during the summer of 1990 compared to the Monte Carlo generated spectrum (dashed line). Both the data and the MC results were smoothed in (a).

Most of the effort from the ' 90 data run has focused on understanding the detector performance compared to MC simulations and determining the modifications which will be needed for the on-line routines that will be used for this summer's run. Thus, not all of the modifications to the fitting routine have been implemented that would improve its performance. Two constraints that we plan to add would greatly reduce the problem of overestimatirig the perpendicular momentum for small circles. One of these is to add a constraint on the fitting routine which requires that the two "first pass" circles intersect nearly tangent to each other in the vicinity of the vertex location. A second constraint would be to limit the size of the circle that we could fit for an event where one member of the pair made it only into the second layer of drift chambers. 
This modification should help to eliminate the problem that we have observed where large circles get fit (incorrectly) to the low momentum side of these events.

Another part of the software effort that our group has undertaken is to move SED to the DECstation. As we noted above, SED was written for a VAX workstation and the program has many VMS system calls embedded in it. In addition to moving the code to the UNIX platform, several enhancements are being made. These include: (1) interfacing the SED and MEGA databases; (2) showing the reconstructed tracks for both the photon and electron arm detectors from either cosmic ray or muon generated events; (3) adding photon layers 2 and 3 to the code. Much of the work in implementing SED and adding the enhancements has already been completed. The task remaining to be completed is fitting straight through tracks (from cosmic rays or muons with the magnet off) through the chambers. Also some sections of the code are being rewritten in order to make it more efficient.

\section{Electronics}

Our group is responsible for all of the electronics necessary for the photon arm scintillators, together with the front-end electronics required to implement the delay line read-outs. A block diagram of the electronics for the photon scintillators is given in Fig. 4. The basic scheme was worked out several years ago, but we finalized the designs of the various modules this past year based on our experiences at LAMPF. The 1990 run was the first time that we had access to the actual ADC's that will be used for MEGA - 32-channel Phillips differential input ADC's. This permitted us to perform realistic checks on our linear fan-ins for the first time. We did tests with three different modules, eventually concluding that we obtained the best performance with a design that used a high-speed Burr-Brown OPA-621 op-amp as a summer, followed by a Mini-Circuits T1-6T transformer to convert the single-ended output of the op-amp into a differential signal. This design choice required us to change the configuration of the linear buffer portion of our dual threshold discriminators.

The first-stage trigger for MEGA, which is under construction at the University of Chicago, takes photon scintillator and MWPC signals as its primary inputs. The MWPC signals have a large arrival time jitter due to the finite drift time in the chamber gas. The timing constraint for the first-stage trigger is that the photon scintillator meantimer outputs must arrive in coincidence with the last of the MWPC signals: For this reason, great efforts were expended prior to the 1990 run to speed up the MWPC signals, both by using the fastest possible electronics and by minimizing cable runs. The photon scintillator signals used much longer cable runs, since they were expected to be eariy in any case. The actual timing tests during 1990

\section{PHOTON SCINTILLATOR ELECTRONICS BLOCK DIAGRAM}

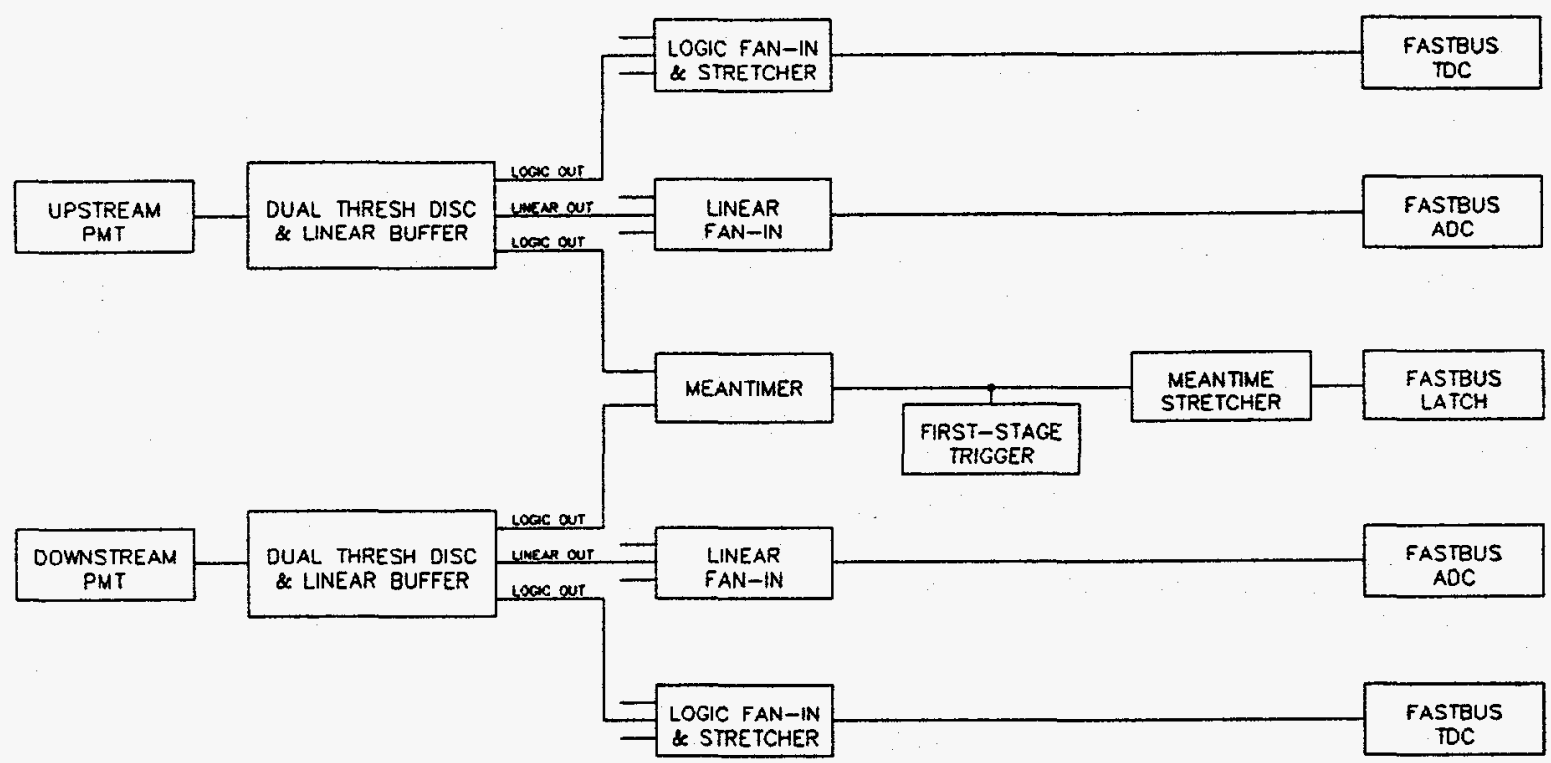

Fig. 4. Block diagram of the photon scintillator electronics. 
demonstrated that the photon scintillator signals arrived at the appropriate time, even with their circuitous routing, but only just barely. Other trigger timing studies indicated that there would be significant benefits to the experiment as a whole if the first-stage trigger could be sped up. Since then, the University of Houston group has studied various alternatives to $\mathrm{Ar}-\mathrm{C}_{2} \mathrm{H}_{6}$ as a chamber gas, in order to minimize the drift time in the MWPC gas. They have since settled on an $\mathrm{Ar}-\mathrm{CH}_{4}$-isobutane gas mixture which is substantially faster than the original gas, thus forcing us to reduce the time that it takes for the photon scintillator signals to reach the first-stage trigger.

We have completely reconfigured the layout of the photon scintillator electronics since 1990 in order to minimize cable delays. The new layout includes redesigned meantimers. Whereas the original meantimers sat in CAMAC crates, the new ones sit in the same card cages with the dual threshold discriminators. This meantimer redesign also permitted us to remove circuitry which has proven to be unnecessary and change components in order to reduce the propagation time from $24 \mathrm{nsec}$ to $15 \mathrm{nsec}$. Unfortunately, the new meantimer design places more stringent constraints on its input signals than did the original design. Previously, inputs from our scintillators could be anywhere from 15-24 nsec wide, whereas the new design requires them to be 15-18 nsec wide. We changed the width controls in the dual threshold discriminators to give us finer channel-bychannel adjustments than previously available and added pulse stretchers to our logic fan-ins to compensate for this.
The prototype delay line $\mathrm{z}$ read-out studies were all performed with differential pre-amps that were fabricaled using standard dual-in-line electronics and through-hole PC board technology. During the past year, we redesigned the pre-amp using surface mount components to reduce the space required. At the same time, we changed the power distribution. The original circuit used $+5 \mathrm{~V},-5 \mathrm{~V}$, and ground all as "working voltages." The final delay line electronics is to be integrated with the difit chamber and MWPC electronics that has been developed at the University of Houston. This requirement forces us to eliminate ground as a working voltage in order to minimize ground loop problems, and implies modest changes in the other power voltages. Figure 5 show: a schematic of the new delay line pre-amp. The entire circuit fits on a board which is only $1.14^{\prime \prime} \times 0.94^{\prime \prime}$, with components and traces on only one side and a solid ground plane on the other. The new design has the same gain as the pre-amps that were used in the prototype tests and a rise-time that is approximately $1.5 \mathrm{nsec}$ faster. Tests have also proved it to be very stable in a brciad range of configurations.

The output of the delay line pre-amps is fed to a circuit that combines the functions of a CFD, a delay, and a logic gate. Figure 6 shows a schematic of this module. The front-end CFD circuitry is identical to that which we used during our prototype tests. The delay and gate circuitry is required by the wire chamber multiplexing scheme for MEGA. The delay allows the first-staige trigger enough time to identify candidate events and oren

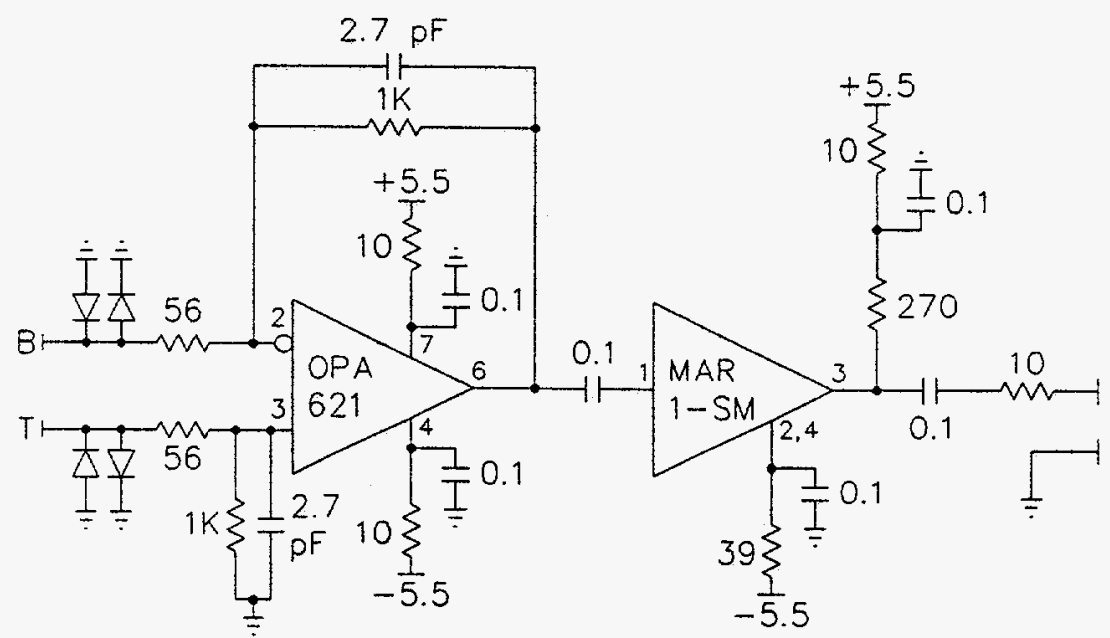

Fig. 5. A schematic diagram of the delay line surface mount differential pre-amplifier. 


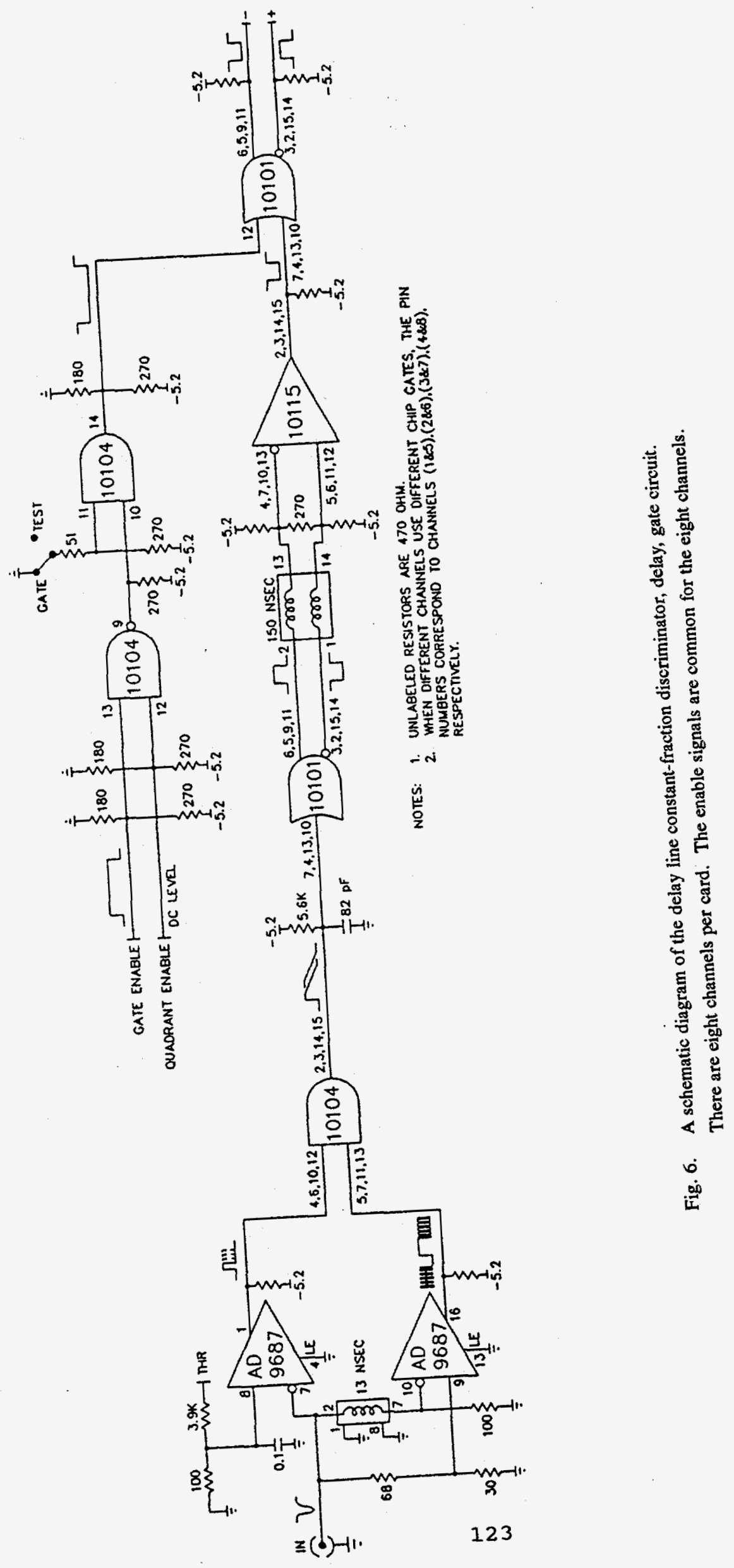


gates that indicate their location in phi around the pair spectrometer. The gates then pass on only those wire chamber hits that are near the first-stage trigger to passive OR circuits that fan them together and send them on to Fastbus TDC's. The delay and gate circuitry was originally developed at the University of Houston for use with the drift chambers and MWPC's. We copied it here for the delay lines.

At present, the scintillator and the delay line electronics for the inner two pair spectrometers are under construction. The scintillator electronics is being fabricated at Texas A\&M. The front-end delay line electronics is being built according to our designs under LAMPF supervision. The back-end delay line electronics, including OR circuits and control modules which drive the gates from the first-stage trigger, are under construction at the University of Houston.

\section{Measurement of $\rho$}

During the past year, the MEGA collaboration proposed an auxiliary experiment to improve the measurement of the Michel parameter, $\rho$ which was approved by the PAC in August ' 91 . We hope to achieve a precision of $\Delta \rho / \rho=0.001$ during the summer of ' 92 using the existing MEGA positron spectrometer. Compared with the present best value, $\rho=0.7518 \pm$ 0.0026 from an experiment performed more than 25 years ago, ${ }^{3}$ the proposed precision will be three times better than the current level. This result will further test the Standard Model and allow us to search for evidence suggesting physics beyond it. A particularly interesting example of a theoretical extension of the Standard Model with reference to $\rho$ is the Left-Right Symmetric Model ${ }^{4}$ based on the gauge group $S U(2)_{L} \times S U(2)_{R} \times U(1)$. In this model the weak charged current is carried by right as well as left handed bosons $\left(W_{L}, W_{R}\right)$. A straightforward derivation, assuming a light $\nu_{R}$, gives the following approximation for $\rho: \rho=(3 / 4)\left(1-2 \zeta^{2}\right)$, where the parameter $\zeta$ is the mixing angle between the two bosons. In this model, the result for $\rho$ is independent of the mass of $W_{R}$. At present, the best limit on $\zeta,|\zeta|<0.043(90 \%$ C.L.), comes from our current knowledge of $\rho$. It is obvious that the proposed accuracy of the $\rho$ measurement will significantly restrict the allowed mixing angle $\zeta$ and thus complement other experiments that test the left-right symmetric model. The present experiment will also permit an informed study that may lead to a second-stage experiment dedicated to improving the precision of $\rho$ to $\Delta \rho / \rho=0.0003$. Such an effort would be undertaken after the MEGA experiment has been completed.
We plan to obtain $\rho$ by measuring the positron energy spectrum in normal muon decay, $\mu \rightarrow$ e $\nu$ i with the muon decaying at rest in the MEGA detector system. A surface $\mu$ beam is produced by $\pi$ decay at rest at the Stoppid Muon Channel target. We plan to use a bearn with a momentum tuned to $25 \mathrm{MeV} / \mathrm{c}$ and a relatively smill momentum spread using the so called Souder tune." Other beam tunes are also being investigated. For example, a muon beam of reversed polarization can be obtained if the SMC is tuned for low momentum muons produced by 70 $\mathrm{MeV} / \mathrm{c}$ pions decaying in flight. It is important to have different beam tunes because they can be used to chesk systematic errors.

The positron spectrometer part of the MEGA detector consists of 176 strips of plastic scintillators and 8 MWPC's. The electron scintillators form two hollow barrels coaxial with the beam, located upstream and downstream from the target. The scintillator strips are each $30 \mathrm{~cm}$ in length and are approximately rhomboidal in cross section with an acute angle of roughly $60^{\circ}$. The MWPCs are cylindrical in shape and $126 \mathrm{~cm}$ long. Seven chambers have diameters of $12 \mathrm{~cm}$; the eighth chamber has a diameter of $22 \mathrm{~cm}$. The chambers are arranged such that the seven smaller ones surround the larger central one which is coaxial with the beam. Each chamber has a layer of anode wires sandwiched by two cathode foils with a wire to cathode foil gap of $1.75 \mathrm{~mm}$. The anode plane consists of $15 \mu$ diameter gold-plated tungsten wires with $1.3 \mathrm{~mm}$ spacing. The cathode foils are $25 \mu$ thick KAPTON with vapor-deposited copper of $200 \mathrm{~nm}$ thickness on one side. The copper surfaces are scribed into $3 \mathrm{~mm}$ wide stripes for independent read-out. These stripes are made to spiral exactly $360^{\circ}$ over the whole length of the chambers with an opposite sense of rotation on inner and outer foils. The central region of the large MWPC is a single cathode surface. The MWPCs are operated with a gas mixture of $80 \% \mathrm{CF}_{4}$ and $20 \%$ isobutane. The whole electron spectrometer is contained within the innermost $60 \mathrm{~cm}$ diameter region of the MEGA magnet. The beam enters along the axis of the solenoicl, stops in a thin vertical target and decays at rest. Under the influence of the magnetic field, a positron from the muon decay is confined to the central region. The trigger for a normal muon decay event is given by the OR of the finely segmented plastic scintillators. The track of the positron, which is a helix, can be reconstructed from the coordinates of chamber hits. The locations of MWPC wires provide the $(x, y)$ pairs, while the $z$-positions are calculated from the intersection of spiral cathode strips and the wires. The momentum of the positron is then 
derived from the parameters of the helix.

Data from the electron chambers and scintillators are encoded and stored in FASTBUS latches, TDC's and ADC's. A block of typically 25 events from a LAMPF macropulse is read from FASTBUS memory and transferred through VME to one of the microprocessors in our work station farm. The events are sorted, filtered and compressed in the workstation farm and then transferred to magnetic tape for off-line analysis.

Since the polarization of the muon beam is greater than $99 \%$, it is desirable to exclude the polarizationdependent term in the general expression for the decay amplitude ${ }^{6}$ to avoid complications caused by other Michel parameters. The high degree of symmetry between the upstream and downstream parts of the detector enables us to eliminate this term by summing the measured spectra at $\pm \cos \theta$. Therefore only the 'unpolarized' portion of the spectrum remains. Once we obtain the energy spectrum, $\rho$ can be extracted by fitting the experimental spectrum to a linear combination of two normalized Monte Carlo spectra $\Gamma_{1}^{\mathrm{MC}}(\mathrm{x})$ and $\Gamma_{2}^{\mathrm{MC}}(\mathrm{x}): \Gamma^{\mathrm{Exp}}(\mathrm{x})=\alpha \Gamma_{1}^{\mathrm{MC}}(\mathrm{x})+\beta \Gamma_{2}^{\mathrm{MC}}(\mathrm{x})$, where $\Gamma_{1}^{\mathrm{MC}}(\mathrm{x})$ is the nominal positron spectrum with $\rho=0.75$, $\Gamma_{2}^{\mathrm{MC}}(\mathrm{x})$ gives the incremental change in the energy spectrum corresponding to a deviation in $\rho$ from 0.75 , and $x=$ $\mathrm{E}_{\mathrm{e}} / \mathrm{E}_{\max }$. By a proper calibration, the Michel parameter $\rho$ can be determined from the ratio $\beta / \alpha$. At a magnetic field of $1.5 \mathrm{~T}$, which is the field used for the search of the rare decay of the muon, the electron spectrometer is sensitive to positrons with energies above $\mathrm{x}=0.57\left(\mathrm{E}_{\mathrm{c}}=30\right.$ $\mathrm{MeV}$ ). However the detector acceptance for the energy range $0.57<\mathrm{x}<0.74$ varies rapidly at this field. Thus we plan to determine $\rho$ from the shape of the spectrum between $0.75<x<1.0$, over which range a subset of the data has a flat acceptance. This is sufficient for our purpose although not ideal. The expected statistical error is $2.75 h \sqrt{\mathrm{N}}$, where $\mathrm{N}$ is the number of events. For the precision we propose to achieve it only takes about 13 hours to accumulate such a data sample. Thus the experiment is not limited by statistical errors but rather by systematic errors as discussed below.

When summing the upstream and downstream spectra it is unavoidable that there may be mismatches between the upstream and downstream detector responses, which include different detector element efficiencies, angular acceptances at the upstream and downstream ends, and different energy offsets for upstream and downstream tracks. These mismatches will lead to an imperfect cancellation of the polarization dependence. Thus a broad class of systematic errors stem from these instrumental shortcomings. Being aware of this, we have looked for additional possible sources of systematic errors. They include the transverse beam spot not centered on the target, the displacement of the target from the center of the detector system, the beam stopping distribution inside the target, the nonuniformities of the magnetic field, the efficiencies of the different detector elements, and their misalignments in both azimuthal and $\mathrm{z}$ directions. Other sources that can cause systematic errors include the accuracy of the reconstruction program, contamination from background, and physical processes such as muon decay in flight, electron-positron annihilation, and secondorder radiative corrections.

An extensive Monte Carlo study has been carried out to obtain the sensitivity of the $\rho$ measurement to these sources of systematic errors. Some of the flaws considered above turn out to make negligible contributions to the systematic error at the level of precision considered here. Those that do make significant contributions can be controlled to our tolerance using various schemes. For energy offsets the sharply falling edge of the spectrum at $52.83 \mathrm{MeV}$ provides excellent sensitivity to a mismatch. We can then correct the shift by sliding the spectra to find the best matches either between the upstream and downstream spectra or between the experimental and Monte Carlo reference spectra. We will map the magnetic field to the needed accuracy. We will use the positron straight line and helix tracks, corresponding to magnet off and on respectively, to locate the positions of the chambers as a whole as well as individual wires and cathode stripes. The chamber efficiency can be also determined using this technique. We will use a trigger requiring a hit in the cathode of the large MWPC to obtain the absolute efficiencies of the scintillators. In our data analysis we will make a fiducial cut on the ends of the scintillators demanding that the upstream and downstream regions are symmetric about the target. The centroid of the beam stopping distribution may be centered in the target by fine tuning the beam momentum to match the upstream and downstream energy shifts. If we choose a reasonably thin target and fit the spectrum with the upper limit well away from the end point, we can reduce the systematic error caused by the energy resolution smearing due to energy loss in the target to a negligible level. The physical processes mentioned above will be included in the revised Monte Carlo program for reference spectra $\Gamma_{1}^{\mathrm{MC}}(\mathrm{x})$ and $\Gamma_{2}^{\mathrm{MC}}(\mathrm{x})$. We will limit the background by utilizing an $\vec{E} \times \vec{B}$ separator to reduce the positrons in the muon beam, installing a collimator upstream from the target to cut off the excess beam and setting a time window in the electronics to reject accidental events. 


\section{REFERENCES}

1. M. Atac et al., Nucl. Instrum. Methods 140, 461 (1977).

2. R. Bosshard et al., Brookhaven National Laboratory Internal Report.
3. Particle Data Group, Phys. Lett. B 239, (1990).

4. M. A. B. Beg, R. V. Bundy, R. Mohapatra, and A. Sirlin, Phys. Rev. Lett. 38, 1252 (1977).

5. R. Holmes et al., Nucl. Instrum. Methods A303, 226 (1991).

6. L. Michel, Proc. Phys. Soc. A63, 514 (1950)

\title{
A $4 \pi$ CsI DETECTOR OF HEAVY ION REACTION PRODUCTS
}

\author{
B.Xiao, G.Derrig, K.Hagel, R.Wada, J.B.Natowitz, \\ J.Li, Y.Lou, D.Miller, D.Utley
}

We have finished the design of a nearly $4 \pi \mathrm{CsI}(\mathrm{TI})$ crystal array which was originally proposed by Vanderbilt University. ${ }^{1}$ It will be used in conjunction with the Heavy Ion Light Ion (HILI) detector built by ORNL which is now located here and with the Texas A\&M University $4 \pi$ Neutron Ball to provide a comprehensive particle detector system for the full event reconstruction of complete and incomplete fusion reactions induced by heavy ion reactions.

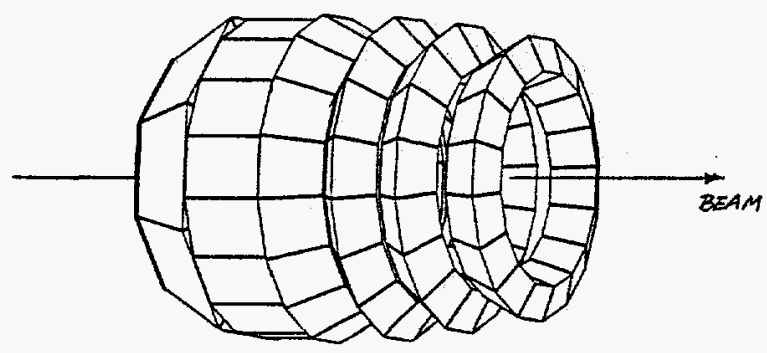

Fig. 1. Schematic three-dimensional view of 96-piece crystal array.

A schematic three-dimensional view of the 96-piece CsI crystal array is shown in Fig. 1. Light guides and PMTs are not included in this picture. Figure 2 shows a half-plane view of the detector in the vertical plane which contains the beam line. The array consists of 7 separate rings coaxial about the beam. Every CsI crystal is backed by a light guide and a PMT. Individual rings are labeled by the numbers from 1 to 7 . We have abandoned the traditional equal solid angle geometries since that approach leads to unaceeptably high multi-hit probabilities in individual charged-particle detector elements for the inverse reaction events. ${ }^{1}$ Since the angular distributions of the emitted particles will be strongly forward peaked, ${ }^{2}$ the most forward ring (ring 1) which sees the highest counting rates subtends the smallest solid angle. We uste much larger solid angles in the 2 backward rings which see much lower counting rates. The number of crystals in each individual ring, the distance from the center of each crystal to the target, the thickness of a crystal in each different ring, the polar angle coverage of each ring and the solid angle coverage of a crystal in each ring are

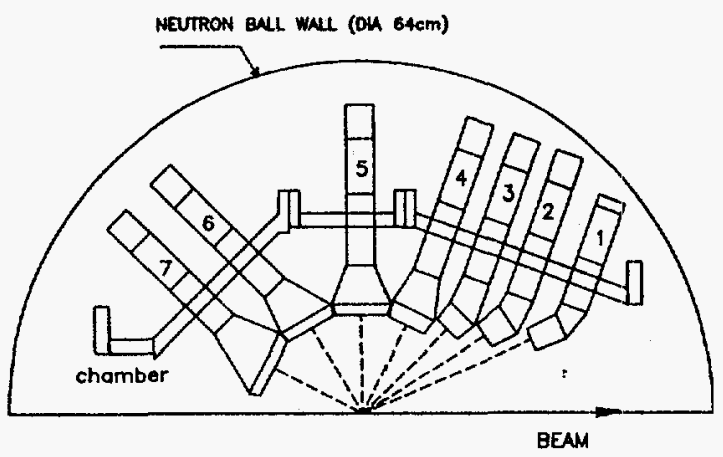

Fig. 2 Half-plane view of the detector.

shown in Table 1. The front face geometries of the individual crystals in different rings are shown in Fig. 3. The back face of each individual crystal is geometrically similar to the front face and subtends the same solid angle with respect to the target location. The opening angle of $20^{\circ}$ for the most forward ring is matched to the HILI counter geometry.

For phototubes, we have chosen the HAMAMATSU R1925 PMT because of its fast time response (typ. rise time $=2 n s)$ and short length $(L=2 \mathrm{in})$. Fig. 4 shows a side view of a crystal backed by light guide and a PMT with base. 


\begin{tabular}{|c|c|c|c|c|c|c|c|}
\hline Ring ID. & 1 & 2 & 3 & 4 & 5 & 6 & 7 \\
\hline$N$ & 16 & 16 & 16 & 16 & 16 & 8 & 8 \\
\hline$D(\mathrm{~mm})$ & 167.57 & 132.16 & 108.80 & 90.00 & 90.00 & 90.00 & 90.00 \\
\hline$T(\mathrm{~mm})$ & 30 & 25 & 20 & 15 & 10 & 10 & 10 \\
\hline$\Delta \theta($ Deg. $)$ & $20^{\circ}-27^{\circ}$ & $27^{\circ}-38^{\circ}$ & $38^{\circ}-52^{\circ}$ & $52^{\circ}-75^{\circ}$ & $75^{\circ}-105^{\circ}$ & $105^{\circ}-135^{\circ}$ & $135^{\circ}-170^{\circ}$ \\
\hline \multirow[t]{2}{*}{$\Delta \Omega(m R a d)$} & 18.675 & 38.145 & 64.322 & 136.516 & 202.939 & 370.664 & 230.089 \\
\hline & $\begin{array}{l}\text { N: } \\
D: \\
T: \\
\Delta \theta: \\
\Delta \Omega:\end{array}$ & \multicolumn{6}{|c|}{$\begin{array}{l}\text { number of crystals in each individual ring } \\
\text { distance from the center of each crystal to the target } \\
\text { thickness of each crystal } \\
\text { polar angle coverage of each ring } \\
\text { solid angle coverage of each crystal }\end{array}$} \\
\hline
\end{tabular}

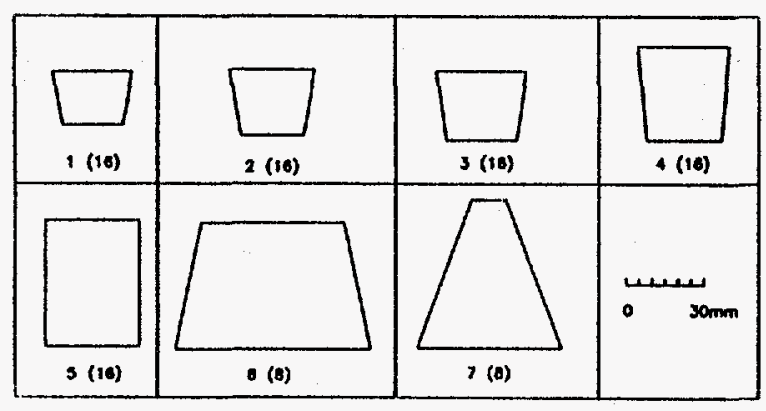

Fig. 3. The front face geometries of the individual crystals in the different rings.

This CsI(Tl) ball will cover approximately $92 \%$ of $4 \pi$. The loss in solid angle reflects: (i) the back hole for beam entrance and the front exit hole ( $\approx 3.7 \%$ of $4 \pi$ ); (ii) the gaps for optical isolation of detectors and allowance for mechanical tolerances $(\approx 2.4 \%$ of $4 \pi$ ); (iii) the removal of one crystal at $90^{\circ}$ to insert target holder $(\approx 1.6 \%$ of $4 \pi$ ).

Prototype Lucite crystal models are now nearly completed at Vanderbilt University. Detailed design of the chamber is being completed at Texas A\&M University. We will put a thin plastic scintillator foil in front each CsI crystal to produce a phoswich detector. In order to calibrate the phoswich detectors more easily and more accurately, we will put one $\Delta \mathrm{E} \mathrm{Si}$ detector in front of one crystal in each ring. Experiments with this detector should take place in the late fall of this year.

\section{REFERENCES}

1. C. F. Maguire, et al., Proposal of A Comprehensive Detector of Heavy Ion Reaction Products.

2. R. T. de Souza, et al., The MSU Miniball $4 \pi$ Fragment Detector Array. (MSU preprint, April, 1990).

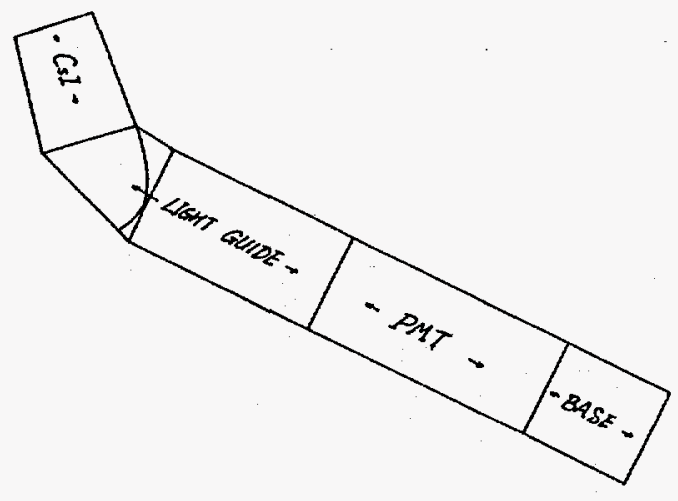

Fig. 4. Side view of a crystal backed by a light guide and a PMT with base. 


\section{SIMULATION OF A BEAM PROFILE MONITOR}

\section{J.Li, R.Wada, K.Hagel, J.B.Natowitz, X.Bin, Y.Lou, D.Miller, D.Utley}

A beam profile monitor, consisting of a thin plastic scintillator and four PMTs, is being designed to monitor the beam profile at low intensity (up $10^{-5} \mathrm{pps}$ ). The structure is shown in Fig. 1. We reconstruct the beam profile from photon numbers detected by four PMTs. The observed photon numbers are proportional to the solid angles (fluctuation must be considered), so

$$
N(o b s .)=\Omega \times N 0 \pm \sqrt{\Omega \times N O}
$$

where $\mathrm{NO}$ is the total photon number created by the scintillator. We assume $0.5 \mu \mathrm{m}$ thick NE102 plastic scintillator and ${ }^{20} \mathrm{Ne}$ beam at $10 \mathrm{Mev} / \mathrm{u}$ which emit about 600 photons (N0).

A simple Monte Carlo simulation program has been made for the simulation. In Fig. 2(a) an input profile used to generate events is shown and the reconstructed profile of Fig. 2(a) is presented in Fig. 2(b). Position resolution $(\Delta \mathrm{X})$ is also given in Fig. 3 .

This simulation indicates that we can reconstruct the beam profile with an order of $\Delta X=5 \mathrm{~mm}$ for ${ }^{20} \mathrm{NE}$ beam and with better resolution for the heavier beam.

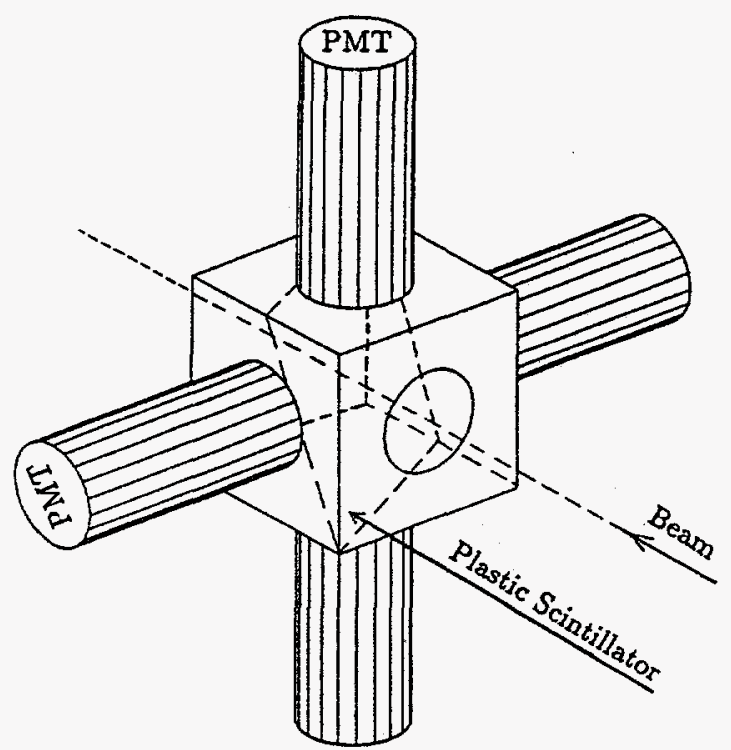

Fig. 1. Schematic view of a beam profile monitor.

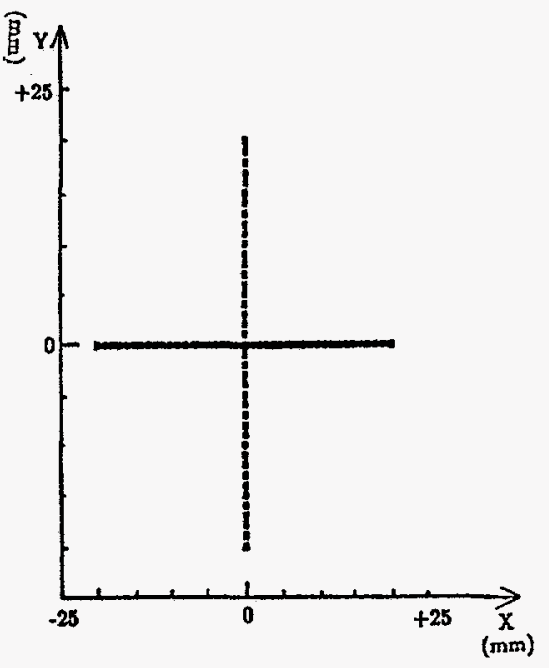

Fig. 2a. Input profile

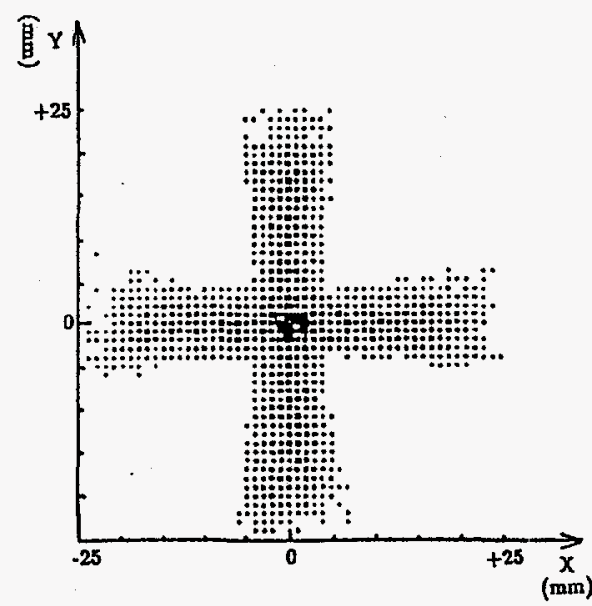

Fig. 2b. Reconstructed profile

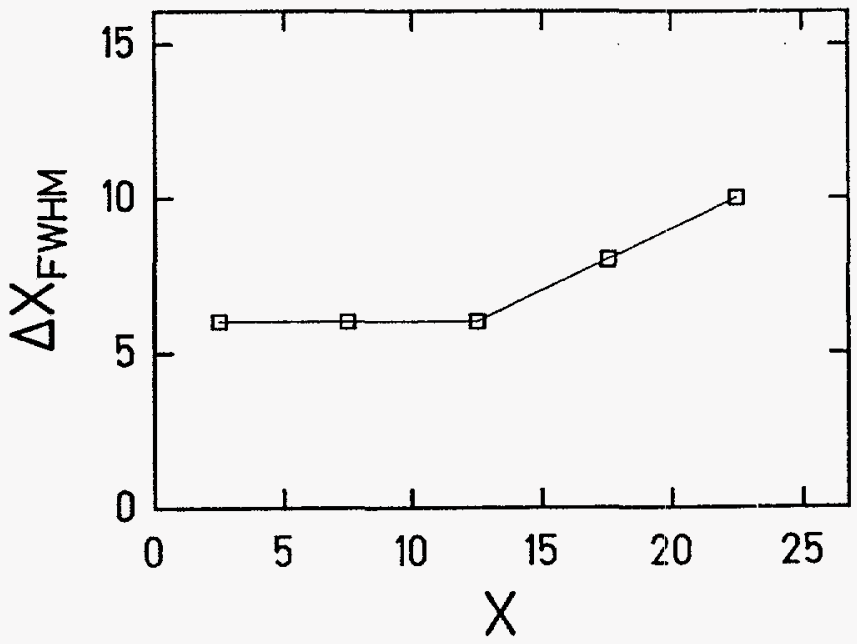

Fig. 3. Position resolution in $\mathrm{X}$ 


\section{PUBLICATIONS}




\section{ABSTRACTS OF PAPERS PUBLISHED}

April, 1991 - March, 1992

\section{PION CONTRIBUTION TO $\boldsymbol{K}^{+}$-NUCLEUS SCATTERING}

S. V. Akulinichev

Phys. Rev. Lett. 68, 290 (January, 1992)

The contribution of virtual pions to the total $\boldsymbol{K}^{+}$nucleus cross section in the $P_{L}=450-1000 \mathrm{MeV} / c$ region is found to be about $10 \%$. With this contribution, the data can be explained without the assumption of nucleons "swelling" in the nucleus.

\section{DISAPPEARANCE OF FLOW IN INTERMEDIATE- ENERGY NUCLEUS-NUCLEUS COLLISIONS}

\author{
Hong Ming Xu \\ Phys. Rev. Lett. 67, 2769 (Nov., 1991)
}

The disappearance of transverse collective flow for ${ }^{40} \mathrm{Ar}+{ }^{27} \mathrm{Al}$ collisions is studied with an improved Boltzmann-Uehling-Uhlenbeck equation. For collisions at impact parameters less than $3 \mathrm{fm}$, the predicted energy of balance, $E_{\mathrm{bal}}$, is very sensitive to the in-medium nucleonnucleon cross section, but insensitive to the equation of state. At larger impact-parameter collisions, the sensitivities to both the in-medium nucleon-nucleon cross section and the equation of state at subnuclear density become comparable. Comparisons with experimental data indicate an in-medium nucleon-nucleon cross section in the range of $25-45 \mathrm{mb}$.

\section{EFFECT OF CHIRAL RESTORATION ON KAON PRODUCTION IN RELATIVISTIC HEAVY-ION COLLISIONS}

\author{
C. M. Ko, Z. G. Wu, L. H. Xia, G. E. Brown \\ Phys. Rev. Lett 66, 2577 (May, 1991)
}

Kaon production from meson-meson annihilation is enhanced significantly because of the decrease of hadron masses in hot and dense matter as a result of the restoration of chiral symmetry. We show that this can lead to enhanced kaon yield in high-energy heavy-ion collisions.

\section{MEAN-FIELD EFFECTS AND APPARENT TEMPERATURES OF NUCLEONS AND ANTI-NUCLEONS}

\author{
V. Koch, G. E. Brown, and C. M. Ko \\ Phys. Lett. B265, 29 (1991)
}

It is shown that collective scalar and vector fields may affect particle spectra in heavy-ion collisions at the AGS energies. In model calculations, we find that in particular the slope parameter of antiprotons is changed considerably leading to a lower apparent temperature than that of protons in qualitative agreement with preliminary experimental data.

\section{PHI MESON PRODUCTION IN HADRONIC MATTER}

\author{
C. M. Ko and B. H. Sa \\ Phys. Lett. B $\underline{6}$ (1991)
}

The decrease of hadron masses in hot and dense hadronic matter as a result of the restoration of chiral symmetry increases substantially the phi meson production cross section from the reactions $\mathrm{KK} \rightarrow \phi \rho$ and $\mathrm{KA} \rightarrow \phi \mathrm{N}$. In the hydrochemical model, we show that this leads to an enhanced production of phi mesons in ultrarelativistic heavy-ion collisions and may be responsible for the large $\phi / \omega$ ratio that has been recently observed in CERN experiments.

\section{SEARCH FOR RESONANT ELECTRON-POSITRON ANNIHILATION-IN-FLIGHT}

\author{
W. H. Trzaska, H. Dejbakhsh, S. B. Dutta, Q. Li, \\ and T. M. Cormier \\ Phys. Lett. B269, 54 (1991)
}

We have studied the energy dependence of the electron-positron annihilation-in-flight cross section for diphoton invariant masses in the range of $1600-2000 \mathrm{keV}$. At the $95 \%$ confidence level, no resonances are observed implying, for example, $\Gamma_{\gamma \gamma} \Gamma_{\infty} / \Gamma<6.6 \mathrm{meV}$ at the location of the anomalous peak in $\mathrm{e}^{+} \mathrm{e}^{-}$sum energy at $\approx 800 \mathrm{keV}$ observed in heavy ion reactions. 
MEASUREMENT OF SPIN-CORRELATION

PARAMETERS $A_{L L}$ AND $A_{S L}$ IN $p-p$ ELASTIC SCATTERING FROM 500 TO $800 \mathrm{MeV}$

G. Glass, T. S. Bhatia, J. C. Hiebert, R. A. Kenefick, S. Nath, L. C. Northcliffe, W. B. Tippens, D. B. Barlow, J. J. Jarmer, J. E. Simmons, R. H. Jeppesen, and G. E. Tripard

Phys. Rev. C. 45, 35 (1992)

The spin-correlation observable $A_{L L}$ for $p-p$ elastic scattering has been measured at energies 589, 640, 692, 743 , and $793 \mathrm{MeV}$, over a c.m. angular range between $20^{\circ}$ and $100^{\circ}$. the spin observalbe $A_{L L}$ was also measured in this angular range at energies 640 and $793 \mathrm{MeV}$. At $488 \mathrm{MeV}$ both the spin observables were measured, but only near the c.m. angle of $90^{\circ}$. The data are compared with the predictions of several phase-shift analyses and previous measurements. The energy dependece of $A_{L L}$ for the c.m. angle of $90^{\circ}$ is also presented and shows no anomalous behavior. These data provide better angular coverage over five of the energies, with comparable or better statistical precision, than previous measurements of $A_{L L}$

\section{PHI MESON IN DENSE MATTER}

C. M. Ko, P. Lévai, and X. J. Qiu

Phys. Rev. C. 45, 1400 (March, 1992)

The effect of the kaon loop correction to the property of a phi meson in dense matter is studied in the vector dominance model. Using the density-dependent kaon effective mass determined from the linear chiral perturbation theory, we find that with increasing baryon density the phi meson mass is reduced slightly while its width is broadened drastically.

\section{TEMPERATURE AND MASS DEPENDENCE OF LEVEL DENSITY PARAMETER}

S. Shlomo and J. B. Natowitz

Phys. Rev. C44, 2878 (Dec., 1991)

We calculate the nuclear level density parameter over a wide range of nuclear mass and temperature, using a recently developed realistic model. The model calculations take into account the effects of finite size of the nucleus, the continuum states, the momentum and frequency dependence of the effective mass, and the variation of these effects with temperature. A reasonable agreement with recent experimental data is obtained. $n p$ ELASTIC SPIN TRANSFER MEASUREMENTS AT $788 \mathrm{MeV}$

M. W. McNaughton, K. Koch, I. Supek, N. Tanaka, K. H. McNaughton, P. J. Riley, D. A. Ambrose, J. [1. Johnson, A. Smith, G. Glass, J. C. Hiebert, L. C. Northcliffe, A. J. Simon, D. L. Adams, R. D. Ransome, D. B. Clayton, H. M. Spinka, R. H. Jeppesion, anid G. E. Tripard

Phys. Rev. C444, 2267 (December 1991)

We have measured the spin-transfer parameters $K_{L}$, $K_{S L}, K_{L S}$, and $K_{S S}$ at $788 \mathrm{MeV}$ from $47^{\circ}$ to $177^{\circ} \mathrm{c.m}$., and also uncovered a $10-16 \%$ nomalization discrepancy which affects all previous $n p$ elastic spin data from LAMPF. Results disagree significantly from previous phase-shift predictions. With the inclusions of these new data, the $N N$ phase shifts and amplitudes (isospin 0 and 1) become well determined for the first time near $800 \mathrm{MeV}$.

\section{MONOPOLE STRENGTH IN ${ }^{58} \mathrm{Ni}$}

D. H. Youngblood and Y.-W. Lui

Phys. Rev. C44, 1878 (1991)

Differential cross section data from $0^{\circ}$ to $8^{0}$ for inelastic scattering of $129 \mathrm{MeV}$ alpha particles exciting ${ }^{58} \mathrm{Ni}$ in the region of $14-22 \mathrm{MeV}$ have been analyzed to explore the existence of monopole strength at approximately $\mathrm{E}_{\mathrm{x}}=17 \mathrm{MeV}$. The angular distribution for a peak at $E_{x}=17.0 \mathrm{MeV}$ with $\Gamma=4.0 \mathrm{MeV}$ is consistent with an EO transition exhausting $19 \pm 10 \%$ of the E0 energy weighted sum rule (EWSR). The angula: distributions for peaks at $E_{x}=16.1$ and $20.4 \mathrm{MeV}$ with $\Gamma=4.7$ and $4.4 \mathrm{MeV}$, respectively, were fit by $52 \pm 10 \%$ of the E2 EWSR and a combination of E2 (6.9 $\pm 2.0 \%$ EWSR $)$ and EO (2.9 $\pm_{-2.9}^{+2.1} \mathrm{~T}$ EWSR).

\section{LEVEL STRUCTURE OF ${ }^{101} \mathrm{Tc}$ INVESTIGATED BY MEANS OF MASSIVE TRANSFER REACTIONS}

\author{
H. Dejbakhsh, G. Mouchaty, and R. P. Schmitt \\ Phys. Rev. C44, 119 (July, 1991)
}

The structure of ${ }^{101} \mathrm{TC}$ has been studied using the ${ }^{100} \mathrm{Mo}(\mathrm{Li}, \alpha 2 n)$ reaction at $49 \mathrm{MeV}$. Both particle- $\gamma$ and particle $-\gamma-\gamma$ coincidence experiments were performed. The intensities of $\gamma$ rays both in and out of the reaction plane were measured to obtain information on the $\Delta I$ of the transitions. A new band based on the $\pi g_{9 \%}$ configuration was identified for the first time. To 
investigate the shape coexistence or configuration dependent deformation in this nucleus, interacting-bosonfermion-model and cranked shell-model calculations have been performed. Cranked shell-model calculations were used to interpret the states at higher excitation energies.

\section{PION CORRELATIONS IN RELATIVISTIC HEAVY ION COLLISIONS FOR THREE SYMMETRIC SYSTEMS}

A. D. Chacon, J. A. Bistirlich, R. R. Bossingham, H. Bossy, H. R. Bowman, C. W. Clawson, K. M. Crowe, T. J. Humanic, M. Justice, P. Kammel, J. M. Kurck, S. Ljungfelt, C. A. Meyer, C. Petitjean, J. O. Rasmussen, and M. A. Stoyer

Phys. Rev. C43, 2670 (June, 1991)

The method of two-pion interferometry was used to obtain source-size and lifetime parameters for the pions produced in heavy ion collisions. Two acceptances (centered at approximately $0^{\circ}$ and approximately $90^{\circ}$, in the center of mass) were used for each of three systems, $1.70 \mathrm{GeV} /$ nucleon ${ }^{56} \mathrm{Fe}+\mathrm{Fe}, \quad 1.82 \mathrm{GeV} /$ nucleon ${ }^{40} \mathrm{Ar}+\mathrm{KCl}$, and $1.54 \mathrm{GeV} /$ nucleon ${ }^{93} \mathrm{Nb}+\mathrm{Nb}$, allowing a search for dependences on nuclear mass and viewing angle. The correlation functions were calculated by comparing data samples to event-mixed reference samples. The effect of the particle correlations on the reference samples were corrected by weighting the events appropriately to remove the residual correlation effect. The source parameters, in the nucleus-nucleus center-ofmass frame, show an oblate source (i.e., $R_{\perp}>R_{\|}$) for the lighter systems and an approximately spherical source for the heaviest system. The dependence on nuclear mass shows that $R_{\perp}$ is essentially constant (under both viewing angles), whereas $R_{\|}$for the $90^{\circ}$ (c.m.) data increases with the nuclear mass. No evidence was found for a dependence of the source size on the pion momentum.

\section{KAON PRODUCTION FROM HOT AND DENSE MATTER FORMED IN HEAVY-ION COLLISIONS}

\author{
G. E. Brown, C. M. Ko, Z. G. Wu, and L. H. Xia \\ Phys. Rev. C433, 1881 (April, 1991)
}

In heavy-ion collisions, kaons can be produced from baryon-baryon, meson-baryon, and meson-meson interactions. Simple meson-exchange models are introduced to study kaon production from these processes in the free space. These models are then extended to determine kaon production in hot, dense nuclear matter by taking into account the decreasing hadron masses as a result of the restoration of chiral symmetry and the condensation of kaons. We find that the cross sections for kaon production from all three processes are enhanced. In particular, the effect of decreasing hadron masses on kaon production from the meson-meson annihilation is most significant. In the hydrochemical model for heavy-ion collisions, we demonstrate that the observed enhancement of kaon yield in high-energy heavy-ion collisions can be explained if the medium effect is included.

\section{ENERGY LEVEL DENSITY OF NUCLEI}

\author{
Shalom Shlomo \\ Nucl. Phys. A539 (1992)
}

We consider the calculation of the nuclear level density parameter $a=\frac{1}{6} \pi^{2} \mathbf{g}_{8}\left(\epsilon_{\mathrm{F}}\right)$, where $\mathrm{g}_{\mathrm{g}}(\epsilon)$ is the smoothed single-particle level density and $\epsilon_{\mathrm{F}}$ is the Fermi energy. We present an exact (Quantum Mechanical) calculation of $g_{8}(\epsilon)$ for bound and continuum states using a Green function approach with Strutinski smoothing. The accuracy and applicability of semiclassical approximations, such as the Thomas Fermi (TF), TF $+\hbar^{2}$ correction and the local density approximation (LDA) are investigated using finite and infinite single-particle potential wells with sharp or smooth surfaces.

\section{RESPONSE OF A SAMPLING CALORIMETER TO LOW ENERGY PARTICLES}

M. W. Rawool-Sullivan, J. Shoemaker, J. Simon, J. P. Sullivan, K. L. Wolf, A. Archuleta, G. Barasch, P. Bennett, D. Bertini, J. Boissevain, L. Champs, B. Doung, D. Fox, A. Gavron, D. Hardekopf, K. Holtzscheiter, B. Jacak, S. Johnson, B. Jones, T. Lopez, R. Ruminer, W. Sondheim, J. Sunier, H. Van Hecke, and B. Wolf Nucl. Phys. A $\underline{525}$, 677C (1991)

A $\mathrm{Pb} /$ scintillator sampling calorimeter has been built for use in relativistic heavy ion experiments. The calorimeter is constructed from 59 layers of $3 \mathrm{~mm}$ scintillator separated by $1.0 \mathrm{~cm}$ layers of $\mathrm{Pb}$, with every 6 th $\mathrm{Pb}$ layer replaced with a $1.6 \mathrm{~cm}$ plate of $\mathrm{Fe}$. The read-out is done via wave-length shifting optical fibers which are connected to photomultipliers in groups. The calorimeter read-out was divided into four depth segments in the direction of the beam. The first depth segment consists of the signals from the first six scintillator layers, the second depth segment corresponds to the next six 
scintillator layers, the third depth segment consists of the next 24 scintillator layers, and the fourth (and last) depth segment corresponds to the remaining 23 scintillator layers. At the low particle energies used in the tests reported here, none of the particles reach the fourth depth segment. The calorimeter is further separated into 16 azimuthal segments with eight segments in the radial direction for a total of 512 channels. The fiber optics system minimizes the dead space within the calorimeter, but is efficient enough to have very little effect on the overall resolution of the device.

\section{THE TEXAS A\&M K500 CYCLOTRON FACILITY}

\author{
D. H. Youngblood \\ Nucl. Instrum. Methods B $\underline{56}, 991$ (1991)
}

A K500 superconducting cyclotron, following the MSU design, has been constructed with funds provided by Texas A\&M University and The Robert A. Welch Foundation. First beam was extracted in June, 1988, and the first experiments were performed shortly afterward in July using a PIG internal ion source. An electroncyclotron resonance ion source was constructed and delivered first beam to the cyclotron in November, 1989, and experiments with injected beams began immediately. Experiments are underway with existing instrumentation and a $4 \pi$ neutron calorimeter constructed for heavy ion reaction mechanism studies. A diproton spectrometer for Gamow-Teller studies is nearing completion, and a recoil mass spectrometer and 57-element $\mathrm{BaF}_{2}$ array are under construction.

\section{MARS: A STATUS REPORT}

\section{R. E. Tribble, C. A. Gagliardi, and W. Liu}

Nucl. Instrum. Methods B $\underline{56}, 956$ (1991)

We are building a momentum achromat recoil spectrometer (MARS) for use with the new K500 superconducting cyclotron at Texas A\&M University. MARS uses a unique optical design utilizing two dispersive planes to combine a momentum achromat with a recoil mass spectrometer. This configuration makes MARS applicable to a broad range of nuclear reaction studies utilizing inverse kinematics. It also leads to a system that is well matched to the range of secondary particle energies that will be produced in reactions with K500 beam. MARS will have a typical mass resolution of $\delta M / M \approx 1 / 300$, with an energy acceptance of $\pm 9 \%$
$\Delta E / E$ and a geometric solid angle of up to $9 \mathrm{msr}$. A beam swinger system will allow reaction products in the angular range $0^{\circ}$ to $30^{\circ}$ to be studied. MARS will be used to study both the excited states and decay properties of very proton- and neutron-rich nuclei. MARS will also be used to provide a reaction mechanism filter to assist investigations of the dynamics of heavy ion collisions and to produce secondary radioactive beams for reaction and spectroscopic studies of particular interest for nuclear astrophysics. We briefly describe the design of MARS, give a status report on its construction and an overview of the scientific program planned for it.

\section{A LARGE SOLID ANGLE HIGH RESOLUTION RECOIL MASS SPECTROMETER FOR USE WITH RADIOACTIVE BEAMS AT GAMMASPHERE}

\author{
T. M. Cormier, J. D. Cole, J. H. Hamilton, \\ and A. V. Ramayya \\ Nucl. Instrum. Methods B56, 546 (1991)
}

A large solid angle, high resolution, recoil mass spectrometer has been designed for use with heavy ion beams from the Holifield Heavy Ion Facility at Oak Ridge National Laboratory. The design has been coordinated with the GAMMASPHERE detector project such that the spectrometer will operate with this 110-element germanium detector array at its target or focal plane positions with minimum loss of gamma ray solid angle. The spectrometer has been designed with the specific intention of using inverse kinematic reactions. The use of inverse kinematics tremendously enhances the capabilities of the spectrometer and selected examples will be discussed including the use of low intensity radioactive beams.

\section{RECOIL-ION KINETIC ENERGIES FOR $96 \mathrm{MeV}$ Ar COLLISIONS}

O. Heber, R. L. Watson, and G. Sampolil

Nucl. Instrum. Methods B56, 232 (1991)

The kinetic energies of $\mathrm{He}, \mathrm{Ne}$, and $\mathrm{Ar}$ recoil-ions produced in single collisions with $96 \mathrm{MeV} \mathrm{Ar}^{4+}$ and $\mathrm{Ar}^{15+}$ projectiles have been determined from high resolution time-of-flight measurements. They were found to vary from the ambient thermal energy for charge 1 recoil-ions to $5 \mathrm{eV}$ for $\mathrm{Ar}^{11+}$ recoil-ions. Average impact parameters were deduced from these results. 


\section{ATOMIC PHYSICS WITH THE TEXAS A\&M ECR ION SOURCE}

R. L. Watson, D. A. Church, R. E. Tribble, L. Yang, B. B. Bandong, and T. Lotze

Nucl. Instrum. Methods B $\underline{56}, 223$ (1991)

An electron-cyclotron-resonance (ECR) ion source has been constructed to provide highly charged ions for injection into the K500 superconducting cyclotron. The source is also available approximately $30 \%$ of the time for use in experiments independent of the cyclotron. An atomic physics beamline has been constructed for this purpose and is now operational. A variety of experiments pertaining to neutralization processes, laser spectroscopy and charge exchange are currently in progress.

\section{K-PLUS L-SHELL IONIZATION OF 4TH ROW ELEMENTS BY $30 \mathrm{MeV} / \mathrm{amu}$ Ar IONS}

\author{
V. Horvat, R. L. Watson, G. Sampoll, T. Lotze, \\ and $B$. Hill
}

Nucl. Instrum. Methods B56, 61 (1991)

Spectra of $\mathbf{K}$-rays produced by collisions of 30 MeV/amu Ar ions with metallic targets of $\mathrm{Ti}, \mathrm{V}, \mathrm{Cr}, \mathrm{Fe}$, $\mathrm{Co}, \mathrm{Ni}, \mathrm{Cu}$, and $\mathrm{Zn}$ were measured with a $\mathrm{Si}(\mathrm{Li})$ detector system. The measured $K \alpha$ and $K \beta$ transition energies were compared to the results of Dirac-Fock calculations to estimate the average number of $L$-shell vacancies at the time of $\mathrm{K} \mathrm{x}$-ray emission. The target double-to-single $\mathrm{K}$ shell ionization probabilities were also determined, along with the relative probabilities of having single and double $\mathrm{K}$-shell vacancies in the projectile during passage through the metal foils.

\section{MULTIPLE IONIZATION OF He, Ne, AND Ar BY HIGH VELOCITY $\mathrm{N}^{7+}$ IONS}

\section{O. Heber, R. L. Watson, G. Sampoll, V. Horvat, B. Hill, and T. Lotze}

Nucl. Instrum. Methods B $\underline{56}, 15$ (1991)

The yields of $\mathrm{He}, \mathrm{Ne}$, and $\mathrm{Ar}$ recoil-ions produced in collisions with $10-40 \mathrm{MeV} / \mathrm{amu} \mathrm{N}^{7+}$ projectiles were measured by the time-of-flight technique. The ratio of the yields for double and single ionization of $\mathrm{He}$ and $\mathrm{Ne}$ were found to be higher than predicted by theoretical and semiempirical calculations. The role of electron correlation in multiple ionization was assessed by comparing the experimental data for $\mathrm{Ne}$ and $\mathrm{Ar}$ with the results of an independent electron approximation analysis.

\section{THE DESIGN \& CONSTRUCTION OF A Pb SCINTILLATOR SAMPLING CALORIMETER}

J. Simon-Gillo, A. Farooq, M. W. Rawool-Sullivan, A.Ray, J. Shoemaker, J. P. Sullivan, K. L. Wolf, E. F. Barasch, J. G. Boissevain, D. Fox, A. Gavron, K. Holzscheiter, B. V. Jacak, T. Lopez, J. Kapustinsky, W. Sondheim, J. W. Sunier, H. Van Hecke, and B. Wolf Nucl. Instrum. Methods A309, 427 (1991)

A $\mathrm{Pb} /$ scintillator sampling calorimeter covering the pseudorapidity interval of $\eta=0.83$ to 4.20 has been designed and constructed for Experiment 814 of Brookhaven National Laboratory. The calorimeter uses wavelength shifting optical fibers for readout. Such fibers allow the construction of a highly granular and longitudinally compact device. A novel scheme for coupling a fiber to a scintillator plate has been designed that yields a high photoelectron response. Longitudinally, the calorimeter has a depth of four interaction lengths divided into two electromagnetic sections and two hadronic sections of $0.4,0.4,1.6$, and 1.6 interaction lengths, respectively.

\section{DISSOCIATION OF MULTICHARGED CO MOLECULAR IONS PRODUCED IN COLLISIONS WITH 97-MeV Ar ${ }^{14+}$ : TOTAL-KINETIC-ENERGY DISTRIBUTIONS}

G. Sampoll, R. L. Watson, O. Heber, V. Horvat, K. Wohrer, and M. Chabot

Phys. Rev. A 45, 2903 (March, 1992)

Transient molecular ions of $\mathrm{CO}^{q^{+}}$(where $q=2-7$ ) were produced in single collisions of $97-\mathrm{MeV} \mathrm{Ar}^{14+}$ projectiles with neutral $\mathrm{CO}$ molecules. The resulting dissociation products were identified by coincidence timeof-flight spectroscopy in which the time of flight of the first ion to reach the detector and the time difference between the first ion and its partner were recorded event by event. An iterative matrix-transformation procedure was employed to convert the time-difference spectra for the prominent dissociation channels into total-kineticenergy distributions. Analysis of the total-kinetic-energy distributions and comparisons with the available data for $\mathrm{CO}^{2+}$ and $\mathrm{CO}^{3+}$ from synchrotron radiation experiments led to the conclusion that ionization by Ar-ion impact populates states having considerably higher excitation energies than those accessed by photoionization. 
THE WIDTH OF THE GIANT DIPOLE RESONANCE BUILT ON EXCITED STATES OF $\mathrm{Cu}$ COMPOUND NUCLEI

B. Fornal, F. Gramegna, G. Prete, R. Burch, G. D'Erasmo, E. M. Fiore, L. Fiore, A Pantaleo, V. Paticchio, G. Viesti, P. Blasi, N. Gelli, F. Lucarelli, M. Anghinolfi, P. Corvisiero, M. Taiuti, A. Zucchiatti, P. F. Bortignon, D. Fabris, G. Nebbia, J. A. Ruiz, M. Gonin, and J. B. Natowitz

Z. Phys. A $\underline{340}, 59$ (1991)

Continuum $\gamma$-ray spectra from the decay of ${ }^{59} \mathrm{Cu}$ formed at an excitation energy of $100 \mathrm{MeV}$ and angular momenta up to $43 \mathrm{~h}$ by means of the reaction $190 \mathrm{MeV}$ ${ }^{32} \mathrm{~S}+{ }^{27} \mathrm{Al}$ have been measured and analyzed. The parameters of the Giant Dipole Resonance (GDR) have been extracted using the statistical model. The derived GDR width confirms the sizable broadening of this resonance in ${ }^{59} \mathrm{Cu}$ already reported in our earlier investigation at $77 \mathrm{MeV}$ excitation energy $\left(J_{\text {crit }}=38 \hbar\right)$. Estimates of the GDR width have been performed in the adiabatic approximation. Predicted values account qualitatively for the experimental data of ${ }^{59} \mathrm{Cu}$ as well as of the heavier isotope ${ }^{63} \mathrm{Cu}$, in which the broadening was not seen up to $77 \mathrm{MeV}$ excitation $\left(J_{\text {crit }}=35 \hbar\right)$. The present analysis demonstrates the strong sensitivity of the GDR to spin effects in this mass region.

\section{STRANGENESS PRODUCTION IN RELATIVISTIC HEAVY-ION COLLISIONS}

\section{G. E. Brown, C. M. Ko, and K. Kubodera}

Z. Phys. A $\underline{341}, 301$ (1992)

Kaon production in relativistic heavy-ion collisions is studied. Particular attention is paid to situations in which high densities are obtained, such as in the Brookhaven AGS experiments with $14.6 \mathrm{GeV} /$ nucleon $\mathrm{Si}$ on $\mathrm{Au}$. Because of the explicit chiral-symmetry breaking terms in chiral Langrangians, kaons acquire an effective mass $m_{K}^{*}$ which goes to zero at the critical baryon density. Well before such densities, $m_{K}^{*}$ is sufficiently reduced to greatly facilitate kaon production through processes like $\pi x \rightarrow K K$. Previous expressions for the decreasing kaon masses were arrived at by linear chiral perturbation theory. Whereas we cannot systematically proceed to higher order, we use physical models to suggest how relevant quantities will behave in higher order. We present arguments that $\mathrm{m}_{\mathrm{K}}^{*}$ effectively goes to zero in the present AGS experiments.

\section{RADIOLUMINESCENCE STUDY IN KI(Eu)}

E. Belmont-Moreno, A. Menchaca-Rocha, M. E. Brandan, J. Hernandez A., M. Gonin, K. Hagel, R. Wada, and J. B. Natowitz

Rad. Eff. Def, in Solids $\underline{118}, 185$ (1991)

The luminous response to $\alpha, \beta$, and $\gamma$ rays from radioactive sources and $E / A \leq 20 \mathrm{MeV}, 3 \leq Z \leq 7$ heavy ions of $\mathrm{KI}(\mathrm{Eu})$ crystals was studied. Measurements include optical spectral response and $\mathrm{Eu}$ concentration dependence. When coupled with a transmission $S$ i surface-barrier $\Delta E$ detector, the light-output response $L$. observed from an optimized $\mathrm{KI}(\mathrm{Eu})$ sample is found to provide adequate $Z$ resolution in a $\Delta E$ vs. $L$ plot. $A$. comparison with the results of a similar study on $\mathrm{KBr}(\mathrm{Eu})$ and $\mathrm{CsI}(\mathrm{Tl})$ crystals is presented. 


\section{ABSTRACTS OF PAPERS SUBMITTED}

$$
\text { April, } 1991 \text { - March, } 1992
$$

\section{THE MULTIFRAGMENTATION OF ${ }^{40} \mathrm{Ca}+{ }^{40} \mathrm{Ca}$}

K. Hagel, M. Gonin, R. Wada, J. B. Natowitz, B. H. Sa, Y. Lou, M. Gui, D. Utley, G. Nebbia, D. Fabris, G. Prete, J. Ruiz, D. Drain, B. Chambon, B. Cheynis, D. Guinet, X. C. Hu, A. Demeyer, C. Pastor, A. Giorni, A. Lleres, P. Stassi, J. B. Viano, P. Gonthier Phys. Rev. Lett. (Submitted, 1991)

The multi-fragment emission of "completely characterized" events in the ${ }^{40} \mathrm{Ca}+{ }^{40} \mathrm{Ca}$ system at 35 $\mathrm{MeV} / \mathrm{u}$ has been compared to the predictions of several models. The observed multifragment emission is not in agreement with models based on conventional statistical binary decay, but is in agreement with both a simultaneous multifragmentation model and a sequential emission model in which expansion is treated.

\section{THE $M^{\mathrm{T}}$-SCALING IN DILEPTON SPECTRUM AS A SIGNATURE FOR QUARK-GLUON PLASMA}

M. Asakawa, C. M. Ko, P. Lévai

Phys. Rev. Lett. (Submitted, 1991)

In general, the spectrum of dilepton pairs produced in nuclear reactions depends on both its invariant mass and momentum. But under a few reasonable assumptions we show that if the quark-gluon plasma is created in ultrarelativistic heavy ion collisions, the dilepton spectrum becomes dependent only on its transverse mass $M^{T}$ and shows thus the $M^{\mathrm{T}}$-scaling.

\section{REACTION DYNAMICS AND DEUTERON PRODUCTION}

M. B. Tsang, P. Danielewicz, D. Bowman, N. Carlin, C. K. Gelbke, Y. D. Kim, W. G. Lynch, L. Phair, R. T. de Souza, H. M. Xu, and F. Zhu Phys. Rev. Lett. (Submitted, 1992)

Proton and deuteron azimuthal distributions with respect to the reaction plane have been measured as a function of associated charged-particle multiplicity for ${ }^{36} \mathrm{Ar}+{ }^{197} \mathrm{Au}$ collisions at $\mathrm{E} / \mathrm{A}=35 \mathrm{MeV}$. Quantitative corrections for the dispersion of experimentally determined reaction planes about the true reaction plane have been applied. The results are compared to predictions of a microscopic transport model.

\section{PION MULTIPLICITY AS A PROBE OF THE DECONFINEMENT TRANSITION IN HEAVY-ION COLLISIONS}

\author{
M. I. Gorenstein, S. N. Yang, C. M. Ko \\ Phys. Lett. B (In Press)
}

The hydrochemical model is used to calculate the pion multiplicity in relativistic heavy-ion collisions. Chemical reactions are explicitly taken into account in the expansion stage of the hadronic phase. It leads to the absence of chemical equilibrium among hadronic particles and a non-zero value of the pion chemical potential at thermal freeze out. We find a specific structure in the incident energy dependence of the pion multiplicity as a result of the formation of the quark-hadron mixed phase in the initial stage of the collision.

\section{ANTILAMBDA ENHANCEMENT IN ULTRARELATIVISTIC HEAVY ION COLLISIONS}

\author{
C. M. Ko, M. Asakawa, and P. Lévai \\ Phys. Lett. B (Submitted, 1991)
}

In the Walecka model, the antilambda mass in dense nuclear matter is smaller than its value in free space. This reduces the threshold for antilambda production in dense matter that forms in the compression stage of ultrarelativistic heavy ion collisions. Because of the large number of mesons produced in the collision, the process $\mathrm{KM} \rightarrow \Lambda \mathrm{N}$, where $\mathrm{M}$ denotes mesons such as the pion and the rho meson, is shown to be important and provides a plausible explanation for the observed enhancement of antilambda yield in recent experiments carried out at CERN SPS with nuclear beams. 


\section{RESIDUE TEMPERATURES AND THE NUCLEAR EQUATION OF STATE}

\section{H. M. Xu, P. Danielewicz, and W. G. Lynch} Phys. Lett. B (Submitted, 1992)

Excitation energies are calculated for heavy residues produced in central ${ }^{40} \mathrm{Ar}+{ }^{124} \mathrm{Sn}$ collisions for a range of incident energies and impact parameters using the BUU transport equation. These excitation energies are evaluated at freezeout times determined from the time dependence of the thermal excitation energy and the nucleon emission rate. Both the thermal excitation energies and temperatures, obtained assuming Fermi gas level densities, are sensitive to the nuclear equation of state and the impact parameter. Suprisingly little sensitivity is observed in the in-medium nucleon-nucleon cross section.

\section{THERMALIZATION IN NUCLEUS-NUCLEUS COLLISIONS}

F. Zhu, W. G. Lynch, D. R. Bowman, R. T. deSouaza, C. K. Gelbke, Y. D. Kim, L. Phair, M. B. Tsang, C. Williams, H. M. Xu, and J. Dinium Phys. Lett. B (Submitted, 1992)

Impact parameter dependent excited state populations of intermediate mass fragments are investigated for ${ }^{36} \mathrm{Ar}$ induced reactions on ${ }^{197} \mathrm{Au}$ at $\mathrm{E} / \mathrm{A}=35 \mathrm{MeV}$. Population inversions, indicative of non-thermal excitation mechanisms, are observed in peripheral collisions characterized by low associated charged particle multiplicities. These population inversions disappear for collisions with larger associated charged particle multiplicities, consistent with a more complete thermaliation for more complex final states. Discrepancies, observed in central collisions, indicate that the limit of local thermal equilibrium has not yet been observed.

\section{DIFFERENTIAL CROSS SECTION FOR $n-p$ ELASTIC SCATTERING IN THE ANGULAR REGION 50 $<\theta$ $<180^{\circ}$ AT $459 \mathrm{MeV}$}

L. C. Northcliffe, Mahavir Jain, M. L. Evans, G. Glass, J. C. Hiebert, R. A. Kenefick, B. E. Bonner,

J. E. Simmons, C. W. Bjork, and P. J. Riley
The differential cross section for $n-p$ elastic scattering at $459 \mathrm{MeV}$ in the c.m. angular region $50^{\circ}<\theta<180^{\circ}$ has been measured with high statistical precision and gcod relative accuracy. The uncertainty in the absolute normalization (based on the simultaneously measured yield of deuterons from the $N P \rightarrow \mathrm{d} \pi^{\circ}$ reaction) w/as initially estimated to be $\approx 7 \%$. The results agree well with back-angle data obtained independently at LAMPF but less well with results from Saclay and PPA and, except for a normalization difference of $10 \%$, are fairly well represented by a phase-shift fit. The poleextrapolation method of Chew was used to extract the pion-nucleon coupling constant $f^{2}$ from the back-angle portion of the data. The value obtained, $f^{2}=0.069$, is somewhat smaller than the values $0.0735-0.0790$ obtained from analyses of pion-nucleon scattering, tending to confirm the need for an upward renormalization of the angular distribution by $\approx 10 \%$.

\section{RHO MESON IN DENSE HADRONIC MATTER}

\author{
M. Asakawa, C. M. Ko, P. Lévai, and X. J. Qiu \\ Phys. Rev. C (Submitted, 1991).
}

The property of a rho meson at rest in dense hadronic matter is studied in the vector dominance model by including the effect of the delta-hole polarization on the pion. With the free rho meson mass in the Lagrangian, we find that both the mass and width of rho meson increase with increasing nuclear density, and that a low mass peak appears at invariant masses around three times the pion mass. Including the decreasing densitydependent rho meson mass in the Lagrangian as suggestied by the scaling law of Brown and Rho leads to smaller rho meson mass and width in dense matter. The relevance of the rho meson property in dense matter to dilepton production in heavy-ion collisions is discussed.

\section{INVARIANT QUADRUPOLE RESONANCE IN Ni ISOTOPES}

D. H. Youngblood, Y.-W. Lui, U. Garg, and R. J. Peterson

Phys. Rev. C (In Press)

Inelastic scattering of $129 \mathrm{MeV}$ alpha particles has been used to excite the giant quadrupole resonance in ${ }^{58,60,62,64} \mathrm{Ni}$. The resonance was found to exhaust $58 \pm$ $12 \%, 76 \pm 14 \%, 78 \pm 14 \%$, and $90 \pm 16 \%$ of the $E_{2}$ energy weighted sum rule, respectively for ${ }^{58,60,62,64} \mathrm{Ni}$. 


\section{$\mathrm{K}^{+}$TOTAL CROSS SECTIONS AND MEDIUM EFFECTS IN NUCLEI}

R. A. Krauss, J. C. Hiebert, Y. Mardor, E. Piasetsky, J. Alster, D. Ashery, M. A. Moinester, R. Weiss, A. I. Yavin, S. Bart, R. E. Chrien, P. H. Pile, R. Sawatta, R. J. Sutter, R. L. Stearns, T. Kishimoto, R. R. Johnson, and R. Olshevsky

Submitted to Phys. Rev. C. (Jan. 1992)

The total cross sections for $\mathrm{K}^{+}$mesons on carbon and deuterium nuclei have been measured in the momentum range $450-740 \mathrm{MeV} / \mathrm{c}$ at the Alternating Gradient Synchrotron at Brookhaven National Laboratory. The $\mathrm{K}^{+}$ meson is the least strongly interacting of available hadronic probes, with a long mean free path in nuclear matter. At low incident momentum the $\mathrm{K}^{+} \mathrm{N}$ interaction is dominated by the $S_{11}$ phase shift and varies slowly with energy. These characteristics make the $\mathrm{K}^{+}$an ideal tool for probing the nuclear volume to reveal nuclear medium effects. Measurements of the ratio of $\mathrm{K}^{+-12} \mathrm{C}$ to $\mathrm{K}^{+}-\mathrm{d}$ total cross sections have been suggested as a way to reveal possible unconventional effects of the nuclear medium. The experimental technique was of the standard transmission type using a sequential set of plastic scintillators of increasing size. The scintillator $\mathrm{K}^{+}$event rates were recorded on a set of scalers, and the scaler readings were supplemented by events recorded in a set of tracking chambers. The measured transmission cross sections were corrected for the Coulomb interaction and extrapolated to zero scattering angle. The resulting total cross section ratios are found to lie significantly above the possible range predicted by conventional optical model calculations. This indicates that novel phenomena, such as nucleon "swelling," are taking place within the nucleus and cannot be reconciled with conventional nuclear medium corrections. The data are compared to the available models and they do not well reproduce the momentum dependence of the predicted effect on the total cross section ratio. $n p$ Elastic Spin Transfer Measurements at
485 and $635 \mathrm{MeV}$

K. H. McNaughton, K. Johnston, P. J. Riley, D. A. Ambrose, P. Coffey, M. W. McNaughton, I. Supec, G. Glass, J. C. Hiebert, L. C. Northcliffe, A. J. Simon, D. J. Mercer, D. L. Adams, H. Spinka, R. H. Jeppesen, G. E. Tripard, H. Woolverton Submitted to Phys. Rev. C. (Feb., 1992)

We have measured the spin transfer parameters $K_{L L}$ $K_{S L}, K_{L S}$, and $K_{S S}$ at $635 \mathrm{MeV}$ from $50^{\circ}$ to $178^{\circ} \mathrm{c} . \mathrm{m}$. and at $485 \mathrm{MeV}$ from $74^{\circ}$ to $176^{\circ} \mathrm{c} . \mathrm{m}$. These new data have a significant impact on the phase shift analyses. There are now sufficient data near these energies to overdetermine the elastic nucleon-nucleon amplitudes.

${ }^{2} \mathrm{H}(p, n) 2 \mathrm{p}$ Spin Transfer from 305 to $788 \mathrm{MeV}$

M. W. McNaughton, I. Supek, G. Glass, J. C. Hiebert, L. C. Northeliffe, A. J. Simon, D. J. Mercer, D. L. Adams, H. Spinka, R. H. Jeppesen, G. E. Tripard, and $\mathrm{H}$. Woolverton

Submitted to Phys. Rev. C. (Feb., 1992)

Measurements of the spin-transfer parameter $\boldsymbol{K}_{L L}$ for ${ }^{2} \mathrm{H}(p, n) 2 \mathrm{p}$ at $0^{\circ}$ to calibrate the neutron beam polarization clarify a normalization discrepancy affecting $n p$ data at LAMPF. The new data are in good agreement with theoretical predictions.

\section{ENERGY DEPENDENT MEASUREMENTS OF THE $p-p$ ELASTIC ANALYZING POWER AND NARROW DIBARYON RESONANCES}

Y. Kobayashi, K. Kobayashi, T. Nakagawa, H. Shimizu, H. Y. Yoshida, H. Ohnuma, J. A. Holt, G. Glass, J. C. Hiebert, R. A. Kenefick, S. Nath, L. C. Northcliffe, A. J. Simon, S. Hiramatsu, Y. Mori, H. Sato, A. Takagi, T. Toyama, A. Ueno, and K. Imai

Submitted to Nucl. Phys. A (October, 1991)

The energy dependence of the $p-p$ elastic analyzing power has been measured using an internal target during polarized beam acceleration. The data were obtained in incident-energy steps varying from 4 to $17 \mathrm{MeV}$ over an energy range from 0.5 to $2.0 \mathrm{GeV}$. The statistical uncertainty of the analyzing power is typically less than 0.01 . A narrow structure is observed around $2.17 \mathrm{GeV}$ in two proton invariant mass distribution. A possible explanation for the structure with narrow resonances is discussed.

\section{ENERGY DEPENDENCE OF THE ANALYZING POWER FOR THE $p p \rightarrow \pi^{+} d$ REACTION IN THE ENERGY REGION $500-800 \mathrm{MeV}$}

H. Y. Yoshida, H. Shimizu, H. Ohnuma, Y. Kobayashi, K. Kobayashi, T. Nakagawa, J. A. Holt, G. Glass, J. C. Hiebert, R. A. Kenefick, S. A. Nath, L. C. Northcliffe, A. Simon, S. Hiramatsu, Y. Mori, H. Sato, A. Takagi, T. Toyama, A. Ueno, and K. Imai 
The energy dependence of the analyzing power $A_{y}$ for the $p p \rightarrow \pi^{+} d$ reaction was measured during polarized beam acceleration from 500 to $800 \mathrm{MeV}$, using an internal target inserted into the beam every acceleration cycle. The measurement was made with the pion laboratory angle fixed at $68^{\circ}$ and incident proton energy bins of 10 to $30 \mathrm{MeV}$ width, the statistical accuracy per bin being $\Delta A_{y}$ $\approx 0.06$.

\section{LIGHT EMISSION FROM A Ag TARGET BY MULTIPLY-CHARGED Ar IONS}

C. Assad, W. Liu, and R. E. Tribble

Nucl. Instrum. Methods (Submitted, 1991)

Light emitted by $\mathrm{Ag}$ atoms. following the bombardment of a $\mathrm{Ag}$ target with $\mathrm{Ar}$ ions has been studied. Light yields that include contributions from both displaced surface and sputtered atoms have been obtained as a function of incident ion beam kinetic energy and with different Ar ion charge states. No change in the light yield was observed as the incident ion charge state was varied. The light yield was found to increase by nearly a factor of 10 as the ion beam kinetic energy changed from $8 \mathrm{keV}$ to $100 \mathrm{keV}$.

\section{NEUTRON PROTON ELASTIC SCATTERING SPIN-SPIN CORRELATION PARAMETER MEASUREMENTS BETWEEN 500 AND $800 \mathrm{MeV}$ I. $C_{S L}$ and $C_{L L}$ at Backward c.m. Angles}

W. R. Ditzler, D. Hill, J. Hoftiezer, K. F. Johnson, D. Lopiano, T. Shima, H. Shimizu, H. Spinka, R. Stanek, D. Underwood, R. G. Wagner, A. Yokosawa, G. R. Burleson, J. A. Faucett, C. A. Fontenla, R. W. Garnett, C. Luchini, M. W. Rawool-Sullivan, T. S. Bhatia, G. Glass, J. C. Hiebert, R. A. Kenefick, S. Nath, L. C. Northeliffe, R. Damjanovich, J. J. Jarmer, J. Vaninetti, R. H. Jeppesen, and G. E. Tripard

Submitled to Phys. Rev. D (March, 1992)

Final results are presented for the spin-spin correlation parameters $C_{S L}$ and $C_{L L}$ for $\mathrm{np}$ elastic scattering with a polarized neutron beam incident on a polarized proton target. The beam kinetic energies are 484,634 , and $788 \mathrm{MeV}$, and the c.m. angular range is $80^{\circ}-180^{\circ}$. These data will contribute significantly to the determination of the isospin- 0 amplitudes in the energy range from $500-800 \mathrm{MeV}$.

\section{S. V. Akulinichev and S. Shlomo Submitted (Nov., 1991)}

We consider a nucleon as a bound system of quarks in the rest frame and in the infinite momentum frame. We show that the space uncertainty of a quark, with any value of the Bjorken scaling parameter $x$, remains confined to the size of the nucleon in any frame. Considering the covariant oscillator model for the quark wave function, we also show that a quark with a larger value of $x$ occupies a correspondingly larger volume of the nucleon. These two results are in direct contradiction to the conjecture thiat quarks and gluons of low $x$ and of different nucleons in a nucleus overlap (fuse). Therefore, our results cast doubt on the applicability of various models for the nuclear shadowing effect which are based on the assurnption of quark fusion at small $x$.

\section{RECOIL EFFECTS IN THE NUCLEAR SPECTRAL FUNCTION}

\section{G. M. Vagradov and S. Shlomo}

(Submitted, 1991)

We present the formal theory for the nuclear spectral function for finite nuclei taking into account the conservation of momentum and energy, i.e., imposing space-time translational invariance. In this formulation, the recoil correction term emerges naturally in the expression for the spectral function and related quantities. The consequences of the recoil effect are discussed.

\section{TRANSVERSE AND LONGITUDINAL MOMENTUM DEPENDENCES IN PAIR PRODUCTION BY AN EXTERNAL FIELD}

\author{
M. Asakawa \\ (Submitted, 1991)
}

The transverse and the longitudinal momentum dependencies of the pair production under an adiabatically exerted uniform Abelian external field are calculated without resort to the WKB method with their importance in models for the production of quark-gluon plasma in ultrarelativistic heavy ion collisions in mind. The importance of the initial condition is revealed. The peculiar nature of the boost invariant system which is expected to be approximately realized in ultrarelativistic nuclear collisions is pointed out. 


\section{VIRTUAL PION CONTRIBUTION IN HEAVY \\ ION REACTIONS}

\section{S. V. Akulinichev}

(Submitted, 1991)

We present evidence that virtual nuclear pions strongly affect the strangeness production in heavy ion reactions at high energies. The $\boldsymbol{K}^{+, o}$-production via interactions of virtual pions may be crucial at AGS energies and can be seen in the kaon rapidity distribution at CERN energies. Virtual pions are also a possible source of low $p_{1}$ enhancement of the produced pions and photons.

\section{DYNAMICAL ASPECTS OF INTERMEDIATE MASS FRAGMENT EMISSION IN THE REACTION OF ${ }^{32} \mathrm{~S}+\mathrm{Ag}$ AT $30 \mathrm{AMeV}$}

R. Wada, M. Gonin, M. Gui, K. Hagel, Y. Lou, D. Utley, B. Xiao, D. Miller, J. B. Natowitz, D. Fabris, G. Nebbia, R. Zanon, B. Chambon, B. Cheynis, A. Demeyer, D. Drain, D. Guinet, X. C. Hu, C. Pastor, K. Zaid, J. Alarja, R. Bertholet, A. Giomi, A. Lleres, C. Morand, P. Stassi, L. Schussler, B. Viano, and P. Gonthier

(Submitted, 1992)

The emission of intermediate mass fragments (IMFs) has been studied using the $4 \pi$ array, "AMPHORA." The energy spectra, the angular distributions, the multiplicities, and the charge distributions are studied inclusively as well as in coincidence with projectile-like fragments (PLFs) and other fragments. The low energy component of the inclusive fragment spectra can be reproduced by a statistical binary decay code GEMINI, although the calculated absolute cross sections are an order of magnitude smaller than the experimental IMF cross sections. At intermediate angles, the fragments are dominated by IMFs with $Z \leq 10$ both for inclusive and coincidence events and the energy spectra of the fragments show hard components which cannot be explained as statistical emission. For this non-equilibrium component strong azimuthal angular correlations are observed in IMF-PLF and IMF-IMF coincidence events. Both the energy spectra and the azimuthal angular correlations of the non-equilibrium component are well reproduced by an extended classical dynamical model. 
APPENDIX 
Rare Muon Decays, C. A. Gagliardi. AIP Conference on Intersections Between Particle and Nuclear Physics, Tucson, Arizona (May, 1991).

Neutron and Gamma-Ray Probes of the Fission Process, R. P. Schmitt, L. Cooke, B. K. Srivastava, B. J. Hurst, D. J. O'Kelly, W. Turmel, H. Utsunomiya, Invited Talk, International Workshop on Dynamical Aspects of Nuclear Fission, Smolenice, CSFR (June, 1991).

Nuclear Reaction Studies with the Texas A\&M Neutron Ball, R. P. Schmitt, T. Botting, L. Cooke, B. Hurst, D. O'Kelly, B. K. Srivastava, W. Turmel, International Conference on Advanced Nuclear Physics with Advance Techniques, Ierapetra, Crete, Greece (June, 1991).

Radioactive Ion Beam Ratio at $0^{\circ}$ from Adverse Reactions, R. E. Tribble, S. Liu, Y-W. Lui, Invited Talk, International Workshop on Unstable Nuclei, Nuclei in Nuclear Astrophysics, Tokyo, Japan (June, 1991).

New Devlopments at the Texas A\&M University Cyclotron Institute, R. E. Tribble, Seminar at IPCR (June, 1991).

Strangeness Production in Heavy-Ion Collisions, C. M. Ko, Invited Talk, International Symposium on High Energy Nuclear Collisions and Quark Gluon Plasma, Kyoto, Japan (June, 1991).

Dynamic and Statistical Properties of Hot Nuclei, J. B. Natowitz, M. Gonin, K. Ilagel, R. Wada, X. Bin, M. Gui, Y. Lou, D. Utley, T. Botting, R. K. Choudhury, L. Cooke, B. Ilurst, D. O'Kelly, R. P. Schmitt, W. Turmel, H. Utsunomiya, G. Nebbia, D. Fabris, J. A. Ruiz, G. Nardelli, M. Poggi, R. Zanon, G. Viesti, R. H. Burch, F. Gramegna, G. Prete, D. Drain, B. Chambon, B. Cheynis, D. Guinet, X. C. Hu, A. Demeyer, C. Pasteur, A. Giorni, A. Lleres, P. Stassi, B. Viano, A. Menchaca-Rocha, M. E. Brandan, and P. Gonthier, Nucleus-Nucleus Collisions IV, Kanazawa, Japan (June, 1991).

Dynamical Origins of Intermediate Velocity Light Particles in ${ }^{64} \mathrm{Zn}+{ }^{58} \mathrm{Ni}$ at $35-79 \mathrm{AMeV}$, R. Wada, M. Gonin, K. Hagel, J. B. Natowitz, M. Gui, Y. Lou, D. Utley, B. Xiao, J. C. Angélique, G. Bizard, R. Brou, D. Cussol, A. Kerambrun, J. P. Patry, J. Péter, R. Régimbart, J. C. Steckmeyer, B. Tamain, E. Vient, G. Auger, C. Cabot, E. Crema, A. Péghaire, F. SaintLaurent, P. Eudes, C. Lebrun, E. Rosato, Y. El Masri. AIP Conference: Towards a Unified Picture of Nuclear Dynamics, Nikko, Japan (1991).

Primordial ${ }^{7} \mathrm{Li}$ Synthesis Studied with Projectile Breakup Reactions, H. Utsunomiya, Conference on Nucleus-Nucleus Collisions, Kanazawa, Japan (1991).

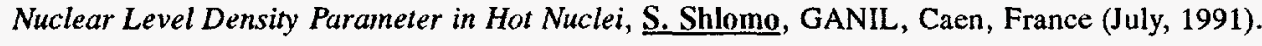

Nuclear Level Density Parameter in Hot Nuclei, S. Shlomo, University of Nantes, France (July, 1991).

Rho Meson in Dense Hadronic Matter, C. M. Ko, P. Lévai, and X. J. Qiu, Invited Talk, Second Rio de Janeiro International Workshop on Relativistic Aspects of Nuclear Physics, Rio de Janeiro, Brasil (August, 1991).

Meson Dynamics in Heavy Ion Collisions, C. M. Ko, Invited Talk, Workshop on Mesons in Nuclei, Seattle, Washington (November 1991).

Review of Dilepton Physics at Bevalac/SIS Energies Experiments, C. M. Ko, Invited Talk, Third Workshop on a Dilepton/Real Photon Program at SIS, Giessen, Germany (November 1991).

Weak Interactions and Nuclear Astrophysics Measurements with MARS, R. E. Tribble, Seminar at Princeton University (November, 1991).

Antilambda Enhancement in Heavy Ion Collisions, C. M. Ko, M. Asakawa, and P. Lévai, Invited Talk, Eighth Winter Workshop on Nuclear Dynamics, Jackson Hole, Wyoming (January 1992). 


\section{CONFERENCE PROCEEDINGS}

April 1, 1991 - March 31, 1992

The Mega Detector and Trigger System, D. D. Koetke, B. Nefkens, B. Tippens, J. Crocker, C. Wright, P. Cooper, M. Barakat, M. Zemidzic, J. Flick, E. Hungerford, K. Lan, B. Mayes, L. Pinsky, L. Tang, W. Von Witsch, J. Szymanski, J. Amann, R. Bolton, S. Carius, M. Cooper, W. Foreman, R. Harrison, G. Hart, G. Hogan, N. June, T. Kozlowski, R. Mischke, F. Naivar, J. Novak, M. Oothoudt, C. Pillai, S. Schilling, S. Stanislaus, J. Sturrock, D. Whitehouse, C. Gagliarti, G. Kim, F. Liu, R. Tribble, L. Van Ausdeln, R. Fisk, R. Manweiler, W. Stephens, D. Wright, K. Ziock, L. Piilonen, J. Markey. Proceedings of AIP Conference on Intersections Between Particle and Nuclear Physics, Tucson, Arizona (May, 1991), 243, 397.

Rare Muon Decays, C. A. Gagliardi. Proceedings of AIP Conference on Intersections Between Particle and Nuclear Physics, Tucson, Arizona (May, 1991), 243, p. 691.

Dynamical Origins of Intermediate Velocity Light Particles in ${ }^{64} \mathrm{Zn}+{ }^{58} \mathrm{Ni}$ at $35-79 \mathrm{AMeV}$, R. Wada, M. Gonin, K. llagel, J. B. Natowitz, M. Gui, Y. Lou, D. Utley, B. Xiao, J. C. Angélique, G. Bizard, R. Brou, D. Cussol, A. Kerambrun, J. P. Patry, J. Péter, R. Régimbart, J. C. Steckmeyer, B. Tamain, E. Vient, G. Auger, C. Cabot, E. Crema, A. Péghaire, F. SaintLaurent, P. Eudes, C. Lebrun, E. Rosato, Y. El Masri. Proceedings of AlP Conference: Towards a Unified Picture of Nuclear Dynamics, Nikko, Japan (1991), 250, p. 452.

Radioactive Ion Beam Ratio at $0^{\circ}$ from Adverse Reactions, R. E. Tribble, S. Liu, Y-W. Lui, Proceedings of International Workshop on Unstable Nuclei, Nuclei in Nuclear Astrophysics, Tokyo, Japan, (June, 1991) World Scientific (in press).

Neutron and Gamma-Ray Probes of the Fission Process, R. P. Schmitt, L. Cooke, B. K. Srivastava, B. J. Hurst, D. J. O'Kelly, W. Turınel, H. Utsunomiya, Invited Talk, Proceedings of International Workshop on Dynamical Aspects of Nuclear Fission, Smolenice, CSFR (June, 1991).

Nuclear Reaction Studies with the Texas A\&M Neutron Ball, R. P. Schmitt, T. Botting, L. Cooke, B. Ilurst, D. O'Kelly, B. K. Srivastava, W. Turmel, Proceedings of International Conference on Advanced Nuclear Physics with Advance Techniques, lerapetra, Crete, Greece (June, 1991).

Radioactive Ion Beam Ratio at $0^{\circ}$ from Adverse Reactions, R. E. Tribhle, S. Liu, Y-W. Lui, Invited Talk, Proceedings of International Workshop on Unstable Nuclei, Nuclei in Nuclear Astrophysics, Tokyo, Japan (June, 1991).

Strangeness Production in Heavy-Ion Collisions, C. M. Ko, Invited Talk, Proceedings of International Symposium on High Energy Nuclear Collisions and Quark Gluon Plasma, Kyoto, Japan (June, 1991), p. 85.

Dynamic and Statistical Properties of Hot Nuclei, J. B. Natowitz, M. Gonin, K. IIagel, R. Wada, X. Bin, M. Gui, Y. Lou, D. Utley, T. Botting, R. K. Choudhury, L. Cooke, B. Hurst, D. O'Kelly, R. P. Schmitt, W. Turmel, II. Utsunomiya, G. Nebbia, D. Fabris, J. A. Ruiz, G. Nardelli, M. Poggi, R. Zanon, G. Viesti, R. H. Burch, F. Gramegna, G. Prete, D. Drain, B. Chambon, B. Cheynis, D. Guinet, X. C. Hu, A. Demeyer, C. Pasteur, A. Giorni, A. Lleres, P. Stassi, B. Viano, A. Menchaca-Rocha, M. E. Brandan, and P. Gonthier, Proceedings of Nucleus-Nucleus Collisions IV, Kanazawa, Japan (June, 1991).

Dynamical Origins of Intermediate Velocity Light Particles in ${ }^{64} \mathrm{Zn}+{ }^{58} \mathrm{Ni}$ at $35-79$ AMeV, R. Wada, M. Gonin, K. Hagel, J. B. Natowitz, M. Gui, Y. Lou, D. Utley, B. Xiao, J. C. Angélique, G. Bizard, R. Brou, D. Cussol, A. Kerambrun, J. P. Patry, J. Péter, R. Régimbart, J. C. Steckmeyer, B. Tamain, E. Vient, G. Auger, C. Cabot, E. Crema, A. Péghaire, F. SaintLaurent, P. Eudes, C. Lebrun, E. Rosato, Y. El Masri. Proceedings of AIP Conference: Towards a Unified Picture of Nuclear Dynamics, Nikko, Japan (1991).

Primordial ${ }^{7}$ Li Synthesis Studied with Projectile Breakup Reactions, II. Utsunomiya, Proceedings of Conference on NucleusNucleus Collisions, Kanazawa, Japan (1991).

Rho Meson in Dense Hadronic Matter, C. M. Ko, P. Lévai, and X. J. Qiu, Invited Talk, Proceedings of Second Rio dle Janeiro International Workshop on Relativistic Aspects of Nuclear Physics, Rio de Janeiro, Brasil (August, 1991). 
Meson Dynamics in Heavy Ion Collisions, C. M. Ko, Invited Talk, Proceedings of Workshop on Mesons in Nuclei, Seattle, Washington (November 1991).

Review of Dilepton Physics at Bevalac/SIS Energies Experiments, C. M. Ko, Invited Talk, Proceedings of Third Workshop on a Dilepton/Real Photon Program at SIS, Giessen, Germany (November 1991).

Antilambda Enhancement in Heavy Ion Collisions, C. M. Ko, M. Asakawa, and P. Lévai, Invited Talk, Proceedings of Eighth Winter Workshop on Nuclear Dynamics, Jackson Hole, Wyoming (January 1992). 
Transport Model for Heavy Ion Collisions, C. M. Ko, National Taiwan University, Taiwan, Japan (May, 1991).

Probing Pion Dynamics in Dense Matter with Dilpetons, C. M. Ko, National Taiwan University, Taiwan, Japan (May, 1991 ).

Hadronic Matter under Extreme Conditions, C. M. Ko, National Taiwan University, Taiwan, Japan (May, 1991 ).

Hadronic Matter under Extreme Conditions, C. M. Ko, National Tsing Hua University, Taiwan, Japan (June, 1991).

Hadronic Matter under Extreme Conditions, C. M. Ko, Academia Sinica, Taiwan, Japan (June, 1991).

New Developments at the Texas A\&M University Cyclotron Institute, R. E. Tribble, IPCR (June, 1991).

Nuclear Level Density Parameter in Hot Nuclei, S. Shlomo, GANIL, Caen, France (July, 1991).

Nuclear Level Density Parameter in Hot Nuclei, S. Shlomo, Univeristy of Nantes, Nantes, France (July, 1991).

Probing Dense Hadronic Matter in Heavy lon Collisions, C. M. Ko, State University of New York at Stony Brook, Stony Brook, NY (November, 1991).

Weak Interactions and Nuclear Astrophysics Measurements with MARS, R. E. Tribble, Princeton University (November, 1991).

Nuclear Reaction Studies with the Texas A\&M Neutron Ball, R. P. Schmitt, Michigan State University, East Lansing, MI (February, 1992). 


\section{FACULTY AND RESEARCH GROUP LEADERS}

Thomas M. Cormier, ${ }^{1}$ Professor of Physics

Carl A. Gagliardi, Associate Professor of Physics

John C. Hiebert, Professor of Physics

Che Ming Ko, Professor of Physics

Joseph B. Natowitz, Professor of Chemistry, Director ${ }^{2}$

Lee Northcliffe, Professor of Physics

Richard P. Schmitt, ${ }^{3}$ Professor of Chemistry

Robert E. Tribble, Professor of Physics

Rand L. Watson, Professor of Chemistry

Kevin L. Wolf, Professor of Chemistry

Dave H. Youngblood, Professor of Physics, Director ${ }^{4}$

\section{VISITING SCIENTISTS}

S. V. Akulinichev, ${ }^{5}$ Institute for Nuclear Research, Moscow, Russia

R. K. Choudhury, ${ }^{6}$ Bhabha Atmomic Research Centre, Bombay, India

\section{RESEARCH STAFF}

Jeff Bronson, Senior Scientist

Haydeh Dejbakhsh, Assistant Research Scientist Sanghamitra Dutta, ${ }^{7}$ Visiting Assistant Professor Yiu-Wing Lui, Research Scientist

John C. Hagel, ${ }^{8}$ Assistant Research Scientist

Don May, Accelerator Physicist

Georges Mouchaty, Accelerator Physicist

Shalom Shlomo, Senior Scientist

John Shoemaker, Research Assistant

Hiroaki Utsunomiya, ${ }^{9}$ Associate Research Scientist

Ryoichi Wada, Associate Research Scientist

\section{ENGINEERING STAFF}

Walter Chapman, Mechanical Engineer

Greg Derrig, Mechanical Engineer

Robert Gutierrez, ${ }^{10}$ Electrical Engineer

Robert C. Rogers, Chief Engineer

Peter Smelser, Cryogenics Engineer

\begin{tabular}{ll}
\hline${ }^{1}$ Through 8-31-91 & ${ }^{7}$ Leave from 9-1-91 \\
${ }^{2}$ From 9-1-91 & ${ }^{8}$ From 11-1-91 \\
${ }^{3}$ From 9-1-91 & ${ }^{9}$ Leave 4-1-91 - 12-31-91 \\
${ }^{4}$ Through 8-31-91 & ${ }^{10}$ From 8-1-91 \\
5From 9-17-91-12-31-91 & ${ }^{11}$ From 9-1-91 \\
${ }^{6}$ From 4-1-91 & ${ }^{12}$ Through 9-1-91
\end{tabular}

\section{RESEARCH ASSOCIATES}

\author{
Masayuki Asakawa ${ }^{11}$ \\ Marin Chabot ${ }^{12}$ \\ Aaron Chacon \\ John C. Hagel ${ }^{13}$ \\ Vladimir Horvat \\ George Kim \\ Damir Latypov ${ }^{14}$ \\ Peter Levails \\ Qingli $\mathrm{Li}^{16}$
}

\author{
Wenzhong Liu \\ R. Parameswaran ${ }^{17}$ \\ Xi-Jun Qiu ${ }^{18}$ \\ Gabriel Sampoll-Ramirez ${ }^{19}$ \\ Wladyslaw Trzaska ${ }^{20}$ \\ Xiao-Lin $\mathrm{Tu}^{21}$ \\ Karine Wohrer ${ }^{22}$ \\ Hongming $\mathrm{Xu}$ \\ Xiao-Gang Zhou ${ }^{23}$
}

\title{
GRADUATE STUDENTS
}

Tye Botting
Larry Cooke
Ronald Erkert
Bradley Hurst
Harry Jabs
Dorothy Miller
Chemistry

\author{
Donna O'Kelly \\ Gabriel Sampoll-Ramirez ${ }^{26}$ \\ Jehanne Simon ${ }^{27}$ \\ William Turmel \\ Dennis Utley \\ Lane Whitesell \\ Serena $\mathrm{Wu}^{28}$
}

Physics

Gulnara Ajuopova ${ }^{29}$
Chahriar Assad
Angela Betker
Xiao Bin
Phil Bowman
Xushan Fang
Stephen Fitzsimmons
Mei Gui $^{31}$
Zurab Khviengia

Fan Liu

Yunian Lou

Ruben Minasian ${ }^{33}$

David Semon ${ }^{34}$

Anthony Simon

Leo Van Ausdeln

Li Xiong 35

Anastasia Zaruba

\begin{tabular}{|c|c|}
\hline${ }^{13}$ Through $10-31-91$ & ${ }^{25}$ From 5-15-91 \\
\hline${ }^{14}$ From 1-1-92 & ${ }^{26}$ Through 2-1-92 \\
\hline${ }^{15}$ Through 1-31-92 & ${ }^{27}$ Through 11-1-91 \\
\hline${ }^{16}$ Through 10-25-91 & ${ }^{28}$ From $9-1-91-1-31-92$ \\
\hline${ }^{17}$ From 3-1-92 & ${ }^{29}$ From $1-16-92$ \\
\hline${ }^{18}$ Through 9-1-91 & ${ }^{30}$ Through 5-31-91 \\
\hline${ }^{19}$ From 2-2-92 & ${ }^{31}$ Through 12-31-91 \\
\hline${ }^{20}$ Through $11-30-91$ & ${ }^{32}$ From $8-19-91-12-31-91$ \\
\hline${ }^{21}$ From 1-1-92 & ${ }^{33}$ From $8-19-91-12-31-91$ \\
\hline${ }^{22}$ Through 9-19-91 & ${ }^{34}$ Through 9-1-91 \\
\hline${ }^{23}$ From $1-1-92$ & ${ }^{35}$ Through $11-30-91$ \\
\hline${ }^{24}$ Through 1-9-92 & \\
\hline
\end{tabular}


April 2

Rene Bellwied, State University of New York, Stony Brook, NY

April 23

Alex Szanto de Toledo, University of Sao Paulo, Sao Paulo, Brazil

May 7

Charles Maguire, Vanderbilt University, Nashville, TN

July 3

D. Guerreau, GANIL, Caen, France

July 18

Hans Feldmeier, Gesellshaft für Schwerionenforschung, Federal Republic of Germany

August 5 Wanbao Gao, Vanderbilt University, Nashville, TN

August 15 Xiao-Lin Tu, Los Alamos National Laboratory, Los Alamos, NM

August 23 W. Kim, University of New Hampshire, Durham, NH

August 30

Koyla Nikolaev, L. D. Landau Institute for Theoretical Physics, Moscow, Former USSR

September 13 Daniel Bes, Comision Nacional de Energia Atomica, Buenos Aires, Argentina

September 13 Marlan O. Scully, Center for Advanced Studies, University of New Mexico, Albuquerque, NM

October 18 Tamas Biro, University of Giessen, Giessen, Germany

November 5 J. Lopez, University of Texas, El Paso, TX

November 25 D. H. Russell, Chemistry Dept., Texas A\&M University
Stopping and Forward Baryons in Relativistic Heavy Ion Collisions

Dissipative and Binary Processes in Light Heavy Ion Reactions

Coincident Multiplicity Constraints in the Fusion Reactions of $630 \mathrm{MeV}{ }^{58} \mathrm{Ni}$ with ${ }^{24} \mathrm{Mg}$

Production of Hot Nuclei in Peripheral and Central Collisions-Toward Nucl. Disassembly

Fermionic Molecular Dynamics

Triple Correlation Study of Continuum Gamma-Rays in ${ }^{130} \mathrm{Ce}$

Extension of the Mass Surface for Exotic Neutron-Rich Nuclei in the $Z=17-26$ Region

High Resolution Electron Scattering at MITBates

Color Transparency and Diffractive Deep Inelastic Scattering

The Treatment of Collective Coordinates in Many-Body Systems, through the BRST Invariance

Laser Accelerators of Charged Particles

Semiclassical Quark Transport Theory

Hot Zone Evolution in Heavy Ion Collisions

Molecular Beam Approaches to Studies of Clusters 
November 26 Tom Cowen, Lawrence Livermore Lab, Berkeley, CA

December 2 J. Gryko, Physics Dept., Texas A\&M University

December 2 L. Csernai, University of Bergen, Bergen, Norway

December 3 S. Austin, National

Superconducting Cyclotron lab, Michigan State University, E.

Lansing, MI

December 9 Bruce Barrett, University of Arizona, Tucson, AR

December 9 R. Parameswaran, Kansas State University, Manhattan, Kansas

December 9 William H. Marlow, Nuclear Engineering Dept., Texas A\&M University

December 10 S. V. Akulinichev, Institute for Nuclear Research, Moscow, Former USSR

December 19 V. Tsarev, Lebedev Institute, Former USSR

January 28 Tetsuo Hatsuda, University of Washington, Seattle, WA

February 3 Peter Sigmund, University of Odense, Denmark

February 4 Toshiki Maruyama, Kyoto University, Kyoto, Japan

February 10 E. Schweikert, Chemistry Dept., Texas A\&M University

February 11 P. Rosen, University of Texas, Arlington, TX

February 17 R. Lucchese, Chemistry Dept., Texas A\&M University
Origin of Anomalous Peaks in Heavy Ion Induced Positron Spectra

Properties of Silicon Clusters

String and Fluid Dynamical Model Predictions for Ultrarelativistic Heavy Ion Collisions

Physics with Radioactive Beams

F-Spin: A New Quantum Number for Nuclear Structure

Resonant Transfer and Excitation in Ion-Atom Collisions

Cluster Dynamics and Thermodynamics for Materials Processing and Climate Change Research

Pionic Effects in High Energy Reactions

Studies of Deuterated Metals

QCD Sum Rules for Hadrons in Nuclear Medium

Cluster Bombardment and Cluster Fusion

Fragment Mass Distribution and Reaction Time Scale

keV Cluster-Solid Interactions

Recent Development in Double $\beta$ Decay

Modeling the Potential Energy Hypersurface of the Hydrogen Bond HCN-HF 
February 18 E. Hungerford, University of Houston, Houston, TX

February 24 Catherine McNeal, Chemistry Dept., Texas A\&M University

February 25 F. Videbaek, Brookhaven National Lab, Upton, Long Island, NY

February 26 Marvin Justice, Lawrence Berkeley Lab, Berkeley, CA

March 2

March 3

March 6

$$
\begin{aligned}
& \text { R. MacFarlane, Chemistry } \\
& \text { Dept., Texas A\&M University }
\end{aligned}
$$

H. Schuessler, Physics Dept., Texas A\&M University

Linhua Xia, Physics Dept., Oregon State University, Corvallis, OR

March 9 George Bertsch, National Superconducting Cyclotron lab, Michigan State University, E. Lansing, MI

March 10

George Bertsch, National Superconducting Cyclotron lab, Michigan State University, E. Lansing, MI

March 26

J. Napolitano, Continuous Electron Beam Accelerator Facility, CEBAF, VA

March $30 \quad$ Graham Peaslee, National Superconducting Cyclotron lab, Michigan State University, E. Lansing, MI
Strange Things are Happening

Structure and Characterization of High Nuclearity Metal Clusters

Results from the AGS Relativistic Heavy Ion Experiments E802/E859

Electromagnetic Dissociation of ${ }^{238} \mathrm{U}$ at 120 $\mathrm{MeV} / \mathrm{A}$

Excited State Properties of $\mathrm{C}_{60}^{+,-}$

On Line Laser Spectroscopy of Short Lived Isotopes

Pions and Deltas in Nuclear Matter

Collective Plasmon Excitations in $\mathrm{C}_{60}$ Clusters

Measuring Strangeness in the Proton

Complex Fragment Production in Heavy Ion Reactions 
May 23

Mei Gui, Texas A\&M

University

August 14 Xi-Jun Qiu, Texas A\&M

University

August 20 Peter Lévai, Texas A\&M

University

September 10 W. H. Trzaska, Texas A\&M University

September 24 John C. Hagel, Texas A\&M University

October 1 Che Ming Ko, Texas A\&M University

October 10 Rand L. Watson, Texas A\&M University

October 22 Li Xiong, Texas A\&M University

October 29 R. K. Choudhury, Texas A\&M University

November 12 R. Wada, Texas A\&M

University

November 16 Leo Van Ausdeln, Texas A\&M University

January 21 Hiroaki Utsunomiya, Texas A\&M University

March 13 Masayuki Asakawa, Texas A\&M University

\section{Nuclear Fission}

A Chiral Dynamical Theory for Hadron System

Nonequilibrium Processes in Relativistic Heavy Ion Collisions

Search for Resonant Electron-Positron Annihilation In-Flight

The Multifragmentation of ${ }^{40} \mathrm{Ca}+{ }^{40} \mathrm{Ca}$

Hadronic Matter under Extreme Conditions

When Molecules Explode

Quasi-Pions in Heavy Ion Collisions

Role of Entrance Channel Mass Asymmetry in Heavy Fusion Reactions

Origin of Non-Equilibrium Particles in Heavy Ion Reactions

The Search for the Rare Decay $\mu \rightarrow \mathrm{e} \gamma$

Current Experimental Status of Coulomb Breakup

Novel Features in Dilepton Spectra from Hot Dense Matter 